\section{CONTINUOUS GLUCOSE MONITORING}

1-OR

Impact of Study Design and Analytic Techniques on the Reported Accuracy of Continuous Glucose Monitoring (CGM) Systems DAVID A. PRICE, TOM PEYSER, PETER SIMPSON, KATHERINE NAKAMURA, AARTHI MAHALINGAM, San Diego, CA

CGM performance is an important factor considered by clinicians recommending a CGM system and commonly used metrics are often compared across systems. However, these comparisons are challenging. CGM performance is often worst on the first and last days of use and studies that minimize data from those days may be misleading. Clinical trials with limited data in hypo- or hyperglycemia can distort the appearance of overall sensor performance. Real time systems for commercial use by patients require a prospectively determined calibration curve using SMBG. However, retrospective calibration optimized for each sensor, increased frequency of calibration, or calibration with reference glucose values are used in some analyses to optimize results and affecting apparent accuracy. Some studies report median, instead of mean differences from reference glucose, negating the impact of outlier data and impacting the accuracy perception. Studies commonly exclude data but the amount of data excluded and the criteria for exclusion often lack transparency. Some studies report combined Clarke Error Grid A and B zones as a way of improving the perceived accuracy. As an example, we demonstrated different methods of analyzing data from a study of Dexcom Seven Plus CGM to show the effects of excluding selected data, retrospective calibration, and frequent calibration on the performance metrics of the system

\begin{tabular}{|c|c|c|c|c|c|c|}
\hline & $\begin{array}{c}\text { Seven } \\
\text { Plus No } \\
\text { exclusion }\end{array}$ & $\begin{array}{l}\text { Exclude } \\
\text { Day } \\
\text { Seven }\end{array}$ & $\begin{array}{l}\text { Exclude } \\
\text { Hypo }\end{array}$ & $\begin{array}{c}\text { Euglycemia } \\
(70-180)\end{array}$ & $\begin{array}{c}\text { Retrospective } \\
\text { Calibration } \\
\text { ( } \leq 4 \mathrm{x} / \text { day) }\end{array}$ & $\begin{array}{c}\text { Retrospective } \\
\text { Calibration } \\
(\geq 4 x / \text { day })\end{array}$ \\
\hline Mean ARD & $16 \%$ & $15 \%$ & $15 \%$ & $16 \%$ & $13 \%$ & $9 \%$ \\
\hline Median ARD & $13 \%$ & $13 \%$ & $12.5 \%$ & $13 \%$ & $10 \%$ & $7 \%$ \\
\hline Clarke A & $74 \%$ & $76 \%$ & $75 \%$ & $71 \%$ & $79 \%$ & $92 \%$ \\
\hline Clarke $A+B$ & $97 \%$ & $97 \%$ & $100 \%$ & $100 \%$ & $99 \%$ & $100 \%$ \\
\hline $\mathrm{N}$ & 1827 & 1272 & 1675 & 927 & 1899 & 2714 \\
\hline
\end{tabular}

Different study designs and methods of analysis confound the ability to compare accuracy of different CGM systems. There is a need for standardized study designs and metrics to allow such comparisons.

A1C and Mean Glucose (MG) in Insulin Treated Diabetes Using the Dexcom SevenPlus Continuous Glucose Monitor (CGM): Correlation and Intra-Patient Consistency Over Time

NICHOLAS B. ARGENTO, KATHERINE NAKAMURA, ROBERT D. SALA, Columbia, MD, San Diego, CA

The relationship between $A 1 C$ and $M G$ is controversial. An estimated $A 1 C$ derived glucose (eAG) is said to be useful, but CGM data shows significant variation between eAG and CGM measured MG (CMG). Little information is available on the consistency of this relationship in an individual patient over time. We report this relationship between eAG and CMG (eAG-CMG) in insulin requiring patients and the consistency of the relationship in a subgroup. Patients using Dexcom SevenPlus were identified by review of clinic downloads, and included if an $\mathrm{A} 1 \mathrm{C}$ value was drawn when the 60 days prior included at least $50 \%$ utilization of CGM ( $n=138)$. A subgroup had between 2 to 6 data points $(n=98)$. The most recent eAG-CMG was used to derive the linear regression model. Within patients, the prior eAG-CMG were examed for consistency. Mean age 48.7 yr (18-75), duration 26 yr (1-58), 61\% male, $39 \%$ female, 91\% T1D, 9\% T2D, 80\% CSII, 20\% MDI.

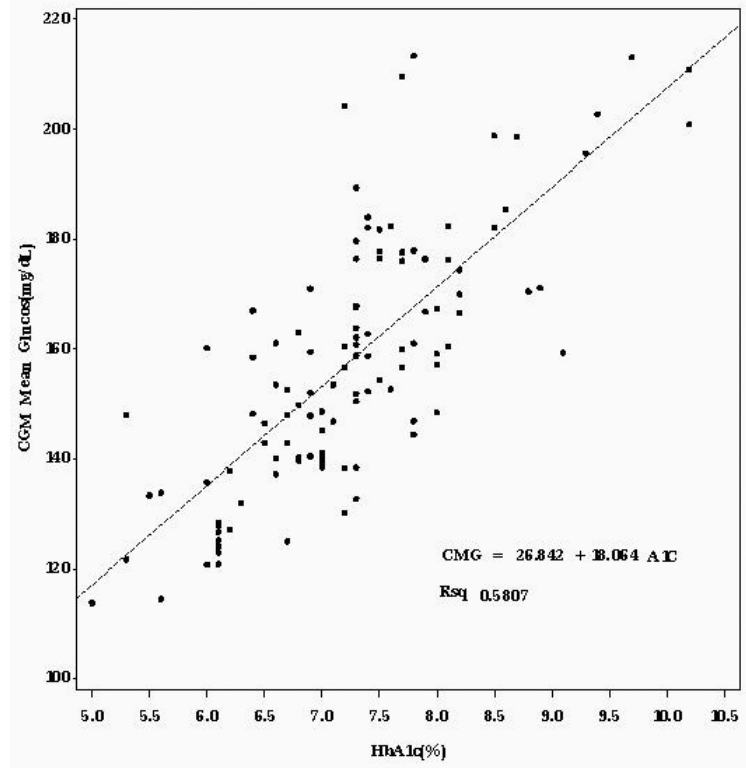

A1C $7.0 \%=$ eAG $153.2 \mathrm{mg} / \mathrm{dl}$ The intra-patient eAG-CMG relationship is generally consistent over time. For example, subj 1: A1C 7.3, CMG 139, eAG 159 (CMG - eAG) /eAG x $100=-12.7 \%$; A1C 7.7, CMG 149, eAG 166, -10.4\%. Subj 2: A1C 7.2, CMG 204, eAG 157,+30.2\%; A1C 7.1, CMG 189, eAG 155, +21.6\%. In conclusion, the Dexcom SevenPlus yields similar eAG-CGM correlation to the JDRF CGM study. There is significant individual patient variability. Some subjects have striking differences between $C M G$ and $\mathrm{eAG}$, which trends consistently over time. Clinicians should consider modification of A1C goals in patients with persistent significant divergence of $\mathrm{CMG}$ and eAG.

3-OR

Accuracy and Large Inaccuracy of Two Continuous Glucose Monitoring (CGM) Systems

LALANTHA LEELARATHNA, MARIANNA NODALE, JANET M. ALLEN, DANIELA ELLERI, KAVITA KUMARESWARAN, AHMAD HAIDAR, KAREN CALDWELL, MALGORZATA E. WILINSKA, CARLO L. ACERINI, MARK L. EVANS, HELEN R. MURPHY, DAVID B. DUNGER, ROMAN HOVORKA, Cambridge, United Kingdom, Montreal, OC, Canada

Sensor inaccuracy can lead to potentially harmful insulin over or under delivery particularly during closed-loop glucose control and has yet to be quantified for commercial CGM systems. We analysed data from 32 adults $(36.2 \pm 9.3 \mathrm{yrs})$ and 20 adolescents $(15.3 \pm 1.5 \mathrm{yrs})$ with type 1 diabetes who attended clinical research facility to undergo testing of closed-loop. Paired CGM and plasma glucose values (PG; measured by YSI 2300 STAT Plus Analyser) were collected every 15 to 60min using Freestyle Navigator (FSN, 1548 hours of data collection, 4218 data pairs) and DexCom Seven Plus (DSP, 1440 hours, 2964 pairs). Median absolute relative difference was 10\% for FSN and $13 \%$ for DSP; $78.4 \%$ of FSN and $70.9 \%$ of DSP values were in Zone A of Clarke Error Grid. Large sensor over-reading by $40 \%, 50 \%$ and $60 \%$ (or 2.4 3.0, and $3.6 \mathrm{mmol} / \mathrm{L}$ at PG below $6 \mathrm{mmol} / \mathrm{L}$ ) occurred 2 - 3 fold more commonly with DSP than FSN (Table 1). At higher error thresholds, duration of sensor over-reading decreased with FSN but remained unchanged with DSP (Table 1). Sensor errors of 1 hour or longer duration were absent with FSN at $50 \%$ and $60 \%$ error levels but one out of ten errors was of 1 hour or longer with DSP. Frequency and duration of large sensor under-reading was comparable. We conclude that FSN and DSP differ little in their overall accuracy but substantial differences in frequency and duration of large inaccuracies favour FSN for use in closed-loop insulin delivery systems. 
Table 1: Incidence and Duration of Large Sensor Inaccuracy

\begin{tabular}{lccccc}
\hline & \multicolumn{3}{c}{$\begin{array}{c}\text { Free Style Navigator } \\
\text { (FSN) }\end{array}$} & $\begin{array}{c}\text { DexCom Seven Plus } \\
\text { (DSP) }\end{array}$ \\
\hline & $\begin{array}{c}\text { Over- } \\
\text { reading }\end{array}$ & $\begin{array}{c}\text { Under- } \\
\text { reading }\end{array}$ & $\begin{array}{c}\text { Over- } \\
\text { reading }\end{array}$ & $\begin{array}{c}\text { Under- } \\
\text { reading }\end{array}$ \\
\hline $40 \%$ error Incidence (per 100 days use) & 40.0 & 27.8 & 83.3 & 26.3 \\
\cline { 2 - 6 } & Duration in min (median, IOR) & $36(20-63)$ & $22(13-44)$ & $28(9-61)$ & $24(9-31)$ \\
\hline $50 \%$ error Incidence (per 100 days use) & 21.8 & 14.1 & 50.0 & 5.0 \\
\cline { 2 - 6 } & Duration in min (median, IQR) & $37(15-44)$ & $23(18-29)$ & $32(22-47)$ & $43(15-54)$ \\
\hline $60 \%$ error Incidence (per 100 days use) & 9.3 & 4.6 & 27.0 & 5.0 \\
\cline { 2 - 6 } & Duration in min (median, IOR) & $14(7-31)$ & $2(1-16)$ & $28(13-43)$ & $29(1-35)$ \\
\hline
\end{tabular}

Supported by: JDRF, Diabetes UK, NDDK and EU Commission (7th FP)

4-OR

A Comparative Analysis of Three Continuous Glucose Monitors: Not All Are Created Equal

EDWARD R. DAMIANO, FIRAS H. EL-KHATIB, KENDRA L. MAGYAR, DAVID M. NATHAN, STEVEN J. RUSSELL, Boston, MA

The goal of this study was to compare three continuous glucose monitoring (CGM) devices in subjects with type 1 diabetes under closed-loop bloodglucose (BG) control. Six subjects with type 1 diabetes lage $52 \pm 14$ years, diabetes duration $32 \pm 14$ years) each participated in two 51-hour closed-loop BG control experiments in the hospital. Venous plasma glucose (PG) measurements (GlucoScout, International Biomedical) obtained every 15 minutes (2,360 values) were paired in time with corresponding CGM glucose (CGMG) measurements obtained from three CGM devices (FreeStyle Navigator, Abbott Diabetes Care; DexCom Seven Plus, DexCom; Guardian RT, Medtronic) worn simultaneously by each subject. Errors in paired PG-CGMG measurements and data reporting percentages were obtained for each CGM device. The Navigator had the best overall accuracy, with an aggregate mean absolute relative difference (MARD) of all paired points of $11.8 \pm 11.1 \%$ and an average MARD across all 12 experiments of $11.8 \pm 3.8 \%$. The DexCom and Guardian produced aggregate MARDs of all paired points of $16.5 \pm 17.8 \%$ and $20.3 \pm 18.0 \%$, respectively, and average MARDs across all 12 experiments of $16.5 \pm 6.7 \%$ and $20.2 \pm 6.8 \%$, respectively. Data reporting percentages, a measure of reliability, were $76 \%$ for the DexCom and nearly 100\% for the Navigator and Guardian. A comprehensive head-to-head-to-head comparison of three CGM devices for $B G$ values from $36-563 \mathrm{mg} / \mathrm{dl}$ revealed marked differences in performance characteristics that include accuracy, precision, and reliability. The Navigator was found to out-perform the other two in these areas.

\section{5-OR}

Glycemic Variability is Higher in Type 1 Diabetic Patients With Microvascular Complications Irrespective of Glycemic Control

JAN SOUPAL, MARTIN PRAZNY, JAN SKRHA, Prague, Czech Republic

Increased glycemic variability (GV) may be associated with higher risk of microvascular complications (MVC). Continuous glucose monitoring systems (CGMS) can provide more data for the assessment of GV. The aim of the study is to compare GV in T1DM patiens with and without MVC. Thirty-two T1DM patients (age $43 \pm 13 \mathrm{yrs}$, duration of DM $19 \pm 11 \mathrm{yrs}$ ), of them 15 patients with and 17 without $\mathrm{MVC}\left(\mathrm{HbA}_{1 \mathrm{C}} 71 \pm 9\right.$ and $\left.66 \pm 13 \mathrm{mmol} / \mathrm{mol}, \mathrm{p}=0.21, \mathrm{NS}\right)$, participated in the study. CGM was recorded for two weeks using blinded CGM systems. GV was analyzed using proprietary software. Among other parameters, standard deviation (SD) and coefficient of variation (CV) were calculated. Vibration perception threshold (VPT) was measured by neurothesiometer and used as an estimate of neuropathy (0-15 V normal and $>15 \mathrm{~V}$ impared VPT). GV was higher in patients with retinopathy $(n=13)$ compared to patients without retinopathy (SD: $4.1 \pm 0.7$ vs. $3.4 \pm 0.9, p=0.03$ ) although there was no significant difference in $\mathrm{HbA}_{1}$. Similarly, GV was higher in patients with impaired VPT ( $n=12, S D: 4.3 \pm 0.7$ vs. $3.4 \pm 0.8, p=0.003$ ). Moreover, a positive association was observed between VPT and SD in all patients $(r=0.53, p=0.002)$. Patients with microalbuminuria $(n=7)$ had higher GV (CV: $0.46 \pm 0.07$ vs. $0.38 \pm 0.07, p=0.02$ ) even though $\mathrm{HbA}_{1 \mathrm{c}}$ was nonsignificantly higher in the group without microalbuminuria $(68 \pm 13$ vs. $67 \pm 8 \mathrm{mmol} / \mathrm{mol})$. In general, patients with any MVC had significantly higher GV compared to patients without MVC (SD: $4.1 \pm 0.7$ vs. $3.4 \pm 0.8, p=0.01$ and CV: $0.44 \pm 0.06$ vs. $0.37 \pm 0.07, p=0.005$ ) although their $\mathrm{HbA}_{1}$ was comparable. T1DM patients with any MVC had significantly higher GV than the patients without complications although they did not differ in glycemic control. This finding supports the hypothesis that high GV may contribute to the development of MVC in $\mathrm{T} 1 \mathrm{DM}$ and that $\mathrm{HbA}_{1 \mathrm{C}}$ may not describe diabetes control completely.
6-OR

Performance of a Microdialysis-Based Continuous Glucose Monitoring (CGM) System

ERIC ZIJLSTRA, TIM HEISE, WOLFGANG KÜNNECKE, Neuss, Germany, Braunschweig, Germany

Combining intravenous microdialysis with on-line glucose analysis for CGM may prove to become an important development to achieve tight glycemic control in hospitalized patients without blood loss, measurement delays or frequent manual interventions. In this study we evaluated the performance of a microdialysis-based CGM system for up to 48 hours use. Twenty-one healthy adult subjects were connected to 1 or 2 CGM systems. Blood glucose was sampled automatically without blood loss by means of microdialysis every 1-2 minutes and the dialysate was analyzed immediately by an on-line glucose sensor. The glucose sensor was calibrated once every 24 hours. Reference blood samples were taken manually and analyzed using a laboratory glucose analyzer every 10-60 minutes. The subjects consumed meals or glucose was administered orally or intravenously to analyze the accuracy of the CGM system over a range of blood glucose concentrations. A total of 1796 paired sensor-reference data points were evaluated. Mean relative deviation was $9.4 \%$ and $91.4 \%$ of data points were accurate according to the IS015197 criteria. Accuracy in the hypoglycemic range was comparable: $94.6 \%$ of the sensor values below $75 \mathrm{mg} / \mathrm{dL}$ were within 15 $\mathrm{mg} / \mathrm{dL}$ of the reference value. Clark Error Grid analysis showed that $99.3 \%$ of the data points were located within the accurate and acceptable zones. This study shows that CGM using microdialysis in blood is feasible for up to 48 hours and provides reasonably accurate glucose results with only a once-daily calibration. Improved calibration and/or referencing strategies may increase the accuracy of the system further in future studies.

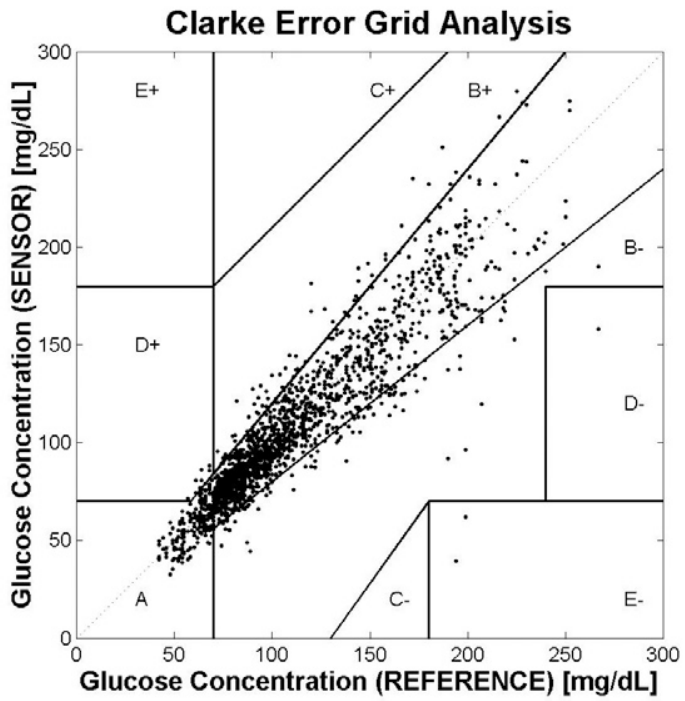

7-0R

Transdermal Continuous Glucose Monitoring Following Skin Permeation

WAYNE MENZIE, JAMES HURLEY, KEITH KRYSTYNIAK, MY-QUYEN TRIEU, JASON BERLIN, KENNETH GARY, Franklin, MA

The skin is an effective barrier, limiting access to interstitial fluid (ISF) for diagnostic monitoring. Invasive techniques such as subcutaneous insertion of sensors are generally required to access ISF. Prelude ${ }^{\circledR}$ SkinPrep System is a non-invasive device for skin permeation to provide ISF access for continuous analyte measurements. This study was performed to evaluate the potential for transdermal continuous glucose measurement in patients with diabetes without requiring needles or other invasive techniques, using Symphony ${ }^{\circledR}$ Transdermal Continuous Glucose Monitoring (tCGM) System on sites pre-treated with Prelude SkinPrep. Twenty adult volunteers with either type 1 or type 2 diabetes were evaluated. Prelude was used to permeate the skin and a tCGM device was applied to each of 2 test sites. Venous reference blood samples were taken from intravenous lines at 15-minute intervals for 24 hours. The reference blood glucose (BG) values were measured with a YSI 2300 Stat+ glucose analyzer. At the conclusion of the study, the skin at the test sites was inspected for redness or any other undesirable effects. Accuracy of the tCGM data relative to the reference $B G$ values was determined 
using Continuous Glucose Error Grid Analysis (CG-EGA) and mean absolute relative difference (MARD). Using over 2,600 Symphony tCGM glucose readings from the 20 study subjects paired with reference BG measurements, CG-EGA showed that $94.4 \%$ of the readings were clinically accurate $(A)$ and $2.5 \%$ were benign (B) errors with a combined $A+B$ of $96.9 \%$. The MARD for the study was $12.6 \%$. Values for BG measurements ranged from 38 to 399 $\mathrm{mg} / \mathrm{dL}$. There were no unanticipated adverse device effects reported from the Prelude skin permeation or the Symphony tCGM biosensor. Continuous glucose measurement with Symphony tCGM was reliably achieved using Prelude SkinPrep for transdermal access to ISF. This approach represents a simple, convenient, needle-free solution for continuous glucose monitoring in patients with diabetes.

\section{8-OR}

Long-Term Effects of Sensor-Augmented Pump Therapy in Type 1 Diabetes: A 3-Year Follow-Up Study

SIGNE SCHMIDT, KIRSTEN NØRGAARD, Hvidovre, Denmark

Sensor-augmented pump (SAP) therapy can improve glycemic control in adults with type 1 diabetes. This has been demonstrated in studies of up to 18 months duration, but the long-term effects remain to be determined. Eurythmics was a 26-week randomized European multi-center trial comparing the effects of SAP with multiple daily injections (MDI). Eighty-three adults with type 1 diabetes participated in the trial; 24 of these were from Denmark. Thirteen of the 24 Danish patients were randomized to receive the study intervention, SAP (Paradigm REAL-Time System), while 11 were randomized to the control group continuing MDI. After completion of the trial, the 11 Danish patients in the control group started SAP and the 13 patients in the intervention group continued using SAP with quarterly follow-up visits. In this study we assessed the metabolic and psychosocial effects of SAP 3 years after therapy start by changes in HbA1C and DTSO, HFS and PAID questionnaire scores in the Danish subgroup of Eurythmics participants. Figure 1 shows mean HbA1c values 3 years before and after SAP start. The mean decrease in $\mathrm{HbA1c}$ from SAP start to 3 years was $1.5 \%(\mathrm{P}<.0001)$. At 3 years, 14 of 16 SAP users were using SAP $>70 \%$ of time. The mean DTSO score increased from 20.4 at SAP start to 32.1 at 3 years of SAP use $(\mathrm{P}<$ $.0001)$, the mean HFS score decreased from 28.2 to $21.4(P=.257)$, and the mean PAID score decreased from 29.6 to $16.2(P=.002)$. In summary, this 3-year follow-up study documents long-term beneficial effects of SAP on $\mathrm{HbA1c}$, treatment satisfaction and magnitude of perceived diabetes-related problems in patients with poorly controlled type 1 diabetes.

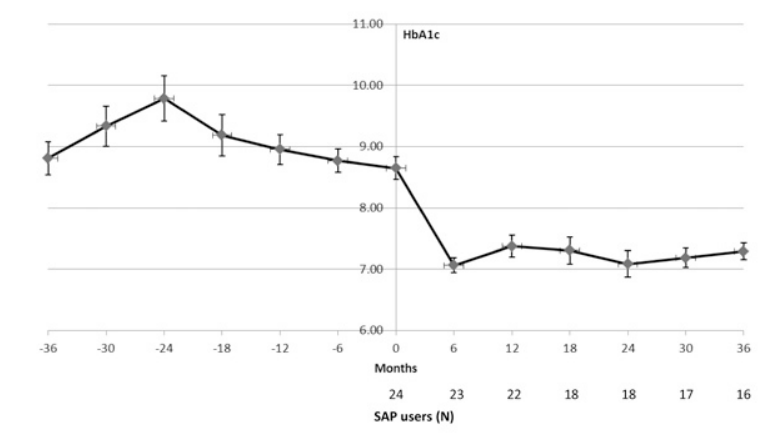

Figure 1. HbAlc 3 years before and after SAP start (0 months). Error bars are SEM.

\section{THE CLINICAL MANAGEMENT OF DIABETES}

9-OR

Increased Glucose Variability is Independently Associated With Longer Length of Stay and Mortality in Non-Critically III Hospitalized Patients

CARLOS E. MENDEZ, KI-TAE MOK, ASHAR ATA, ROBERT J. TANENBERG, Albany, NY, Greenville, NC

Increased glucose variability (GV) has been associated in vitro with oxidative stress damage. In the critical care setting, patients with high GV have been found to have increased mortality. Studies assessing the correlation between GV and length of stay (LOS) or mortality in the non-critically ill are limited. In this retrospective study, we included hospitalized patients admitted to medicine or surgery from 2008 to 2010 . We analyzed 56,777 glucose values from a total of 4,262 inpatient encounters. Those with daily glucose monitoring averaging a minimum of two values per day were selected. ICU, long term care, and patients with a hospital stay greater than 60 days were excluded. Glucose Standard Deviation (GSD) was calculated as a surrogate for GV. Linear and logistic regression analyses were used to assess the effects of GSD on LOS and mortality. There was a positive association between GSD and LOS. For every10 units increment in the GSD, LOS increases by $6.03 \%$. There were no significant differences between diabetic and nondiabetic, or in between medical and surgical cohorts. This correlation was demonstrable even when stratifying the sample by mean glycemia and hypoglycemia occurrence. GSD was also positively associated with mortality, increasing the odds of dying by $8 \%$ per every 10 units increment in GSD. These results suggest that GV is independently associated with increased LOS and mortality in non-critically ill patients. Prospective studies with precise measurement of GV are necessary to confirm these results and to elucidate factors that may influence GV.

\begin{tabular}{llrcc}
\hline \multicolumn{4}{l}{ Summary of Results } \\
\hline Effect of GSD on LOS & $\begin{array}{l}\text { Effect } \\
(\mathbf{l o g} \text { scale) }\end{array}$ & P value & 95\% Confidence Interval \\
\hline All Patients ( $\mathrm{n}=962)$ & 6.03 & $<0.001$ & 4.20 & 7.85 \\
\hline With Diabetes $(\mathrm{n}=818)$ & 6.31 & $<0.001$ & 4.28 & 8.33 \\
\hline Without Diabetes $(\mathrm{n}=144)$ & 5.94 & 0.008 & 1.59 & 10.29 \\
\hline Surgical ( $\mathrm{n}=179)$ & 6.82 & 0.008 & 1.77 & 11.87 \\
\hline Medical (783) & 6.04 & $<0.001$ & 4.08 & 7.99 \\
\hline Effect of GSD on Mortality & $\mathbf{0 d d s}$ Ratio & $\mathbf{P}$ value & $\mathbf{9 5 \%}$ Confidence Interval \\
\hline All Patients ( $\mathrm{n}=962)$ & 1.08 & $<0.001$ & 1.04 & 1.13 \\
\hline
\end{tabular}

10-0R

Hospital Discharge Algorithm Based on Admission HbA1C for the Management of Patients With Type 2 Diabetes

KATHIE HERMAYER, DAWN SMILEY, DARIN E. OLSON, AMNA KHAN, CHRISTOPHER NEWTON, VIVIAN FONSECA, SOL JACOBS, LIMIN PENG, INGRID PINZON, MARIA FEREIRA, ASHWINI GORE, VICKIE HUNT, DAVID REYES, MONICA RIZZO, GUILLERMO UMPIERREZ, Charleston, SC, Atlanta, GA, New Orleans, LA

Few studies have focused on the optimal management of patients with type 2 diabetes (T2DM) after hospital discharge. Accordingly, we conducted a prospective, multicenter trial to determine the efficacy and safety of a hospital discharge algorithm based on admission $\mathrm{HbA1c}$ to guide outpatient therapy in medicine and surgery patients with T2DM. Discharge algorithm: patients with admission $\mathrm{HbA1c}<7 \%$ were discharged on the same outpatient therapy. Those with an $\mathrm{HbA1c}$ between $7 \%$ and $9 \%$ were discharged on glargine at $50-80 \%$ of total daily dose (TDD) in combination with their outpatient oral antidiabetic (OAD) agents. Those with an $\mathrm{HbA1c}>9 \%$ were discharged on glargine at $80-100 \%$ of hospital TDD in combination with OAD or with basal bolus regimen with glargine and glulisine. A total of 224 patients (age: $58 \pm 12 \mathrm{yr}$, duration DM: $9.3 \pm 8 \mathrm{yr}$ ) were followed after discharge every 4 weeks with treatment adjusted to achieve a target $\mathrm{HbA1c}<7 \%$. The primary outcome was change in $\mathrm{HbA1C}$ at 4 and 12 weeks after discharge. Pharmacologic therapy and treatment response during follow-up is shown in the table. In summary, the proposed HbA1c-based hospital discharge algorithm was found to be effective and safe for the management of general medicine and surgical patients with T2DM.

\begin{tabular}{|c|c|c|c|c|c|c|}
\hline \multicolumn{7}{|c|}{ Outcomes by Discharge Treatment } \\
\hline & $\begin{array}{c}\text { All } \\
\text { patients }\end{array}$ & OAD & $O A D+$ Glargine & $\begin{array}{l}\text { Glargine + } \\
\text { Glulisine }\end{array}$ & Glargine & Diet \\
\hline Number of patients, $n(\%)$ & 224 & $81(36)$ & $61(27)$ & $54(24)$ & $20(9)$ & $8(4)$ \\
\hline HbA1c on admission, $\%$ & $8.7 \pm 2.5$ & $6.9 \pm 1.5$ & $9.2 \pm 1.9$ & $11.1 \pm 2.3$ & $8.2 \pm 2.2$ & $7.0 \pm 1.2$ \\
\hline HbA1c 4 weeks of F/U, \% & $7.9 \pm 1.7$ & $7.0 \pm 1.4^{\dagger}$ & $8.0 \pm 1.4^{\dagger}$ & $8.8 \pm 1.8$ & $7.7 \pm 1.7$ & 5.9 \\
\hline HbA1c 12 weeks of F/U, \% & $7.3 \pm 1.5$ & $6.6 \pm 1.1^{\ddagger}$ & $7.5 \pm 1.6^{\ddagger}$ & $8.0 \pm 1.6^{\ddagger}$ & $6.7 \pm 0.8$ & $7.1 \pm 1.3$ \\
\hline $\mathrm{BG}<70 \mathrm{mg} / \mathrm{dL}, \mathrm{n}(\%)$ & $62(29)$ & $17(22)$ & $17(30)$ & $23(44)$ & $5(25)$ & $0(0)$ \\
\hline $\mathrm{BG}<40 \mathrm{mg} / \mathrm{dL}, \mathrm{n}(\%)$ & $7(3)$ & $3(4)$ & $0(0)$ & $3(6)$ & $0(0)$ & $0(0)$ \\
\hline
\end{tabular}

${ }^{{ }^{\circ}} \mathrm{p}<0.0001: \mathrm{HbA} 1 \mathrm{c}$ at admission vs $4 \mathrm{wks} ;{ }^{\ddagger} \mathrm{p}<0.001: \mathrm{HbA} 1 \mathrm{c}$ at admission vs 12 wks

Supported by: sanofi-aventis 
11-0R

Real-World Outcomes of Initiating Injectable Therapy With Insulin Glargine or Liraglutide Among Patients With Type 2 Diabetes

PHILIP LEVIN, WENHUI WEI, ALEKSANDRA VLAJNIC, CHUNSHEN PAN, LIN XIE, JAY LIN, ONUR BASER, Baltimore, MD, Bridgewater, NJ, Boca Raton, FL, Ann Arbor, MI, Flemington, NJ

To date, no real-world comparative data has been published on initiation of injectable therapy with insulin glargine disposable pen (GLA-P) or glucagonlike peptide-1 agonist liraglutide (LIRA) among patients with type 2 diabetes mellitus (T2DM). Using the IHCIS national managed care claim database, this study assessed 1-year clinical outcomes and plan-paid health care costs of T2DM patients failing oral antidiabetic drugs (OADs) and initiating LIRA or GLA-P. Included were adult T2DM patients who were previously on $\geq 10 A D s$, had $A 1 C>7 \%$, initiated GLA-P or LIRA between $01 / 2010$ and 06/2010, and had 1 year follow-up. Stringent 1:1 propensity score matching was applied to remove observed differences in baseline demographic and clinical characteristics between the two cohorts. Analysis was conducted among 336 matched patients ( $n=168 /$ cohort; $45 \%$ women; mean baseline age 53 years, baseline A1C 8.9\%, number of OADs 2.32, Charlson comorbidity index 0.32). During follow-up, there were no statistically significant differences between GLA-P and LIRA cohorts in treatment persistence $(51.7 \%$ vs $47.6 \%, P=$ nonsignificant [NS]), A1C reduction from baseline $(-1.02 \%$ vs $-0.95 \%, P=N S)$ and $\%$ patients achieving $\mathrm{A} 1 \mathrm{C}<7 \%(23.3 \%$ vs $28.5 \%, P=\mathrm{NS})$ among those with follow-up A1C data available ( $n=77 /$ cohort). Hypoglycemia-related event rates were low in both cohorts (overall: 10.7 vs 6.6 per 100 patient/year, $P=N S$; hospital/emergency room-related events: 1.1 vs 1.1 per 100 patient/ year, $P=N S)$. This database did not have information on weight. GLA-P patients, compared to LIRA patients, had significantly lower study drug costs $(\$ 1,198$ vs $\$ 2,784 ; P<0.001)$, resulting in lower diabetes-related health care costs $(\$ 5,653$ vs $\$ 7,976 ; P=0.02)$. This real-world comparative study suggested that among T2DM patients who failed OADs and initiated injectable therapy, GLA-P may be a more cost-effective option than LIRA with similar clinical outcomes yet lower diabetes care cost.

Supported by: sanofi-aventis

12-OR

Comparison of 24-Week Treatment With Exenatide, Insulin and Pioglitazone in Newly Diagnosed and Drug-Naïve T2DM

WEN XU, JIN LI, LONGYIZENG, DALONG ZHU, ZILIN SUN, LIXIN GUO, TAO YANG, GE WU, CAIPING LI, LIXIN SHI, QIFU LI, OIANG LI, XIAOHUI GUO, ZUOJIE LUO, JUMING LU, ZHONGYAN SHAN, WENYING YANG, OIUHE JI, LI YAN, HONG LI, XUEFENG YU, SHAOQING LI, ZHIGUANG ZHOU, XIAOFENG LV, GUOCHUN LUO, LINONG JI, SHAODA LIN, JIANPING WENG, Guangzhou, China, Nanjing, China, Beijing, China, Zhanjiang, China, Inner Mongolia, China, Guiyang, China, Chongqing, China, Harbin, China, Nanning, China, Shenyang, China, Xian, China, Kunming, China, Wuhan, China, Qingyuan, China, Changsha, China, Shenzhen, China, Shantou, China

Exenatide has been demonstrated to have $\beta$-cell protective effect. To date no studies compare the effects of exenatide with other $\beta$-cell protecting hypoglycemic agents, like insulin and pioglitazone, in newly diagnosed and drug-naivve T2DM. Hence, the CONFIDENCE trial was designed to evaluate the effects of exenatide, insulin and pioglitazone on glycemic control and $\beta$-cell function in these patients. 416 newly diagnosed and drug-naïve T2DM with $\mathrm{HbA}_{1} \mathrm{C}$ between $7 \%$ and $10 \%$ were enrolled from 25 centers in China and randomly assigned to exenatide, insulin or pioglitazone group for a predictive followup period of 48 weeks from August 2010. HbA1c, glucose and insulin during fixed breakfast and IVGTT are measured at baseline, 24 weeks and 48 weeks to assess glycemic control. $\beta$-cell function was evaluated at baseline and 48 weeks. By November 2011, 127 patients completed 24- week treatment. These 127 patients (mean age 52 years, BMl $26 \mathrm{Kg} / \mathrm{m}^{2}$ ) were allocated in exenatide group (40), insulin group (43) and pioglitazone group (44). Baseline characteristics were similar among three groups. After 24-week therapy, $\mathrm{HbA}, \mathrm{c}, \mathrm{FPG}$ and $2 \mathrm{hPG}$ after fixed breakfast decreased in all patients. But change of $\mathrm{HbA}_{1} \mathrm{C}$ from baseline to 24 week was more significant in exenatide and insulin group (exenatide: $-1.8 \pm 1.1 \%$, insulin: $-1.8 \pm 1.3 \%$, pioglitazone: $-1.3 \pm 1.0 \%, P=0.036$ ). Greater weight change was found in exenatide group (exenatide: $-5.0 \pm 2.6$ $\mathrm{Kg}$, insulin: $-0.7 \pm 2.7 \mathrm{Kg},-2.6 \pm 3.2 \mathrm{Kg}, \mathrm{P}<0.01$ ). No severe hypoglycemic episodes were reported in all patients. The most common adverse events in exenatide group were gastrointestinal reactions (60\%). One case of acute pancreatitis was reported in exenatide group. This preliminary data from CONFIDENCE trial demonstrated that 24-week exenatide treatment is more effective on glycemic control with regard to $\mathrm{HbA}_{1} \mathrm{c}$ reduction at 24 weeks, accompanied with more distinct weight reduction, compared with insulin and pioglitazone in newly diagnosed and drug-naïve T2DM.

Supported by: Project of Clinical Discipline of Hospital Affiliated to Ministry of Health

For author disclosure information, see page 797.
13-OR

Association of Treatment Persistence and Adherence With RealWorld Outcomes Among Insulin-Treated Patients With Type 2 Diabetes Mellitus (T2DM)

WENHUI WEI, CHUNSHEN PAN, LIN XIE, ONUR BASER, Bridgewater, NJ, Boca Raton, FL, Ann Arbor, MI

Administrative data are often used to measure treatment persistence/ adherence and the associated outcomes in real-world patients; however, they are difficult to measure for insulin-treated T2DM patients due to non-fixed dosing and titration. This study pooled patient-level data from 3 published observational studies-all used IMPACT ${ }^{\circledR}$, a national managed care database, and had similar study designs-to examine T2DM patients previously on oral anti-diabetes drugs (OADs) and/or glucagon-like peptide 1 (GLP1) analogs and initiating insulin glargine (IG) or insulin detemir (ID). Endpoints included the correlations of 1-year follow-up treatment persistence (remaining on treatment without discontinuation) and adherence (medication possession ratio $\geq 80 \%$ ) with $\mathrm{A} 1 \mathrm{C}, \mathrm{A} 1 \mathrm{C}$ reduction from baseline $(\triangle \mathrm{A} 1 \mathrm{C})$, hypoglycemia-related event rate, health care utilization and costs. Included were 4804 patients (IG disposable pen: 2257, IG vial: 1915, ID disposable pen: $632 ; 43 \%$ women; baseline mean age 56 years; baseline A1C 9.37\%; baseline \# OADs 2.05). Overall, higher treatment persistence/adherence was associated with significantly lower $\mathrm{A} 1 \mathrm{C}$, greater $\triangle \mathrm{A} 1 \mathrm{C}$, lower hospitalization and emergency room (ER) visit rates, and higher drug costs (Table). This study showed that real-world administrative data is a viable source for measuring treatment persistence/adherence among insulintreated T2DM patients. Improved 1-year follow-up persistence/adherence was associated with better clinical outcomes and higher drug costs, but offset by lower hospitalization/ER rates.

Table: Pearson correlation coefficients between 1-year follow-up treatment persistence ( $r$-persistence )/adherence ( $r$-adherence) and health outcomes. ${ }^{*} \mathrm{P}<0.05$; ${ }^{* *} \mathrm{P}<0.01 ;{ }^{* * *} \mathrm{P}<0.001$.

\begin{tabular}{|c|c|c|c|}
\hline $\begin{array}{l}\text { 1-Year follow-up } \\
\text { health outcome }\end{array}$ & $n$ & $r_{\text {persistence }}$ & $r_{\text {adherence }}$ \\
\hline$\overline{\mathrm{A} 1 \mathrm{C}(\Delta \mathrm{A} 1 \mathrm{C})}$ & 1867 (1489) & $-0.054^{*}\left(-0.069^{* *}\right)$ & $-0.081^{* * *}(-0.034)$ \\
\hline $\begin{array}{l}\text { Hypoglycemia-related } \\
\text { event rate }\end{array}$ & 4804 & $-0.031^{*}$ & -0.006 \\
\hline $\begin{array}{l}\text { Overall rate of } \\
\text { hospitalizations (rate } \\
\text { of diabetes-related } \\
\text { hospitalizations) }\end{array}$ & 4804 (4804) & $-0.080^{* * *}\left(-0.046^{* *}\right)$ & $-0.047^{* *}\left(-0.040^{* *}\right)$ \\
\hline Rate of ER visits & 4804 & $-0.039^{* *}$ & $-0.032^{*}$ \\
\hline Total health care costs & 4804 & 0.022 & 0.011 \\
\hline Drug costs & 4804 & $0.129^{* * *}$ & $0.178^{* * *}$ \\
\hline
\end{tabular}

Supported by: sanofi-aventis

14-0R

Clinical Characteristics and Outcomes of T2DM Patients Adding 1 to 3 Stepwise Prandial Insulin Doses to Basal Insulin and Oral Therapy: Identifying a Problematic Subgroup

MATTHEW C. RIDDLE, LING GAO, ALEKSANDRA VLAJNIC, Portland, OR, Hillsborough, NJ, Bridgewater, NJ

Stepwise addition of prandial insulin to basal insulin in T2DM theoretically allows all patients to reach $7.0 \%$ A1C, but studies suggest adding 1 prandial dose may be as effective. We studied subgroups of patients in an arm of the All to Target trial (NCT00384084). Titrated insulin glargine was added to oral agents $(\mathrm{OAs})$, followed by 1 prandial dose of insulin glulisine if A1C was not improved at wk-12, and up to 3 doses if $A 1 C$ was $>7.0 \%$ at wk24,36 , or 48 . 191 patients began treatment (mean age $55 \mathrm{y}$; T2DM duration 9.5 y; BMI 32.7; A1C 9.4\%; all used 2-3 OAs) and 150 completed 60 weeks. At endpoint (wk-60 or LOCF) $38 \%$ took insulin glargine only $(\mathrm{G}+0)$, and 24 , 21 , and $18 \%$ took insulin glargine $+1(\mathrm{G}+1),+2(\mathrm{G}+2)$, or $+3(\mathrm{G}+3)$ insulin glulisine doses respectively. Groups did not differ in gender, age, T2DM duration, $\mathrm{BMI}$, or $\mathrm{OA}$ use; but differed in mean baseline $\mathrm{A} 1 \mathrm{C}: \mathrm{G}+0,9.0 \%$; $\mathrm{G}+1,9.2 \% ; \mathrm{G}+2,9.6 \% ; \mathrm{G}+3,10.4 \%(\mathrm{P}<0.01 ; \mathrm{Fig} A)$. Mean $\mathrm{A} 1 \mathrm{C}$ was higher in the $\mathrm{G}+3$ group at endpoint: $\mathrm{G}+0,6.8 \%$; $\mathrm{G}+1,6.9 \% ; \mathrm{G}+2,7.3 \%, \mathrm{G}+3,8.3 \%$ $(P<0.001 ;$ Fig A). Weight gain was similar $(6.1,6.5,7.8,7.4 \mathrm{~kg})$ but insulin dose higher in multiple-dose regimens $(0.54,0.73,1.01,1.39 \mathrm{U} / \mathrm{kg} ; \mathrm{P}<0.01$; Fig B). G+0 and $\mathrm{G}+1$ groups comprised $62 \%$ of patients and achieved mean A1C of 6.8 and $6.9 \%$, respectively. Patients in the G+3 group had higher baseline $A 1 C$ and along with $\mathrm{G}+2$ patients generally failed to reach $7.0 \%$ despite higher total insulin dose. In conclusion, patients unsuccessful with 
insulin glargine alone or with 1 prandial dose pose a therapeutic problem not routinely solved by a full basal-prandial insulin regimen.

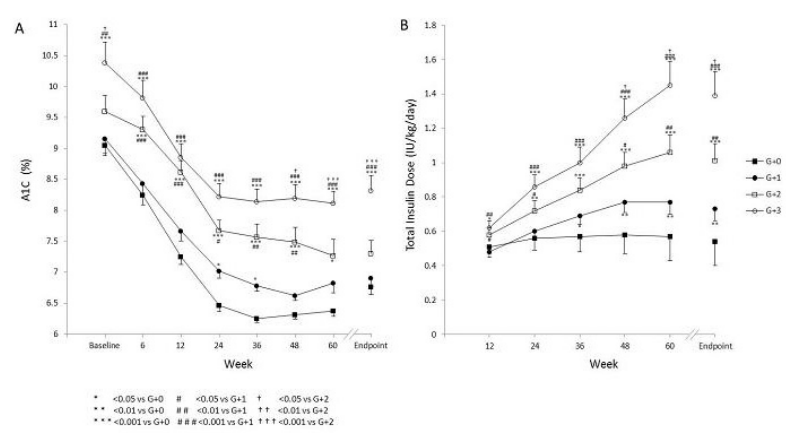

Supported by: sanofi-aventis

\section{NOVEL RISK FACTORS FOR DIABETES}

$15-0 R$

Relationship Between Obstructive Sleep Apnea and Diabetes Risk-A Meta-Analysis

KAZUYA FUJIHARA, SATORU KODAMA, CHIKA HORIKAWA, YORIKO HEIANZA AYUMI SUGAWARA, MIHO MAKI, YOKO YACHI, HITOSHI SHIMANO, KAZUMI SAITO, HIROHITO SONE, Mito, Ibaraki, Japan, Tsukuba, Ibaraki, Japan

Obstructive sleep apnea (OSA) and diabetes mellitus (DM) are hypothesized to be mutually associated. However, epidemiological data to elucidate this hypothesis remain inconsistent. Our aim of this meta-analysis is to clarify the strength and causal direction of the association between OSA and DM. MEDLINE- and EMBASE-based electronic literature searches were conducted for studies that examined the relationship between OSA and DM using a cross-sectional or cohort design. The risk of or odds ratios for diabetes for the highest or lowest group of OSA grade or that of OSA for DM or non-DM were pooled by an inverse-variance method. Twenty-four eligible studies were included in this meta-analysis. The pooled odds ratio $195 \%$ confidence interval [CI]) of 17 cross-sectional studies examining DM risk related to severe OSA was 1.92 [1.55-2.39] (Fig. 1). In 6 cohort studies, OSA was also related to future DM risk (pooled risk estimate [95\% CI], 2.06 [1.77-2.40]). However, according to the pooled analysis of 5 cohort studies examining future OSA risk related to DM, DM did not significantly predict OSA risk (pooled risk estimate [95\% CI], 1.03 [0.89-1.19]) (Fig. 2). These results suggest that OSA causes incident DM, but that the reverse is not true.

Fig 1. Pooled odds ratio with 95\% confidence interval $(95 \%$ CD) for diabetes related to severe obstructive sleep apnea of 17 cros\%

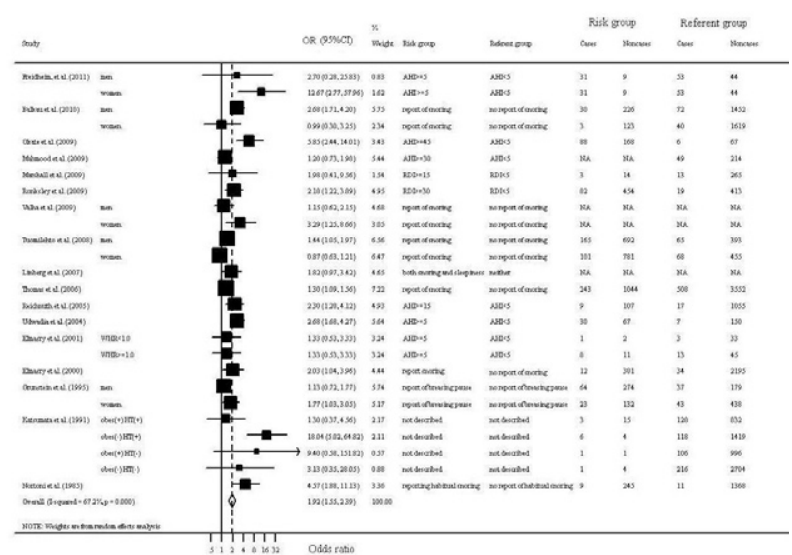
Fig. 2 Pooled risk estimate (i.e., odds ratio (OR) or risk ratio (RR)) with $95 \%$ confidence interval $(95 \%$
CI) of diabetes (DM) related to severe obstructive sleep apnea (OSA) [top] and that of OSA related to DM [bottom]. Size of each data point is proportional to study weight
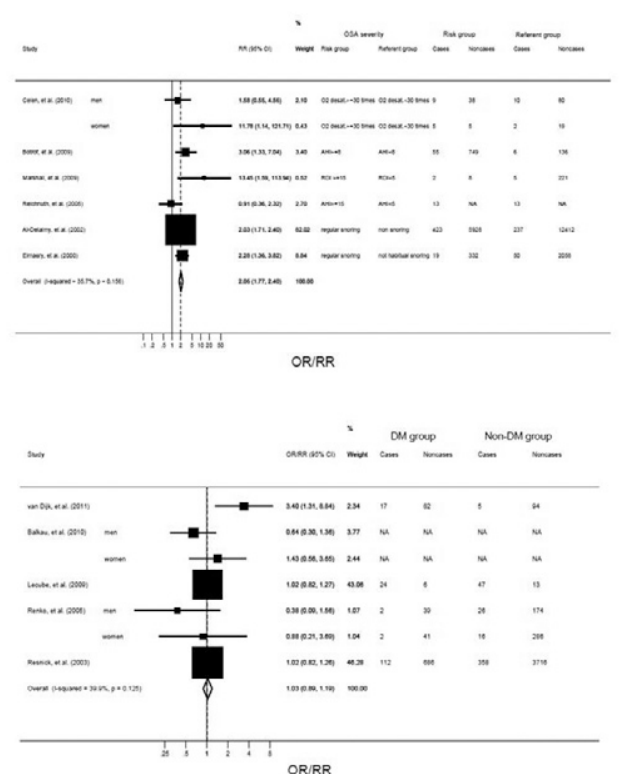

OR/RR

16-0R

The Association of Serum Cotinine Level and Diabetes in Never Smokers

OMAYMA 0. ALSHAARAWY, JIE XIAO, ANOOP SHANKAR, Morgantown, WV

Several studies have shown that smoking is associated with an increased risk of developing diabetes. However, there are no studies investigating the relationship between environmental tobacco smoking (ETS or passive smoking), measured objectively by serum cotinine levels, and diabetes in never smokers. We examined $n=3151$ never smokers from the National Health and Nutrition Examination Survey (NHANES) 2005-08. Our exposure of interest was ETS estimated by serum cotinine level and our outcome was diabetes $(n=404)$, defined based on the guidelines of the American Diabetes Association. We found that in never smokers, higher serum cotinine levels were positively associated with diabetes. Compared to those with serum cotinine levels $\leq 0.025 \mathrm{ng} / \mathrm{mL}$, the multivariable odds ratio (OR) $195 \%$ confidence interval $[\mathrm{CI}]$ ) of diabetes among those with cotinine levels of $0.026-0.053 \mathrm{ng} /$ $\mathrm{mL}$ was 1.27 (0.84-1.92), among those with cotinine levels of 0.054-0.223 $\mathrm{ng} / \mathrm{mL}$ was $1.39(0.99-1.95)$ and among those with cotinine level $\geq 0.224 \mathrm{ng} /$ $\mathrm{mL}$ was 1.52 (1.01-2.29); $p$-trend=0.0284. Higher ETS exposure measured objectively by serum cotinine was found to be associated with diabetes in never smokers.

A ADA-Funded Research

For author disclosure information, see page 797. 
17-0R

Risk of Type 2 Diabetes and the Use of Second Generation Antipsychotics in a Large Pediatric Medicaid Population

AVNISH TRIPATHI, JEANETTE M. JERRELL, ALI A. RIZVI, Columbia, SC

Second generation antipsychotics (SGAs) have been associated with increased risk of metabolic disorders. Our objective was to examine the association of some SGAs with the incidence of type 2 diabetes mellitus (T2DM) after accounting for other risk factors in a large cohort of children and adolescents. The study cohort included patients $\leq 17$ years old who were served through the South Carolina State Medicaid Program and were newly prescribed at least one of five SGAs including aripiprazole, ziprasidone, quetiapine, risperidone, and olanzapine sometime over the 11-year study period of 1996-2006. At least 24 months of service encounters before the start date of SGAs were captured as a 'SGAs free' baseline period. The incidence of T2DM was defined as $\geq 2$ initial service encounters, at least 30-days apart, with ICD-9 codes of T2DM. Other comorbid metabolic conditions such as obesity, dyslipidemia, and hypertension were similarly defined. Of the total cohort $(N=4140)$ with median age of 11 years (IOR: 4-11 year), 176 (4.3\%) developed new-onset T2DM. Cox's proportional hazards analysis with time-dependent covariates suggested a higher risk of T2DM incidence associated with females (aHR 1.78; 95\% Cl 1.30-2.44): African Americans (aHR 1.52; 95\% Cl 1.07-1.25); overweight/obesity (aHR 4.19; 95\% Cl 2.876.11) and dyslipidemia (aHR 3.33; 1.76-6.30); use of aripiprazole (aHR 2.92; $95 \% \mathrm{Cl} 1.08-7.90$ ) or ziprasidone (aHR 3.56; $95 \% \mathrm{Cl} 1.61-7.89$ ) vs. 'no SGA'; and use of mood stabilizers (aHR 1.64; 95\% $\mathrm{Cl} 1.13-2.37$ ) versus no use. Multivariable marginal structural model suggested a higher causal association of overweight/obesity (aRR 5.59; 95\% Cl 2.71-11.51) and ziprasidone (aRR 5.12; $95 \% \mathrm{Cl} 1.15-22.70)$. The results suggest that SGAs, especially ziprasidone, may independently increase the risk of T2DM in children and adolescents. This warrants increased awareness in healthcare providers about the use of SGAs in pediatric patients, especially in those with other risk factors for diabetes.

18-OR

Plasma Levels of Fetuin-A and Hepatic Enzymes as Predictors of Type 2 Diabetes Risk in U.S. Women

OI SUN, MARILYN C. CORNELIS, JOANN MANSON, FRANK B. HU, Boston, MA

Context: Animal experiments have demonstrated that fetuin-A may interfere with insulin action, although data regarding the association between fetuin-A and risk of type 2 diabetes in humans are sparse. Objective: To prospectively examine fetuin-A, as well as alanine transaminase (ALT) and gamma-glutamyltranspeptidase (GGT), in relation to type 2 diabetes risk in US women. Design and Setting: The Nurses' Health Study (NHS). Participants: Among the 18,717 NHS participants who provided blood samples in 2000-2001, we prospectively identified and confirmed 470 incident diabetes cases through 2006 and matched the cases with the same number of controls who were free of diabetes at blood draw. Plasma levels of fetuin-A, ALT, and GGT and a wide array of covariates were measured for the cases and controls. Results: After multivariate adjustment for covariates, including age, body mass index, alcohol use, and C-reactive protein, levels of fetuinA were significantly associated with increased risk of type 2 diabetes: the odds ratio (OR) [95\% confidence interval (CI)] comparing extreme quintiles was $1.88(1.14,3.09 ; \mathrm{P}$ for trend=0.006) for fetuin-A. Further adjustment for ALT and GGT levels did not alter this association: the OR (95\% CI) was 1.84 (1.10, 3.06; $P$ for trend=0.006). In addition, the association of fetuin-A was not modified by liver enzymes (P for interaction=0.89 for ALT and 0.64 for GGT), although strong joint associations were found between fetuin-A and liver enzymes. In comparison to women with both fetuin-A and ALT levels in the lowest tertiles, women with both markers in the highest tertile had an OR $(95 \% \mathrm{CI})$ of $3.75(2.02,6.97)$. This joint association was $8.30(3.73,18.50)$ for GGT. Conclusions: Plasma fetuin-A levels were prospectively associated with higher risk of type 2 diabetes, independent of body mass index, liver enzymes, and other diabetes risk factors. The combination of elevated fetuin-A and GGT is associated with a particularly high risk of developing diabetes.

Supported by: NIH Grants CA87969, DK58845, DK58785, and K99HL098459
Racial/Ethnic Differences in Risk Factors for Type 2 Diabetes: Northern Manhattan Study (NOMAS)

TIFFANY L. GARY-WEBB, YESEON P. MOON, TANJA RUNDEK, RALPH L. SACCO, MYUNGHEE PAIK, MITCHELL S. ELKIND, New York, NY, Miami, FL

Disparities in diabetes for African Americans and Hispanics compared to whites are well known, but the role of risk factors among minority populations remains uncertain. We conducted an analysis to determine if risk factors for diabetes differ for three racial/ethnic groups. Among 2,498 participants free from diabetes at enrollment into the Northern Manhattan Study (NOMAS), a prospective cohort study among a tri-ethnic, urban population aged $>40$ yrs, diagnosis of diabetes was assessed annually (median 9 yrs; data collected 1993-2009). Weibull regressions for interval censoring were used to estimate hazard ratios (HR) and $95 \%$ confidence intervals (Cl) for the effect of risk factors at baseline: waist-hip ratio, physical activity, alcohol use, smoking, hypertension, and HDL with interaction terms specified for race and each risk factor in separate models. At baseline, participants were mean age $69 \pm 10$ yrs; $52 \%$ Hispanic, $23 \%$ black, and $23 \%$ white. By 2009 , $351(14.1 \%)$ developed diabetes. Waist-hip ratio predicted diabetes among whites (HR per SD 1.68, 95\% Cl 1.28-2.19) and Hispanics (HR per SD 1.31, 1.14-1.50), but not blacks (HR per SD 0.97, 0.76-1.25) (p for interaction=0.01). Moderate alcohol use was inversely associated with risk of diabetes among whites (HR 0.30, 0.13-0.71), but much less strongly among blacks (HR 0.86) and Hispanics (HR 0.92) ( $p$ for interaction=0.035). Furthermore, current smoking was a strong risk factor for diabetes among whites (HR 5.22, 1.99-13.69), but not for blacks or Hispanics ( $p$ for interaction=0.017). After adjusting for baseline risk factors, Hispanics (HR 2.09, 1.32-3.32) and blacks (HR 1.54, $0.97-2.45)$ still had greater risk of diabetes than whites. These results suggest that the risk of diabetes was higher among minorities than whites for those living in a single urban community. Risk factors for diabetes also differed by race-ethnicity, with several risk factors that were important among whites playing a small or insignificant role among minorities.

Supported by: NIH

$\Delta$ NT-pro BNP and Incident Diabetes

20-0R

MARIANA LAZO, FREDERICK L. BRANCATI, JOSEPH CORESH, CHIADI NDUMELE, RON C. HOOGEVEEN, CHRISTIE M. BALLANTYNE, ELIZABETH SELVIN, Baltimore, MD, Houston, $T X$

Natriuretic peptides are produced by cardiac myocytes and are critical to homeostatic control of blood pressure. In clinical practice they are used for the diagnosis and prognosis of heart failure. Recently, it has been postulated that the natriuretic peptide axis influences lipid and glucose metabolism. While a number of cross-sectional studies have shown that natriuretic peptide levels are lower in people with diabetes, the direction of the association is unclear. Our objective was to determine the prospective association of N-terminal b-type natriuretic peptide (NT-proBNP) with the incidence of diagnosed diabetes in a community-based sample without cardiovascular disease or diabetes at baseline. We conducted a prospective analysis of 7657 participants (80\% white, mean age 62 years) from the Atherosclerosis Risk in the Communities (ARIC) Study. Over a median follow up of 11 years, 1443 participants reported a diagnosis of diabetes. In Cox proportional hazards models, quartiles of NT-proBNP were inversely associated with diabetes risk, even after multivariable adjustment (Table and Figure). Our results highlight a metabolic role of the natriuretic peptide axis, and suggest that upstream factors (low levels of natriuretic peptide) predispose to insulin resistance.

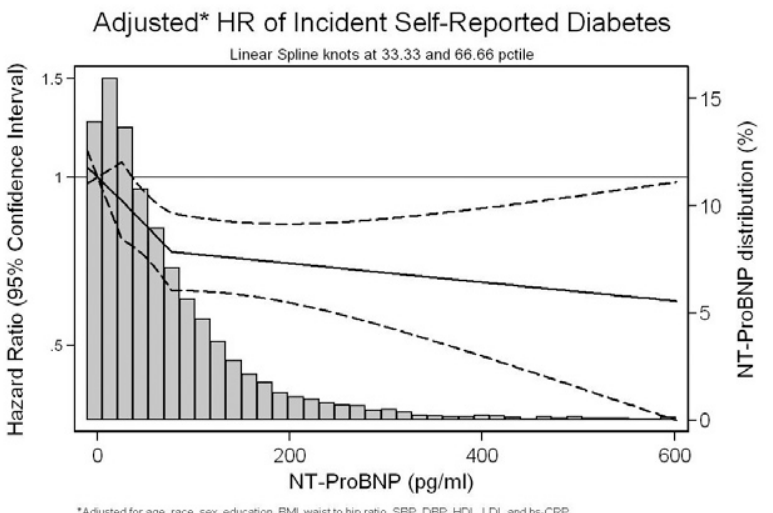


GLUCOSE TRANSPORT AND INSULIN RESISTANCE

Table. Adjusted Hazard Ratios (95\%Cls) for Incident Diabetes by Quartiles of NT-ProBNP at Baseline

\begin{tabular}{lccccc}
\hline NT-proBNP & Unadjusted & Model 1 & Model 2 & Model 3 & Model 4 \\
\hline 01 [21.2(14.3-28.1)] & 1.0 (Ref) & 1.0 (Ref) & 1.0 (Ref) & 1.0 (Ref) & 1.0 (Ref) \\
\hline 02 [48.6 (41.5-57.3)] & 0.83 & 0.87 & 0.88 & 0.91 & 0.89 \\
& $(0.72-0.95)$ & $(0.75-0.99)$ & $(0.77-1.01)$ & $(0.79-1.05)$ & $(0.78-1.02)$ \\
\hline 03 [89.6 (76.7-104.8)] & 0.69 & 0.72 & 0.76 & 0.79 & 0.75 \\
& $(0.59-0.79)$ & $(0.62-0.84)$ & $(0.66-0.89)$ & $(0.68-0.92)$ & $(0.65-0.87)$ \\
\hline 04 [183.5(146.2-263.7)] & 0.64 & 0.68 & 0.74 & 0.78 & 0.70 \\
& $(0.55-0.75)$ & $(0.58-0.80)$ & $(0.63-0.87)$ & $(0.66-0.92)$ & $(0.59-0.83)$ \\
\hline
\end{tabular}

Model 1: age, race-center, sex, education Model 2: Model 1+BMI Model 3 . Model 2+ Waist to Hip Ratio Model 4: Model 3 + SBP, DBP, Smoking, LDL, $\mathrm{HDL}$, hs-CRP and eGFR.

21-0R

Plasma Stearoyl Co-A Desaturase-1 Activity and Risk of Incident Type 2 Diabetes: The Atherosclerosis Risk in Communities (ARIC) Study

LISA CHOW, LYNN E. EBERLY, ELIZABETH SEAQUIST, JOHN H. ECKFELDT, RON C. HOOGEVEEN, DAVID COUPER, LYN M. STEFFEN, JAMES S. PANKOW, Minneapolis, MN, Houston, TX, Chapel Hill, NC

Stearoyl Co-A desaturase (SCD-1), the rate limiting enzyme in monounsaturated fatty acid synthesis, is critical for lipid synthesis, lipid oxidation and possibly type 2 diabetes. This study characterized SCD-1 activity through fatty acids ratios in baseline plasma measurements and established their association with incident diabetes in the Atherosclerosis Risk in Communities (ARIC) study. In 2738 Caucasian adults (age $45-64,47 \%$ men) free of diabetes, baseline SCD-1 activity was estimated by plasma fatty acid ratios in cholesterol esters (SCD16c-16:1/16:0, SCD18c-18:1/18:0) and plasma phospholipids (SCD16p-16:1/16:0, SCD18p-18:1/18:0). Incident diabetes was ascertained during three follow up visits. Cox proportional hazards regression was used to evaluate the relationship between estimated SCD-1 activity and incident diabetes. 207 (7.6\%) participants developed incident diabetes (mean 8 years \pm SD 2.1 yrs). The estimates of SCD-1 activity correlated with each other $(p<0.001)$. After adjusting for age and gender, higher levels of SCD16c and SCD16p were positively associated with incident diabetes, but higher levels of SCD18p was inversely associated. With further adjustment for parental history of diabetes, smoking, blood pressure, activity, carbohydrate intake, fiber intake, fat (saturated, monounsaturated, polyunsaturated) intake, lipid composition, Waist-hip ratio, education, alcohol, and bodymass-index (BMI), only SCD16c remained positively associated with incident diabetes (RR=1.1: 95\%Cl 1.01-1.30; $p=0.03$ ) which persisted when adjusting for insulin resistance ( $p=0.05)$. Estimated SCD-1 activity by SCD16c was associated with incident diabetes, even when considering risk factors for type 2 diabetes. Refinement of SCD-1 activity measurement and exploration of its role in humans is recommended.

22-0R

Alternative Markers of Glycemia and Risk of Diabetes in the Atherosclerosis Risk in Communities (ARIC) Study

STEPHEN P. JURASCHEK, MICHAEL W. STEFFES, EDGAR R. MILLER, ELIZABETH SELVIN, Baltimore, MD, Minneapolis, MN

Despite growing interest in glycated albumin, fructosamine, and 1,5-anhydroglucitol (1,5-AG) as alternative markers of glycemic control, little is known about their utility as risk factors for the development of diabetes. We examined the association between quartiles of these markers and incident diabetes in a subset of 1,325 participants from the ARIC Study (2004-2005) who had no history of diabetes or diabetes medication use. Incident diabetes was self-reported during annual telephone calls to all participants. During a median follow-up of 2.6 years, there were 94 new cases of diabetes. The upper quartiles of glycated albumin and fructosamine and the lowest quartile of 1,5-AG were each associated with diabetes risk even after adjustment for baseline A1c (Table). There was evidence for dose-response relationships for both glycated albumin and fructosamine with diabetes risk (Figure). Our results suggest that elevated concentrations of alternative markers of glycemia are risk factors for diabetes independently of baseline A1c. Future studies should examine the utility of these markers for predicting long-term complications of diabetes, particularly in populations where glucose and A1c are unavailable or not valid.

ג ADA-Funded Research
Table. Adjusted hazard ratios (95\% confidence intervals) of incident diagnosed diabetes by quartiles of each glycemic marker at baseline

Quartile of Glycated Albumin (\%)

\begin{tabular}{|c|c|c|c|c|c|}
\hline & $9.7-12.6$ & $12.7-13.4$ & $13.5-14.4$ & $14.5-34.8$ & Pfor trend* \\
\hline Model 1 & 1.0 [Ref] & $1.71(0.71,4.14)$ & $1.89(0.81,4.41)$ & $6.57(2.87,15.06)$ & $<0.001$ \\
\hline Model 2a & $1.0[$ Ref] & $1.63(0.68,3.91)$ & $1.68(0.73,3.84)$ & $4.20(1.89,9.34)$ & $<0.001$ \\
\hline \multirow[t]{3}{*}{ Model 2b } & 1.0 [Ref] & $1.71(0.71,4.15)$ & $1.77(0.74,4.21)$ & $5.73(2.49,13.21)$ & $<0.001$ \\
\hline & \multicolumn{5}{|c|}{ Quartile of Fructosamine $(\mu \mathrm{mol} / \mathrm{L})$} \\
\hline & $163.1-216.1$ & $216.2-228.0$ & $228.2-242.0$ & $242.1-463.4$ & Pfor trend* \\
\hline$\overline{\text { Model } 1}$ & 1.0 [Ref] & $1.64(0.62,4.32)$ & $3.21(1.44,7.17)$ & $5.44(2.33,12.69)$ & $<0.001$ \\
\hline$\overline{\text { Model 2a }}$ & 1.0 [Ref] & $1.58(0.61,4.13)$ & $2.92(1.34,6.40)$ & $3.56(1.57,8.09)$ & 0.001 \\
\hline \multirow[t]{3}{*}{ Model 2b } & 1.0 [Ref] & $1.44(0.53,3.90)$ & $2.69(1.18,6.13)$ & $5.04(2.15,11.84)$ & $<0.001$ \\
\hline & \multicolumn{5}{|c|}{ Quartile of 1,5-Anhydroglucitol ( $\mu \mathrm{g} / \mathrm{mL}) \dagger$} \\
\hline & $22.1-39.5$ & $18.0-22.0$ & $14.0-17.9$ & $1.0-13.9$ & Pfor trend* \\
\hline Model 1 & 1.0 [Ref] & $3.66(1.66,8.07)$ & $2.20(0.94,5.14)$ & $4.53(2.08,9.86)$ & 0.001 \\
\hline Model 2a & 1.0 [Ref] & $3.65(1.64,8.09)$ & $2.28(0.97,5.36)$ & $3.73(1.70,8.16)$ & 0.004 \\
\hline$\overline{\text { Model } 2 \mathrm{~b}}$ & 1.0 [Ref] & $3.45(1.58,7.53)$ & $1.55(0.61,3.94)$ & $4.17(1.94,8.95)$ & 0.002 \\
\hline
\end{tabular}

Model 1: Adjusted for age, sex, race, total cholesterol, high density lipoprotein cholesterol, body mass index, average systolic blood pressure, family history of diabetes, smoking status

Model 2a: Model 1 + baseline fasting glucose concentration

Model 2b: Model 1 + baseline hemoglobin A1c

*P-value for trend evaluated using an ordinal variable based on the median value in each quartile.

†Note 1,5-AG quartiles are presented in reverse order for symmetry. Serum 1,5-AG is lowered in the presence of hyperglycemia.

Figure. Incidence Rate (per 100 person-years) of diagnosed diabetes by baseline quartiles of glycemic markers

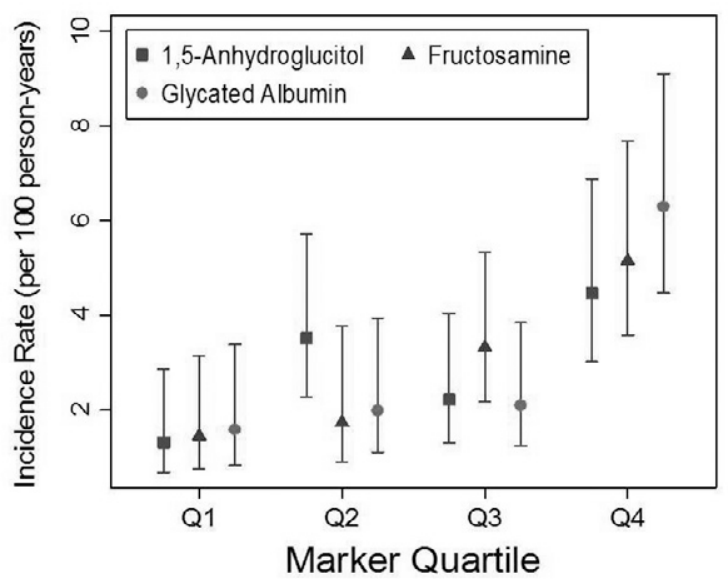

Note: 1,5-AG quartiles are presented in reverse order.

Supported by: NIH/NIDDK K01 DK076595 and R01 DK089174; NIH/NHLBI T32HLOO7024

\section{GLUCOSE TRANSPORT AND INSULIN RESISTANCE}

23-0R

Characterization of Unique Regulatory Mechanism of Tbc1d1 in GLUT4 Trafficking by Single Molecule Analysis of GLUT4 Behavior HIROYASU HATAKEYAMA, MAKOTO KANZAKI, Sendai, Japan

GLUT4 translocation facilitated by insulin and exercise is suggested to be mediated by TBC1D family Rab GTPase-activating proteins AS160 and Tbc1d1. Due to technical limitations of conventional assays, it is still unclear how these proteins regulate GLUT4 trafficking and how their actions differ among each other. We recently developed a single molecule approach for investigating intracellular GLUT4 behavior and demonstrated systematization of the insulin-responsive GLUT4 trafficking property. Specifically, "static GLUT4 retention" and "insulin-responsive GLUT4 liberation" are critical distinct features, and the former is generated by sortilin. Here, we analyzed the detailed roles of AS160 and Tbc1d1 in GLUT4 trafficking. In undifferentiated

For author disclosure information, see page 797 
3T3L1 fibroblasts expressing neither sortilin nor AS160 endogenously, we successfully reconstituted "insulin-responsive GLUT4 liberation" essentially the same as in differentiated 3T3L1 adipocytes by exogenously expressing these two proteins. In contrast, fibroblasts exogenously expressing sortilin and Tbc1d1 showed no insulin-responsive liberation. Instead, these cells exhibited GLUT4 liberation in response to AICAR, an AMPK activator. After AICAR removal, GLUT4 was efficiently recycled back to the "static" compartment, and importantly, the restored static GLUT4 now exhibited marked "insulin-responsive liberation" even several hours after AICAR removal, indicating that Tbc1d1 gains insulin-responsiveness by pre-AICAR/restoration treatment. In addition, we revealed the importance of a rise in intracellular $\mathrm{Ca}^{2+}$ level for the temporal acquisition of "insulin-responsive" activity of Tbc1d1. Thus, our findings demonstrate the unique regulatory mechanism of Tbc1d1 in the GLUT4 liberation process, and suggest that combined and/ or sequential stimulation with multiple signals (i.e. AMPK, $\mathrm{Ca}^{2+} \rightarrow \mathrm{Akt}$ ) is essential for "insulin-responsive GLUT4 liberation" activity of Tbc1d1.

\section{4-0R}

Phosphorylation of Tmod3 by Akt2 Regulates GLUT4 Translocation by Remodeling Cortical Actin

CHUN YAN LIM, XUEZHI BI, WANJIN HONG, WEIPING HAN, Singapore, Singapore

Insulin stimulates glucose uptake into muscle and fat cells by promoting the translocation of glucose transporter 4 (GLUT4) from intracellular storage compartment to the plasma membrane (PM) via the activation of PI3K/Akt signaling pathway. Most notably, Akt2 is essential for directing the insulin action by phosphorylating a number of downstream substrates that are required in multiple discrete steps of vesicle trafficking and exocytosis. In this study, we identified Tropomodulin 3 (Tmod3 or UTmod), a ubiquitously expressed actin-capping protein, as an interacting partner of constitutively active Akt2 in differentiated 3T3-L1 adipocytes by a proteomic screening. Using biochemical and RNAi-functional analyses in combination with TIRF imaging, we demonstrated that $\operatorname{Tmod} 3$ was a novel Akt2-specific substrate, and was required for insulin-stimulated glucose transport, and GLUT4 translocation. Tmod3 proteins were found to be associated with cortical actin, and clustered around reorganized actin in response to insulin stimulation. We further identified that S71 residue of Tmod3 was the target site of Akt phosphorylation, and critical for actin monomer-binding and -nucleating activities. A phospho-defective Tmod3 mutant showed stronger potency of actin-stabilizing activity in vitro, and significantly inhibited insulin-induced GLUT4 translocation when overexpressed in adipocytes; while a phosphomimetic Tmod3 mutant facilitated F-actin rearrangements, a necessary process for translocation and fusion of GLUT4 vesicles at the cell surface. Taken together, these findings establish cortical actin remodeling as a novel molecular link between insulin signaling pathway and GLUT4 translocation from intracellular storage to the PM, and suggest that defects in membrane cytoskeletal-based mechanism could contribute to GLUT4 dysregulation in insulin-resistant state.

$\Delta$

25-0R Rabs 10, 14, and 8a/b are Required for Glut4 Translocation in Adipocytes, but Affect Different Trafficking Steps

CYNTHIA C. MASTICK, PAUL D. BREWER, ESTIFANOS N. HABTEMICHAEL, IRINA ROMENSKAIA, Reno, NV

AS160 regulates the conversion of sequestered Glut4 Storage Vesicles (GSVs) into vesicles that are competent to fuse to the plasma membrane (PM), but does not directly regulate the fusion step itself. AS160 is a RabGTPase activating protein that inhibits translocation by reversing the GTP loading of Rabs required for Glut4 trafficking. Four Rabs that are substrates for AS160 in vitro co-localize with cycling Glut4 in adipocytes: Rabs 10,14, $8 \mathrm{a}$, and $8 \mathrm{~b}$. The effect of shRNA-mediated knockdown (KD) of these Rabs on the trafficking kinetics of Glut4 and LRP1 were measured in 3T3-L1 adipocytes. LRP1 co-localizes with Glut4 in GSVs, although a significant fraction $(50-60 \%)$ is also constitutively cycling through the endocytic pathway in these cells. The effects of KD were also measured in fibroblasts to determine if they act through the constitutive or specialized insulin-regulated trafficking pathways (there are no GSVs in these cells). Knockdown of Rabs $10,14,8 \mathrm{~b}$, or $8 \mathrm{a}$ decreased PM Glut4 (65\%, $47 \%, 29 \%$, or $24 \%$, respectively). KD of Rab10 decreased exocytosis ( $\mathrm{k}_{\mathrm{ex}}$ ) of both Glut4 and LRP1 in adipocytes, but not fibroblasts, consistent with a role in specialized GSV trafficking. KD of Rab14 or 8a also decreased the $\mathrm{k}_{\text {ex }}$ of Glut4 in adipocytes, but surprisingly, it increased the $\mathrm{k}_{\mathrm{ex}}$ of LRP1 2-3-fold. This was not observed in fibroblasts. Mathematical modeling/simulations support the following model: Rab10 is required to make GSVs competent for fusion to the PM; AS160 inhibits the accumulation of Rab10-GTP on GSVs in basal cells. In contrast, Rabs14 and $8 \mathrm{a}$ are required for the formation of GSVs from the endosomes/TGN, a step that is also regulated by AS160. KD of either Rabs14 or 8a leads to accumulation of Glut4 and LRP1 in the pre-GSV sorting compartment. LRP1 is efficiently trafficked from this compartment through the constitutive cycling pathway (so exocytosis increases in KD cells), while Glut4 is trafficked slowly from this compartment (so exocytosis decreases in KD cells).

GLUT4 Endosomal Trafficking, but Not Signaling is Altered During Insulin Resistance in 3T3-L1 Cells

ANN L. OLSON, KANDICE TESSNEER, Oklahoma City, OK

Glucose transporter isoform 4 (GLUT4) is the insulin-responsive glucose transporter mediating peripheral glucose uptake by skeletal muscle and adipose. Insulin-mediated recruitment from intracellular storage vesicles to the plasma membrane is the rate-limiting step in peripheral glucose disposal making GLUT4 an important regulator of glucose homeostasis. In white adipose tissue (WAT), GLUT4 protein expression and recruitment to the cell surface are decreased during insulin resistance and Type II diabetes mellitus (T2DM). These conditions are accompanied by fasting hyperinsulinemia and hyperglycemia. We hypothesize that chronic hyperinsulinemia may cause the alterations in GLUT4 regulation observed during insulin resistance in vivo. To test this hypothesis, we have characterized GLUT4 endosomal trafficking in 3T3-L1 adipocytes treated with $100 \mathrm{mM}$ insulin for 24 hours (CHI). This treatment reduced insulin-mediated redistribution of an exogenous HA-GLUT4-GFP reporter by $60 \%$. Using a GLUT4 trafficking mutant in combination with mutant Rab5, we next demonstrated that chronic insulin treatment targets GLUT4 to an endosomal compartment that has a reduced capacity to equilibrate with the cell surface in response to acute insulin treatment. Co-expression of dominant negative Rab5 S34N prevented CHI-dependent sequestration of GLUT4, as did an EE499/501AA mutation in the T498ELEY motif of the GLUT4-C-terminus. Overexpression of wild type Rab5, increased sequestration of GLUT4 in the moderately insulin responsive compartant. Proximal insulin signaling through phosphorylation of AS160 was not altered by $\mathrm{CHI}$, however, basal phosphorylation of AKT and AS160 was elevated by CHI. The results suggest that $\mathrm{CHI}$ sequesters GLUT4 into a rab5-dependent intracellular compartment, leading to reduced equilibration of GLUT4 with the plasma membrane in response to insulin. This may be an adaptive response to nutrient excess to buffer glucose clearance by an expanding adipose mass.

Supported by: OCAST

27-0R

CaMKKalpha Stimulates Skeletal Muscle Glucose Uptake in Insulin Resistant Mice In Vivo

JAMES M. HINKLEY, LAURA A. GILLIAM, JEFFREY J. BRAULT, CAROL A. WITCZAK, Greenville, NC

Signaling via the $\mathrm{Ca}^{2+}$-activated, serine/threonine kinase, $\mathrm{Ca}^{2+} / \mathrm{calmodu}-$ lin-dependent protein kinase kinase $\alpha(\mathrm{CaMKK} \alpha)$ stimulates skeletal muscle glucose uptake in healthy, insulin-sensitive mice. The goal of this study was to determine whether CaMKK $\alpha$ could stimulate glucose uptake in muscles from insulin-resistant mice. Male mice (C57BL6/J strain; 6 wks old) were fed either a low fat (14\% kcal fat; control) or a high fat (60\% kcal fat) diet for 12 wks. To assess insulin resistance, in vivo muscle $\left[{ }^{3} \mathrm{H}\right]$-2-deoxyglucose uptake was examined following an intravenous injection of glucose $11 \mathrm{mg} / \mathrm{g}$ body $\mathrm{wt}$ ) to induce a physiological insulin response. In control mice, insulin increased muscle glucose uptake 2-fold over basal and this was significantly impaired in the high fat fed mice (1.6-fold increase over basal), indicating that the high fat fed mice were insulin resistant. To stimulate CaMKK $\alpha$ signaling, tibialis anterior muscles were transfected with plasmid DNA (100 $\mu \mathrm{g})$ containing constitutively active CaMKK $\alpha$ or empty vector using in vivo electroporation. Two weeks later, active CaMKK $\alpha$ protein levels were robustly increased over endogenous CaMKK $\alpha$ levels, and were not different between control and insulin-resistant mice. Basal and insulin-induced muscle glucose uptake were assessed in vivo as described above. In control mice, uptake was increased 1.6-fold by active CaMKK $\alpha$ and 2.6-fold by active CaMKK $\alpha$ + insulin, demonstrating for the first time that CaMKK $\alpha$ and insulin have additive effects on muscle glucose uptake. In high fat fed mice, uptake was increased 2-fold by active CaMKK $\alpha$, and 3 -fold by active CaMKK $\alpha+$ insulin, indicating that CaMKK $\alpha$ is effective at stimulating glucose uptake in insulin resistant muscles. In conclusion, these results show that activation of CaMKK $\alpha$ signaling can stimulate glucose uptake in both insulin-sensitive and insulin-resistant muscles, and suggest that CaMKK $\alpha$ may be a novel therapeutic target for the treatment of impaired muscle glucose uptake in type 2 diabetes.

Supported by: NIH R00AR056298 
$\Delta$

28-0R

Genetic Deletion of Matrix Metalloproteinase 9 (MMP9) in C57BL/6J Mice Exacerbates Diet-Induced Muscle Insulin Resistance (IR) LI KANG, WESLEY H. MAYES, FREYJA D. JAMES, DEANNA P. BRACY, DAVID H. WASSERMAN, Nashville, TN

Increased deposition of extracellular matrix (ECM) collagens is a characteristic of muscle IR. MMPg is the primary enzyme that degrades collagen IV (ColIV), a major component of the basement membrane which plays a key role in ECM remodeling. The hypotheses that genetic deletion of MMP9 in mice 1) increases muscle CollV, 2) induces IR in lean mice and 3) worsens diet-induced muscle IR were tested. Wildtype $\left(\mathrm{mmp}^{+/ t}\right)$ and MMP9 nul $(\mathrm{mmpg}-)_{\text {mice }}$ were chow fed (CF) or high fat (HF) fed (HFF) for 16wks. The hyperinsulinemic $(4 \mathrm{mU} / \mathrm{kg} / \mathrm{min})$ euglycemic clamp (IC) was used to assess insulin action in conscious mice with indwelling arterial (sampling) and venous (infusion) catheters. A muscle glucose metabolic index (Rg) was assessed using $2\left[{ }^{14} \mathrm{C}\right]$ deoxyglucose. mmp $9 \%$ increased muscle CollV by $1.7 \pm 0.3$ fold in CF mice. HF feeding increased CollV by $1.4 \pm 0.1$ fold in $m m p 9^{+t}$. CollV in HFF $m m p 9 \%$ was further increased compared to HFF $m m p 9^{+\neq}(1.3 \pm 0.1$ fold $)$. Body weight and composition did not differ between CF $m m p g^{+/ 4}$ and $\mathrm{mmp}^{-} \%$. HFF female $m m p 9^{-}$weighed less than HFF female $m m p 9^{+/+}(27 \pm 1$ vs $35 \pm 2 \mathrm{~g})$ primarily due to less fat mass. HFF male $\mathrm{mmp}^{+{ }^{+/}}$and $\mathrm{mmp} \mathrm{g}^{-/}$were of equal weight. IC's were performed in weight-matched mice. $\mathrm{mmp} 9 \%$ did not affect fasting insulin or glucose in CF or HFF mice. Glucose infusion rate (GIR) and muscle Rg were the same in CF $m m p 9^{+t+}$ and $\mathrm{mmpg}^{-\%}$. In contrast, HFF mmpgdecreased GIR (Females: $40 \pm 1$ vs $45 \pm 2$; Males: $27 \pm 3$ vs $38 \pm 3 \mathrm{mg} / \mathrm{kg} / \mathrm{min}$ ) and muscle Rg (Females: $14 \pm 2$ vs $27 \pm 1$; Males: $11 \pm 2$ vs $20 \pm 2 \mu \mathrm{mol} / 100 \mathrm{~g}_{\text {tissue }} / \mathrm{min}$ ). In conclusion, genetic deletion of MMPg is insufficient to induce IR in CF mice despite a marked increase in CollV, demonstrating that impaired ECM remodeling is not a significant barrier to insulin-stimulated glucose uptake in otherwise healthy mice. However, muscle IR in HFF mmpg- is considerably worse highlighting the importance of ECM remodeling in determining IR in the presence of the metabolic and inflammatory challenges posed by HF diet.

Supported by: NIDDK54902

29-0R

Insulin-Stimulated Glucose Transport Defects are More Resilient than Phosphorylation Defects in Type 2 Diabetes: A Dose Response Study With Dynamic PET Imaging

JASON M. NG, ALESSANDRA BERTOLDO, DAVNEET S. MINHAS, NICOLE L. HELBLING, PAUL M. COEN, JULIE C. PRICE, CLAUDIO COBELLI, DAVID E. KELLEY, BRET H. GOODPASTER, Pittsburgh, PA, Padova, Italy, Rahway, NJ

Prior PET imaging studies employing sequential triple tracers revealed dual defects in skeletal muscle glucose transport and phosphorylation under physiological hyperinsulinemia can more clearly define specific loci in muscle insulin resistance. Normal weight (NW; $N=6)$, obese ( $\mathrm{OB} ; \mathrm{N}=7$ ) and obese type 2 diabetes (T2D; $\mathrm{N}=6$ ) subjects were studied with $\mathrm{PET}$ imaging under separate 6-hour euglycemic clamps at 40 (Mid) and $120 \mathrm{mU} / \mathrm{min}^{-\mathrm{m}^{2}}$ (Hi) levels of hyperinsulinemia. Compartmental modeling with arterial input function of $\left.\left[{ }^{15} \mathrm{O}\right]-\mathrm{H}_{2} \mathrm{O},{ }^{[11} \mathrm{C}\right] 3-\mathrm{OMG}$, and $\left[{ }^{18} \mathrm{~F}\right] \mathrm{FDG}$ PET analogs provided quantitative rates of glucose delivery, transport, and phosphorylation, respectively, within soleus muscle. Rates of glucose delivery $\left(k_{1}\right)$ did not increase with higher insulin dose stimulation, nor was $\mathrm{k}_{1}$ significantly different across groups. Insulin-stimulated inward glucose transport rates $\left(k_{3}\right)$ were significantly higher $(p<0.05)$ in NW vs. T2D during Mid, and were also higher $(p<0.01)$ in $\mathrm{NW}$ vs. $\mathrm{OB}$ and $\mathrm{T} 2 \mathrm{D}$ during $\mathrm{Hi}$. The fractional phosphorylation rate of glucose $\left(k_{5}\right)$ trended higher in NW ( $p=0.07$ ) vs. T2D (Mid) although this difference was not evident in Hi. These PET imaging studies provide novel in vivo human data indicating that greater insulin stimulation reduces the defect in glucose phosphorylation in T2D. Defects in glucose transport, however, persist in both $\mathrm{OB}$ and T2D. Further PET imaging studies could determine how specific defects in glucose metabolism are rectified by interventions designed to improve insulin resistance in obesity and $\mathrm{T} 2 \mathrm{D}$.

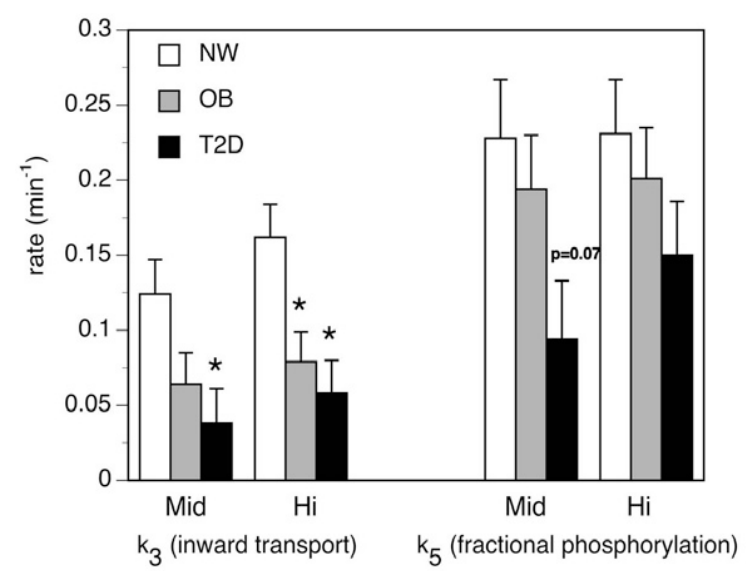

Supported by: NIH/NIDDK R01

30-0R

Capillary Density is Blunted in the Postprandial State in Men With T2DM or Metabolic Syndrome

RENATE E. VAN GENUGTEN, ERIK H. SERNÉ, DANIËL H. VAN RAALTE, MICHAELA

DIAMANT, Amsterdam, The Netherlands

Hyperinsulinemia during a euglycemic-hyperinsulinemic clamp increases capillary density, which contributes to overall glucose uptake. We investigated whether similar responses occur after meal-ingestion and hypothesized that postprandial capillary recruitment, compared to controls, is impaired in metabolic syndrome (MetS) and type 2 diabetes (T2DM), and associates with insulin sensitivity and postprandial hyperglycemia. We recruited 12 healthy men $(\mathrm{HC}), 13$ with MetS (IDF-criteria) without T2DM and 12 with uncomplicated T2DM. Microvascular function was assessed by skin capillary videomicroscopy. Capillary density at baseline and during post-occlusive peak reactive hyperemia (PRH) was measured fasting (TO), 60 (T60) and $120 \mathrm{~min}$ (T120) after ingestion of a mixed-meal. Oral glucose insulin sensitivity (OGIS) and postprandial hyperglycemia (4h-AUCgluc) were calculated. Groups were matched for age (mean $\pm S D: 56.6 \pm 5.7 y$ ) and differed for BMI (HC 26.9 \pm 2.1 ; MetS $32.1 \pm 2.7 ;$ T2DM 30.4 $\pm 14.7 \mathrm{~kg} / \mathrm{m} 2$ ), fasting glucose (HC 5.3 \pm 0.2 ; MetS 5.8 \pm 0.6 ; T2DM 8.0 $\pm 2.0 \mathrm{mmol} / \mathrm{l}$ ) and $\mathrm{HbA1c}$ (HC 5.4 \pm 0.2 ; MetS 5.6 $\pm 0.2 ;$ T2DM 6.7 $\pm 1.0 \%$ ). As expected, OGIS and 4h-AUCgluc differed between groups. Fasting baseline capillary density was similar among

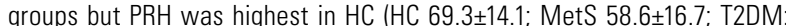
$56.6 \pm 7.3 \mathrm{n} / \mathrm{mm} 2 \mathrm{P}<0.05)$. Postprandial capillary recruitment was blunted in MetS and T2DM (PRH T60 vs. TO P $<0.05$ for HC only: T120 vs. TO $\mathrm{P}<0.05$ for all groups). In all groups, postprandial AUC for PRH correlated with OGIS (r0.492 $\mathrm{P}<0.05)$ and with 4h-AUCgluc $(\mathrm{r}-0.350 \mathrm{P}<0.05)$. In summary, both MetS and T2DM individuals are characterized by decreased capillary density during PRH and impaired meal-related effects on capillary recruitment. Postprandial PRH associated with both insulin sensitivity and postprandial hyperglycemia. These data suggest that microvascular dysfunction might contribute to postprandial hyperglycemia and may already be present in those at high risk to develop T2DM.

\section{MECHANISMS OF INSULIN RESISTANCE IN HUMANS - INFLAMMATION AND LIPIDS}

31-0R

Relationship Between Body Fat Depots and Spillover of Fatty Acids from Dietary Fat in Type 2 Diabetes: Effect of Weight Loss

JAIME ALMANDOZ, EKTA SINGH, KAREN GROTHE, LISA HOWELL, DANIELLE VLAZNY, ALMIRA SMAILOVIC, BRIAN IRVING, ROBERT H. NELSON, JOHN M. MILES, Rochester, MN

Spillover (SO) of lipoprotein lipase-generated fatty acids from chylomicrons into the plasma free fatty acid (FFA) pool is an important source of FFA and reflects inefficiency in dietary fat storage. The present study was conducted to examine the relationship between SO and various body fat depots, and to determine whether weight loss (WL) reduces SO in people with type 2 diabetes (T2DM) on oral agents ( $n=13,9$ men, 4 women, age $52 \pm 2$ $y$, BMI $\left.33.4 \pm 1.0 \mathrm{~kg} / \mathrm{m}^{2}, \mathrm{HbA1c} 7.9 \pm 0.3 \%\right)$. We measured SO with infusions of a $\left[{ }^{3} \mathrm{H}\right]$ triolein-labeled lipid emulsion and $\left[\mathrm{U}-{ }^{13} \mathrm{C}\right]$ oleate during ingestion of liquid mixed meal aliquots q $15 \mathrm{~min} \times 6.5 \mathrm{~h}$ to produce steady-state chylo- 
micronemia and suppression of lipolysis. Subjects were studied at baseline and again 5 months after starting a lifestyle modification program (diet and daily exercise). Body fat was measured with DXA and CT. At baseline, there was a strong negative correlation between $\mathrm{SO}$ and both total body fat $\mathrm{r}=$ $-0.78, P=0.002)$ and gynoid fat $(r=-0.75, P=0.003)$, whereas the negative correlation between $\mathrm{S} 0$ and android fat was borderline $(r=-0.52, P=0.07)$. SO was positively correlated with truncal:leg fat ratio $(R=0.56, P=0.047)$, but there was no correlation between SO and visceral fat area. Multivariate analysis revealed that leg fat was a stronger negative predictor of $S 0$ than truncal fat ( $\beta=-0.8$ vs. -0.3 ). All significant correlations disappeared after a $>13 \% \mathrm{WL}$, which decreased BMI to $29.2 \pm 1.1 \mathrm{~kg} / \mathrm{m}^{2}$ and $\mathrm{HbA1c}$ to $6.3 \pm 0.2 \%$ (both $\mathrm{P}<0.0001$ ). During WL, 11 of 12 subjects discontinued sulfonylureas. There was a decrease in both meal-suppressed FFA concentration (186 \pm 17 to $115 \pm 10 \mu \mathrm{mol} / \mathrm{L}, P<0.0001)$ and oleate flux $(65 \pm 9$ to $50 \pm 7 \mu \mathrm{mol} / \mathrm{min}, P=0.007)$. Fractional SO did not change $(28 \pm 3 \%$ to $31 \pm 4 \%, P=0.23)$. These results indicate that in T2DM SO relates negatively to fat storage capacity, and specifically that there is an inverse association between $\mathrm{SO}$ and lower body fat. WL results in marked improvement in meal-suppressed lipolysis, but not in SO.

$\Delta$ 32-0R Effect of Aging on JNK Phosphorylation and Insulin Resistance in Human Skeletal Muscle

SANGEETA GHOSH, RAWEEWAN LERTWATTANARAK, RALPH A. DEFRONZO, NICOLAS MUSI, San Antonio, TX

Aging is an important risk factor for glucose intolerance and type 2 diabetes. Mitogen activated protein kinases (MAPK), such as JNK and ERK, have been implicated in the development of insulin resistance in the elderly. We hypothesized that (i) MAPK signaling (phosphorylation of JNK and ERK) would be increased and insulin signaling (IRS1-tyrosine, AKT, AS160 and GSK3 phosphorylation) would be decreased in skeletal muscle from older subjects; and (ii) physical activity would reduce MAPK signaling and improve insulin action (signaling and sensitivity). Insulin sensitivity (M/I) was measured with a hyperinsulinemic ( $40 \mathrm{mU} / \mathrm{mg}$.min) euglycemic clamp and vastus lateralis muscle biopsies were performed in 13 lean, young, normal glucose tolerant (NGT) (age $=25 \pm 1 \mathrm{y}, \mathrm{BMl}=23.5 \pm 0.7 \mathrm{~kg} / \mathrm{m}^{2}, \quad V_{2}$ max $=27.3 \pm 2.5 \mathrm{ml} /$ kg.min, $\mathrm{M} / \mathrm{l}=17.8 \pm 2.1 \mathrm{mg} / \mathrm{kg} \mathrm{FFM.min} / \mathrm{mU} / \mathrm{ml} \times 100$ ) and 12 lean, older, NGT lage $=74 \pm 2 y, B M I=24.1 \pm 1 \mathrm{~kg} / \mathrm{m}^{2}, V_{2} \max =16.3 \pm 0.8, \mathrm{M} / \mathrm{l}=13.0 \pm 1.7 \mathrm{mg} / \mathrm{kg}$ FFM. $\mathrm{min} / \mathrm{mU} / \mathrm{ml}$ x100) subjects. In 11 older subjects, these measurements were done before and after a 16-week aerobic exercise program. At baseline, JNK phosphorylation was significantly elevated (2.4 fold, $\mathrm{P}<0.05)$ in muscle from older subjects. There was no difference in ERK between the groups. Before training, insulin stimulated AKT phosphorylation was significantly lower in the older group ( $50 \%$ of younger, P<0.05), however, IRS-1, GSK3 and AS160 phosphorylation were not different. The exercise training program did not affect phosphorylation of JNK, ERK or the phosphorylation of insulin signaling molecules (IRS-1, Akt, GSK3, AS160). Summary: (1) Aging is associated with increased MAPK (JNK) signaling; (2) Activation of JNK is associated with impaired insulin stimulation of proximal insulin signaling molecules (Akt); and (3) training does not reverse aging related changes in MAPK and insulin signaling in muscle from older subjects, suggesting that physical activity improves insulin sensitivity through a different mechanism.

Supported by: NIH (AG030979, DK080157) and American Federation for Aging Research

33-OR

High Oxidative Muscle is not Protected From Acute Lipid-Induced Insulin Resistance

JOHN J. DUBE, NICOLE L. HELBLING, MAJA STEFANOVIC-RACIC, PAUL M. COEN, KAZANNA C. HAMES, ALEX A. DESPINES, BRET H. GOODPASTER, Pittsburgh, PA

Excess accumulation of deleterious lipids, diacylglycerol (DAG) and ceramide, is associated with insulin resistance (IR). Exercise increases muscle oxidative capacity and improves lipid partitioning as evidenced by increased intramuscular triglyceride and decreased ceramide. We hypothesized that high oxidative muscle (exercise trained) would be protected from acute lipid-induced IR compared to sedentary subjects. Eleven endurance-trained (ET), 11 obese (OS) and 11 lean sedentary (LS) subjects completed two 6-h insulin clamps with a co-infusion of saline or Intralipid. IMCL, DAG and ceramide, insulin signaling and glucose metabolism proteins and ex vivo mitochondrial respiration were measured in biopsy samples in a fasted and insulin-stimulated condition. ET $(17.5 \pm 4.3)$ were more insulin sensitive $(\mathrm{mg} /$ $\mathrm{kgFFM} / \mathrm{min}$ ) at baseline compared to OS (10.7 \pm 3.9$)$. Baseline IMCL tended to be higher in ET compared to OS and LS, while DAG, ceramide or other sphingolipids we not different. In response to Intralipid: All subjects were equally insulin resistant $(-41.0 \pm 1.9 \%)$. IMCL increased similarly in all subjects
$(23.4 \pm 7.2 \%)$, while DAG and ceramide were unchanged. Dihydroceramide increased in LS $(27.0 \pm 27.5 \%)$, but not in ET $(-23.0 \pm 10.3 \%)$ and 0 S $(-26.5 \pm 9.0 \%)$. In all subjects, carbohydrate supported ex vivo mitochondrial respiration decreased $(-16.6 \pm 5.7 \%, P<0.05)$, P-Akt was unchanged, while PDK4 decreased $(-16.3 \pm 10.1 \%, P<0.05)$. Whole-body fat oxidation increased in $E T$ and $O S$ to a greater degree than LS. Carbohydrate oxidation was decreased to a greater degree in ET, while non-carbohydrate disposal was less effected. In summary, acute lipid-induced IR is not attenuated by high oxidative capacity muscle, nor associated with increased DAG or ceramide, decreased Akt activation or increased PDK4. Rather, key substrate pathways (whole-body fat oxidation and non-oxidative glucose disposal) handle the excess substrate at the expense of "insulin sensitivity" in exercise-trained muscle.

Supported by: NIH K01 DK084213-01

34-0R

Mechanisms of Lipid-Induced Insulin Resistance by Oral and Parental Application in Humans

BETTINA NOWOTNY, LEJLA ZAHIRAGIC, PETER J. NOWOTNY, JULIA SZENDROEDI, TORU YOSHIMURA, DONGYAN ZHANG, CHRISTIAN HERDER, NANETTE SCHLOOT, PETER SCHADEWALDT, GERALD I. SHULMAN, MICHAEL RODEN, Düsseldorf, Germany, New Haven, CT, Bad Homburg, Germany

Increased lipid availability and subclinical inflammation are involved in the development of insulin resistance. Increased free fatty acids (FFA) impair insulin action in skeletal muscle and liver. Besides direct cellular effects, FFA may activate immune cells, stimulate inflammatory processes and thereby affect insulin sensitivity. This study compared the effects of identical amounts and composition of lipids given i.v. or p.o. on muscular insulin sensitivity and systemic inflammation in humans. Healthy glucosetolerant subjects ( $5 \mathrm{f} / 11 \mathrm{~m}$; age $24 \pm 2$ years; body mass index $22.7 \pm 1.2 \mathrm{~kg} / \mathrm{m}^{2}$ ) were given in random order for 6 hours: i.v. Intralipid ${ }^{\circledR}$ infusion (IVL; $1.5 \mathrm{ml} /$ $\mathrm{min})$, p.o. corresponding amount of soy bean oil (POL; $100 \mathrm{ml}$ ) and i.v. glycerolinfusion (CON; $2.5 \%$ glycerol, $1.5 \mathrm{ml} / \mathrm{min}$ ). Insulin sensitivity was assessed by hyperinsulinemic-euglycemic clamps combined with isotopic dilution ([ $\left.{ }^{2} \mathrm{H}_{2}\right]$ glucose). Skeletal muscle biopsies were taken after 5 hours in 7 subjects. Time courses of metabolic, endocrine and inflammatory markers were analyzed with repeated blood sampling. Whole body insulin sensitivity (M value) was lower during IVL and POL at $5.7 \pm 0.4$ and $6.5 \pm 0.6 \mathrm{mg} / \mathrm{kg}^{*} \mathrm{~min}$ (all $p<0.01)$ compared with CON $\left(9.7 \pm 0.7 \mathrm{mg} / \mathrm{kg}^{*} \mathrm{~min}\right)$. Endogenous glucose production (EGP) was suppressed by insulin only after IVL $(-0.7 \pm 0.1$ vs. $-1.6 \pm 0.3$ $\mathrm{mg} / \mathrm{kg}^{*}$ min for CON; $\left.\mathrm{p}<0.05\right)$. IVL and POL were associated with a tendency to increased PKC theta levels in skeletal muscle $11.50 \pm 0.20$ and $1.49 \pm 0.23$ $p=0.046$ and 0.08 respectively) compared to CON. FFA and triglyceride levels were increased 1.7 fold after IVL, but not POL or CON $(p<0.01)$. Systemic levels of inflammatory markers (IL-6, TNF-alpha, IL-1ra) remained unchanged. In conclusion, increased lipid availability by both oral and intravenous routes results in comparable degree of peripheral insulin resistance which is likely mediated by novel PKC isoform activation, but does not seem not involve inflammatory pathways.

Supported by: EFSD

35-0R

Role of Diacylglycerol Activation of PKC $\theta$ in Lipid-Induced Muscle Insulin Resistance in Humans

JULIA SZENDRÖDI, TORU YOSHIMURA, ESTHER PHIELIX, MELISSA MARCUCCI, DONGYAN ZHANG, CHRISTIAN HERDER, PETER NOWOTNY, GERALD I. SHULMAN, MICHAEL RODEN, Düsseldorf, Germany, New Haven, CT

Multiple mechanisms have been invoked to explain muscle insulin resistance associated with obesity and type 2 diabetes (T2D) including inflammation and increased myocellular lipid metabolites [ceramides, diacylglycerols (DAGs)]. To examine which mechanism might underlie lipid-induced muscle insulin resistance in humans we measured circulating cytokines (TNF $\alpha$, IL-6, sICAM, adiponectin, RBP-4), intramuscular DAG and ceramide content, activities of protein kinase $C$ isoforms (PKC $\beta, \Delta, \theta$ ) before, at $2.5 \mathrm{~h}$ and $4 \mathrm{~h}$ lipid or glycerol infusion in healthy humans (CON, $n=16,30 \pm 5$ years, BMI: $24 \pm 2$ $\mathrm{kg} / \mathrm{m}^{2}$ ). In addition we assessed these parameters in obese, insulin resistant but glucose tolerant subjects $\left(O B, n=5,26 \pm 2\right.$ years, $\left.45 \pm 3 \mathrm{~kg} / \mathrm{m}^{2}\right)$ and patients with T2D (T2D, $n=4,61 \pm 1$ years, $\left.35 \pm 3 \mathrm{~kg} / \mathrm{m}^{2}\right)$. Hyperinsulinemic-euglycemic clamps combined with $\left[6,6-{ }^{2} \mathrm{H}_{2}\right]$ glucose were performed to measure insulin sensitivity. Whole-body insulin-stimulated glucose disposal was $57 \%$ lower during lipid infusion $\left(\mathrm{P}<10^{-6}\right)$ than during glycerol infusion. Myocellular total DAG increased $\sim 2$ fold $(P<0.005)$ at $2.5 \mathrm{~h}$ and remained $\sim 1.4$ fold higher $(P<0.05)$ at $4 \mathrm{~h}$ lipid infusion. Consistent with increasing muscle DAG content PKC $\theta$ activity increased by $64 \%(P<0.005)$ at $4 \mathrm{~h}$ lipid infusion. Circulating cytokines and myocellular ceramide content did not change. Insulin-stimulated 
glucose disposal was $78 \%$ and $88 \%$ lower in $\mathrm{OB}$ and $\mathrm{T} 2 \mathrm{D}$ (both $\mathrm{P}<0.005$ vs. CON). Cytosolic DAGs were $180 \%$ and $220 \%$ greater in OB and T2D than in $\mathrm{CON}$ (both $\mathrm{P}<0.0001$ vs. CON) while muscle ceramide content in $\mathrm{OB}$ and T2D were not increased compared to CON. In conclusion these data support the concept that DAG-induced activation of PKC $\theta$ is responsible for muscle insulin resistance during lipid infusion as well as in obese and type 2 diabetic individuals.

Supported by: DFG, DZD e.V., EFSD, Lilly, NIH (DK-49230, DK-045735)

\section{$\Delta$}

36-OR

Effect of Macronutrients on Markers of Oxidative Stress, Proinflammatory Cytokines, Adipokines, CV Risk Factors and Lipid Peroxidation in Obese Non-Diabetic Women

ABBAS E. KITABCHI, FRANKIE B. STENTZ, EBENEZER A. NYENWE, KRISTIN A. MCDANIEL, FRANCES A. TYLAVSKY, JIM Y. WAN, CHRISTOPHER W. SANDS, Memphis, TN

Various dietary macronutrients influence weight loss but metabolic advantages of high-protein (HP) diet (30\% protein, $30 \%$ fat, and $40 \% \mathrm{CHO}$ ) vs high-carbohydrate (CHO) diet (HC) (15\% protein, 30\%fat, $55 \% \mathrm{CHO}$ ) are not established. We assessed the effect of HP vs HC diets on biomarkers of insulin sensitivity, oxidative stress, cardiovascular risk (CVR), lipid peroxidation and weight loss for 6 months. Diets were designed as a $500 \mathrm{Kcal}$ reduction from each subject's resting energy expenditure and provided by prepackaged foods on a weekly basis. The subjects were non-diabetic, obese, premenopausal females ages 20 to 50 years with $\mathrm{BMI} \geq 30$ to $\leq 55$ who were randomized to the HP or HC diet. An Oral Glucose Tolerance Test (OGTT) and Meal Tolerance Test (MTT) (HP or HC) were done with sampling at 0,30, 60,90 , and 120 minutes at baseline and 6 months. To date $23(12 \mathrm{HP}, 11 \mathrm{HC})$ women were studied in a randomized prospective protocol. Proinflammatory cytokines (TNF $\alpha$, IL6), markers of oxidative stress (dichlorofluorescein - DCF), lipid peroxidation (MDA), insulin sensitivity (HOMA IR), CVR (BP,BMI, CRP, Eselectin), adiponectin and leptin were measured at baseline and 6 months.

\begin{tabular}{|c|c|c|c|c|c|c|c|}
\hline \multirow[b]{2}{*}{ Parameters } & \multicolumn{3}{|c|}{$\mathrm{HP}(\mathrm{n}=12)$} & \multicolumn{3}{|c|}{$\mathrm{HC}(\mathrm{n}=11)$} & \multirow[b]{2}{*}{$\mathrm{p}^{* *}$} \\
\hline & Baseline & 6 months & $p^{*}$ & Baseline & 6 months & $p^{*}$ & \\
\hline$\overline{B M I}\left(\mathrm{~kg} / \mathrm{m}^{2}\right)$ & $41.3 \pm 1.8$ & $37.3 \pm 1.9$ & $<0.001$ & $37.4 \pm 1.7$ & $34.1 \pm 1.6$ & 0.002 & 0.391 \\
\hline \% Weight Loss & & $9.8 \pm 1.4$ & $<0.001$ & & $8.7 \pm 1.8$ & $<0.001$ & 0.692 \\
\hline$\overline{\mathrm{BP} \text { (sys/diast) }}$ & $130 / 84 \pm 4 / 3$ & $118 / 73 \pm 3 / 3$ & $.01 / .01$ & $129 / 83 \pm 3 / 3$ & $119 / 74 \pm 3 / 3$ & $.01 / .01$ & $.70 / 81$ \\
\hline $\mathrm{TG}(\mathrm{mg} / \mathrm{dl})$ & $113 \pm 16$ & $86 \pm 11$ & 0.01 & $114 \pm 13$ & $95 \pm 10$ & 0.01 & 0.02 \\
\hline hCRP (mg/L) & $5.6 \pm 4.8$ & $3.83 \pm 2.1$ & 0.01 & $5.4 \pm 4.7$ & $4.0 \pm 3.8$ & 0.03 & 0.04 \\
\hline E-Selectin (ng/ml) & $42.6 \pm 1.3$ & $37.4 \pm 1.2$ & 0.02 & $43.4 \pm 1.4$ & $39.7 \pm 1.6$ & 0.07 & 0.03 \\
\hline HOMA IR & $4.1 \pm 0.8$ & $1.6 \pm 0.2$ & 0.01 & $3.9 \pm 0.5$ & $2.7 \pm 0.3$ & 0.02 & 0.04 \\
\hline$\overline{\mathrm{DCF}(\mu \mathrm{M})}$ & $3.4 \pm 0.2$ & $2.3 \pm 0.1$ & 0.01 & $3.3 \pm 0.3$ & $2.8 \pm 0.1$ & 0.04 & 0.02 \\
\hline$\overline{\mathrm{MDA}(\mu \mathrm{M})}$ & $1.3 \pm 0.07$ & $0.6 \pm 0.04$ & 0.01 & $1.2 \pm 0.08$ & $0.9 \pm 0.07$ & 0.04 & 0.04 \\
\hline$\overline{\mathrm{TNF} \alpha(\mathrm{pg} / \mathrm{ml})}$ & $5.0 \pm 2.8$ & $4.08 \pm 2.4$ & 0.03 & $6.0 \pm 3.0$ & $5.5 \pm 2.6$ & 0.05 & 0.02 \\
\hline IL-6 (pg/ml) & $6.2 \pm 4.2$ & $5.8 \pm 1.8$ & 0.63 & $5.7 \pm 4.4$ & $5.4 \pm 1.3$ & 0.68 & 0.08 \\
\hline Adiponectin (ng/ml) & $5556 \pm 81$ & $5840 \pm 87$ & 0.01 & $5531 \pm 77$ & $5471 \pm 69$ & 0.6 & 0.01 \\
\hline Leptin (ng/ml) & $43.4 \pm 3.7$ & $29.6 \pm 3.1$ & 0.01 & $44.6 \pm 3.9$ & $33.1 \pm 3.4$ & 0.02 & 0.07 \\
\hline \% Compliance & & 94 & & & 89 & & 0.646 \\
\hline
\end{tabular}

The * indicates Wilcoxon Signed Rank Test and ${ }^{* *}$ indicates Wilcoxon Rank Sum Test for 6 months HPvs HC Although both diets demonstrated significant but similar improvement in weight loss, and BP; with similar compliance, the HP diet exhibited greater improvement in insulin sensitivity, CV Risk Factors, adipokines, lipid peroxidation and inflammatory parameters than the $\mathrm{HC}$ diet.

37-0R

Acute Endotoxemia Reduces Insulin Sensitivity and Glucose Effectiveness in Healthy Caucasians, but not in African Americans

RHIA Y. SHAH, JANE F. FERGUSON, RACHANA SHAH, MICHAEL R. RICKELS, NEHAL N. MEHTA, MUREDACH P. REILLY, Philadelphia, PA

Previous studies have shown marked variation in metabolic responses to endotoxemia and have supported the importance of insulin resistance in cardiometabolic disease. We hypothesized that the change in insulin sensitivity induced by endotoxemia may differ by race and gender. Healthy volunteers (ages 18-45, BMI (body mass index) 18-30, 47\% female, African American $\mathrm{N}=30$ and Caucasian $\mathrm{N}=69$ ), recruited as part of the Genetics of Evoked-Responses to Niacin and Endotoxemia (GENE) Study, were subjected to an inpatient endotoxin challenge (1 $\mathrm{ng} / \mathrm{kg}$ lipopolysaccharide (LPS)). Insulin and glucose values obtained during a frequently sampled intravenous glucose tolerance test (FSIGTT) pre- and post-LPS were analyzed using the minimal model (MINMOD). At baseline, African Americans had significantly lower insulin sensitivity ( $S_{\text {.) }}$ ) than Caucasians (2.5 vs. $4.4 \mathrm{P}<0.0001$ ), however pancreatic beta cell function as measured by the acute insulin response to glucose (AIR) , was significantly higher in African Americans (1010 vs. 405 , $\mathrm{P}<0.0001)$. BMl did not differ between groups, and there were no within-race differences by gender. Remarkably, while $\left(S_{i}\right)$ and glucose effectiveness $\left(S_{g}\right)$ declined significantly in Caucasians post-LPS (4.4 to 2.8 and 0.02 to 0.016 , $P<0.0001$ ), there was no significant change in African Americans (2.5 to 2.2 and 0.019 to 0.018). While baseline insulin sensitivity was lower in African Americans than in Caucasians, this appeared to be compensated for by a higher pancreatic beta cell function, indicating race-specific differences in maintaining glucose and insulin homeostasis. LPS induced a robust reduction in insulin sensitivity in Caucasians, not observed in African Americans. The specific effect of inflammation on insulin sensitivity may thus be different in African Americans compared to Caucasians, providing insight into mechanisms of race differences in cardiometabolic traits.

38-OR

Combined Training Improves Ex-vivo Skeletal Muscle Substrate Metabolism in Type 2 Diabetics

LAUREN M. SPARKS, NEIL M. JOHANNSEN, TIMOTHY S. CHURCH, ESTHER MOONEN-KORNIPS, MATTHIJS K. HESSELINK, STEVEN R. SMITH, PATRICK SCHRAUWEN, Maastricht, The Netherlands, Baton Rouge, LA, Orlando, FL

Type 2 diabetes (T2D) is a disease of disordered lipid and glucose metabolism. The current study investigated effects of 9-months of aerobic (AT), resistance (RT) or combined aerobic and resistance (ATRT) exercise training on mitochondrial content and ex vivo myocellular lipid oxidation in individuals with T2D. Fifty-two individuals $\left(57.5 \pm 7.6 y, 34.8 \pm 5.7 \mathrm{~kg} / \mathrm{m}^{2}\right)$ from the HART$D$ study were randomized to 9 -months of either AT $(n=13)$, RT ( $n=17)$, ATRT $(n=12)$ or non-exercise Control $(n=10)$. Muscle biopsies were taken before and after intervention, and ex vivo palmitate, octanoate, pyruvate and acetate oxidation $\left({ }^{14} \mathrm{C}\right.$ labeled $\mathrm{CO}_{2}$ production) were measured. Mitochondrial DNA (mtDNA) copy number was assessed by qPCR. Data were not normally distributed and natural log transformed. All variables were similar across groups at baseline. Mitochondrial content was higher in RT and ATRT after intervention compared with Control (mean $\pm 95 \% \mathrm{Cl} ; 1104 \pm 163$ and $1106 \pm 192$ vs. $793 \pm 150$, resp; $P<0.05$ for both). Complete palmitate oxidation was higher after intervention in all exercise groups compared with Control $(0.89 \pm 0.37$, $1.07 \pm 0.40$ and $1.27 \pm 0.55$ vs. $0.35 \pm 0.16 \mathrm{nmol} / 2 \mathrm{~h} / \mathrm{mg}$ protein for AT, RT, ATRT vs. Control, resp; $\mathrm{P}<0.05)$; however, only AT and ATRT had higher complete to incomplete palmitate ratios compared with Control $(0.26 \pm 0.10$ and $0.56 \pm 0.29$ vs. $0.06 \pm 0.03$, resp; $P<0.05$ ). Complete pyruvate and acetate oxidation were significantly higher after all 3 exercise modalities compared with Control $(\mathrm{P}<0.05$ for all), while complete octanoate oxidation was significantly higher after AT and ATRT compared with Control $(1.20 \pm 0.53$ and $1.16 \pm 0.52$ vs $0.34 \pm 0.16$, resp; $P<0.05$ ). Nine months of RT and ATRT significantly improves mitochondrial content in T2Ds. While AT and RT alone improve some aspects of ex vivo lipid oxidation, only ATRT improves all measures of lipid and carbohydrate oxidation indicating a combined exercise program as a more effective lifestyle therapy to improve muscle substrate metabolism in T2Ds.

Supported by: NIH Grant DK068298 and VICI Grant 918.96 .618

\section{STIMULUS-SECRETION COUPLING IN ISLET CELLS}

\section{9-0R}

Functional Evaluation of Islets from Infants With KATP Hyperinsulinism

PUJA PATEL, CHANGHONG LI, STEPHANIE GIVLER, FRANZ M. MATSCHINSKY, CHARLES A. STANLEY, DIVA D. DE LEON, Philadelphia, PA

Loss-of-function mutations in the $\mathrm{K}_{\text {ATP }}$ channel are the most common cause of congenital hyperinsulinism $\left(\mathrm{K}_{\mathrm{ATP}} \mathrm{HI}\right)$. The clinical phenotype of $\mathrm{K}_{\mathrm{AT}}$ ${ }_{\mathrm{p}} \mathrm{HI}$ is characterized by severe fasting and protein-induced hypoglycemia. In contrast, glucose-stimulated insulin secretion is impaired. Islets from the $\mathrm{K}_{\mathrm{ATP}} \mathrm{HI}$ mouse model (SURT\%) have elevated cytosolic calcium and respond to stimulation with amino acids but not glucose. Our goal is to examine the fuel responsiveness and cytosolic calcium changes in $\mathrm{K}_{\text {ATP }} \mathrm{HI}$ human islets. We hypothesize that $\mathrm{K}_{\mathrm{ATP}} \mathrm{HI}$ islets will have similar responses as $\mathrm{SURT} \%$ islets. Islets from 27 diffuse $\mathrm{HI}$ cases (age $5.9 \pm 2.3 \mathrm{~m}$ ) were collected and compared to islets from cadaver donors. $21 \mathrm{HI}$ cases have confirmed diseasecausing mutations in $A B C C 8(\mathrm{n}=19)$ or $K C N J 11(\mathrm{n}=2) .6$ cases were negative for mutations in either gene. Islet fuel responsiveness and calcium influx were examined by perifusion and static incubations. Basal insulin secretion in perifused $\mathrm{K}_{\mathrm{ATP}} \mathrm{HI}$ islets was greater than in controls (3.0 $\pm 0.5 \mathrm{vs} .0 .4 \pm$ 
$0.1 \mathrm{ng} / 150$ islets/min, $\mathrm{p}<0.01$ ). Basal $\left[\mathrm{Ca}^{2+}\right]_{i}$ was higher compared to controls $(1.68 \pm 0.07$ vs. $0.86 \pm 0.03-340 / 380$ ratio, $p<0.01)$. Opposite to controls, $\left[\mathrm{Ca}^{2+}\right]_{\mathrm{i}}$ and insulin secretion in $\mathrm{K}_{\mathrm{APP}} \mathrm{HI}$ islets increased after stimulation with amino acids but not glucose. Exendin (9-39), a GLP-1 receptor antagonist, inhibited amino acids-stimulated insulin secretion in $\mathrm{K}_{\mathrm{ATP}} \mathrm{HI}$ islets. Islets from 3 cases with clinical phenotype of $\mathrm{K}_{\mathrm{ATP}} \mathrm{HI}$ but without confirmed mutations exhibited the same fuel responsiveness as islets from genetically confirmed $\mathrm{K}_{\mathrm{ATp}} \mathrm{HI}$ cases. In summary, $\mathrm{K}_{\mathrm{ATp}} \mathrm{HI}$ islets replicate the islet phenotype of the SUR1\% mouse and are congruent with the clinical phenotype. The stimulatory effect of amino acids in $\mathrm{K}_{\mathrm{ATP}} \mathrm{HI}$ islets may be mediated by the GLP-1 receptor. Overall, the clinical and islet phenotype may help elucidate the pathophysiology in cases without confirmed mutations. Further studies may reveal specific genotype-phenotype correlations.

Supported by: The Goldsmith Foundation

$\Delta$

40-0R

Plasma Membrane Electron Transport Regulates Redox Status and Insulin Secretion in Pancreatic Beta Cells

JOSHUA P. GRAY, GARY W. CLINE, REBECCA F. FOLLMER, EMMA A. HEART, New London, CT, New Haven, CT, Woods Hole, MA

Plasma Membrane Electron Transport (PMET) mediates the oxidation of intracellular reducing equivalents and the transfer of their electrons to extracellular targets. PMET, a plasma membrane analog of the mitochondrial electron transport chain, consists of a family of cytosolic and plasma membrane oxidoreductases, electron carriers and terminal acceptors. PMET activity, measured by the degree of reduction of extracellular membrane impermeable electron acceptors (ferricyanide and WST-1), is proportional to the concentration of glucose in beta cells. Using INS-1 832/13 cells and isolated islets from N001 knockout mice, we have investigated role of N001, a NAD(P)H-dependent cytosolic oxidoreductase and component of PMET, on beta cell intermediary metabolic pathways and glucose-stimulated insulin secretion (GSIS). Adenoviral-mediated over-expression of NQ01 increased PMET activity in parallel to a decrease in the NADH/NAD ratio in INS-1 832/13 cells, in accordance with PMET-dependent re-oxidation of NADH. Since the NADH/NAD+ ratio regulates glycolytic flux, the effect of NOO1 over-expression on glucose oxidation and utilization was measured. NQ01 over-expression increased glucose oxidation and utilization and enhanced insulin secretion in response to stimulatory glucose levels. In contrast, INS$1832 / 13$ cells with adenoviral-mediated N001 knock-down and N001-KO islets had decreased PMET, elevated NADH/NAD+ ratio, decreased glucose oxidation and utilization and decreased GSIS. These data demonstrate a critical role for the PMET/NQO1 axis in the regulation of beta cells NADH redox status, glycolytic flux and insulin secretion.

Supported by: R56DK088093

41-0R

Lipolysis-Derived Long Chain Saturated Monoacylglycerol as a Metabolic Coupling Factor in Glucose-Stimulated Insulin Secretion SHANGANG ZHAO, JOSE IGLESIAS, YVES MUGABO, VIVIANE DELGHINGAROAUGUSTO, IAN BOUDREAU, ROXANE LUSSIER, MARCO PINEDA, MARIE-LINE PEYOT, ERIK JOLY, MURTHY MADIRAJU, MARC PRENTKI, Montreal, QC, Canada

Glycerolipid/free fatty acid cycle and its lipolytic segment are essential components of the lipid amplification pathway of glucose stimulated insulin secretion (GSIS) by $\beta$-cells. However, little is known about the molecular nature of the lipid metabolic coupling factor(s) involved in this process. We now provide evidence that monoacylglycerol (MAG) acts as a metabolic coupling factor in GSIS. Obliterated GSIS, due to lipolysis inhibition in $\beta$-cells with orlistat, is restored by exogenous MAG. In rat islets and INS832/13 $\beta$-cells MAG levels increase in the presence of high glucose concentration. Inhibition of the major membrane-bound MAG hydrolase, ABHD6, in INS832/13 cells and islets with WWL70 leads to accumulation of MAG with concomitant increase in GSIS. The predominant MAG species elevated by high glucose as well as by WWL70 addition are the saturated long chain MAGs 1-stearoylglycerol (C18:0) and 1-palmitoylglycerol (C16:0), which strongly potentiate GSIS in vitro, unlike monounsaturated and polyunsaturated MAGs. Overexpression and RNAi-knockdown of ABHD6 in INS832/13 cells, resulted in decreased and increased GSIS, respectively. Administration of WWL70 (i.p.) in normal CD-1 mice enhance GSIS, and it greatly improves glucose tolerance by increasing insulin secretion in the low-dose streptozotocin type 2 diabetes mouse model. We also show that MAGs can bind the C1-domain of the exocytotic effector protein, Munc13-1 more efficiently than diacylglycerol, and as efficiently as the phorbol ester PMA, an established activator of Munc13-1. Collectively, the results provide strong evidence that the lipid amplification arm of GSIS in $\beta$-cells is mediated by lipolysis-derived long chain saturated MAGs that activate insulin exocytosis via Munc 13-1. ABHD6 is the major MAG hydrolase in the $\beta$-cell and is a new target for the development of anti-diabetic drugs.

\section{2-0R}

Gck-Mody Due to Protein Misfolding, Cellular Self-Association and Degradation

MARIA NEGAHDAR, BENTE B. JOHANSSON, INGVILD AUKRUST, JANNE MOLNES, JØRN SAGEN, KNUT DAHL-JØRGENSEN, ANDERS MOLVEN, FRANZ M. MATSCHINSKY, ROHIT N. KULKARNI, ODDMUND SØVIK, TORGEIR FLATMARK, PÅL R. NJØLSTAD, LISE BJøRKHAUG, Bergen, Norway, Oslo, Norway, Philadelphia, PA, Boston, MA

Heterozygous inactivating mutations in the glucokinase (GK) encoding gene (GCK) generally increase the threshold for glucose-stimulated insulin secretion, resulting in mildly elevated fasting plasma glucose levels (5.5$8.0 \mathrm{mmol} / \mathrm{L}$ ) and maturity-onset diabetes of the young (GCK-MODY). The purpose of the present study was to address the question whether protein misfolding and instability could be the molecular basis for the previously unexplained GCK-MODY mutations S263P, G264S and R275C. These mutations, when expressed as recombinant mutant proteins, have demonstrated near-normal (wild-type) enzyme kinetics and normal interactions with known regulatory proteins. Here, an instability of the recombinant mutant proteins was observed by limited proteolysis (S263P and G264S) and by an in vitro ubiquitin-proteasomal degradation system (all three mutants). The recovery and molecular properties of the mutant proteins were investigated in stably transfected MIN6 $\beta$-cells and HEK293 cells. All three mutants revealed an increased rate of cellular degradation with a contribution of both the lysosomal and proteasomal quality control systems. Moreover, the mutations lead to misfolded and conformationally unstable proteins causing dimerization and aggregation (R275C>G264S>S263P), and were recovered both in the cytosol and in a subcellular fraction enriched in lysosomes. Our structural analyses demonstrate that the residues S263, G264 and R275 contribute to the stability of the F260-L270 loop structure, and that the single point mutations elicit conformational changes in the loop structure which is considered to promote the dimerization of their mutant proteins by a domain swapping mechanism. Our data underline the importance of a cell biological approach to fully characterize the GCK-MODY mutant enzymes, and suggests a possible new therapeutic approach by prevention of self-association (conformational rescue) of some mutant proteins using bioactive small molecules.

43-0R

The Roles of Voltage-Gated Potassium Channels Kv2.1 and Kv2.2 in the Regulation of Insulin and Somatostatin Release from Pancreatic Islets

XIAOYAN NINA LI, Rahway, NJ

Objectives: To define the role(s) of voltage-gated potassium channel Kv2.1 and Kv2.2 in the regulation of islet hormone secretion. Research Design and Methods: Using genetic and pharmacological approaches, we examined the effects of Kv2 inhibition on insulin and somatostatin release in isolated islets and perfused pancreata, as well as in glucose tolerance in vivo. Results: Pancreatic $\beta$-cells from Kv2.1\% mice possess reduced Kv current and display greater glucose-stimulated insulin secretion (GSIS) relative to wild-type $\beta$-cells. Inhibition of Kv2 channels with peptidyl (GxTX-1E) or selective small molecule (RY796) inhibitors enhanced GSIS in isolated mouse and human islets. However, in wild-type mice neither inhibitor improved glucose tolerance in vivo, and this lack of glucose lowering effect was associated with an increase in plasma somatostatin levels. GXTX-1E and RY796 enhanced somatostatin release in isolated human and mouse islets and perfused pancreata from WT and Kv2.1\% mice; Moreover, Kv2.2 silencing in mouse islets by adenovirus-shRNA specifically enhanced islet somatostatin, but not insulin, secretion. In mice lacking somatostatin receptor 5, GXTX-1E significantly elevated plasma insulin and improved glucose tolerance. Conclusions: Kv2.1 and Kv2.2 channels play distinct roles in pancreatic islets - with Kv2.1 primarily regulating insulin secretion in $\beta$-cells and Kv2.2 modulating somatostatin release in $\delta$-cells. Paracrine somatostatin is a powerful inhibitor of $\beta$-cell function and can counteract mechanisms that normally would enhance insulin secretion, such as Kv2.1 inhibition. Development of selective Kv2.1 inhibitors may provide new avenues to promote GSIS for the treatment of type 2 diabetes. 
Glucose Dependent Down Regulation of GLP-1 Receptor SINDHU RAJAN, ELIZABETH MATHEW, BARTON WICKSTEED, LOUIS H. PHILIPSON, Chicago, IL

Glucagon Like Peptide-1 based therapy is central to the treatment of Type 2 diabetes. Activation of GLP-1 receptor (GLP-1R) results in rapid elevation of cAMP and enhanced insulin secretion. However, the incretin effect may be significantly reduced in diabetes. We show for the first time that forward trafficking of GLP-1R to the plasma membrane in pancreatic beta cells is impaired in hyperglycemia and Protein Kinase A (PKA) dependent posttranslational modification by SUMO (Small Ubiquitin-like Modifier protein) is an important contributing mechanism. Pancreatic islets and insulinoma cells exposed to high glucose conditions showed diminished GLP-1R density at the plasma membrane. Hyperglycemia augmented expression levels of SUMO and SUMO conjugating enzyme, Ubc-9. Elevated SUMO levels resulted in impaired cell surface trafficking of the GLP-1R and attenuated receptor signaling. In addition, elevated expression of SUMO also inhibited insulin secretion and caused reduced insulin content. The role of hyperglycemia induced SUMO modification in GLP-1R function was evaluated in mouse islets by partial knock-down of SUMO conjugating enzyme Ubc-9 by retroviral shRNA GLP-1R agonist stimulated insulin secretion was significantly enhanced in Ubc-9 shRNA transduced mouse islets exposed to high glucose concentrations. A dynamic FRET based real-time protein interaction assay between GLP-1R-CFP and YFP-SUMO showed that SUMO modification of GLP-1R is dependent on PKA activity. Increasing CAMP levels or expression of constitutively active PKA resulted in intracellular retention of the GLP-1R, both in high and low glucose. Furthermore, down-regulation of PKA activity by expressing a dominant negative PKA subunit was able to reverse SUMO mediated glucose induced intracellular retention of the receptor. We conclude that a glucose induced "phospho SUMIOyl switch" modulates the function of GLP-1R. Understanding the mechanisms that regulate GLP-1 receptor will help improve drug efficacy of GLP-1 based treatments.

Supported by: NIDDK, Merck (IISP)

45-0R

WFS1 Protein Regulates Insulin Granule Exocytosis via Maintenance of Intragranular Acidification and Modulation of the SNARE Proteins

MANABU KONDO, MASAYUKI HATANAKA, KATSUYA TANABE, YASUHARU OHTA, YUKIO TANIZAWA, Ube, Japan

Wolfram syndrome (WFS) is an autosomal recessive disorder characterized by juvenile-onset diabetes mellitus and optic atrophy. The gene responsible for the disorder, WFS1, encodes a transmembrane protein. Previous studies suggested that WFS1 protein localizes to endoplasmic reticulum (ER) and is related to ER stress responses. Recently we found that WFS1 protein also localizes to insulin secretory granules and determines granule acidification. Intragranular acidification is essential for priming of insulin secretory granule. However, the role of WFS1 protein in glucose-stimulated insulin secretion (GSIS) is still unknown. Moreover, detailed analyses of insulin dynamics of WFS1-deficient beta cell have not been performed yet. In the present study, we conducted in vivo and in vitro experiments to elucidate the role of WFS1 protein in GSIS. All the experiments were performed with young WfS1-null mice (8-10 weeks of age), because both beta cell mass and pancreatic insulin content were similar to those of wild type mice at this age. In perfusion experiments with $16.7 \mathrm{mM}$ glucose, GSIS, especially in the first phase, was markedly blunted in Wfs1-null mice. Stimulatory effect of high $\mathrm{K}^{+}$ was also impaired in these experiments. Exendin- 4 remained stimulatory in Wff1-null mice (first phase $=3.2$ fold increase; second phase $=4.4$ fold increase), but stimulation was nevertheless reduced relative to wild type (3.8 fold and 10.5 fold, respectively). These results were consistent with static incubation study using isolated islets, and ipGTT. Western blot analysis of WFS1-deficient islets revealed significantly decreased expression level of Syntaxin1A and SNAP25, both of them are reported to be involved in the first phase insulin secretion. Taken together, these results suggest that WFS1 protein play an important role in granule exocytosis, especially in the triggering pathway, via maintenance of granular acidification and modulation of SNARE proteins
Phosphatidylinositol 4,5-Biphosphate Modulates Interaction of Syntaxin-1A With Sulfonylurea Receptor 1 to Regulate Pancreatic Beta-Cell ATP-Sensitive Potassium Channels

TAO LIANG, CHRISTIN CHAO, YOUHOU KANG, TAIRAN OIN, LI XIE, HERBERT Y. GAISANO, Toronto, ON, Canada

Pancreatic beta-cell regulates glucose-stimulated insulin secretion through association with ATP-sensitive potassium (KATP) channels. We previously demonstrated that syntaxin (Syn)-1A, a SNARE protein mediating exocytotic fusion, binds and inhibits the KATP channels at its sulfonylurea receptor (SUR) 1 regulatory subunit. Phosphatidylinositol 4,5-biphosphate (PIP2) participates in a wide variety of cellular functions including regulation of KATP channel by its direct action on the Kir6.2 subunit, and also from interactions with various exocytotic fusion proteins, including syn-1A. In this study, we have hypothesized that these actions of PIP2 on Syn-1A could influence Syn-1A interactions with SUR1 to affect KATP channel activities in rat islet beta-cells, using FRET assay and patch clamp electrophysiology. FRET data showed that PIP2 can disrupt the intimate FRET interactions of full length SUR1 and Syn-1A on the plasma membrane in living cells. To examine whether PIP2 could block Syn-1A inhibition of SUR1/KATP channel current, we performed inside-out patch-clamp recordings in INS-1E cells and found that exposure to PIP2 can fully recover the Syn-1A-inhibitory. To delineate the physiological relevance of our findings in INS-1E, we employed rat islet beta-cells using whole-cell recordings. Dialysis of GST-Syn-1A with PIP2 gave a maximum KATP current density equal to GST control, compared to much reduced current by GST-Syn-1A alone. Taken together, our results demonstrate that application of PIP2 could disrupt the molecular interaction between Syn-1A and SUR1, which abolished Syn-1A inhibitory on KATP channels. Our findings suggest the effect of membrane lipid composition on beta-cell insulin secretion is contributed by its profound consequences on membrane electrical activity through several modes of actions, now including regulating Syn-1A interactions with SUR1.

Supported by: CIHR

\section{DIABETIC DYSLIPIDEMIA}

47-0R

Long-Term Atorvastatin Administration Improves Baroreflex Sensitivity in Subjects With Type 2 Diabetes and Dyslipidaemia PINELOPI GRIGOROPOULOU, IOANNA ELEFTHERIADOU, CHRISTOS ZOUPAS, DESPOINA PERREA, STAUROS LIATIS, KONSTANTINOS MAKRILAKIS, NICHOLAS TENTOLOURIS, Athens, Greece

Baroreflex sensitivity (BRS) is reduced in patients with type 2 diabetes mellitus (T2DM), even in the absence of clinically apparent autonomic neuropathy. Statins improve endothelial function and BRS in subjects without diabetes. However, no data exist on the effect of treatment with statins on BRS in individuals with T2DM. This study examined the effect of chronic administration of low dose of atorvastatin on BRS in subjects with T2DM. A total of 76 patients with T2DM and dyslipidemia were recruited. The main inclusion criteria were age between $40-75$ years, low density lipoprotein cholesterol $(\mathrm{LDLC}) \geq 100 \mathrm{mg} / \mathrm{dl}$ and absence of clinically apparent macrovascular disease.. Forty-three patients (15 men/28 women) were assigned to atorvastatin (10 mg per day) and low-fat diet and 33 age-matched patients (15 men/18 women) to low-fat diet only. Both groups were comparable regarding baseline serum lipids, HbA1c, arterial blood pressure and $B R S$ values. BRS was measured by the sequence method using the BaroCor system (Atcor Medical, Australia). All patients underwent 3 more visits, at 3,6 and 12 months, during which the same blood tests and BRS estimation were repeated. Atorvastatin significantly reduced total cholesterol by $27.30 \%$ (from $235.88 \pm 28.41 \mathrm{mg} / \mathrm{dl}$ to $170.05 \pm 21.33 \mathrm{mg} / \mathrm{dl}, \mathrm{p}<0.001$ ), LDLc by $36.9 \%$ (from $154.91 \pm 26.41 \mathrm{mg} / \mathrm{dl}$ to $96.42 \pm 22.65 \mathrm{mg} / \mathrm{dl}, \mathrm{p}<0.001$ ) and TG by $12.86 \%$ (from $147.30 \pm 71.46 \mathrm{mg} / \mathrm{dl}$ to $117.42 \pm 56.03 \mathrm{mg} / \mathrm{dl}, \mathrm{p}=0.001$ ) after 12 months.nBRS was significantly improved in atorvastatin treated patients after 12 months therapy from $6.38 \pm 2.90 \mathrm{msec} / \mathrm{mmHg}$ to $8.08 \pm 4.35$ $\mathrm{msec} / \mathrm{mmHg}(\mathrm{p}=0.007)$. Diet had no influence on BRS values. No difference in $\mathrm{BMI}$, arterial blood pressure, heart rate, $\mathrm{HbA1c}$, and fasting glucose was observed at both groups compared with baseline. Long-term administration of low dose of atorvastatin, beyond the beneficial effects on plasma lipids, improves BRS in dydlipidaemic subjects with T2DM. 


\section{DIABETIC DYSLIPIDEMIA}

$\Delta$

48-0R

Favorable Effect of Intensive Glucose Lowering on Lipoprotein Particle Profiles

JURAJ KOSKA, ARAMESH SAREMI, GIDEON BAHN, PETER D. REAVEN, FOR VET-

ERANS AFFAIRS DIABETES TRIAL (VADT), Phoenix, AZ, Hines, IL

Intensive glucose-lowering therapy (INT) did not reduce cardiovascular (CV) risk outcomes in randomized trials, possibly because INT did not improve, or worsened, lipid profiles. Increased prevalence of small dense particles within both low- and high-density lipoprotein (LDL and HDL) fractions is common in diabetes and is associated with greater CV risk. Standard plasma lipids and cholesterol content of lipid subfractions (via the vertical auto profile assay) were therefore determined in 262 subjects from a substudy of the VA Diabetes Trial (VADT) at baseline and after 9 months of INT or standard therapy (STD). Despite greater increases in BMI (medians, $4 \%$ INT vs. $1 \%$ STD, $p<0.001$ ), INT reduced triglycerides (11\% vs. $0 \%$, $p<0.01)$, did not change total LDL cholesterol but increased total HDL cholesterol (9\% vs. $4 \%, p<0.05)$. INT also caused greater decreases in pro-atherogenic dense LDL4, and larger increases in less dense LDL2 and HDL2 subfractions, while STD slightly increased LDL1 only (Figure).

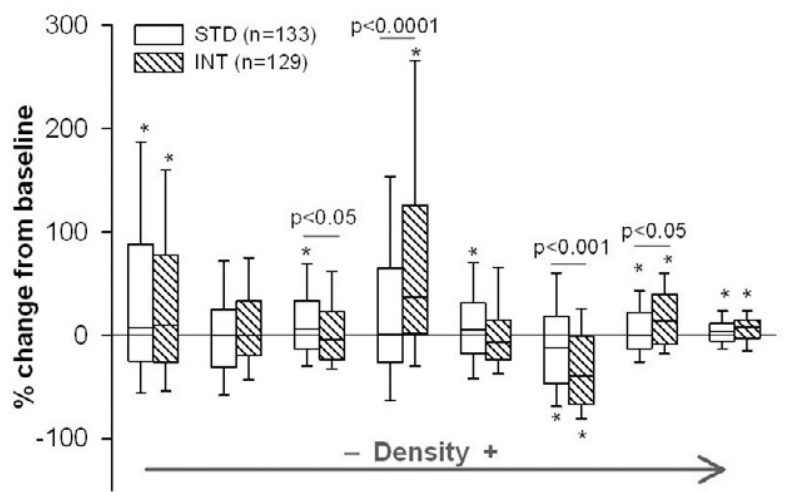

IDL Lp(A) LDL1 LDL2 LDL3 LDL4 HDL2 HDL3

Figure: Changes in LDL and HDL subclasses after 9 months. Plot outlines (top to bottom): $95^{\text {th }}, 75^{\text {th }}, 50^{\text {th }}, 25^{\text {th }} \& 5^{\text {th }}$ percentile; ${ }^{*} p<0.05,9$ months vs. baseline.

In conclusion, in addition to lowering glucose, INT was associated with improved lipid levels and a favorable shift in LDL and HDL particle profile patterns. The lack of benefit of INT on CV risk reported in the VADT despite these lipid changes may be due to (1) their relatively modest magnitude, (2) a limited role for TG, LDL and HDL particle size in modulating CV risk in advanced type 2 diabetes and/or (3) factors (e.g. BMI) that were not improved or possibly worsened with INT.

Supported by: VA CSP465, NIH R01067690, P01HL076491, P01HL77107, P01HL70621

49-0R

Exenatide, a Glucagon-Like Peptide Receptor Agonist, Acutely Inhibits Intestinal Lipoprotein Production in Healthy Humans

CHANGTING XIAO, ROBERT H. BANDSMA, SATYA DASH, LINDA SZETO, GARY F. LEWIS, Toronto, ON, Canada, Groningen, The Netherlands

Incretin-based therapies for the treatment of type 2 diabetes improve plasma lipid profiles and postprandial lipemia, but their exact mechanism of action remains unclear. Here we examined the acute effect of the GLP1 receptor agonist, exenatide, on intestinal and hepatic triglyceride-rich lipoprotein (TRL) production and clearance in healthy humans. Fifteen normolipidemic, normoglycemic men underwent two studies each (s.c. $10 \mathrm{ug}$ exenatide vs placebo), 4-6 weeks apart, in random order, in which TRL particle kinetics were examined with a primed, constant infusion of deuterated leucine and analyzed by multicompartmental modeling, under pancreatic clamp conditions. A fed state was maintained during each study by infusing a high fat, mixed macronutrient, liquid formula at a constant rate directly into the duodenum via a nasoduodenal tube. Exenatide significantly suppressed the plasma concentration (exenatide $1.83 \pm 0.30$ vs placebo $1.24 \pm$ $0.19 \mathrm{mg} / \mathrm{L}, \mathrm{P}<0.05$ ) and production rate (exenatide $0.12 \pm 0.02$ vs placebo 0.08 $\pm 0.02 \mathrm{mg} / \mathrm{kg} / \mathrm{day}, \mathrm{P}<0.05)$ of TRL-apoB-48. TRL-apoB-100 concentrations and production rates were not significantly different between exenatide and placebo. These results suggest a possible direct effect of exenatide on intestinal lipoprotein particle production, independent of changes in body weight and satiety as seen in chronic studies, and independent of changes in gastric emptying. This finding expands our understanding of the effects of exenatide in metabolic regulation beyond its primary therapeutic role in regulation of glucose homeostasis.

Supported by: CIHR

50-0R

Glycine Normalizes Hepatic Triglyceride-Rich VLDL Secretion by Triggering the CNS in High-Fat Fed Rats

JESSICA T. YUE, PATRICIA I. MIGHIU, MARK NAPLES, KHOSROW ADELI, TONY K. LAM, Toronto, ON, Canada

Dysregulation of hepatic triglyceride-rich very low-density lipoproteins (VLDL-TG) in obesity and type 2 diabetes contributes to the dyslipidemia that leads to cardiovascular morbidity. The hypothalamus regulates hepatic lipid metabolism, but it is unknown whether extrahypothalamic regions, such as the dorsal vagal complex (DVC), regulate VLDL-TG. Given that glycine enhances CNS N-methyl-D-aspartate receptor (NMDAr)-mediated transmission, we here tested whether glycine regulates hepatic VLDL-TG secretion by potentiating NMDAr-mediated transmission in the DVC. Using 10-h fasted male Sprague-Dawley rats implanted with stereotaxic cannulae into the DVC and vascular catheters to enable direct DVC infusion and blood sampling, respectively, the rate of hepatic VLDL-TG secretion was measured following tyloxapol injection. Direct DVC glycine infusion lowered VLDL-TG secretion (saline, $n=5: 0.051 \pm 0.002$ vs. glycine, $n=12: 0.041 \pm 0.002 \mathrm{mmol} /$ L.min; $P<0.01)$. DVC NR1 subunit of NMDAr antagonist 7-chlorokynurenic acid $(\mathrm{n}=11,0.053 \pm 0.003 ; P<0.02$ vs. glycine), DVC injection of NR1 shRNA ( $\mathrm{n}=9$, $0.052 \pm 0.002 ; P<0.01$ vs. glycine), and hepatic vagotomy $(n=8,0.053 \pm 0.003$; $P<0.02$ vs. glycine) nullified glycine's effect. Finally, DVC glycine normalized the hypersecretion of VLDL-TG induced by high-fat feeding (HFF+saline, $n=9$ : $0.061 \pm 0.003$ vs. HFF+glycine, $\mathrm{n}=8: 0.049 \pm 0.003 ; P<0.03)$. In summary, molecular and pharmacological inhibition of the NR1-containing NMDAr in the DVC negated the ability of glycine to inhibit hepatic secretion of VLDL-TG in vivo. Importantly, the hypersecretion of hepatic VLDL-TG induced by high-fat feeding was restored by the hepatic lipid control of DVC glycine sensing. These findings suggest that glycine or a glycine analogue may have therapeutic benefits in lowering plasma lipid levels in diabetes and obesity by triggering the CNS.

Supported by: CIHR (TKT Lam, MOP-86554)

$51-0 R$

Genome-Wide Association Study of Genetic Determinants of LDL-c Response to Atorvastatin Therapy: Importance of $L p(a)$

HELEN M. COLHOUN, HARSHAL DESHMUKH, TOBY JOHNSON, PAUL M. MCKEIGUE, D. JOHN BETTERIDGE, PAUL DURRINGTON, JOHN FULLER, SHONA LIVINGSTONE, VALENTINE CHARLTON-MENYS, ANDREW NEIL, NEIL POULTER, PETER SEVER, DENIS SHIELDS, ALICE STANTON, AUROBINDO CHATTERJEE, CRAIG HYDE, ROBERTO CALLE, DAVID DEMICCO, MARK CAULFIELD, GRAHAM HITMAN, Dundee, United Kingdom, London, United Kingdom, Edinburgh, United Kingdom, Manchester, United Kingdom, Oxford, United Kingdom, Dublin, Ireland, New York, NY

We carried out a genome wide association study of LDL-c response to statin using data from participants in the CARDS trial $(n=1156)$, ASCOT trial $(n=895)$ and the observational phase of ASCOT $(n=651)$, all prescribed atorvastatin $10 \mathrm{mg}$. Following genome wide imputation, we combined data from the three studies in a meta-analysis. We found associations of $\mathrm{LDL}-\mathrm{c}$ response to atorvastatin that reached genome-wide significance at rs10455872 ( $p=6.13 \times 10-9)$ within the LPA gene and at two SNPs within the APOE region ( $r$ 4445925; $p=2.22 \times 10-16$ and $r s 4420638 ; p=1.01 \times 10-11$ ) that are proxies for $\varepsilon 2$ and $\varepsilon 4$ variants in APOE respectively. Using CARDS data we further showed that atorvastatin therapy did not alter Lp(a) and that $L p(a)$ levels, accounted for all of the association of SNPS in LPA gene and apparent LDL-c response levels. However statin therapy had a similar effect in reducing CVD in patients in the top quartile for serum Lp(a) levels $(H R=0.60)$ compared with those in the lower three quartiles $(H R=0.66)$ $(p=0.8$ for interaction). Lp(a) levels, accounted for all of the association of SNPS in LPA gene and apparent LDL-c response. The data emphasise that high $L p(a)$ levels affect the measurement of LDL-c and the clinical estimation of LDL-c response. Therefore, an apparently lower LDL-c response to statin therapy may indicate a need for measurement of $L p(a)$. However statin therapy seems beneficial even in those with high Lp(a).

Supported by: Pfizer 
Alterations in Intestinal Insulin Signaling in Obese Subjects and their Effect on Lipid and Lipoprotein Metabolism

ALAIN VEILLEUX, ÉMILIE GRENIER, ANDRÉ CARPENTIER, DENIS RICHARD, ÉMILE

LEVY, Montreal, OC, Canada, Sherbrooke, OC, Canada, Quebec, OC, Canada

Insulin resistance, a central feature of type 2 diabetes (T2D), is commonly associated with atherogenic dyslipidemia. Impaired hepatic VLDL production and clearance are known to play a key role, but mounting evidence indicates the active implication of the small intestine in this hyperlipidemia. The present study aimed to establish whether alterations in small intestine insulin sensitivity modify intestinal lipid and lipoprotein homeostasis in obese insulin-resistant compared to obese insulin-sensitive subjects. Markers of insulin sensitivity, oxidative stress and inflammation as well as of lipid, cholesterol, apolipoproteins (apo) and lipoprotein homeostasis were analyzed in small intestine sections obtained from obese subjects undergoing bariatric surgery. In obese insulin-resistant subjects, we observed: (a) a defect in insulin signaling as pointed out by a decreased phosphorylation of insulin receptor, insulin receptor substrate-1 and Akt as well as an increased phosphorylation of p38 MAPK; (b) a higher level of lipid peroxidation markers, including malondiadehyde, which support the occurrence of oxidative stress; (c) a higher TNF- $\alpha$ and IL-6 levels, suggesting a local inflammatory state; (d) an increased protein levels of fatty acid-binding proteins, SR-B1 cholesterol transporter, SAR1B and microsomal triglyceride transfer protein; and finally (e) an elevated lipogenesis rate and apo B-48 synthesis along with triglyceride-rich lipoprotein production. Accordingly, higher expression levels of transcription factors (SHREBP and LXR) were noticed in small intestine of obese insulin-resistant subjects. In conclusions, the small intestine could be classified as an insulin-sensitive organ. Its deregulation, caused by oxidative stress and inflammation, may lead to the amplification of lipid and lipoprotein synthesis, which could therefore contribute to atherogenic dyslipidemia observed in metabolic syndrome and T2D in humans.

53-0R

Signaling Through GPR119 Increases HDL Cholesterol and Reduces Post-Prandial Triglycerides

KATHLEEN K. BROWN, MANDY L. BERGQUIST, MELANIE K. SHADOAN, DEREK J. NUNEZ, ANDREW A. YOUNG, ANDREW J. CARPENTER, PAUL L. FELDMAN, Research Triangle Park, NC

In addition to its previously described activity in the endocrine pancreas and on enteroendocrine cells in the gastrointestinal tract, GPR119 is also a sensor for a range of endogenous fatty acid ligands. The receptor is likely to have a functional role in the integration and coordination of the systemic appearance of organic nutrients in part by releasing peptides necessary for their absorption and assimilation. However, additional biology with effects on lipid and lipoprotein metabolism has not been described. Using a randomized crossover study design with a 4 wk washout period, we investigated the effects of chronic dosing with a potent and selective GPR119 agonist GSK1292263 $(6 \mathrm{mg} / \mathrm{kg})$ or vehicle in canine model $(\mathrm{n}=9)$, acclimatized to a diet high in saturated fat. Serum chemistries (glucose, FFAs, glycerol, triglycerides [TG], total and HDL Cholesterol [TC, HDLc]) were measured weekly. Lipoprotein profiles were evaluated by FPLC at the end of each treatment period and a 2 meal tolerance test was performed. By the $2^{\text {nd }} w k$ of dosing with agonist, there was a significant increase in TC and HDLc $121.5 \%$ and $16.4 \%, p=0.0008$ and 0.0025 , respectively) and the differences persisted throughout the active treatment period. The significant differences by FPLC were an increase in the $\mathrm{C}$ content of $\mathrm{HDL}$ particles with remodeling of the particles and the appearance of "big HDL" on the chromatogram. In the 2 meal tolerance test, evaluation of paired data showed an average of $61 \%$ reduction in the $\mathrm{TG}$ area under the curve for the $1^{\text {st }}$ meal and a $57 \% \%$ reduction in the AUC of the $2^{\text {nd }}$ meal ( $p=0.0379$ and $p=0.0582$ respectively). There was considerable variability in the TG AUC between the dogs. Fasting serum TGs in this study were not different between the treatments but as there was a significant lowering in the prandial period, some aspects of the HDL remodeling may have been driven by the triglyceride response. Other mechanisms such as effects on Apolipoprotein A1, phospholipid transfer protein and LCAT may also be implicated.
Hypertriglyceridemic Waist Does Not Predict Metabolic Triad in Men of African Descent

SOPHIA S. YU, NATALIE L. RAMSEY, DARLEEN C. CASTILLO, MADIA RICKS, ANNE E. SUMNER, Bethesda, MD

Hypertriglyceridemic waist (HTGW) identifies the Metabolic Triad, a set of three factors highly associated with cardiovascular disease (CVD). These three factors are: hyperinsulinemia, hyperapoB and sdLDL. As HTGW consists of only 2 criteria, waist circumference $(W C) \geq 90 \mathrm{~cm}$ and triglyceride (TG) $\geq 177 \mathrm{mg} / \mathrm{dL}$, it is simple, inexpensive, and has potential for worldwide use as a screening test. However, HTGW has never been validated in Africans or African Americans. Of particular concern is that TG levels are lower in blacks than other races and ethnicities. Therefore, we determined in 146 black men (71 Africans, 75 African American) (age: $35 \pm 8 y, ~ B M l: ~ 28.5 \pm 5.4 \mathrm{~kg} /$ $\mathrm{m} 2$ ) the ability of HTGW to predict the Metabolic Triad. While the prevalence of Metabolic Triad was 42\%, only 7\% of black men had both HTGW and Metabolic Triad (Table). Hence, HTGW failed to predict Metabolic Triad because less than $10 \%$ of black men met the TG threshold of $\geq 177 \mathrm{mg} / \mathrm{dL}$ (Figure). In fact, the mean TG level in black men with Metabolic Triad was only $97 \pm 44 \mathrm{mg} / \mathrm{dL}$. Therefore, screening tests such as HTGW which require hypertriglyceridemia to detect CVD risk are unlikely to be effective in African or African American men.

Table: Characteristics

\begin{tabular}{lccr}
\hline & $\begin{array}{c}\text { Metabolic Triad } \\
\text { Absent }(\mathrm{n}=85)\end{array}$ & $\begin{array}{c}\text { Metabolic Triad } \\
\text { Present }(\mathrm{n}=61)\end{array}$ & $\boldsymbol{P}$-value \\
\hline \% HTGW & $0 \%$ & $7 \%$ & 0.02 \\
\hline $\mathbf{W C}(\mathrm{cm})$ & $89 \pm 12$ & $101 \pm 14$ & $<0.01$ \\
\hline TG $(\mathrm{mg} / \mathrm{dL})$ & $64 \pm 28$ & $97 \pm 44$ & $<0.01$ \\
\hline Insulin $(\mathrm{mU} / \mathrm{mL})$ & $3.9 \pm 3.8$ & $8.4 \pm 4.8$ & $<0.01$ \\
\hline ApoB $(\mathrm{mg} / \mathrm{dL})$ & $72 \pm 21$ & $100 \pm 24$ & $<0.01$ \\
\hline LDL Size $(\mathrm{nm})$ & $21.3 \pm 0.6$ & $20.6 \pm 0.5$ & $<0.01$ \\
\hline
\end{tabular}

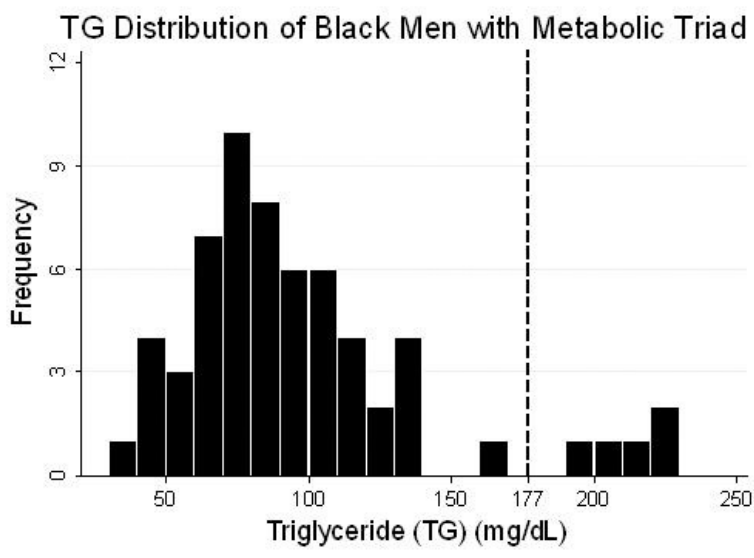

\section{INCRETIN THERAPIES}

55-0R

Once-Weekly GLP-1 Receptor Agonist Albiglutide vs. Titrated Prandial Lispro Added on to Titrated Basal Insulin Glargine in Type 2 Diabetes (T2D) Uncontrolled on Glargine Plus Oral Agents: Similar Glycemic Control With Weight Loss and Less Hypoglycemia JULIO ROSENSTOCK, BO AHREN, FRANCIS CHOW, VIVIAN FONSECA, JORGE GROSS, ROBERT RATNER, SUSAN JOHNSON, MURRAY STEWART, FRED YANG, LAWRENCE LEITER, Dallas, TX, Lund, Sweden, Hong Kong, China, New Orleans, LA, Rio Grande do Sul, Brazil, Hyattsville, MD, Research Triangle Park, NC, King of Prussia, PA, Toronto, ON, Canada

GLP-1 receptor agonists (RA) and prandial insulin are 2 options for advancing T2D uncontrolled on insulin glargine (GL) + oral agents. Harmony 6 was a randomized, open label, active controlled trial with primary endpoint at week 26 and continued treatment to week 52 that tested once weekly (OW) albiglutide (ALBI), a GLP-1 RA, vs prandial insulin lispro (LIS) both combined with titrated GL. Basal insulin-treated patients (mean A1C 8.4\%, FPG 152 


\section{INCRETIN THERAPIES}

$\mathrm{mg} / \mathrm{dL}$, weight $92 \mathrm{~kg}$ ) with A1C 7-10\% \pm oral agents (no sulfonylurea) entered a run-in standardization GL period followed by randomization to ALBI $30 \mathrm{mg}$ $\mathrm{OW}(\mathrm{n}=282)$ or LIS $(\mathrm{n}=281)$ and continued on metformin and/or TZD. GL was titrated to a target FPG $80-130 \mathrm{mg} / \mathrm{dL}$. LIS was adjusted per prespecified algorithm based on BG monitoring. ALBI could be uptitrated to $50 \mathrm{mg}$ $\mathrm{OW}$. A1C was reduced from baseline by $-0.82 \%$ and $-0.66 \%$ with $\mathrm{ALBI}$ and LIS, respectively, meeting the primary non-inferiority endpoint at week 26 with further A1C decreases by week 52 among completers. Weight changes were sustained to week 52: ALBI, -0.96 kg; LIS, $+1.66 \mathrm{~kg}$ (Table). Mean GL dose increased in both groups. Adverse events of interest with ALBI/LIS were: nausea (13.0\%/2.1\%); vomiting $(7.0 \% / 1.4 \%)$, injection site reactions $(9.5 \% / 5.3 \%)$, and hypoglycemia (32.6\%/49.8\%). Once weekly ALBI may become a therapeutic option in $\mathrm{T} 2 \mathrm{D}$ inadequately controlled on basal insulin to improve glucose control with a lower risk of hypoglycemia and significant weight loss vs thrice daily LIS.

\begin{tabular}{|c|c|c|c|c|c|c|c|c|}
\hline & \multicolumn{4}{|c|}{ Week 26 (LOCF) } & \multicolumn{4}{|c|}{ 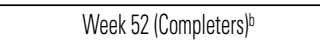 } \\
\hline & $\begin{array}{c}\text { ALBI } \\
(n=279)^{c}\end{array}$ & $\begin{array}{c}\text { LIS } \\
\text { (n }=278)^{\circ}\end{array}$ & $\begin{array}{l}\text { Treatment } \\
\text { Difference } \\
(95 \% \text { Cl) }\end{array}$ & $\begin{array}{lc} & p \\
\text { e Value }\end{array}$ & $\begin{array}{c}\text { ALB } \\
(n=121)^{\circ}\end{array}$ & $\begin{array}{c}\text { LIS } \\
(n=141)^{c}\end{array}$ & $\begin{array}{l}\text { Treatment } \\
\text { Difference } \\
(95 \% \text { Cl) }\end{array}$ & $\begin{array}{c}P \\
\text { Value }\end{array}$ \\
\hline $\begin{array}{l}A 1 C, M A \Delta \\
\text { from } B L, \% \pm \\
\text { SE }\end{array}$ & $\begin{array}{c}-0.82 \pm \\
0.06\end{array}$ & $\begin{array}{c}-0.66 \pm \\
0.06\end{array}$ & $\begin{array}{c}-0.16^{d} \\
(-0.32 \\
0.00)^{\prime}\end{array}$ & $\begin{array}{c}<.0001 \\
\text { non- } \\
\text { inferiority }\end{array}$ & $\begin{array}{c}-1.01 \pm \\
0.07\end{array}$ & $\begin{array}{c}-0.85 \pm \\
0.06\end{array}$ & $\begin{array}{c}-0.17^{d} \\
(-0.35, \\
0.02)^{\prime}\end{array}$ & .0861 \\
\hline $\begin{array}{l}\mathrm{A} 1 \mathrm{C}<6.5 \%, \\
\mathrm{n}(\%)\end{array}$ & $\begin{array}{c}31 \\
(11.1)\end{array}$ & $\begin{array}{c}23 \\
(8.3)\end{array}$ & $\begin{array}{l}1.372 \mathrm{e} \\
(0.73 \\
2.57)\end{array}$ & .3518 & $\begin{array}{c}18 \\
(14.9 \%)\end{array}$ & $\begin{array}{c}17 \\
(12.1 \%)\end{array}$ & $\begin{array}{l}1.057 \mathrm{e}^{-} \\
(0.48 \\
2.34)^{\prime}\end{array}$ & .7694 \\
\hline $\begin{array}{l}\mathrm{A} 1 \mathrm{C}<7.0 \%, \\
\mathrm{n}(\%)\end{array}$ & $\begin{array}{c}83 \\
(29.7)\end{array}$ & $\begin{array}{c}70 \\
(25.2)\end{array}$ & $\begin{array}{l}1.21 e^{\prime} \\
(0.78, \\
1.88)\end{array}$ & .3977 & $\begin{array}{c}54 \\
(44.6 \%)\end{array}$ & $\begin{array}{c}42 \\
(29.8 \%)\end{array}$ & $\begin{array}{l}1.487 e^{\prime} \\
(0.80, \\
2.78)\end{array}$ & .2301 \\
\hline $\begin{array}{l}\text { FPG, MA } \Delta \\
\text { from BL at } \\
\text { week } 26 \\
\mathrm{mg} / \mathrm{dL} \pm \mathrm{SE}\end{array}$ & $\begin{array}{l}-17.9 \pm \\
2.95\end{array}$ & $\begin{array}{c}-12.9 \pm \\
2.96\end{array}$ & $\begin{array}{c}-4.9^{d} \\
(-13.2, \\
3.3)^{\prime}\end{array}$ & .2390 & $\begin{array}{c}-26.7 \pm \\
3.65\end{array}$ & $\begin{array}{c}-15.5 \pm \\
3.39\end{array}$ & $\begin{array}{l}-11.2^{d} \\
\left(-21.2^{2}\right. \\
-1.2)^{\prime}\end{array}$ & .0278 \\
\hline $\begin{array}{l}\text { Weight, MA } \Delta \\
\text { from BL at } \\
\text { week } 26, \mathrm{~kg} \pm S E\end{array}$ & $\begin{array}{c}-0.73 \pm \\
0.19\end{array}$ & $\begin{array}{c}+0.81 \pm \\
0.20\end{array}$ & $\begin{array}{l}-1.54^{d} \\
(-2.09 \\
-1.00) \\
\end{array}$ & $<.0001$ & $\begin{array}{c}-0.96 \pm \\
0.36\end{array}$ & $\begin{array}{c}+1.66 \pm \\
0.34\end{array}$ & $\begin{array}{l}-2.61^{\mathrm{d}} \\
(-3.61 \\
-1.62) \\
\end{array}$ & $<.0001$ \\
\hline $\begin{array}{l}\text { GL dose, } U \\
\text { ( } \Delta \text { from BL) }\end{array}$ & $\begin{array}{c}52 \\
(+5) \\
\end{array}$ & $\begin{array}{c}50 \\
(+7) \\
\end{array}$ & & & $\begin{array}{c}54 \\
(+7) \\
\end{array}$ & $\begin{array}{c}52 \\
(+8) \\
\end{array}$ & & \\
\hline $\begin{array}{l}\text { LS dose, U } \\
\text { ( } \Delta \text { from BL) }\end{array}$ & & $\begin{array}{c}30 \\
(+14)\end{array}$ & & & & $\begin{array}{c}35 \\
(+19) \\
\end{array}$ & & \\
\hline $\begin{array}{l}\text { Hyperglycemia } \\
\text { rescue, } n(\%)\end{array}$ & $\begin{array}{c}61 \\
(21.6)\end{array}$ & $\begin{array}{c}66 \\
(23.5)\end{array}$ & & & $\begin{array}{c}123 \\
(43.6)^{\mathrm{a}}\end{array}$ & $\begin{array}{c}109 \\
(38.8)^{3}\end{array}$ & & $.3260^{f}$ \\
\hline
\end{tabular}

A1C, glycosylated hemoglobin; ALBI, albiglutide; BL, baseline; $\mathrm{Cl}$, confidence interval; FPG, fasting plasma glucose; LIS, insulin lispro; LOCF, last observation carried forward; MA, model-adjusted; SD, standard deviation. ${ }^{a}$ Intent-totreat population with last observation carried forward. ${ }^{b}$ Observed cases population with the exception of hyperglycemia rescue. ${ }^{\mathrm{C}} \mathrm{n}$ listed is that of $\mathrm{A} 1 \mathrm{C} ; \mathrm{n}$ varies slightly for different parameters. ${ }^{\mathrm{d}} \mathrm{ALBI}$ minus LIS. ${ }^{\mathrm{e}}$ Odds ratio for ALBI vs LIS. ${ }^{\dagger}$ Log-rank test for overall hyperglycemia rescue for 1 year.

56-OR

Cardioprotective Effect of Chronic Treatment With Lixisenatide in an In Vivo Rat Model of Myocardial Ischemia/Reperfusion-Induced Injury

WOLFGANG LINZ, DOMINIK LINZ, PAULUS WOHLFART, ULRICH WERNER, THOMAS HÜBSCHLE, HARTMUT RUETTEN, Frankfurt, Germany, Homburg/Saar, Germany

Effects of GLP-1R agonists on the cardiovascular system have been described recently. In isolated rat hearts, the GLP-1R agonist lixisenatide significantly reduced infarct size after ischemia-reperfusion (I/R) ex vivo. In this study, the effect of chronic lixisenatide treatment vs placebo (PBO) on cardiovascular outcome after ischemia (30 min) and long-term reperfusion of the left coronary artery was investigated in vivo. Male Wistar rats (250-300 g) were randomized into 4 groups: 1 . sham-operated controls without I/R (Sham); 2. I/R PBO; 3. I/R ramipril (1 mg/kg/d in chow; I/R RA); 4. I/R lixisenatide (10 $\mu \mathrm{g} / \mathrm{kg} / \mathrm{d}$ s.c. via osmotic minipump). After $10 \mathrm{wk}$ of treatment, heart function was investigated via tip catheter, an autopsy performed and lung weighed. Plasma brain natriuretic peptide (BNP) was determined as a marker of chronic heart failure. Lixisenatide significantly attenuated I/R-induced deterioration of diastolic function (Left Ventricular end-diastolic Pressure) and Tau Weiss, a measure of myocardial relaxation (Figure). Lung weight was significantly increased with PBO (a measure of congestion), but was normalized by lixisenatide and ramipril (Figure). Moreover, BNP levels were significantly $(p<0.05)$ reduced by either agent. Thus, deterioration of left ventricular func- tion following ischemia-reperfusion was significantly prevented by chronic lixisenatide treatment. In conclusion, lixisenatide has shown, in a rat model of myocardial ischemia/reperfusion-induced injury, cardioprotective effects comparable to the ACE inhibitor ramipril.
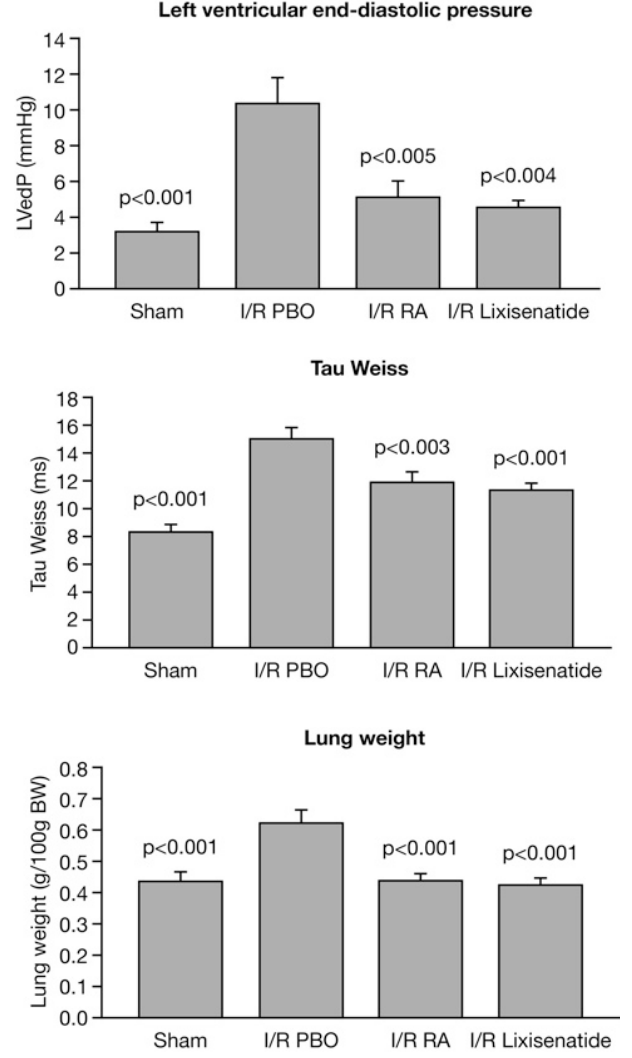

Supported by: sanofi-aventis

57-0R

ZYOG1, A Novel Orally Efficacious Peptidomimetic GLP-1 Receptor Agonist

MUKUL R. JAIN, RAJESH BAHEKAR, AMIT JOHARAPURKAR, DEBDUTTA BANDHYOPADHYAY, RAJESH SUNDAR, HARILAL PATEL, VISHWANATH PAWAR, Ahmedabad, India

GLP-1 (Glucagon-like Peptide) agonists have emerged as effective therapeutic agents for managing diabetes and obesity. Currently available GLP-1 agonists are all parenteral products. While these agents are effective in managing diabetes, they cause nausea \& vomiting. Using peptidomimetic approach, we have developed ZYOG1, a novel orally efficacious GLP-1 receptor agonist. ZYOG1 is a potent and selective agonist of GLP-1 receptor. In an in vitro assay using $\mathrm{CHO}$ cells stably transfected with hGLP1R, ZYOG1 showed concentration-dependent cAMP production with an EC50 of $6.6 \mathrm{nM}$. The agonistic activity of ZYOG1 was blocked by GLP-1 antagonist, Exendin 9-39. ZYOG1 dose-dependently increased glucose-stimulated insulin release in isolated mouse islets. When given orally to C57 mice, ZYOG1 showed dose-dependent reduction in glucose excursion during intraperitoneal glucose tolerance test, with an ED50 of $2.6 \mathrm{mg} / \mathrm{kg}$. In ob/ob and $\mathrm{db} / \mathrm{db}$ mice, the animal models of diabetes, acute dosing of ZYOG1 at $2.6 \mathrm{mg} / \mathrm{kg}$ or $10 \mathrm{mg} / \mathrm{kg}$ significantly reduced fed serum-glucose and glucose excursion during IPGTT with a concomitant elevation of plasma insulin levels. In a type 2 diabetic C57 mice model prepared by high fat diet feeding and low doses of streptozotocin injection, oral ZYOG1 not only lowered glucose during a 8 week treatment, but also reduced $\% \mathrm{HbA1c}$, indicating a long term glycemic control with this compound. ZYOG1 also reduced the body weight, adiposity and improved glucose homeostasis in a diet-induced obesity model in C57 mice and in genetically obese $\mathrm{db} / \mathrm{db}$ mice, after a 4 weeks oral treatment. ZYOG1 is eliminated mainly via non-renal pathways. Repeated-dose toxicity studies in Wistar rats and Beagle dogs revealed good safety profile, and absence of any immunogenicity. ZYOG1 was devoid of any nausea or vomiting effects in relevant models including Beagle dogs. In conclusion, ZYOG1 represents a novel and orally efficacious GLP-1 agonist for the safe and effective treatment of Type 2 Diabetes. 
Exenatide Treatment for 6 Months Improves Insulin Sensitivity in Adults With Type 1 Diabetes Mellitus

GAYATRI SARKAR, MAY ALATTAR, REBECCA J. BROWN, MICHAEL J. QUON, DAVID

M. HARLAN, KRISTINA I. ROTHER, Bethesda, MD, Baltimore, MD, Worcester, MA

Adjunctive exenatide treatment improves blood glucose control in adults with type 2 diabetes and reduces post-prandial hyperglycemia in adolescents with type 1 diabetes mellitus. We studied effects of exenatide on glucose metabolism in 14 adult patients with long-standing type 1 diabetes mellitus using a cross-over study of 6 months on exenatide $(10 \mathrm{mcg}$ four times daily) and 6 months off. We assessed changes in fasting and postprandial blood glucose, daily insulin requirements, and changes in insulin sensitivity (hyperinsulinemic euglycemic clamps) before and after each study arm. Exenatide reduced post-prandial blood glucose but was associated with higher fasting glucose levels without change in hemoglobin A1C (patients lost weight as previously reported). Insulin requirements were significantly reduced and insulin sensitivity increased after 6 months of exenatide therapy by amounts that exceeded those expected from weight loss per se. Thus, in patients with well controlled type 1 diabetes mellitus at baseline, high dose exenatide therapy increased fasting and decreased postprandial glucose levels without effecting a net change in hemoglobin A1C. Exenatide decreased daily insulin requirements and increased insulin sensitivity beyond effects expected due to weight reduction per se. We conclude that exenatide is a promising adjunctive agent to insulin therapy in adult patients with type 1 diabetes mellitus that reduces postpandrial blood glucose and improves insulin sensitivity.

Effects of 6-month Exenatide Use on Weight,Plasma Glucose, A1C, Insulin Requirement, SI

\begin{tabular}{lccc}
\hline Variable & On Exenatide & Off exenatide & P value \\
\hline Weight $(\mathrm{kg})$ & $74.2 \pm 12.6$ & $78.4 \pm 12.1$ & $\mathbf{0 . 0 0 0 3}$ \\
\hline A1c $(\%)$ & $6.6 \pm 0.6$ & $6.8 \pm 0.6$ & $\mathbf{0 . 3 8 8}$ \\
\hline Fasting plasma glucose $(\mathrm{mg} / \mathrm{dl})$ & $136.9 \pm 3.19$ & $129.7 \pm 3.19$ & $\mathbf{0 . 0 0 0 2}$ \\
\hline Postpandrial plasma glucose $(\mathrm{mg} / \mathrm{dl})$ & $135.5 \pm 4.43$ & $142.5 \pm 4.42$ & $\mathbf{0 . 0 0 0 5}$ \\
\hline Insulin requirement (unit/kg/day) & $0.48 \pm 0.11$ & $0.55 \pm 0.13$ & $\mathbf{0 . 0 0 6 2}$ \\
\hline $\begin{array}{l}\text { Insulin Sensitivity Index/SI) } \\
\text { (mg/m2/min per } \mathrm{mU} / \mathrm{ml} \text { ) }\end{array}$ & $7.15 \pm 2.046$ & $5.21 \pm 1.642$ & $\mathbf{0 . 0 0 3 9}$
\end{tabular}

Supported by: NIDDK

59-0R

Vildagliptin Reduces Glucagon During Hyperglycemia and Sustains Glucagon Counter-Regulation in Type 1 Diabetes

BO AHREN, JOHAN FARNGREN, MARGARETHA PERSSON, ANJA SCHWEIZER, JAMES FOLEY, Lund, Sweden, Basel, Switzerland, East Hanover, NJ

The DPP-4 inhibitor, vildagliptin, improves glycemia in type 2 diabetes with low risk of hypoglycemia. Vildagliptin inhibits glucagon secretion at hyperglycemia but appears to improve glucagon counter-regulation during hypoglycemia in type 2 diabetes. We hypothesized that vildagliptin also improves alpha-cell function in type 1 diabetes (T1D) and examined its effect on glucagon levels at hyper- and hypoglycemia in patients with T1D. The study was a single-center, double-blind, randomized, placebo-controlled crossover study involving 28 patients with $\mathrm{T} 1 \mathrm{D}(21 \mathrm{M}, 7 \mathrm{~F}$, mean age $30 \mathrm{yrs}$, diabetes duration $11 \mathrm{yrs}, \mathrm{HbA} 1 \mathrm{c} 60 \mathrm{mmol} / \mathrm{mol}(7.7 \%)$, BMI $\left.24.7 \mathrm{~kg} / \mathrm{m}^{2}\right)$ treated with long acting (30U/day) and short-acting (29U/day) insulin. Patients received vildagliptin (50 mg BID) or placebo as add-on to insulin for four weeks in random order with a four week washout in-between. On day 28 of the respective treatment, patients were served a standard meal (500 kcal) followed by a hyperinsulinemic hypoglycemic clamp at $2.5 \mathrm{mmol} / \mathrm{l}$. Vildagliptin but not placebo reduced HbA1c $(-3.6 \mathrm{mmol} / \mathrm{mol}(-0.3 \%), \mathrm{P}<0.001)$. During the meal, glucagon levels were lower with vildagliptin than with placebo $(8.4 \pm 0.6$ vs. $9.5 \pm 0.7 \mathrm{ng} / \mathrm{ml} \times \mathrm{min}, \mathrm{P}=0.022)$. In contrast, during hypoglycemia, the glucagon counter-regulation was not reduced by vildagliptin ( $5.5 \pm 3.7 \mathrm{vs}$. $6.2 \pm 3.0 \mathrm{pg} / \mathrm{ml}$, NS). Also the counter-regulatory responses in epinephrine, norepinephrine and cortisol were not different. Interestingly, during the recovery from hypoglycemia, glucose levels reached $6.4 \pm 0.3 \mathrm{mmol} / \mathrm{l}$ with vildagliptin and $7.6 \pm 0.6 \mathrm{mmol} / \mathrm{l}$ with placebo $(P=0.040)$. The study shows that vildagliptin improves glycemia, reduces glucagon during meal and preserves glucagon counter-regulation during hypoglycemia in T1D. The study therefore suggests that action of vildagliptin via alpha-cells maybe an appropriate add-on to insulin therapy in T1D.
Effects of Sitagliptin and Metformin Treatment on GLP-1 Responses to Oral Glucose and on the Incretin Effect in Patients With Type 2 Diabetes

IRFAN VARDARLI, ELISABETH ARNDT, CAROLYN F. DEACON, JENS J. HOLST, LARS D. KÖTHE, MICHAEL A. NAUCK, Bad Lauterberg, Germany, Copenhagen Denmark

DPP-4 inhibitors (sitagliptin; S) prevent inactivation of incretin hormones (GLP-1 and GIP). Metformin (M) may also increase GLP-1 levels. The present study was designed to study the influence of $\mathrm{M}, \mathrm{S}$, or their combination on GLP-1 responses after oral glucose and on the incretin effect. 20 patients with type 2 diabetes (age: $59 \pm 7$ yrs., duration of diabetes $5.1 \pm 2.7 \mathrm{yrs}$. $\mathrm{HbA}_{1 \mathrm{c}}: 7.0 \pm 0.6 \%$, BMI $30.6 \pm 3.0 \mathrm{~kg} / \mathrm{m}^{2}$, means $\pm S D$, drug-naïve $/ 0 \mathrm{AD}$ monotherapy, 6 weeks wash-out) entered a 4-period crossover trial with placebo $S(100 \mathrm{mg} / \mathrm{d}), \mathrm{M}$ (escalation to $2000 \mathrm{mg} / \mathrm{d}$ ), and M/S combination for 6 days (order randomized). On day 5 , an oral glucose challenge $(75 \mathrm{~g})$ and on day 6 , an "isoglycaemic" intravenous glucose infusion was performed. Glucose, insulin, C-peptide, insulin secretion rates (deconvolution), and GLP-1 (total and intact) were determined. Statistics: Repeated-measures ANOVA. Total GLP-1 in the fasting state was increased by M $(p=0.010)$ and not changed by $S(p=0.23)$. After oral glucose, $M$ increased $(p=0.0018)$ and $S$ decreased $(p=0.0055)$ total post-load GLP-1 (AUC). Intact GLP-1, fasting and post-load, increased with $S$ (both $p<0.0001)$ and $M(p=0.06$ and 0.0081 , respectively). After oral glucose, only $S(p=0.0021)$, but not $M(p=0.39)$ augmented insulin secretion. Insulin secretion in response to oral glucose was higher than with "isoglycaemic" intravenous glucose with all treatments $(p<0.01)$. Numerically, the incretin effect (placebo: $26 \pm 22 \%$ ) did not change with any treatment ( $S: p=0.33, M: p=0.48$, interaction 0.46 ). In conclusion, sitagliptin increases intact GLP-1 through DPP-4 inhibition, but reduces total increments in post-load GLP-1 (L-cell feedback). Metformin increases fasting GLP-1 and post-load GLP-1 (total and intact). Against expectations, although raising intact GLP-1 levels, neither treatment significantly increased the numerical contribution of the incretin effect to insulin secretion after oral glucose.

Supported by: Merck Research Labs

61-0R

The DPP-4 Inhibitor Linagliptin Protects from Experimental Diabetic Retinopathy

NADINE DIETRICH, STEPHANIE BUSCH, VALERIE SCHWARZ, PETRA BUGERT, ULRIKE KAISER, JIHONG LIN, THOMAS KLEIN, ANGELIKA BIERHAUS, HANS-PETER HAMMES, Mannheim, Germany, Ingelheim, Germany, Heidelberg, Germany

Dipeptidyl peptidase (DPP)-4 inhibition improves glycemic control in type 2 diabetes. GLP-1 receptor agonists are heart- and brain-protective, but the effects of DPP-4 inhibitors on the diabetic vasculature are still unclear. To assess the effect of DPP-4 inhibition on vascular damage in the diabetic retina, streptozotocin-induced $(35 \mathrm{mg} / \mathrm{kg})$ diabetic male Wistar rats $(\mathrm{n}=25)$ were treated with the DPP-4 inhibitor linagliptin $(0.083 \mathrm{~g} / \mathrm{kg}$ chow) for 26 weeks. Blood glucose and $\mathrm{HbA1c}$ were increased by diabetes $(\mathrm{p}<0.001)$ and were reduced by approximately $10 \%$ in the linagliptin-treated diabetic group, while body weight remained unchanged. Linagliptin reduced DPP-4 enzyme activity by $75 \%$ and increased active levels of GLP-1 by more than 12 fold. Methylglyoxal levels increased by 4 fold in the retina of diabetic animals ( $p<0.001$ vs non-diabetic controls), and were reduced by more than $60 \%$ in the linagliptin-treated diabetic group. IL-1b, ICAM-1, CXC-R4, and MCP-1 expression were all significantly upregulated in the diabetic group $(p<0.05$ or less), whereas only IL-1b expression was significantly reduced in the linagliptin group $(p<0.01)$. The GLP-1 receptor was localized in neuroglial cells of the inner retina and the photoreceptors. p-AKT was predominantly found in cells of the ganglion layer, and was reduced in diabetic rats. Linagliptin treatment induced a substantial increase in the number of $p$-AKT-positive cells in diabetic animals. Most importantly, in retinal digest preparations, linagliptin significantly reduced the number of diabetes-induced acellular capillaries by $75 \%(p<0.01)$. In conclusion, our data suggest that DPP-4 inhibition by linagliptin has a protective effect on the microvasculature of the diabetic retina most likely due to a combined effect on the neurovascular unit.

Supported by: Boerhinger Ingelheim, DFG GRK 880 
62-0R

Efficacy and Safety of Once-Daily Lixisenatide Added on to Titrated Glargine plus Oral Agents in Type 2 Diabetes: GetGoal-Duo 1 Study JULIO ROSENSTOCK, THOMAS FORST, RONNIE ARONSON, LEOBARDO SAUQUE-REYNA, ELISABETH SOUHAMI, LIN PING, MATTHEW RIDDLE, Dallas, TX, Mainz, Germany, Toronto, ON, Canada, Cuernavaca, Mexico, Frankfurt, Germany, Portland, $O R$

Lixisenatide (LIXI) is a once-daily GLP-1R agonist with robust postprandial effects that could complement basal insulin therapy. This randomized, double-blind, multicenter trial in T2D inadequately controlled on metformin \pm $\mathrm{SU} \pm \mathrm{TZD}$ assessed the efficacy and safety of LIXI added on to titrated insulin glargine [NCT00975286]. Glargine was initiated and titrated in a 12-wk runin phase to achieve FPG $80-100 \mathrm{mg} / \mathrm{dL}$. Subjects with $\mathrm{A} 1 \mathrm{C} \geq 7 \%$ were then randomized to AM once-daily LIXI $20 \mu \mathrm{g}(\mathrm{n}=223)$ or placebo (PBO; $n=223)$ for 24 wk while glargine titration continued. At randomization, SU therapy was stopped and all subjects were on metformin and $12 \%$ on a TZD. Patient demographics were similar in both groups (mean age 56.2 y, T2D duration 9.2 $y, B M I 31.8 \mathrm{~kg} / \mathrm{m}^{2}$ ). Primary endpoint was change in A1C from randomization. A1C decreased during run-in from $8.6 \%$ to $7.6 \%$ and LIXI further reduced A1C vs PBO (LS mean difference: $-0.32 \% ; p<0.0001$ ), with more LIXI achieving $\mathrm{A} 1 \mathrm{C}<7.0 \%$ (56\% vs $39 \%$, respectively). LIXI significantly improved 2-h PPG after a standardized breakfast and had a beneficial effect on body weight vs PBO (LS mean difference: - $0.89 \mathrm{~kg} ; \mathrm{p}=0.0012$ ) despite glargine titration. AEs occurred in $80 \%$ on LIXI vs $68 \%$ on PBO; nausea/vomiting were more common with LIXI (27.4\%/9.4\% vs $4.9 \% / 1.3 \%)$ as was discontinuation due to $\mathrm{AEs}$ (8.5\% vs $3.6 \%$ ). More LIXI vs PBO had symptomatic hypoglycemia $(22.4 \%$ vs $13.5 \%$ ). In conclusion, LIXI added to optimally titrated glargine and oral agents significantly improved $\mathrm{A} 1 \mathrm{C}$, reduced PPG and had a beneficial effect on weight with an expected GLP-1 AE profile that subsided over time.

\begin{tabular}{|c|c|c|c|}
\hline \multicolumn{2}{|c|}{ Efficacy parameters in mITT population } & \multirow{2}{*}{$\begin{array}{c}\begin{array}{c}\text { Lixisenatide } \\
(\mathrm{N}=\mathbf{2 2 3})\end{array} \\
8.60 \pm 0.80 \\
7.56 \pm 0.54 \\
6.96 \pm 0.81 \\
-0.71 \pm 0.09 \\
-0.32[-0.463 \text { to } \\
-0.171] ; \mathrm{p}<0.0001\end{array}$} & \multirow{2}{*}{$\begin{array}{r}\begin{array}{r}\text { Placebo } \\
\text { (N=223) }\end{array} \\
8.60 \pm 0.80 \\
7.60 \pm 0.54 \\
7.30 \pm 0.85 \\
-0.40 \pm 0.09\end{array}$} \\
\hline$\overline{\mathrm{A} 1 \mathrm{C}(\%)}$ & $\begin{array}{l}\text { Mean screening } \pm \text { SD } \\
\text { Mean baseline* } \pm \text { SD } \\
\text { Mean Week } 24 \pm \text { SD } \\
\text { LS mean } \pm \text { SE change from baseline } \\
\text { LS mean difference vs placebo }\end{array}$ & & \\
\hline $\begin{array}{l}\text { Proportion achieving } \\
\text { A1C }<7.0 \%\end{array}$ & $\begin{array}{l}n(\%) \\
p \text { value vs placebo }\end{array}$ & $\begin{array}{c}121(56.3 \%) \\
p=0.0001\end{array}$ & $85(38.5 \%)$ \\
\hline $\begin{array}{l}\text { Proportion achieving } \\
\text { A1C } \leq 6.5 \%\end{array}$ & \begin{tabular}{|l}
$n(\%)$ \\
$p$ value vs placebo
\end{tabular} & $\begin{array}{c}69(32.1 \%) \\
p<0.0001\end{array}$ & $36(16.3 \%)$ \\
\hline 2-h PPG (mmol/L)** & $\begin{array}{l}\text { Mean baseline* } \pm \text { SD } \\
\text { Mean Week } 24 \pm \text { SD } \\
\text { LS mean } \pm \text { SE change from baseline } \\
\text { LS mean difference vs placebo }\end{array}$ & $\begin{array}{c}13.02 \pm 3.83 \\
9.87 \pm 4.24 \\
-3.09 \pm 0.48 \\
-3.16[-3.951 \text { to } \\
-2.375] ; p<0.0001\end{array}$ & $\begin{array}{c}12.85 \pm 3.75 \\
13.04 \pm 3.94 \\
0.08 \pm 0.48\end{array}$ \\
\hline $\begin{array}{l}\text { Average 7-point SMPG } \\
\text { (mmol/L) }\end{array}$ & $\begin{array}{l}\text { Mean baseline } *^{*} \text { SD } \\
\text { Mean Week } 24 \pm \text { SD } \\
\text { LS mean } \pm \text { SE change from baseline } \\
\text { LS mean difference vs placebo }\end{array}$ & $\begin{array}{c}8.20 \pm 1.45 \\
7.75 \pm 1.51 \\
-0.47 \pm 0.18 \\
-0.39[-0.680 \text { to } \\
-0.107] ; p=0.0071\end{array}$ & $\begin{array}{r}8.29 \pm 1.52 \\
8.21 \pm 1.72 \\
-0.08 \pm 0.18\end{array}$ \\
\hline$\overline{F P G}(\mathrm{mmol} / \mathrm{L})$ & $\begin{array}{l}\text { Mean baseline* } \pm \text { SD } \\
\text { Mean Week } 24 \pm \text { SD } \\
\text { LS mean } \pm \text { SE change from baseline } \\
\text { LS mean difference vs placebo }\end{array}$ & $\begin{array}{c}6.56 \pm 1.74 \\
6.70 \pm 1.79 \\
0.34 \pm 0.21 \\
-0.12[-0.463 \text { to } \\
0.232] ; p=0.5142\end{array}$ & $\begin{array}{l}6.69 \pm 1.98 \\
6.86 \pm 1.88 \\
0.46 \pm 0.21\end{array}$ \\
\hline
\end{tabular}

Supported by: sanofi-aventis

\section{FRONTIERS OF THE DIAGNOSIS AND TREATMENT OF NEURAL DYSFUNCTION}

63-0R

Impact of Glycemic Control Strategies on the Incidence of Diabetic Peripheral Neuropathy in the Bypass Angioplasty Revascularization Investigation 2 Diabetes (BARI 2D) Cohort

RODICA POP-BUSUI, JIANG LU, ALBERT STEWART, BRUCE PERKINS, JENNIFER GREEN, JORGE ESCOBEDO, PASQUALE PALUMBO, FRED WHITEHOUSE, TERESA L. JONES, Ann Arbor, MI, Pittsburgh, PA, St. Louis, MO, Toronto, ON, Canada, Durham, NC, Mexico City, Mexico, Rochester, MN, Detroit, MI, Bethesda, MD

The BARI 2D trial demonstrated similar long-term clinical effectiveness of insulin-sensitizing (IS) versus insulin-providing (IP) treatments for type 2 diabetes (T2DM) on cardiovascular outcomes in a cohort with documented coronary artery disease. We evaluated the effects of randomized glycemic control strategy (IS vs.IP) on the prevalence and incidence of diabetic peripheral neuropathy (DPN) in this cohort. DPN was assessed at baseline and yearly thereafter during 4 years of follow-up and defined as a clinical examination score $>2$ on the Michigan Neuropathy Screening Instrument (MNSI). DPN prevalence and incidence were compared by intention-to-treat, i.e. IS vs. IP, modeling by logistic (GEE) models for prevalence, Kaplan-Meier esti- mates and Cox regression models for incidence rate.Results are reported for 2159 BARI 2D participants with valid baseline and at least one follow-up MNSI score (mean age $62 \pm 9$ years, mean HbA1c $7.7 \pm 1.6 \%$, diabetes duration $10 \pm 9$ years, $70 \%$ males). There were no baseline clinical differences between groups. The prevalence of DPN throughout the 4 years of follow-up was similar ( $p=0.83$ ). However, for the 1075 BARI 2D participants with no DPN at baseline, the 4-year cumulative incidence rate of DPN was significantly lower in the IS $(54 \%)$ compared to the IP group $(63 \%)(p=0.022)$. After adjusting for the in-trial hemoglobin A1c values, the hazard ratio (HR) of new DPN for IS vs. IP was 0.81 ( $p=0.009)$. In subgroups analyses, the effects of the IS strategy in preventing DPN were higher compared to the IP strategy, in participants who were $<65$ years ( $H R=0.75, p=0.004)$, were males ( $H R=0.74$, $p=0.003)$ and had higher triglycerides ( $>=150 \mathrm{mg} / \mathrm{dl}$ ) at baseline (HR=0.74, $\mathrm{p}=0.009$ ). Among patients with T2DM followed for up to 4 years during BARI $2 \mathrm{D}$, a glycemic control therapy with IS significantly reduced the cumulative incidence of DPN compared with IP therapy

Supported by: NHLBI (U01 HL061746, U01 HL061748, U01 HL063804) and NIDDK (HLO61744)

64-0R

New Collagen-Linked Advanced Glycation Endproducts (AGEs) Predict Microvascular (MV) Disease Progression Risk in Type 1 Diabetes-16 Years after the Diabetes Control and Complication Trial (DCCT)

VINCENT M. MONNIER, WANJIE SUN, DAVID R. SELL, CHRISTOPHER M. STRAUCH, PATRICIA A. CLEARY, JOHN M. LACHIN, SAUL GENUTH AND THE DCCT/EDIC RESEARCH GROUP, Cleveland, OH, Rockville, MD

We previously reported that 6 collagen-linked AGEs measured in skin obtained near DCCT closeout may in part explain the "metabolic memory" phenomenon of the DCCT/EDIC study, i.e. the persistent effect of glycemic exposure (DCCT A1c) on progression of retinopathy (RET) and nephropathy (NEPH) 10 years post DCCT. We now report 4 new added AGEs, glucosepane (GSPNE), methylglyoxal (MG-H1) and glyoxal (G-H1) hydroimidazolones, and carboxyethyl-lysine (CEL), enhances prediction of incident RET, NEPH and neuropathy (NEU) events during 16 years post DCCT. The AGEs were measured by LC/MS in collagen digest. Multivariate logistic regression was used to assess AGE effects on risk of MV progression in EDIC in those free of events at DCCT closeout. The complete 10 AGE panel predicts 3 step ETDRS worsening of RET ( $p \leq 0.002)$, independent of either mean DCCT or EDIC A1c. In multivariate models, GSPNE and fructose-lysine (as furosine, FUR) play dominant roles in RET progression, and the RET dependence on A1c loses significance when adjusted for these 2 AGEs. The 10 AGE panel also predicts microalbuminuria ( $p=.008$ ), despite adjustment for A1c. FUR is the dominant AGE for NEPH independent of A1c ( $p \leq 02$ ). Notably, the 10 AGE panel predicts NEU (peripheral and/or autonomic neuropathy) in EDIC despite adjustment for DCCT and EDIC mean A1c ( $p \leq 0.005)$. Of these, MG-H1 and FUR are dominant, independent of $A 1 c(p<.0001)$ whereas A1c loses predictive significance after adjustment for them. Overall, the new 4 AGEs add to the predictive effect of the original 6 in RET and NEU $(p<0.04)$ but not in NEPH. These results further implicate collagen AGEs as one factor in the pathogenesis of complications. GSPNE and methylglyoxal derived MG-H1 emerge as the principal new risk factors associated with NEU development. The AGEs were predictive up to 16 years of follow-up, arguing for early and sustained implementation of intensive therapy.

Supported by: JDRF

65-0R

Peroxynitrite and Protein Nitration in the Pathogenesis of Diabetic Peripheral Neuropathy

SERGEY LUPACHYK, HANNA SHEVALYE, ROMAN STAVNIICHUK, JOHN T. GROVES, IRINA OBROSOVA, Baton Rouge, LA, Princeton, NJ

Peroxynitrite, a product of the reaction of superoxide anion radical with nitric oxide, is the most potent oxidant formed in a variety of pathological conditions including diabetes. Peroxynitrite injury causes oxidative stress with concomitant inactivation of enzymes, poly(ADP-ribosyl)ation, mitochondrial dysfunction, impaired stress signaling, as well as protein nitration. This study evaluated the role for peroxynitrite injury in toto vs protein nitration in advanced diabetic peripheral neuropathy (DPN). Male control and streptozotocin (STZ)-diabetic C57BI6/J mice were treated with the potent peroxynitrite decomposition catalyst $\mathrm{Fe}(\mathrm{III})$ tetramesitylporphyrin octasulfonate (FeTMPS, $10 \mathrm{mgkg}^{-1} \mathrm{~d}^{-1}$ ) or protein nitration inhibitor (-)-epicatechin (Epi, $20 \mathrm{mg}^{-1} \mathrm{~d}^{-1}$ i.p.) for 4 wks, after initial 7 mo without treatment. Dose selection for both agents was performed in short-term studies in STZ- 
diabetic C57BI6/J mice in which FeTMPS counteracted both nitration and poly(ADP-ribosyl)ation in the peripheral nerve, whereas Epi blunted nitration without any effect on oxidative stress. C57BI6/J mice with 7-mo duration of STZ-diabetes developed $13 \%$ motor and $20 \%$ sensory nerve conduction velocity deficits, clearly manifest thermal and mechanical hypolagesia, tactile allodynia, and $20 \%$ loss of intraepidermal nerve fibers. Both FeTMPS and Epi partially corrected sensory nerve conduction slowing and small sensory nerve fiber dysfunction without alleviation of hyperglycemia. Correction of MNCV deficit and increase in intraepidermal nerve fiber density were found with FeTMPS treatment only. In conclusion, peroxynitrite injury in toto and its component, protein nitration, are implicated in the development of DPN. The findings indicate that both structural and functional changes of DPN can be reversed at a very advanced stage, and provide rationale for the development of a new generation of antioxidants, peroxynitrite decomposition catalysts, for treatment of this complication.

Supported by: NIH DK081147

66-0R

The Impact of Depression on Central Pain Processing in Painful Diabetic Peripheral Neuropathy-A Functional Magnetic Resonance Study

DINESH SELVARAJAH, JENNIFER DAVIES, ADITHYA SANKAR, ELAINE CACHIA,

IAIN WILKINSON, SOLOMON TESFAYE, Sheffield, United Kingdom

We have reported a high prevalence of mood disorders in painful diabetic neuropathy (DN) that influence response to pharmacotherapy. It is also well recognised that antidepressants are effective at relieving neuropathic pain. However, as most trials exclude depressed patients it is unknown whether any pain relieving properties are partly mediated by easing depression. We sought to explore the complex interaction between painful DN and depression by looking at regional brain activation during pain processing using fMRI. Methods: 15 painful DN and 12 healthy volunteers (HV) underwent neurophysiological assessment. Mood disorders were assessed using the Hospital Anxiety and Depression Scale (HADS). All subjects underwent fMRI whilst heat pain was applied to the thigh (non-neuropathic region) at a pain level of at least 7 (11 point Likert scale). MR data was analysed using SPM5. Results In painful DN, there was significantly ( $<<0.001$, uncorrected) greater activation in the ventromedial prefrontal cortex (BA 9), stereotactic coordinates: $x=28, y=34, z=24)$ and cingulate gyrus $(-8,2,30)$ compared with HV. Subjects with painful DN were then divided into two groups based on HADS-D. Painful DN subjects with depression (HADS-D $>8, n=9$ ) had significantly greater activation $(p<0.001$, uncorrected) in the prefrontal cortex (BA 9, 8,46,52) compared with those without (HADS-D<8,n=6). Discussion Increased activation in BA9 and the cingulate gyrus suggests a greater perception of suffering in painful-DN as these regions are involved in emotional pain processing. Increased activation in BA9 in subjects with depressive symptoms suggests a neurocortical link between mood disorders and abnormal acute pain processing. Further studies are required to investigate the impact of antidepressant treatment on this abnormal activation. Using this novel technique, we report new insights into abnormal pain processing which maybe amenable to targeted treatments.

Supported by: JDRF

67-0R

Frontal-Temporal White Matter Microstructural Alterations in Type 1 Diabetes Mellitus

ALAN M. JACOBSON, INKYOON LYOO, SUJUNG YOON, NICOLAS R. BOLO, DONALD C. SIMONSON, GAIL F. MUSEN, PERRY F. RENSHAW, SOO M. LIM, JAEUK HWANG, JI-HYUN KIM, JIEUN E. KIM, SOJIN LEE, DO-UN JEONG, CHRISTOPHER RYAN, Mineola, NY, Seoul, Republic of Korea, Boston, MA, Salt Lake City, UT, Pittsburgh, $P A$

Structural deficits in specific brain regions may account for emotional and cognitive changes in T1DM. Frontal and temporal white matter may be especially vulnerable because it becomes myelinated later than other regions during childhood and adolescence when active brain development occurs. To identify regional abnormalities in white matter tracts in T1DM patients and examine their contributions to cognitive and emotional dysfunction, diffusion tensor (DT) images were obtained from 34 T1DM patients (age $=32$ $\pm 4.8 \mathrm{yrs}$, Male/Female $=16 / 18, \mathrm{HbA1c}=7.7 \pm 1.2 \%$, DM duration $=21 \pm 3.4$ yrs, BP mean $=122 / 76 \mathrm{mmHg}$, Total Cholesterol $=193 \pm 60 \mathrm{mg} / \mathrm{dL}$ ) and 16 age and sex-matched control subjects lage $=31 \pm 5.5 \mathrm{yrs}, \mathrm{HbA} 1 \mathrm{c}=5.1 \pm 0.3 \%$, $B P$ mean $=116 / 72 \mathrm{mmHg}$, Total Cholesterol $=176 \pm 35 \mathrm{mg} / \mathrm{dL}$ ). Images were analyzed using tract-based spatial statistics. All analyses were corrected for multiple comparisons. Fractional anisotropy (FA), which is highly sensitive to white matter integrity, was the primary outcome variable. Global mean
FA values were substantially reduced in T1DM patients relative to control subjects $\left(F_{1.46}=7.49, P=0.007\right)$. Whole-brain voxelwise analyses showed that T1DM-related FA reductions were particularly salient in the fronto-temporal regions. Lower FA values in bilateral superior longitudinal fasciculi, where group differences were prominent, correlated with decreased performance on working memory in T1DM patients (left, $r=0.57, P<0.001$; right, $r=0.44$, $P=0.009$ ). No subjects met criteria for current major depression. However, subsyndromal depressive symptoms, assessed using the Hamilton Depression Rating Scale, were also associated with lower FA values in the right inferior fronto-occipital fasciculus $(r=-0.49, P=0.004)$. Fronto-temporal white matter microstructural abnormalities may lead to both emotional and cognitive dysregulation, thereby serving as mechanisms underlying T1DM-related CNS complications that compromise the quality of life of T1DM patients.

Supported by: NIH/NIDDK, National Research Foundation of Korea

68-0R

The Effect of DA-9801 on the Peripheral Nerve Protection in Streptozotocin Induced Diabetic Rats

HEUNG YONG JIN, TAE SUN PARK, KYUNG AELEE, HONG SUN BAEK, JAE MOON KIM, SUN HEE KIM, Jeon Ju, Republic of Korea

DA-9801, a mixture extract of Dioscorea japonica and Dioscorea nipponica, was reported to have neurotrophic activity. Therefore, we investigated the effect of DA-9801 on the peripheral nerve protection in diabetic rats. Animals were divided into 6 groups $(\mathrm{N}=8-10)$ and designated as follows according to the DA-9801 dose: Normal, Normal+DA-9801-100(mg), Diabetes(DM), DM+DA-9801-10(mg), DM+DA-9801-50(mg), and DM+DA-9801-100(mg). Statistical analyses for functional parameters and neuronal quantification were performed after 16 weeks treatment. In results, blood glucose levels were not affected, however thermal withdrawal latency, and mechanical allodynia using von frey filament were improved in the DA-9801 treated DM group as shown in the [Figure1]. Intraepidermal nerve fiber (IENF) density less reduced significantly in the DM+DA-9801 groups than DM group although dose dependant effect was not observed $(9.12 \pm 0.96$ vs $8.96 \pm 0.60$ vs $9.30 \pm 0.70$ vs 5.81 $\pm 0.52 / \mathrm{mm}, \mathrm{DM}+\mathrm{DA}-9801-10, \mathrm{DM}+\mathrm{DA}-9801-50, \mathrm{DM}+\mathrm{DA}-9801-100$, and $D M$, respectively, $P<0.05$ [ [Figure1]. Mean myelinated axonal area in the sciatic nerves was also higher significantly in the DM+DA-9801 treated groups than DM group $\left(70.2 \pm 3.46\right.$, vs $71.5 \pm 2.15$, vs $72.1 \pm 1.57$, and $59.1 \pm 2.91 \mu \mathrm{m}^{2}$, DM+DA-9801-10, DM+DA-9801-50, DM+DA-9801-100, and DM, respectively, $P<0.05$ [ [Figure1]. Our results demonstrated that DA-9801 is beneficial in the respect of peripheral nerve preservation in diabetes. Therefore, this agent can be used in the pathogenic treatment for diabetic peripheral neuropathy in the future.
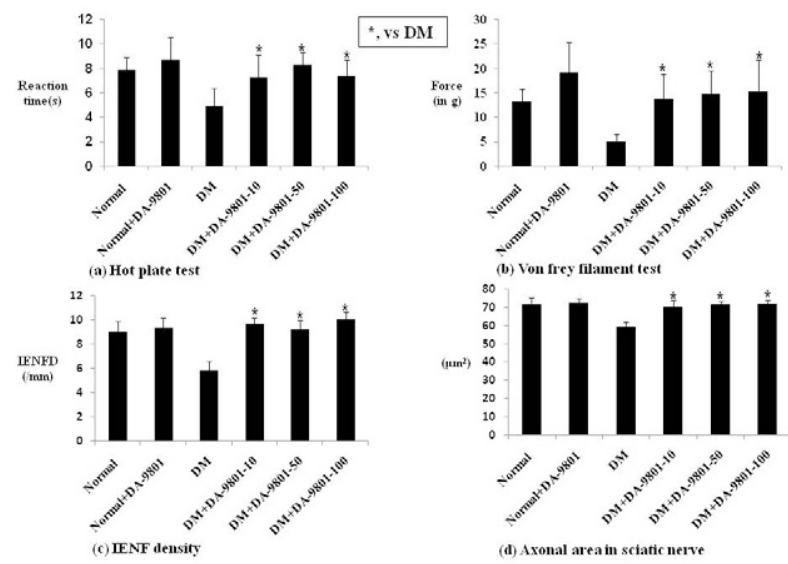

Figure 1.

69-0R

Can Simple Function Tests for Cardiovascular Autonomic Neuropathy (CAN) Predict Coronary Artery Disease (CAD) in Type 1 Diabetes (T1D)?

GEORGIA PAMBIANCO, TREVOR ORCHARD, Pittsburgh, PA

CAN is thought to confer a high risk of mortality, CAD, heart failure and tachycardia. Since there is no agreement on the optimal screening for autonomic neuropathy in TID, the long term predictive power of two simple tests, $E / I$ ratio and 30:15 ratio, were assessed with regard to $C A D$ incidence in the Pittsburgh Epidemiology of Diabetes Complications (EDC) study of childhood onset T1D cohort. At study entry (1986-1988, $n=658)$, the mean age was 
28 years and mean diabetes duration was 19 years. Follow up (mean=10.3 years) was determined from the time of first assessment of the 30:15 ratio test (1994) in 248 participants. The E/I ratio was measured by means of heart rate response to deep breathing and dichotomized as $>=1.1$ (normal) and $<1.1$ (abnormal). The 30:15 ratio was measured by the longest RR interval around the $30^{\text {th }}$ heartbeat and the shortest around the $15^{\text {th }}$ heart beat after standing up and dichotomized as $>1.04$ (normal) and $<=1.04$ (abnormal). CAD was defined as clinic physician diagnosed angina, ischemic ECG changes, revascularization, confirmed $\mathrm{MI}$, or CAD death. Risk factors examined were diabetes duration, age, HbA1c, hypertension, smoking, total cholesterol, HDL, triglycerides and LDL cholesterol, waist/hip ratio (WHR), gender, WBC, estimated glomerular filtration rate (GFR), and albumin excretion rate (AER). Forty seven $(19 \%)$ out of 248 participants developed CAD in the follow up period. Univariately, E/I ratio (HR 2.8, 95\% Cl 1.5, 5.1) but not 30:15 ratio (HR 1.3, 95\% Cl .7, 2.3) predicted CAD. In the fully adjusted model, E/I ratio remained independently predictive (HR 1.9, 95\% $\mathrm{Cl} 1.1,3.5$ ), along with diabetes duration (HR 1.1, Cl 1.0, 1.1), WBC (HR 1.2, Cl 1.0, 1.4), and HbA1c (HR 1.3, Cl 1.1, 1.7). The study indicates that the $\mathrm{E} / \mathrm{I}$, but not the $30: 15$, is an independent predictor of $\mathrm{CAD}$, and thus the preferred simple measure.

\section{0-0R}

Transplantation of Neural Progenitor-Like Cells Derived from Spheroidal Dermal Cells Improves Diabetic Polyneuropathy in Mice MASAKI KONDO, HIDEKI KAMIYA, TATSUHITO HIMENO, JIRO KATO, TETSUJI OKAWA, ATSUSHI FUJIYA, KEIKO NARUSE, YOJI HAMADA, NAOMI NISHIO, SACHIKO ITO, YUTAKA OISO, KEN-ICHI ISOBE, JIRO NAKAMURA, Nagoya, Japan

Background and Aims: We have identified and established spheroidal dermal cells (sDCs) from skin that possess pluripotency, and succeeded to derive neural progenitor-like cells (NPLCs) from sDCs. Here we investigated the effects of NPLC transplantation on diabetic polyneuropathy (DPN) in mice. Materials and Methods: We cultured dermal cells from 5-week old EGFPC57BL/6 male mice and formed 1x104 dermal cells into spheroids (sDCs) on U-bottom 96-well plates. NPLCs were induced from sDCs by co-culturing with PA 6 for 10 days. Diabetes was induced by intraperitoneal injection of STZ to 8-week old C57BL/6 male mice. Then, NPLCs (1x103 cells per cluster) or saline were injected into the right or left hindlimb muscles (10 clusters/limb) of 12-week STZ diabetic (D) and age-matched normal (N) mice, respectively. Four weeks later, thermal planter test (TPT), current perception thresholds (CPTS), and nerve conduction velocities (MNCV and SNCV) were evaluated. Result: Induced NPLCs were positive for p75 and beta-III tubulin. Four weeks after transplantation, NPLCs resided in the injected muscles and did not produce teratoma or other tumors. D showed impaired thermal sensation (TPT; N: $5.5 \pm 1.7 s, D: 9.6 \pm 3.6$ ) indicating hypoalgesia, and delayed MNCV (N: $53.6 \pm 6.9 \mathrm{~m} / \mathrm{s}, \mathrm{D}: 36.9 \pm 6.7$ ) and SNCV (N: $32.9 \pm 4.9 \mathrm{~m} / \mathrm{s}, \mathrm{D}: 21.9 \pm 2.7$ ), which were significantly ameliorated by NPLC transplantation (TPT: $5.2 \pm 1.1$, MNCV: 48.1 \pm 5.5 , SNCV: $33.7 \pm 5.8$ ). Conclusion: These results indicate that transplantation therapy with NPLCs would be effective for DPN.

\section{EXPANDING THE DOMAINS OF DIABETES EDUCATION}

\section{1-0R}

\section{Games are a Preferred Method to Improve Diabetes Knowledge and} Self-Efficacy in Adults

CIRCE W. TSUI, JANE M. CAUDLE, ADRIENNE R. HERRON, IRMGARD U. WILLCOCKSON, CATHERINE S. BARNES, DAVID C. ZIEMER, Atlanta, GA, Conroe, TX

Digital gaming promotes positive health outcomes in juvenile diabetes patients, but its usefulness for adults with diabetes is unknown. We conducted a randomized pilot to examine the effectiveness of a custom-designed computer game, Wheel of Diabetes (WOD), on diabetes management among adults. WOD took a 'repetition and reward' approach to teaching patients about hemoglobin A1c, cholesterol and blood pressure. Playing WOD $(n=60)$ was compared to reading a pamphlet $(n=55)$. A knowledge test and a 4-question self-efficacy survey were administrated before and after. After posttesting, all subjects completed the alternative intervention and preference survey. Subjects were recruited in the Grady Diabetes Center, a hospitalbased outpatient clinic previously shown to have a high prevalence of low literacy. The population was 91\% African American, $71 \%$ female, with mean age 55 (20-85) yr and diabetes duration 11 yr; $69 \%$ had not or rarely played computer games before. Pre-Post outcomes were analyzed by a mixed effect model for repeated measures. The interventions had significant and positive effect on knowledge $(P<0.001)$, self-efficacy for controlling blood glucose $(P=0.001)$, controlling cholesterol $(P=0.007)$, as well as living a normal life $(P=0.008)$. There was a trend effect on efficacy for controlling blood pressure $(P=0.08)$. The two interventions were equally effective (between intervention $P=0.637, P=0.983, P=0.771, P=0.429, P=0.734$, respectively). A strong majority of the subjects found that the game was more fun (71\%), more interesting $(60 \%)$ and made learning easier (55.7\%). Seventy-four percent would replay the game rather than read the pamphlet again. While both digital gaming and reading improve diabetes management knowledge and self-efficacy among adults, gaming is overwhelmingly favored by the subjects. Their expressed willingness to replay the games rather than read more, provides a viable strategy for diabetes education and reinforcement in a low-literacy adult population.

72-0R

How Do You Get from Here to There With Diabetes?

BLAKE E. ELKINS, MARK W. TRUE, MARCUS M. CRANSTON, Lackland AFB, TX, Keesler AFB, MS

Despite available travel advice for patients with diabetes mellitus, little is known regarding the practice of medical providers or the level of patient knowledge. This study examined provider and patient beliefs and behaviors regarding travel medicine recommendations during routine diabetic visits. This cross-sectional study surveyed 228 diabetic patients at Wilford Hall Medical Center, San Antonio, Texas using a self-report questionnaire. Survey questions addressed provider practices and patient behaviors and knowledge. The study population was predominantly older patients with established diabetes mellitus, type $2(87.3 \%>50$ years-old; $81.5 \%$ type 2; $85.6 \%$ had diagnosis $>5$ years). Only $23.5 \%$ of subjects had ever been asked about travel by a provider and only $18.5 \%$ had ever inquired about glucose monitoring during travel. Among those using insulin, only $27.8 \%$ had ever inquired about insulin dosing during travel. Patients that sought advice most often obtained information from Nurse Educators $(35.5 \%)$ or written materials $(32.2 \%)$, while only $16.5 \%$ obtained information from the ADA. When traveling outside the United States only $27.9 \%$ stated they would pre-arrange a medical facility, in case of illness. The majority would depend on the U.S. Embassy or hotel staff for recommendations for medical care $(72.1 \%)$ or prescription medication replacement (63.0\%). Finally, when given travel scenarios crossing multiple time zones, subjects rarely stated they would anticipate a need to adjust medications, $25.0 \%$ for westward and $24.6 \%$ for eastward travel. Adults with diabetes mellitus are not commonly asked about travel during routine diabetes clinic appointments. In addition, few patients inquire about care prior to travel and most do not prepare for medication dose changes, illness or lost medications. This study reveals a significant gap in patient knowledge and provider practice in which a concerted effort is needed to ensure safe travel for patients with diabetes mellitus.

73-0R

Diabetes Self-Management Education Patterns Associated With Glycemic Control in Youth With Type 1 Diabetes

LINDSAY M. JAACKS, RONNY A. BELL, DANA DABELEA, RALPH B. D'AGOSTINO, JR., LAWRENCE M. DOLAN, GIUSEPPINA IMPERATORE, GEORGEANNA J. KLINGENSMITH, JEAN M. LAWRENCE, SHARON SAYDAH, JOYCE YI-FRAZIER, ELIZABETH MAYER-DAVIS, FOR THE SEARCH FOR DIABETES IN YOUTH STUDY GROUP, Chapel Hill, NC, Winston-Salem, NC, Denver, CO, Cincinnati, OH, Atlanta, GA, Pasadena, CA, Seattle, WA

Poor glycemic control increases the risk of diabetes-related complications. The specific components of diabetes self-management education associated with glycemic control in youth with type 1 diabetes (T1D) have not been fully elucidated. SEARCH for Diabetes in Youth is a study of youth $<20$ yrs old at diagnosis in the US. This analysis includes cross-sectional data from 1,368 participants from 5 centers in the 2002-2005 incident cohorts (mean \pm SD age and A1C: $14.6 \pm 4.4 \mathrm{yrs}$ and $8.8 \pm 1.8 \%$ ). Principal component analysis was used to identify patterns of diabetes education. Associations between resulting factor scores and glycemic control (A1C $\geq 9.5 \%$ versus $<$ $9.5 \%)$ were evaluated using multivariable logistic regression adjusted for age, diabetes duration, parental education and family composition. Four factors were identified using eigen value-one criteria and a scree plot. Of these factors, Direct Communication about Care was associated with lower odds of $\mathrm{A} 1 \mathrm{C} \geq 9.5 \%$ and Medical Nutrition Therapy and Attendance at Visits were associated with greater odds of $\mathrm{A} 1 \mathrm{C} \geq 9.5 \%$ while Receiving DiabetesRelated Information was not significantly associated with glycemic control. A limitation of these results is the potential for reverse causality: youth with $\mathrm{A} 1 \mathrm{C} \geq 9.5 \%$ may be more likely to be referred to attend visits or for receipt of additional nutrition recommendations. Prospective research is needed to further understand this relationship and patterns of diabetes education for improving glycemic control in youth with T1D. 


\begin{tabular}{|c|c|c|c|}
\hline Factor & Variable & Factor Loading & g $\quad \mathrm{OR}(95 \% \mathrm{Cl})^{*}$ \\
\hline $\begin{array}{l}\text { Direct Communica- } \\
\text { tion about Care }\end{array}$ & $\begin{array}{l}\text { Received info in-person } \\
\text { Received a copy of laboratory } \\
\text { results Received appointment } \\
\text { reminder Received info via tele- } \\
\text { phone }\end{array}$ & $\begin{array}{l}0.680 .68 \\
0.610 .43\end{array}$ & $0.78(0.68,0.90$ \\
\hline $\begin{array}{l}\text { Medical Nutrition } \\
\text { Therapy }\end{array}$ & $\begin{array}{l}\text { Calorie tracking recommenda- } \\
\text { tion Fat gram tracking recom- } \\
\text { mendation Dietary exchanges } \\
\text { recommendation Low glycemic } \\
\text { index recommendation }\end{array}$ & $\begin{array}{l}0.760 .76 \\
0.760 .69\end{array}$ & $1.22(1.06,1.40$ \\
\hline Attendance at Visits & $\begin{array}{l}\text { Met with dietician or nutritionist } \\
\text { in past } 12 \text { months Met with dia- } \\
\text { betes educator or nurse in past } \\
12 \text { months }\end{array}$ & 0.820 .79 & $1.20(1.04,1.39$ \\
\hline $\begin{array}{l}\text { Receiving Diabetes- } \\
\text { Related Information }\end{array}$ & $\begin{array}{l}\text { Received info about diabetes } \\
\text { support groups Received info } \\
\text { about camps Received written } \\
\text { info about diabetes, such as } \\
\text { pamphlets Received info on how } \\
\text { to find diabetes info online } \\
\text { Received info about other } \\
\text { research studies }\end{array}$ & $\begin{array}{l}0.730 .63 \\
0.530 .53 \\
0.40\end{array}$ & $1.02(0.88,1.19$ \\
\hline
\end{tabular}

*Odds ratio (OR) and $95 \%$ confidence interval $(95 \% \mathrm{Cl})$ for a $1 \mathrm{SD}$ change in factor scores predicting $\mathrm{A} 1 \mathrm{C} \geq 9.5 \%$ compared to $<9.5 \%$, adjusted for age, diabetes duration, parental education and family composition.

Supported by: The CDC with support from the NIDDK

74-0R

Knowledge Application (KA) Predicts Reproductive Health Attitudes and Behaviors in Teens With Diabetes

DENISE CHARRON-PROCHOWNIK, SUSAN SEREIKA, DOROTHY BECKER, NEIL H. WHITE, ANDREA R. FISCHL, WILLIAM HERMAN, A.B. POWELL, PATRICIA SCHMITT, ANA DIAZ, JACKIE JONES, MONICA DINARDO, FENG GUO, LAURA MCEWEN, JULIE DOWNS, Pittsburgh, PA, St. Louis, MO, Ann Arbor, MI

ADA recommends that preconception counseling (PC) start at puberty. READY-Girls is a self-administered PC program (DVD, book) for teens with type 1 (T1D) or type 2 (T2D) diabetes. We used a diabetes and reproductive health knowledge assessment to evaluate the long-term effectiveness of READY-Girls. At 2 sites, 113 teens [mean age=16.2yrs (13.3-19.9yrs)] with T1D or T2D, were randomized to a standard care control group (CG; $\mathrm{N}=59$ ) or a READY-Girls intervention group (IG; $\mathrm{N}=54$ ). $17 \%$ were African American and $8 \%$ had T2D. READY-Girls was given over 3 visits. At baseline, about $20 \%$ $(n=11)$ in each group were sexually active with mean sexual debut of $15.4 y r s$ (12-18yrs); $50 \%$ had unsafe sex. The KA measure has multiple choice problem-solving vignettes and 7 subscales: PC, pregnancy, contraception, sexuality, puberty, general family planning, and general diabetes; scored $100 \%$ correctness. Attitudes and behaviors included: self-efficacy, benefits and barriers to birth control $(\mathrm{BC})$ and $\mathrm{PC}$; severity and susceptibility to unplanned pregnancy/complications; and initiating discussion with health professionals. Baseline mean total $\mathrm{KA}$ score for $\mathrm{IG}=65 \%$ and $\mathrm{CG}=68 \%(\mathrm{p}=.10)$. Over $12 \mathrm{mos}$, IG had improvements in KA with significant group-by-time effects for total score $(p<.0001), P C(p=.0005)$, pregnancy $(p<.0001)$, and sexuality $(p=.0041)$. CG had no improvements. Total knowledge was significantly associated with self-efficacy $(p=.05)$, benefits $(p<.0001)$, barriers $(p<.0001)$, severity $(p<.05)$, susceptibility $(p<.05)$, and intention to use family planning $(p=.059)$, but not with initiating discussion ( $p>05)$. We conclude that the READY-Girls program has significant and sustained beneficial effects on knowledge, and that knowledge is positively associated with reproductive health attitudes and behaviors.

Supported by: NIH/NICHD (R01 HD44097)

75-OR

Educator Use of Masked Continuous Glucose Monitoring Device (CGM) in a Clinic Population of Youth With Type 1 Diabetes (T1D)

KERRY M. MILASZEWSKI, JOYCE KEADY, LISA M. SCHMIDT, LISA E. RASBACH, ASHLEY E. ATKINS, LISA K. VOLKENING, LORI M. LAFFEL, Boston, MA

Despite advanced diabetes technologies, many youth with T1D fail to meet target A1c goals. We assessed use of a 3-day, masked CGM and evaluated its impact on glycemic control in a diabetes clinic caring for $>2000$ children, teens, and young adults. Over $\sim 2$ years, 122 patients with T1D were referred for CGM use to evaluate elevated A1c, unexplained hypoglycemia, post-prandial BG excursions, and/or impact of activity on BG. Youth $(47 \%$ male, $61 \%$ pump-treated) had a mean age of $14.3 \pm 3.9$ years (range $7-28$ ) and T1D duration of $7.5 \pm 4.7$ years. A1c was $8.5 \pm 1.1 \%$ (range $5.8-12.6 \%$ ) at CGM insertion. Experienced RNs/NPs evaluated CGM tracings and provided Rx recommendations (RECs). Post CGM use (median 76 days, inter-quartile range $50-99$ ), 115 youth (7 not done) had A1c of $8.4 \pm 1.2 \%$. CGM tracings for 122 youth (3 required reinsertion) yielded CGM mean glucose of $181 \pm 34$ and SD glucose of $75 \pm 16 \mathrm{mg} \%$ with an average of $894 \pm 136$ readings (range 435-1151) per youth; CGM mean glucose and SD were correlated with insertion A1c $(r=0.43, p<.0001 ; r=0.24, p<.009)$. Patients received 3.1 \pm 1.1 RECs after CGM use. RECs were to: give pre-meal/snack boluses (81\%), change bolus doses (64\%), change basal/intermediate doses $(51 \%)$, use advanced boluses/attend to active insulin (39\%), adjust exercise plan (35\%), count carbs/macronutrients (15\%), adjust hypoglycemia Rx $(12 \%)$, and increase BG monitoring (6\%). Post CGM use, 39 youth (34\%) improved A1c by $\geq 0.5 \%$. Those who improved were older ( $15.5 \pm 4.4$ vs $14.0 \pm 3.6)$, had higher insertion A1c ( $8.9 \pm 1.0$ vs $8.3 \pm 1.1 \%)$, and received more RECs $(3.5 \pm 1.1$ vs $3.0 \pm 1.1)$ than those who did not (all $p<.05)$; mode of insulin delivery was not associated with improvement. Those who received REC to use advanced boluses/attend to active insulin were 3.5 times $(p<.002)$ more likely to improve A1c by $\geq 0.5 \%$. Educator use of masked CGM in youth with $\mathrm{T} 1 \mathrm{D}$ is clinically practical. Patients amenable to advanced bolus dosing and attending to active insulin appear likely to improve A1c after CGM use.

76-OR

Hospital Survival Skills DSME DVD Improves Post Discharge Medication Adherence

MICHELLE F. MAGEE, NAWREEN H. KHAN, CARINE M. NASSAR, Washington, DC

Diabetes self-management education (DSME) improves knowledge, clinical outcomes, e.g. A1C, and costs. While the hospital may be considered a suboptimal environment in which to provide DSME, admissions present an opportunity to deliver education. The ADA (Standards of Care 2012) suggest "survival skills" education as a feasible approach to inpatient DSME. Evidence is needed to define strategies for hospital DSME delivery. We have piloted a diabetes survival skills instructional DVD entitled "Diabetes-ToGo" in an urban teaching hospital. Content was aligned with ADA suggested areas for hospital DSME. The primary goal was to reduce readmissions. Patients with uncontrolled diabetes $(B G \geq 200 \mathrm{mg} / \mathrm{dL}$ or $\leq 40 \mathrm{mg} / \mathrm{dL}$ ) admitted to the hospital were eligible for inclusion. Knowledge and diabetes medication adherence were assessed at baseline, 2 and 3 months post-discharge. Patients were directed to watch DVD sections corresponding to survey identified knowledge deficits prior to discharge. Patients, $n=125$, were consented and enrolled in this IRB approved study. Interim data analysis has been performed for 72 program completers. Mean age $59 \pm 13$ years; $68 \%$ female; $86 \%$ African American; Mean BG $282 \pm 141 \mathrm{mg} / \mathrm{dL}$; Mean A1C $9.7 \pm 2.8 \%$.

\begin{tabular}{lccc}
\hline ITEM & \multicolumn{3}{c}{ TIME PERIOD } \\
\hline Knowledge Test & Baseline & Post-, $\mathrm{p}$ value \\
\hline $\mathbf{5}$ out of $\mathbf{8}$ questions correct & & $64.29 \%$ & $85.71 \%, \mathrm{p}=0.006$ \\
\hline Medication Adherence & Baseline to & Baseline to & 2 weeks to \\
& 2 weeks & 3 months & 3 months \\
\hline Change in Morisky Scale* & -2.83, & -2.9, & 0.23, \\
Adherence Scores & $\mathrm{p}<0.0001$ & $\mathrm{p}<0.0001$ & $\mathrm{p}<0.8195$ \\
\hline Hospital Admission(s) & & 3 months & 3 months \\
& & before study & after study \\
\hline Did NOT require hospitalization & 82.4\% & $91.2 \%, \mathrm{p}=0.1797$ \\
\hline${ }^{*}$ Morisky Scale used with permission from David Morisky
\end{tabular}

A targeted survival skills DSME DVD offered in the hospital demonstrated preliminary evidence of knowledge improvement, an increase in medication adherence from baseline to 2 weeks which persisted until 3 months, and a trend toward reduction in hospital readmissions.

Supported by: sanofi-aventis

77-0R

Impact of a Pocket Insulin Dosing Guide on Utilization of Basal/Bolus Insulin by Internal Medicine Resident Physicians

MICHAEL G. JAKOBY, MOHAMMED ALNIJOUMI, SHERYLL SORIANO, DANXUAN LONG, DOMINIKA JANOWSKI, SOO AH KIM, OWAISE MANSURI, EDWARD RICO, CHAITANYA MAMILLAPALLI, DEEPIKA NALLALA, JENNIFER BOND, Springfield, IL

Basal/bolus insulin (BBI) is superior to sliding scale insulin (SSI) for diabetes patients admitted to general medicine and surgery services, but little is published on strategies to promote utilization of BBI by resident physicians. We completed a prospective study with historical controls to evaluate the impact of a pocket insulin dosing guide on diabetes management practices of internal medicine resident physicians at the SIU School of Medicine rotat- 


\section{SGLT-INHIBITORS}

ing on general medicine. Pocket insulin dosing cards with instructions for initiation of $\mathrm{BBI}$ and daily insulin adjustments were provided to all internal medicine residents November 2010. BBI utilization rates were monitored November 2010-February 2011 and compared to the corresponding four month period the previous academic year (November 2009-February 2010) before pocket insulin dosing cards were introduced. Study patients ( $N=212$ ) and historical controls $(\mathrm{N}=223)$ were generally well matched. $\mathrm{BBI}$ orders increased from $12.8 \%$ of all resident insulin orders November 2010 to $58.1 \%$ of all orders February 2011 ( $\mathrm{P}<0.0001$ for trend) compared to $\mathrm{BBI}$ insulin order rates of $0-8 \%$ November 2009-February 2010. Overall, BBI as a proportion of all insulin orders was $35.7 \%$ during the four month study window, a sixfold increase over the previous academic year $(6 \%)$ that was highly and statistically significant $(\mathrm{P}<0.0001)$. In February 2011, when BBI utilization was maximal, proportion of capillary blood glucose (CBG) measurements 70-140 $\mathrm{mg} / \mathrm{dL}$ for BBI managed patients was $42.8 \%$ compared to $35.9 \%$ for SSI managed patients, a significant improvement $(\mathrm{P}=0.005)$ that occurred without an increase in CBG measurements $<70 \mathrm{mg} / \mathrm{dL}$ (2.8\% for BBI patients, $2.4 \%$ for SSI patients, $\mathrm{P}=0.73$ ). The SIU pocket insulin dosing guide significantly increased utilization of BBI, decreased SSI orders, and improved hospital glycemic control for patients with diabetes mellitus.

Behavior Score Instrument

78-OR

AMPARO GONZALEZ, SANDRA BURKE, ROBERTO MEJIA, RUTH LIPMAN, GUILLERMO UMPIERREZ, Atlanta, GA, Chicago, IL, Medellin, Colombia

The Behavior Score Instrument (BSI) developed by the American Association of Diabetes Educators (AADE) provides quantitative assessment on behavior changes during Diabetes Self-Management Education/Training (DSME/T). The BSI tool covers healthy eating, being active, monitoring, medications, problem-solving, healthy coping and reducing risks (7 AADE Self-Care Behaviors $\left.{ }^{\mathrm{T}}\right)$. Three questions for each behavior evaluate frequency, confidence and conviction of patient's intervention acceptance. We report the first prospective evaluation of the BSI with 92 diabetes patients from the Grady Health System (Atlanta, GA). Participants were assessed using the BSI, SF-12v2 for quality of life (OOL) measures, and clinical indicators (A1C, blood pressure and BMI) at baseline and 3-6 months. Demographics lage $55 \pm 11$ yr, female $63 \%$, high school education $53.3 \%$, household income $<\$ 15,000 /$ year $71.7 \%$ ). BSI overall scores: $2.64 \pm 0.19$ baseline and $2.7 \pm 0.27$ (6 mo). A1C: $9.0 \% \pm 2$ baseline, $8.0 \% \pm 2$ (6 mo). BMl: $34.5 \pm 7$ initial, $35.7 \pm 14$ (6 mo). Systolic BP: $133.4 \pm 2$ baseline, $127.8 \pm 20$ (6 mo). Pearson correlation showed significant correlation between overall initial and 3 mo BSI score $(p<0.01)$ suggesting internal consistency. BSI score significantly correlated with A1C ( $p<0.01)$ level and with $00 \mathrm{~L}$ aggregate SF-12v2 mental component at baseline and $6 \mathrm{mo}(\mathrm{p}<0.01)$. The trend over time for overall BSI was driven by change in healthy eating and monitoring. Among each of the AADE Self-Care Behaviors ${ }^{\mathrm{TM}}$, only healthy eating $(\mathrm{p}=0.08)$ and monitoring $(\mathrm{p}=0.09)$ trended toward improvement at $3 \mathrm{mo}$ and were significant at $6 \mathrm{mo}(\mathrm{p}=0.001$ and 0.004 , respectively). Factor analysis suggests that $80 \%$ of the variance in the model is accounted for by two integrated factors. The first factor is being active and taking medication. The second factor is the combination of healthy coping and monitoring behaviors. In summary, the BSI appears to be a valuable tool to access patient readiness to initiate behavior change and may also have use in redirecting patient's goal setting to facilitate behavior change.

Supported by: Johnson and Johnson Diabetes Institute

\section{SGLT-INHIBITORS}

79-0R

Canagliflozin Lowers Postprandial Glucose and Insulin by Delaying Intestinal Glucose Absorption in Addition to Increasing Urinary Glucose Excretion

DAVID POLIDORI, SUE SHA, SUNDER MUDALIAR, THEODORE P. CIARALDI, ATALANTA GHOSH, NICOLE VACCARO, KRISTIN FARRELL, PAIVI M. BURKE, PAUL ROTHENBERG, ROBERT R. HENRY, San Diego, CA, Raritan, NJ

Canagliflozin (CANA), a potent sodium glucose co-transporter 2 (SGLT2) inhibitor, is also a low-potency SGLT1 inhibitor; intestinal CANA levels post-dose may be sufficiently high to transiently inhibit intestinal SGLT1. A 2-period crossover study was performed in 20 healthy subjects (mean $\pm S D$ age $=26 \pm 6 \mathrm{yr}, B W=78 \pm 10 \mathrm{~kg}$ ) to assess effects of a single $300 \mathrm{mg}$ CANA dose on intestinal glucose (G) absorption using a dual tracer method (IV ${ }^{3} \mathrm{H}-\mathrm{G}$ and oral ${ }^{14} \mathrm{C}-\mathrm{G}$ ). Placebo (PBO) or CANA was given 20 min prior to a 600 $\mathrm{kcal}$ mixed-meal tolerance test containing $75 \mathrm{~g}$ of G. Plasma G, ${ }^{3 \mathrm{H}}-\mathrm{G},{ }^{14} \mathrm{C}-\mathrm{G}$, and insulin were measured frequently for $6 \mathrm{~h}$; these values were used to calculate the rate of appearance in plasma of oral $G\left(R_{2} 0\right)$, endogenous $G$ production, and $G$ disposal. CANA was well tolerated. CANA delayed $R_{a} 0$ (Figure $\mathrm{A}$ ) and reduced cumulative oral $\mathrm{G}$ appearance (AUC $\mathrm{R}, 0$ ) by $31 \%$ at $1 \mathrm{~h}$ (geometric mean $\mathrm{PBO}=381 \mathrm{vs}$. CANA $=264 \mathrm{mg} / \mathrm{kg}, \mathrm{p}<.001$ ) and by $20 \%$ at $2 \mathrm{~h}(\mathrm{PBO}=723 \mathrm{vs}$. CANA $=576 \mathrm{mg} / \mathrm{kg}, \mathrm{p}=.002)$; this was nearly matched by increased $R_{a} 0$ over 2-6 $h$, so that AUC $R_{a} 0$ over $0-6$ h was $<6 \%$ lower for CANA than $\mathrm{PBO}(\mathrm{PBO}=1018 \mathrm{vs}$. CANA $=960 \mathrm{mg} / \mathrm{kg}, \mathrm{p}=.003$ ), consistent with the lack of notable malabsorption. CANA also increased urinary glucose excretion (UGE) over $0-2 \mathrm{~h}(\mathrm{PBO}<0.2 \mathrm{~g}$; $\mathrm{CANA}=6 \pm 3 \mathrm{~g})$, and $2-6 \mathrm{~h}(\mathrm{PBO}<0.1 \mathrm{~g}$; CANA $=12 \pm 4 \mathrm{~g}$ ). Total $\mathrm{G}$ disposal over $0-6 \mathrm{~h}$ was similar for $\mathrm{PBO}$ and CANA. In conclusion, CANA reduces postprandial plasma glucose and insulin (Figures $B$ and $\mathrm{C}$ ) by both increasing UGE (due to renal SGLT2 inhibition) and delaying $\mathrm{R}_{\mathrm{a}} \mathrm{O}$ (likely due to intestinal SGLT1 inhibition).
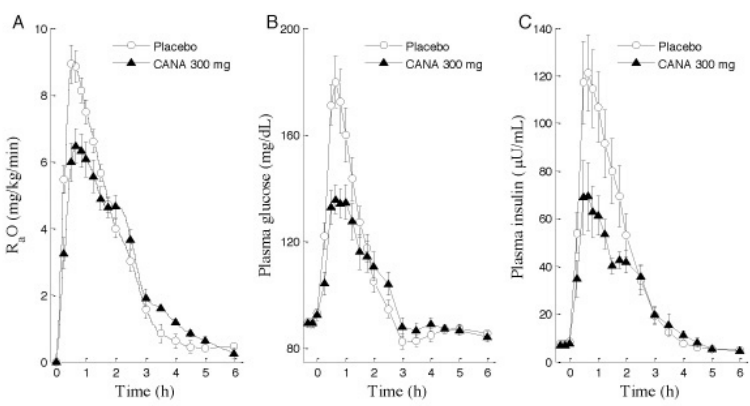

Supported by: Janssen Research and Development, L.L.C.

80-0R

Tofogliflozin, a Novel and Selective SGLT2 Inhibitor Improves Glycemic Control and Lowers Body Weight in Patients With Type 2 Diabetes Mellitus Inadequately Controlled on Stable Metformin or Diet and Exercise Alone

TAKASHI KADOWAKI, SACHIYA IKEDA, YASUKI TAKANO, OSAMU CYNSHI, ANDREAS D. CHRIST, VIKTOR BOERLIN, ULRICH BEYER, ANDREAS BECK, TokYo, Japan, Basel, Switzerland

Urinary glucose excretion (UGE) induced by selective inhibition of sodiumglucose cotransporter 2 (SGLT2) elicits a complex set of metabolic changes and ameliorates type 2 diabetes mellitus (T2DM). Tofogliflozin (TOFO) is a novel and highly selective SGLT2 inhibitor. Safety, tolerability and efficacy of TOFO was evaluated in a double-blind, randomized, placebo (PBO)-controlled 12-week dose finding study. After 4-week PB0 run-in period, a total of 398 T2DM patients (pts), either (1) treated with diet and exercise (D\&E) and a stable dose of metformin or (2) treated with D\&E alone were randomized to TOFO 2.5, 5, 10, 20, $40 \mathrm{mg}$ or PBO (qd, $15 \mathrm{~min}$ before breakfast, approx. $60 \%$ of D\&E+Met). Significant (except for $2.5 \mathrm{mg}$ ) and dose-dependent reductions of $\mathrm{HbA1c}$ were shown with a maximum lowering of $0.56 \%$ (PBO-adjusted, $40 \mathrm{mg}$ ), along with increased UGE. No clear difference was found between two treatment backgrounds. Dose-dependent BW reduction was also observed (Table), glucose intolerance was improved accompanied with plasma adiponectin increase and there was a trend for median blood pressure to decrease by $3-4 \mathrm{mmHg}$. Once daily doses of TOFO for 12 weeks were well tolerated and did not increase the incidence of hypoglycemia, urinary or genital tract infection, or cardiovascular events relative to placebo.

Table

\begin{tabular}{lcccccc}
\hline $\begin{array}{l}\text { Change from baseline at } \\
\text { week-12 (LOCF) }\end{array}$ & $\begin{array}{c}\text { PBO } \\
(\mathrm{n}=65)\end{array}$ & $\begin{array}{c}2.5 \mathrm{mg} \\
(\mathrm{n}=64)\end{array}$ & $\begin{array}{c}5 \mathrm{mg} \\
(\mathrm{n}=65)\end{array}$ & $\begin{array}{c}10 \mathrm{mg} \\
(\mathrm{n}=66)\end{array}$ & $\begin{array}{c}20 \mathrm{mg} \\
(\mathrm{n}=64)\end{array}$ & $\begin{array}{c}40 \mathrm{mg} \\
(\mathrm{n}=66)\end{array}$ \\
\hline $\mathrm{BL} \mathrm{HbA1c}(\%)$ & 7.87 & 7.94 & 8.01 & 8.00 & 7.92 & 7.93 \\
\hline BL Body weight (BW; kg) & 84.0 & 85.5 & 82.1 & 83.4 & 84.9 & 81.6 \\
\hline$\Delta \mathrm{HbA1c}(\%)$ & -0.27 & -0.44 & $-0.62^{* *}$ & $-0.69^{* *}$ & $-0.77^{* *}$ & $-0.83^{* *}$ \\
\hline$\Delta \mathrm{BW}(\mathrm{kg})$ & -0.7 & $-1.6^{*}$ & $-1.9^{* *}$ & $-2.2^{* *}$ & $-2.6^{* *}$ & $-2.8^{* *}$ \\
\hline$\Delta$ 24h UGE $(\mathrm{mmol} / 24 \mathrm{~h})$ & 16.9 & $217.9^{* *}$ & $272.3^{* *}$ & $346.2^{* *}$ & $396.0^{* *}$ & $402.9^{* *}$ \\
\hline$\Delta$ Adiponectin $(\mu \mathrm{g} / \mathrm{mL})$ & -0.22 & -0.22 & 0.22 & 0.36 & 0.10 & $0.53^{*}$ \\
\hline unadjusted p-value: ${ }^{*}<0.05,{ }^{* *}<0.01$ & & & &
\end{tabular}




\section{SGLT-INHIBITORS}

81-0R

Canagliflozin, a Sodium Glucose Co-Transporter 2 Inhibitor, Improves Glycemic Control and Lowers Body Weight in Subjects With Type 2 Diabetes Inadequately Controlled With Diet and Exercise KAJ STENLÖF, WILLIAM T. CEFALU, MARIA ALBA, KEITH USISKIN, YUE ZHAO, WILLIAM CANOVATCHEL, Gothenburg, Sweden, New Orleans, LA, Raritan, NJ

Canagliflozin (CANA), a novel inhibitor of the sodium-glucose co-transporter 2, is in development for the treatment of patients with type 2 diabetes mellitus (T2DM). This randomized, double-blind, placebo (PBO)-controlled, Phase 3 study evaluated CANA 100 or $300 \mathrm{mg}$ versus PBO in subjects with T2DM inadequately controlled with diet and exercise $(N=584$; age 55.4 years; A1c 8.0\%; fasting plasma glucose [FPG] $9.5 \mathrm{mmol} / \mathrm{L}$; body mass index $\left.31.7 \mathrm{~kg} / \mathrm{m}^{2}\right)$. At 26 weeks, A1c was significantly reduced with CANA $100 \mathrm{mg}$ (8.1\% to $7.3 \%$ ) and CANA $300 \mathrm{mg}(8.0 \%$ to $7.0 \%)$ versus PBO (8.0\% to $8.1 \%$; $P<0.001)$. Both CANA doses significantly improved FPG, 2-hr postprandial glucose, and percentage of subjects reaching A1c $<7 \%$ versus PBO. CANA also reduced body weight, reduced systolic blood pressure, and improved $\mathrm{HDL}-\mathrm{C}$ versus PBO. The overall incidence of adverse events (AEs) was modestly higher with CANA 100 and $300 \mathrm{mg}$ ( $61 \%$ and $60 \%$, respectively) versus PBO $(49 \%)$. Rates of serious AEs and AE-related discontinuations were low and similar among groups. Incidences of AEs consistent with genital mycotic infections were higher with CANA in women and men. A slightly higher rate of urinary tract infections was seen with CANA as well as AEs consistent with osmotic diuresis (eg, pollakiuria); these were generally mild and led to few discontinuations. The incidence of hypoglycemia was similar among groups. In summary, CANA improved glycemic control, reduced body weight, and was well tolerated in subjects with T2DM inadequately controlled with diet and exercise.

Table. Summary of Efficacy Endpoints (PBO-Adjusted) at Week 26 (LOCF)

\begin{tabular}{l|c|c}
\hline Parameter & CANA $100 \mathrm{mg}$ & CANA $300 \mathrm{mg}$ \\
\hline$\% \Delta \mathrm{A} 1 \mathrm{c}$ & $-0.91^{\mathrm{a}}$ & $-1.16^{\mathrm{a}}$ \\
\hline$\%$ of subjects reaching A1c $<7 \%$ & $23.9^{\mathrm{a}}$ & $41.7^{\mathrm{a}}$ \\
\hline$\Delta \mathrm{FPG}, \mathrm{mmol} / \mathrm{L}$ & $-1.97^{\mathrm{a}}$ & $-2.41^{\mathrm{a}}$ \\
\hline$\Delta$ 2-hr PPG, mmol/L & $-2.67^{\mathrm{a}}$ & $-3.55^{\mathrm{a}}$ \\
\hline$\% \Delta$ Body weight & $-2.2^{\mathrm{a}}$ & $-3.3^{\mathrm{a}}$ \\
\hline$\Delta$ Systolic BP, mmHg & $-3.71^{\mathrm{a}}$ & $-5.42^{\mathrm{a}}$ \\
\hline$\% \Delta$ HDL-C & $6.8^{\mathrm{a}}$ & $6.1^{\mathrm{b}}$ \\
\hline$\% \Delta$ Triglycerides & -5.4 & -10.2 \\
\hline LOCF, last observation carried forward; ANCOVA, analysis of covariance. $\Delta=$ PBO-adjusted least
\end{tabular}

LOCF, last observation carried forward; ANCOVA, analysis of covariance. $\triangle=P B O$-adjusted le
squares mean changes from baseline using ANCOVA. ${ }^{P} P<0.001$ vs $P B O,{ }^{b} P<0.01$ vs PBO

Supported by: Janssen Research and Development, L.L.C.

82-0R

Efficacy Increases With Increasing Baseline HbA1c Category With Dapagliflozin Therapy

ELISE HARDY, AFSHIN SALSALI, VERONIKA HRUBA, TRACI MANSFIELD, KATJA ROHWEDDER, CATRIN WESSMAN, JENNIFER SUGG, LI WEI, AGATA PTASZYNSKA, SHAMIK PARIKH, Wilmington, DE, Princeton, NJ, Prague, Czech Republic, Wedel, Germany, Molndal, Sweden

For patients with type 2 diabetes (T2DM) baseline HbA1c is an important factor in the choice of therapy and the magnitude of effect of antidiabetic agents. Dapagliflozin (DAPA) is a selective SGLT2 inhibitor that reduces hyperglycemia through the inhibition of renal glucose reabsorption and a resulting increase in urinary glucose excretion (UGE). Data from 5 phase III studies were pooled to assess the impact of baseline $\mathrm{HbA1c}$ on the efficacy of DAPA $(2.5 \mathrm{mg}, 5 \mathrm{mg}$ or $10 \mathrm{mg})$ QD or matched placebo (PBO) for 24 weeks. $\mathrm{HbA1c}$ levels were reduced with all doses of DAPA vs. PBO and across all baseline $\mathrm{HbA1c}$ levels. Higher baseline values were associated with greater reductions in $\mathrm{HbA1C}$ at 24 weeks (Figure). An increase in the proportion of patients with $\mathrm{HbA1c}$ levels reduced by $\geq 0.5 \%$ was observed with DAPA vs. PBO across all baseline HbA1c categories and was greatest in patients with the highest HbA1c levels at baseline (Table). Higher baseline HbA1c levels were also associated with greater increases in UGE/creatinine ratio as early as week 4 . In conclusion, higher baseline $\mathrm{HbA1c}$ levels were associated with greater improvements in glycemic efficacy and greater increases in UGE with DAPA in T2DM patients with inadequate glycemic control.

\begin{tabular}{|c|c|c|c|c|c|}
\hline \multirow{2}{*}{ Baseline $\mathrm{HbA} 1 \mathrm{c}$} & \multicolumn{5}{|c|}{ DAPA (mg/qd) } \\
\hline & & PBO & 2.5 & 5 & 10 \\
\hline \multicolumn{6}{|c|}{ Proportion of patients with a reduction in $\mathrm{HbA} 1 \mathrm{c} \geq 0.5 \% \mathrm{n}(\%) 24$ weeks (LOCF) } \\
\hline & $\mathrm{N}$ & 689 & 558 & 695 & 690 \\
\hline \multirow[t]{2}{*}{$<8 \%$} & $\mathrm{N \#}$ & 287 & 232 & 272 & 290 \\
\hline & & $96(33.4)$ & $108(46.6)$ & $123(45.2)$ & $175(60.3)$ \\
\hline \multirow[t]{2}{*}{$8 \%$ to $<9 \%$} & N\# & 245 & 220 & 230 & 242 \\
\hline & & $98(40.0)$ & $151(68.6)$ & $153(66.5)$ & $179(74.0)$ \\
\hline \multirow[t]{2}{*}{$\geq 9 \%$} & $\mathrm{~N} \#$ & 143 & 99 & 184 & 147 \\
\hline & & $67(46.9)$ & $79(79.8)$ & $150(81.5)$ & $127(86.4)$ \\
\hline \multicolumn{6}{|c|}{ Mean UGE/creatinine ratio g/g (SD) 4 weeks } \\
\hline & $\mathrm{N}$ & 689 & 625 & 763 & 766 \\
\hline \multirow[t]{2}{*}{$<8 \%$} & $\mathrm{~N} \#$ & 111 & 110 & 99 & 107 \\
\hline & & $1.4(5.1)$ & $14.9(18.0)$ & $25.9(23.4)$ & $32.3(21.5)$ \\
\hline \multirow[t]{2}{*}{$8 \%$ to $<9 \%$} & $\mathrm{~N} \#$ & 116 & 135 & 109 & 128 \\
\hline & & $3.0(9.3)$ & $19.9(24.7)$ & $28.9(22.9)$ & $40.4(24.2)$ \\
\hline \multirow[t]{2}{*}{$\geq 9 \%$} & $\mathrm{~N} \#$ & 62 & 57 & 84 & 60 \\
\hline & & $9.3(20.5)$ & $34.2(28.9)$ & $36.4(29.9)$ & $52.7(37.5)$ \\
\hline
\end{tabular}

$\mathrm{N}$ is the number of subjects in the Full Analysis Set. N\# is the number of subjects with non-missing baseline and Week 24 (LOCF) values in the Randomized Subjects/Full Analysis Set.
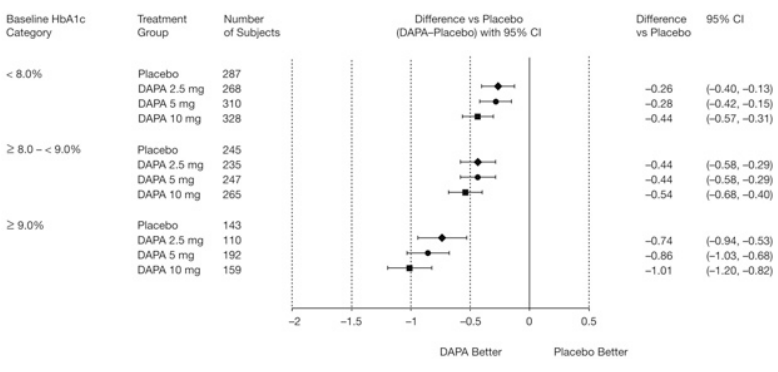

Supported by: AstraZeneca and Bristol-Myers Squibb

\section{Renal Glucose Kinetics in Response to Dapagliflozin}

RALPH A. DEFRONZO, MARCUS HOMPESCH, SREENEERANJ KASICHAYANULA, XIAONI LIU, YING HONG, MARC PFISTER, LINDA A. MORROW, BRUCE R. LESLIE, DAVID W. BOULTON, AGATHA CHING, STEVEN C. GRIFFEN, San Antonio, TX, Chula Vista, CA, Princeton, NJ

Dapagliflozin (DAPA) is a selective SGLT2 inhibitor that reduces hyperglycemia in type 2 diabetes (T2D) by promoting urinary glucose excretion. This study (NCT01165268) examined the effect of DAPA on renal glucose kinetics in 12 healthy, age-, BMI -, gender -, and ethnicity-matched control $(\mathrm{HbA1c}$ $\left.5.5 \%, 41 \mathrm{y}, 27 \mathrm{~kg} / \mathrm{m}^{2}, 7 \mathrm{M} / 5 \mathrm{~F}\right)$ and $12 \mathrm{~T} 2 \mathrm{D}\left(\mathrm{HbA} 1 \mathrm{c} 6.5 \%, 53 \mathrm{y}, 30 \mathrm{~kg} / \mathrm{m}^{2}\right.$, $7 \mathrm{M} / 5 \mathrm{~F}$ ) subjects. All subjects had an estimated glomerular filtration rate (eGFR) $\geq 60$ and $\leq 160 \mathrm{~mL} / \mathrm{min} \cdot 1.73 \mathrm{~m}^{2}$ by the Modification of Diet in Renal Disease equation. Subjects underwent a pancreatic stepped hyperglycemic clamp (SHC) (plasma glucose range 100-550 mg/dL) at baseline (BL) and after $7 \mathrm{~d}$ of DAPA, $10 \mathrm{mg} / \mathrm{d}$. The SHC was performed with octreotide and basal insulin/glucagon/growth hormone replacement. T2D subjects continued on $\mathrm{BL}$ therapy, unless taking metformin, which was held for $48 \mathrm{~h}$ before SHC. A model was developed to describe maximum tubular glucose transport (TmG), renal glucose threshold (for onset of glucosuria), and splay of the glucose titration curve for healthy and T2D populations and then used to estimate these parameters from individual titration curves. At BL, the TmG (443 vs $326 \mathrm{mg} / \mathrm{min}, P<0.04$ ) and splay (28650 vs $14248 \mathrm{mg}^{2} / \mathrm{min}^{2}, P<0.0001$ ) were significantly increased in T2D vs controls. DAPA treatment reduced TmG by $58 \%$ in T2D subjects and $53 \%$ in healthy subjects. The splay declined similarly by $37 \%$ for both groups. The BL threshold was similar in T2D (196 mg/ $\mathrm{dL}$ ) and control (171 mg/dL) subjects. Following DAPA, the modeled threshold was markedly reduced in T2D patients by $89 \%(21 \mathrm{mg} / \mathrm{dL})$ and in healthy subjects by $78 \%(37 \mathrm{mg} / \mathrm{dL})$. These modeled threshold values were well below the plasma glucose levels evaluated in this study. eGFR was reduced by $14 \%$ in both groups with DAPA. In summary, T2D subjects have a higher TmG than that of healthy controls. DAPA treatment for $7 \mathrm{~d}$ reduced $\mathrm{TmG}$ and 
splay. However, the markedly decreased renal glucose threshold was the primary mechanism responsible for the induction of glucosuria with DAPA in both $\mathrm{T} 2 \mathrm{D}$ and healthy subjects.

Supported by: AstraZeneca and Bristol-Myers Squibb

84-0R

Canagliflozin (CANA), a Sodium Glucose Co-Transporter 2 (SGLT2) Inhibitor, Reduces Post-meal Glucose Excursion in Patients With Type 2 Diabetes Mellitus (T2DM) by a Non-Renal Mechanism PETER P. STEIN, JOLENE K. BERG, LINDA MORROW, DAVID POLIDORI, EUNICE ARTIS, SARAH RUSCH, KIRK WAYS, NICOLE VACCARO, DAMAYANTHI DEVINENI, Raritan, NJ, San Antonio, TX, Chula Vista, CA, San Diego, CA, Beerse, Belgium

In early studies of CANA, doses $\geq 200 \mathrm{mg}$ lowered post-prandial glucose excursions (PPGE) more than lower doses studied, despite similar urinary glucose excretion (UGE), raising the potential for an additional, non-renal effect of CANA on PPGE. This study examined this by separating CANA's effect to lower PPGE by increased UGE vs non-renal effects during a meal tolerance test (MTT): 37 subjects with T2DM inadequately controlled (A1c 7-9\%) on metformin (mean age $55 \mathrm{yrs}, 62 \%$ male, mean BMI $31.2 \mathrm{~kg} / \mathrm{m}^{2}$, mean A1c $8.0 \%$ ) were randomized to a 4-period crossover study with 3-day inpatient stays (+ 2 wk wash-out). Treatments (TRT) administered on the morning of Day 2/Day 3 were: A=Placebo (PBO)/PBO; B=CANA $300 \mathrm{mg} /$ PBO; C=CANA $300 \mathrm{mg} /$ CANA $300 \mathrm{mg}$; D=CANA $300 \mathrm{mg} / \mathrm{CANA} 150 \mathrm{mg}$. The MTT was started 20 min post-dose on Day 3. CANA $300 \mathrm{mg}$ provides nearmaximal effects on UGE for 24h so TRTs B-D were expected to have similar UGE during MTT so differences in PPGE between $C$ or D vs B should reflect a non-renal mechanism, while differences between $B-D$ vs A reflect both UGE and non-renal effects. Thirty-six subjects completed all periods. CANA 300 mg lowered FPG on Day 3: mean (95\% CI): 171 (161; 181) mg/dL for A, 146 (141; 152) $\mathrm{mg} / \mathrm{dL}$ for $B$ and similar for $C$ and D. During MTT, TRT $C$ lowered total $(t)$ GLU AUC $\mathrm{C}_{0-2 \mathrm{~h}}$ vs $\mathrm{A}$ by $16 \%(\mathrm{p}<0.001)$ and incremental $(\Delta) \mathrm{AUC}_{0-2 \mathrm{~h}}$ by $19 \%$ vs $\mathrm{A}$, with TRT C lowering $\triangle A U C_{0-2 h}$ by $12 \%$ vs $B(p=0.012)$. Similar results were observed for GLU AUC : TRT C lowered t-AUC by $17 \%$ vs A and lowered $\triangle A U C$ by $15 \%$ vs $B$. There was no effect of $D$ vs $B$ on $\triangle A U C$. UGE over 2 and $4 \mathrm{~h}$ was similar for B, C, and D. CANA was generally well tolerated. Thus, 1) single doses of CANA $300 \mathrm{mg}$ lower FPG and 2) an additional, non-renal mechanism contributes to the PPGE-lowering observed with CANA $300 \mathrm{mg}$ (but not $150 \mathrm{mg}$ ). The non-renal effect on CANA to lower PPGE may be due to local / transient intestinal SGLT1 inhibition from high intraluminal CANA levels during drug absorption.

Supported by: Janssen Research and Development, L.L.C.

\section{HUMAN IMMUNOLOGY AND DIABETES}

85-OR

Teplizumab Preserves Insulin Production in Type 1 Diabetes After the New Onset Period

KEVAN C. HEROLD, STEPHEN GITELMAN, STEVEN WILLI, PETER GOTTLIEB, JAMES DZIURA, JEFFREY BLUESTONE, New Haven, CT, San Francisco, CA, Philadelphia, PA, Aurora, CO

Successful immune therapy trials in T1D have generally enrolled patients soon after onset but loss of insulin production continues for years afterwards. To determine whether immune modulation affects C-peptide responses after onset, we performed a randomized placebo (pcbo) controlled trial of anti-CD3 mAb (teplizumab) in patients with T1D for 4-12 mos duration. A total of 58 patients with T1D (mean age 12.3 $2.01 \mathrm{yrs)} \mathrm{of} \mathrm{4-12}$ mos duration (mean=7.11 \pm 0.31 mos) were enrolled at 4 sites and received a 14 day course of teplizumab or pcbo. At baseline, subjects were taking $0.40 \pm 0.022$ units $/ \mathrm{kg} / \mathrm{d}$ of insulin. The baseline C-peptide AUC $(\ln (A U C+1))$ in drug and pcbo tx'd groups were $0.466 \pm 0.032 \mathrm{pmol} / \mathrm{ml} / \mathrm{min}$ and $0.441 \pm 0.045$ $\mathrm{pmol} / \mathrm{ml} / \mathrm{min}$ respectively. The drug was well tolerated: a total of 6 SAEs in 5 subjects were reported. Teplizumab treatment preserved C-peptide at 12 months $(0.379 \pm 0.041$ vs $0.300 \pm 0.044 \mathrm{pmol} / \mathrm{ml} / \mathrm{min}, \mathrm{p}=0.03)$ and had a significant effect on $\mathrm{C}$-peptide responses over 1 year in a mixed model corrected for baseline $\mathrm{C}$-peptide and duration of diabetes ( $p=0.045$ ). Of the 58 randomized subjects, five lost detectable levels of C-peptide at 12 months and all were in the pcbo group $(\mathrm{p}=0.02)$. Insulin use was also decreased in drug treated subjects (average of $0.438 \pm 0.24$ vs $0.522 \pm 0.025$ units $/ \mathrm{kg} / \mathrm{d}$, $p=0.019$ ). A drug-dependent decrease in the CD4:CD8 T cell ratio $(p=0.049)$ at 2 wks and an increase in the absolute number of $C D 8^{+} C D 45 R O^{+} C D 62 L^{+}$ central memory cells $(p=0.018)$ at 2 mos distinguished responders from nonresponders. The effects on subjects enrolled within 4-8 mos after $\mathrm{dx}$ were greater ( $95 \% \mathrm{Cl}$ : $0.32 / 0.40$ vs $0.25 / 0.35$ ) than in individuals treated $9-12$ mos after $\mathrm{dx}(0.33 / 0.45 \mathrm{vs} 0.27 / 0.40)$ but the test of treatment effect of duration was not significant $(\mathrm{p}=0.4)$. In addition, subjects age $8-14$ showed better responses $(p=0.017)$ compared to older subjects $(p=0.36)$. We conclude that immune therapy can attenuate the decline in C-peptide in T1D even 8 mos after diagnosis with children showing greater responses to this therapy.

86-0R

Administration of CD4+CD25 high CD127- T Regulatory Cells as a Therapy for Remission in Type 1 Diabetes in Children

NATALIA MAREK-TRZONKOWSKA, MALGORZATA MYSLIWIEC, ANITA DOBYSZUK, MARCELINA GRABOWSKA, ILONA TECHMANSKA, JOLANTA JUSCINSKA, MAGDALENA A. WUJTEWICZ, PIOTR WITKOWSKI, WOJCIECH MLYNARSKI, ANNA BALCERSKA, JOLANTA MYSLIWSKA, PIOTR TRZONKOWSKI, Gdansk, Poland, Chicago, IL, Lodz, Poland

Type 1 diabetes (DM1) is a condition in which pancreatic islets are destroyed by autoreactive T cells. The process is facilitated by deficits in the number and suppressive activity of T regulatory cells (Tregs). Here, we show for the first time that the infusion of ex vivo expanded Tregs may prolong a clinical remission in children with recently diagnosed DM1. The study was conducted according to the ethically approved protocol (NKEBN/8/2010). Written Informed consent was obtained from parents and children over 16 yo prior to the study inclusion. We administered Tregs in ten DM1 children within two months since the diagnosis. Autoimmunity was confirmed with anti-GAD, ICA and, IAA. The preparation consisted of autologous $\mathrm{CD}^{+}{ }^{+} \mathrm{CD}{ }^{+} \mathrm{CD} 25^{\text {high }} \mathrm{CD} 127$ - Tregs sorted with single-use-sample-line FACSsorter and expanded under GMP-conditions. The number of infused Tregs was up to $20 \times 10^{6} / \mathrm{kg}$ b.w. No adverse effects were observed during and after Tregs infusion. A significant increase in the percentage of Tregs in the peripheral blood was recorded after the treatment. Half a year after the diagnosis of DM1 all patients treated with Tregs were still in the remission defined as daily dose of insulin (DDI) less than $0.5 \mathrm{UI} / \mathrm{kg}$ b.w. Study patients were followed along with ten DM1 patients not treated with Tregs. This control group matched Tregs-treated group in regards to DDI and C-peptide levels at the time of DM1 diagnosis, as well as age, sex and BMI. Significant differences between the groups were apparent six months after the diagnosis. The remission was over at this time in the group not treated with Tregs. In addition, plasma C-peptide levels were significantly higher in the Treg treated group as compared to those not treated [median(minmax) $0.65(0.46-2.11) \mathrm{ng} / \mathrm{ml}$ versus $0.40(0.15-0.74) \mathrm{ng} / \mathrm{ml}$; U-Mann-Whitney test, $p=0.04)$. During the ADA Congress the results of 1 -year follow up will supplement the presentation.

Supported by: Polish Ministry of Science and Kosciuszko Foundation

LADA is not a Distinct Form of Autoimmune Diabetes

87-0R

RICHARD D. LESLIE, MOHAMMED I. HAWA, HUBERT KOLB, NANETTE SCHLOOT, HURIYA BEYAN, STAVROULA A. PASCHOU, RAFFAELLA BUZZETTI, DIDAC MAURICIO, ALBERTO DE LEIVA, KNUD YDERSTRAEDE, HENNING BECK-NIELSEN, CINZIA SARTI, JAAKKO TUOMILEHTO, CHARLES THIVOLET, DAVID HADDEN, STEVEN HUNTER, SCHERNTHANER GUNTRAM, WERNER A. SCHERBAUM, SINEAD BROPHY, RHYS WILLIAMS, PAOLO POZZILLI, London, United Kingdom, Düsseldorf, Germany, Rome, Italy, Lleida, Spain, Barcelona, Spain, Odense, Denmark, Helsinki, Finland, Lyon, France, Belfast, Ireland, Vienna, Austria, Swansea, United Kingdom

Type 1 diabetes is characterized by specific autoantibodies, also found in adult-onset diabetes even when initially non-insulin requiring i.e. in latent autoimmune diabetes (LADA). Our aim was to characterise adult-onset autoimmune diabetes in Europe. Consecutive diabetes patients within 5 years of diagnosis (age range 30-70 years) attending European clinics were examined clinically and for autoantibodies to glutamic acid decarboxylase (GADA), insulinoma-associated antigen-2 (IA-2A) and zinc-transporter8 (ZnT8A) using established radio-immunoprecipitation assays. Autoantibody-positive patients were designated type 1 diabetes (insulin-treated at diagnosis), and LADA, (6 months insulin-free). Of 6,156 patients, 541 (8.8\%) had GADA alone or in combination with the other autoantibodies. GADA titers showed two modes. An additional 57 (0.9\%) had IA-2A and/or ZnT8A alone. Of autoantibody positive patients, those classified as type 1 diabetes compared with LADA had a lower age of onset and BMI, ( $p<0.001$ for both), but similar number with high GADA titre ( $80 \%$ vs $76 \%$ ). Autoimmune diabetes patients with high titre ( $>200$ WHO IU) GADA ( $n=403$ ), in contrast to low titre GADA $(n=138)$, were more likely to be female, younger, with lower $B M I(p<0.01$ for all) with lower triglycerides $(p<0.001)$ and more insulin treated $(54.6 \%$ versus $39.7 \% ; p=0.005$ ). Compared to GADA negative patients, the low GADA titre group also had lower $B M I(p<0.01)$ with more insulin treated $(p<0.001)$. Among all GADA positive patients, GADA titre was positively correlated 


\section{HUMAN IMMUNOLOGY AND DIABETES}

with age at diagnosis $(r=0.129, p=0.010)$. European patients with adultonset autoimmune diabetes, whilst usually non-insulin requiring with GADA, have a broad clinical spectrum. Between type 1 diabetes and LADA there were some quantitative, but no categorical, immunophenotypic differences apart from initial insulin therapy. This largest study of adult-onset autoimmune diabetes indicates a gradation in features so, insulin treatment apart, LADA is not a distinct form of autoimmune diabetes.

88-0R LADA China Study-Frequency, Immunogenetics and Clinical Characteristics of Latent Autoimmune Diabetes in China

ZHIGUANG ZHOU, YUFEI XIANG, LINONG JI, WEIPING JIA, GUANG NING, GAN HUANG, LIN YANG, JIAN LIN, ZHENOI LIU, WILLIAM A. HAGOPIAN, RICHARD D. LESLIE, LADA CHINA STUDY GROUP, Changsha, China, Beijing, China, Shanghai, China, Charlottesville, VA, Seattle, WA, London, United Kingdom

We sought to determine features of adult-onset autoimmune diabetes among recently-diagnosed diabetes patients with glutamic acid decarboxylase antibodies (GADA) in 46 hospital-based clinics across China. We screened ketosis-free diabetes patients, within one year of diagnosis, who were independent of insulin therapy for $>6$ months and aged $>15$ years, for GADA and for HLA-DQ genotype measured centrally and clinical data collected locally. Latent Autoimmune Diabetes of Adult-onset (LADA) was identified in GADA-positive patients aged $>30$ years at diagnosis. Of 5128 patients, $6.2 \%$ had GADA, most with LADA (5.9\%). LADA showed a north-south gradient (6.5\% vs. $5.4 \%$ respectively; $P=0.040)$. Compared with GADA-negative type 2 diabetes, LADA patients were leaner [body mass index(BMI): $23.9 \pm 3.7$ vs. $24.8 \pm 3.4 \mathrm{~kg} / \mathrm{m} 2, \mathrm{P}<0.001]$, with lower fasting $C$-peptide [0.47 (0.01-3.34) vs. $0.64(0.01-4.96) \mathrm{ng} / \mathrm{ml}, \mathrm{P}<0.001)]$, and less Metabolic Syndrome $(62.0 \%$ vs. $75.6 \%, P<0.001)$. These differences were even greater in LADA patients with higher GADA titers. HLA diabetes-susceptible haplotypes were more frequent in LADA [63.9\% (115/180)], than in both type 2 diabetes [47.1\% (82/174); $P<0.010)]$ and controls [43.2\% (214/495); $P<0.001]$. HLA diabetesprotective haplotypes were less frequent in LADA [22.8\% (41/180)], than in both type 2 diabetes [33.3 \% (58/174), $P<0.05]$ and controls [32.7\% (162/495), $\mathrm{P}<0.05]$. Among patients with the clinical characteristics of type 2 diabetes, GADA-positive and LADA diabetes was prevalent in China $(6.2 \%$ and $5.9 \%$, respectively) with north-south frequency gradient. The immunogenetic and clinical features of LADA in China are strikingly similar to those in Europe, some differences are noted. Our study implicates universal immunogenetic effects, with some ethnic differences, associated with adult-onset autoimmune diabetes.

Supported by: EFSD

89-0R

\section{WITHDRAWN}

Glucagon-Like Peptide-1: A Key Regulator of Innate Immune Function With Clinical Efficacy in a Range of Inflammatory Diseases ANDREW E. HOGAN, OBESITY IMMUNOLOGY RESEARCH GROUP, DONAL O'SHEA, OBESITY IMMUNOLOGY RESEARCH GROUP, Dublin, Ireland

Invariant natural killer T (iNKT) cells are key innate immune cells implicated in the pathogenesis of many diseases. Glucagon-like peptide-1 (GLP-1) is an incretin hormone implicated in the regulation of glucose metabolism and body weight. We recently demonstrated that psoriasis is improved in patients treated with GLP-1 analogue therapy and that GLP-1 is a regulato of iNKT cell cytokine production. We proposed that GLP-1 therapy would improve other inflammatory conditions, both with and without co-morbid diabetes, and that GLP-1 regulates other innate immune cells, including monocytes and macrophages. Two diabetes patients, one with rheumatoid arthritis (RA) and one with ulcerative colitis (UC), were treated with the GLP1 analogue liraglutide. Twelve weeks of therapy resulted in improvements in the symptoms and signs of RA (28-joint Disease Activity Score reduced from 5.5 to 3.0), and a reduction of bowel frequency from 5-6 bloody motions/day to 2-3 motions/day, respectively. This was associated with a significant reduction in pathogenic cytokine production by peripheral blood mononuclear cells in each patient (TNF $\alpha$ : 200 to $98 \mathrm{pg} / \mathrm{ml}$ in RA; IL-13: 82 to $32 \mathrm{pg} / \mathrm{ml}$ in UC). GLP-1 analogue therapy in standard non-diabetic animal models of UC and RA led to a reduction in colitis disease severity, accelerated repair of colon inflammation, and a decrease in serum amyloid levels in the UC model, and amelioration of the severity of arthritis-like disease by multiple parameters in the RA model. GLP-1 receptor activation on innate immune cells (iNKT cells, monocytes and macrophages) in vitro activated the anti-inflammatory transcription factor CREB and inhibited the classic proinflammatory transcription factor NFKB. We conclude that GLP-1 therapy is immunomodulatory and may impact on the clinical course of a range of inflammatory diseases. Future work should investigate the range of beneficial, and potentially adverse, immune effects of incretin therapies.

Supported by: National Children's Research Centre, $\mathrm{OLCH}$

91-0R

Proinsulin/Insulin Autoantibodies Measured With Electrochemiluminescent Assay Are the Earliest Indicator of Prediabetic Islet Autoimmunity

ANDREA K. STECK, LIPING YU, FRAN DONG, DONGMEI MIAO, JANET WENZLAU, JOHN HUTTON, MARIAN J. REWERS, GEORGE S. EISENBARTH, Aurora, CO

The pre-clinical phase of type 1 diabetes is marked by the presence of persistent islet autoantibodies. The Diabetes Autoimmunity Study in the Young (DAISY) follows prospectively for development of islet autoimmunity (defined as presence of autoantibodies to insulin, GAD65, IA-2 or ZnT8) and type 1 diabetes children at increased genetic risk (general population children and first-degree relatives). Serial serum samples from subjects that progressed to type 1 diabetes $(\mathrm{N}=63$ ), most followed from birth, were tested using a novel electrochemiluminescent assay for insulin/proinsulin autoantibodies (ECL-IAA). The ECL-IAA detected onset of islet autoimmunity earlier than any of the four traditional biochemical antibodies in $15(24 \%)$ children. ECL-IAA antedated the onset of islet autoimmunity in these children by a mean of 2.6 years (range: 0.3-7.6 years). At the first positive autoantibody visit, ECL-IAA was the most frequent autoantibody present (95\%), followed by mIAA (48\%), GAD65 (41\%), ZnT8 (22\%), and IA-2 (19\%) autoantibodies ( $<<0.0001)$. This new ECL-IAA assay defines more precisely the onset of prediabetic autoimmunity and may help identify events triggering islet autoimmunity as well as allow earlier intervention for type 1 diabetes.

Supported by: JDRF (11-2010-206)

92-0R

The Identification of Islet Autoantibodies in Patients With Monogenic Diabetes

JANA URBANOVÁ, ČERA, M. ANDĚL, B. RYPÁČKOVÁ, P. HENEBERG, Z. BAZALOVÁ P. KU, Prague, Czech Republic

Introduction: The incidence of islet autoantibodies (iAb) is considered as manifestation of autoimmune insulitis and diagnostic criteria of T1DM. On the other hand absence of iAb at the time of diagnosis, especially with the evidence of preserved insulin production outside the honeymoon period at lean adults with at least 1 diabetic parent increases the susceptibility for monogenic diabetes (MDM). However, cases of patient with MDM and posi- 
tive titre iAb has been published. Aims: The aim of this research was to determine the incidence of islet autoantibodies in patients with MDM. Methods: GADA and IA2A were currently determined or retrospectively searched at 24 patients with molecular genetically verified MDM (HNF4A diabetes: $n=3$, HNF1A diabetes: $n=12$, GCK diabetes: $n=9$ ). GADA / IA2A were determined by RIA. Genetic testing was performed by direct sequencing $(A B \mid$ PRISM 3130), evaluated in the program SeqScape. Results: We have shown a significant presence of specific $i A b$ in almost one third of the patients with the most common subtypes of monogenic diabetes $(7 / 24 ; 29.16 \%)$, especially at HNF1A diabetes $(4 / 8 ; 16.66 \%)$, small group of HNF4A diabetics $(2 / 1 ; 8.33 \%)$, and rather exceptionally at GCK diabetes $(1 / 6 ; 4.16 \%)$. The most prevalent antibody was GADA $(6 / 7 ; 85,71 \%)$. iAb were present at the time of manifestation of diabetes and also many years later. In most of the $i A b$ positive patients sulfonyulurea derivates have been found not sufficient for good glycemic control and these patients lately needed also insulin regardless of the sufficient level of $\mathrm{C}$-peptide. Conclusion: It is difficult to interpret simultaneous presence of $\mathrm{iAb}$ and mutation in HNF1A / HNF4A gene. One possibility could be explained as rare coexistence of autoimmune insulitis and MDM. However, the iAb may also reflect an increased destruction of beta-cells (progressive beta-cell loss possibly due to impaired cell turnover in the course of MDM).

\section{INSULIN SIGNAL TRANSDUCTION AND ACTION}

$\triangle$

93-0R

Regulation of Insulin on Tyrosine Phosphorylation in Human Skeletal Muscle

DANJUN MA, XIANGMIN ZHANG, MICHAEL CARUSO, ZHENGPING YI, Detroit, MI, Tempe, $A Z$

Tyrosine phosphorylation plays a fundamental role in many cellular processes such as growth and insulin signaling. In insulin resistant skeletal muscle, aberrant tyrosine phosphorylation of a few proteins has been reported. Here, we report the 1st tyrosine phosphoproteomic study of human skeletal muscle from a lean, healthy volunteer. Hyperinsulinmic, euglycemic clamp was performed, and biopsies at basal, $0.5 \mathrm{~h}$ and $2 \mathrm{~h}$ after insulin infusion were obtained. Trypsinized muscle proteins were subjected to immunoprecipitation of phosphortyrosine peptides and HPLC-ESI-MS/MS. 52 distinct tyrosine phosphorylation sites in 35 proteins were identified, 8 major glycolytic enzymes (such as EN01 \& ALDOA), 6 enzymes regulating glycogen metabolism (such as GYS1 \& GSK3), 8 sarcomeric proteins (such as MYH1/2/7 \& MYL3), as well as proteins involved in MAP kinase signaling (MAPK12 \& PTPN11) and phosphocreatine resynthesis (CKM \& CKMT2). Among the identified phosphorylation sites, 23 were not listed in the largest phosphorylation site database, www.phosphosite.org, and thus appeared to be novel. One of the sites is pY140 in creatine kinase M-type (CKM) detected with 5 spectra in the basal, 2 in the $0.5 \mathrm{~h}$, and 5 in the $2 \mathrm{~h}$ biopsies, indicating that pY140 level decreased after $0.5 \mathrm{~h}$ insulin infusion and came back to the basal level after $2 \mathrm{~h}$ insulin infusion. On the other hand, pY539 of phosphoglucomutase-1(PGM1) was identified with 2 spectra each in the basal, $0.5 \mathrm{~h}$, and $2 \mathrm{~h}$ biopsies, suggesting little effect of insulin on this site. Besides, pY353 in phosphorylase b kinase gamma catalytic chain (PHKG1) was not detected in the basal biopsy, while detected with 2 spectra in the $0.5 \mathrm{~h}$ and 1 spectrum in the $2 \mathrm{~h}$ biopsies, implying pY353 was an insulin stimulated site. More experiments are on-going to confirm these results. These results provided the 1st evidence that multiple novel tyrosine phosphorylation sites have distinct response to insulin in vivo in a time dependent manner, and may lead to new insights into the pathogenesis of $\mathrm{T} 2 \mathrm{D}$.

94-0R Investigating Global IRS1 Ser/Thr Phosphorylation Using a Phosphospecific Monoclonal Antibody Approach NANCY HANCER, WEI QIU, MORRIS WHITE, Boston, MA

Insulin Receptor Substrate 1 (Irs1) is an important adapter protein that lies immediately downstream of the insulin receptor. IRS1 coordinates insulin signaling upon tyrosine phosphorylation; however, phosphorylation of serine and threonine residues in Irs1 is generally thought to have a negative regulatory role. Mass spec analysis of insulin-stimulated IRS1 reveals more than 50 phosphorylated Ser/Thr residues, but quantitative analysis is difficult to accomplish. We have solved this problem by generating phosphospecific monoclonal antibodies that span the length of IRS1 and using Luminex technology to study Irs1 Ser/Thr phosphorylation. Using CHO cells, L6 myoblasts or Fao hepatoma cells, insulin stimulates or inhibits with distinct time-dependent profiles the phosphorylation of most of the detected sites. Moreover, insulinstimulated Ser/Thr phosphorylation displayed similarities and difference in each cell type. Using small molecule inhibitors of PI3 kinase, AKT and mTOR we found a close inverse relation between insulin-stimulated Ser/Thr and tyrosine phosphorylation, but the contribution of individual sites to insulin resistance was difficult to resolve. Moreover, agonists of cellular stressanisomycin, thapsigargin, and tunicamycin-also revealed distinct phosphorylation pattern and a close inverse relation between multisite Ser/Thr phosphorylation and insulin-stimulated tyrosine phosphorylation. Together our results suggest that multisite Ser/Thr phosphorylation — rather than the phosphorylation of any single residue-modulates the insulin signal.

Supported by: NIH, HHMI

95-0R

Par14 Associates With IRS-1, Thereby Enhancing Insulin-Induced IRS-1 Phosphorylation and Metabolic Actions

JUN ZHANG, YUSUKE NAKATSU, TSUYOSHI YAMAMOTOYA, YING GUO, MIDORI FUJISHIRO, AKIFUMI KUSHIYAMA, HIDEYUKI SAKODA, TOMOICHIRO ASANO, Hiroshima City, Japan, Tokyo, Japan

Pin1 and PAR14 are parvulin-type peptidyl prolyl cis/trans isomerases. While numerous proteins have been identified as Pin1 substrates, the target proteins of PAR14 remain largely unknown. We previously reported the association of Pin1 and IRS-1, and found that the PAR14 expression level increased in Pin1 KO mouse tissues or Pin1 siRNA-treated HepG2 cells, suggesting a compensatory relationship between the functions of Pin1 and PAR14. Herein, we demonstrated that PAR14 associates with IRS-1, employing a pull-down experiment with GST-PAR14 protein and HepG2 cell lysates. The association of PAR14 with IRS-1 was confirmed in not only overexpression experiments using HepG2 cells, but also endogenously in the mouse liver. The analysis using deletion-mutated PAR14 and IRS-1 constructs revealed the DBD domain located in the $\mathrm{N}$-terminal side of PAR14 and the two relatively C-terminal portions of IRS-1 to be involved in their association, in contrast to the WW domain of Pin1 and the SAIN domain of IRS-1. The PAR14 overexpression in HepG2 markedly enhanced insulin-induced IRS-1 phosphorylation and its downstream events; PI 3-kinase binding with IRS-1 and Akt phosphorylation. In contrast, treating HepG2 cells with PAR14 siRNA suppressed these events. In addition, overexpression of PAR14 in the ob/ob mouse liver by adenoviral transfer significantly improved hyperglycemia with normalization of hepatic PEPCK and G6Pase mRNA levels. Therefore, Pin1 and PAR14 associate with different portions of IRS-1, but it is very likely that the cis-trans conformational change in these proline residues enhances the efficiency of insulin receptor-induced IRS-1 phosphorylation. This process constitutes a potential therapeutic target for novel insulin-sensitizing agents.

96-0R

Genetic or Pharmacologic Inhibition of the STARS-SRF Pathway Increases AMPK Phosphorylation and Oxidative Metabolism WEN KONG, ALISON BURKART, MICHAEL CHEN, WANZHU JIN, MICHAEL HIRSHMAN, LAURIE GOODYEAR, RICHARD NEUBIG, SCOTT LARSEN, PAUL ALLEN, C.G. TEG PIPES, RHONDA BASSEL-DUBY, ERIC OLSON, MARY-ELIZABETH PATTI, Boston, MA, Wuhan, China, Ann Arbor, MI, Dallas, TX

We recently reported expression of the serum response factor (SRF) regulator STARS (striated muscle activator of Rho signaling) was increased by 2.5-fold in skeletal muscle of humans with T2D and correlated with insulin resistance $(r=-0.42)$. To identify the role of STARS in vivo, we analyzed metabolic phenotypes in mice with whole-body ablation of STARS (STARS $\mathrm{KO}$ ) and wild type (WT) littermates. STARS KO mice are resistant to dietinduced obesity, with a $41 \%$ reduction in body weight ( $p<0.01$ vs. WT) despite unchanged food intake. STARS KO mice have a $30 \%$ increase in energy expenditure ( $\mathrm{p}<0.05 \mathrm{vs}$. WT) and increased fitness, as indicated by increased running duration ( 2 -fold increase, $p<0.05)$, and increased V02max $(30 \%$ increase, $p<0.05$ ). These results indicate that inhibition of the STARS-SRF pathway can have beneficial effects on systemic metabolism. To determine potential mechanisms mediating these effects, we isolated primary myoblasts from WT and STARS KO mice. STARS KO myotubes had increased AMP kinase (AMPK) phosphorylation ( $53 \%$ increase, $p<0.05$ ) with no change in AMPK protein. This occurred in parallel with an $18 \%$ reduction in $\mathrm{mTOR}$ phosphorylation $(\mathrm{p}<0.05)$. Moreover, STARS K0 myotubes had a $77 \%$ reduction in mitochondrial DNA content $(p=0.01)$, a $63 \%$ decrease in OXPHOS complex II protein $(\mathrm{p}<0.05)$ and a 4 -fold increase in UCP3 mRNA and protein expression ( $p<0.05$ for both). To determine whether effects of STARS ablation could be recapitulated by inhibition of SRF, we treated C2C12 myotubes with the SRF inhibitors CCG203471 at 3uM overnight. CCG203471 increased AMPK phosphorylation (4 fold, $\mathrm{p}<0.05$ ), decreased mTOR phosphorylation 
(33\% decrease, $\mathrm{p}<0.05$ ), and in parallel increased UCP3 expression (4.7 fold, $\mathrm{p}<0.05)$. Together, our data indicate that STARS can modulate cellular and whole body-energy metabolism, potentially via SRF-dependent alterations in mitochondrial metabolism and activation of AMPK-dependent pathways.

Supported by: China Scholarship Council Fund, NIH-DK-062948, 5T32DK07260-34, Lilly Foundation

97-0R

Mitochondrial Akt1 Signaling Enhanced Reprogramming to Induced Pluripotent Stem Cells-A Novel Gateway of Cell Fate Decision HANK CHEN, YUMAY CHEN, HSIN-BANG LEU, WU DENG, LESLIE LOCK, RYAN LIM, MATTHEW KAYALA, PIERRE BALDI, PETER DONOVAN, PING H. WANG, Irvine, $C A$

This project was to test a novel hypothesis that activation of Akt1 in mitochondria plays a role in somatic cell reprogramming and stem cell differentiation. Generation of induced pluripotent stem cells (iPSC) via reprogramming is a promising path toward cell replacement therapy for diabetes. We have identified mitochondria as a major location where Akt1 translocated into upon growth factor stimulation. A mitochondria-targeting constitutively active Akt1 (Ad-mito-Akt) was expressed in human embryonic stem cells (hESC) and up-regulated genes controlling stem cell stemness and pluripotency, and down-regulated genes promoting differentiation. To confirm its role in hESC stemness, a mitochondria-targeting dominant negative Akt (mito-dnAkt) was introduced to hESC via a Tet-on lentiviral vector. Compared to the controls, mito-dnAkt induced rapid in vitro differentiation and embryoid body formation, thus indicated a critical role in the regulation of hESC differentiation. To further verify its role in cell fate decision, we have studied the efficiency of fibroblasts reprogramming to iPSC by the four factors (Sox2/KIf4/Oct4/c-Myc), with Ad-mito-Akt or Ad-GFP. Ad-mito-Akt significantly increased the number of IPSC colonies and SSEA1/4-positive cells during reprogramming, whereas mito-dnAkt inhibited iPSC induction. Demethylation of Oct4 and Nanog promoter is a key regulatory step in reprogramming. Activation of mitochondrial Akt1 during reprogramming was associated with complete demethylation of Nanog and Oct4 prsomoter, similar to hESCs. Four factors alone, without mitochondrial Akt1 activation, achieved only partial demethylation of Nanog and Oct4 promoters. The Ad-mito-Akt-iPSC lines are pluripotent and capable of developing into pancreatic islet-like cells expressing insulin. Mitochondria Akt1 signaling presents a new opportunity to develop novel strategies to modulate somatic cell reprogramming and stem cell differentiation.

Supported by: Oxnard Foundation

Hepatic mTORC2 Activates Glycolysis and Lipogenesis through Akt Glucokinase and SREBP1c

ASAMI HAGIWARA, MARION CORNU, NADINE CYBULSKI, PAZIT POLAK, CHARLES BETZ, FRANCESCA TRAPANI, LUIGI TERRACCIANO, MARKUS H. HEIM, MARKUS A. RÜEGG, MICHAEL N. HALL, Basel, Switzerland

The liver plays a key role in insulin-mediated glucose and lipid homeostasis. Mammalian target of rapamycin complex 2 (mTORC2) is one of the key components of insulin signaling pathway as it phosphorylates hydrophobic motif of Akt. To assess the role of hepatic mTORC2 in metabolism, we generated liver-specific rictor knockout (LiRiKO) mice. Fed LiRiKO mice displayed loss of Akt Ser473 phosphorylation in the liver, whereas phosphorylation of Akt Thr308, a site in the catalytic loop phosphorylated by PDK1, was normal. The Akt substrates Fox01 and GSK3 were hypophosphorylated, while phosphorylation status of S6K and S6, mTORC1 substrates that act as Akt downstream effectors, was normal. The aberrant Akt signaling in LiRiKO liver led to unsuppressed gluconeogenesis and reduced glycogen synthesis. Insulin-induced activation of glucokinase and SREBP1c was also impaired in LiRiKO liver, leading to reduced glycolysis and lipogenesis. These liver specific defects resulted in impaired systemic glucose homeostasis, as indicated by significantly higher blood glucose and insulin levels in both fasted and fed states, and higher glucose area under the curve (AUC) after intraperitoneal glucose tolerance test (IPGTT). To investigate further the role of mTORC2-mediated Akt phosphorylation, we introduced phosphomimetic mutant Akt2 in which Ser474 (equivalent to Ser473 in Akt1) is substituted by aspartic acid (Akt2-S474D) in mTORC2 deficient hepatocytes. Expression of Akt2-S474D restored insulin signaling pathway and suppressed defects in glucose uptake and lipogenesis in LiRiKO hepatocytes. In conclusion, mTORC2-mediated Akt phosphorylation is essential for hepatic glucose and lipid metabolism to control whole body metabolic homeostasis.
p70S6-Kinase is a Novel AMPK Kinase that Suppresses Hypothalamic AMPK Activity to Inhibit Food Intake

YOSSI DAGON, Boston, MA

The hypothalamic mammalian Target of Rapamycin (mTOR)-p70S6-kinase (S6K) pathway responds to hormonal and nutrient signals, suppresses food intake and is necessary for leptin's anorexigenic effect. We aimed to identify S6K downstream targets that mediate these effects in hypothalamus. Bioinformatic analysis of the S6K consensus motif RXXS identified a potential phosphorylation site on serine 491 in the catalytic a2 subunit of AMPK (AMPK $\alpha 2$ ). A proline at -5 is likely to impede phosphorylation by other AGC kinases. Expression of constitutively active S6K1 in hypothalamic GT1-7 neurons stimulated AMPK phosphorylation on serine 491 but not on threonine 172. In vitro, recombinant S6K1 directly phosphorylated recombinan AMPK $\alpha 2$ on serine 491. Immunoprecipitation of S6K from brains and GT1-7 neurons revealed an AMPK $\alpha 2$-S6K1 complex, suggesting a direct interaction. Modulation of hypothalamic AMPK activity is a central mechanism for food intake regulation and is necessary for leptin's anorexigenic effect. S6K-/mice do not respond to leptin's anorectic action and exhibit increased AMPK activity. Leptin injection increased AMPK serine 491 phosphorylation by $47 \%$ in arcuate and $53 \%$ in ventromedial/dorsomedial hypothalamus but had no effect in S6K-/- mice. To test the effect of S6K dependent phosphorylation of AMPK serine 491, we expressed a phosphodefective AMPKa2-S491A mutant (S491A) in basomedial hypothalamus in normal mice. S491A expression increases food intake by $48 \%$ /day compared to WT-AMPK $(p<0.05)$ and blocks leptin's effects to inhibit AMPK $\alpha 2$ activity and to reduce food intake (WT-AMPK $-42 \%$ vs $\$ 491$ A $-6 \% / 24 \mathrm{~h} p<0.05$ ) and body weight. Thus, AMPK serine 491 phosphorylation is necessary for leptin's effects on food intake and body weight. Conclusion: S6K is a novel inhibitory AMPK-kinase and AMPK can be a substrate for mTOR-S6K. This has broad implications since mTOR-S6K and AMPK have multiple and generally opposing cellular effects in metabolism, cell growth and development.

The Role of Inositol Polyphosphate in Energy Homeostasis 100-0R SOOKHEE BANG, SEYUN KIM, MEGAN J. DAILEY, YONG CHEN, TIMOTHY H. MORAN, SOLOMON H. SNYDER, SANGWON F. KIM, Philadelphia, PA, Baltimore, MD

Energy homeostasis is essentially a balancing act between energy intake and energy expenditure. Obesity by the excess energy intake is strongly associated with type 2 diabetes. AMP-activated protein kinase (AMPK), a phylogenetically conserved serine/threonine protein kinase, acts as an integrator of regulatory signals monitoring systemic and cellular energy status. Moreover, AMPK in the hypothalamus plays an important role in food intake control. Hence, AMPK pathway has become one of the major targets to treat type 2 diabetes and the metabolic syndromes. We have previously identified that inositol polyphosphate multikinase (IPMK), one of the enzymes involved in the inositol pathway, is regulated by nutrient availability in the hypothalamus. Therefore, we explored whether IPMK plays any role in nutrient sensing in the hypothalamus. We find that IPMK physically interacts with AMPK and this interaction is enhanced by glucose supplementation. Importantly, a dominant negative peptide which interferes interaction between IPMK and AMPK attenuates glucose-mediated regulation of AMPK. Moreover, we found that IPMK regulates glucose signaling to AMPK in a pathway whereby glucose activates phosphorylation of IPMK at tyrosine-174 enabling the enzyme to bind to AMPK and regulate its activation. Furthermore, we observed that genetic deletion of IPMK in hypothalamus reduces food intake measured under fasting/refeeding paradigm. In conclusion, IPMK appears to play a major role regulating AMPK signaling pathway. Therefore these findings imply that IPMK will be a new therapeutic target for the treatment of obesity and type 2 diabetes.

Supported by: R01DK084336 and NARSAD

\section{INSULIN ACTION IN ANIMALS}

101-0R

Physical Inactivity and High Fat Diet Synergically Induce Insulin Resistance in Skeletal Muscle Through PTP1B Activation

SAORI KAKEHI, YOSHIFUMI TAMURA, SHIN-ICHI IKEDA, KAGEUMI TAKENO, MINAKO KAWAGUCHI, TAKAHIRO WATANABE, TAKASHI FUNAYAMA, FUMIHIKO SATO, RYUZO KAWAMORI, HIROTAKA WATADA, Tokyo, Japan

Both physical inactivity and high fat diet (HFD) are associated with insulin resistance. In rodent model, not only very short-term ( 24h) physical inactivity by hind-limb cast immobilization ( $\mathrm{HCl}$ ), but also long-term (more than 
4wk) HFD induce insulin resistance in skeletal muscle. Although physical inactivity is generally coincident with HFD in real world, combined effect of these factors has not been clarified yet. To elucidate this combined effect, C57BL6J mice were randomly assigned to four groups; control group, 24h HCl group, short-term (2wk) HFD group, 24h HCl after 2wk HFD group. Then, we evaluated insulin sensitivity (M-IS) and insulin signal transduction in plantaris muscle by ex-vivo insulin-stimulated 2-deoxy glucose uptake and Western blot analysis, respectively. The $24 \mathrm{~h} \mathrm{HCl}$ significantly decreased M-IS by $\sim 30 \%$. Interestingly, although 2 wk HFD did not change M-IS, $24 \mathrm{~h}$ $\mathrm{HCl}$ after 2 wk HFD dramatically decreased M-IS by $\sim 70 \%$. In parallel with impaired M-IS, we observed decreased insulin stimulated phosphorylation levels of insulin receptor (IR), IR substrate (IRS)-1 and Akt after $24 \mathrm{~h} \mathrm{HCl}$, which were more exacerbated in $\mathrm{HCl}$ after $\mathrm{HFD}$ group, while only 2wk HFD did not change those phosphorylation states. According to this mechanism, it has been shown that protein tyrosine phosphatase 1B (PTP1B) impair insulin action by dephosphorylation of IR. Thus, we examined association between IR and PTP1B and found that the association was increased by $\mathrm{HCl}$, which was more robust in $\mathrm{HCl}$ after HFD group. Finally, PTP1B inhibitor treatment normalized the association between IR and PTP1B and completely cancelled both impaired insulin signal transduction and decreased M-IS induced by $\mathrm{HCl}$ combined with/without HFD. These results suggested that physical inactivity and HFD synergically induce insulin resistance in skeletal muscle. In addition, impaired insulin stimulated IR phosphorylation by activated PTP1B is, at least in part, involved in this mechanism.

$\Delta$ 102-0R De Novo Lipogenesis in Skeletal Muscle Promotes Insulin Resistance

KATSUHIKO FUNAI, LI YIN, TREY COLEMAN, HAOWEI SONG, IRFAN J. LODHI, CLAY F. SEMENKOVICH, St. Louis, MO

High fat diets and obesity are linked to insulin resistance and diabetes. Endogenous fat production is not thought to be relevant to insulin resistance in muscle, a major site of glucose disposal. Unexpectedly, fatty acid synthase (FAS), necessary for de novo lipogenesis, was elevated in skeletal muscle from high fat diet (HFD)-fed mice. We generated FAS KnockOut in Skeletal muscle (FASKOS) mice to determine if de novo lipogenesis promotes skeletal muscle insulin resistance. This intervention did not affect HFD-induced weight gain. Glucose/insulin tolerance testing, hyperinsulinemic euglycemic clamp studies, and measurements of insulin-stimulated glucose uptake in isolated skeletal muscles showed that FASKOS mice were protected from HFD-induced skeletal muscle insulin resistance. Insulin-stimulated Akt phosphorylation was not altered in FASKOS skeletal muscle. However, AMP-activated protein kinase (AMPK) activity and phosphorylation of Akt substrate of $160 \mathrm{kD}$ (AS160, a Rab GTPase-activating protein that regulates GLUT4 vesicular trafficking) were increased in FASKOS skeletal muscle. In $\mathrm{C}_{2} \mathrm{C}_{12}$ myocytes, FAS knockdown also promoted AMPK activation and phosphorylation of AS160. AMPK is known to be activated by $\mathrm{Ca}^{2+} / \mathrm{calmodulin}$ dependent protein kinase kinase $\beta$ (CaMKK $\beta$ ), and knockdown of CaMKK $\beta$ eliminated the increase in AMPK activity induced by FAS knockdown. FAS knockdown also increased cytosolic calcium detected by a fluorophore. Subcellular fractionation showed that fractions containing sarco/endoplasmic reticulum (SR) are highly enriched in FAS. Mass spectrometry and coimmunoprecipitation experiments demonstrated an association between SR Ca ${ }^{2+}$-ATPase 1/2 (SERCA1/2) and FAS in skeletal muscle. These results suggest that the endogenous production of lipid by FAS in skeletal muscle modulates calcium flux to alter insulin-stimulated glucose transport through effects on CaMKK and AMPK

103-OR

Transcriptional Regulation of Fasting Inducible Hormone FGF21 by Retinoic Acid Receptor $\beta$ in the Liver

YU LI, KIMBERLY WONG, AMBER GILES, MENGWEI ZANG, Boston, MA

All-trans retinoic acid (RA), a natural ligand of retinoic acid receptor (RAR) ameliorates glucose tolerance and hepatic steatosis in ob/ob mice. Fibroblast growth factor 21 (FGF21) is the hepatocyte-derived hormone that plays a role in lipid homeostasis with beneficial effects on diabetes. However, whether there is a potential link between RAR and FGF21 remain unknown. Here we define the mechanism for RAR $\beta$ to stimulate FGF21 transcription during fasting. RAR activity was stimulated by RA in HepG2 hepatocytes, as determined by RARE-Luc reporter activity. Consequently, a FGF21 promoterdriven luciferase reporter and expression of FGF21 mRNA were increased by RA. FGF21 induction by RA was unaffected by selective RAR $\alpha$ or $\gamma$ antagonists. RA-induced FGF21 expression was mimicked by RAR $\beta$ overexpression and diminished by RAR $\beta$ knockdown, suggesting that FGF21 is selectively upregulated by RAR $\beta$. Deletion analysis of FGF21 promoter showed that various lengths of FGF21 promoter from -2090 to -650 displayed a robust response to RA. Conversely, each of 5'-deletion FGF21 promoters from -600 to -70 abrogated the inducibility by RA. We functionally dissected FGF21 promoter and characterized that a putative RA responsible element (RARE) was primarily located between positions -650 and -600 . Disruption of the RARE sequence abolished RA responsiveness in HepG2 cells. Importantly, chromatin immunoprecipitation assays revealed that hepatic RAR $\beta$ was recruited to the FGF21 promoter in a fasting inducible manner in vivo, subsequently driving FGF21 transcription and increasing hepatic and plasma FGF21 levels in fasted mice. Induction of FGF21 by RAR $\beta$ promoted expression of key enzymes governing fatty acid oxidation and ketogenesis in the liver of fasted mice. These results unravel RAR $\beta$ as a novel component to control hepatic FGF21 in response to nutrient deprivation. Hepatic RAR $\beta$-dependent activation of FGF21 provides a potential druggable target for the treatment of metabolic disorders.

Supported by: NIH/NIDDK RO1

$\Delta$ 104-0R Losartan Increases Muscle Insulin Delivery via Microvascular Recruitment

NASUI WANG, WEIDONG CHAI, LINA ZHAO, EUGENE J. BARRETT, ZHENOI LIU, Charlottesville, VA

To exert its biological actions on muscle cells, insulin first has to be delivered to the microvascular beds perfusing the muscle cells and then transverse the endothelial layer to reach the interstitium, two discrete steps that limit insulin action. We have previously reported that angiotensin II receptors modulate basal microvascular tone and insulin responses in muscle. While angiotensin II type 1 receptor (AT1R) blockade increases muscle microvascular recruitment, it is unclear whether AT1R blockade increases muscle insulin delivery. After an overnight fast, male SD rats received a bolus injection of losartan (AT1R blocker, $0.3 \mathrm{mg} / \mathrm{kg}$, i.v.) \pm infusion of L-NAME (NOS inhibitor). Additional rats received saline as control. Muscle microvascular blood volume (MBV), an index of microvascular surface area and perfusion, was measured using contrast-enhanced ultrasound before and $30 \mathrm{~min}$ after losartan injection. Muscle insulin delivery was quantified at the end of the study using 125I-insulin as tracer. Losartan increased muscle MBV by 2.5 -fold $(p<0.02)$ without affecting plasma insulin and glucose levels. This was associated with a significant increase in muscle insulin delivery $(20.5 \pm$ 3.6 vs. $32.7 \pm 3.8 \mathrm{fmol} / \mathrm{g}$ tissue, $p=0.03$ ). Simultaneous infusion of L-NAME abolished losartan-induced increase in MBV and brought muscle insulin delivery back to the control level $(19.3 \pm 1.9 \mathrm{fmol} / \mathrm{g})$. To assess whether AT1R modulates endothelial cell insulin uptake, bovine aortic endothelial cells were treated with vehicle or losartan $(10 \mu \mathrm{M})$ for 15 min and 125I-insulin (200 $\mathrm{pM})$ internalization was assessed. Losartan \pm angiotensin II did not alter endothelial internalization of insulin $(97 \pm 11 \%$ of control, $n=6)$. We conclude that the AT1R blockade increases insulin delivery to muscle mainly via microvascular recruitment without altering endothelial uptake of insulin. This may contribute to the clinical findings of decreased cardiovascular events and new onset of diabetes in patients receiving AT1R blockers.

Supported by: NIH

Tribbles 3 (TRB3) Mediates Endoplasmic Reticulum (ER) StressInduced Decreases in Insulin-Stimulated Glucose Uptake in Mouse Skeletal Muscle

HO-JIN KOH, NICOLAS MUSI, MICHAEL F. HIRSHMAN, LAURIE J. GOODYEAR, Boston, MA, San Antonio, TX

Insulin stimulation of glucose uptake in peripheral tissues is critical for glucose homeostasis and people with type 2 diabetes have increased insulin resistance in skeletal muscle. ER stress has been linked to the development of insulin resistance in multiple tissues. Using C2C12 muscle cells, we found that ER stress induced by thapsigargin (THAP) and tunicamycin (TUNI) treatment increased TRB3 expression, and that knockdown of TRB3 reversed the effects of ER stress to inhibit insulin signaling (IRS1 Y612 and AKT T308) and glucose uptake. We next determined if ER stress causes insulin resistance via induction of TRB3 in adult skeletal muscle. Mice fed a high fat diet for 6 wks had a 1.9 fold increase in TRB3 protein expression in tibialis anterior (TA) muscle $(p<0.05)$. Overexpression of TRB3 in mouse TA by electroporation (4 fold) decreased insulin-stimulated IRS1 Y612 phosphorylation by $22 \%$ $(P<0.05)$ and tended to decrease Akt $T^{308}$ phosphorylation (23\%; $\left.P=0.12\right)$. Incubation of extensor digitorum longus (EDL) muscles with THAP and TUNI significantly increased TRB3 mRNA (3.6 to 4.0 fold; $P<0.001$ ) and protein expression (10 fold; $\mathrm{P}<0.01)$. THAP and TUNI also resulted in insulin resistance 
as determined by decreased insulin-stimulated glucose uptake $(P<0.05)$. The THAP and TUNI-induced decrease in insulin-stimulated glucose uptake was prevented in EDL and soleus muscles from TRB3 knockout mice. Finally, vastus lateralis muscle biopsies from insulin resistant human subjects with obesity and type 2 diabetes had a 1.7 to 1.9 fold increase in TRB3 $(p<0.01)$ accompanied by an increase in multiple ER stress markers (XBP1: 1.2-1.5 fold; CHOP: 1.2-1.4 fold; PERK: 2.6-3.0 fold; and Bip: 1.2-1.5 fold vs. lean subjects; $\mathrm{p}<0.05)$, raising the possibility that ER stress and TRB3 may be involved in muscle insulin resistance in humans. In conclusion, ER stress impairs insulinstimulated glucose uptake through induction of TRB3 expression in mouse skeletal muscle.

\section{Muscle-Infiltrating Macrophages in Type 2 Diabetes}

106-OR

LISBETH N. FINK, ANDREAS OBERBACH, THOMAS E. JENSEN, ANETTE SAMS, MATTHIAS BLÜHER, AMIRA KLIP, Gentofte, Denmark, Toronto, ON, Canada, Leipzig, Germany

Muscle insulin sensitivity is critical for optimal whole body metabolism as muscle is the major site of glucose disposal. Recent detection of macrophages in adipose tissue connects inflammatory inputs to insulin resistance. We hypothesize that skeletal muscle may also be susceptible to immune cell infiltration, and thus our aim was to explore whether immune cells are present in muscle of T2D humans and in mice fed a high fat diet (HFD). By isolating free cells from quadriceps muscle of C57BI6 mice fed HFD, we observed a clear increase in the number of CD11c-positive pro-inflammatory macrophages ( $48 \%$ increase by 1 week HFD, $77 \%$ by 10 weeks). A comparable increase in this macrophage subtype in mouse adipose tissue correlates with insulin resistance. In contrast to epididymal fat, T cell counts in muscle were low and unchanged by HFD. In muscle biopsies of humans, we measured by qRT-PCR the expression of genes characteristic of macrophage subsets, and quantified infiltrating CD68-positive macrophages by immunohistochemistry. CD11c expression was 2.5-fold higher in T2D compared to obese normo-glucotolerant individuals and correlated positively with $\mathrm{HbA1c}$ and negatively with glucose disposal rate measured by euglycemichyperinsulinemic clamps, whereas the anti-inflammatory marker TGF $\beta$ correlated negatively with $\mathrm{HbA1c}$. The number of CD68-positive macrophages was $2 \mathrm{X}$ higher in T2D muscle, and CD11c-positive cells were also detected. With 1 year of exercise intervention, expression of two anti-inflammatory macrophage markers (macrophage galactose binding lectin and mannose receptor) correlated strongly with glucose disposal rate. We conclude that, in addition to the disease risk associated with 'inflamed fat', there is a link between CD11c-positive macrophages in muscle and insulin resistance. Conversely, anti-inflammatory macrophages track with insulin sensitivity. The occurrence of macrophages in muscle may afford the opportunity to resolve muscle insulin resistance leading to T2D by locally targeting inflammation.

\section{$\Delta$}

\section{AS160 Regulates Glucose Uptake in the Heart}

107-OR

DING AN, TARO TOYOTA, KRISTEN HITCHCOX, MIN-YOUNG LEE, KRISTEN GETCHELL, LILI GUO, MICHAEL F. HIRSHMAN, SUSANNA KELLER, LAURIE J. GOODYEAR, Boston, MA, Charlottesville, VA

Maintaining glucose utilization is critical for normal cardiac function, yet the distal signaling mechanisms mediating myocardial glucose uptake are not known. Akt substrate of $160 \mathrm{kDa}$ (AS160) is a Rab GTPase-activating protein (GAP) that contains consensus Akt phosphorylation sites (PAS) and other phospho-sites including Ser ${ }^{711}$, an AMPK consensus sequence. In adipocytes and skeletal muscle, insulin causes AS160 PAS phosphorylation, inactivating the GAP domain, resulting in GLUT4 translocation. There have been no studies of AS160 phosphorylation or function in the heart. Here we found that insulin stimulation in vivo increased AS160 phosphorylation at PAS and Ser ${ }^{711}$ sites in the heart, an effect partially inhibited in Akt2 knockout mice. Exercise and ischemia did not increase AS160 PAS phosphorylation, but did increase Ser ${ }^{711}$ phosphorylation; an effect fully inhibited in muscle-specific AMPKo2 inactive TG mice. To study AS160 function in vivo we used wild type and global AS160 knockout (KO) mice. Basal glucose uptake was not altered in KO hearts, while insulin-stimulated glucose uptake was completely abolished. Insulin-stimulated IRSY612 and AktT ${ }^{308}$ phosphorylation were normal. Interestingly, KO hearts had a 60\% decrease in GLUT4 protein with no change in GLUT4 mRNA. Immunostaining confirmed the reduction in total GLUT4, which was due to depletion of the intracellular compartment. To study the effects of AS160 overexpression, HL-1 cardiomyocytes were transfected with wild type AS160 (70\% increase vs control). AS160 overexpression had no effect on basal glucose uptake, but inhibited insulin and phenformin-induced glucose uptake, effects reversed by disruption of the
Rab-GAP domain. These data suggest that overexpression of AS160 suppresses GLUT4 translocation consistent with AS160 functioning as a "brake", while lack of AS160 may alter GLUT4 recycling and protein stability, leading to impaired insulin-stimulated glucose uptake in the heart. In conclusion, AS160 plays a key role in regulating glucose metabolism in the heart.

108-0R

Histone Deacetylase Inhibition (HDACi), a Promising Approach to Enhance Energy Metabolism and Mitochondrial Function in Skeletal Muscle

VIDHI GAUR, SEAN MCGEE, KEN WALDER, Waurn Ponds, Australia

Impaired energy metabolism and mitochondrial function in skeletal muscle contributes to the pathogenesis of metabolic diseases such as type 2 diabetes. The class Ila histone deacetylases (HDACs) contribute to this response, by repressing metabolic genes in muscle (McGee et al., unpublished data). These enzymes induce chromatin remodelling through histone deacetylation, which favours transcriptional silencing. Therefore, HDAC inhibitors (HDIs) could potentially be used to enhance skeletal muscle metabolism and mitochondrial function and could represent a novel therapeutic intervention for metabolic diseases. We have developed and screened novel class Ila specific HDIs analogues (MRU062, MRU059, MRU047, MRU066) along with commercially available broad spectrum HDIs (Scriptaid, TSA) for their efficacy to regulate energy metabolism and mitochondrial function in vitro using the Seahorse bioanalyser. Initial analyses showed that all of these compounds, except MRU66, induced histone 3 acetylation. L6 myotubes were then treated with each HDI for 1 hour, twice per day, on day 4 and day 5 of differentiation. The mitochondrial function analyses on the following day demonstrated that MRU047 and MRU 059 significantly improved maximal mitochondrial respiration whereas Scriptaid and MRU062 significantly increased basal (by $\sim 17.6 \%$ and $\sim 49.5 \%$ respectively) and maximal mitochondrial respiration (by $23.4 \%$ and $\sim 71 \%$ respectively), with MRU062 showing the maximum response (data are mean \pm SEM, ${ }^{*} p<0.05, n=6-14$ ). The increase in basal respiration was due to increased ATP turnover, but not uncoupled respiration. In contrast, TSA reduced the maximal respiration $(p<0.05, n=6-7)$. These studies show that selective HDI(s) improve mitochondrial function and muscle metabolism. We are currently examining the efficacy of HDAC inhibition as a therapeutic strategy for metabolic diseases in vivo.

Supported by: Diabetes Australia Research Trust Viertel Award

\section{SIGNALING IN THE ISLET}

109-0R

Insulin Signaling Regulates Insulin Processing and Modulates ER Stress in Pancreatic $\beta$-Cells

CHONG WEE LIEW, ANKE ASSMANN, KATHRYN L. LIPSON, SINDHU RAJAN, JIANG HU, IRIS LINDBERG, LOUIS H. PHILIPSON, ANDREW T. TEMPLIN, RAGHAVENDRA G. MIRMIRA, NAHUM SONENBERG, ALLISON B. GOLDFINE, FUMIHIKO URANO, ROHIT N. KULKARNI, Boston, MA, Worcester, MA, Chicago, IL, Baltimore, MD, Indianapolis, IN, Montreal, QC, Canada

Hyperproinsulinemia is among the early features observed in the pathogenesis of Type 2 Diabetes (T2D). The $\beta$-cell is unique in its ability to process immature proinsulin into bioactive insulin using prohormone convertases PC1/3, PC2 and Carboxypeptidase E (CPE). Insufficient action of either of these enzymes leads to accumulation of unprocessed proinsulin. To explore the role of insulin action in proinsulin processing we examined mice lacking functional insulin receptors in $\beta$-cells ( $\beta \mid R K O)$. $\beta$ IRKO mice, primary islets and $\beta$-cell lines exhibited increased circulating and cellular proinsulin content. This was associated with reduced ( 60\%) expression of CPE. Concomitantly, we observed that $\beta$ IRKO $\beta$-cells exhibit dilated endoplasmic reticulum (ER) and concordant alterations in expression of unfolded protein response including phospho- and total IRE $\alpha, B i P$, spliced and total XBP1, suggesting compensation for the increased proinsulin biosynthesis. Reduced expression of CPE was due to a decrease in the biosynthesis of CPE protein and not due to alterations in transcripts or protein stability. Polyribosome profile analysis from $\beta$-cell treated with thapsigargin to mimic ER stress revealed a shift in CPE mRNA from polyribosomes to monoribosomes, indicating a block in translational initiation of the mRNA. Interestingly, we observed downregulation of the translational initiation scaffolding protein eIF4G1 in $\beta$ IRKO cells, which was confirmed by acute knockdown of the insulin receptor in control $\beta$-cells. We provide evidence, for the first time to our knowledge, for direct regulation by insulin signaling of the key proinsulin processing enzyme, CPE, via the scaffolding protein elF4G1. Re-expression of the insulin receptor, 
knocking down proinsulin, or restoring the levels of CPE each independently reversed the phenotype. Together these data provide compelling evidence for a novel link between insulin signaling, translational initiation, insulin processing and ER stress in $\beta$-cells.

\section{$\Delta$}

110-0R

Loss of Canonical Wnt Signaling in the Beta-Cell Results in Decreased Beta-Cell Insulin Content and Secretion Without Changes in Beta-Cell Mass

ERICA V. GONZALEZ, RENUKA T. MENON, VIJAY YECHOOR, LAYLA ABU-SHAMAT,

SUSAN L. SAMSON, Houston, TX

Genetic variations of transcription factor 7-like 2 (TCF7L2) are associated with risk for T2D. TCF7L2 communicates signaling from the canonical Wnt pathway, working with co-factor beta-catenin (beta-cat) to alter gene expression. Wnt signaling has a role in neonatal beta-cell proliferation in rodents, but contributions in the adult are not established. We knocked out canonical Wnt signaling in beta-cells using a tamoxifen (Tam) inducible rat insulin promoter II driven Cre recombinase (Ins2Cre-ERT) to delete floxed beta-cat in adult C57BL/6J mice (8 weeks). The mice received a High Fat/ High Sucrose diet to determine if beta-cat was necessary for beta-cell compensation if challenged with obesity and insulin resistance. We previously reported that this model displays marked impairment of glucose tolerance and insulin secretion, and here we have investigated the underlying mechanisms. After 20 weeks on diet, Tam+ mice have decreased pancreatic insulin content compared to control mice (Tam $+252 \pm 68$ vs Tam- $649 \pm 77 \mathrm{ng} / \mathrm{mg}$ protein, $p=0.004, n=5$ each group), but this was due to reduced islet insulin content (Tam $+665 \pm 64$ vs $1266 \pm 22 \mathrm{ng} / \mathrm{mcg}$ nucleic acid, $\mathrm{p}=0.012$ ) rather than reduced beta-cell mass. Also, there were no differences in islet expression of Wnt-regulated cell cycle genes (cyclin D1, D2, c-myc, cdk4). Islets from Tam+ mice had $79 \%(p=0.001)$ reduction in basal insulin secretion at $2.8 \mathrm{mM}$ glucose, with a $52 \%$ reduction at $11 \mathrm{mM}$ glucose $(\mathrm{p}=0.02)$. KCl stimulated insulin release also was reduced $48 \%(p=0.002)$ indicating there were less primed insulin granules without beta-cat. Expression of genes for insulin processing (prohormone convertases $1 / 3$ and 2) and granule priming and exocytosis (ZnT8, Munc 18-1, Rab3A) were decreased. These results indicate that canonical Wnt signaling is required for compensation of the beta-cell for insulin resistance by regulating granule maturation and insulin secretion rather than beta-cell proliferation.

Supported by: Alkek Foundation, NIH (P30DK079638)

111-0R

Role of Tcf7l2-Mediated Pathway in Pancreatic Beta Cell In Vivo ISEKI TAKAMOTO, NAOTO KUBOTA, KATSUYOSHI KUMAGAI, ATSUSHI OBATA, TETSUYA KUBOTA, KOHJIRO UEKI, TAKASHI KADOWAKI, Tokyo, Japan

Tcf7/2 was identified as one of the major type 2 diabetes susceptibility genes, though the molecular mechanisms by which Tcf7l2 affect insulin secretion in vivo are still unclear. To elucidate the pathophysiological role of Tcf7I2-mediated pathway in vivo, we generated genetically engineered mice in which the function of Tcf7/2 was impaired in pancreatic beta cell. We constructed an expression vector of murine dominant-negative form of Tcf7l2 (DNTcf) under the rat insulin promoter (RIP) and generated beta-cell specific DNTcf transgenic mice (RIP-DNTcf-Tg mice). 3 independent RIP-DNTcf-Tg mice lines were established, in the islets of which exogenous expression level of DNTcf showed more than 10-fold increase compared with endogenous expression level of Tcf7|2. In all 3 lines, RIP-DNTcf-Tg mice and their littermate wild-type mice exhibited the same level of body weight. Though both mice showed similar degree of insulin resistance in insulin tolerance test, RIP-DNTcf-Tg mice developed overt diabetes with impaired insulin secretion in oral and intraperitoneal glucose tolerance test. Interestingly, exogenous administration of GLP1-agonist exendin-4 ameliorated intraperitoneal glucose tolerance with improved insulin secretion in RIP-DNTcf-Tg mice, indicating that incretin effect was still preserved. Histological analysis of RIP-DNTcf-Tg mice revealed that beta-cell mass was markedly decreased with low BrdU incorporation rate, and total insulin content of the whole pancreas was also diminished. Consistently, quantitative RT-PCR analysis of isolated islets from RIP-DNTcf-Tg mice showed decreased gene expression of insulin 1, insulin 2, Cyclin D2 and MafA. Moreover, similar phenotypes of adult RIP-DNTcf-Tg mice described above were observed in the weaning age and neonatal state. Taken together, these findings provide new evidence that Tcf7l2-mediated pathway in pancreatic beta cell plays a crucial role in glucose metabolism through regulation of beta-cell mass and total insulin amount in vivo.
$\Delta$

112-OR

A Complex Integration of Distinct $\beta$-Cell Adaptation Phases over 12 Weeks of HFD in Normal Mice

THOMAS L. JETTON, DHANANJAY GUPTA, NAVJOT MONGA, KYLA LAROCK, JAMES LAUSIER, BASANTHI SATISH, MINA PESHAVARIA, JACK L. LEAHY, Colchester, VT

High energy fat-enriched diets in rodents are useful to study adaptive changes in $\beta$-cell mass (BCM) and function in response to obesity and insulin resistance. However the mechanistic details and signaling pathways are poorly known, as is their relationship to impaired insulin sensitivity. We previously showed a key role for PPAR-g and its transcriptional regulation of $p d x-1$, pyruvate carboxylase (PC), and GIPR following a loss of BCM from a partial pancreatectomy (Px). We have now studied B6 mice fed on a HFD $(60 \%$ fat) or control chow for 12 wks to identify $\beta$-cell adaptive responses. Within 1 wk, HFD mice became glucose intolerant and mildly hyperinsulinemic. However, insulin sensitivity was normal until 3-4 wks. At the same time, hyperinsulinemia that had been maintained at $\sim$-fold over the control diet mice rapidly rose to $\sim 25$-fold by 12 weeks. Also, the BCM was unchanged until 4 weeks, and then progressively doubled by non-proliferative mechanisms. We focused on the time point before insulin resistance ( $<3$ weeks), and found hyperexpression of a gene profile that was nearly identical to the pattern onserved post Px - ppar-g, mafa, pdx1, nkx6.1, PC, gipr, ins1/2. At 4 weeks, this surge was followed by increased levels of $\beta$-cell activated CREB, Irs2 and cytoplasmic Fox01, suggesting that enhanced growth and survival underlie the BCM increase. Another wave in gene expression occurred at 8 weeks with enhanced neuroD, ins1/2, and glp1r. In summary, the $\beta$-cell adaptive responses to fat feeding in normal mice are complex and multifaceted. Early on, before detectable insulin resistance, there was activation of the ppar-g/Pdx-1 pro-differentiation pathway. By 4 weeks, insulin resistance was present in tandem with enhancements of $\mathrm{BCM}$, survival, and function as reflected in marked hyperinsulinemia. These data collectively point to a complex integration of distinct $\beta$-cell responses that ensure the progressively increasing insulin demands are met in the context of nutrient overabundance and obesity.

$\Delta$ 113-OR Direct Transcriptional Regulation of the Histone Methytransferase Set7/9 Gene By Peroxisome Proliferator-Activated Receptor-Gamma (Ppary) in Islet Beta Cells

DHANANJAY GUPTA, THOMAS JETTON, MINA PESHAVARIA, JACK LEAHY, Colchester, VT

We previously reported PPAR $\gamma$ signaling in islet $\beta$-cells transcriptionally regulates the pro-differentiation gene pdx-1 and the incretin receptor GIPR during states of metabolic stress such as partial pancreatectomy (Px) or changes in insulin sensitivity. An islet enriched SET domain-containing histone methyltransferase Set7/9 promotes euchromatin modifications through methylation of the histone3-lysine-4 residue. Activation of PPAR $\gamma$ signaling in $\beta$-cells is reported to enhance Set7/9 expression, but the mechanism is unknown. We now have investigated the molecular mechanism of PPAR $\gamma$ mediated transcriptional regulation of Set7/9. Sequence analysis of the mouse Set7/9 promoter revealed a putative PPAR response element (PPRE) (-578 to $-590 \mathrm{bp}$ ) that was conserved both in rat and human orthologue sequences. PPAR $\gamma$ specific binding on the Set7/9 PPRE was confirmed by electrophoretic mobility shift/super-shift and ChIP (chromatin immuno-precipitation) assays. In INS-1 cells transfected with a Set7/9 promoter driven luciferase reporter construct, 48-hour stimulation with the PPAR $\gamma$ agonist troglitazone (TZD, $10 \mu \mathrm{M})$ increased the reporter activity 2.0-fold $(p<0.001)$ compared to vector alone-transfected INS-1 cells. A 72-hour TZD treatment of INS-1 cells and primary mouse islets resulted in a 2-fold $(p<0.004 ; p<0.008$ respectively) increase in Set7/9 protein levels. Also, in the normoglycemic model of $\beta$-cell adaptation in rats $(60 \% \mathrm{Px})$, 14-day post-Px islets had a 2.1 fold increase in Set7/9 protein levels compared to islets from sham-operated rats corresponding to the adaptive phase when islet PPAR $\gamma$, Pdx-1, and GIPR levels are upregulated $(p<0.0001)$. Combined together, our data provide evidence for a novel mechanism of direct transcriptional regulation of the Set7/9 gene by PPAR $\gamma$, plus that interaction may be an important element of the adaptive $\beta$-cell transcriptional machinery to metabolic stresses. 
114-OR PAS Kinase Regulates PDX-1 Stability via GSK3 Beta in Pancreatic Beta-Cells

MERIEM SEMACHE, GHISLAINE FONTÉS, SARAH FOGARTY, CHINTAN KIKANI, JARED RUTTER, VINCENT POITOUT, Montreal, OC, Canada, Salt Lake City, UT

PAS kinase (PASK) is a serine/threonine kinase implicated in the regulation of insulin gene expression via modulation of the transcription factor pancreas-duodenum homeobox-1 (PDX-1). However, the precise mechanisms by which PASK regulate PDX-1 expression and activity are unknown. The aim of this study was to test the hypothesis that PASK phosphorylates and thereby inactivates glycogen synthase kinase-3 beta (GSK3 $\beta$ ), to alleviate PDX-1 protein degradation. In beta-cells, GSK3 $\beta$ is known to phosphorylate PDX-1 on Ser residues at low glucose and trigger its proteasomal degradation. First, in vitro we observed that PASK directly phosphorylates GSK3 $\beta$ on Ser9 (a known inactivating phosphorylation site of GSK3 $\beta$ ). Second, we examined the effects of PASK on GSK3 $\beta$ and PDX-1 phosphorylation in isolated rat islets and HIT-T15 insulin secreting lines by adenoviral transduction or transient transfection, respectively, of a wild-type (WT) or kinase-dead (KD, acting as a dominant-negative) form of PASK. We observed that overexpression of KD-PASK completely blocks the ability of glucose to phosphorylate GSK3 $\beta$ on Ser9. Third, WT-PASK mimics the effect of glucose in inhibiting PDX-1 serine phosphorylation, as assessed by immunoprecipitation. Fourth, overexpression of WT-PASK protects PDX-1 protein from degradation in the presence of cycloheximide. We conclude that in beta-cells PASK phosphorylates and inactivates GSK3 $\beta$, resulting in a decrease in PDX-1 serine phosphorylation and stabilization of PDX-1 protein.

Supported by: NIH (R01 DK-58096), FROS

115-OR

Molecular Characterization of Store-Operated $\mathrm{Ca}^{2+}$ Influx in Pancreatic Beta Cells: Role of Stim1

COLIN A. LEECH, RICHARD KOPP, OLEG CHEPURNY, GEORGE G. HOLZ, MICHAEL W. ROE, Syracuse, NY

Store-operated $\mathrm{Ca}^{2+}$ entry (SOCE) is known to occur in pancreatic $\beta$ cells but the molecular basis, regulation and function of this current remain uncertain. Here we demonstrate that SOCE is activated following endoplasmic reticulum (ER) $\mathrm{Ca}^{2+}$ store depletion by the ER $\mathrm{Ca}^{2+}$ sensor Stim1. ER $\mathrm{Ca}^{2+}$ stores in the MIN6 or INS-1 cell lines were depleted by bathing cells in $\mathrm{Ca}^{2+}$-free media containing carbachol and cyclopiazonic acid. Subsequent re-introduction of $\mathrm{Ca}^{2+}$ to the bathing solution produces a pronounced nifedipine-insensitive rise of cytosolic [ $\left.\mathrm{Ca}^{2+}\right]$ as a result of SOCE. Cells were then transfected with either a scrambled control or short hairpin RNA (shRNA). A robust SOCE remained in cells treated with scrambled control RNA but SOCE was substantially inhibited by treatment with shRNA targeted against Stim1. Transient knockdown of Stim1 using shRNA inhibited glucose-stimulated insulin secretion. Stable cell lines expressing scrambled control or shRNA were generated for studies using patch clamp whole cell recording (WCR). These studies revealed that the shRNA did not significantly change voltage-dependent $\mathrm{Ca}^{2+}$ currents or $\mathrm{Ca}^{2+}$-induced exocytosis, determined by capacitance measurements. WCR using a $\mathrm{Cs}^{+}$pipette solution containing 1 $\mathrm{mM} \mathrm{Mg}^{2+}$ and $200 \mu \mathrm{M}$ EGTA, to passively deplete ER stores, revealed an inward current in cells held at $-60 \mathrm{mV}$ that had a linear current-voltage relation and was tolbutamide-sensitive. This current was significantly smaller in cells treated with shRNA against Stim1. Store depletion under these recording conditions was confirmed in cells expressing an ER-targeted $\mathrm{Ca}^{2+}$ reporter We propose that Stim1 acts as a sensor of the filling state of $\mathrm{ER} \mathrm{Ca}^{2+}$ stores in $\beta$ cells and regulates SOCE. This current is likely to play a role in regulating cell excitability and cytosolic $\mathrm{Ca}^{2+}$ dynamics and therefore influences glucose-induced insulin secretion.

Supported by: NIH

116-OR

The FoxM1 Pathway is Implicated in Nutrient-Induced Beta-Cell Proliferation in Rats

BADER ZARROUKI, GHISLAINE FONTÉS, ONDREJ SEDA, VINCENT POITOUT,

Montreal, OC, Canada

A compensatory increase in beta-cell mass occurs in several physiological and pathophysiological situations, such as pregnancy, obesity and insulin resistance. We have previously observed that a 72 -h infusion of glucose (GLU) + Intralipid (IL) in 6-mo-old rats causes insulin resistance and beta-cell dysfunction, despite a marked increase in beta-cell proliferation and mass. To further examine the effects of nutrient excess on islet function in vivo, 6-mo old rats were infused with saline (SAL), GLU, IL, or GLU+IL for 72h, and gene expression profiles were examined using Affymetrix GeneChip 1.0 ST.
Using Bonferroni analysis, we found 700 genes significantly modulated by GLU vs. SAL, 20 modulated by IL vs. SAL, and 791 by GLU+IL vs. SAL. Gene set enrichment analysis showed that the most highly regulated pathways in GLU+IL vs. SAL were related to cell cycle and mitosis, consistent with the increase in beta-cell proliferation. Of these, many targets of the transcription factor FoxM1, including FoxM1 itself, were significantly upregulated in the GLU+IL group. This was confirmed by qRT-PCR analysis which showed increased expression of FoxM1 $(3.32 \pm 0.35$ fold vs. $S A L, N=5, P<0.05)$ and its targets Polo-like kinase $1(9.41 \pm 0.92$ fold vs. $S A L, N=5, P<0.01)$ and Aurora kinase $B(24.68 \pm 1.80$ fold vs. $S A L, N=5, P<0.001)$. FoxM1 protein expression was also increased by $2.63 \pm 0.03$ fold in $G L U+I L$ vs. $S A L(N=4, P<0.01)$. We conclude that the FoxM1 pathway is implicated in beta-cell proliferation in response to nutrient excess in this model.

Supported by: NIH (R01-DK58096), Eli Lilly

\section{DIABETIC FOOT DISEASE- WHAT CONTRIBUTES TO HEALING?}

117-0R

Mesenchymal Stem Cells Improve Impaired Foot Ulcer Healing in Diabetic Rats

JIRO KATO, HIDEKI KAMIYA, TATSUHITO HIMENO, TAIGA SHIBATA, TETSUJI OKAWA, MASAKI KONDO, ATSUSHI FUJIYA, YUSUKE SEINO, SHIN TSUNEKAWA, YOJI HAMADA, KEIKO NARUSE, YUTAKA OISO, JIRO NAKAMURA, Nagoya, Japan

Aim: This study was conducted to evaluate the therapeutic effects of mesenchymal stem cells (MSCs) transplantation on the delayed foot ulcer healing under the diabetic condition using STZ-diabetic rats. Methods: 1) MSCs were isolated from bone marrow of 5-week old SD rats, and their characters were analyzed by FACS and RT-PCR. 2) Human keratinocytes (HKCs) were cultured in $6 \mathrm{mM}(\mathrm{NG}), 12 \mathrm{mM}(\mathrm{HG})$ or $25 \mathrm{mM}$ (HHG) glucose concentrations with or without MSC-conditioned media (MSC-CM) for 72 hrs. Cell viabilities were evaluated by MTS assay. The abilities of migration and cytokine production were evaluated by RT-PCR. 3) Diabetic foot ulcer model: diabetes was induced by intraperitoneal injection of STZ to 6-week old SD rats. After 8 weeks of diabetes, a full thickness $4 \mathrm{~mm}$ diameter punch holes were created in the hind paw of normal (N) and diabetic rats (D). MSCs $\left(1 \times 10^{6}\right)$ labeled with PKH were injected around the wounds. Wounds were photographed every other day. Wound tissues were excised and histological evaluations were performed. Results:1) MSCs were positive for CD29/ CD90 and negative for CD34/ CD45. mRNA expressions of VEGF, bFGF, NGF, EGF and KGF were detected. 2) HKC viabilities were significantly impaired by $\mathrm{HG}$ or $\mathrm{HHG}$, which was improved by MSC-CM. mRNA expression of MMP-2, MMP-9, EGF and IGF-1 were decreased by HG or HHG. And MSC-CM restored these HG-induced deficits. 3) MSCs survived in dermal and subcutaneous tissues even 2 weeks after transplantation. The duration of wound healing was significantly prolonged in $\mathrm{D}(\mathrm{N}: 15.8 \pm 2.5$ days, $\mathrm{D}: 21.2 \pm 1.9)$, which was significantly ameliorated by MSC transplantation (D+MSC: 18.3 \pm 2.1 ). Conclusion:These observations indicate that MSC transplantation would be useful for treatment of diabetic foot ulcer through improving impaired keratinocyte functions.

118-OR

Expression of Cytokines and Growth Factors in Patients With Healing versus Non Healing Foot Wounds

STEPHANIE C. WU, EYAYU BELAY, DANIEL PETERSON, Chicago, IL

The percent change in foot ulcer area after 4 weeks is typically used as a predictor of healing. Wound healing is regulated by a network of complex cellular signaling cascades involving numerous growth factors, cytokines, and chemokines, however, the level of growth factor and cytokine distribution in healing versus non healing wounds are ill-defined. The purpose of this initial study was to assess the gene expression profiles of healing versus nonhealing foot ulcers Patients with chronic foot ulcers were recruited for the study. All wounds were cleansed prior to debridement, tissue specimens obtained, and the gene expression profile of key factors involved in wound healing analyzed. Factors included Interleukin-1beta (IL-1 $\beta$ ), Interleukin-8 (IL-8), Tumor Necrosis Factor alpha (TNF- $\alpha$ ), Vesicular Endothelial Growth Factor (VEGF) and Platelet Derived Growth Factor (PDGF). Wounds were followed on a weekly basis for twelve weeks to tract clinical healing. Chronic wounds have decreased expression levels of the cytokines and growth factors. Wounds that did not heal in 12 weeks have a $10 \%$ lower expression level than wounds that healed over time. The expression level of TNF- $\alpha$, IL-1 $\beta$ and IL-8 was up to $25 \%$ less in the nonhealing wounds (both diabetic 
and non-diabetic) relative to levels of VEGF and PDGF. In the group of wounds that did not heal in 12 weeks, there was a 10 to $20 \%$ decrease in all the cytokines and growth factor expression level in the diabetic wound compared to non-diabetic wound. There appeared to be no marked spatial variation of the expression level of these factors around the wound. Wound expression levels of cytokines and growth factors can affect the fate of an ulcer. Further research in this area may lead to individualized supplementation of wound healing factors to facilitate healing.

\section{$\Delta$}

119-OR

Depression and Not Specific Emotional Distress Predicts Diabetic Foot Ulcer Incidence

LORETTA VILEIKYTE, JEFFREY S. GONZALEZ, ANDREW J. BOULTON, Manchester, United Kingdom, Bronx, NY

Prior diabetic foot ulceration (DFU) does not increase risk for depression. However, depression is associated with increased DFU risk. We re-examined these relationships substituting depression (Hospital and Anxiety Depression Scale; HADS-D) with a measure of foot problem-Specific Emotional Distress (NeuroQoL-SED). Data from an 18-month prospective cohort study of 333 patients with diabetic peripheral neuropathy, DPN $71 \%$ male; age 62yrs; 73\% type 2 diabetes; Neuropathy Disability Score 7.4) showed that those with prior DFU had significantly $(\mathrm{p}<.001)$ greater Neuro0oL-SED ( $\mathrm{M}=$ $2.70, S D=1.19)$ at baseline than those without $(M=2.00, S D=1.10)$. This difference persisted $(p=.003)$ in multivariate regression models controlling for demographics, comorbidities, DPN-severity, symptoms and functional impairment, and was enhanced, rather than attenuated, when HADS-D was controlled for $(p<.001)$. However, longitudinal multivariate models showed that NeuroQoL-SED did not predict DFU over time. Controlling for NeuroQoLSED did not attenuate previously reported association between HADS-D and DFU risk. Item-level analyses showed HADS-D items assessing positive affect were not significantly associated with DFU risk. Only 'feeling slowed down' $(p=.030)$ and 'losing interest in appearance' $(p=.003)$ were significantly associated with increased risk of DFU. Controlling for DPN-related DFU risk factors and subjective health ratings (general or DPN-specific) did not attenuate the relationship between HADS-D and DFU risk. These results suggest that while prior DFU is associated with increased specific emotional distress and not depression, it is depression, particularly negative affect, that is associated with increased risk for the development of new DFU over time. While the mechanism for this relationship is unclear, it does not appear to be accounted for by confounding with specific emotional distress or with objective or subjective indicators of poor health.

120-OR

High Mortality Rates from Foot Complications in Diabetic Patients on Dialysis

AGBOR NDIP, ANAND VARDHAN, KAREN BREISLIN, ANDREW J. BOULTON, Manchester, United Kingdom

Diabetes is the commonest cause of end-stage renal disease (ESRD), requiring renal replacement therapy. Lower extremity complications in diabetes can result in significant morbidity and mortality further compounding the poor survival of the dialysis population. A prospective observational study involving diabetic patients with ESRD on dialysis was undertaken to evaluate the impact of foot ulcers and amputations on mortality. We studied 192 diabetic patients receiving dialysis at the Manchester Royal Infirmary, UK. These included 17 (8.9\%) African-Caribbeans, 38 (19.8\%) Indo-Asians and $137(71.4 \%$ ) White Europeans aged (mean \pm SD) $59 \pm 14$ years, with a male/ female ratio of $121 / 71$. Comprehensive foot assessment was performed at baseline including evaluating for neuropathy, peripheral arterial disease, foot deformities, and foot ulcers/amputation. Patients were followed up for a two-year period. Overall mortality at the end of two years was high $(102 / 192,53.1 \%)$. Patients who had a foot ulcer at baseline (35/59, 59.3\%) had a higher mortality after 2 years of follow-up, compared with patients without $(55 / 133,41.4 \%, p=0.028)$. Similarly, patients who had had an amputation at baseline had higher mortality at 2 years $(26 / 35,74.3 \%)$ compared to patients without amputation $(64 / 157,40.8 \%, p<0.0001)$. These data highlight the excess mortality in dialysis-treated diabetic patients resulting from foot ulcers and amputations, therefore underpinning the importance of integrating foot care and amputation prevention within the dialysis population.

Supported by: Diabetes UK
Plasma Adiponectin, IL-6, hsCRP and TNF- $\alpha$ Levels in Subject With Diabetic Foot and its Correlation With Clinical Variables in a North Indian Tertiary Care Hospital

MOHAMMAD ZUBAIR, ABIDA MALIK, JAMAL AHMAD, Aligarh, India

Pro-and-anti-inflammatory processes are crucial in different phases of wound healing and its disturbances interfere with tissue homeostasis after the manifestation of ulcers leading to chronic non-healing wounds. However, data on the association between inflammation and acute foot syndrome are scarce. Circulating levels of acute-phase reactant and cytokines were measured in diabetic patients with an ulcer ( $n=162)$ and without ulcer ( $n=162)$ in a case-control study. Every subject with diabetic foot was matched for age, sex and BMI. Of the patients, $85.1 \%$ had type 2 diabetes. Subjects with diabetic foot ulcer showed lower median plasma level of adiponectin [8.4(7.1-9.2) vs $13.4(12.1-14.2)] \mathrm{ng} / \mathrm{ml}$, higher median plasma levels of IL-6 [32.5(9.4-44.8) vs 6.7(4.6-14.6)] ng/ml, hsCRP [12.6(11.2-13.6) vs 8.4(7.1-9.2)] $\mathrm{mg} / \mathrm{ml}$ and TNF- $\alpha$ [99.4(79.9-121.5) vs $4.9(4.5-5.6)] \mathrm{ng} / \mathrm{ml}$. A positive correlation was found between BMI and retinopathy for adiponectins, for IL-6, between grades of ulcer, BMI, LDL-C, triglycerides, retinopathy, nephropathy \& smoking, for hsCRP: grades of ulcer, BMI, LDL-C, triglycerides, retinopathy, nephropathy \& smoking, while total cholesterol and neuropathyfor TNF- $\alpha$. This study demonstrates that diabetic subjects with various grades of diabetic foot ulcer showed a higher plasma IL-6, hsCRP, TNF- $\alpha$ and lower adiponectin levels in comparison with diabetes without foot ulcer independent of the concomitant infections. It would be interesting to find out whether an activation of immune system precedes the development of foot ulcer and whether anti-inflammatory therapies will be effective in improving the outcome in such patients.

122-0R

A Retrospective Analysis of Risk of Acute Kidney Injury among People With Diabetic Foot Disease Undergoing Surgical Interventions JUNAID IQBAL, ELAINE HIGGINS, ANDREW FISHER, JANE ASTLE, IRFAN AKHTAR, KHALID MAKDHOOMI, DEVAKA J. FERNANDO, Sutton in Ashfield, United Kingdom

Acute Kidney injury (AKI) is a significant cause of in patient morbidity and mortality. Renal impairment is often associated with foot ulceration. Patients with diabetic foot disease are exposed to nephrotoxic antibiotics, radiological investigations involving potentially nephrotoxic contrast media and surgical interventions. Hence they are at higher risk of developing AKI. We reviewed the medical records of 45 consecutive people (Mean age 68.1 SD 10.2 years, 30 males) with diabetes (HBa1c 8.1 SD 1.5\%) admitted with diabetic foot problems to a district general hospital retrospectively during a six month period. The frequency of Chronic Kidney Disease (CKD) and progression to AKI if any, during their in patient stay was determined. $23(51 \%)$ satisfied criteria for CKD on admission. 12 (26.7\%) developed AKI during in patient stay. $10(43.5 \%)$ of those with CKD developed AKI (Likelihood ratio 7.2, $p=0.007)$ compared to $2(9.1 \%)$ in whom AKI developed de novo. $5(41.7 \%)$ of those receiving contrast media, developed AKI compared to $7(21.2 \%)$ of those who did not (Likelihood ratio $69.2, p=0.001)$ while 11 $(18.3 \%)$ of those undergoing surgery developed AKI compared to $8(25 \%)$ of those who did not (Likelihood ratio 67.4, $p=0.001$ ). Those who did not receive surgery had a higher frequency of co morbidities and were hence deemed unsuitable for surgical treatment. Patients admitted with diabetic foot problems are at high risk of deteriorating renal function requiring inputs from specialist nephrologists. Service provision for this patient group should include early assessment of risk of AKI, access to renal care and acute renal support services.

\section{HYPOGLYCEMIA AND THE BRAIN}

\section{3-OR}

Hypothalamic GABA Drops in Response to Acute Hypoglycemia in Healthy Humans

GULIN OZ, AMIR MOHEET, UZAY E. EMIR, MELISSA TERPSTRA, ANJALI KUMAR, LYNN E. EBERLY, ELIZABETH R. SEAQUIST, Minneapolis, MN

Hypothalamic GABA signaling has been shown to play an important role in regulating the counterregulatory response (CR) to hypoglycemia (HG) in animal models. While a fall in hypothalamic GABA levels appears necessary for activation $\mathrm{CR}$ to $\mathrm{HG}$ in rodents, the role of GABA in regulating the $\mathrm{CR}$ to $\mathrm{HG}$ in humans is uncertain. Here we used ultra-high field magnetic resonance spectroscopy (MRS) to determine if hypothalamic GABA levels decrease in response to hypoglycemia in healthy volunteers. Subjects underwent a 


\section{HYPOGLYCEMIA AND THE BRAIN}

2-step hyperinsulinemic $(2.0 \mathrm{mU} / \mathrm{kg} / \mathrm{min})$ clamp in a 7 tesla scanner where blood glucose was first maintained at $90 \mathrm{mg} / \mathrm{dL}$ by the IV infusion of dextrose, then allowed to drop to $50 \mathrm{mg} / \mathrm{dL}$ by the discontinuation of the dextrose infusion, and then maintained at the $\mathrm{HG}$ plateau by a variable dextrose infusion. GABA levels in the hypothalamus (voxel size $13 \times 12 \times 10 \mathrm{~mm}^{3}$ ) were measured using short-echo single voxel spectroscopy (STEAM, TE=8ms, TR=5s) with a 16 channel transceiver array coil and $B_{1}$ shimming. Concentrations were corrected for cerebrospinal fluid in the voxel. Four subjects returned for a subsequent scanning session without IV infusions to assess the test-retest coefficient of variance (CV) of the measure. Eight subjects completed the hypoglycemia experiment $(4 \mathrm{~F} / 4 \mathrm{M}$, age $28.2 \pm 4.5 \mathrm{yrs}, \mathrm{BMI} 22.5 \pm 2.5 \mathrm{~kg} / \mathrm{m} 2$, mean \pm SD). Hypothalamic GABA concentration significantly decreased from mean $3.6 \pm$ SD 0.4 to $3.1 \pm 0.4 \mu \mathrm{mol} / \mathrm{g}(p=0.02)$ as glucose fell from mean 89 $\pm \mathrm{SD} 6 \mathrm{mg} / \mathrm{dl}$ at baseline to $61 \pm 3 \mathrm{mg} / \mathrm{dl}$ within $10-20$ minutes of stopping the glucose infusion. This $13 \%$ decrease in GABA was greater than the $9 \%$ CV determined for the GABA measurement in a subset of the subjects. Our experiments demonstrate that GABA concentration can be measured in the human hypothalamus and decrease significantly in response to an abrupt fall in blood glucose. These experiments suggest that GABA signaling may be important in regulating the $\mathrm{CR}$ to $\mathrm{HG}$ in humans.

124-0R

Hypothalamic Glucosensing Neurons Display Blunted Electrical Response to Hypoglycemia After Recurrent Low Glucose Exposure and Antecedent KATP Activation

ELIZABETH A. HAYTHORNE, CRAIG BEALL, D. LEE HAMILTON, MICHAEL L. ASHFORD, RORY J. MCCRIMMON, Dundee, United Kingdom

Recurrent Hypoglycemia (RH) results in the attenuation of the Counterregulatory Response (CRR) to subsequent hypoglycemic episodes in both humans and rodents. Hypothalamic Glucosensing (GS) neurons are thought to be involved in hypoglycemia detection and activation of the CRR. We have recently established that GT1-7 cells, a population of mouse hypothalamic GS neurons, display characteristic features of a GS system. The electrical activity of these cells is directly regulated by glucose metabolism, including glucokinase, AMPK and ATP-sensitive K+ channels (KATP). The aim of the current study was to determine whether the response to hypoglycemia of GS neurons is altered following $\mathrm{RH}$, and whether this defect is driven by prior KATP activation. Patch-clamp electrophysiology was used to examine the electrical activity of GT1-7 cells after exposure to RH ( 3 hours of $0.1 \mathrm{mM}$ glucose for 3 days) or antecedent KATP opener NN414 (3 hours during the previous day) versus appropriate controls. Radiolabeled metabolic assays were used to investigate if any change occurred in glucose oxidation capacity or uptake kinetics. After exposing GT1-7 cells to RH, the magnitude of the $\Delta V \mathrm{~m}$ hyperpolarizing response to $0.5 \mathrm{mM}$ glucose was reduced by $66.7 \%$ compared to control $(\Delta \mathrm{VRH}=4.9 \pm 1.0 \mathrm{mV}$ vs. $\Delta V$ Cont $=14.7 \pm 0.9 \mathrm{mV}, p<0.0001$; $\mathrm{n}=7-10)$. Antecedent NN414 exposure $(5 \mu \mathrm{M})$ also produced a defect in the ability of the cells to hyperpolarize to $0.5 \mathrm{mM}$ glucose by $73.1 \%$ compared to control ( $\Delta \mathrm{VNN} 414=3.8 \pm 1.7 \mathrm{mV}$ vs. $\Delta \mathrm{V}$ Cont $=14.2 \pm 1.4 \mathrm{mV}, \mathrm{p}<0.01 ; \mathrm{n}=6)$. Maximum KATP conductance and glucose uptake and oxidation were unaffected by RH and antecedent NN414 treatments. Thus exposure of GT1-7 cells to $\mathrm{RH}$ induces defective GS, which was mimicked by antecedent direct activation of KATP, independently of any change in glucose uptake or oxidation. This suggests that defective GS, following $\mathrm{RH}$, may be dependent on prior KATP activation.

$\Delta$

125-OR

Increased Brain Uptake of Lactate in Type 1 Diabetic Patients With Hypoglycemia Unawareness

HENK M. DE FEYTER, GERALD I. SHULMAN, DOUGLAS L. ROTHMAN, KITT F PETERSEN, New Haven, CT

Previous studies have reported that brain metabolism of acetate is increased more than twofold in intensively treated type 1 diabetic (T1DM) subjects with hypoglycemia unawareness. These data support the hypothesis that upregulation of blood-brain barrier monocarboxylic acid (MCA) transport activity may contribute to the maintenance of brain energetics during hypoglycemia in T1DM patients with hypoglycemia unawareness. In contrast to acetate, which circulates in plasma at relatively low concentrations ( 0.1 mM), plasma lactate concentrations are approximately tenfold higher making it a primary candidate as an alternative brain fuel during hypoglycemia. We therefore examined this hypothesis by measuring $13 \mathrm{C}$ label incorporation from infused [3-13C] lactate [10 $\mu \mathrm{mol} /(\mathrm{kg}-\mathrm{min})]$ into brain lactate by $13 \mathrm{C}$ magnetic resonance spectroscopy in T1DM patients $(n=5)$ with hypoglycemia unawareness and non-diabetic control subjects $(n=6)$ during a hypoglycemic ( $3 \mathrm{mM}$ ) clamp. Plasma lactate concentrations were approximately $40 \%$ higher in control subjects compared to T1DM subjects $(1.8 \pm 0.4$ vs. $1.3 \pm 0.3 \mathrm{mmol} / \mathrm{L} ; P=0.05)$, while plasma lactate fractional $13 \mathrm{C}$ enrichments were not different ( $26.2 \pm 4.0$ vs. $31.6 \pm 6.7 \%$; $P=0.92)$. Despite the lower plasma lactate concentrations, brain lactate concentrations were approximately twofold higher in the T1DM subjects compared to the control subjects $(2.0 \pm 0.9 \mathrm{mmol} / \mathrm{L}$ vs. $1.0 \pm 0.6 \mathrm{mmol} / \mathrm{L} ; P=0.04)$. Taken together these data support the hypothesis that upregulation of the MCA transporter promotes increased brain lactate uptake in T1DM patients with hypoglycemic unawareness. During hypoglycemia brain lactate may serve as an important alternative fuel to support brain energetics and be responsible for hypoglycemic unawareness in patients with T1DM.

Supported by: AICR10A847, NIHR24DK085638, R01AG034953, R01AG23686, R01DK49230, R01NS051854, HHM

126-OR

Enhanced Brain Alternate Fuel Metabolism Following Recurrent Antecedent Hypoglycemia and STZ Diabetes

RAIMUND I. HERZOG, LIHONG JIANG, ROBERT S. SHERWIN, New Haven, CT

Intensive insulin therapy increases the risk of recurrent hypoglycemia. It blunts central counterregulatory responses to low blood glucose magnifying the risk of severe hypoglycemia and brain injury. Here we show in rats exposed to 3 days of recurrent hypoglycemia and others exposed to three weeks STZ-diabetes that brain energy substrate and neurotransmitter homeostasis undergo significant changes favoring the uptake and oxidation of fuels other than glucose. Isotopically labeled [2 13C] acetate infused into separate groups of animals allowed characterization of key metabolic rates: Exposure to antecedent recurrent hypoglycemia led to differential changes of neuronal and glial glucose and acetate utilization. Comparing the relative contributions of acetate and glucose when going from eu- to hypoglycemia (CMRac/CMRgl), control animals showed a $12 \%$ decrease from baseline $(p<0.06)$, whereas recurrently hypoglycemic rats revealed a $24 \%$ increase from baseline $(p<0.006)$, indicating a higher relative contribution of acetate to brain metabolism in 3dRH animals under hypoglycemic conditions. We observed a similar trend in STZ diabetic animals during NMR spectroscopy at euglycemia, where the glial $13 \mathrm{C}$-glutamine enrichment rate was significantly higher than in healthy control animals (Figure 1). In conclusion, a higher metabolic activity in STZ animals supports the concept that acetate as a metabolic substrate is more readily metabolized in the brain of diabetic animals than in controls and that alternate fuels could better support brain metabolism of diabetic and recurrently hypoglycemic animals.

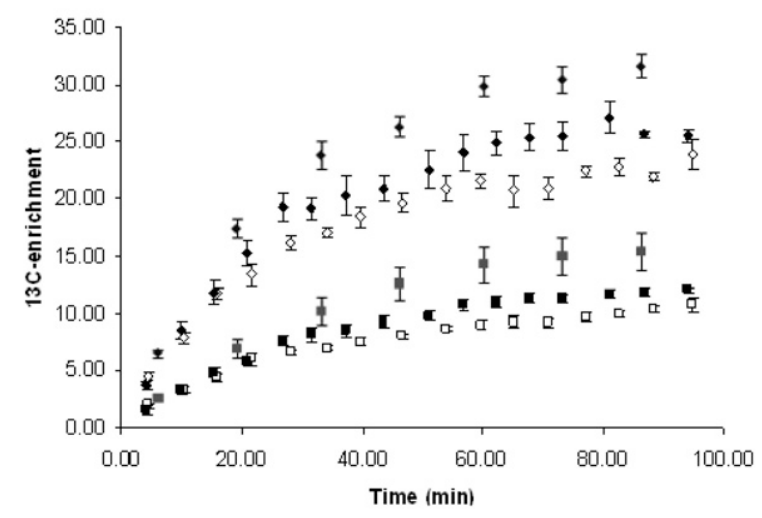

Figure 1: Representative brain glutamine (diamonds) and glutamate (squares) enrichment time courses from nourmal control (black symbols), recurrently hypoglycemic (white symbols) and STZ diabetic (blue and pink) animals. DERC 
It appears that the human brain does not ordinarily utilize physiological concentrations of circulating alternative fuels aside from glucose effectively; nonetheless, some data suggest that hypoglycemia per se increases brain acetate transport in patients with diabetes. Thus, this study was undertaken to determine if there is an increase in blood-to-brain transport of lactate as an alternative brain metabolic fuel in humans during acute hypoglycemia using a novel $\left[3-{ }^{11} \mathrm{C}\right]$ lactate PET method. We measured global blood-to-brain lactate transport using $\left[3-{ }^{11} \mathrm{C}\right]$ lactate PET in 5 healthy subjects. PET scans were performed during hyperinsulinemic euglycemic ( 92 $\mathrm{mg} / \mathrm{dL})$ and hypoglycemic $(\sim 55 \mathrm{mg} / \mathrm{dL})$ clamps. Dynamic PET acquisition started immediately after $\left[3-{ }^{11} \mathrm{C}\right]$ lactate injection. $\mathrm{T} 1$ weighted anatomical magnetic resonance (MR) data were acquired with $1 \mathrm{~mm}$ isotropic voxels, and time-of-flight (TOF) MR angiography (MRA) images were obtained with $0.3 \mathrm{~mm}$ in-plane resolution and $0.6 \mathrm{~mm}$ slice thickness. The PET data and the MR data were co-registered for quantitative analysis. Two approaches were taken to estimate the blood-to-brain lactate transport rate. In the first approach, area-under-curve ratio (AUCR) of tissue and blood time activity profiles was calculated. In the second approach, a one-compartmental model was used to fit the data and rate constant $\mathrm{K} 1$ was used as the measurement of blood-to-brain transfer rate. For both approaches, the first 200 seconds of data were used to minimize the impact of tracer metabolism and deviation from a one-compartmental model. Hypoglycemia per se did not significantly increase global lactate uptake (AUCR: $0.11 \pm 0.05$ (Euglycemia) vs. $0.09 \pm 0.04$ (Hypoglycemia), $\mathrm{p}=0.11 ; \mathrm{K} 1: 0.051 \pm 0.029 \mathrm{~min}^{-1}$ (Euglycemia) vs. $0.041 \pm 0.022$ $\mathrm{min}^{-1}$ (Hypoglycemia), $\mathrm{p}=0.13$ ). Better understanding of the central integration of the hypoglycemic responses may provide insight into the problem of recurrent hypoglycemia in diabetes.

Supported by: Children's Discov. Inst. 1KL2RR024994-01

128-OR

Hypothalamus Functional Connectivity is Altered in Type 1 Diabetes NICOLAS R. BOLO, GAIL MUSEN, LISA NICKERSON, RICHARD MCCARTNEY, PERRY F. RENSHAW, DONALD C. SIMONSON, ALAN M. JACOBSON, Boston, MA, Belmont, MA, Salt Lake City, UT, Mineola, NY

The hypothalamus is functionally connected to a network of brain regions. This functional connectivity (FC) may be altered by hypoglycemia. To examine this question, we employed a novel, model-free approach to obtain individual maps of resting state networks (RSN) while subjects were exposed to gradually declining blood glucose levels. We used blood oxygen level dependent (BOLD) functional MRI (fMRI) during a hyperinsulinemic clamp to compare FC in type 1 diabetes patients (T1DM, $\mathrm{n}=16$ ) and age-matched controls $(n=10)$ while blood glucose declined from euglycemia (EU, $5.0 \mathrm{mmol} / \mathrm{l}, 5$ $\mathrm{min}$ ) to hypoglycemia (HY, $2.8 \mathrm{mmol} / \mathrm{l})$ over $40 \mathrm{~min}$. Independent component analysis (ICA) was applied to the 45-min time courses (TC) of all subjects' fMRI data to obtain 30 maps of RSNs with temporally coherent BOLD signals. The first and last $10 \mathrm{~min}$ of individual TCs were extracted to define EU and HY TCs. We first computed subject/condition-specific TCs for each map by spatial regression. A second regression used these TCs to compute individual RSNs for each condition. We also computed between-network correlations. The initial ICA revealed 2 RSNs containing the hypothalamus and 7 other RSNs of interest, including several that have been associated with feeding behavior and obesity. During EU, a RSN connecting hypothalamus, brainstem, thalamus, caudate and insula showed increased FC with basal ganglia in T1DM vs. controls $(p<0.05)$. During HY, controls increased FC in this RSN, with no change in FC for T1DM. There were widespread alterations of between-network FC from EU to HY in T1DM, but not in controls. The hypothalamus-related RSNs have not been reported previously, and may activate only during hypoglycemia. RSNs previously associated with interoception, insulin sensitivity and hunger showed larger changes in betweennetwork connectivity in T1DM than controls during HY. These FC alterations in T1DM suggest that ICA may be a useful tool for understanding clinical conditions related to diabetes such as hypoglycemia unawareness.

Supported by: NIH (5R01-DK073843-03, DK-60754, DK-62218-01A1)

The AMP-Activated Protein Kinase (AMPK)-Uncoupling Protein 2 Pathway Controls Hypothalamic Glucosensing and Whole Body Glucose Counterregulation

CRAIG BEALL, D. LEE HAMILTON, LISA LOGIE, FIONA ASHFORD, MARC P. SOUTAR, JENNIFER GALLAGHER, XIAONING FAN, MICHAEL L. ASHFORD, SABRINA DIANO, RORY J. MCCRIMMON, Dundee, United Kingdom, New Haven, CT

Hypoglycemia is a limitation to intensive insulin therapy. Defining pathways of hypoglycemia detection may expose therapeutic targets aimed at reducing hypoglycemia risk. AMPK plays an important role in hypoglycemia detection within the hypothalamus, an important glucosensing region of the brain, although the mechanism by which AMPK controls glucosensing remains largely unknown. We have previously reported that lentiviral knockdown of $\alpha 2 A M P K$ in hypothalamic glucose-excited GT1-7 cells reduces their glucose sensitivity, which was mimicked by the UCP2 inhibitor, genipin. We now show that knockdown of $\alpha 1$ AMPK, despite representing $>90 \%$ of total AMPK activity, does not alter the threshold for GT1-7 cell glucose sensing, whereas $\alpha 2 A M P K$ knockdown reduced the glucose threshold to lower than $<0.25 \mathrm{mM}$ glucose before we could observe significant changes in membrane potential. $\alpha 2 A M P K$ knockdown also led to a significant reduction in UCP2 mRNA. To further examine the role of UCP2, hyperinsulinemic-hypoglycemic clamp studies were performed in whole body UCP2 null mice. Loss of UCP2 significantly increased the mean glucose-infusion rate, which was mediated by reduced glucagon and epinephrine responses to hypoglycaemia (Table 1). The putative link between AMPK and UCP2 appears to be important for the maintenance of glucosensing machinery at the cellular and whole body level.

\begin{tabular}{lrr}
\hline & Control & UCP2-/- \\
\hline Mean GIR $(\mathrm{mg} / \mathrm{kg} / \mathrm{min})$ & $8.3 \pm 2.4$ & $16.7 \pm 2.6^{*}$ \\
\hline Glucagon $(\mathrm{ng} / \mathrm{l})$ & $648.1 \pm 157$ & $193.5 \pm 31^{*}$ \\
\hline Epinephrine $(\mathrm{pg} / \mathrm{ml})$ & $2128 \pm 233$ & $636 \pm 74^{*}$ \\
\hline
\end{tabular}

Supported by: JDRF, Diabetes UK, Anonymous Trust

Serotonergic Signaling in the Ventromedial Hypothalamus Modulates the Counterregulatory Response to Hypoglycemia

OLEG OTLIVANCHIK, AMBROSE A. DUNN-MEYNELL, BARRY E. LEVIN, Newark, NJ, East Orange, NJ

Serotonin (5HT) selective reuptake inhibitors (SSRIs) enhance the counterregulatory response (CRR) to insulin-induced hypoglycemia (IIH) and reverse the blunted CRR to recurrent hypoglycemia in rats and humans. To assess the role of hypothalamic $5 \mathrm{HT}$ activity in modulating the CRR to IIH in adult rats ( $\mathrm{n}=12 / \mathrm{group})$, we first found that a single bout of IIH (30-40mg/dl over $2 \mathrm{~h}$ after $4.5 \mathrm{U} / \mathrm{kg}$ insulin, i.v.) increased $5 \mathrm{HT}$ turnover $(5 \mathrm{HIAA} / 5 \mathrm{HT}$; $I \mathrm{H}: 2.14 \pm 0.20$ vs. saline: $1.69 \pm 0.11 ; p=0.05)$ selectively in the ventromedial, $(\mathrm{VMH})$ but not in the dorsomedial, paraventricular or lateral hypothalamus. We then selectively depleted VMH $5 \mathrm{HT}$ by bilateral stereotaxic injection of $4 \mu \mathrm{g}$ (in $1 \mu \mathrm{L}$ per side) 5,7-dihydroxytryptamine (57DHT) in desmethylimipramine (25 $\mathrm{mg} / \mathrm{kg}$, i.p) pre-treated rats ( $\mathrm{n}=6-8 / \mathrm{group})$. Across all rats, there were negative correlations between: VMH $5 \mathrm{HT}$ and 60 min epinephrine (Epi; $r=-0.628$; $P=0.009)$, Epi $2 h$ AUC $(r=-0.744 ; P=0.002)$ and norepinephrine (NE) 2h AUC $(r=-0.541 ; P=0.031)$. Compared to saline controls, rats with complete 57DHT VMH 5 HT ablations, had increased baseline plasma glucose (104 \pm 2 vs. $118 \pm 4$ $\mathrm{mg} / \mathrm{dL} ; \mathrm{p}=0.012$ ), but similar glucose and corticosterone levels over $2 \mathrm{~h}$ of $\mathrm{IIH}$; increased 90 min plasma Epi $(1313 \pm 195$ vs. $2594 \pm 510 \mathrm{pg} / \mu \mathrm{L} ; \mathrm{p}=0.041)$ and $2 \mathrm{~h}$ AUC (181 \pm 17 vs. $276 \pm 21 \mathrm{ng} / 2 \mathrm{~h} ; \mathrm{p}=0.006) ; 60 \mathrm{~min}(255 \pm 40$ vs. $436 \pm 59 \mathrm{pg} / \mu \mathrm{L}$; $\mathrm{p}=0.030$ ) and $90 \mathrm{~min}(343 \pm 33 \mathrm{vs} .449 \pm 31 \mathrm{pg} / \mu \mathrm{L} ; \mathrm{p}=0.043)$ plasma NE and $2 \mathrm{~h}$ AUC (18.7 \pm 0.8 vs. $28.1 \pm 3.12 \mathrm{ng} / 2 \mathrm{~h} ; \mathrm{p}=0.017)$; baseline $(66 \pm 4$ vs. $84 \pm 4 \mathrm{pg} / \mathrm{mL}$; $\mathrm{p}=0.013)$ and $60 \mathrm{~min}$ glucagon levels $(112 \pm 13 \mathrm{vs} .228 \pm 38 \mathrm{pg} / \mathrm{mL} ; \mathrm{p}=0.017)$. Therefore, a single bout of IIH selectively increases VMH $5 \mathrm{HT}$ turnover while selective depletion of VMH $5 \mathrm{HT}$ increases the Epi, NE, glucagon but not corticosterone responses to $\mathrm{IH}$. This suggests that SSRI enhancement of the CRR is either not mediated by VMH $5 \mathrm{HT}$ or that systemic SSRI therapy may decrease VMH 5HT turnover and/or VMH $5 \mathrm{HT}$ receptor sensitivity.

Supported by: NIDDK DK53181; VA Research Svc 


\section{CAN WE REIN IN THE COSTS OF DIABETES WITH BETTER DIABETES CARE?}

131-0R

Medical Expenditures Associated With Diabetes: The Change in Last Two Decades

XIAOHUI ZHUO, PING ZHANG, KAI BULLARD, RUI LI, EDWARD GREGG, Atlanta, GA

Advances in anti-diabetic medications and medical technologies have substantially changed the way diabetic patients are treated, but the impact of the changes on medical expenditures has not been well documented. Using 1987 National Medical Expenditure Survey ( $N=22538)$, and the Medical Expenditure Panel Survey in years 1997-98 (N=57652) and 2007-08(N=44815), we produced nationally representative estimates of the medical utilization and expenditures attributed to diabetes in persons age $\leq 90$ years at 3 different times. A two-part model was used to estimate medical expenditures and a zero-inflated Poisson model was used to estimate the numbers of outpatient visits, hospital admissions, and prescription drugs taken for persons with and without self-reported diabetes. Excess expenditures and utilizations due to diabetes were calculated as the incremental effects of having diabetes in the regressions. We also estimated excess medical expenditures for those with and without macrovascular complications. The total expenditure declined from 1987 to 1998 and then remained near the 1998 level (table). From 1987 to 2008, the share of inpatient costs decreased due to reduced utilization. However, the share of prescription drugs substantially increased due to increased use of drugs per patient. Annual expenditure decreased by $29 \%$ in patients without macrovascular compliations but increased $13 \%$ in those with macrovascular complications.

Table, $^{*}: p<0.05$, reference is year 1987 . No difference was found between 2007-08 and 1997-98.

\begin{tabular}{lccc}
\hline Medical Expenditures (Year 2008 \$) & Year 1987 & Year 1997-98 & Year 2007-08 \\
\hline Total & 5692 & $4560^{*}$ & $4621^{*}$ \\
\hline Inpatient & $3967(70 \%)$ & $1913^{*}(42 \%)$ & $1500^{*}(32 \%)$ \\
\hline Outpatient & $1023(18 \%)$ & $901^{*}(20 \%)$ & $742^{*}(16 \%)$ \\
\hline Prescription Drug & $474(8 \%)$ & $1391^{*}(31 \%)$ & $2121^{*}(46 \%)$ \\
\hline Other & $227(4 \%)$ & $355^{*}(8 \%)$ & $258(6 \%)$ \\
\hline $\begin{array}{l}\text { Persons with no macrovascular } \\
\text { complications }\end{array}$ & 3856 & $2662^{*}$ & $2735^{*}$ \\
\hline $\begin{array}{l}\text { Persons with macrovascular } \\
\text { complications }\end{array}$ & 7939 & $8334^{*}$ & $8986^{*}$ \\
\hline
\end{tabular}

In conclusion, from 1987 to 2008, the medical expenditure attributed to diabetes among persons without macrovascular complications declined in the first decade and changed little in the second decade.

132-OR

Self-Monitoring Blood Glucose Test Strip Utilization in Canada JASON YEAW, TORSTEN E. CHRISTENSEN, DANIELLE GROLEAU, MICHAEL L. WOLDEN, WON C. LEE, Redwood City, CA, Søborg, Denmark, Mississauga, ON, Canada, Alexandria, VA

Self-monitoring blood glucose (SMBG) tests are a recommended part of diabetes management for patients on insulin therapy, in order to assess glycemic control and optimize treatment. However, little real-world information is currently available on the utilization and associated cost impact of SMBG use. The aim of this study was to investigate the annual utilization and per-patient cost of SMBG tests as a proportion of diabetes-related pharmacy costs in Canada. A retrospective cohort study was conducted using the IMS Brogan Inc. drug plan database for the period of July 12006 to June 302010 . The database contains patient-level medical claims data from private and public sources. A total of 142,551 patients with type 1 or type 2 diabetes who had at least two prescriptions of insulin (basal only, basal-bolus, bolus only or premix) were identified. Overall mean utilization for blood-glucose strips across all patients, regardless of the type of insulin, was approximately 1094 strips per patient per year, with an average cost per strip of approximately CAD $\$ 0.79$. SMBG testing constituted $41.6 \%$ of the total annual diabetes-related pharmacy costs (Table). The mean numbers of test strips (by regimen) per patient per year were: 935 , basal only; 1324 , basal-bolus; 890 , premix; and 1413, bolus only. The proportion of diabetesrelated pharmacy costs were: $33.7 \%$, basal only; $41.5 \%$, basal-bolus; $41.4 \%$, premix; and $33.7 \%$, bolus only. SMBG test strips account for more than $40 \%$ of the total diabetes-related pharmacy cost among patients with type 1 and insulin-treated type 2 diabetes in Canada.

\begin{tabular}{lcccc}
\hline & $\begin{array}{c}\text { Number of } \\
\text { subjects }\end{array}$ & $\begin{array}{c}\text { Number of test } \\
\text { strips per patient } \\
\text { per year }\end{array}$ & $\begin{array}{c}\text { Mean annual test } \\
\text { strip cost } \\
\text { per patient }\end{array}$ & $\begin{array}{c}\text { Test strips as proportion } \\
\text { total diabetes-related } \\
\text { pharmacy cost }\end{array}$ \\
\hline Overall & 142,551 & 1094 & CAD $\$ 860$ & $41.6 \%$ \\
\hline Basal & 45,003 & 935 & CAD $\$ 740$ & $33.7 \%$ \\
\hline Basal-bolus & 38,553 & 1324 & CAD $\$ 1056$ & $41.5 \%$ \\
\hline Bolus only & 43,470 & 1413 & CAD $\$ 1112$ & $52.8 \%$ \\
\hline Premix & 15,525 & 890 & CAD $\$ 678$ & $41.4 \%$ \\
\hline
\end{tabular}

133-OR

Chronic Kidney Disease Progression and Associated Medical Costs in Type 2 Diabetes

SUMA VUPPUTURI, TERESA M. KIMES, MICHAEL O. CALLOWAY, JENNIFER B. CHRISTIAN, DAVID BRUHN, ALAN A. MARTIN, GREGORY A. NICHOLS, Atlanta, $G A$, Portland, OR, Research Triangle Park, NC

Chronic kidney disease (CKD) is a common and costly complication of diabetes. We estimated the rate of progression to CKD and calculated associated medical costs. We identified 25,583 members of the Kaiser Permanente Northwest and Georgia regions who had type 2 diabetes and a serum creatinine measurement in 2005. We used estimated glomerular filtration rate (GFR) to assign patients to baseline stages of kidney function (stage $0 / 1, \geq 90 \mathrm{ml} / \mathrm{min} / 1.73 \mathrm{~m}^{2}, \mathrm{n}=8,647$; stage 2, 60-89, $n=12,371$; stage 3, 30-59, $n=3,882$; stage $4,15-29, n=683$ ). CKD was considered present at stage 3 or higher (GFR $<60$ on two occasions). We examined all subsequent GFRs through 2010 to assess progression of kidney disease. Inpatient, outpatient, pharmacy and total medical costs before and after progression to CKD were calculated and compared. Mean age of patients was 60 years, $51 \%$ men, and mean diabetes duration was 5.1 years. Patients in higher stages were older with longer diabetes duration and more likely to be women. At baseline $17.9 \%$ of patients with T2D also had CKD. Of the 21,018 patients with stages 1 or 2 at baseline, $11.6 \%(n=2,439)$ subsequently progressed to CKD (stage 3 or greater) over mean follow-up of 4.5 years (incidence rate $26.0 / 1,000$ person-years, $95 \% \mathrm{Cl} 24.3-27.8$ ). Among patients who progressed to CKD (stage 3 or greater), annual total medical costs approximately doubled after progression compared to pre-progression costs, even when dialysis costs were not included. Progression to CKD drives substantial medical care costs. Interventions designed to minimize progressive kidney damage would reduce the burden of CKD in type 2 diabetes.

\begin{tabular}{|c|c|c|c|c|c|c|}
\hline \multicolumn{7}{|c|}{ Stages of Kidney Disease at Baseline and Over Mean Follow-up of 4.5 Years } \\
\hline & $\begin{array}{l}\text { Follow-up } \\
\text { Stage 0/1 }\end{array}$ & $\begin{array}{l}\text { Follow-up } \\
\text { Stage } 2\end{array}$ & $\begin{array}{l}\text { Follow-up } \\
\text { Stage } 3\end{array}$ & $\begin{array}{l}\text { Follow-up } \\
\text { Stage } 4\end{array}$ & $\begin{array}{l}\text { Follow-up } \\
\text { Stage } 5\end{array}$ & Total \\
\hline Baseline Stage 0/1 & 3,534 & 4,860 & 197 & 39 & 17 & $\begin{array}{c}8,647 \\
(33.8 \%) \\
\end{array}$ \\
\hline Baseline Stage 2 & 563 & 9,622 & 1,839 & 288 & 59 & $\begin{array}{c}12,371 \\
(48.4 \%) \\
\end{array}$ \\
\hline Baseline Stage 3 & 0 & 554 & 2,181 & 930 & 217 & $\begin{array}{c}3,882 \\
(15.2 \%)\end{array}$ \\
\hline Baseline Stage 4 & 0 & 65 & 122 & 310 & 186 & $\begin{array}{c}683 \\
(2.7 \%)\end{array}$ \\
\hline Total & $\begin{array}{c}4,097 \\
(16.0 \%)\end{array}$ & $\begin{array}{c}15,101 \\
(59.0 \%)\end{array}$ & $\begin{array}{c}4,339 \\
(17.0 \%)\end{array}$ & $\begin{array}{l}1,567 \\
16.1 \%\end{array}$ & $\begin{array}{c}479 \\
(1.9 \%)\end{array}$ & 25,583 \\
\hline
\end{tabular}

134-OR

Intensive Glucose, Blood Pressure, or Lipid Control had No Significant Negative Impact on Health Utility or Quality-Adjusted Life Years in the ACCORD Trial

PING ZHANG, LANEY LIGHT, PATRICK OCONNOR, DENNIS RAISCH, ALI MOHAMMED, K.M. VENKAT NARAYAN, WENKE HWANG, ROGER ANDERSON, JOANN SPERL-HILLEN, ROBERT COHEN, LOIS KATZ, MARK SULLIVAN, Atlanta, GA, Winston-Salem, NC, Bloomington, MN, Albuquerque, NM, Hershey, PA, Minneapolis, $M N$, Cincinnati, $\mathrm{OH}$, New York, NY, Seattle, WA

ACCORD study is a multicenter randomized trial to compare cardiovascular disease (CVD) outcomes in 10,251 high-CVD risk participants with type 2 diabetes receiving intensive or standard glucose, blood pressure (BP), and lipid control strategies. Here we report the impact of the ACCORD interventions on health-related quality of life (HROOL), measured by Health Utilities Index Mark 3(HUI-3) and the Feeling Thermometer (FT) and quality adjusted life years (QALY)s, calculated as the product of survival time and HUI or FT score. HUI-3 and FT were measured the baseline and repeated at 12,36, 48 month visit, and the end of the study. We analyzed the change in HUI-3 and 
FT measures as a function of follow-up time, and of which glucose, BP, and lipid treatment strategies the patient was randomly assigned to. Tests of significance were done using general linear models for repeated measures. Differences in QALYs by treatment strategy were assessed using analysis of variance model. During a mean follow up of 5 years (range 3.4 to 8.5 years), no difference could be detected between groups in HUI and FT scores at the baseline and the end of the study ( $\mathrm{P}$ values for testing all group difference were greater than 0.10 ). No significant difference was detected in total QALYs calculated using either HUI-3 scores or FT scores (P values for testing all group difference were greater than 0.20 ). These results indicate that intensive glucose, BP, and lipid control as implemented in the ACCORD trial in subjects with established type 2 diabetes did not significantly alter patientreported measures of health utility or QALYs. While, no adverse effect of intensive glucose, BP, or lipid control were observed on measures of HROOL or QALYs in the ACCORD Trial, the resources needed for implementing the interventions in relation to benefits as measured by other clinical outcomes should be taken into consideration for policy formulation.

Supported by: NIH, CDC

\section{5-OR}

Health-Related Quality of Life Associated With Daytime and Nocturnal Hypoglycemic Events: a Time Trade-Off Survey

MARC EVANS, KAMLESH KHUNTI, MUHAMMAD MAMDANI, STEWART HARRIS, CLAUS GALBO-JØRGENSEN, JENS GUNDGAARD, METTE BØGELUND, Cardiff, United Kingdom, Leicester, United Kingdom, Toronto, ON, Canada, London, ON, Canada, Holte, Denmark, Søborg, Denmark

Hypoglycemic events (hypos) can affect health-related quality of life (HROOL) via acute symptoms, altered behavior and fear of future hypos. Nocturnal hypos, in particular, are unpredictable and difficult to avoid, and may affect HROOL more than daytime hypos. We examined if HROLL differs between daytime and nocturnal, severe and non-severe hypos, using time trade-off (TTO) methods. HROoL was quantified via an online TTO survey with 10,087 respondents from the general population in the UK, USA, Canada, Germany and Sweden. Descriptions of hypo health states were derived from a survey of 247 UK patients with diabetes. HROoL was measured on a utility scale: 0 (dead), 1 (perfect health). Respondents traded-off length of life for improving health states and evaluated health states of wellcontrolled diabetes and diabetes with non-severe/severe and daytime/nocturnal hypos. Table 1 shows the results. Non-severe nocturnal hypos were associated with 0.0026 (95\% Cl: 0.0016; 0.0034) higher disutility compared with non-severe daytime hypos (equivalent to a $63.4 \%$ increase in negative impact); severe nocturnal hypos were associated with $0.0057(95 \% \mathrm{Cl}$ : $-0.0001 ; 0.0114$ ) higher disutility compared with severe daytime hypos (not statistically significant). The study estimates the disutility associated with diabetes, uniquely illustrating the detrimental effect of hypos on HROOL and in particular demonstrating the adverse effect of nocturnal hypos on HROoL compared with daytime hypos. These results highlight the clinical imperative of avoiding hypos, particularly nocturnal hypos.

Table 1 Utility estimates from the time trade-off survey

\begin{tabular}{lccccc}
\hline Type of hypoglycemia & $\mathbf{n}$ & $\begin{array}{c}\text { Average } \\
\text { utility }\end{array}$ & $\begin{array}{c}\text { Standard } \\
\text { error }\end{array}$ & $\begin{array}{c}95 \% \text { Cl } \\
\text { Lower }\end{array}$ & $\begin{array}{c}95 \% \text { CI } \\
\text { Upper }\end{array}$ \\
\hline Diabetes with no hypoglycemia & 7642 & 0.8440 & 0.0022 & 0.8395 & 0.8484 \\
\hline Non-severe daytime event & 7435 & -0.0041 & 0.0003 & -0.0035 & -0.0047 \\
\hline Non-severe nocturnal event & 7347 & -0.0067 & 0.0004 & -0.0059 & -0.0074 \\
\hline Severe daytime event & 3603 & -0.0565 & 0.0021 & -0.0527 & -0.0606 \\
\hline Severe nocturnal event & 3647 & -0.0622 & 0.0021 & -0.0582 & -0.0663
\end{tabular}

136-OR

Validation and Evaluation of the Risk-To-Benefit Ratio of Glucose Lowering Therapies in High Cardiovascular Risk Type 2 Diabetes Patients; Projections using the IMS CORE Diabetes Model VOLKER FOOS, NICK FREEMANTLE, JAMES PALMER, DAVID GRANT, ADAM LLOYD, MARC EVANS, PHIL MCEWAN, Basel, Switzerland, London, United Kingdom, Cardiff, United Kingdom, Swansea, United Kingdom

Meta-analyses of recent outcomes trials in type 2 diabetes (T2DM) have demonstrated an overall reduction in coronary events without increased risk of mortality. The aim of this study is to demonstrate the predictive validity of a diabetes simulation model to 5 -year meta-analyzed trial data and assess the long term expected outcomes of intensive versus conventional glycemic control in high cardiovascular risk T2DM patients. The IMS CORE Diabetes Model (CDM) is a validated and widely used simulation model designed to predict the healthcare costs and benefits associated with diabetes management. The CDM was initialized to baseline characteristics consistent with ACCORD and predicted 5-year relative risks of cardiovascular morbidity and all cause mortality was compared to random effects meta-analysis of ACCORD, ADVANCE and VADT. Results were extrapolated over a lifetime to obtain life and quality-adjusted life expectancy (OALE). Healthcare benefits were discounted at $3.5 \%$. Five-year relative risk for non-fatal myocardial infarction (MI), non-fatal stroke, heart failure and all cause mortality were $0.76,0.83,0.93$ and 0.94 for intensive versus conventional therapy respectively; all within $95 \%$ confidence intervals of the meta analysis results. Lifetime relative risks were 0.90 (non-fatal MI), 0.88 (non-fatal stroke), 0.89 (heart failure) and 0.91 (diabetes specific mortality), favoring intensive glycemic control. Life expectancy was 11.49 and 11.13 years, QALE was 7.4 and 7.2 years in the intensive versus conventional arms respectively. The CDM relative risk reductions associated with intensive glycemic control in high-risk type 2 diabetes patients were consistent with recent meta-analysis results. Long-term projections suggest that intensive therapy contributes to both increased life and quality-adjusted life expectancy in high cardiovascular risk patients.

137-0R

Implications of Key Biomarkers Trends on Health Outcomes in Patients With Type 2 Diabetes Mellitus (T2DM) in the U.S.

MICHAEL WILLIS, CHERYL NESLUSAN, JIANMING HE, Lund, Sweden, Raritan, NJ

The objectives of this study were to examine trends in key biomarkers among patients with T2DM in the US since 2000 and to estimate their impact on the cumulative incidences (Cls) of micro- and macrovascular events and survival over a 10 -year period. Data on $\mathrm{HbA1C}$ and Total Cholesterol to HDL ratio (TC/HDL) were obtained from IMS LifeLink ${ }^{\mathrm{T}}$, a database of privately insured patients with linked laboratory results. Data on BMI and systolic blood pressure (SBP) were taken from a recent publication that used the National Health and Nutrition Examination Survey (NHANES) over 2000 to 2008. To estimate the impact of these trends on future health outcomes, we utilized a validated micro-simulation model, the Economic and Health Outcomes in T2DM (ECH0-T2DM) model. In the Lifelink ${ }^{\text {TM }}$ database, most of the improvement in $\mathrm{HbA1c}$ had occurred by 2005 (mean values were $8.1 \pm 1.6 \%$ in $2000,7.1 \pm 1.5 \%$ in 2005 , and $7.2 \pm 1.3 \%$ in 2010 ), while the mean TC/HDL ratio decreased ( $4.4 \pm 1.3$ to $3.8 \pm 1.2$ ) from 2000 to 2010 . Mean BMI increased from $31.9 \pm 1.1 \mathrm{~kg} / \mathrm{m}^{2}$ in 2000 to $33.4 \pm 0.51 \mathrm{~kg} / \mathrm{m}^{2}$ in 2005 , and decreased to $32.0 \pm 0.51 \mathrm{~kg} / \mathrm{m}^{2}$ in 2008 . Mean values of SBP did not change over this period. Simulations indicated that had patients in 2000 been treated to the biomarker values observed in $2005,4.4 \%$ more patients would have survived, and the rates of micro- and macrovascular disease would have been substantially lower over a 10 year period (e.g., the Cls of background diabetic retinopathy, microalbuminuria and myocardial infarction would have been respectively, $27 \%, 23 \%$ and $16 \%$ lower). The lower Cls of macrovascular events were primarily driven by trends in $\mathrm{HbA1c}$ and the TC/HDL ratio, whereas the lower Cls of microvascular events were largely driven by the trends in $\mathrm{HbA1c}$ alone. The improvements in biomarkers after 2005 were smaller, resulting in smaller predicted improvements in patient outcomes. These results suggest that although these trends have likely resulted in better health outcomes, further progress is needed.

Supported by: Janssen Global Services, LLC

$\Delta$

The Cost-Effectiveness of MODY Genetic Testing

138-OR

ROCHELLE N. NAYLOR, PRIYA M. JOHN, AARON N. WINN, LOUIS H. PHILIPSON, SIRI ATMA W. GREELEY, GRAEME I. BELL, ELBERT S. HUANG, Chicago, IL, Boston, MA

Maturity-onset diabetes of the young (MODY) is caused by mutations in genes important to beta cell function. Mutations in HNF1A, HNFAA and GCK account for the majority of MODY cases and frequently allow for treatment with sulfonylureas (SU) (HNF1A, HNF4A) or no therapy (GCK). However, MODY diagnosis requires genetic testing, which is not routinely obtained. Assessing the economic value of routine genetic screening may inform provider behavior and coverage decisions. The aim of this study was to evaluate the societal cost-effectiveness of a genetic testing policy for HNF1A, HNF4A and GCK-MODY in individuals diagnosed with type 2 diabetes (T2D) between the ages of 20-50 years. We used a simulation model of T2D complications based on the UKPDS, and also accounted for the natural history of disease by genetic subtype, to compare a screening policy of genetic testing at diagnosis versus a policy of no testing. Under the screening policy we assumed $75 \%$ of patients with HNF1A or $4 A-M O D Y$ would transition to SU therapy with a resultant glycemic benefit of $0.8 \%$ and that all patients with GCK- 
MODY would discontinue therapy. We estimated a 2\% prevalence of MODY in the screened population with genetic testing costs of $\$ 2000$ per individual screened. Outcomes included costs and quality-adjusted life years (QALYS), based on lifetime risk of complications and treatments, expressed as the incremental cost-effectiveness ratio (ICER, \$/QALY). The testing policy yielded an average lifetime gain of 0.02 QALYs. The base case resulted in an ICER of $\$ 91,389 /$ QALY. In sensitivity analysis the testing policy became cost-savings if screening was done in a more limited population where the expected prevalence of MODY was $\geq 8 \%$. Reducing genetic testing costs in the base case to $\$ 500$ would also make the screening policy cost-savings. In the United States context, routine screening for MODY subtypes in incident cases of T2D is a cost-effective use of personalized genetic medicine, and may be seen as a compelling argument for routine coverage of genetic testing in diabetes.

\section{APPLICATIONS OF HIGH-THROUGHPUT SEQUENCING}

139-0R

Search for Novel Type 2 Diabetes Susceptibility Loci Using GenomeWide Association Studies Imputed From a 1000 Genomes Reference Panel

INGA PROKOPENKO, CLEMENT MA, REEDIK MÄGI, HAN CHEN, BENJAMIN F. VOIGHT, LU OI, NATALIE VAN ZUYDAM, HARALD GRALLERT, LOIC YENGO, CHRISTIAN DINA, GUDMAR THORLEIFSSON, CHRISTIAN FUCSHBERGER, LIMING LIANG, MARTINA MUELLER-NURASYID, SARA M. WILLEMS, LINDA KAO, PAU NAVARRO, VALGERDUR STEINTHORSDOTTIR, CORNELIA M. VAN DUIJN, MICHAEL BOEHNKE, JOSÉE DUPUIS, MARK I. MCCARTHY, LAURA J. SCOTT, ON BEHALF OF DIAGRAM+ CONSORTIUM, Oxford, United Kingdom, Ann Arbor, MI, Tartu, Estonia, Boston, MA, Philadelphia, PA, Dundee, United Kingdom, Neuherberg, Germany, Lille, France, Nantes, France, Reykjavik, Iceland, Rotterdam, The Netherlands, Baltimore, MD, Edinburgh, United Kingdom

To date more than 50 common variant associations have been described for type 2 diabetes (T2D); however these account for $<10 \%$ of inherited susceptibility. Most T2D-associated variants have minor allele frequency (MAF) $>10 \%$. Our goal was to identify causal variants at known T2D loci and to uncover novel sites by performing imputation from a large reference panel generated by genome resequencing. We imputed SNP variants using the 1000 Genomes European reference panel (566 CEU haplotypes, December/2010 release) within each of 12 European descent cohorts with genome-wide association (GWA) data. Following T2D association analysis, we performed a fixed-effects inverse variance meta-analysis for 14,890 individuals with $\mathrm{T} 2 \mathrm{D}$ and 62,932 controls. 7.9M SNPs were present in at least 4 studies; $2.4 \mathrm{M}$ of these SNPs had MAF $<0.05$. We observed genome-wide significant $\left(p\right.$-value $\left.<5 \times 10^{-8}\right)$ associations at 17 and suggestive ( $p$-value $\left.<1 \times 10^{-5}\right)$ associations at 16 more known T2D loci. Within 3 of these loci (TCF7L2, CDKN2A/2B and CDKAL1), we identified common variants (rs35198068, rs7451008, rs10965246, respectively) which on imputation demonstrated more significant association than previously detected variants. In TCF7L2, we also observed association (OR=1.6[1.4-1.7], $p$-value $\left.=6 \times 10^{-16}\right)$ at a low frequency (rs116369954; MAF=0.03) variant, $35 \mathrm{~kb}$ from the known signal $\left(p\right.$-value $\left.=5 \times 10^{-76}\right)$ : conditional tests of independence are in process. We observed novel association at a common variant near VPS13C (rs12437560, $\mathrm{OR}=1.11[1.07-1.16]$, $p$-value $\left.=2 \times 10^{-8}\right)$ not well-tagged in previous imputations, and suggestive associations at/near ATXN7L1 (p-value $\left.=2 \times 10^{-7}\right)$ and $\angle R C H 1(\mathrm{p}-$ value $=3 \times 10^{-7}$ ). Imputation with the current 1000 Genomes reference panel has not revealed novel association signals driven by low MAF variants. However, imputation for many low-MAF variants remains suboptimal given the size of the current reference panel and the density of much existing GWAs data.

140-OR

Detecting Functional Rare Variants Relating to Type 2 Diabetes Using Deep Whole Genome Sequencing

GOO JUN, MARCIO ALMEIDA, PABLO CINGOLANI, ANDREW R. WOOD, CHRISTIAN FUCHSBERGER, TANYA M. TESLOVICH, THOMAS D. DYER, JOANNE CURRAN, JASON GRUNSTAD, THOMAS W. BLACKWELL, DONNA M. LEHMAN, ROBERT GROSSMAN, STEPHEN E. LINCOLN, JASON LARAMIE, MICHAEL BOEHNKE, MARK MCCARTHY, TIMOTHY M. FRAYLING, ROBERT SLADEK, RAVINDRANATH DUGGIRALA, JOHN BLANGERO, GONCALO ABECASIS, T2D-GENES CONSORTIUM, Ann Arbor, MI, San Antonio, TX, Montreal, OC, Canada, Exeter, United Kingdom, Chicago, IL, Mountain View, CA, Oxford, United Kingdom

The contribution of low frequency, rare, and private genomic variants to type 2 diabetes risk is unknown. Recent advances in sequencing technology now make it possible to carry out whole genome sequencing on a scale large enough to enable complex disease gene discovery. As part of the T2D-GENES Consortium, we aim to identify causal variants influencing type 2 diabetes risks and related traits. We examined richly phenotyped individuals from 20 Mexican American pedigrees from San Antonio with a high prevalence $(30 \%)$ of diabetes. Our current data includes 483 sequenced by Complete $\mathrm{Ge}-$ nomics (CGI) at average 60x coverage. Sequenced samples were optimally selected to provide for highly accurate family-based imputation. We used a novel multi-sample SNP filtering pipeline to collect quality measures across all samples, and applied support vector machine classifiers to filter out low quality SNPs. Modified MACH software was used to fully impute complete WGS data to all 959 individuals. We identified a total of 26.8M SNPs in 483 individuals. Quality control eliminated in 17 outlier individuals and a cleaned set of 24M SNPs. $>69 \%$ of the quality controlled SNPs are not in dbSNP 129 and the overall transition to transversion ratio (Ti/Tv) is 2.18 , while SNPs in dbSNP has Ti/Tv=2.19. $>51 \%$ have minor allele frequency $<1 \%$. We discovered 37 non-synonymous SNPs affecting known MODY genes, including an HNF1B/G370S transition (MODY5) transmitted in one parent child pair. Of particular relevance for diabetes, we provide, for the first time in this important ethnic group, exhaustive frequency spectra for all major positional candidate genes previously supported for influencing type 2 diabetes risk. As expected, these genes show large amounts of rare variation, much of which is privately limited to specific lineages. The identification of likely functional regulatory variants in these well-established diabetes-related genes will aid our quest for understanding the causal state space of inherited risk.

141-OR

Whole Genome Sequencing to Discover Type 2 Diabetes Risk Genes in Mexican American Pedigrees: T2D-GENES Consortium Project 2 MARCIO ALMEIDA, GOO JUN, TANYA M. TESLOVICH, ANDREW R. WOOD, TIMOTHY M. FRAYLING, CHRISTIAN FUCHSBERGER, PABLO CINGOLANI, THOMAS W. BLACKWELL, THOMAS D. DYER, JOANNE CURRAN, DONNA M. LEHMAN, ROBERT SLADEK, JASON GRUNSTAD, ROBERT GROSSMAN, JASON M. LARAMIE, STEPHEN E. LINCOLN, MARK MCCARTHY, MICHAEL BOEHNKE, RAVINDRANATH DUGGIRALA, GONCALO ABECASIS, JOHN BLANGERO, T2D-GENES CONSORTIUM, San Antonio, TX, Ann Arbor, MI, Exeter, United Kingdom, Montreal, OC, Canada, Chicago, IL, Mountain View, CA, Oxford, United Kingdom

The T2D-GENES Consortium is a collaborative international effort to identify genes involved in type 2 diabetes risk in multiple ethnic groups using next generation sequencing. In the T2D-GENES Project 2, deep coverage $(60 x)$ whole genome sequencing has been performed by Complete Genomics on 483 individuals from 20 large Mexican American pedigrees from San Antonio. Family-based imputation based on a high density GWAS SNP framework provided comprehensive WGS data on 959 individuals with known diabetes status in these pedigrees. We observed $\sim 2 \mathrm{M}$ variants (many previously undiscovered) that have passed quality control and frequency filtering. Initial analyses focused on a variety of rational prior hypotheses based on QTL information from existing linkage and GWA studies, potential functional consequences of coding or regulatory variants, and evolutionary conservation or selective constraint information. Association analysis was performed using a variance component-based approach as implemented in SOLAR allowing for residual correlation amongst relatives under a classical threshold normal probit model. Several existing linkage-derived QTL regions observed in Mexican American families yielded associations with novel variants. These included rare variants in the NCAPD3 gene $\left(p=1 \times 10^{-8}\right)$ on chromosome 11 and in the DMRT3 gene $\left(p=1 \times 10^{-7}\right)$ on chromosome 9 . Similarly, examination of comprehensive sequence variation in/near the TCF7L2 gene (a well-replicated type 2 diabetes risk region) identified an associated novel intragenic rare variant $\left(p=9 \times 10^{-5}\right)$. Our preliminary results already suggest that the chance accumulation of rare, even private, variants in large pedigrees permits variant-specific statistical inference on a whole genome sequence scale. These rare variants of moderate to large effect may lead to more rapid causal gene identification.

142-0R

Whole-Exome Sequencing of Overweight Individuals With and Without Type 2 Diabetes

MARGARIDA C. LOPES, OVERWEIGHT DIABETES EXOME STUDY, Hinxton, United Kingdom

To identify low frequency and rare exonic variants associated with the ill effects of excess weight, we performed whole-exome sequencing of 416 overweight individuals, 221 of whom had type 2 diabetes (T2D) and 195 were normoglycaemic. Subjects were drawn from the CoLaus population-based Swiss cohort of $\sim 6,000$ individuals with genome-wide single nucleotide vari- 
ant (SNV) data available. Exomes were captured using either the NimbleGen or Agilent CCDS solution capture products, in which over $90 \%$ of the samples achieved more than $10 x$ coverage over at least $80 \%$ of the bait regions and sequenced at an average depth of 65x. In total, 317,664 autosomal SNVs passed quality control filtering (call rate $\geq 90 \%$ and Hardy-Weinberg equilibrium $\left.p \geq 1 \times 10^{-6}\right)$. Seven samples were excluded on the basis of deviation from observed autosomal heterozygosity, genome-wide call rate $<50 \%$ and genotype concordance failure; 218 cases and 191 controls remained in the study. We searched for known disease-causing variants in the 409 exome dataset by comparing calls with the Human Gene Mutation Database. After excluding overlapping variants that were found in the homozygous state in the 1000 Genomes pilot dataset or had a Condel score $<0.99$, we identified 286 candidate disease variants, over two-thirds of which were singletons. Among these were Ser94Asn in MCR4 and Arg143Term in UCP3 which have been linked to relevant phenotypes in literature reports. Moreover, we tested for association with T2D through single point tests and methods testing for an aggregation of rare variants. We have prioritized 23 candidate genes for follow-up, including VEGFA, TNKS and VCAM1, which are interesting as they achieved aggregate test $p<0.01$ across all methods and/or single-point $p<10^{-4}$, were in the top three of each individual aggregate analysis and had strong biological credentials. This is one of the largest exome sequencing studies for a common disease that we are aware of, raising both challenges and exciting opportunities for further investigation.

Supported by: Wellcome Trust Grant 098051

$\Delta$ 143-OR Exome Sequencing to Identify Variants that Contribute to Type 2 Diabetes in Pima Indians

LESLIE J. BAIER, KE HUANG, YUNHUA MULLER, SAYUKO KOBES, MELISSA DEL ROSARIO, MARYAM ABDUSSAMAD, ALEJANDRA MARINELARENA, SHEENA CARBAUGH, VENKATA YELLAPANTULA, CLIFTON BOGARDUS, Phoenix, AZ

To identify rare or novel variants that affect risk for type 2 diabetes in Native Americans, we obtained exome sequence data on 177 Pima Indians. These unrelated individuals had been metabolically characterized for predictors of type 2 diabetes when they were nondiabetic, which included measures of insulin secretion, insulin action, and percent body fat (pfat). A subset $(\mathrm{N} \approx 50)$ had undergone abdominal fat and/or skeletal muscle biopsies for genome-wide gene expression profiling. Exome sequencing was performed by ShanghaiBio Corp. SNP validation showed that variants with a quality score of $\geq 100$, that met HWE expectations, were unlikely to be artifacts. Among SNPs that met these criteria $(N=408,487)$ only 88,005 mapped within exons; of these, 71,367 were coding (35,195 nonsynonymous), while 3,913 and 12,725 were in $5^{\prime}$ and $3^{\prime}$ UTRs, respectively. There were also 1,359 coding indels (698 predicted a frameshift). Variants occurring in $\geq 5$ subjects were preliminarily analyzed for association with a metabolic trait in the 177 individuals, and those SNPS with the strongest associations are currently being genotyped in a sample of 3500 Pima Indians informative for type 2 diabetes and BMI. SNPs detected by sequencing are also being analyzed for association with cis-acting gene expression levels in subjects with expression data. In the preliminary analyses, 3 of the 8 top signals for pfat $\left(P=10^{-5}-10^{-6}\right)$ resulted from novel variants. Five of these signals were in genes unknown to be associated with obesity (LYSMD4, ANKRD36, USP5, DHX32, AGPAT1) and require validation which is ongoing. Another top signal (CYB5A) had been detected in our prior GWAS for $\mathrm{BMI}$, and the $\mathrm{BMI}$ association replicated in two independent samples (total $\mathrm{N}=7285, \mathrm{P}=10^{-7}$ ). Another signal (PARP1) is an obesity gene in mice. SNPs in several biologically relevant genes were associated with other traits that predict type 2 diabetes (e.g. SNPs in LMX1 and NEDD4 were associated with acute insulin secretion and insulin action, respectively; both $\left.P \approx 10^{-5}\right)$.

144-0R

Exome Sequencing Identifies a New Diabetes Susceptibility Mutation in a Japanese Family With Highly Aggregated Type 2 Diabetes DAISUKE TANAKA, KAZUAKI NAGASHIMA, MAYUMI SASAKI, SHOGO FUNAKOSHI, AKIO KOIZUMI, NOBUYA INAGAKI, Kyoto, Japan

In most Japanese families with highly aggregated type 2 diabetes, familial clustering cannot be attributed to known genetic factors. The aim of this study was to investigate the genetic background of familial clustering of diabetes using genome-wide linkage analysis with exome sequencing. We recruited a family with 3 -generation history of diabetes in Japan. The family contained 16 members and 13 of them had been diagnosed with diabetes. Nine affected members had been diagnosed before the age of 40 . For the family, we performed linkage analysis assuming an autosomal dominant model. Linkage regions were observed on chromosomes $4 q 34,5 q 11-q 13$, and 12p11-q22 and the LOD scores were 1.80. To identify the susceptibility mutation, we used the Agilent SureSelect Human All Exon 50Mb Kit to enrich all exons from the genomic DNA of an affected family member. We sequenced the DNA using an Illumina Genome Analyzer IIx sequencer. We aligned the sequence reads to the hg19 human genome data. We predicted the familial clustering of diabetes is caused by a rare non-synonymous variant, and therefore we focused our analysis on non-synonymous variants absent in the dbSNP131. In the linkage regions exome sequencing identified 10 such variants. Seven of them were concordant with the affection status in the family. We genotyped 105 normal subjects and 67 diabetes subjects for the 7 variants. The only variant that was significantly more frequent in the diabetes subjects than in the normal subjects was the E1072K variant of EEA1 gene ( 0 in the normal subjects, 4 in the diabetes subjects, $p=0.022$ ). We propose that the E1072K variant of EEA1 gene is a susceptibility mutation in Japanese population. Although further study is required to evaluate the biological function of the mutation, family-based linkage analysis with exome sequencing may be a promising strategy to elucidate the complex genetic background of type 2 diabetes.

\section{5-0R}

Analysis of Coding Variants from Exome Sequencing in Diabetic Nephropathy (DN) Genes in African Americans

JESSICA N. COOKE, PAMELA J. HICKS, NICHOLETTE D. PALMER, MAGGIE C. NG, JESSICA M. HESTER, LIJUN MA, CARL D. LANGEFELD, BARRY I. FREEDMAN, DONALD W. BOWDEN, Winston-Salem, NC

Type 2 diabetes (T2D) and T2D-associated end-stage renal disease (T2DESRD) disproportionately affect African Americans (AAs). While prior studies have identified common variants, rare coding variants likely contribute additional risk in complex diseases such as T2D-ESRD. We identified 55 non-synonymous coding SNPs in 23 genes with prior evidence of association with nephropathy or ESRD in the NHLBI Exome Sequencing Project ESP_GO database. These variants were genotyped in 1024 AA T2D-ESRD cases and 1104 non-T2D-ESRD controls. Fourteen SNPs were trending $(P<0.1)$ or associated $(P<0.05)$ with disease in a model adjusting for African ancestry. These 14 SNPs were genotyped in a replication set of 786 AA T2D-ESRD cases and 1331 non-T2D-ESRD controls. In joint analysis of all samples, 7 were associated $(P<0.05)$ with $T 2 D-E S R D$. The most strongly associated SNPs were in ACACB (acetyl-CoA carboxylase beta; rs113576948, P=5.9x10-3, $\mathrm{OR}=2.6$ [1.3-5.1]), $A G E R$ (advanced glycosylation end product-specific receptor; rs77170610, $\mathrm{P}=1.6 \times 10-3, \mathrm{OR}=0.67$ [0.52-0.86]), APOL2 (apolipoprotein 2; rs7285167, 8.6x10-5, OR=1.2 [1.1-1.3]), APOL3 (apolipoprotein 3; rs116147257, 3.1x10-3, OR=0.72 [0.58-0.90]), and C6orf167 (rs9481410, 7.6x10-3, OR=0.89 [0.81-0.97]) (all additive model). When the 14 SNPs were tested conditioning on the APOL1 G1 and G2 non-T2D ESRD risk variants, association of 5 SNPs was strengthened (rs116147257 in APOL3, rs9492393 in C6orf191, rs4925583 and rs4478844 in 0R2L13, and rs34585936 in UNC5C) while association of3 SNPs weakened but retained significance $(P<0.05)$ (rs77170610 in AGER, rs9481410 in C6orf167, and rs113576948 in ACACB). These results suggest some influence from non-T2D ESRD variants. Thus, studies of coding variants complement and extend common variant analyses although odds ratios are not striking and recessive models, a likely model for rare variants, are difficult to test.

Supported by: NIH (DK66358)

146-OR

mRNA-seq of the Mouse Pancreatic Beta Cell Reveals a Large Class of Long Intergenic Non-Coding RNAs

GREGORY M. KU, HAIL KIM, IAN VAUGHN, MATTHEW HANGAUER, MICHAEL S. GERMAN, MICHAEL T. MCMANUS, San Francisco, CA, Daejeon, Republic of Korea

The pancreatic beta cell is critical for the maintenance of glycemic control. Knowing the compendium of genes expressed in beta cells will further our understanding of this critical cell type and will allow the identification of future anti-diabetes drug targets. While previous studies have used microarrays to measure gene expression in beta cells, these studies are limited to those printed on the arrays and may have difficulty confidently identifying genes expressed at low levels. One novel class of RNAs that might not have been detected by existing microarrays are long intergenic non-coding RNAs (lincRNAs). Several lincRNAs have now been demonstrated to be functionally important in biological processes ranging from epigenetic silencing to cell cycle control. Here, we report the use of next generation sequencing to interrogate the transcriptome of the mouse pancreatic beta cell. We have obtained nearly 500 million reads from the poly-adenylated RNAs of beta cells from mouse insulin promoter-GFP mice. Indeed, the expression of known beta cell specific genes is enriched in the sorted beta cell popula- 


\section{DIABETES PREVENTION AND LIFESTYLE RISK FACTORS}

tion as compared to total mouse islet while known non-beta cell specific genes are substantially depleted, demonstrating that our sorting strategy is robust and capable of identifying beta cell specific genes. Besides beta cell specific genes, we identified known and novel beta cell specific splice forms and promoter use. We then capitalized on the unbiased nature of mRNAseq and assembled a de novo transcriptome to identify potential long intergenic non-coding RNAs (lincRNAs). After removing transcripts shorter than 200 nucleotides and those with poor conservation, we identified over 1,100 unique potential lincRNAs expressed in mouse beta cells. The majority of these lincRNAs are beta cell specific and we hypothesize that this large set of novel RNAs may play a functional role in beta cell function.

\section{DIABETES PREVENTION AND LIFESTYLE RISK FACTORS}

147-OR

The Impact of Lifestyle Intervention on All-Cause and CardiovascuIar Mortality in Adults With Impaired Glucose Tolerance: 23-Year Follow-Up of the Da Oing Diabetes Prevention Study

GUANGWEI LI, PING ZHANG, JINPING WANG, YALI AN, OIUHONG GONG, EDWARD GREGG, GOJKA ROGLIC, WENYING YANG, MICHEAL ENGELGAU, YANYAN CHEN, YINGHUA HU, PETER BENNETT, Beijing, China, Atlanta, GA, Daqing, China, Geneva, Switzerland, Phoenix, AZ

Lifestyle interventions in people with impaired glucose tolerance (IGT) reduce the incidence of diabetes, but the impact on cardiovascular disease and all-cause mortality is unclear. We aimed to determine the long-term effects of lifestyle intervention on all-cause and cardiovascular (CVD) mortality in adults with IGT. In 1986, 576 adults with IGT from 33 clinics were randomly assigned by clinic to control or one of three lifestyle intervention groups (diet, exercise, and diet plus exercise). The intervention took place from 1986 to 1992. In 2009, 23 years after randomization, study participants were traced to determine the long-term effects of the intervention on all-cause and CVD mortality. We compared mortality rates in women and men in the control group to those in the combined intervention groups using intent-totreat analyses. Over the 23 year follow-up period, 47 women and 127 men died. In women, intervention reduced all-cause mortality by $53 \%$ (Hazard rate ratio $0.47 ; 95 \% \mathrm{Cl} 0.25-0.86$ ) with a cumulative all-cause mortality of $29.3 \%(95 \% \mathrm{Cl} 17.5-48.0)$ in the control arm and $16.2 \%(95 \% \mathrm{Cl} 11.2-21.2)$ in the intervention arm $(\mathrm{p}=0.02)$. The reduction in all-cause mortality in women was mainly due to differences in CVD mortality (heart disease and stroke) (HRR 0.30; 95\% Cl 0.12-0.68) with 23-year cumulative mortality of $18.8 \%$ $(95 \% \mathrm{Cl} 8.8-28.8)$ in the control and $6.8 \%(95 \% \mathrm{Cl} 3.4-10.2)$ in the intervention arm ( $p=0.006)$. Among men, there was no significant difference between the intervention and control groups in cumulative all-cause mortality $(41.1 \%$; $95 \% \mathrm{Cl} 34.7-47.5$ versus $46.7 ; 95 \% \mathrm{Cl} 35.4-58.0)(\mathrm{P}=0.41)$ or $\mathrm{CVD}$ mortality (26.4\%; $95 \% \mathrm{Cl} 21.1-31.6$ versus $27.4 \% ; 95 \% \mathrm{Cl} 18.6-32.2)(P=0.47)$. Over the 23-year follow-up, lifestyle intervention resulted in significantly lower allcause and CVD mortality among women with IGT but not in men.

Supported by: CDC, WHO, China-Japan Friendship Hospital

148-0R

Delay in Progression to Type 2 Diabetes among High-Risk Spanish Individuals Following Lifestyle Intervention in Real-Life Primary Care

XAVIER COS, BERNADO COSTA, FRANCISCO BARRIO, JOAN JOSEP CABRÉ, JOSEP LLUIS PIÑOL, SONIA SARRET, CLAUSTRE SOLÉ, XAVIER MUNDET, TERESA MUR, MONTSERRAT COT, JACINT CAULA, FRANCESC PUJOL, JAANA LINDSTRÖM, JAAKKO TUOMILEHTO, THE DE-PLAN-CAT RESEARCH GROUP, BarceIona, Spain, Helsinki, Finland, Seinajoki, Finland, Krems, Austria

To assess the feasibility and effectiveness of an active real-life primary care lifestyle intervention in preventing type2 diabetes within a high-risk Mediterranean population. Longitudinal cohort assessment conducted in primary healthcare. White European individuals without diabetes aged $45-75$ years ( $n=2054$ ) were screened using the FINRISC and a subsequent 2-h OGTT. High-risk non-diabetic screenees $(n=552)$ participated in lifestyle intervention: either general information on diet and cardiovascular health (standard-care intervention group, $\mathrm{n}=219$ ) or intensive reinforced DE-PLAN (Diabetes in Europe-Prevention using Lifestyle, Physical Activity and Nutritional intervention) programme (intensive intervention group, $n=333$ ). The primary outcome was the development of diabetes (WHO criteria). Analyses were performed based on the intention-to-treat principle. The standardcare(SC) and the intensive intervention groups(II) were comparable in terms of age (62.0/62.2 years), sex (64.4/68.2\% women), BMI (31.3/31.2 kg/m2),
FINDRISC score (16.2/15.8 points), fasting (5.3/5.2 mmol/l), 2-h (7.1/6.9 $\mathrm{mmol} / \mathrm{Il}$ plasma glucose and motivation to make lifestyle changes at baseline. Diabetes was diagnosed in 124 subjects-63(28.8\%) in the SC group and $61(18.3 \%)$ in the II group. During a 4.2-year median follow-up, the incidence of diabetes was 7.2 and 4.6 cases per 100 person-years, respectively $(p<0.005$, $36.5 \%$ relative risk reduction). Similarly, the incidence of diabetes in subjects with prediabetes was 13.4 cases per 100 person-years in the SC group and 8.9 cases per 100 person-years year in the II group $(p<0.02,33.6 \%$ relative risk reduction). The number needed to be treated for 4 years to reduce one case of diabetes by intensive intervention was 9.5. A substantial reduction in diabetes incidence can be achieved in real-life primary healthcare settings among high-risk individuals by a feasible intensive lifestyle intervention.

Supported by: University of Helsinki, Instituto Salud Carlos III, Generalitat Catalunya

149-OR

Tianjin Gestational Diabetes Mellitus Prevention Program: Study Design, Methods, and 1-Year Interim Report on the Feasibility of Lifestyle Intervention Program

GANG HU, HUIGUANG TIAN, FUXIA ZHANG, HUIKUN LIU, CUIPING ZHANG, SHUANG ZHANG, GONGSHU LIU, ZHIJIE YU, XILIN YANG, LU QI, CUILIN ZHANG, HUA WANG, MIN LI, JUNHONG LENG, LEISHEN WANG, YI LI, LING DONG, JAAKKO TUOMILEHTO, Baton Rouge, LA, Tianjin, China, St. Catharines, ON, Canada, Boston, MA, Rockville, MD, Helsinki, Finland

Whether lifestyle management can prevent type 2 diabetes among women with gestational diabetes mellitus (GDM) shortly after delivery has not been systematically tested. The aims of the Tianjin Gestational Diabetes Mellitus Prevention Program are to assess whether lifestyle intervention can reduce the incidence of type 2 diabetes in women with a history of $\mathrm{GDM}$, and to test the gene-intervention interactions in relation to type 2 diabetes related metabolic changes among such women. A total of 1,180 GDM women diagnosed with GDM from 2005 to 2009 were randomly assigned to either a lifestyle intervention $(n=586)$ or control group $(n=594)$. The control women will receive general information about the lifestyle changes necessary to prevent diabetes at the start of the trial $(26.7$ months after delivery) and at annual follow-up visits. Major elements of the intensified intervention include five face-to-face meetings with study dietitians in the first year, and two additional sessions and two telephone calls at each subsequent year. During the first year, body weight loss in the first 404 women entering the trial was $1.40(2.05 \%)$ vs $0.21 \mathrm{~kg}(0.27 \%)$ in the intervention and control group, respectively $(\mathrm{P}=0.001)$; the decrease was greater among women overweight at baseline $\left(B M I>=24 \mathrm{~kg} / \mathrm{m}^{2}\right)$ in the intervention group $(2.91 \mathrm{~kg} / 4.15 \%)$ and in the control group $(0.51 \mathrm{~kg} / 0.71 \%)(P<0.001)$. Women in the intervention group, compared with those in the control group, also had decreased body mass index, body fat, waist circumference, serum insulin and insulin resistance (HOMA), and had improved health behavior indicators including increased leisure time activity, vegetable and fiber intakes, and decreased fat consumption and sitting time. These interim results have shown the efficacy and feasibility of the lifestyle intervention for the prevention of type 2 diabetes among women with a history of GDM.

Supported by: EFSD

\section{Maternal Glucose in Pregnancy and After Delivery in GDM Mot} on Obesity Status of Their Children

SHUANG ZHANG, HUIKUN LIU, CUIPING ZHANG, ZHEYING TANG, NAN LI, SHUTING WANG, GONGSHU LIU, GANG HU, Tianjin, China, Baton Rouge, LA

Gestational diabetes mellitus (GDM) is a strong risk factor for obesity in GDM offspring, but maternal glucose in pregnancy and after delivery in GDM mothers on obesity status of their children are unknown. The aim of the present study is to examine the relation of glycemia during pregnancy and after delivery with anthropometry in offspring of GDM mothers. A total of 1,263 GDM women diagnosed with GDM from 2005 to 2009 and their children finished baseline survey from August 2009 to July 2011 for the Tianjin GDM Prevention Program. These women had a 75-g glucose 2-h 0GTT at 26-30 gestational weeks. We have been using the WHO's criteria to define GDM. Of 1263 GDM women, 9.1\% were diagnosed as diabetes and $90.9 \%$ were diagnosed as impaired glucose tolerance (IGT) based on their OGTT glucose concentrations at 26-30 gestational weeks. The offspring of GDM mothers who were diagnosed as diabetes during pregnancy had higher prevalence of overweight (body mass index $Z$ score $\geq$ the $85^{\text {th }}$ percentile for age- and gender-specific distribution using the World Health Organization growth reference) and obesity (body mass index $Z$ score $\geq$ the $95^{\text {th }}$ ), and higher mean values of $Z$ scores of weight for age, weight for height, and BMl for age than 
the offspring of GDM mother who were diagnosed as IGT. After a mean 2.26 years (27.2 months) delivery of $1263 \mathrm{GDM}$ mothers, 83 were diagnosed as type 2 diabetes $(6.6 \%)$. The offspring of GDM mothers who were diagnosed as diabetes after delivery had higher prevalence of overweight and obesity, higher mean values of $\mathrm{Z}$ scores of weight for age, weight for height, and BMI for age, and higher mean values of birth weight and birth length than the offspring of GDM mothers who were not diagnosed as diabetes after delivery. This study suggests that offspring of GDM mothers with diagnosed diabetes during pregnancy or after delivery are associated with an increased risk of childhood obesity compared with those of GDM mothers with prediabetes or high risk of diabetes during pregnancy or after delivery.

Supported by: EFSD

151-0R

Smoking Cessation, Weight Gain, and Risk of Type 2 Diabetes Mellitus Among Post-Menopausal Women

KAREN MARGOLIS, JUHUA LUO, ELISA TONG, GARY A. GIOVINO, CATHY LEE, CHU CHEN, JUDITH K. OCKENE, LIHONG OI, JACOUES ROSSOUW, Minneapolis, MN, Morgantown, WV, Sacramento, CA, Buffalo, NY, Los Angeles, CA, Seattle, WA, Worcester, MA, Bethesda, MD

Recent studies have found higher short-term risk of type 2 diabetes after smoking cessation, but the mechanism and duration of the risk need further investigation. We assessed the relationship between smoking cessation, weight gain and diabetes risk in 115,092 post-menopausal women enrolled in the Women's Health Initiative, in whom 11,056 cases of incident diabetes occurred over 8.5 years of follow-up after year 3 . The risk for diabetes was elevated in continuing smokers compared with never smokers (HR=1.20, 95\% Cl: 1.10 - 1.32), and women who quit smoking between enrollment and year 3 also had an elevated risk of diabetes ( $\mathrm{HR}=1.43,95 \% \mathrm{Cl}$ : 1.26-1.63). Among new quitters, diabetes risk among women who gained $<5 \mathrm{~kg}$ was similar to the risk in continuing smokers, but among new quitters who gained $\geq 5 \mathrm{~kg}$, there was a $67 \%$ excess risk of diabetes compared to non-smokers ( $\mathrm{p}$ for interaction $=0.02$ ). Among former smokers, years since quitting was significantly inversely associated with risk of diabetes, and the risk was reduced to that of never smokers 10 years after quitting (Figure). We conclude that the increased risk of diabetes associated with smoking cessation is confined to a subgroup which gains at least $5 \mathrm{~kg}$ and returns to that of a never smoker after about 10 years.

Table. Hazard ratios (HRs) and $95 \%$ confidence intervals ( $\mathrm{Cls}$ ) for incident diabetes since year 3 follow-up in relation to smoking status at baseline and year 3 visit

\begin{tabular}{|c|c|c|c|c|c|c|c|}
\hline \multirow[b]{2}{*}{ osure } & \multirow[b]{2}{*}{$N$} & \multirow[b]{2}{*}{ cases } & \multirow[b]{2}{*}{$\begin{array}{l}\text { Multivariable- } \\
\text { adjisted } \\
\text { HR }(95 \% \mathrm{CI})^{t}\end{array}$} & \multicolumn{2}{|c|}{ Weight gain $<5 \mathrm{~kg}$} & \multicolumn{2}{|c|}{ Weight gain $>=5 \mathrm{~kg}$} \\
\hline & & & & cases & \begin{tabular}{|l|} 
Multivariable- \\
adjusted \\
HR $(95 \% \mathrm{Cl})^{t}$
\end{tabular} & cases & \begin{tabular}{|l|} 
Multivariable- \\
adjinsted \\
HR $(95 \% \mathrm{CI})^{t}$
\end{tabular} \\
\hline er smokers & 59904 & 5735 & 1 & 4609 & 1 & 803 & 1 \\
\hline mer smokers & 47799 & 4532 & $1.00(0.96-1.04)$ & 3528 & $1.00(0.96-1.05)$ & 714 & $0.96(0.86-1.07)$ \\
\hline $\begin{array}{l}\text { itinumg } \\
\text { kers }\end{array}$ & 5335 & 535 & $1.20(1.09-1.31)$ & 399 & $1.16(1.04-1.29)$ & 95 & $1.32(1.06-1.64)$ \\
\hline quitters & 2054 & 254 & $1.43(1.26-1.63)$ & 122 & $1.17(0.98-1.41)$ & 110 & $1.67(1.36-2.05)$ \\
\hline
\end{tabular}

${ }^{1}$ Multivariable model adjusted for age at enrollment $(<55,55-59,60-64$, $65-69,70-74,75$ ), ethnicity (American Indian or Alaska Native, Asian or Pacific Islander, Black or African-American, Hispanic/Latino, nonHispanic white, and other), education (high school or less, some collegeitechnical training, college or some post-college, and master's degree or higher), body mass index $(<18.5,18.5-24.9,25.0-29.9,30,0-34.9$, $35.0-39.9,>=40$ ), waist circumference (in continuous), physical activity as (metabolic equivalent tasks [METs] per week: $<5,5-<10,10-<20,20-<30$, 30 or more), alcohol intake (non-drinker, past drinker, <1drink per month, 1 drink/month- $<1$ drink/wk, $1<<$ drinks/wk, $7+$ drinks/wk), hypertension (yes, no) and high cholesterol requiring pills (yes, no)
Effect of duration since quitting (years) on diabetes risk

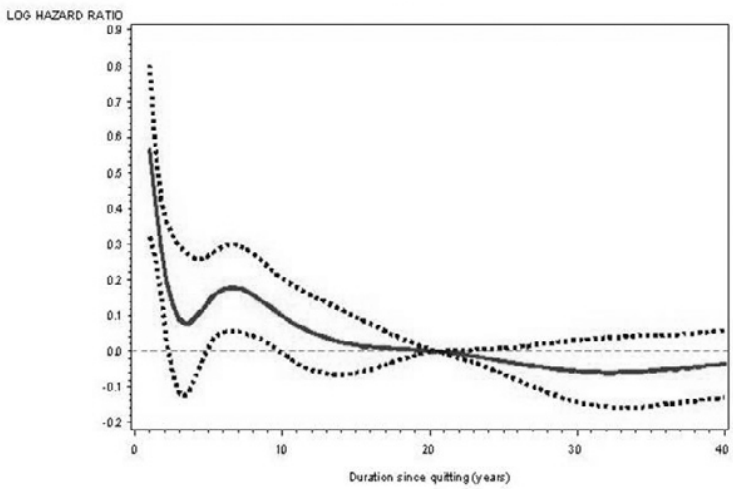

Figure. Effects of duration since quitting by year 3 follow-up on diabetes risk among 115,092 women (red line is point estimator, and two blue lines are $95 \%$ upper and lower confidence limits).

Supported by: Women's Health Initiative

152-0R

Overweight and Diabetes Among Asian Americans: A Systematic Review

LISA R. STAIMEZ, MARY BETH WEBER, K.M. VENKAT NARAYAN, REENA OZAFRANK, Atlanta, GA, Columbus, $\mathrm{OH}$

This systematic review summarizes and synthesizes data published in the past 20 years on mean $\mathrm{BMI}$ and prevalence of overweight/obesity and type 2 diabetes mellitus among Asian groups in the U.S. We conducted systematic searches in PubMed between September 2009 and August 2010 for peerreviewed, English-language citations published between 1988 and 2009 that reported mean $\mathrm{BMI}$, percent (\%) overweight, obesity, and diabetes among South Asians, Chinese, Filipino, Korean, and Vietnamese. We identified 647 database citations and 23 citations from hand-searching. After screening titles, abstracts, and full-text publications, 572 citations were excluded. Among the 98 remaining citations, none were published between 1988 and 1992, 28 between 1993 and 2003, and 70 between 2004 and 2009. Publications were identified for the following Asian groups: South Asian $(n=8)$, Asian Indian $(n=19)$, Chinese $(n=45)$, Filipino $(n=22)$, Korean $(n=8)$, and Vietnamese $(n=3)$. The observed sample sizes ranged from 30 to 4245 subjects with mean ages ranging from 23 to 78 years. Only $20 \%$ of all articles reported adjusted data. Among samples of men and women, the lowest reported mean BMI was in South Asians $\left(22.1 \mathrm{~kg} / \mathrm{m}^{2}\right)$, whereas the highest was in Filipinos $\left(26.8 \mathrm{~kg} / \mathrm{m}^{2}\right)$. Across studies, different definitions for obesity and overweight were used, resulting in variable estimates for overweight (12.3 $46.4 \%)$ and obesity $(2.1-59 \%)$. Reported prevalence of diabetes ranged from 1 - 11.6\% in South Asians, $6.6-32.9 \%$ in Asian Indians, $2.2-15.2 \%$ in Chinese, $3.7-36.4 \%$ in Filipinos, $10-22.7 \%$ in Koreans, and $5.3-15.6 \%$ in Vietnamese. This review suggests heterogeneity among US Asian populations in cardiometabolic risk factors, yet comparisons are limited due to the variability in study populations, methods, and definitions used in published reports. Future endeavors should investigate heterogeneity across Asian groups using standardized methods to understand obesity and diabetes risk in this growing US ethnic population.

153-OR

Physical Activity and Lower Risk of All-Cause Death and Cardiovascular Disease in Diabetes: A Meta-Analysis

SATORU KODAMA, CHIKA HORIKAWA, RYOKO TAJIMA, YORIKO HEIANZA, AYUMI SUGAWARA, MIHO MAKI, YOKO YACHI, KAORUKO T. IIDA, KAZUMI SAITO, HIROHITO SONE, Ibaraki, Japan, Tokyo, Japan

Our aim of this meta-analysis is to clarify the relationship between daily physical activity (PA) and risk of all-cause mortality (ACM) or cardiovascular disease (CVD) in patients with diabetes. Electronic literature search using MEDLINE and EMBASE was conducted for cohort studies that examined daily total or leisure-time PA and future risk of ACM or CVD (defined as including coronary heart disease among major study outcomes). Relative risk (RR) for the highest (H) vs. lowest (L) (nearly corresponding to sedentary) PA group in each study was pooled with a random-effects model. For studies that allowed quantitative estimation of energy expenditure (EE), we also conducted linear and cubic spline regression analyses to determine the relationship between the amount of PA and ACM or CVD risk. Our meta-analysis included 


\section{CLINICAL ISLET TRANSPLANTATION}

17 studies, 13 of which investigated ACM risk and 11 CVD risk. Qualitatively, the $\mathrm{H}$ group had a lower RR [95\% confidence interval] for ACM (0.61 [0.52$0.70])$ and CVD (0.65 [0.59-0.72]) than the L group. Linear regression analysis indicated that a $1 \mathrm{MET} x \mathrm{~h} / \mathrm{d}$ increase in PA was associated with ACM and CVD risk reduction by $9.5 \%(P=0.001)$ and $7.9 \%(P=0.001)$, respectively. The cubic spline regression curve suggested that daily PA yielding more than 5.6 METs $x \mathrm{~h}$ of EE accelerated the preventive effect of ACM although the benefit of additional PA was smaller for CVD than for ACM (Figure). The metaanalysis suggests an association between lower mortality or CVD risk and more PA in diabetic patients.

Figure Relationship between weekly PA and all-cause mortality and CVD risk in patients with diabetes. Solid line indicates the linear relationship. Dashed line and the area surrounded by the dotted line indicate the cubic spline regression
curve and its accompanying 050 confidence region, respectively. Size of each data point is proportional to its statistical weight.
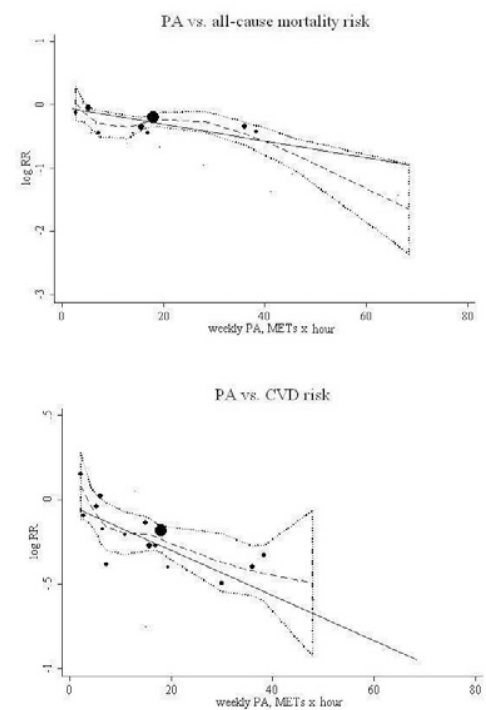

154-0R

Endogenous Sex Hormone Changes in Postmenopausal Women in the Diabetes Prevention Program (DPP)

CATHERINE KIM, SHENGCUN KONG, GAIL A. LAUGHLIN, SHERITA H. GOLDEN, KIEREN J. MATHER, BIN NAN, SHARON L. EDELSTEIN, JOHN R. RANDOLPH, FERNAND LABRIE, ELIZABETH OSBORNE, ELIZABETH BARRETT-CONNOR, Ann Arbor, MI, San Diego, CA, Baltimore, MD, Indianapolis, IN, Washington, DC, Quebec, OC, Canada

Few studies have examined changes in endogenous sex hormones or ESH [sex hormone binding globulin (SHBG), estradiol (E2), testosterone (T), and dehydroepiandrosterone (DHEA)] in response to intensive lifestyle change (ILS) and subsequent effects on glucose. We examined if randomization to ILS or metformin changed ESH compared to placebo among postmenopausal glucose-intolerant women. The DPP was a randomized trial that assessed the impact of ILS and metformin upon diabetes incidence in glucose intolerant adults. This report includes 382 postmenopausal women not using estrogen therapy. The main outcome measures were intervention-related ESH changes between baseline and 1 year and associations between ESH changes and glucose changes. Linear regression models determined the association between study arm and ESH changes and the associations between ESH changes and glucose changes after adjustment for age, race/ ethnicity, follicle stimulating hormone, and changes in 1/fasting insulin and waist circumference (WC). ILS increased SHBG by $10.4 \mathrm{nmol} / \mathrm{l}$ and decreased DHEA by $-0.31 \mathrm{ng} / \mathrm{ml}$, and these ESH changes were significantly different from changes in the placebo arm after adjustment for all covariates. SHBG increases were associated with fasting plasma glucose (FPG) decreases $(p<0.01)$ and 2-hour glucose $(2 \mathrm{HG})$ decreases $(p=0.04)$. DHEA decreases were associated with FPG decreases $(p=0.02)$ and $2 H G$ decreases $(p=0.01)$ after adjustment for variables including changes in WC and 1/fasting insulin. ILS did not decrease E2 or T, although E2 decreases were associated with FPG decreases after adjustment for all variables $(p=0.02)$. Metformin did not significantly impact ESH or glucose. Among postmenopausal glucose intolerant women, ILS increases SHBG and decreases DHEA, and these ESH changes were associated with FPG and $2 \mathrm{HG}$ independent of $\mathrm{WC}$ and fasting insulin.

Supported by: NIDDK
CLINICAL ISLET TRANSPLANTATION

155-OR

\section{WITHDRAWN}

$\beta$-Cell Secretory Capacity in Human Islet Transplantation According to the CITO7 Protocol

MICHAEL R. RICKELS, CARISSA FULLER, CORNELIA DALTON-BAKES, ALLEN J. CHIOU, EILEEN MARKMANN, MARAL PALANJIAN, SCOTT A. SOLEIMANPOUR, RICHARD D. SHLANSKY-GOLDBERG, KUMAR VIVEK, CHENGYANG LIU, ALI NAJI, Philadelphia, PA

The multi-center Clinical Islet Transplantation 07 (CIT07) protocol utilizes anti-thymocyte globulin \& etanercept induction, islet culture, heparinization and intensive insulin therapy, with the same low-dose tacrolimus and sirolimus maintenance immunosuppression as in the Edmonton protocol. To determine whether CITO7 improves engrafted islet $\beta$-cell mass, our center measured $\beta$-cell secretory capacity from glucose-potentiated arginine tests 
75 days post-transplant $(n=11)$, and compared the results to those achieved by our group with the Edmonton protocol $(n=5)$, and to normal $(n=11)$. CITO7 subjects received fewer islets $(9,097 \pm 803$ vs. $14,313 \pm 2237 \mathrm{IE} / \mathrm{kg}, \mathrm{p}<0.05)$ from a median 1 vs. 2 donors in Edmonton with all subjects off insulin. The acute insulin and C-peptide responses to $5 \mathrm{~g}$ iv arginine were measured fasting $\left(\mathrm{AlR}_{\mathrm{arg}} \& A C \mathrm{R}_{\text {arg }}\right)$ and during a $230 \mathrm{mg} / \mathrm{dl}$ glucose clamp $\left(\mathrm{AlR}_{\text {pot }} \& A C R_{\text {pot }}\right)$. $\mathrm{AlR}_{\text {arg }}$ was not different across CIT07, Edmonton and normal ( $28 \pm 4$ vs. $15 \pm 2$ vs. $28 \pm 3 \mu \mathrm{U} / \mathrm{ml}$ ), while $\mathrm{ACR}_{\mathrm{arg}}$ was greater in CIT07 than Edmonton and not less than normal ( $1.3 \pm 0.2$ vs. $0.5 \pm 0.1$ vs. $1.5 \pm 0.2 \mathrm{ng} / \mathrm{ml}, p<0.01$ for both). $\mathrm{AlR}_{\text {pot }}$ was greater in CIT07 than Edmonton with both transplant groups less than normal $(57 \pm 8$ vs. $29 \pm 3$ vs. $143 \pm 15 \mu \mathrm{U} / \mathrm{ml}, \mathrm{p}<0.05$ between transplant groups \& $p<0.01$ for both vs. normal), with similar findings for $\mathrm{ACR}_{\text {pot }}(3.0$ \pm 0.6 vs. $1.1 \pm 0.2$ vs. $6.4 \pm 0.8 \mathrm{ng} / \mathrm{ml}, \mathrm{p}<0.05$ between transplant groups \& $p<0.01$ for both vs. normal). Six CIT07 subjects have completed reassessment at 365 days post-transplant with a trend toward increasing $\operatorname{AlR}_{\text {pot }}(64$ \pm 11 vs. $88 \pm 15 \mu \mathrm{U} / \mathrm{ml}, p<0.1)$. These data indicate that while still less than normal, engrafted islet $\beta$-cell mass is markedly improved with the CIT07 vs. Edmonton protocol, especially given the smaller number of islets transplanted. While several peri-transplant differences may have each contributed to this improvement, the lack of deterioration in $\beta$-cell secretory capacity over time in CITO7 suggests that low-dose tacrolimus and sirolimus may not be toxic to islets.

Supported by: PHS (U01DK070430, UL1RR024134, and P30DK19525)

157-OR

Sitagliptin Plus Pantoprazole Enhances Graft Function and Temporarily Restores Insulin Independence in Clinical Islet Transplantation PETER A. SENIOR, JONATHAN YAU, PARASTOO DINYARI, SHARLEEEN IMES, ANDREW J. MALCOLM, JAMES SHAPIRO, ANGELA KOH, Edmonton, AB, Canada

Maintenance of insulin independence after islet transplantation is challenging. Incretins can augment insulin secretion and may increase beta cell mass. The combination of GLP-1 and gastrin can restore normoglycemia in NOD mice. Therefore we tested a combination of pantoprazole and sitagliptin in islet transplant recipients with early graft dysfunction. 7 male and 1 female subjects (mean age $55 \pm 8$ years, 2 months to 12 years since last transplant), with early islet graft dysfunction (A1c $>6 \%$ or fasting glucose $>7 \mathrm{mmol} / \mathrm{L}$ or random glucose $>10 \mathrm{mmol} / \mathrm{L}$ and insulin use $<10 \mathrm{U} /$ day) were recruited. Treatment was with sitagliptin $100 \mathrm{mg}$ daily and pantoprazole $40 \mathrm{mg}$ bid for 6 months. Glycemic control and graft function were assessed using A1c, insulin dose, mixed meal test, intravenous arginine stimulation test and continuous glucose monitoring system (CGMS) at baseline, 3 and 6 months, and after a 3 month washout. 5 of 8 subjects became insulin independent after 6 months of treatment. At baseline the mean HbA1c was $6.3 \pm 0.65 \%$, which decreased to $6.1 \pm 0.46 \%$ after 1 month of treatment ( $P=0.043)$ with no further change during treatment. $\mathrm{HbA1C}$ increased when treatment was withdrawn $(6.4 \pm 0.59 \%$ to $6.8 \pm 0.59 \%(P=0.032)$. Measures of graft function were stable during the study (mean fasting and stimulated $\mathrm{C}$-peptide/glucose ratios: $0.080 \pm 0.030$ and $0.20 \pm 0.10 ; 0.10 \pm 0.050$ and $0.20 \pm 0.16 ; 0.080 \pm 0.010$ and $0.21 \pm 0.08$, at baseline, 6 and 9 months respectively. There were no statistically significant changes in CGMS data, or acute insulin response to arginine. The study drugs were well tolerated, and there were no increases in hypoglycemia in any subjects. Insulin independence can be regained and glycemic control improved with the use of sitagliptin and pantoprazole, but these improvements may not be maintained when drugs are withdrawn. These data suggest that beta cell function is augmented by the study drugs, but theres is no evidence of significant beta cell regeneration.

Supported by: JDRF

\section{Use of Liraglutide in Islet Transplant Recipients}

EDUARDO M.L. PEIXOTO, LEONOR G. MIRELES-ZAVALA, NUJEN C. BOZKURT, VALENTINA DELMONTE, ANDREA CORRALES, CAMILLO RICORDI, RODOLFO ALEJANDRO, Miami, FL

Glucagon Peptide Like-1(GLP-1) analogs have been used in our center as an adjuvant therapy for islet transplantation. This population benefits from the unique GLP-1 actions such as optimization of glucose control by promoting glucose dependent insulin secretion and beta cell protection. Recently, our center switched subjects from a short acting GLP-1 analog (exenatide) to a long acting (liraglutide) in the attempt to improve metabolic outcomes and minimize side effects. A prospective study was done in a subset of islet transplant recipients $(n=6)$. Data on insulin, glucose, c-peptide, acetaminophen, glucagon and GLP-1 in response to a 300 minute Mixed Meal Tolerance Test (MMTT) were collected at 0 and 6 months, in addition to weight and $\mathrm{HbA1c}$. All subjects discontinued exenatide at least 3 days prior to base- line then started liraglutide $0.6 \mathrm{mg}$ daily, increasing to $1.2 \mathrm{mg}$ after 1 week. Data were analyzed using ANOVA and T-tests. The GLP-1 Omin mean was $1.65 \pm 1.19 ; 8.65 \pm 3.5 \mathrm{pM} / \mathrm{L}$ at 0 and 6 months respectively (P>0.05). The GLP1 mean peak at baseline was $13.95 \pm 17.43 \mathrm{pM} / \mathrm{L}$ and at 6 months $42.73 \pm 42.02$ $\mathrm{pM} / \mathrm{L}(\mathrm{P}<0.01)$. Glucose; $\mathrm{c}$-peptide, glucagon response and $\mathrm{HbA1c}$ remained stable $(6.0 \pm 0.30$ to $6.3 \pm 0.39 \%)(P>0.05)$. No difference was observed in the acetaminophen absorption. Study dose(1.2mg) was achieved in $100 \%(6 / 6)$ of patients, and sustained in $66.67 \%(4 / 6)$. Dose was decreased due to constipation and diarrhea. No nausea was observed and appetite was increased in $66.67 \%(4 / 6)$ of patients. Weight loss was reported in $66.67 \%(4 / 6)$ (mean:2.85 $\pm 2.32 \mathrm{Kg}$ ). Liraglutide was tolerated in the majority of subjects. Its action improved glucose response to MMTT, decreased weight. Liraglutide improved GLP-1 physiologic response to MMTT in these subjects, decreasing time to peak and increasing levels after a meal. The chronic higher GLP-1 levels (basal and stimulated) might lead to longer islet survival. Controlled studies are needed to confirm these observations.

159-0R

Islet Autotransplant to Prevent Diabetes in Children Undergoing Total Pancreatectomy for Chronic Pancreatitis

VISHA PARNERKAR, DAVID E. SUTHERLAND, GREGORY J. BEILMAN, TY B. DUNN, TIMOTHY L. PRUETT, SRINATH CHINNAKOTLA, SARAH J. SCHWARZENBERG, BERNHARD J. HERING, A.N. BALAMURUGAN, MELENA D. BELLIN, Minneapolis, MN

For children with severe chronic pancreatitis (CP), total pancreatectomy (TP) with islet autotransplant (IAT) can alleviate pain and avoid or lessen surgical diabetes. The objective of this analysis was to report metabolic outcomes in a large cohort of children who underwent TPIAT at a single center from 1989 -2011. Medical records were reviewed for insulin use, HbA1c levels, and mixed meal tolerance tests. 53 patients ( 29 female) underwent TPIAT at a mean age of $14 \pm 4$ years (range $5-18 y$ ). Mean islet yield was $213,854 \pm 166,990$ islet equivalents (IE) or 4,054 $\pm 3,295 \mathrm{IE} / \mathrm{kg}$, but was lower in the 12 pts with prior distal resection or Puestow drainage $(2,417 \pm 1,981 \mathrm{IE} /$ $\mathrm{kg}, \mathrm{p}=0.01)$. Most $(\mathrm{n}=50)$ were non-diabetic before TPIAT, with mean HbA1c $5.1 \pm 0.4 \%$. In all, 24 patients (44\%) achieved insulin independence (at $0.2-3$ years post-IAT), with 5 returning to insulin use 1-6 years after IAT. Of those with $>1$ year follow up, $17(45 \%)$ were insulin independent, $6(16 \%)$ required once daily insulin, and $15(39 \%)$ were fully insulin dependent at 1 year. Factors associated with insulin independence included younger patient age $(p=0.007)$ and greater IEQ/kg ( $p=0.001) .85 \%(10 / 12)$ of patients aged $<10$ years were insulin independent. For each $2500 \mathrm{IEO} / \mathrm{kg}$ in islet yield, odds of insulin independence increased 3.5 fold $(95 \% \mathrm{Cl} 1.8,8.5)$. $\mathrm{HbA1} \mathrm{c}$ was $<7 \%$ in $75 \%$ of all post-IAT measurements, and in $>94 \%$ of insulin independent and high islet yield $(>5,000 \mathrm{IE} / \mathrm{kg})$ recipients. $\mathrm{HbA1c}$ was lower and C-peptide higher in those who achieved insulin independence vs dependent: HbA1c $5.6 \pm 0.5 \%$ vs $7.1 \pm 1.6 \%$, peak C-peptide $3.5 \pm 1.6$ vs $1.7 \pm 1.4 \mathrm{ng} / \mathrm{mL}$, and AUC C-peptide $5.2 \pm 1.7$ vs $2.7 \pm 2.1 \mathrm{ng} / \mathrm{mL}$ hr ( $p<0.001$ for all). HbA1c was lower, and $\mathrm{C}$-peptide higher in high yield recipients $(\mathrm{p}<0.001)$. In conclusion, IAT permits goal glycemic control in the majority of children undergoing TPIAT for severe CP. Younger age and high islet yield were associated with greater likelihood of insulin independence and lower $\mathrm{HbA1c}$.

$160-0 R$

\section{WITHDRAWN}


$161-0 R$

Islet Autotransplantation After About 50\% Partial Pancreatectomy in Patients Without Diffuse Parenchymal Disease of PancreasImportance of Effectiveness of Islet Isolation

SANG-MAN JIN, SUN-MI PARK, JI CHEOL BAE, JAE HYEON KIM, MYUNG-SHIK LEE, MOON KYU LEE, KWANG-WON KIM, Seoul, Republic of Korea

There has been only little experience in islet autotransplantation (IAT) in patients without diffuse parenchymal disease of pancreas, unless the extent of pancreatic excision was more than $80 \%$. We aimed to identify the factors associated with earlier diabetes development in the patients without diffuse parenchymal disease of pancreas, who underwent IAT after about $50 \%$ partial pancreatectomy. Among the 37 patients without pre-operative diabetes and diffuse parenchymal disorders of pancreas, 20 underwent IAT after about $50 \%$ partial pancreatectomy with informed consent. In these 20 patients, annual oral glucose tolerance test was performed. Factors associated with earlier development of diabetes were identified. The median follow-up period was 61 months (range, 16-126). Higher preoperative levels of fasting plasma glucose (FPG, $p=0.005$ ), higher preoperative insulin resistance ( $p=0.040$, QUICKI index), less total transplanted islet mass $(p=0.012$, less islet isolation yield in islet equivalent per resected pancreas weight in gram (IEQ/g, $p=0.039$ ) were associated with diabetes development in 12 months post-operation $(n=5)$. Receiver-operator characteristic analysis revealed that islet isolation yield per resected pancreas mass of $<5154$ $\mathrm{IEQ} / \mathrm{g}$, but not the total transplanted islet mass, predicted the development of diabetes in 12 months (sensitivity $87 \%$, specificity $100 \% ; p=0.005$ ). In Kaplan-Meier survival analysis, diabetes-free survival was significantly prolonged when islet isolation yield was $>5154 \mathrm{IEO} / \mathrm{g}(p=0.0179)$. In patients without diffuse parenchymal disease of pancreas, preoperative FPG, insulin resistance, and islet isolation yield per pancreas weight predicted earlier diabetes development. A clinical trial designed to control these factors could prove the benefit of IAT in this population.

162-0R

Insulin Independence After Islet Autotransplant is Associated With Subtle Differences in Early Post-Transplant Glycemic Control

MELENA D. BELLIN, DAVID E. SUTHERLAND, SRINATH CHINNAKOTLA, TY DUNN, YONG KIM, JEREMIAH JOHNSON, ASAD SAEED, CAROL MANCHESTER, A.N. BALAMURUGAN, BERNHARD J. HERING, TIMOTHY L. PRUETT, GREGORY J. BEILMAN, Minneapolis, MN

Beta cell apoptosis after islet transplant is reduced when frank hyperglycemia is avoided. However, the impact of small deviations from normoglycemia is unclear. We reviewed early glycemic control in 255 islet autotransplant (IAT) recipients since 2006. In 2009, the hospital insulin drip shifted from strictly targeting euglycemia (80-110 mg/dL, "Era 1") to more liberal goals (100-120 mg/dL, "Era 2"). All patients were maintained on an insulin drip for $>3$ days. Average blood glucose (BG) was calculated for each of the first three post-operative days and for the first week. Insulin use was known for 171 cases, 1 year C-peptide for 70. Mean BG were lower in Era 1 vs Era 2: $113 \pm 11 \mathrm{mg} / \mathrm{dL}$ vs $119 \pm 12 \mathrm{mg} / \mathrm{dL} 119 \pm 14$ vs $126 \pm 18 \mathrm{mg} / \mathrm{dL}$ over the first 3 and 7 days respectively $(p<0.001)$, but insulin independence did not differ between Era 1 and Era 2 (40\% vs 39\%). In logistic regression, lower BG was associated with higher likelihood insulin independence (fig 1, $p<0.001$ ). Mean $\mathrm{BG}$ was lower in patients who achieved insulin independence (figure 2), an effect which was strongest in patients with $<5000 \mathrm{IE} / \mathrm{kg}$ transplanted. There was a modest correlation between peak $\mathrm{C}$-peptide at 1 year and 7 day BG average only in those with low islet yield, $(<2500 \mathrm{IE} / \mathrm{kg}, \mathrm{r}=0.54, \mathrm{p}=0.04)$. These data suggest a relationship between small $(6-10 \mathrm{mg} / \mathrm{dL})$ differences in early glycemic control and long-term IAT outcomes, which is strongest in those with marginal mass grafts. Lower BG in the insulin independent patients may mediate better graft survival, or conversely, may be a marker of better quality islets allowing fewer glycemic excursions.
Average BG in First Week Post-IAT

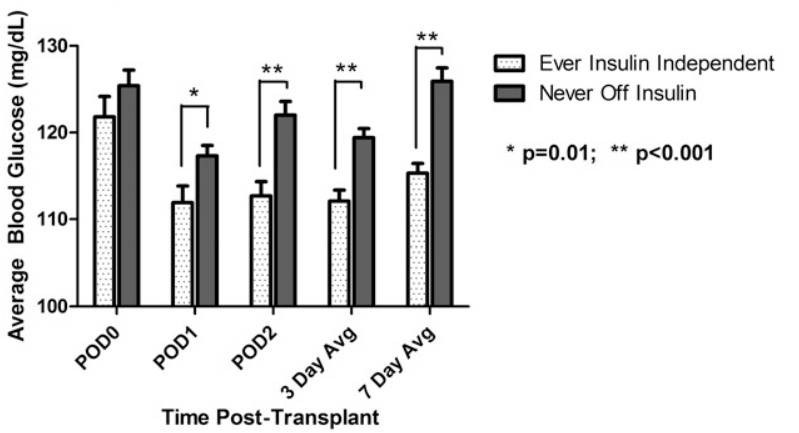

$\mathrm{POD} 0=$ Post-operative day $0, \mathrm{POD} 1=$ Post-operative day 1 POD2= Post-operative day 2, 3 Day Avg= Avg over first 3 days,

7 Day Avg= Avg over first 7 days post-operatively

\section{HOT TOPICS IN HUMAN OBESITY}

163-0R

Expression of Mir-17-92 Cluster in Adipose Tissue: Potential Role in the Regulation of Fibrosis and Angiogenesis

LATHA MUNIAPPAN, RESAT UNAL, BRIAN S. FINLIN, CHARLOTTE A. PETERSON, PHILIP A. KERN, Lexington, KY

A prominent feature of adipose tissue with obesity is an increase in fibrosis and a decrease in capillaries. Thrombospondin (TSP1) is pro-fibrotic and anti-angiogenic, and TSP1 expression in adipose is increased with obesity and insulin resistance. Because the mechanism for TSP1 up-regulation is unknown, we examined the functional significance of the microRNA (miR) 1792 cluster, which is composed of 7 miRs (miR 17-3p, 17-5p, 18a, 19a, 19b, 20a and 92) in tandem. In this cluster, miR-19a and 19b are known to decrease TSP1 and CTGF expression in cancer and other cell types, but no studies have examined adipose tissue. We hypothesized that miR-17-92 plays an important role in the development of obesity associated insulin resistance through regulation of TSP1 and other targets. Adipose tissue biopsies from 14 obese (BMI: $33.5 \pm 1.2, \mathrm{~S}_{\mathrm{I}}: 1.87 \pm 0.2$ ) and 14 lean human subjects (BMI: $24.2 \pm 1.2, \mathrm{~S}_{\text {: }}$ $4.57 \pm 1.2$ ) were examined for miR-17-92 cluster expression using a cluster plate assay and were confirmed by qRT-PCR experiments. Expression of all miR-17-92 cluster members was significantly lower in obese subjects, compared to lean, by $30-80 \%$ (all $p<0.05$ ). Overexpression of miR-17-92 cluster members miR-19a/b and 18a in cultured 3T3L1 adipocytes decreased TSP1 and CTGF expression by $80 \%$ and $75 \%$, respectively $(p<0.05)$. In addition, the knockdown of miR-19a/19b with siRNA increased the abundance of TSP1 and CTGF mRNA by $65 \%$ and $59 \%$, respectively $(p<0.05)$. TSP1 is expressed by both macrophages and adipocytes, and the coculture of macrophages with adipocytes induces TSP1 expression by both cells, but especially M2c polarized macrophages. Adipocyte conditioned medium (ACM) increased TSP1 expression, and the knockdown of miR-19a and 19b in M2c macrophages in the presence of ACM further increased TSP1 mRNA. These results suggest that down-regulation of miR-17-92 in obese subjects may provide a mechanism for the increase in TSP1, and hence the increased fibrosis and decreased angiogenesis.

Supported by: R01 DK71349

164-0R MicroRNAs in the Liver and Visceral Adipose Tissue in Non-Alcoholic Fatty Liver Disease (NAFLD)

JARKKO SORONEN, JULIA PERTTILÄ, HANNELE YKI-JÄRVINEN, VESA OLKKONEN, Helsinki, Finland

MicroRNAs (miRNAs) are small endogenous RNA molecules which regulate gene expression by reducing mRNA stability and translational activity. We aimed to determine whether miRNAs are abnormally expressed in NAFLD vs. normal human liver and in visceral adipose tissue in subjects with NAFLD compared to visceral adipose tissue in those with no NAFLD. Total RNA was extracted from liver and visceral adipose tissue (VAT) of patients who had either severe NAFLD (liver fat $>30 \%$, mean $38.3 \pm 4.5 \%$, $n=15$ ) or no NAFLD (liver fat $<5 \%$, mean $3.0 \pm 1.2 \%, n=15$ ). The groups were matched for age ( $49 \pm 2$ vs. $48 \pm 3$ yrs), gender ( 9 females and 6 males vs. 11 females and 4 males) and BMI ( $48.8 \pm 1.2$ vs. $44.4 \pm 1.7 \mathrm{~kg} / \mathrm{m} 2)$. RNA was subjected to miRNA profiling on Exiqon mercury LNATM microRNA arrays detecting 1438 
human miRNAs. The VAT and liver miRNA profiles were found to be distinct but overlapping. In liver of NAFLD, 8 miRNAs (miR-3663-5p, miRPlus-1137*, miR-576-5p, miR-3924, miR-892a, miR-106b, miR-103a-2*, miR-1282) were significantly increased compared to liver in no NAFLD. In VAT of NAFLD, one miRNA, miR-192*, was significantly up-regulated compared to VAT in no NAFLD. Analysis of predicted miRNA target genes suggests that the differential miRNA expression may downregulate insulin and IGF-1 signaling routes. These human data describe miRNAs in human liver and VAT and show that expression of miRNAs is altered in human NAFLD both in the liver and in VAT compared to subjects with no NAFLD. Studies in cultured hepatocytes and adipocytes are currently underway to explore the functional role of the miRNAs identified.

165-0R

Obese Women Display Inflammatory Changes in Liver Which Are Not Correlated to Hepatic Insulin Sensitivity or Liver Fat Content BARBARA A. DE WEIJER, M. VAN EIJK, M.T. ACKERMANS, I. JANSSEN, F. BERENDS, A. VAN DE LAAR, A.P. HOUDIJK, E. FLIERS, A.J. NEDERVEEN, M.J. SERLIE, Amsterdam, The Netherlands, Alkmaar, The Netherlands

Obesity is associated with hepatic steatosis, hepatic insulin resistance and low grade inflammation. Although liver inflammatory markers, insulin resistance and liver fat were shown to be associated in animal models, this is less clear in humans. We studied liver fat content using ${ }^{1} \mathrm{H}-\mathrm{MRS}$ and insulin sensitivity using the hyperinsulinemic euglycemic clamp technique in obese women undergoing bariatric surgery. We compared the expression of hepatic inflammatory markers between these women and a lean control group. We included 16 women (BMI 45 [39 - 51] $\mathrm{kg} / \mathrm{m} 2$ ) scheduled for RYGB and 6 lean healthy women (BMI 22 [20 - 24] kg/m2) scheduled for elective cholecystectomy. In the obese women, basal EGP was 13.6 (10.3-18.1) $\mu \mathrm{mol} / \mathrm{kgFFM} /$ min, insulin-mediated suppression of EGP was 79 (55 - 99)\% peripheral glucose uptake was $25.9(11.6-42.5) \mu \mathrm{mol} / \mathrm{kg} / \mathrm{min}$ and liver fat content was 8.5 $(0-23.3) \%$. Basal EGP and Rd were not correlated with liver fat content while hepatic insulin sensitivity showed a significant negative correlation. In liver biopsies, mRNA expression of the pro-inflammatory markers TNF $\alpha$, CCL2, the anti-inflammatory markers IL-10 and MR and macrophage markers CD68 and MIP1- $\beta$ were all significantly increased compared to the lean controls (fig.1). Within the obese group no correlation was seen between the expression profiles and liver fat content, EGP, Rd or hepatic insulin sensitivity. In conclusion, morbidly obese women are characterized by an inflamed liver which is independent of liver fat content. Moreover, this inflammatory state is not correlated to measures of insulin sensitivity.

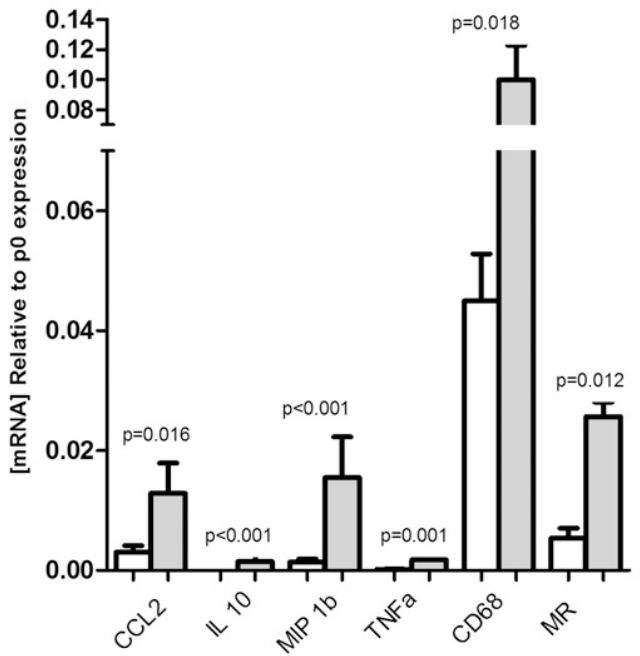

Fig. 1 Inflammatory gene expression (mean $\pm \mathrm{sd}$ ) in liver tissue from obese (grey columns, $n=16$ ) and lean (white columns, $n=6$ ) women. $p<0.05$, Mann-whitney test.
166-OR Enhanced Adipo/Lipogenic Potential of Human Adipose Tissue Stem Cells in Obesity

SEBASTIO PERRINI, PASQUALE NIGRO, ANGELO CIGNARELLI, MARIA BARBARO, ROMINA FICARELLA, ALESSANDRO PESCHECHERA, MICHELE DE FAZIO, FRANCESCO PUGLISI, ANNALISA NATALICCHIO, LUIGI LAVIOLA, FRANCESCO GIORGINO, Bari, Italy

An impaired adipo/lipogenic capacity of human adipose stem cells (ASCs) may contribute to obesity and the related metabolic abnormalities. Aim of this study was to investigate whether obesity is associated with changes in the adipogenic and/or lipogenic potential of ASCs from the subcutaneous (SC) and visceral (V) fat tissue depots. ASCs were isolated from SC and $V$ fat biopsies, obtained from 8 lean (L) (BMI $24.1 \pm 2.3 \mathrm{~kg} / \mathrm{m} 2)$ and 8 obese (Ob) (BMI $37.4 \pm 3.2 \mathrm{~kg} / \mathrm{m} 2)$ subjects. Cells were differentiated in vitro with adipogenic inducers in the absence or presence of $100 \mathrm{nM}$ insulin. Staining with Nile Red or Oil-Red-0 was used to quantify adipocyte conversion and the lipid droplet size and number, respectively. mRNA levels of lipogenic and adipogenic genes were measured by qRT-PCR. No significant differences in the conversion to mature adipocytes were seen in SC-ASCs from $\mathrm{L}$ and $\mathrm{Ob}$ subjects, whereas adipogenesis was about $60 \%$ higher in V-ASCs from Ob compared to $L$ donors. Following differentiation of both V-ASCs and SC-ASCs into mature adipocytes in the absence of insulin, there was a 70-80\% higher number of lipid droplets, with no apparent change in size, in Ob compared to $L$ cells $(p<0.05)$. Similar results were obtained in the presence of insulin, and insulin-induced Akt and Erk-1/2 phosphorylation was similar in L and $0 \mathrm{~b}$ ASCs. When the expression of 14 lipogenic and adipogenic genes was assessed in both ASCs and adipocytes from the experimental subjects, larger differences were found in V compared to $S C$ cells $(p<0.05)$. Specifically, the SIRT1/2 mRNA levels were lower in Ob compared to L V-ASCs and adipocytes. A trend to an inverse relationship between SIRT1/2 mRNA expression and BMI was also found in $V$ fat biopsies $(R=-0.145)$. In conclusion, Ob ASCs, particularly from the $V$ fat depot, exhibit lower SIRT1/2 gene expression and possess an enhanced inherent adipo/lipogenic potential, which is largely insulin-independent.

167-OR

Mitochondrial Function of Human Subcutaneous and Omental Adipocytes

XIAO YIN, JAMES SWAIN, MICHAEL SARR, IAN LANZA, K.S. NAIR, MICHAEL D. JENSEN, Rochester, MN

Some investigators suggest that mitochondrial (MITO) dysfunction, including adipocyte MITO, contributes to obesity-related metabolic complications. However, obese humans have larger adipocytes and the reported MITO defects might be related to cell size vs. obesity per se. Aim: To determine whether obesity, cell size and fat depot determine MITO dysfunction in white adipose tissue. Methods: Omental (OM) and/or abdominal subcutaneous (SQ) adipose tissue was collected from 20, age matched, non-diabetic men and women undergoing elective abdominal surgery or research needle biopsy. By separating large from small adipocytes (differential floatation) we isolated populations of large $0 \mathrm{M}$ and SO $(0.50 \pm 0.21$ and $0.86 \pm 0.18 \mu \mathrm{g}$ lipid/ cell) from lean adults that were not different from small populations from obese $\mathrm{OM}$ and $\mathrm{SO}(0.46 \pm 0.03$ and $0.61 \pm 0.06 \mu \mathrm{g} \mathrm{lipid} /$ cell $)$. $\mathrm{mtDNA}$-encoded NADH dehydrogenase 1 genes (ND1/28S) for both populations of cells was were measured using qPCR. MITO were isolated from each cell population for measurement of protein content, stage 2, 3, 4 and uncoupled MITO oxygen consumption rates (OCR) using a Clark-type oxygen electrode and citrate synthase (CS) activity. OCR and CS activity were normalized to MITO protein to allow comparison across groups. Results: mtDNA abundance was less $(P=0.02)$ in cells from obese vs. lean adults. SQ CS activity was greater in MITO from lean than obese $(1.2 \pm 0.18$ vs. $0.56 \pm 0.06 \mu \mathrm{mole} / \mathrm{min} / \mathrm{mg}$ protein, respectively, $P=0.007)$ as was $0 \mathrm{M} \mathrm{CS}$ activity $(1.0 \pm 0.14$ vs. $0.67 \pm 0.11$, respectively, $P=0.09$ ) when matched for cell size. MITO OCR across all measured phases of respiration were greater (all $\mathrm{P}<0.05)$ in lean than obese $0 \mathrm{M}$ and SO when matched for fat cell size. MITO function and content were not significantly different between small and large cell groups, between different depots or between men and women. Conclusions: Adipocyte MITO oxidative capacity and mtDNA copy number is decreased in non-diabetic obese vs. lean adults, which cannot be attributed to fat cell size differences.

Supported by: NIH 
168-OR

High Serum Leptin Concentration is an Independent Feature of Individuals With Lower than Expected Resting Energy Expenditure (REE) GUIDO LATTUADA, FRANCESCA RAGOGNA, ELISABETTA DEVECCHI, GIANLUCA PERSEGHIN, Milan, Italy

Adipocytokines may play a role in the regulation of energy metabolism. To test this hypothesis we established whether lower than expected REE was associated with altered fasting serum adipocytokines concentrations in humans.We performed indirect calorimetry and measured serum leptin adiponectin, resistin in 296 white normal-weight $(n=129)$, over-weight $(n=88)$ and obese $(n=79)$ eu-thyroid individuals. The measured REE was normalized to the expected REE (Harris Benedict equation based on age, sex, body weight, height and ethnic group) and study subjects were segregated in tertiles of $\%$ of expected REE. Cut-offs were: < 97\% (tertile 1), > 97\% but $<102 \%$ (tertile 2) and $>102 \%$ of expected REE (tertile 3 ). Habitual physical activity index (PAl) at work, during sport and leisure time was assessed using a questionnaire. Individuals in tertile 1 were more likely women, younger, and with higher fasting serum leptin and adiponectin concentrations with respect to tertiles 2 and 3 ( $p<0.0001$ for all). They had lower sport PAI $(p<0.0001)$, higher BMI $(p<0.02)$, and in spite of these features lower fasting glucose $(p<0.01)$, LDL- chol $(p=0.04)$, triglycerides $(p<0.02)$ and intra-hepatic fat content ('HMR-Spectroscopy) with respect to tertile 3 . No difference in PAl at work and leisure time, respiratory quotient, insulin, HOMA-IR, ghrelin, resistin, FFA and TSH were found. Stepwise regression analyses selected the sport PAI ( $r=0.34 ; 12 \%)$ and sport PAI + leptin $(r=0.40 ; 16 \%)$ as independent predictors of the \% of expected REE. In conclusion low resting energy expenditure is independently associated with higher serum leptin reflecting a compensatory adaptation for the low metabolic rates; whether this may be also responsible for the lower fasting serum glucose, LDL-chol and triglycerides that characterized these subjects theoretically at greater risk of obesity-related disorders is yet to be clarified.

Supported by: Musazzi and Stellato families

169-OR

A Variant in ACACB Associates With BMI and Gene Expression LIJUN MA, MARIANA MUREA, JAMES A. SNIPES, PAMELA J. HICKS, ALEJANDRA MARINELARENA, JACQUELINE KRÜGER, KURT A. LANGBERG, MEREDITH A. BOSTROM, JESSICA N. COOKE, DAISUKE SUZUKI, TETSUYA BABAZONO, TAKASHI UZU, SYDNEY C. TANG, ASHIS K. MONDAL, NEERAJ K. SHARMA, PETER A. ANTINOZZI, LAWRENCE L. RUDEL, MATTHEW DAVIS, SWAPAN K. DAS, JOHN S. PARKS, SAYUKO KOBES, MATTHIAS BLÜHER, MICHAEL STUMVOLL, DONALD W. BOWDEN, SHIRO MAEDA, ROBERT L. HANSON, PETER KOVACS, LESLIE J. BAIER, STEVEN C. ELBEIN, BARRY I. FREEDMAN, Winston-Salem, NC, Phoenix, AZ, Leipzig, Germany, Tokyo, Japan, Otsu, Japan, Hong Kong, China, Yokohama, Japan

The single nucleotide polymorphism (SNP) rs2268388 in the acetyl coenzyme $A$ carboxylase $B$ gene $(A C A C B)$ is reproducibly associated with type 2 diabetic (T2D) nephropathy (DN) in multiple populations. ACACB knock-out mice are protected against obesity when fed a high-fat diet. This study explored relationships between rs2268388, BMI and gene expression. In a meta-analysis containing 4905 initially non-T2D individuals, rs2268388 (T allele) was associated with higher BMI (Stouffer $p=0.0056$ ), comprised of 512 non-T2D European American (EA) subjects from Utah and Arkansas ( $p=$ 0.096), 1146 German Caucasians ( $\mathrm{p}=0.055)$, and 3247 Pima Indians (mixed model $p=0.042$ from 19718 longitudinal exams). Rs2268388 was not associated with BMI in a large sample of T2D cases of Japanese $(n=2720)$ and $E A$ ( $n=1136)$ descents; however, the risk allele T for DN was associated with higher BMI in the subset of these subjects with $\mathrm{BMI}>30 \mathrm{~kg} / \mathrm{m}^{2}: 568$ EAs (additive $p=0.049$ ) and 196 Japanese (additive $p=0.049$ ) adjusting for age, gender, and diabetes duration. The association was strengthened in a meta-analysis that included additional 763 African Americans (AAs, $p=0.09$ ) and 48 Hong Kong Chinese $(p=0.81)$ with T2D and BMI $>30 \mathrm{~kg} / \mathrm{m}^{2}$ (Stouffer additive $p=0.004 ; n=1575)$. We next assessed the effect of rs2268388 on gene expression in available tissue /cell samples. The T allele was associated with higher gene expression in transformed lymphocytes $(p=0.05$, Sanger HapMap in silico, $n=162$ ), adipose tissue in $41 \mathrm{EAs}$ and $20 \mathrm{AAs}$ with $\mathrm{BMI}>30 \mathrm{~kg} / \mathrm{m}^{2}$ (adjusted additive $\mathrm{p}=0.03$, Illumina $\mathrm{H} 12$ array), and liver tissue (mixed model $p=0.03,25$ bariatric surgery EA subjects examined by 75 Western blot tests, BMI $>30 \mathrm{~kg} / \mathrm{m}^{2}$ ). The T allele was also associated with higher hepatic triglyceride levels (adjusted additive $p=0.03, n=38$ ). These data support a role for $A C A C B$ in obesity and a conditional role for altered lipid metabolism in susceptibility to DN.
$170-0 R$ Finding Obesity Genes by Whole Exome Sequencing in a UK Cohort of Severely Obese Children

AUDREY E. HENDRICKS, Cambridge, United Kingdom

Within the UK10K project, we are undertaking whole exome sequencing (WES) in 1,000 patients from the Severe Childhood Onset Obesity Project (SCOOP). SCOOP consists of UK white patients with severe obesity (BMI Standard Deviation (SD) Score $>3$ ) of early onset $(<10$ years), a design that is enriched for rare, highly penetrant causes of obesity. Patients were selected for WES after known causes of obesity had been excluded on the basis of clinical phenotype, measurement of serum leptin, insulin/proinsulin, and sequencing of the MC4R gene, the most common monogenic form of obesity. Using Agilent SureSelect Human All Exon 50Mb array and Illumina HiSeq sequencing a mean target coverage of $\sim 50 \mathrm{x}$ was achieved, $90 \%$ of the samples had $\geq 10 x$ coverage for more than $80 \%$ of the target bases. We took the union of variants after calling with both GATK and SAMtools. We then filtered by several quality control (OC) measures (including $4 \leq$ depth $\leq$ 2000 , mapping quality $>25$, strand bias $\leq-0.1)$. After this initial $Q C, \sim 50,000$ non-reference, good quality variants were produced per subject. As an initial analysis, we focused on rare variants residing in genes in one of three candidate lists: known to cause human obesity syndromes, strong biological candidates (disruption/overexpression associated with obesity in mice), and associated with obesity through hypothesis free approaches such as genome-wide association study meta-analyses. We confirmed subject specific variants that we found previously in this cohort using Sanger sequencing (including variants in MC4R, LEPR, SIM1), and identified novel variants that are predicted to be deleterious; we are following these up in the probands and family members. Within Uk10K we are also undertaking WES in BMI extremes from UK population based cohorts. These parallel approaches will provide a large set of data on which further analyses, such as case-control association analysis, are planned.

Supported by: Wellcome Trust 091310/B/10/Z

\section{TARGETING HYPOGLYCEMIA}

171-0R

Coronary Flow Reserve During Acute Hypoglycemia in Type 1 Diabetes

RADZI M. NOH, NINIAN N. LANG, ALEX J. GRAVELING, AUDREY C. WHITE, CHIM C. LANG, DAVID E. NEWBY, BRIAN M. FRIER, Edinburgh, United Kingdom, Aberdeen, United Kingdom, Dundee, United Kingdom

Hypoglycemia exerts a profound hemodynamic effect through sympathoadrenal stimulation. Progressive microvascular dysfunction associated with increasing duration of type 1 diabetes may compromise myocardial blood flow at times of stress. The present study evaluated coronary blood flow reserve during hypoglycemia in people with type 1 diabetes of differing duration. Coronary blood flow velocity was evaluated in the left anterior descending coronary artery using trans-thoracic Doppler echocardiography before and during adenosine-induced microvascular hyperemia. Coronary Flow Reserve (CFR) is the maximum hyperemic blood flow velocity divided by baseline blood flow velocity and is a well-validated non-invasive measure of coronary microvascular function. Ten non-diabetic control subjects and 16 adults with type 1 diabetes ( $6<5$ years; $10>15$ years duration) were studied (age range 20-46 years). CFR was measured (a) during euglycemia $(4.5 \mathrm{mmol} / \mathrm{L} ; 81 \mathrm{mg} / \mathrm{dL}$ ) and (b) during hypoglycemia $(2.5 \mathrm{mmol} / \mathrm{L} ; 45 \mathrm{mg} / \mathrm{dL})$ using a glucose-insulin clamp method. During euglycemia, there appeared to be a trend toward lower CFR in the diabetic group (diabetic vs. control group $3.69 \pm 0.6$ vs. $4.00 \pm 0.87, p=0.07)$. The long-duration diabetic group had the lowest CFR during euglycemia $(3.56 \pm 0.66)$. During hypoglycemia, CFR appeared to rise slightly in normal controls and fall further in those with diabetes such that CFR was lower in those with diabetes compared to non-diabetic controls (diabetic vs. control group $3.53 \pm 0.48$ vs. $4.08 \pm 0.81$, $\mathrm{p}<0.05)$. In conclusion, diabetes is associated with impaired coronary microvascular function especially during acute hypoglycemia. This may enhance the propensity for myocardial ischemia in people with diabetes at times of hypoglycaemia and could explain the tendency to poorer cardiovascular outcomes observed in large randomised trials examining the effects of strict glycemic control.

Supported by: Scottish Chief Scientist Office 
172-OR

Optimal Timing of Terbutaline Release has the Potential to Prevent Insulin-Induced Nocturnal Hypoglycemia

LESZEK NOSEK, PATRICIA IBARRA, KARIM BAGATE, JEAN-MICHEL CARDOT, DAVID R. OWENS, ANNELIE FISCHER, TIM HEISE, Neuss, Germany, Muttenz, Switzerland, Clermont Ferrand, France, Cardiff, United Kingdom

Terbutaline has been shown to protect against nocturnal hypoglycemia but can also lead to morning hyperglycemia. In this randomized, cross-over trial we studied the effect of $5 \mathrm{mg}$ immediate release terbutaline (TER) and placebo (PLA) in comparison to $2.5 \mathrm{mg}$ and $5 \mathrm{mg}$ of SKP-1052 (SKP), a formulation using the Geoclock technology to delay the release of terbutaline for about 2 hours post-dosing, on nocturnal blood glucose (BG) levels in 30 subjects with type 1 diabetes. On each treatment study day, BG was lowered to $90 \mathrm{mg} / \mathrm{dL}$ before injection of insulin glargine at 18:30 hrs. TER, SKP or PLA were given at $21.00 \mathrm{hrs}, \mathrm{BG}$ and pharmacokinetics (PK) followed until the next day. Carbohydrates $(10 \mathrm{mg})$ were given when hypoglycemia $(B G<$ $50 \mathrm{mg} / \mathrm{dL}$ ) occurred. Maximum PK levels were reached 2-2.5h later with SKP than with TER confirming the delayed release with the Geoclock technology, whereas dose normalized PK showed bioequivalence between SKP and TER. TER and both SKP doses showed a 41-67\% reduction in hypoglycemic events vs. placebo ( $p<0.01$ for TER and SKP $5 \mathrm{mg}$, (table). Whereas TER and $5 \mathrm{mg}$ SKP showed slightly higher nocturnal and fasting BG than placebo, 2.5 mg SKP only exhibited a modest and transient (non-significant) increase in BG 3-8 hours post-dose. All terbutaline formulations were well tolerated without relevant increase in adverse events or changes in electrolytes or ECGs. In conclusion, this study confirms the potential of preventing nocturnal hypoglycemia with terbutaline. SKP-1052 when used in an optimized dose may be able to prevent fasting hyperglycemia seen with the normal formulation of terbulatine.

\begin{tabular}{|c|c|c|c|c|}
\hline \multicolumn{5}{|c|}{ Mean results (SD) } \\
\hline Parameter & Placebo & $\begin{array}{c}\text { SKP-1052 } \\
2.5 \mathrm{mg}\end{array}$ & $\begin{array}{c}\text { SKP-1052 } \\
5 \mathrm{mg}\end{array}$ & $\begin{array}{c}\text { Terbutaline } \\
5 \mathrm{mg}\end{array}$ \\
\hline $\begin{array}{l}\text { Mean BG (0-10h postdosing) } \\
\text { [mg/dL] }\end{array}$ & $100(35)$ & $100(32)$ & $121(44)$ & $129(49)$ \\
\hline $\begin{array}{l}\text { Mean BG (2-10h postdosing) } \\
{[\mathrm{mg} / \mathrm{dL}]}\end{array}$ & $103(40)$ & $105(38)$ & $130(52)^{*}$ & $137(58)^{*}$ \\
\hline $\begin{array}{l}\text { Number of hypoglycemic } \\
\text { events }(11 \mathrm{pm}-7 \mathrm{am})\end{array}$ & 27 & 16 & $9^{*}$ & $10^{*}$ \\
\hline $\begin{array}{l}\text { Number of hypoglycemic } \\
\text { events (1 am - } 5 \mathrm{am})\end{array}$ & 13 & 5 & 3 & 4 \\
\hline Fasting BG (7 am) [mg/dL] & $113(57)$ & $111(57)$ & $144(73)$ & $143(70)$ \\
\hline
\end{tabular}

${ }^{*} p$

173-OR

Role of Carotid Bodies as Novel Integrative Metabolic Sensors in Humans

SIMMI DUBE, ANANDA BASU, ERICA WEHRWEIN, TIMOTHY B. CURRY, MICHAEL J. JOYNER, RITA BASU, Rochester, MN, East Lansing, MI

The carotid body receptors are known to sense arterial oxygen, carbon dioxide and blood pressure changes. Studies have suggested that glucose regulation is affected in carotid body denervated dogs. The present studies were conducted to evaluate the role of these sensors in glucose regulation in humans. Seven healthy volunteers lage $=27 \pm 0.4$ years, $\mathrm{BMl}=25 \pm 0.3$ $\mathrm{kg} / \mathrm{m}^{2}, \mathrm{FFM}=53 \pm 2 \mathrm{Kg}$ ) were subjected to two 180 min hyperinsulinemic (2 $\mathrm{mU} / \mathrm{kg} \mathrm{FFM} / \mathrm{min})$, hypoglycemic $(3.33 \mathrm{mmol} / \mathrm{L})$ clamps one week apart with randomized exposure to normoxia (arterial P02 ( $\mathrm{PaO} 2) 111 \pm 6.3 \mathrm{mmHg}$ ) or hyperoxia ( $\mathrm{PaO2} 345 \pm 80.6 \mathrm{mmHg}$ ). Results were compared using means of last forty minutes of baseline and clamp data. Plasma glucose concentrations were no different during normoxia and hyperoxia at baseline $(5.52$ \pm 0.15 vs. $5.55 \pm 0.13 \mathrm{umol} / \mathrm{ml}, \mathrm{p}=\mathrm{ns})$ and during clamp ( $3.4 \pm 0.05$ vs. 3.3 $\pm 0.05 \mathrm{umol} / \mathrm{ml}, p=n s)$. Glucose infusion rate required to maintain hypoglycemia was higher $(p<0.001)$ during hyperoxia $(38.80 \pm 3.5$ vs. $25.0 \pm 3.6$ $\mathrm{umol} / \mathrm{kgFFM} / \mathrm{min}$ ) than normoxia. Counterregulatory hormones were lower $(p<0.01)$ during hyperoxia (norepinephrine $50.7 \pm 5.2 \%$, epinephrine $62.6 \pm$ $3.3 \%$, cortisol $63.2 \pm 2.1 \%$, growth hormone $53.1 \pm 2.7 \%$, glucagon $48.6 \pm$ $2.1 \%)$ compared to normoxia (table1). Endogenous glucose production (EGP) was completely suppressed during hyperoxia than to normoxia $(-0.90 \pm 1.7$ vs. $10.0 \pm 1.2 \mathrm{umol} / \mathrm{kgFFM} / \mathrm{min}, \mathrm{p}<0.001)$. These data suggest that hyperoxia turns off "carotid bodies" and suppresses hormonal counterregulation to hypoglycemia thus reducing EGP. Future studies are required to determine role of carotid bodies in glucose counterregulation in other diseased states.

\begin{tabular}{l|l|l|l|l|l|l}
\hline HORMONES & $\begin{array}{l}\text { NORMOXIA } \\
\text { Baseline }\end{array}$ & $\begin{array}{l}\text { HYPEROXIA } \\
\text { Baseline }\end{array}$ & $\begin{array}{l}\text { p } \\
\text { value }\end{array}$ & $\begin{array}{l}\text { NORMOXIA } \\
\text { Clamp }\end{array}$ & $\begin{array}{l}\text { HYPEROXIA } \\
\text { Clamp }\end{array}$ & $\begin{array}{l}\text { palue } \\
\text { valu }\end{array}$ \\
\hline $\begin{array}{l}\text { Insulin } \\
\text { (pmo/L) }\end{array}$ & $31 \pm 6$ & $28 \pm 4$ & $\mathrm{p}=\mathrm{ns}$ & $816 \pm 75$ & $791 \pm 65$ & $\mathrm{p}=\mathrm{ns}$ \\
\hline $\begin{array}{l}\text { C-peptide } \\
\text { (nmo/L) }\end{array}$ & $0.6 \pm 0.4$ & $0.6 \pm 0.4$ & $\mathrm{p}=\mathrm{ns}$ & $0.08 \pm 0.00$ & $0.08 \pm 0.00$ & $\mathrm{p}=\mathrm{ns}$ \\
\hline $\begin{array}{l}\text { Glucagon } \\
\text { (pg/ml) }\end{array}$ & $60 \pm 5$ & $56 \pm 5$ & $\mathrm{p}=\mathrm{ns}$ & $109 \pm 9$ & $91 \pm 8$ & $\mathrm{p}<0.001$ \\
\hline $\begin{array}{l}\text { Cortisol } \\
\text { (ug/dl) }\end{array}$ & $8.0 \pm 1.0$ & $9.0 \pm 1.0$ & $\mathrm{p}=\mathrm{ns}$ & $20 \pm 2$ & $13 \pm 2$ & $\mathrm{p}<0.01$ \\
\hline $\begin{array}{l}\text { Growth } \\
\text { hormone } \\
\text { (ng/ml) }\end{array}$ & $1.0 \pm 0.4$ & $1.0 \pm 0.4$ & $\mathrm{p}=\mathrm{ns}$ & $8.0 \pm 2$ & $4.0 \pm 1$ & $\mathrm{p}<0.001$ \\
\hline $\begin{array}{l}\text { Nor } \\
\text { epinephrine } \\
\text { (pg/ml) }\end{array}$ & $188 \pm 21$ & $202 \pm 20$ & $\mathrm{p}=\mathrm{ns}$ & $348 \pm 37$ & $285 \pm 43$ & $\mathrm{p}<0.001$ \\
\hline $\begin{array}{l}\text { Epinephrine } \\
\text { (pg/ml) }\end{array}$ & $21 \pm 3$ & $25 \pm 4$ & $\mathrm{p}=\mathrm{ns}$ & $736 \pm 126$ & $485 \pm 103$ & $\mathrm{p}<0.001$ \\
\hline
\end{tabular}

Supported by: DK 090541

174-0R

Relationship Between Hypoglycemic Episodes, Glycemic Fluctuations and Arrhythmias in Patients With Type 2 Diabetes and Cardiovascular Diseases

ANNETT STAHN, MARKOLF HANEFELD, CARSTA KOEHLER, Dresden, Germany

Patients with type 2 diabetes and cardiovascular disease (CVD) may represent a high risk group for hypoglycemic episodes associated with arrhythmias and sudden death. Little is known on frequency of asymptomatic hypoglycemia, in particular nocturnal hypoglycemia, frequency of silent arrhythmias and its relationship to glycemic fluctuations. In a high risk cohort we recorded in parallel interstitial glucose (i. G.) concentrations with continuous glucose measurement system (CGMS) and long-term ECG (LT-ECG). 23 patients with type 2 diabetes, $\mathrm{HbA} 1 \mathrm{c}<9 \%$, age 56 - 80 years, with proven CVD, treated with insulin and/ or sulfonylurea, were considered. CGMS with Medtronic MiniMed Gold ${ }^{\mathrm{TM}}$ system and ECG with Amedtec ECG pro ${ }^{\circledR}$ were recorded in parallel for 5 days. Hypoglycemia by CGMS was a) modest: 3.1$3.9 \mathrm{mmol} / \mathrm{l}$ i.G. b) serious: $<3.1 \mathrm{mmol} / \mathrm{l}$ i.G. Patients noticed symptoms of hypoglycemia and arrhythmias in a predefined protocol. 69 episodes with i.G. $<3.9 \mathrm{mmol} / \mathrm{I}$ were recorded by CGMS. However only 14 mild symptomatic hypoglycemias $(20.2 \%)$ and 1 serious hypoglycemia were noticed by the patients. We found 35 hypglycemic periods with a duration of more than 30 minutes. These hypoglycemias occured more at night (24 episodes at night vs. 11 at daytime). No arrhythmias were noticed by the patients. However we observed multiple ventricular arrhythmias (Couplets: 14 patients (60.9\%), Triplets: 10 patients ( $43.5 \%)$, Ventricular tachycardias: 5 patients (21.7\%). Correlation analysis did not show a direct relationship between standard deviation, MAGE (mean amplitude of glucose excursions) and arrhythmias. Patients with CVD on insulin or sulfonylurea treatment represent a high risk for silent hypoglycemia and arrhythmias, especially at night. Avoidance of silent hypoglycemic episodes in patients with insulin and/or sulfonylurea treatment seems to be of importance to protect patients with CVD from arrhythmias.

175-OR

Adrenergic Blockade Prevents Life Threatening Cardiac Arrhythmias and Sudden Death Due to Severe Hypoglycemia

CANDACE M. RENO, DORIT DAPHNA-IKEN, SIMON J. FISHER, St. Louis, MO

Severe hypoglycemia can be lethal. We hypothesized that blocking the actions of the markedly elevated catecholamines during severe hypoglycemia would decrease associated cardiac arrhythmias and mortality. Therefore, 9 week old, pre-cannulated, awake, unrestrained, Sprague-Dawley rats were infused with saline (CON, $n=8$ ) or combined $\alpha$ - and $\beta$-adrenergic receptor blockers (prazosin $1.2 \mathrm{ng} / \mathrm{min}$ and propranolol $\mathrm{ng} / \mathrm{min}, \mathrm{n}=9$ ) during a 3 hour hyperinsulinemic $\left(0.2 \mathrm{U} \cdot \mathrm{kg}^{-1} \cdot \mathrm{min}^{-1}\right)$ severe hypoglycemic $(10-15 \mathrm{mg} /$ dl) clamp. During severe hypoglycemia, $\alpha / \beta$ blocker treated rats, as compared to $\mathrm{CON}$ rats, had an increased glucose infusion rate $(5 \pm 0.3$ vs $3 \pm 0.6$ 
$\mathrm{mg} / \mathrm{kg} / \mathrm{min}, \mathrm{p}<0.008)$, decreased heart rate $(244 \pm 5$ vs $358 \pm 5$ BPM, $p<0.001)$, and increased OTc to a lesser extent $(134 \pm 3$ vs $163 \pm 2 \mathrm{~ms}, p<0.001)$. Potas sium levels fell to a similar degree in $\mathrm{CON}$ and $\alpha / \beta$ blocker rats $(3.1+0.2 \mathrm{vs}$. $2.8 \pm 0.1 \mathrm{mmol}, \mathrm{p}=\mathrm{NS}$ ). Severe hypoglycemia induced mortality was $50 \%$ in CON rats, and remarkably, was $0 \%$ in $\alpha / \beta$ blocker treated rats $(p<0.029)$ Continuous EKG analysis during severe hypoglycemia demonstrated that $\alpha / \beta$ blocker rats displayed no premature ventricular contractions, nor $1^{\text {st }}$ or $2^{\text {nd }}$ degree heart block arrhythmias frequently observed in CON rats. In summary, blocking the actions of catecholamines during severe hypoglycemia provided a considerable survival advantage and prevented pre-terminal cardiac arrhythmias. We conclude that under studied conditions, lethal cardiac arrhythmias and sudden death induced by severe hypoglycemia were mediated by the counterregulatory sympathoadrenal response.

\section{Adrenergic blockade improves survival and reduces cardiac arrhythmias during severe hypoglycemia}

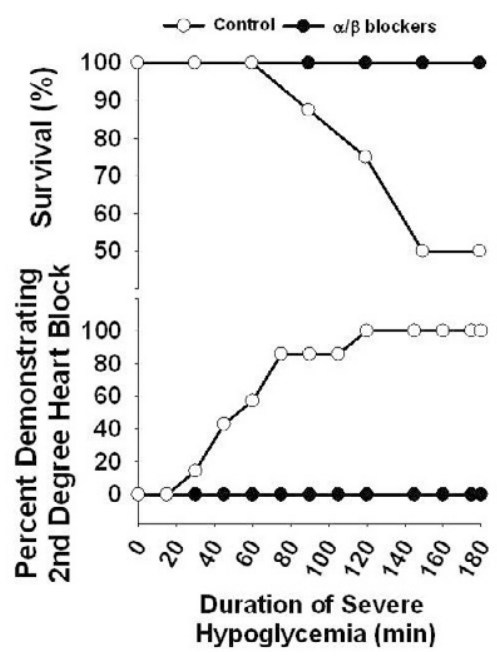

176-OR

Somatostatin Receptor Type 2 Antagonism Improves Glucagon Counterregulatory Response to Hypoglycemia in Type 1 Diabetes BB Rats

TAIRAN QIN, NEGAR KARIMIAN, MAYOWA OSUNDIJI, YACHI HUANG, DAVID H. COY, MLADEN VRANIC, HERBERT Y. GAISANO, Toronto, ON, Canada, New Orleans, LA

Impaired counterregulation during hypoglycemia in type 1 diabetes (T1D) is partly due to inadequate islet alpha-cell glucagon secretion, and this is a major limiting factor of intensive insulin treatment in diabetes. One of the reasons could be that the normal intra-islet somatostatin suppression of hypoglycemia-stimulated alpha-cell glucagon release becomes accentuated during T1D. We hypothesized that hypoglycemia can be prevented in autoimmune T1D by selective somatostatin receptor type 2 (SSTR2) antagonism of alpha cells to relieve SSTR2 inhibition thereby increasing glucagon secretion. We have employed the Biobreeding diabetes prone (BBDP) rats, a model that closely mimics human autoimmune T1D. In the in vivo study, non-diabetic $\mathrm{BBDP}(\mathrm{N})$ and diabetic BBDP(D) rats underwent 3-h infusion of vehicle or SSTR2a during insulin-induced hypoglycemia clamped at $2.5 \pm 0.5 \mathrm{mmol} / \mathrm{I}$. D rats treated with SSTR2a needed $95 \%$ less glucose infusion than untreated $D$ rats $(p<0.05)$ to attain hypoglycemia, implying that attenuated glucagon response to hypoglycemia in diabetic BB rats was restored by SSTR2a. To monitor real-time glucagon secretory response directly, we developed a technique of thin slices of pancreas subjected to perifusion to changing conditions, specifically with control vehicle vs SSTR2a, then subjected to sequential non-stimulatory $7 \mathrm{mM}$ glucose (G). This was followed by stimulatory $1 \mathrm{mM} \mathrm{G}$ and $20 \mathrm{mM}$ arginine (7mM G). 30 uM SSTR2a treatment similarly enhanced glucagon secretion from both $\mathrm{N}$ and D BBDP rat slices: $7 \mathrm{mM} \mathrm{G}$ (by 1-2 fold), $1 \mathrm{mM} \mathrm{G}$ (2-3 fold), and $20 \mathrm{mM}$ arginine (in $7 \mathrm{mM} \mathrm{G}$, by 2 fold) $(p<0.001)$. Thus, somatostatin may contribute to impaired glucagon responsiveness to hypoglycemia in autoimmune T1D. We conclude that SSTR2 antagonism can enhance hypoglycemia-stimulated glucagon release sufficient for normoglycemic control.

\section{$\Delta$} 177-OR

The Counterregulatory Response to Hypoglycemia is Positively Correlated to Thalamic Activation

SILVIA MANGIA, AMIR MOHEET, FEDERICO DE MARTINO, NOLA TESFAYE, ANJALI KUMAR, PETE KOLLASCH, LYNN EBERLY, ELIZABETH SEAQUIST, Minneapolis, MN

The thalamus has been identified as a brain region involved in the sensing and coordination of the counterregulatory (CR) response to hypoglycemia $(\mathrm{HG})$. Activation of this region is reduced during $\mathrm{HG}$ in patients with type 1 diabetes (T1DM) and $\mathrm{HG}$ unawareness relative to controls. However, the relationship between reduced activation and blunting of $\mathrm{HG}$ induced $\mathrm{CR}$ hormone secretions is unknown. Here we test the hypothesis that the magnitude of thalamic activation during $\mathrm{HG}$ correlates with the magnitude of the CR response. Fifteen controls ( $7 \mathrm{M} / 8 \mathrm{~F}$, age $\left.43 \pm 15 \mathrm{yrs}, \mathrm{BMI} 25 \pm 5 \mathrm{~kg} / \mathrm{m}^{2}\right)$ and 15 patients $\left(6 \mathrm{M} / 9 \mathrm{~F}\right.$, age $\left.43 \pm 14 \mathrm{yrs}, \mathrm{BMI} 27 \pm 4 \mathrm{~kg} / \mathrm{m}^{2}\right)$ with well controlled T1DM (A1c $6.7 \pm 0.4 \%)$ underwent a two step hyperinsulinemic $(2.0 \mathrm{mU} / \mathrm{kg} /$ $\mathrm{min}$ ) clamp while cerebral blood flow (CBF) weighted images were acquired at $3 \mathrm{~T}$ using arterial spin labeling (ASL) technique to monitor cerebral activation. Blood glucose was first held at $95 \mathrm{mg} / \mathrm{dl}$ and then allowed to drop to $50 \mathrm{mg} / \mathrm{dl}$. CBF weighted images were collected over $10 \mathrm{~min}$, as soon as target glycemia was reached. Blood was collected at baseline and every 10 min during $\mathrm{HG}$ for measurement of CR hormones. Relative difference of CBF weighted ASL signals between euglycemia and $\mathrm{HG}$ were measured in the thalamus of each subject, and then correlated with the average values of $\mathrm{CR}$ hormones (e.g., epinephrine in figure) during $\mathrm{HG}$. Our results demonstrate a positive correlation between thalamic response and epinephrine, growth hormone and cortisol responses to $\mathrm{HG}(r=0.62, p=0.0003 ; r=0.48, p=0.0079$; $r=0.46, p=0.011$, respectively), confirming that this region plays role in the coordination of the $\mathrm{CR}$ response to $\mathrm{HG}$

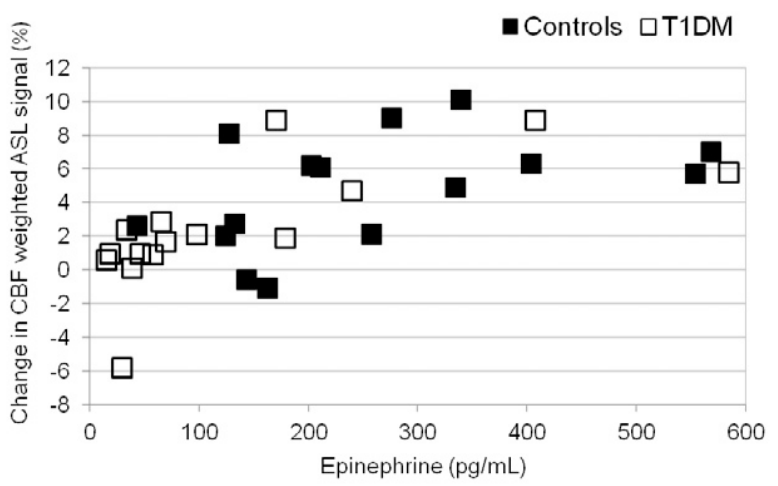

Supported by: NIH (BTRR-P41RR008079, P30 NS057091, R01 DK62440)

178-0R Comparable Regional Brain Responses to Hypoglycemia by ASL and PET

ANA MARIA ARBELAEZ, YI SU, JEWELL B. THOMAS, BEAU M. ANCES, TAMARA HERSHEY, St. Louis, MO

As plasma glucose falls, different brain regions sense and modulate counterregulatory responses to changes in circulating glucose. The aim of this study was to determine if pulsed arterial spin labeling magnetic resonance imaging (PASL-MRI) provides a method for measuring absolute regional cerebral blood flow (rCBF) responses throughout the brain to changes in circulating glucose levels as reliably as positron emission tomography (PET). Nine non-diabetic volunteers underwent a hyperinsulinemic euglycemic $(92 \pm 3 \mathrm{mg} /$ $\mathrm{dL}$ ) - hypoglycemic $(53 \pm 1 \mathrm{mg} / \mathrm{dL})$ clamp in which 2 runs of ASL and 4 of [ ${ }^{150}$ water PET were used to measure rCBF at each glucose level. Plasma glucose and counterregulatory hormones were measured during both study sessions and ROls were defined for both methodologies using the FreeSurfer program. rCBF in the thalamus, medial prefrontal cortex, and globus pallidus significantly increased during hypoglycemia by both PASL and PET methodology. Our data suggest that PASL is reliable and feasible for measuring blood flow responses to hypoglycemia compared to PET. These data also underscore the presence of a brain network involved in the response to hypoglycemia that includes the thalamus, medial prefrontal cortex, and globus pallidus. 
$\wedge$

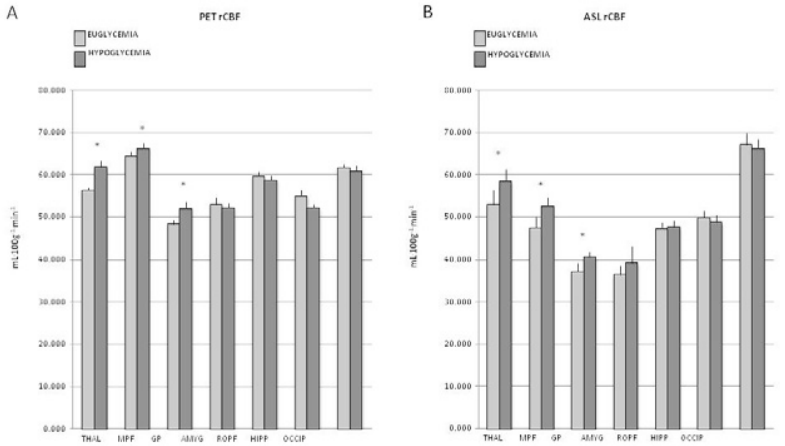

Quantitative regional cerebral blood flow $\left(\mathrm{mL} \cdot 100 \mathrm{~g}^{-1} \cdot \mathrm{min}^{-1}\right)$ responses between euglycemia and hypoglycemia by PET and ASL. ${ }^{*} p<0.05$; THAL=thalamus, $M P F=$ medial prefrontal cortex, GP=globus pallidus, $A M Y G=a m y g d a l a$, ROPF=right orbitoprefrontal, HIPP=hippocampus, OCCIP=occipital.

Supported by: Children's Discov. Inst. 1KL2RR024994-01 and WU Mallinckrodt Inst. Radiol. Pilot

\section{IMPACT OF EXERCISE IN TYPE 1 AND TYPE 2 DIABETES}

179-OR

Increased Skeletal Muscle Capillarization After Aerobic Exercise Training and Weight Loss Predicts Improved Insulin Sensitivity and Glucose Tolerance in Older Adults With IGT

STEVEN J. PRIOR, LYNDON JOSEPH, JACOB B. BLUMENTHAL, LESLIE I. KATZEL, ANDREW P. GOLDBERG, ALICE S. RYAN, Baltimore, MD

Transcapillary transport of insulin is one determinant of glucose uptake in skeletal muscle; thus, a reduction in capillary density (CD) may worsen insulin sensitivity and contribute to impaired glucose tolerance (IGT). Skeletal muscle CD is reduced in older adults with IGT compared to normal adults and may be modifiable through aerobic exercise training and weight loss $(A E X+W L)$. We tested the hypothesis that 6-mo AEX+WL increases CD to increase insulin sensitivity (M) and improve glucose tolerance in older adults with IGT. Before and after $6-\mathrm{mo} \mathrm{AEX}+\mathrm{WL}, 16$ sedentary $\left(\mathrm{VO}_{2 \max }=1.8 \pm 0.1\right.$ $\mathrm{L} / \mathrm{min})$, obese (BMI $32 \pm 1 \mathrm{~kg} / \mathrm{m}^{2}$; mean \pm SEM), older $(63 \pm 2 \mathrm{yrs})$ men $(n=8)$ and women $(n=8)$ with IGT underwent hyperinsulinemic-euglycemic clamps (3-hr, $\left.80 \mathrm{mU} / \mathrm{m}^{2} / \mathrm{min}\right), 0 \mathrm{GTTs}$, exercise and body composition testing, and vastus lateralis skeletal muscle biopsies to determine CD. ANCOVA tested for differences before and after AEX+WL; regression analyses tested associations between $C D$ and metabolic variables. At baseline, 120 -min postprandial glucose $\left(G_{120}\right)$ inversely correlated with $C D(r=-0.68, p<0.01)$ and $M$ directly correlated with $C D(r=0.69, p<0.01)$. After $A E X+W L, V O_{2 \max }$ increased $12 \%(p=0.02)$ and both weight and fat mass decreased by $>7.5 \%$ ( $p$ $<0.02$ ). Fat-free mass did not change ( $54.4 \pm 3$ vs. $53.8 \pm 3 \mathrm{~kg}$ ). CD increased $15 \%$ (264 \pm 11 vs. $\left.304 \pm 12 \mathrm{cap} / \mathrm{mm}^{2}, \mathrm{p}<0.01\right)$, M increased $20 \%$ (43.2 \pm 4 vs. $51.7 \pm 4 \mu \mathrm{mol} / \mathrm{kgFFM} / \mathrm{min}, \mathrm{p}<0.05)$, and $\mathrm{G}_{120}$ decreased $16 \%(9.3 \pm 0.4 \mathrm{vs} .7 .8$ $\pm 0.5 \mathrm{mmol} / \mathrm{L}, \mathrm{p}=0.02$ ). The $A E X+W L$-induced increase in $\mathrm{CD}$ independently predicted the increase in $\mathrm{M}(\mathrm{r}=0.63 p<0.05)$ as well as the decrease in $G_{120}(r=-0.51, p<0.05)$ in regression analyses accounting for age, sex, and baseline $C D$. We conclude that $6-m o A E X+W L$ increases skeletal muscle $C D$ in older adults with IGT, and that the increase in CD contributes in part to improvements in insulin sensitivity and glucose tolerance.

180-OR

Regular Exercise Reduces the Risk and Severity of Non-Alcoholic Fatty Liver Disease Independent of Obesity

JI CHEOL BAE, JAE HYEON KIM, JI YOUNG JOUNG, KWANG-WON KIM, SUN-MI PARK, SANG-MAN JIN, YOON YOUNG CHO, MOON KYU LEE, Seoul, Republic of Korea

Non-alcoholic fatty liver disease (NAFLD) is associated with insulin resistance and obesity. We evaluated the role of physical activity in relation to obesity and insulin resistance as a primary contributor in the development and progression of NAFLD. A cross-sectional analysis was conducted in 72,359 healthy adults without diabetes who participated in a comprehensive health check-up. All subjects were categorized into deciles based on their BMI level. We estimated the odds ratios (ORs) for having NAFLD according to physical activity in deciles. Among all subjects, patients with NAFLD $(n=19,921)$ proven by ultrasonography were analyzed separately to evaluate ORs for having a high liver enzyme based on physical activity. The risk for NAFLD was significantly reduced in physically active groups with age- and sex-adjusted ORs of 0.62-0.78 for all BMI deciles except at BMI groups of $<19.6$ and $20.7-21.6 \mathrm{~kg} / \mathrm{m}^{2}$. No difference was seen in BMI between physically active and inactive subjects across the BMI deciles. However, the values of body fat percentage and metabolic risk factors differed between physically active and inactive subjects across the BMI categories. Among NAFLD patients, physically active subjects had a lower risk for having elevated liver enzymes with multivariable adjusted OR of $0.85(95 \% \mathrm{Cl}$ 0.74-0.99, for AST) and 0.74 (95\% Cl 0.67-0.81, for ALT) than did physically inactive subjects. Regular exercise was associated with a reduced risk for having NAFLD and decreased severity of disease, and this relationship was also independent of obesity.

A)

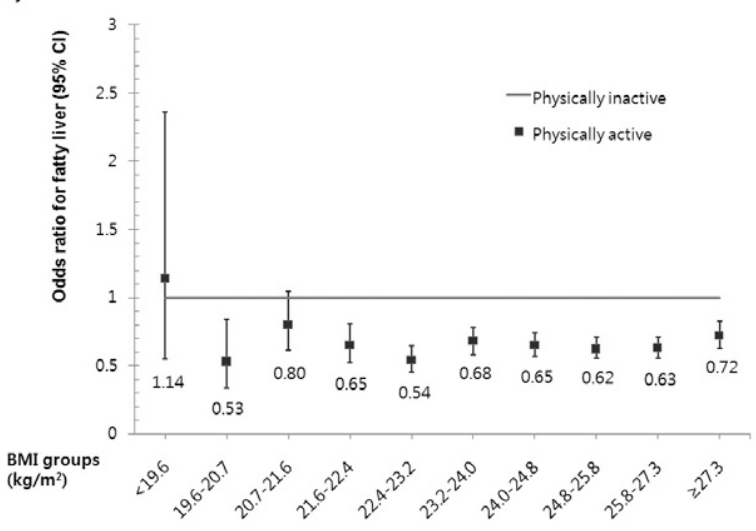

B)

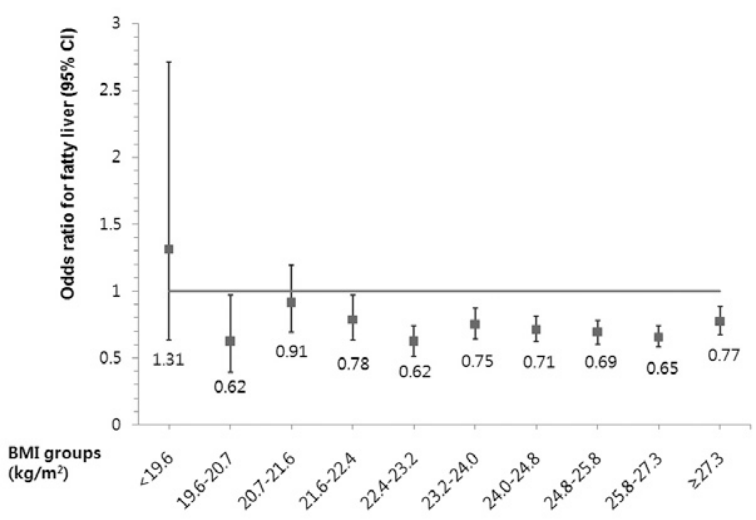

$\Delta$

181-OR

Impact of Lifestyle Intervention on Sedentary Time in Subjects at High-Risk for Diabetes

ANDREA KRISKA, BONNY ROCKETTE-WAGNER, GEORGE A. BRAY, HERMES FLOREZ, SHARON EDELSTEIN, DEEPTI REDDY, MARY LOU CARRION-PETERSEN, DANA DABELEA, LINDA DELAHANTY, PAUL FRANKS, MARIA G. MONTEZ, RICHARD R. RUBIN, ELIZABETH M. VENDITTI, DPP RESEARCH GROUP, Pittsburgh, PA, Baton Rouge, LA, Miami, FL, Rockville, MD, La Jolla, CA, Aurora, CO, Boston, MA, Malmö, Sweden, San Antonio, TX, Baltimore, MD

Sedentary behavior, often estimated as time spent in activities with low energy expenditure such as watching TV and sitting at work, has been shown to be related to risk factors for diabetes and metabolic syndrome. Whether a lifestyle intervention effective in increasing physical activity would also impact on two of the key components of sedentary time is not known. The Diabetes Prevention Program examined this question in 3234 participants randomized to lifestyle intervention, metformin or placebo. The activity goal of the lifestyle intervention was to achieve 150 minutes per week of moderate-intensity activity. Both physical activity and sedentary behavior were estimated by questionnaire, with the latter being assessed 
as reported hours per day spent watching TV (Sit-TV) and sitting at work (SitWork). At study end (average 3.2 yrs), both Sit-TV and Sit-Work decreased $(p<0.05)$ in the lifestyle arm for all age $(<40 ; 40-49 ; 50-59 ; 60+y r s)$ and sex groups except the youngest age group. The range of means for all age and sex specific lifestyle groups decreased $0.4-0.5 \mathrm{hrs} /$ day from baseline values of 2.15- $2.93 \mathrm{hrs} /$ day for Sit-TV; and 0.1-0.4 hrs/day from baseline values of 3.99-4.98 hrs/day for Sit-Work. Compared with the other two arms, the lifestyle group increased over time in moderate physical activity levels and had greater decreases in both Sit-TV and Sit-Work $(p<0.05)$. However, in the oldest age group, significant differences were noted only after eliminating those visits during which physical limitations to movement were reported. The DPP lifestyle intervention focused on increasing moderate intensity activity but also caused a residual decrease in sedentary behavior. Future research should investigate the impact of an intervention that more directly addresses sedentary behavior change.

Supported by: NIDDK (U01-DK048489)

182-OR

Decreased Fetuin-A Correlates With Improved Glucose Tolerance After Short-Term Exercise Training in Adults With NAFLD

STEVEN K. MALIN, CIARAN FEALY, JACOB M. HAUS, MANGESH PAGADALA AMANDA R. SCELSI, HAZEL HUANG, ARTHUR MCCULLOUGH, CHRIS FLASK, JOHN P. KIRWAN, Cleveland, $\mathrm{OH}$

Fetuin-A is a hepatokine associated with non-alcoholic fatty liver disease (NAFLD) and type 2 diabetes. Reductions in body weight, hepatic triglyceride content (HTGC) and skeletal muscle insulin resistance are related to lower circulating fetuin-A levels. However, the effect of exercise on fetuin-A independent of weight loss and lower HTGC is unknown. The purpose of this study was to determine the effect of short-term exercise training, during which we expected no change in body weight or HTGC, on plasma fetuinA concentrations. Thirteen $(50.5 \pm 3.4 \mathrm{yr})$ obese adults $(\mathrm{BMI}=33.3 \pm 0.9$ $\mathrm{kg} / \mathrm{m} 2$ ) with NAFLD underwent 7-days of exercise training (60 $\mathrm{min} / \mathrm{d}$ at $~ 85 \%$ HRmax). After an overnight fast, an oral glucose tolerance test was conducted and blood was collected at $0,30,60,90,120$ and 180 min before and after the intervention. The Matsuda Index and area under the curve (AUC) was used to assess insulin action and glucose tolerance. Magnetic Resonance Spectroscopy was used to determine HTGC before and after the intervention (subset $n=12$ ). Exercise had no effect on body weight ( $97.1 \pm 4.5$ vs. $97.7 \pm 4.6$ $\mathrm{kg} ; \mathrm{p}<0.12),(21.8 \pm 4.3$ vs. $21.2 \pm 4.1 \% ; p<0.73)$, or aerobic fitness $(24.9 \pm 1.9$ vs. $26.3 \pm 1.8 \mathrm{mg} / \mathrm{kg} / \mathrm{min} ; \mathrm{p}<0.14)$. Exercise increased insulin action by $25 \%$ $(1.6 \pm 0.3$ vs. $1.9 \pm 0.3 ; p<0.05)$ and decreased glucose AUC by $13 \%(34798.5 \pm$ 2068.4 vs. $31530.5 \pm 1656.5 \mathrm{mg} / \mathrm{dl}-180 \mathrm{~min} ; \mathrm{p}<0.01)$. Importantly, exercise decreased fetuin-A concentrations by $10 \%(0.66 \pm 0.05 \mathrm{vs} .0 .56 \pm 0.03 \mathrm{~g} / \mathrm{L}$; $p<0.02)$ and this change correlated with reduced glucose AUC $(r=0.58 ; p<$ 0.04 ). Independent of weight loss and HTGC, our findings indicate that lowering fetuin-A may, in part, contribute to the exercise-induced improvement in glucose tolerance. Based on our ongoing work, we speculate that decreased fetuin-A may improve glucose regulation by accentuating skeletal muscle insulin signaling.

Supported by: NIH (R01 AG12834 and UL1-RR024989)

183-0R

Effect of Acute Induction of Insulin Resistance on Exercise Parameters

IRENE E. SCHAUER, LEAH HERLACHE, JUDITH G. REGENSTEINER, JANE E. REUSCH, Aurora, CO

People with type 2 diabetes are sedentary despite the known association between fitness and mortality. One factor may be the decreased functional exercise capacity associated with insulin resistance (IR) observed in people with diabetes. We hypothesized that acute induction of IR in lean young individuals would reproduce the exercise defects in diabetes attributable to IR. We recruited 18 young $(29.4 \pm 6.1$ yo), lean (BMI $23.3 \pm 4.4)$, and sedentary to moderately active subjects with no evidence of IR. Subjects received infusions of saline vs low $(45 \mathrm{ml} / \mathrm{hr})$ and/or high dose $(90 \mathrm{ml} / \mathrm{hr})$ $20 \%$ lipid emulsion/heparin to induce IR. Insulin sensitivity (hyperinsulinemic euglycemic clamp), endothelial function (brachial artery flow-mediated dilation), blood flow (venous plethysmography), and exercise parameters (bicycle ergometry) were measured after 6-8 hours of infusion. Lipid infusion elevated fatty acid levels ( $381 \pm 162$ vs $1094 \pm 222$ vs $2496 \pm 425 \mu \mathrm{M}$ ) and induced IR (glucose infusion rate: $8.7 \pm 2.9$ vs $6.5 \pm 2.2$ vs $5.4 \pm 1.8 \mathrm{mg} / \mathrm{kg} /$ $\min , p<0.0001)$ as expected. No significant defects were noted in blood flow or endothelial function after lipid infusion. Peak exercise capacity was also unaffected $\left(\mathrm{VO}_{2}\right.$ peak $30 \pm 6$ vs $31 \pm 5$ vs $\left.31 \pm 6 \mathrm{ml} / \mathrm{kg} / \mathrm{min}, \mathrm{p}=0.91\right)$. However, oxygen uptake kinetics were slowed (tau2 $=30 \pm 9$ vs $35 \pm 12 \mathrm{sec}$ (combined lipid data), $\mathrm{p}=0.027$ ) and heart rate at submaximal exercise was increased (at lactate threshold: $115 \pm 15$ vs $125 \pm 17, p=0.007 ; 127 \pm 14, p=0.001$ ). Exercise parameters were significantly correlated with GIR at baseline. $\mathrm{VO}_{2}$ peak correlated positively $\left(R^{2}=0.44, p=0.016\right)$ and tau2 negatively $\left(R^{2}=0.41\right.$, $p=0.019$ ). In summary, acute induction of IR with lipid infusion is insufficient to fully recapitulate the substantial exercise defects observed in diabetes. IR does however impair submaximal exercise performance, cardiac efficiency (increased HR/workload) and the ability to transition to increased oxygen demand. These data support a relationship between IR and specific defects in exercise performance.

Supported by: VA Merit Award

184-OR

Resistance Exercise in Already-Active Type 1 Diabetic Individuals: The READI Trial

RONALD J. SIGAL, GLEN P. KENNY, BRUCE A. PERKINS, MICHAEL C. RIDDELL, GARY S. GOLDFIELD, REJEANNE GOUGEON, LOIS DONOVAN, JANINE MALCOLM, FARAH KHANDWALA, THE READI TRIAL INVESTIGATORS, Calgary, $A B$, Canada, Ottawa, ON, Canada, Toronto, ON, Canada, Montreal, OC, Canada, Winnipeg, MB, Canada

The Resistance Exercise in Already-active Diabetic Individuals (READI) trial was undertaken to determine the effects of resistance exercise (weight lifting) on A1C (primary outcome), body composition, lipids and hypoglycemia in physically active type 1 diabetic (T1D) adults. We recruited subjects who habitually performed $\geq 90 \mathrm{~min} /$ wk of vigorous aerobic exercise, or $\geq 150$ $\mathrm{min} / \mathrm{wk}$ of moderate-to-vigorous aerobic exercise. Before randomization, we stabilized subjects on therapy including multiple-dose insulin or insulin pump, carbohydrate $(\mathrm{CHO})$ counting and frequent glucose monitoring. 131 subjects [49M/82F, age $37 \pm 12(S D) y r, A 1 C 7.9 \pm 0.8 \%]$, were randomized to 22 wks of resistance exercise training (RT, $n=71$ ), 3 sets of 8 exercises at 8 rep max $3 X /$ wk or control $(C$, no resistance exercise, $n=60)$; both groups continued their usual aerobic exercise. Exercise training took place at local gyms, supervised by personal trainers. Pre-exercise $\mathrm{CHO}$ supplements and post-randomization insulin adjustments were done according to prespecified protocols. Analyses were intention-to-treat, using mixed model repeated measures. RESULTS: Strength increased significantly more in RT than $C(p<0.001$ for each strength exercise). There were no significant intergroup differences in $\mathrm{A} 1 \mathrm{C}$ change $(-0.10 \%$ in $\mathrm{RT},-0.07 \%$ in $\mathrm{C})$, lipids, or hypoglycemia frequency. There were 5 cases of hypoglycemia requiring assistance during the intervention period ( 3 in RT, 2 in control). The RT group had a significantly greater decrease in waist circumference than $C(-2.0 \mathrm{~cm}$ vs. $-0.5 \mathrm{~cm}, p=0.023$ between groups). Therefore, in people with T1D who were already aerobically active, the addition of resistance training increased strength and decreased waist circumference, but did not change A1C, lipids, or frequency of hypoglycemia.

Supported by: CIHR (MCT-80682)

185-OR

\section{WITHDRAWN}

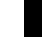


186-OR

Contraction Stimulates IL-6 Release from Skeletal Muscle Fibers via an $A M P K \alpha 2$ Independent Mechanism

HANS P. LAURITZEN, JOSEF BRANDAUER, PETER SCHJERLING, HENRIK GALBO, LAURIE J. GOODYEAR, Boston, MA, Gettysburg, PA, Copenhagen, Denmark

Recent studies suggest that Interleukin 6 (IL-6) is released from contracting muscles during exercise. However, the precise cellular origins of IL-6 within the muscle, the secretion kinetics, and the signaling mechanisms regulating secretion are not known. The aims of this study were to: 1) determine IL-6 protein level and localization in rested and contracted intact mouse muscle fibers; 2) determine the kinetics of contraction-induced IL-6 secretion in living muscle fibers in situ; and 3) determine if AMPK activation and increased intracellular calcium cause IL-6 secretion in living muscle fibers in situ. Confocal imaging was used to visualize endogenous IL-6 protein in fixed muscle fibers. In addition, intact quadriceps muscles were transfected in situ with EGFP-tagged IL- 6 and the kinetics of IL-6 secretion was determined in response to 25 min of in situ contractions, AICAR (AMPK activator), or caffeine (Ca2+ release agent). Under basal conditions IL-6 protein was found in small vesicle structures distributed throughout the muscle fibers. Contractions reduced IL- 6 containing vesicles by $62 \%(p<0.05)$. Kinetic image analysis of IL-6-EGFP vesicles in muscle fibers in vivo showed that contraction-induced IL-6 reduction occurred gradually over a 25 min contraction period. Similar to contraction, AICAR (iv: $1 \mathrm{gr} / \mathrm{kg}$ ) resulted in a $45 \%$ decrease of IL-6-EGFP vesicles; however, contraction-mediated IL-6-EGFP reduction was normal in muscle specific AMPK $\alpha 2$ inactive transgenic mice. Caffeine had no effect on IL-6-EGFP reduction. In conclusion, resting skeletal muscle fibers contain a depot of IL-6 containing vesicles. AICAR and muscle contractions gradually reduce IL- 6 protein in the muscle fibers, although the effect of contraction to release IL-6 occurs through an AMPK $\alpha 2$-independent mechanism.

Supported by: NIH (R01AR45670 and R01DK68626)

\section{PREGNANCY AND NEW METABOLIC MARKERS- IMPACT ON MATERNAL FETAL HEALTH}

187-OR

Higher Carbohydrate vs. Higher Fat Diet in Gestational Diabetes: A Randomized Study

TERI L. HERNANDEZ, RACHAEL E. VANPELT, MOLLY A. KRAUSE, MELANIE S. REECE, WILLIAM T. DONAHOO, ARCHANA MANDE, JACOB E. FRIEDMAN, LINDA A. BARBOUR, Aurora, CO, Denver, CO

The practice of advising a low carbohydrate (carb), thus higher fat, diet in the treatment of gestational diabetes mellitus (GDM) is controversial given data that maternal lipids influence fetal growth. We tested the hypothesis that a higher complex carb/lower fat diet (HC/LF) would result in higher postprandial (PP) glucose (BG), but an overall 24-hr BG area-under-the-curve (AUC) similar to a low carb/higher fat diet (LC/HF), with lower postprandial lipids. In a randomized cross-over study, women with diet-controlled GDM consumed a prepared $\mathrm{HC} / \mathrm{LF}(60 \% \mathrm{CHO}, 25 \%$ fat, $15 \%$ protein) and a LC/HF $(40 \%, 45 \%, 15 \%)$, equivalent in calories, for 3 days each (washout in between) while wearing a continuous glucose monitor (CGM). On day 4 of each, PP lipemia was measured hourly after breakfast ( $30 \%$ of total daily calories). In 10 women (Mean \pm SEM; BMI $33.6 \pm 1.5 \mathrm{~kg} / \mathrm{m}^{2} ; 30.4 \pm 0.5$ wks gestation), the $\mathrm{HC} / \mathrm{LF}$ resulted in modestly higher 1- and 2-hr PP BG compared to the LC/HF (CGM; $115 \pm 3$ vs $106 \pm 3, p=0.009$, and $108 \pm 5$ vs $98 \pm 3 \mathrm{mg} / \mathrm{dL}, p=0.01$, respectively, Fig 1A); all values were lower than recommended targets. The 24-hr BG AUC was slightly higher on the HC/LF diet $(p<0.005)$, but there was no difference in nocturnal or fasting $B G$ and the $B G$ patterns were remarkably similar. Although both diets were low in saturated fat, the PP 5-hr FFA AUC was higher on the $\mathrm{LC} / \mathrm{HF}$ diet ( $p=0.005$, Fig $1 \mathrm{~B}$ ) potentially worsening maternal insulin resistance and providing excess fuel for fetal growth. These pilot data from the first RCT of its kind require extension until delivery to discern whether the conventional GDM diet could have unintended metabolic consequences in the mother and offspring.
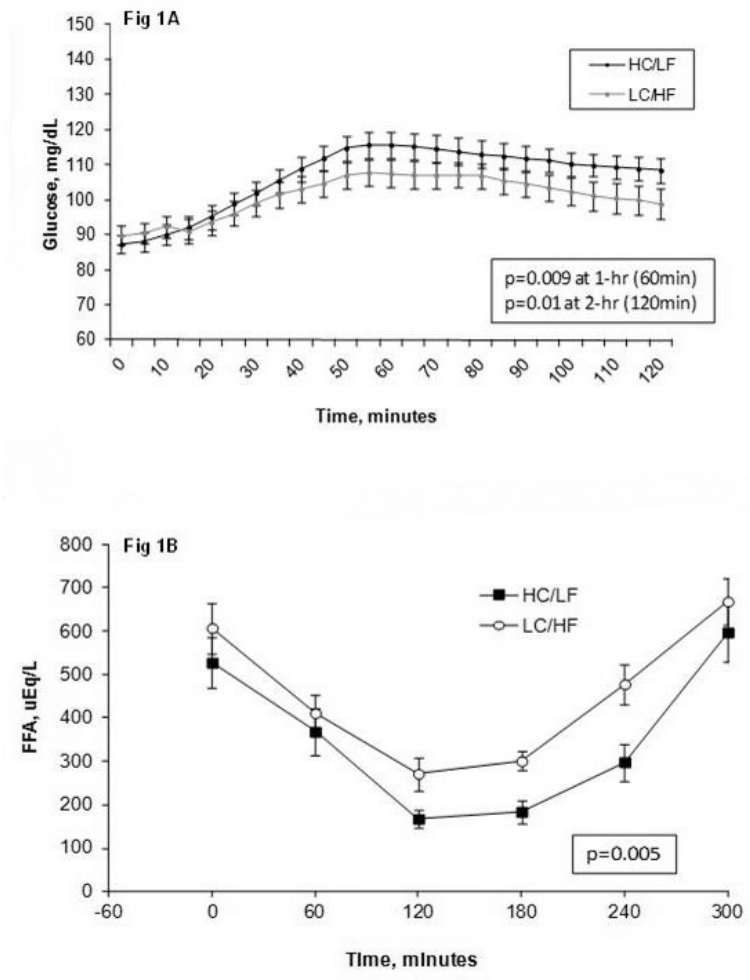

188-OR

Plasma HDL is Associated With Endothelium-Dependent Microvascular Function in Pregnancy and is Reduced in Maternal Obesity DILYS J. FREEMAN, ELEANOR M. JARVIE, MURIEL J. CASLAKE, BRUCE A. GRIFFIN, Glasgow, United Kingdom, Guildford, United Kingdom

Maternal vascular function is improved in healthy pregnancy and compromised in obesity and obesity-associated pregnancy complications such as gestational diabetes mellitus. Cardiovascular research shows that HDL protects vascular function via anti-oxidant and anti-inflammatory mechanisms. Our aim was to assess whether HDL might protect vascular function in pregnancy and whether this is compromised in maternal obesity in a longitudinal study of 55 mothers with a range of BMI $(18-46 \mathrm{~kg} / \mathrm{m} 2)$ sampled at 12,25 \& 35 weeks' gestation and 17 weeks post partum. HDL cholesterol levels were $0.19 \mathrm{mmol} / \mathrm{L}$ higher by 12 weeks compared to non-pregnant levels $(P<0.001)$ and were maintained throughout pregnancy. Maternal BMI had a significant impact on $\mathrm{HDL}$ levels $(\mathrm{P}<0.001)$ with lean individuals reaching a maximum of 1.51 [0.42] and obese 1.36 [0.29] mmol/L. We have previously shown in these subjects that endothelium-dependent microvascular function (EDMVF) as assessed by laser Doppler iontophoresis is significantly lower in obese compared to non-obese women. Across all time points HDL was correlated with EDMVF $(r=0.24, P<0.001)$ and the gestational change in HDL explained $18 \%(P=0.013)$ of the variance in gestational change in EDMVF independent of maternal age, BMI, parity and smoking. Plasma markers of inflammation (IL-6, TNFalpha, CRP) and endothelial activation (sICAM-1, sVCAM-1, PAI-1) were not associated with plasma HDL levels. The gestational change in lipoprotein lipase (LPL) mass was positively correlated with the change in HDL $(r=0.55, P<0.001)$ and negatively with change in very low density lipoprotein (VLDL)-2 triglyceride $(r=-045, P=0.010)$ consistent with current knowledge that LPL activity generates much of the lipid that forms HDL. The relationship between plasma HDL levels and markers of oxidation are under investigation. HDL may afford vascular protection during pregnancy but we provide no evidence that this is via anti-inflammatory mechanisms.

Supported by: Wellbeing of Women, BMA OHRT 
189-0R

Assessment of Insulin and Adiponectin in Human Milk in Relation to Gestational Hyperglycemia and Insulinemia and to Milk Cortisol SYLVIA H. LEY, DEBORAH L. O'CONNOR, MATHEW SERMER, BERNARD ZINMAN, ANTHONY J. HANLEY, Toronto, ON, Canada

Insulin and adiponectin are known to be present in human milk, but the impact of maternal metabolic status during pregnancy on these milk hormones is unclear. Adiponectin concentrations in cord blood are 2-3 fold higher than those in normal-weight non-pregnant adults although the role of adiponectin in the early postpartum is unknown. Recent in vitro evidence demonstrates that adiponectin stimulates steroidogenic gene expression and cortisol secretion. We, therefore, aimed to investigate the associations of gestational metabolic status with insulin and adiponectin in human milk and to study the correlations of these hormones with milk cortisol. Pregnant women aged $\geq 20$ years, with intention to breastfeed and without pre-existing type 1 or type 2 diabetes were recruited. Participants $(n=170)$ underwent a 3-hour oral glucose tolerance test at $30(95 \% \mathrm{Cl} 25,33)$ weeks gestation and donated early (1-week postpartum) and mature (3-month postpartum) milk. Gestational hyperglycemia (beta $\pm S E 0.218 \pm 0.087, p=0.01)$, insulin resistance $(0.255 \pm 0.047$, $p<0.0001)$, lower insulin sensitivity $(-0.521 \pm 0.108, p<0.0001)$, and higher serum adiponectin $(0.116 \pm 0.029, p<0.0001)$ were associated with higher insulin concentrations in mature milk with covariate adjustment. Obstetrical measures including nulliparity $(0.171 \pm 0.058, p=0.004)$, longer duration of gestation $(0.546 \pm 0.146, p=0.0002)$, and unscheduled C-section $(0.387 \pm 0.162$, $\mathrm{p}=0.02$ ) were associated with higher adiponectin in early milk. In early postpartum, milk adiponectin was positively correlated with milk cortisol $(r=0.53$, $p<0.0001)$, while it was inversely correlated with glucose $(r=-0.47, p<0.0001)$ and statistically not correlated with insulin in milk. In conclusion, gestational metabolic abnormalities are associated with higher insulin in mature milk. In the early postpartum, milk adiponectin is associated with obstetrical parameters and is positively correlated with milk cortisol.

Supported by: CDA, CFDR, CIHR

190-OR

Infants of Women With Gestational Diabetes (GDM) are more Insulin Resistant as Compared With Infants from a Matched Control Group PATRICK CATALANO, LARRAINE PRESLEY, ALICIA THOMAS, SAEID AMINI, Cleveland, $\mathrm{OH}$, Washington, $\mathrm{DC}$

Offspring of women with GDM are at increased risk for childhood obesity and metabolic dysfunction. The purpose of this study was to determine whether at birth the neonates of women with GDM, in addition to increased adiposity, have increased insulin resistance in comparison to neonates of a matched group of women with normal glucose tolerance (NGT). Thirty women with GDM were matched in a 1 to 3 with women $(n=90)$ with NGT for age, pre-pregnancy BMI, race, gestational weight gain, gestational age at delivery and neonatal gender at the time of scheduled cesarean delivery. Maternal and umbilical cord blood was obtained for measures of insulin resistance. Neonatal body composition was estimated using anthropometrics and air densistometry (Pea Pod). There was no significant difference in (mean \pm SD) maternal age $(28.3 \pm 5.8$ vs. $28.9 \pm 5.8$ yrs, $p=0.59)$, pre-pregnancy BMI $(34.1+8.2$ vs. $34.4+7.9, p=0.93)$, race $(47 \%$ Black, $37 \%$ White $16 \%$ Hispanic, $p=1.0)$, gestational weight gain $(14.3 \pm 7.7$ vs. $14.1 \pm 6.8 \mathrm{~kg}$ $p=0.89)$ and gestational age $(38.8 \pm 0.7$ vs. $38.5 \pm 0.7$ wks, $p=0.09)$ between NGT and GDM groups. GDM mothers had greater HOMA-IR compared to the NGT $(8.6 \pm 7.4$ vs. $4.6 \pm 3.1, p=0.0001)$. The neonates of the GDM had greater birth weight ( $3637 \pm 498$ vs. $3369 \pm 553 \mathrm{~g}, \mathrm{p}=0.02$ ), lean mass (3082 \pm 315 vs. $2917 \pm 373 \mathrm{~g}, p=0.03)$, fat mass $(555 \pm 206$ vs. $452 \pm 196 \mathrm{~g}, \mathrm{p}=0.02)$ percent body fat $(14.8 \pm 3.9$ vs. $13.6 \pm 3.7 \%, p=0.02)$ and HOMA IR $(4.2 \pm$ 4.7 vs.1.5 $\pm 1.0, p=0.0001$ ) as compared with the NGT. When adjusted for neonatal birth weight, fat and lean mass, the infants of the GDM persisted in having greater HOMA IR $(\mathrm{p}=0.0001)$ as compared with the neonates of the NGT. In conclusion, although maternal obesity is in itself a risk factor for neonatal adiposity and insulin resistance as compared with neonates of normal weight women, GDM is an independent and additional risk factor for both neonatal adiposity and insulin resistance when compared with a matched control NGT group.

Supported by: NICHD 22965
191-OR

The Determinants of Insulin Resistance in Infants at 1 Year of Age: Impact of Gestational Diabetes Mellitus (GDM)

CARLA A. BORGONO, JILL K. HAMILTON, CHANG YE, ANTHONY J. HANLEY, PHILIP W. CONNELLY, MATHEW SERMER, BERNARD ZINMAN, RAVI RETNAKARAN, Toronto, ON, Canada

The offspring of women with GDM display a propensity for the early accrual of cardio-metabolic risk factors, including insulin resistance, by as early as age 5 years. Thus, we sought to identify early life determinants of insulin resistance in infants of women with GDM and their peers. In this study, 104 full-term, singleton infants born to women with $(n=36)$ and without GDM $(n=68)$ underwent fasting bloodwork at age 1-yr, with insulin resistance measured by homeostasis model of assessment (HOMA-IR). At 1-yr, HOMAIR did not differ between infants born to mothers with and without GDM (median 0.21 vs $0.21, p=0.74$ ). On Spearman correlation (Table), birthweight was associated with HOMA-IR in infants of women without GDM but not in the GDM group. Conversely, weight gain in the $1^{\text {st }}$ year was associated with HOMA-IR in infants whose mothers had GDM but not in the nonGDM group. On multiple linear regression analyses (with covariates age, gender, ethnicity, breastfeeding, and maternal BMI, insulin sensitivity and adipokines), the sole independent predictor of infant HOMA-IR in the nonGDM group was birthweight $(t=3.3, p=0.002)$. In contrast, weight gain in the $1^{\text {st }}$ year emerged as the only independent predictor of HOMA-IR in GDM infants $(t=2.2, p=0.04)$. In summary, unlike in their peers, weight gain in the $1^{\text {st }}$ year in infants born to women with GDM is associated with insulin resistance. This relationship between growth and insulin resistance potentially could contribute to their early accrual of cardio-metabolic risk.

\begin{tabular}{lllll}
\hline \multicolumn{4}{l}{ Spearman correlations with infant HOMA-IR } \\
\hline & NonGDM & GDM & \\
\hline & $\mathrm{r}$ & $\mathrm{p}$ & $\mathrm{r}$ & $\mathrm{p}$ \\
\hline Birthweight & 0.32 & 0.02 & -0.18 & 0.34 \\
\hline 1st year weight gain & 0.04 & 0.78 & 0.44 & 0.02 \\
\hline Duration breastfed & 0.03 & 0.81 & 0.02 & 0.93 \\
\hline Maternal prepreg BMI & 0.08 & 0.55 & -0.23 & 0.24 \\
\hline Maternal adiponectin & 0.09 & 0.50 & 0.17 & 0.37 \\
\hline Maternal CRP & 0.08 & 0.54 & -0.20 & 0.31 \\
\hline
\end{tabular}

Supported by: CIHR

192-OR

Evaluation of 1,5-Anhydroglucitol as Marker of Glycemic Control and Birth Weight in Pregnancy Complicated by Type 1 Diabetes Mellitus NATALIA NOWAK, KATARZYNA CYGANEK, BARTLOMIEJ MATEJKO, MACIEJ T. MALECKI, Krakow, Poland

1,5-anhydrogluctiol (1,5-AG) is a marker of short-term glycemic control in diabetes - it reflects a period of 1-2 weeks. Its excretion rate depends on the renal glucose threshold and thus it is not clear whether it may be used in women with type 1 diabetes mellitus (T1DM) during pregnancy. We tested pregnant women with T1DM to anwser a) what is the relationship between 1,5-AG level and glycemic control assessed by continuous glucose monitoring (CGM); b) whether this particle is a predictor of birth weight. We examined 79 pregnant women with T1DM (mean age: 29.3 years; $S D=4.2$, mean diabetes duration: $11.3 \mathrm{yrs} ; \mathrm{SD}=7.7)$. In each trimester, 1,5-AG concentration was measured with an immunoenzymatic assay. The data from CGM device were collected in 57 pregnancies for a 7-day period before blood collection for 1,5-AG and $\mathrm{HbA1C}$ analysis. The data were analized with Sperman rank correlation coefficients and multivariate regression. Receiver operating characteristic (ROC) curve analysis was used to evaluate third-trimester 1.5AG level as a predictor of large for gestational age (LGA) birth $\left(\geq 90^{\text {th }}\right.$ percentile). 1,5-AG correlated significantly with glycemic indices recorded by CGM: area under the curve at $140 \mathrm{mg} / \mathrm{dl}\left(\mathrm{r}=-0.68, \mathrm{p}=1 \times 10^{-7}\right)$, average maximal glucose $\left(r=-0.57, p=3.3 \times 10^{-5}\right)$, glucose standard deviation $(r=-0.61, p=2 \times 10$ $\left.{ }^{6}\right)$ and mean glucose $\left(r=-0.52, p=5.6 \times 10^{-5}\right)$. In multivariate linear regression, $1,5-A G$ was independently associated with the newborn weight $\left(p=1 \times 10^{-4}\right)$, this remained highly significant also after the incorporation of $\mathrm{HbA} 1 \mathrm{c}$ into the model $\left(p=8 \times 10^{-5}\right)$. The area under the 1,5-AG ROC curve in the LGA prediction was 0.76 (95\% Cl:0.63-0.86) at a criterion of $4.65 \mu \mathrm{g} / \mathrm{ml}$. In conclusion, 1,5$\mathrm{AG}$ is an accurate indicator of glycemic control as assessed by CGM in pregnant women with T1DM. In addition, its level in the $3^{\text {rd }}$ trimester could be prognostic for LGA. Thus, 1,5-AG use in glycemic control monitoring should be considered in this population.

Supported by: Grant of Polish Ministry of Science No. N N407 414436 
193-OR

Human Placental LPL Activity is Strongly Associated With Infant Adiposity at Birth

MARGARET J. HEERWAGEN, BECKY A. DELAHOUSSAYE, MOLLY A. KRAUSE, ANITA KRAMER, BARBRA FISHER, MELANIE S. REECE, VIRGINIA D. WINN, RACHEL VANPELT, DALAN R. JENSEN, JACOB E. FRIEDMAN, TERI L. HERNANDEZ, LINDA A. BARBOUR, Aurora, CO

The role of maternal lipids as a major contributor to fetal fat accretion is becoming increasingly recognized, but little is known about mechanisms regulating lipid transport across the placenta. Placental lipoprotein lipase $(\mathrm{LPL})$ is thought to be the major lipase involved in the uptake and delivery of maternal triglyceride (TG) to the fetus, however its activity in relation to infant adiposity is unstudied. Immediately after term delivery, placental samples were obtained in 8 normal glucose tolerant women (pre-pregnancy BMI 20-34) and villous heparin releasable LPL activity was measured using a ${ }^{14} \mathrm{C}$-triolene substrate. Results were normalized to an internal standard, and adjusted for gestational age. Infant adiposity at birth was determined by skin fold thickness at 2 days of age. Placental LPL activity, as determined by villous activity per gram multiplied by placental weight, was strongly associated with infant adiposity $\left(R^{2}=0.84 ; p=0.002\right)$, but not infant birth weight (BW). Placental weight alone was also associated infant adiposity $\left(R^{2}=0.71\right.$, $p=0.02)$, and as expected correlated with infant $B W(r=0.72, p=0.04)$. Villous LPL activity was most strongly associated with the change in maternal fasting insulin and the change in maternal insulin resistance (IR) (HOMA-IR) from early (14-16 wk) to late (37 wk) gestation $\left(R^{2}=0.83, p=0.02 ; R^{2}=0.89, p=0.017\right.$ respectively). This increase in maternal IR was also strongly correlated with late gestation levels of maternal fasting $T G(r=0.73,0.006)$. Our pilot data suggest that increased maternal insulin resistance and plasma insulin concentrations from early to late gestation may not only drive lipid substrate availability to the placenta, but may also increase the rate of substrate delivery to the fetus via placental TG hydrolysis. Corroboration of these findings in a larger group of women with obesity or gestational diabetes may provide a novel mechanism to explain excess infant adiposity in pregnancies characterized with and without nutritional excess.

Supported by: NIH (RO1 DK078645)

$\Delta$

194-OR

Genome-Wide Significant Associations With Maternal Glycemia During Pregnancy in Two Novel Genes: HKDC1 and BACE2

M.G. HAYES, DOUGLAS A. SCHEFTNER, JEAN MORRISON, DAVID LEVINE, CAITLIN P. MCHUGH, LYNN P. LOWE, ANNA PLUZHNIKOV, MARGRIT URBANEK, LOREN L. ARMSTRONG, CHRISTINE M. ACKERMAN, DANIEL B. MIREL, KIMBERLY F. DOHENY, ALAN R. DYER, BOYD E. METZGER, NANCY J. COX, WILLIAM L. LOWE, FOR THE HAPO STUDY COOPERATIVE RESEARCH GROUP, Chicago, IL, Seattle, WA, Cambridge, MA, Baltimore, MD

The multicenter, multiethnic and international Hyperglycemia and Adverse Pregnancy Outcome (HAPO) study examined associations of maternal glucose levels with fetal growth and outcomes in $>25,000$ pregnant women. Using Illumina 610 and $1 \mathrm{M}$ platforms, we conducted a genome-wide association study in 1,382 Northern European-ancestry, 847 Mexican American, 1,087 Afro-Caribbean, and 1,200 Thai HAPO mothers to determine genetic associations with maternal glycemia (fasting [FPG], 1-hr [1HPG], 2-hr [2HPG] plasma glucose) and insulin secretion (fasting [FCP] C-peptide) from an oral glucose tolerance test (OGTT) at $\sim 28$ weeks gestation. Associations were assessed with linear regression under an additive genetic model adjusting for confounders (mother's age, BMI, height, mean arterial pressure and gestational age at OGTT, parity, field center and ancestry) using genotyped and imputed SNPs (cosmopolitan HapMap II reference panel). Results from cohorts were then combined through meta-analyses weighting each stratum by sample size. We replicated known associations of PCSK1 (FPG), PPP1R3B (FPG, FCP), MTNR1B (FPG, 1HPG), and GCKR (FCP) and identified two novel genes associated at a genome-wide significance level of $p<5 \times 10^{-8}$. The lead SNP in HKDC1 (hexokinase domain containing 1), rs5030938, was associated with increased 2HPG (0.16-0.20 mg/dL per T allele; $\left.p=5.7 \times 10^{-15}\right)$, and found in a region of high $L D$ upstream from the first intron of $H K D C 1$. Based on sequence homology, $H K D C 1$ is predicted to have hexokinase activity. The other novel finding was rs6517656 in BACE2, which was associated with increased FCP $\left(0.05-0.15 \mu \mathrm{g} / \mathrm{L}\right.$ per $\mathrm{G}$ allele; $\left.\mathrm{p}=3.1 \times 10^{-8}\right)$. BACE2 has been shown to modulate glucose stimulated insulin secretion in human islets. These data demonstrate two new loci for metabolic traits and raise the possibility that some genetic loci different from those published in nonpregnant populations may impact glucose metabolism in pregnancy.

For author disclosure information, see page 797.
TYPE 1 DIABETES - 21ST CENTURY UPDATE ON INCIDENCE, CENTRAL NERVOUS SYSTEM EFFECTS, AND METABOLISM

195-OR

Trends in Incidence of Type 1 Diabetes Among Non-Hispanic White (NHW) Youth in the US, 2002-2009

JEAN M. LAWRENCE, RONNY A. BELL, DANA DABELEA, LAWRENCE M. DOLAN, GIUSEPPINA IMPERATORE, GEORGEANNA J. KLINGENSMITH， BARBARA LINDER, ELIZABETH MAYER-DAVIS, DAVID J. PETTITT, CATHERINE PIHOKER, SHARON SAYDAH, DEBRA A. STANDIFORD, JENNIFER W. TALTON, JOAN THOMAS, RALPH B. D'AGOSTINO JR., FOR THE SEARCH FOR DIABETES IN YOUTH STUDY GROUP, Pasadena, CA, Winston-Salem, NC, Aurora, CO, Cincinnati, OH, Atlanta, GA, Bethesda, MD, Chapel Hill, NC, Santa Barbara, CA, Seattle, WA, Hyattsville, MD

Although type 1 diabetes (T1D) incidence is increasing in several countries, US data are limited. The SEARCH Study identified persons newly-diagnosed with diabetes from 2002 onward using multiple sources and a consistent timeframe. We report trends in incidence of T1D among NHW youth age < 20 years in the US from between 2002 and 2009. Cases are NHW youth with physician-diagnosed T1D from centers in Ohio, Colorado, Washington, South Carolina, or California; denominators are age- and sex-specific estimates of year-end population using US census data $(\mathrm{OH}, \mathrm{CO}, \mathrm{WA}, \mathrm{SC})$ and membership counts (CA). Age- and sex- specific T1D incidence rates per 100,000 and $95 \%$ $\mathrm{Cl}$ were calculated from pooled data. Poisson regression was used to test for trends in incidence. In total, 5,796 NHW youth with T1D (range 668-780/ year) were identified from $\approx 2.9$ million NHW youth annually. Case completeness was $>96 \%$ and stable over time. The incidence of T1D was $24.1 \mathrm{l} 95 \% \mathrm{Cl}$ 22.4-25.9) in 2002 and 27.2 (95\% Cl 25.4-29.2) in 2009 (Figure). After adjusting for age and sex, a significant increase in T1D incidence was observed (2.7\% relative increase/year, $p<0.002$ ). In age-specific models adjusted for sex, incidence increased in $5-9(p<0.02)$ and $10-14$ year olds $(p<0.001)$ but not in 0-4 $(p<0.20)$ or $15-19$ year olds $(p<0.08)$. Among 5-9 year olds, the trend was significant for males $(p<0.008)$ but not females $(p<0.06)$; for $10-14$ year olds, it was significant for both sexes $(p<0.02$ and $p<0.001$, respectively). More time may be needed to detect trends for lower incidence age groups. Our findings suggest an overall increase in T1D incidence from 2002-2009 among US NHW youth.

Trends in Incidence of Type 1 Diabetes among NHW Youth, by Age Group, 2002-2009

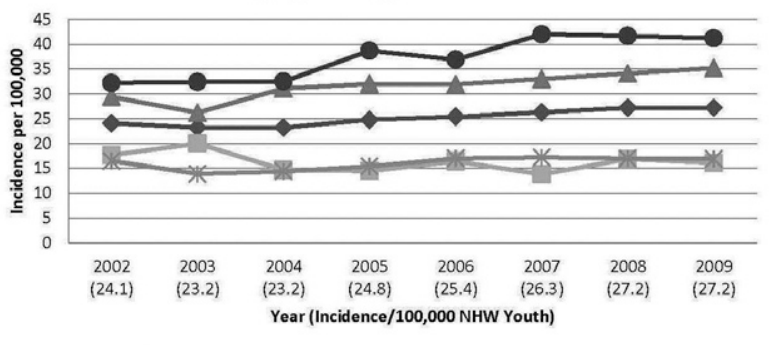

$\rightarrow$ All Youth $\rightarrow-0-4$ yrs $\rightarrow 5-9$ yrs $\rightarrow$ 10-14yrs $\rightarrow-15-19$ yrs

Supported by: The CDC with support from the NIDDK

196-OR

Dysregulation of $\alpha$-cell Function in Youth During the First 2 Years of T1D: Reciprocal Abnormalities in Glucagon Responses to Mixed Meal Feedings and Hypoglycemia

JENNIFER SHERR, EVA TSALIKIAN, LARRY FOX, BRUCE BUCKINGHAM, STUART WEINZIMER, WILLIAM TAMBORLANE, NEIL H. WHITE, ANA MARIA ARBELAEZ, CRAIG KOLLMAN, DONGYUAN XING, KATRINA RUEDY, DIABETES RESEARCH IN CHILDREN NETWORK (DIRECNET), New Haven, CT, lowa City, IA, Jacksonville, FL, Stanford, CA, St. Louis, MO, Tampa, FL

Inappropriate increases in glucagon with meals and reduced glucagon responses to hypoglycemia play important roles in the wide swings in glucose in youth with Type 1 diabetes (T1D). Little is known about the evolution of these reciprocal abnormalities in $\alpha$-cell function early in the course of T1D. Mixed meal tolerance tests (MMTT) and hypoglycemic clamps (glucose lowered to $55 \mathrm{mg} / \mathrm{dl}$ ) were performed in 25 youth with T1D (9-18 yrs) who had a 1.5-12 months duration of T1D(Year 1); studies were repeated 1 year 
later (Year 2) in 17 subjects. MMTT were performed in 20 age and gendermatched non-T1D children and hypoglycemic clamps in 12 non-T1D adults (19-25 yrs). A response was defined as a rise in plasma glucagon $\geq 11 \mathrm{pg} /$ $\mathrm{ml}$ (the lowest value in non-T1D). MMTT-stimulated increases in glucagon were greater in T1D than in non-T1D subjects during Year 1 and 2 with twice as many T1D subjects achieving increases of $\geq 11 \mathrm{pg} / \mathrm{ml}(\mathrm{p} \leq 0.001$ in Year 1 and 2 vs. ND)(Table). Conversely, glucagon responses to hypoglycemia in T1D subjects was much lower than in non-diabetic controls ( $p=0.02$ in Year 1 vs. $\mathrm{ND}, \mathrm{p}=0.004$ in Year 2 vs. ND). By Year 2, only $60 \%$ of T1D subjects were able to achieve $\mathrm{a} \geq 11 \mathrm{pg} / \mathrm{ml}$ rise in glucagon. The rise in glucagon during the MMTT in the T1D subjects in Year 2 approached the response to hypoglycemia in adult controls. These data suggest that progressive dysregulation of $\alpha$-cell function contributes to increasing problems with hyper-, as well as hypoglycemia with increasing duration of T1D.

\begin{tabular}{|c|c|c|c|c|c|c|}
\hline $\begin{array}{l}\text { Plasma } \\
\text { Glucagon } \\
\text { Levels } \\
\text { (pg/ml) }\end{array}$ & $\begin{array}{l}\text { MMTT } \\
\text { T1D } \\
\text { Year1 } \\
\mathrm{N}=25\end{array}$ & $\begin{array}{c}\text { MMTT } \\
\text { T1D } \\
\text { Year } 2 \\
\mathrm{~N}=17\end{array}$ & $\begin{array}{c}\text { MMTT } \\
\text { Non-diabetic } \\
\text { Children } \\
\mathrm{N}=20\end{array}$ & $\begin{array}{c}\text { Hypo Clamp } \\
\text { C1D } \\
\text { Year } 1 \\
\mathrm{~N}=22\end{array}$ & $\begin{array}{c}\text { Hypo Clamp } \\
\text { T1D } \\
\text { Year } 2 \\
\mathrm{~N}=15\end{array}$ & $\begin{array}{c}\text { Hypo Clamp } \\
\text { Non-diabetic } \\
\text { Adults } \\
\mathrm{N}=12\end{array}$ \\
\hline $\begin{array}{l}\text { Baseline } \\
\text { median } \\
\left(25^{\text {th }}, 75^{\text {th }}\right)\end{array}$ & $44(34,53)$ & $43(41,51)$ & $50(46,57)$ & $34(24,38)$ & $36(31,47$ & $43(35,54)$ \\
\hline $\begin{array}{l}\text { Peak median } \\
\left(25^{\text {th }}, 75^{\text {th }}\right)\end{array}$ & $59(52,79)$ & $64(59,81)$ & $64(52,73)$ & $50(37,72)$ & $49(42,66)$ & $93(60,111)$ \\
\hline $\begin{array}{l}\text { Rise Median } \\
\left(25^{\text {th }}, 75^{\text {th }}\right)\end{array}$ & $21(16,30)$ & $25(16,27)$ & $9(5,16)$ & $19(7,32)$ & $12(8,18)$ & $38(19,66)$ \\
\hline $\begin{array}{l}\% \text { subjects } \\
\text { with rise in } \\
\text { glucagon } \\
\geq 11 \mathrm{pg} / \mathrm{ml}\end{array}$ & 88 & 94 & 40 & 73 & 60 & 100 \\
\hline
\end{tabular}

197-0R

Estimated Insulin Sensitivity and Cardiovascular Disease Risk Factors in Adolescents With and Without Type 1 Diabetes

BRIAN J. SPECHT, R.P. WADWA, JANET K. SNELL-BERGEON, FRANZISKA K BISHOP, DAVID M. MAAHS, Aurora, CO

Cardiovascular disease (CVD) is the primary cause of death in type 1 diabetes (T1D), with many risk factors beginning in adolescence, including reduced insulin sensitivity (IS). We tested the hypotheses that: 1) CVD risk factors are similar in non-DM as compared to T1D adolescents in the most IS tertile and 2) CVD risk factors are more atherogenic with increasing IS in T1D adolescents. IS for adolescents with T1D ( $n=290$; age $15.4 \pm 2.1$ years; duration $8.8 \pm 3.0$ years, $A 1 \mathrm{c}=8.9 \pm 1.6 \%$ ) and non-DM controls ( $\mathrm{n}=89$; age $15.4 \pm 2.1$ years) was determined using the model: $\log _{\mathrm{e}} \mathrm{IS}=4.64725-0.02032$ (waist, cm) - 0.09779(A1c, \%) - 0.00235(TG, mg/ dI). The T1D adolescents were divided into IS tertiles (IS $\leq 6.84$, IS 6.84 8.64 , and IS $\geq 8.64$ ). CVD risk factors were compared between all non-DM and T1D adolescents in the most IS tertile and then examined for a linear trend by IS tertile in T1D adolescents, adjusted for sex, race/ethnicity and Tanner Stage. IS was significantly different in non-DM vs T1D adolescents (non-DM=11.5 $\pm 2.9, T 1 D=7.8 \pm 2.4 ; \mathrm{p}<0.0001$ ). $\mathrm{CVD}$ risk factors were similar for non-DM as compared to the most IS T1D adolescents, except for higher HDL-c and DBP and lower TG in T1D adolescents $(p<0.05)$. Among adolescents with T1D, all CVD risk factors except for HDL-c, were more atherogenic in T1D adolescents in decreasing IS tertiles in linear regression (Figure).

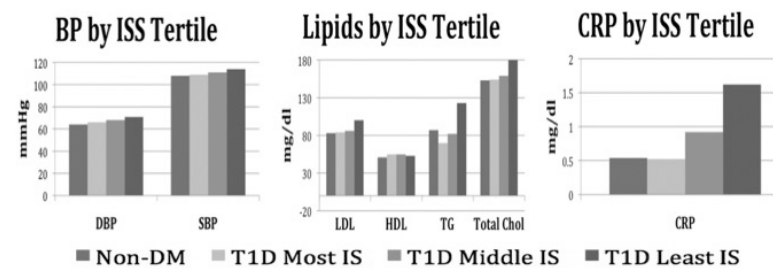

T1D adolescents who are the most IS have similar CVD risk factors compared to non-DM adolescents. CVD risk factors are inversely associated with IS in T1D adolescents. IS may be an important therapeutic target for reducing CVD risk in adolescents with T1D.

Supported by: NIDDK, JDRF
198-OR

Cognitive and Behavioral Differences in Young Children With Type 1 Diabetes Mellitus: Preliminary Results

TAMARA HERSHEY, ALLISON CATO, AIDEN BONDURANT, TANDY AYE, AMY CONRAD, JODIE AMBROSINO, NEIL H. WHITE, NELLY MAURAS, CRAIG KOLLMAN, KATRINA RUEDY, DONGYUAN XING, DIABETES RESEARCH IN CHILDREN NETWORK (DIRECNET), St. Louis, MO, Jacksonville, FL, Stanford, CA, lowa City, IA, New Haven, CT, Tampa, FL

Retrospective studies of cognitive function in type 1 diabetes mellitus (T1DM) commonly find that diagnosis during early childhood is associated with poorer neuropsychological outcome. The objective of this preliminary analysis was to determine whether there are neuropsychological differences between young children with T1DM and non-diabetic controls. Five Direc Net sites tested 128 children aged 4 to 9 years (T1DM, $n=73$, median (interquartiles) diabetes duration $=2.3(1.1,4.2)$ years; Controls, $n=55)$ on standard neuropsychological tests. Age and gender distribution were not different across the two groups (T1DM: mean (SD) age=6.5(1.2), 51\% female; Control: $6.4(1.4), 58 \%)$. Twelve (22\%) controls were siblings of T1DM participants. A parent filled out questionnaires about their child's behavior and performed a brief intelligence test. Cognitive domain z scores (Delayed Memory, Executive Function, Working Memory \& Attention, Processing Speed, I0) were calculated from individual tests and Internalizing and Externalizing scores were derived from the parent questionnaires. The T1DM group had significantly lower IO ( $p=0.01)$, and Executive Function, Working Memory \& Attention ( $p=0.02$ ) than the non-diabetic control group, and had more Internalizing behavioral problems than Controls $(p=0.001)$. These preliminary findings suggest that certain cognitive and behavioral differences can appear quite early in T1DM. Future work will determine if these differences change over time and whether or not they are related to exposure to severe hypoglycemia or chronic hyperglycemia.

\begin{tabular}{llcc}
\hline & $\begin{array}{c}\text { T1DM } \\
\mathbf{N}=73 \\
\text { Mean } \pm \text { SD }\end{array}$ & $\begin{array}{c}\text { Control } \\
\mathbf{N = 5 5} \\
\text { Mean } \pm \text { SD }\end{array}$ & $\begin{array}{c}\text { P-value } \\
\text { (P-value from } \\
\text { repeated measures } \\
\text { least square } \\
\text { regression model, } \\
\text { adjusted for sib- } \\
\text { lings from same } \\
\text { family, age, gender } \\
\text { and parent I0.) }\end{array}$ \\
\hline Delayed Memory & $+0.08 \pm 1.00$ & $-0.11 \pm 0.93$ & 0.15 \\
\hline Executive function/ & $-0.11 \pm 0.88$ & $+0.14 \pm 1.07$ & 0.02 \\
Working memory/Attention & $-0.04 \pm 1.01$ & $+0.05 \pm 0.93$ & 0.85 \\
\hline Processing Speed & $-0.20 \pm 0.98$ & $+0.27 \pm 0.90$ & 0.01 \\
\hline Child IO & $+0.12 \pm 0.91$ & $-0.17 \pm 1.03$ & 0.12 \\
\hline $\begin{array}{l}\text { BRIEF (Behavior } \\
\text { Rating by Parent) }\end{array}$ & $+0.04 \pm 0.94$ & $-0.05 \pm 1.02$ & 0.73 \\
\hline $\begin{array}{l}\text { Externalizing (Behavior } \\
\text { Assessment by Parent) }\end{array}$ & $+0.21 \pm 0.87$ & $-0.29 \pm 1.03$ & 0.001 \\
\hline Internalizing (Behavior & & & \\
Assessment by Parent) & & & \\
\hline
\end{tabular}

199-OR

Glucose Variability is Associated With Simultaneous EEG Changes in Youth With Type 1 Diabetes Mellitus

MARIANNA RACHMIEL, MICHAL COHEN, MIRIT LEZINGER, DORIT INBAR, ELI HEYMEN, SHLOMO GILAT, RONY BRAUNSHTEIN, ELI LAHAT, DANA EKSTEIN, Zerifin, Israel, Tel Aviv, Israel, Jerusalem, Israel

Type 1 diabetes mellitus (T1DM) may cause various neurologic abnormalities. Electroencephalographic (EEG) abnormalities in diabetic children were found to be acquired, and apparently produced by a metabolic disturbance. Controversy remains about whether abnormalities result from severe hypoglycemic episodes or rather due to effect of chronic hyperglycemia. Our aim was to assess simultaneously and continuously the glucose and EEG variability, in youth with T1DM. Ten youth, mean age $14.2 \pm 1.9$ years, with T1DM for an average $5.57 \pm 3.54$ years were recruited. They were monitored simultaneously clinically and continuously by EEG recording and interstitial glucose monitoring by CGMS, for 48 hours. The parts of the recordings that included artifacts and those corresponding to times when the subject's activity was unknown were deleted. A total of 1253 combined readings of glucose levels and 16 electrodes EEG comprise the study data. Mean glucose concentration was $206.95 \pm 94.8 \mathrm{mg} / \mathrm{dl}$. Analysis of variance (ANOVA) 
demonstrated that during sleep, glucose $>200 \mathrm{mg} / \mathrm{dl}$ was associated with higher power of delta, theta, alpha and beta EEG frequencies in temporal and central areas $(p=0.005)$. Glucose $<70 \mathrm{mg} / \mathrm{dl}$ was associated with lower delta and theta power $(p<0.001)$, suggesting a decrease in slow-wave (deep) sleep, when compared to glucose 70-199 mg/dl. During wakefulness, lower glucose was associated with increase in fast brain activity, in the gamma range $(p=0.012)$. Glucose levels $>200 \mathrm{mg} / \mathrm{dl}$ were associated with EEG slowing, as suggested by lower beta and gamma power, and higher delta power $(p=0.005)$. This study is the first to assess simultaneously and continuously diurnal and nocturnal glucose variability, clinical state and EEG recording, in youth with T1DM. We demonstrated that both asymptomatic hypoglycemia and hyperglycemia change the electrical activity in various brain regions and may therefore influence cognitive function, in a subtle but clinically important way.

Supported by: Chief Scientist Office of the Ministry of Health, Israel

$\Delta$ 200-OR Identification of Clinical Predictors of Insulin Resistance in $\mathrm{Pa}$ tients With Type 1 Diabetes

MEGAN M. KELSEY, DANA DABELEA, JERI E. FORSTER, KRISTEN J. NADEAU, Aurora, CO

The presence of relative insulin resistance (IR) in patients with type 1 diabetes (T1D) has been thought to be related to glucotoxicity, although evidence for this is limited in pediatrics. We have shown that lean adolescents with T1D are more IR than lean controls, but do not show typical markers for IR. Recently, an equation was proposed for clinical identification of IR using patients with both T1D and T2D. Factors included in this equation were: fasting triglycerides (TG), waist circumference (WC) and HbA1c. The goal of the current study was to identify which of these factors contributes most to IR in T1D youth alone. We studied 62 adolescents (age $=15.5+2.2 \mathrm{yrs}$, BMI $z$-score $=0.33+0.95$ ) with T1D. Insulin sensitivity was measured by an $80 \mathrm{mU} /$ $\mathrm{m} 2$ /min-1 hyperinsulinemic euglycemic clamp to assess glucose disposal rate (GDR). Regression analysis was used to determine associations among TG, WC, HbA1c, BMl and GDR, with GDR (mg/m2/min-1) as the outcome variable. We found that, of the three equation variables, only WC was significantly associated with GDR, such that for every $1 \mathrm{~cm}$ increase in WC, there was a $0.13 \mathrm{mg} / \mathrm{m} 2 / \mathrm{min}-1$ decrease in $\mathrm{M}$-value ( $p=0.0001)$. When $B M I$ was added into the regression equation, WC remained significant, whereas BMI was not significantly associated with GDR. In summary, we did not find a significant association between $\mathrm{HbA1C}$ and IR, suggesting that glucotoxicity may not be the driving force behind IR in T1D youth. In contrast, WC significantly predicted IR in T1D youth independently from BMI and, therefore, may be a simple clinical measure of IR in these patients, who lack other typical IR risk markers. Analysis of hepatic, abdominal and muscle fat distribution as measured by magnetic resonance is currently underway to further assess underlying pathophysiology. Further studies are also needed to evaluate the additional impact of obesity on insulin sensitivity in children with T1D.

Supported by: 5K12HD057022-04 NIH BIRCWH K12; NIH/NCRR UL1 RR025780

201-0R

Ascorbic Acid Prevents Adverse Effects of Hyperglycemia on Endothelial Function in Adolescents With Type 1 Diabetes

KATHRYN STEPHENS, AMANDA S. DYE, HONG HUANG, JOHN A. BAUER, ROB-

ERT HOFFMAN, Columbus, $\mathrm{OH}$

Hyperglycemia $(H)$ induced oxidative stress plays a key pathophysiological role in altering endothelial function (EF) in type 1 diabetes (T1D). Children with T1D have evidence of increased oxidative stress and reduced oxidative defense. It has been shown that ascorbic acid (AA) may reverse the effects of $\mathrm{H}$ on $\mathrm{EF}$ in adults with well controlled or newly diagnosed diabetes. We have found that $\mathrm{H}$ adversely impacts $\mathrm{EF}$ in adolescents with T1D. To determine if $A A$ infusion prevents $H$ induced changes in $E F$ in adolescents with $\mathrm{T1D}$, reactive hyperemic changes ( 5 minutes of upper arm vascular occlusion) in forearm vascular resistance (FVR) were measured using venous occlusion plethysmography with (1) continuous infusion of $A A(3 \mathrm{mg} / \mathrm{min})$, and (2) without AA during (a) euglycemic (eugly) state ( $90 \mathrm{mg} / \mathrm{dL}$; using 40 $\mathrm{mU} / \mathrm{m} 2 /$ insulin infusion), followed by induction of (b) $\mathrm{H}$ state $(\sim 200 \mathrm{mg} / \mathrm{dL}$ with insulin infusion) in 6 adolescents with T1D (age 14 $\pm 1, \mathrm{BMI} 22 \pm 4$, HgA1C $7.9+0.9$, duration of diagnosis $4+2.5 y$ rs, mean $+S D$ ). EF was assessed as percent change in FVR pre and post upper arm vascular occlusion. Soluable intracellular adhesion molecules (SICAM) was measured at each stage of glycemia as a marker of endothelial activation. Repeated measures ANOVA revealed that the decrease in FVR from eugly to $\mathrm{H}$ was eliminated with $A A$ treatment $(p=0.043)$. Figure 1 displays our results. sICAM levels were lower in both arms during treatment with $A A(p<0.001)$. These results indicate that the adverse effects of acute $\mathrm{H}$ on $\mathrm{EF}$ in adolescents with T1D can be prevented with $A A$, possibly by reducing oxidative stress.

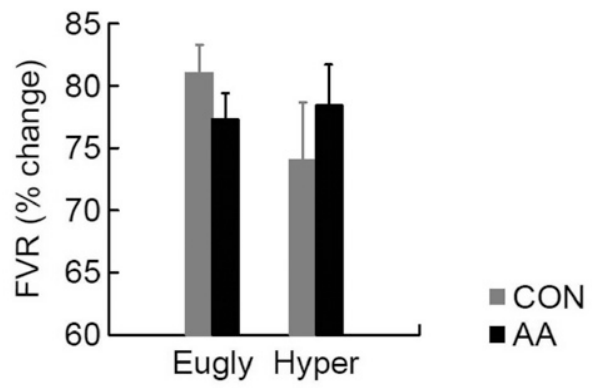

202-0R

The Prediction of Type 1 Diabetes by Multiple Autoantibody Levels and Their Incorporation into an Autoantibody Risk Score in the TrialNet Natural History Study

JAY SOSENKO, JAY SKYLER, GEORGE EISENBARTH, JEFFREY KRISCHER, LIPING YU, JEFFREY MAHON, CRAIG BEAM, DAVID BOULWARE, LISA RAFKIN, DESMOND SCHATZ, JERRY PALMER, THE TYPE 1 DIABETES TRIALNET STUDY GROUP, Miami, FL, Denver, CO, Tampa, FL, London, ON, Canada, Gainesville, FL, Seattle, WA

We studied whether levels of multiple autoantibodies add to the prediction of type 1 diabetes (T1D) beyond designations of positivity and negativity in the TrialNet Natural History Study (TNNHS). All participants were tested for 3 autoantibodies (GADA, IA-2A, and mIAA) at their initial screening. Samples from those positive for at least one autoantibody $(n=784$; age: $19.4 \pm 13.8$ years) were subsequently tested for ICA and ZnT8A. An autoantibody risk score (ARS) was developed on the basis of a proportional hazards model that included levels of each autoantibody along with the designations of positivity and negativity. The ARS was strongly predictive of T1D [hazard ratio (with 95\% CI): $2.72(2.23,3.31) ; p<0.001]$. Receiver operating characteristic curve (ROC) areas (with $95 \% \mathrm{CI}$ ) for the ARS were: $0.84(0.78,0.90)$ at 2 years of follow-up and $0.81(0.74,0.89)$ at 3 years $(p<0.001$ for both). The ROC areas for autoantibody number were: $0.79(0.72,0.86)$ at 2 years and $0.79(0.73,0.86)$ at 3 years (both $p<0.001)$. Compared to the ROC areas for autoantibody number, the ROC area for the ARS was significantly higher at 2 years $(p<0.05)$, but not at 3 years. When another autoantibody risk score was developed on the basis of a model excluding ICA information, the ROC areas were virtually identical to those of the ARS. After adjusting for the pattern of positivity, the composite measure of the multiple autoantibody levels from the ARS model was predictive of T1D $(p<0.001)$. Among individuals positive for the same autoantibodies, risk estimates (from the ARS) varied substantially due to differences in their composite autoantibody levels. For example, 3-year risks ranged from $6 \%$ to $36 \%$ among those with GADA/ ZnT8A $(n=30)$. These findings show the importance of considering levels of multiple autoantibodies in assessing the risk of T1D. Moreover, levels of multiple autoantibodies can be incorporated into an autoantibody risk score that accurately predicts $\mathrm{T} 1 \mathrm{D}$.

Supported by: NIDDK

\section{GLUCAGON AND GLUCAGON-LIKE PEPTIDES- ANIMALS}

203-OR

The Role of XBP1 in Endoplasmic Reticulum Stress in Pancreatic $\boldsymbol{\alpha}$-cell

MASARU AKIYAMA, CHONG WEE LIEW, RACHAEL MARTINEZ, BEN HAMBRO, ROHIT N. KULKARNI, Boston, MA

Type 2 diabetes (T2D) is characterized by insulin resistance, hyperglucagonemia and $\beta$-cell failure while $\alpha$-cell mass is largely unaltered. Although endoplasmic reticulum (ER) stress has been suggested to promote loss of $\beta$-cells in T2D its role in $\alpha$-cells is virtually unknown. XBP1 (X-box binding protein 1 ) is a transcription factor that plays a crucial role in the unfolded protein response (UPR). Spliced XBP1 promotes ER biogenesis and activates the expression of ER chaperones to facilitate protein secretion. XBP1 deficiency impairs secretion of pancreatic $\beta$-cells leading to glucose intolerance. To evaluate the role of XBP1 in $\alpha$-cells, we created complementary in vivo ( $\alpha$-cell specific Xbp1 knockout, $\alpha$ XbpKO mice) and in vitro (stable Xbp1 knock 
down $\alpha$-cell line, $\alpha$ XbpKD) models. $\alpha$ XBPKO mice exhibited mild glucose intolerance on oral glucose gavage ( $\alpha$ XbpK0: $249 \mathrm{mg} / \mathrm{dl}$ vs. control $195 \mathrm{mg} / \mathrm{dl}$ at $15 \mathrm{~min} ; \mathrm{p}<0.05 ; n=6-12$ ). Thapsigargin treatment of $\alpha X B P K D$ cells activated IRE1 (pIRE1/IRE1; 1.4 fold increase compared to control; $p<0.05 ; n=3$ ), an upstream activator of XBP1, while reducing the expression of downstream targets of XBP1 (Bip and Chop) and cleaved caspase 3. Interestingly, insulin treatment of $\alpha \mathrm{XBPKD}$ cells exhibited reduced tyrosine phosphorylation of IRS-1 (pY896) (IRS-1-pY/IRS-1; 30\% decrease; $p<0.001 ; n=3$ ), and phosphorylation (Ser ${ }^{473}$ ) of Akt (pAkt/Akt; $75 \%$ decrease: $p<0.001 ; n=3$ ) leading to activated JNK (pJNK/JNK; 3.5 fold increase: $p<0.01 ; n=3$ ) and serine phosphorylation of IRS1 (IRS-1-pS/IRS1; 2.2 fold increase: $p<0.05 ; n=3$ ). Consequent to the impaired insulin signaling, the $\alpha X B P K D$ cells showed enhanced glucagon secretion following insulin treatment (2.0 fold increase: $p<0.01 ; n=3-6$ ), in agreement with our earlier report of hyperglucagonemia in $\alpha$-cell insulin receptor knockout mice. Together, these data suggest that XBP1 cross talks with proteins in the insulin signaling pathway and plays a crucial role in the regulation of $\alpha$-cell secretory function and survival.

Supported by: Sunstar Foundation for Oral Health Promotion

\section{4-0R}

Hypothalamic Glucagon Signaling Regulates Glucose Production PATRICIA I. MIGHIU, JESSICA T. YUE, BEATRICE M. FILIPPI, TONY K. LAM, Toronto, ON, Canada

Hypothalamic insulin action regulates hepatic glucose production in normal rodents, but little is known in regards to a potential role of glucagon. We here first demonstrated that direct administration of glucagon into the mediobasal hypothalamus (MBH) of rats under pancreatic (basal-insulin) euglycemic clamp conditions surprisingly lowered glucose production (GP) from $13.6 \pm 0.8 \mathrm{mg} \mathrm{kg}^{-1} \mathrm{~min}^{-1}$ to $4.8 \pm 1.0 \mathrm{mg} \mathrm{kg}^{-1} \mathrm{~min}^{-1}$ (vs $10.4 \pm 0.9 \mathrm{mg} \mathrm{kg}^{-1}$ $\min ^{-1}[\mathrm{MBH}$ saline], $\mathrm{P}<0.05)$, but did not alter the rate of peripheral glucose uptake compared to MBH saline $\left(11.3 \pm 0.8 \mathrm{mg} \mathrm{kg}^{-1} \mathrm{~min}^{-1} \mathrm{vs} .11 .8 \pm 0.8 \mathrm{mg} \mathrm{kg}^{-1}\right.$ $\left.\mathrm{min}^{-1}\right)$. This GP-lowering effect was prevented when glucagon receptor signaling was blocked via $\mathrm{MBH}$ infusion of a mouse-monoclonal anti-glucagon antibody $\left(9.3 \pm 0.6 \mathrm{mg} \mathrm{kg}^{-1} \mathrm{~min}^{-1}\right)$ or a glucagon receptor antagonist (Des-His [Glu']glucagon amide) $\left(10.6 \pm 1.7 \mathrm{mg} \mathrm{kg}^{-1} \mathrm{~min}^{-1}\right)$ or PKA-inhibitor Rp-cAMPS $\left(9.5 \pm 0.8 \mathrm{mg} \mathrm{kg}^{-1} \mathrm{~min}^{-1}\right)$. Conversely, direct activation of hypothalamic PKA signalling via MBH PKA-activator Sp-cAMPS administration was sufficient to lower GP from $12.2 \pm 0.9 \mathrm{mg} \mathrm{kg}^{-1} \mathrm{~min}^{-1}$ to $5.2 \pm 0.7 \mathrm{mg} \mathrm{kg}^{-1} \mathrm{~min}^{-1}$. We next evaluated in a non-clamp condition whether MBH glucagon signaling is necessary for circulating glucagon to regulate plasma glucose levels and GP. Intravenous (i.v.) injection of glucagon increased plasma glucose levels by $23 \%$ and GP by $27 \%$ within 10 minutes of injection in normal rats. When $\mathrm{MBH}$ CAMP-PKA signaling was disrupted, i.v. glucagon injection increased plasma glucose levels and GP to a significantly greater extent compared to normal rats with intact MBH CAMP-PKA signaling. In summary, these data unveil a novel function of glucagon action in the MBH that stimulates MBH cAMPPKA signaling and inhibits GP, and counteracts the direct hepatic stimulatory effect of circulating glucagon on GP in normal rodents.

Supported by: CDA, OGS and BBDC

\section{Oxyntomodulin Lowers Plasma GIP in DIO mice}

JULIE LAO, XIAOBING DU, XIAOLAN SHEN, EDWARD KOWALIK, WENYU LI, DAVID E. KELLEY, ALESSANDRO POCAI, Rahway, NJ

Oxyntomodulin (OXM) is a peptide secreted postprandially from the L-cells of the gut that has a weak affinity for both the glucagon-like receptor-1 (GLP1R) and the glucagon receptor (GCGR). OXM administration causes weight loss in humans and rodents. Glucose-dependent insulinotropic polypeptide (GIP) is another peptide known to improve glucose-stimulated insulin secretion in postprandial state. Recent studies also provide evidences for an important role of GIP on adipose tissue lipogenesis and possibly in the pathogenesis of obesity. Because glucagon has been reported to decrease fasting GIP levels in humans, we examined whether short-term infusion of OXM lowers plasma GIP levels and if this effect is distinct from that of a GLP-1R-selective agonist. Comparisons of the effects of GLP-1 and OXM are confounded by the different pharmacokinetics and receptor affinities of the two peptides. We identified an equipotent GLP-1R-selective peptide agonist that differs from OXM by only one residue ( $\mathrm{O3} \rightarrow \mathrm{E}, \mathrm{OXM03E}$ ), but has no significant GCGR agonist activity. We compared the effects of intravenous infusion of OXM and OXMO3E on GIP levels in DIO mice. Vehicle, OXM and OXMO3E were infused for $180 \mathrm{~min}$ in chronically catheterized mice. Free fatty acids (FFA) levels were significantly elevated in OXM but not in OXMO3E treated mice. Interestingly, OXM but not OXM03E treatment lowered GIP levels to the values observed in lean mice ( $9.4 \pm 0.5$ vs. $2.5 \pm 0.3 \mathrm{pg} / \mathrm{mL}$; DIO vs. lean mice $p<0.05$ ). In summary, short-term pharmacological infusion with OXM lowers the incretin hormone GIP relative to a GLP-1R-selective agonist and this difference was associated with higher plasma FFA. These findings indicate differential engagement of a gut-adipocyte axis by OXM compared to a GLP-1R-selective agonist that is mediated by differing effect in modulation of GIP secretion. We postulate that this difference could contribute to the greater weight loss observed with chronic OXM treatment compared to chronic GLP-1 treatment.

206-0R

Exendin-4 (Ex4) Attenuates Cardiomyocyte Apoptosis by Inhibiting Oxidative Stress-Induced Endoplasmic Reticulum (ER) Stress

CRAIG YOUNCE, MELISSA BURMEISTER, JULIO AYALA, Orlando, FL

Cardiomyocyte apoptosis is a contributor to diabetic cardiomyopathy. We show that activation of the GLP1 Receptor (GLP1R) with Ex4 prevents hyperglycemia-induced cardiomyocyte apoptosis. This is associated with reduced expression of ER stress markers. Oxidative stress is a key trigger of hyperglycemia-induced ER stress in cardiomyocytes. We therefore tested the hypothesis that Ex4 attenuates hyperglycemia-induced ER stress in cardiomyocytes by reducing oxidative stress. Neonatal rat ventricular myocytes (NRVM) were incubated in $5 \mathrm{mM}$ glucose (LG), $33 \mathrm{mM}$ glucose (HG) or $33 \mathrm{mM}$ glucose $+30 \mathrm{nM} \mathrm{Ex4}$ (HGEx4) for 1, 6, 12 and $24 \mathrm{~h}$. Reactive $\underline{0} x y g e n$ Species (ROS) levels were assessed using dihydrorhodamine 123 (DHR123) and were used as a marker of oxidative stress. ROS levels peaked at $12 \mathrm{~h}$ but were not different between $\mathrm{HG}$ and HGEx4 (2.4 40.2 vs. $2.1 \pm 0.3$ fold above $L G$ ). In response to the oxidative stressor $\mathrm{H}_{2} \mathrm{O}_{2}(250 \mu \mathrm{M})$, Ex4 also failed to reduce ROS levels $\left(\mathrm{H}_{2} \mathrm{O}_{2}=4.8 \pm 0.5\right.$ vs. $\mathrm{H}_{2} \mathrm{O}_{2}+\mathrm{Ex} 4=5.1 \pm 0.04$ fold over control), but as with hyperglycemia, Ex4 attenuated $\mathrm{H}_{2} \mathrm{O}_{2}$-mediated apoptosis (Control = $12 \pm 2 ; \mathrm{H}_{2} \mathrm{O}_{2}=25 \pm 2 ; \mathrm{H}_{2} \mathrm{O}_{2}+\mathrm{Ex} 4=16 \pm 1 \%$ death assessed by Trypan Blue). Ex4 also reduced $\mathrm{H}_{2} \mathrm{O}_{2}$-induced protein levels of the ER stress chaperone GRP78 and proapoptotic CHOP. This suggests that Ex4 directly inhibits ER stress. To test this, NRVM were treated with the ER stress inducers thapsigargin $(\mathrm{Tg}, 300 \mathrm{nM})$ or Tunicamycin $(\mathrm{Tm}, 10 \mathrm{ug} / \mu \mathrm{L})$ with or without Ex4. Ex4 attenuated Tg- (Control $=10 \pm 2 ; \mathrm{Tg}=39 \pm 4 ; \mathrm{Tg}+\mathrm{Ex} 4=22 \pm 4 \%$ death) but not Tm-induced (Control $=9 \pm 3 ; \mathrm{Tm}=33 \pm 6 ; \mathrm{Tm}+\mathrm{E} 4=28 \pm 2 \%$ death) apoptosis Ex4 also reduced $\mathrm{Tg}$ - but not Tm-induced protein levels of GRP78 and CHOP. In sum, these data show that Ex4 prevents cardiomyocyte apoptosis by directly inhibiting ER stress independently of effects on oxidative stress. This proposes a mechanism by which Ex4 attenuates hyperglycemia-induced cardiomyocyte loss further supporting a potential role in the treatment of diabetic cardiomyopathy.

$\Delta$

207-0R

Glucagon-Like Peptide 1 (GLP-1) Recruits Microvasculature and Increases Glucose Extraction in Muscle via a PKA-Dependent Pathway ZHENGHUA DONG, WEIDONG CHAI, LINA ZHAO, ZHUO FU, EUGENE J. BARRETT, WENHUI WANG, ZHENQI LIU, Charlottesville, VA

GLP-1 causes vasodilation and increases muscle glucose uptake independent of insulin. We have recently shown that GLP-1 recruits muscle microvasculature and increases glucose use via a nitric oxide (NO)-dependent mechanism. Protein kinase A (PKA) is a major signaling intermediate downstream of GLP-1 receptors. To examine whether PKA mediates GLP-1's microvascular action in muscle, overnight fasted male SD rats were given a systemic infusion of GLP-1 (7-36) amide ( $30 \mathrm{pmol} / \mathrm{kg} / \mathrm{min})$ for $120 \mathrm{~min} \pm$ systemic infusion of $\mathrm{H} 89$ (PKA inhibitor, $160 \mathrm{pmol} / \mathrm{kg} / \mathrm{min}$ ) which began $30 \mathrm{~min}$ before starting GLP-1 infusion. Hindleg muscle microvascular blood volume (MBV), microvascular blood flow velocity (MFV) and microvascular blood flow (MBF) were determined using contrast-enhanced ultrasound. GLP-1 infusion acutely increased muscle MBV $(p<0.05)$ within 30 min without altering MFV and blood pressure. This effect persisted throughout the $120 \mathrm{~min}$ infusion period, leading to an increase in muscle MBF $(\sim 2$-fold, $p<0.05)$. These changes were paralleled by increases in plasma NO levels $(\sim 2$-fold, $p<0.05)$ and hindleg glucose extraction $(\sim 2$-fold, $p<0.05)$. Systemic infusion of H89 blocked GLP-1-mediated increases in muscle MBV, MBF, glucose extraction and NO production. To ascertain that GLP-1 can directly stimulate NO production, bovine aortic endothelial cells were treated with GLP-1 $(1 \mathrm{ng} / \mathrm{ml})$ for 20 min with or without $\mathrm{H} 89(10 \mu \mathrm{M})$ pre-treatment. Endothelial N0 synthase (eNOS) phosphorylation, NO production and PKA activity were determined. GLP-1 acutely stimulated eNOS (Ser1177) phosphorylation and NO production. Inhibition of PKA abolished GLP-1-induced eNOS phosphorylation and NO production. We conclude that GLP-1 expands microvascular volume and increases substrate exchange in muscle via a PKA-eNOS dependent pathway in vascular endothelium. This may contribute to postprandial glycemic control and diabetes complication prevention in diabetes.

Supported by: NIH 
$\Delta$

208-0R

Superimposing Glucagon-Like Peptide 1 on Insulin Expands Muscle Microvascular Surface Area and Enhances Insulin Action WEIDONG CHAI, XINGXING ZHANG, EUGENE J. BARRETT, ZHENOI LIU, Charlottesville, VA

Insulin recruits muscle microvasculature and this action contributes $40 \%$ to insulin-mediated glucose disposal during insulin clamp. We have recently reported that glucagon-like peptide 1 (GLP-1) acutely recruits muscle microvasculature, increases muscle delivery of insulin and enhances muscle use of glucose independent of its effect on insulin secretion. To examine whether GLP-1 modulates insulin's microvascular and metabolic actions, overnight fasted adult Sprague-Dawley rats received a euglycemic hyperinsulinemic clamp ( $3 \mathrm{mU} / \mathrm{kg} / \mathrm{min}$ ) for $180 \mathrm{~min}$ with a systemic infusion of GLP-1 (7-36) amide (30 pmol/ $\mathrm{kg} / \mathrm{min}$ ) superimposed between 60 to 120 $\mathrm{min}$. Hindleg muscle microvascular blood volume (MBV), a measure of microvascular surface area and perfusion, and microvascular flow velocity (MFV) were determined using contrast-enhanced ultrasound. Microvascular blood flow (MBF) was calculated as the product of MBV and MFV. Glucose infusion rates (GIR) were calculated and plasma insulin and nitric oxide (NO) levels were measured. Insulin infusion increased GIR to $9.1 \pm 0.5 \mathrm{mg} / \mathrm{kg} / \mathrm{min}$ at 60 min. This was associated with a significant increase in muscle MBV, MBF, and plasma NO levels at 30 and $60 \mathrm{~min}$ ( $p<0.05$ for all). Superimposition of GLP-1 further increased GIR by $23 \%$, MBV by $35 \%$, MBF by $37 \%$, and plasma NO levels by $27 \%(p<0.05$ for all). These changes persisted throughout the GLP-1 infusion period. There were no concurrent changes in plasma insulin levels, muscle MFV, or mean arterial blood pressure. Discontinuation of GLP1 infusion resulted in a return within 30 min in GIR, muscle MBV and MBF, and plasma NO concentrations back to the levels seen with insulin infusion alone. We conclude that addition of GLP-1 to insulin infusion further expands muscle microvascular surface area and enhances insulin-mediated glucose disposal. Thus, GLP-1 may afford potential to improve muscle insulin action and postprandial glycemic control via microvascular recruitment in muscle. Supported by: $\mathrm{NIH}$

209-0R

The Role of GLP-1 Receptor Signaling in Metabolic Changes after Roux-en-Y Gastric Bypass in Diet Induced Obese Rats ZHIBO AN, ERIC P. SMITH, TODD GREER, JOSE BERGER, DAVID A. D'ALESSIO, Cincinnati, $\mathrm{OH}$

Roux-en-Y gastric bypass (RYGB) is the gold-standard for bariatric surgery, and leads to sustained weight loss and rapid resolution of diabetes. RYGB is associated with an elevated postprandial secretion of glucagon-like peptide-1 (GLP-1), which has been proposed to mediate some of the metabolic improvements following RYGB. The present study sought to determine whether GLP-1 receptor signaling is required for the effects of RYGB on glucose homeostasis, food intake, and weight loss. Diet-induced obese rats had RYGB or sham surgeries, and were randomly allocated to chronic blockade of GLP-1 action by minipump infusion of the GLP-1 receptor antagonist Exendin-9,39 (Ex-9; $2 \mathrm{pmol} / \mathrm{kg} / \mathrm{min})$, or vehicle (Vh). Minipumps were implanted subcutaneously at the time of RYGB or sham operation and delivered treatment for 6 weeks. The four groups, sham-Vh $(n=5)$, sham-Ex9 $(n=4)$, RYGB-Vh $(n=6)$ and RYGB-Ex9 $(n=8)$ were tracked for food intake and body weight daily for 6 weeks. At 6 weeks, an oral glucose tolerance test (OGTT; $1.5 \mathrm{~g}$ glucose/ $\mathrm{kg}$ b.w.) was performed. RYGB led to a $11 \%$ and $18 \%$ reduction of body weight and accumulative food intake, respectively, and these effects did not differ between Vh and Ex-9 treated rats. In sham rats, Ex-9 treatment led to a $\sim 19 \%$ increase in the glucose AUC during the OGTT. In contrast, after RYGB, Ex-9 caused a $~ 99 \%$ increase $(p<0.05)$ in glucose AUC. These results demonstrate that GLP-1 action has a disproportionate effect on the maintenance of glucose homeostasis following RYGB in diet induced obese rats. In contrast, increased postprandial GLP-1 is less likely to play a role in alteration of food intake and body weight after this procedure.

Supported by: NIH R01 DK57900, EES Research Grant

210-OR

The GLP-1-Gastrin Dual Agonist ZP3022 Increases Beta-Cell Mass in $\mathrm{db} / \mathrm{db}$ Mice

DORTHE L. ALMHOLT, LOUISE S. DALBØGE, JAKOB L. TOLBORG, CHRISTIAN GRøNDAHL, KELD FOSGERAU, TRINE S. NEERUP, Glostrup, Denmark, Hørsholm, Denmark

Combination treatment of diabetic $\mathrm{db} / \mathrm{db}$ mice with exendin- 4 and gastrin has previously been shown to preserve beta-cell mass by stimulating beta-cell growth and differentiation. Here we investigate the effects of a novel and potent GLP-1-gastrin receptor dual agonist (ZP3022, invented by
Zealand Pharma A/S) that acts on the GLP-1 and CCKB (gastrin) receptors. Equimolar doses $(2 \times 50 \mathrm{nmol} / \mathrm{kg} /$ day) of ZP3022, liraglutide (Lira), or vehicle (Veh) were administered by twice daily subcutaneous injection to male $\mathrm{db} /$ $\mathrm{db}$ mice for 2, 4 or 8 weeks. HbA1c and fasting blood glucose levels were determined at treatment start and at termination. Pancreata were removed and fixed in formalin for stereological determination of beta-cell mass. After 2,4 , and 8 weeks of treatment, both ZP3022 and liraglutide caused a significant improvement in glycemic control as measured by HbA1c (8 weeks: Veh, $7.4 \pm 0.5 \%$; Lira, $5.3 \pm 0.4 \%$; ZP3022, $5.6 \pm 0.6 \%, p<0.001$ for ZP3022 and Lira vs. Veh) as well as fasting blood glucose levels. Similarly, both ZP3022 and liraglutide caused a significant increase in beta-cell mass compared to vehicle following 4 weeks of treatment (Figure, $p<0.05$ ). However, after 8 weeks of treatment only ZP3022 resulted in a significant increase in betacell mass, compared to both vehicle- and liraglutide-treated mice (Figure, $p<0.001$ and $p<0.05$, respectively). The study demonstrates that treatment of $\mathrm{db} / \mathrm{db}$ mice with the GLP-1-gastrin receptor dual agonist ZP3022 causes a sustained increase in the beta-cell mass accompanied by an improvement in glycemic control. In contrast, liraglutide displayed a transient effect on beta-cell mass.

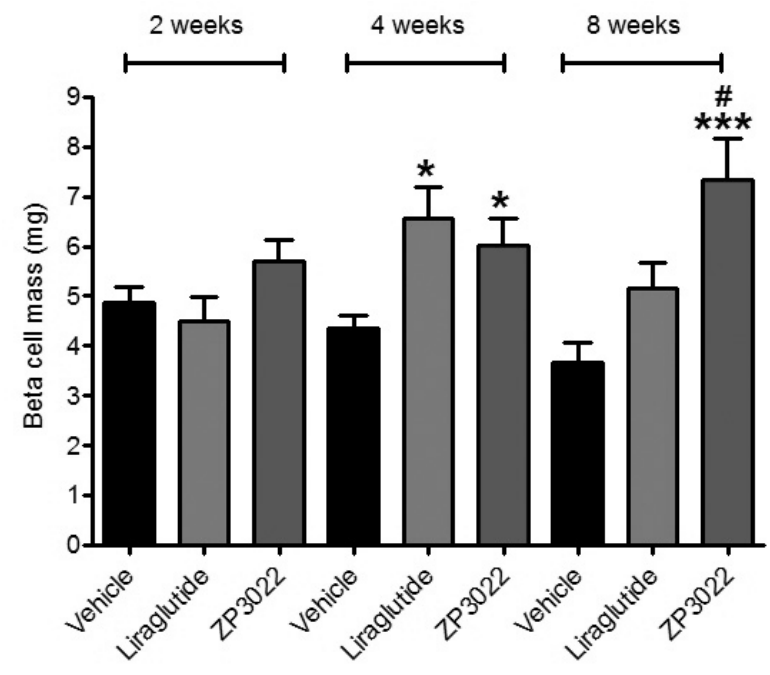

\section{DIABETES AND ATHEROSCLEROSIS- NEW INSIGHTS FROM ANIMAL MODELS}

$\Delta$ 211-OR

FGF21 Expresses in Diabetic Hearts and Protects from Palmitateand Diabetes-Induced Cardiac Cell Death In Vitro and In Vivo via Erk1/2-Dependent p38 MAPK-AMPK Signaling Pathways

CHI ZHANG, XIAO MIAO, YANG BAI, QIANG CHEN, XIAOKUN LI, LU CAI, Louisville, $K Y$, Changchun, China, Wenzhou, China

Fibroblast growth factor (FGF) 21 plays important role in lipid metabolism. The present study examined whether FGF21 mRNA expresses in the heart of diabetic mice and also protects from fatty acid (palmitate)- and diabetesinduced cardiac cell death. Type 1 diabetes, induced by streptozotocin (STZ), up-regulated FGF21 expression about 40 folds at 2 months and $3-1.5$ folds at 4 and 6 months of diabetes. To define if the up-regulated cardiac FGF21 expression offers a protective effect on fatty acid- or diabetes-induced cardiac damage, H9C2 cells were exposed to palmitate at $62.5 \mathrm{nM}$ for 15 $\mathrm{h}$, which induced apoptosis, examined by DNA fragmentation and cleaved caspase-3. Pre-incubation of palmitate-treated cells with FGF21 significantly reduced the apoptosis at a dose range of $25-250 \mathrm{nM}$ with a maximal protection at 50 to $100 \mathrm{nM}$. Mechanistically palmitate down-regulated, and FGF21 up-regulated, phosphorylation levels of Erk1/2, p38 MAPK and AMPK. Via application of each specific inhibitor of these kinases, FGF21 was found to prevent palmitate-induced PTEN activation and Akt inactivation via up-regulating Erk1/2-dependent p38 MAPK/AMPK signaling pathways. More importantly in vivo administration of FGF21 to STZ-induced diabetic mice once hyperglycemia was onset significantly prevented diabetes-induced cardiac apoptosis along with a prevention of diabetes-inhibited Erk1/2, p38 MAPK and AMPK phosphorylation. In addition, in vivo administration of FGF21 to acute fatty acid-treated mice also protected fatty acid-induced cardiac cell death. These results suggest that the early stage increase in FGF21 mRNA 
expression may represent an early protective mechanism; administration of exogenous FGF21 to diabetic mice or acute fatty acid-treated mice can prevent cardiac damage. The cardioprotection by FGF21 from diabetes is mediated by ERK1/2-dependent p38 MAPK and AMPK signaling pathway.

212-0R

Arachidonic 12/15-Lipoxygenase-Induced Inflammation Promotes the Development of Diabetic Cardiomyopathy

MASAYA SAKAMOTO, HIROFUMI SUZUKI, YOSUKE KAYAMA, KAZUNORI UTSUNOMIYA, Tokyo, Japan

Diabetic mellitus increases the risk of heart failure independently of underlying coronary artery disease, and many believe that diabetes leads to cardiomyopathy. The underlying pathogenesis is partially understood, but precise mechanism underlying diabetic cardiomyopathy is still unknown. To investigate the molecular mechanisms of diabetic cardiomyopathy, we created a diabetic cardiomyopathy model (streptozotocin (STZ) injection rat) and performed microarray analysis. Echocardiography showed that STZ injection rat developed diastolic and systolic dysfunction and cardiac fibrosis was increased in STZ injection rat. Among the genes analyzed, arachidonic 12-lipoxygenase (12-LOX) was upregulated in the heart of STZ rat. mRNA level of 12-LOX and production of 12(s)-HETE, a major metabolite of 12-LOX were upregulated in the heart of STZ rat. Until recently, 12-LOX has been reported to play an important role in the development of atherogenesis and neurogenerative disease. However, the role of 12-LOX in diabetic cardiomyopathy has not been examined. To determine the role of 12-LOX in STZ injection heart, we created STZ injection mice (WT-STZ) and compared to those of 12-LOX KO (KO-STZ) mice. Disruption of 12-LOX significantly reduced cardiac fibrosis and improving diastolic and systolic dysfunction. Moreover the expression of inflammatory cytokine genes such as TNF- $\alpha$ and STZ-induced reactive oxygen species were significantly inhibited by disruption of 12-LOX. In vitro experiment reveal that hyperglycemia induced expression of 12-LOX as well as TNF- $\alpha$ by neonatal cardiomyocyte. Treatment with Cinnamy1-3,4dihydroxy- $\alpha$-cyanocinnamate (CDC) , a inhibitor of 12-LOX, decreased the expression of TNF- $\alpha$ by cardiomyocyte under high glucose stimulation. Our results suggest that cardiac 12-LOX-induced inflammation is involved in the development of diabetic cardiomyopathy and that inhibition of 12-LOX could be a novel treatment for this condition.

213-0R

AdipoR1 in Macrophages and AdipoR2 in Endothelial Cells via PPARgamma/SOD Play the Protective Roles against Atherosclerosis In Vivo

MIKI O. IWABU, TOSHIMASA YAMAUCHI, MASATO IWABU, TETSUYA KUBOTA, NAOTO KUBOTA, TAKASHI KADOWAKI, Bunkyo-ku, Japan

The adipocyte-derived hormone adiponectin (Ad) has been proposed to play the central roles as antidiabetic and antiatherogenic adipokine. However, whether Ad receptors AdipoR1 (R1) and AdipoR2 (R2) have the protective roles against atherosclerosis in vivo are still undetermined. Ad has been shown to be downregulated in obesity. Here we showed that in mice, ApoE deficiency and obesity induced by high-fat diet or leptin deficiency resulted in significantly decreased expression of R2 in aorta. We showed that R1 knockout (KO) mice exhibited significantly more neointimal formation in response to external vascular cuff injury than wild-type (WT) mice (intima to media

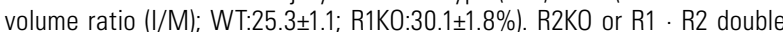
$\mathrm{KO}$ mice exhibited much more neointimal formation in response to cuff injury than R1KO mice (I/M; R2K0:42.8 $\pm 4.0 ;$ R1R2K0:49.6 $\pm 2.6 \%$ ). Interestingly, neointimal formation induced by cuff injury was increased by transplantation of bone marrow from R1KO but not increased by transplantation of bone marrow from R2KO mice. Importantly, the extent of neointimal formation observed in endothelial cells-specific R2KO mice was almost the same as that in conventional R2KO mice. Moreover, adenovirus-mediated supplement of R2 in cuff injury region or endothelial cells-specific R2 upregulation significantly attenuated neointimal proliferation. Adenovirus-mediated expression of R2 in aorta resulted in increased PPARgamma and increased oxidative stress-detoxifying enzymes such as SOD1. Conversely, targeted disruption of R2 in endothelial cells resulted in decreased PPARgamma and SOD1. This study provides the direct evidence that R2 in endothelial cells and R1 in macrophages play the protective roles against atherosclerosis. Moreover, this study raises the possibility that a therapeutic strategy to increase AdipoRs in injured artery could be useful in preventing vascular diseases such as restenosis after angioplasty.
214-OR

Metformin and Saxagliptin Combination Treatment Reduces Nitroxidative Stress and Restores Nitric Oxide Release in the Endothelium of Obese Rats

R. PRESTON MASON, ROBERT F. JACOB, J. JOSE CORBALAN, LU-LIN JIANG, TADEUSZ MALINSKI, Boston, MA, Beverly, MA, Athens, $\mathrm{OH}$

Endothelial cell (EC) dysfunction in diabetes is characterized by reduced nitric oxide (NO) and increased peroxynitrite (ONOO-) levels, an imbalance causally related to atherothrombotic disease. In this study, we hypothesized that EC dysfunction in diabetic rats is reversed with combination treatment using a DPP4 inhibitor (saxagliptin) and metformin due to their distinct and beneficial effects on endothelial NO synthase (eNOS) activity. To test this hypothesis, obese Zucker rats were fed a high-fat diet and treated with saxagliptin $(10 \mathrm{mg} / \mathrm{kg} / \mathrm{day})$, with or without metformin $(300 \mathrm{mg} / \mathrm{kg} /$ day) for 4 weeks. Aortic and glomerular ECs were then assayed ex vivo for NO and $\mathrm{ONOO}$ release using amperometric nanosensors. Changes in EC function were correlated with fasting glucose levels. Saxagliptin was found to increase aortic and glomerular endothelial NO release by 20\% $299 \pm 57$ $\mathrm{nM}$ to $360 \pm 29 \mathrm{nM})$ and $21 \%(110 \pm 14 \mathrm{nM}$ to $133 \pm 24 \mathrm{nM})$, respectively, while decreasing 0NO0- release by $11 \%$ and $16 \%$, respectively, as compared to vehicle-treated controls. Combination treatment with metformin increased aortic and glomerular N0 release by $45 \%(435 \pm 81 \mathrm{nM})$ and $53 \%$ (168 $\pm 32 \mathrm{nM}$ ), respectively, with comparable reductions in 0N00- levels. The NO/ONOO ratio, an indicator of eNOS coupling, increased more than two-fold with the combination treatment and was significantly greater than that observed with saxagliptin alone. Reversal of EC dysfunction with the combination was observed prior to reductions in elevated fasting glucose levels (156 $\pm 14 \mathrm{mg} / \mathrm{dL}$ ). These data indicate that saxagliptin and metformin combination therapy has direct effects on arterial and renal EC function in obese rats, including enhanced NO bioavailability and reduced nitroxidative stress, independent of changes on fasting glucose levels.

215-OR

TIMP3 Regulates Fatty Acid Metabolism, Atherosclerosis and Survival in ApoE Knockout Mice

MICHELE CAVALERA, STEFANO MENINI, VIVIANA CASAGRANDE, MARIA MAVILIO, ROSSELLA MENGHINI, STEFANO RIZZA, GIUSEPPE PUGLIESE, DAVIDE LAURO, RENATO LAURO, MASSIMO FEDERICI, Rome, Italy

TIMP3 is reduced in the muscle and carotid plaques from patients with Type 2 Diabetes but if TIMP3 alters glucose and lipid metabolism is unclear.

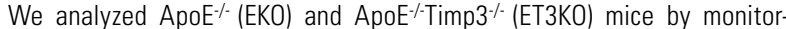
ing the survival rate; lipids, metabolites and atherosclerotic plaques with LC-MS metabolomics and conventional staining; physiological values by calorimetric cages. ET3KO mice revealed a significant higher rate of sudden death compared to EKO mice during a follow up of 12 months ( $\mathrm{N}=18$ per group; $p<0.001)$; Sudan-IV and Oil-Red staining of ET3KO aorta and aortic root displayed increased positive staining $(40 \%$ increase, $p<0.01)$ compared to EKO. Moreover, ET3KO mice showed higher levels of total cholesterol, $\mathrm{HDL}$-cholesterol and triglycerides ( $\mathrm{p}<0.05$ for all). Furthermore, ET3KO mice exhibited lower heart rate $(p<0.01)$, higher systolic and diastolic pressures $(\mathrm{p}=0.06 ; \mathrm{p}<0.05)$, higher $\mathrm{O}_{2}$ consumption and $\mathrm{CO}_{2}$ production, and lower respiratory exchange ratio compared to EKO mice ( $p<0.0001$ for all). These data suggested a different use of metabolic substrates; specifically we found increased levels of $\mathrm{C} 14: \mathrm{OH}, \mathrm{C16}: \mathrm{OH}$ and $\mathrm{C} 18: \mathrm{OH}(\mathrm{p}<0.01$ for all) in the blood of ET3KO mice, together with higher amount of arginine $(p<0.05)$ and histidine $(p<0.01)$. No differences in food and water intake were found. Western blot analysis showed reduced pThr172 AMPK phosphorylation and increased pSer473 AKT phosphorylation in skeletal muscle from ET3KO compared to EKO mice ( $p<0.05$ for all), suggesting that lipid oxidation may be dysfunctional in ET3KO mice. Loss of timp3 accelerates atherosclerosis and causes higher amount of atherosclerotic plaques, increased sudden death, plus defects in intermediary metabolism. The latter results in accumulation in the circulation of hydroxylated long-chain fatty acids that may complicate atherosclerotic plaque composition or perturb heart metabolism.

Supported by: JDRF, Fondazione Roma, Telethon

$\Delta$

216-OR

Obesity-Related Hypertension via Hypothalamic InflammationInduced Glutamatergic Activation

SUDARSHANA PURKAYASTHA, DONGSHENG CAI, Bronx, NY

Obesity-related hypertension is an integral component of metabolic syndrome and diabetic cardiovascular complications. In our recent study (Nature Medicine 2011), we discovered that obesity-related hypertension is caused by proinflammatory IKK-beta activation in the hypothalamic arcuate 
nucleus (ARC) and particularly the comprised proopiomelanocortin (POMC) neurons; however, the underlying mechanism is still unknown. In this study, we explored if brain excitatory glutamatergic system could be involved and how. We found that the hypertensive response following hypothalamic TNF-alpha injection in mice was normalized by prior hypothalamic injection of glutamatergic inhibitor kynurenic acid (mean blood pressure: 121.7 \pm 5.1 vs. $94.97 \pm 6.3 \mathrm{mmHg}, p<0.001)$. To dissect the underlying neurocircutry, we found that an intra-ARC injection of TNF-a led to neuronal activation (indicated by c-fos) evidently in the hypothalamic paraventricular nucleus (PVN) and promotion of glutamate release from the PVN, but these effects were prevented in POMC neuron-specific IKK-beta knockout mice (offspring from crossing POMC-Cre mice and IKK-beta lox/lox mice). These data indicate that IKK-beta in POMC neurons employs a glutamatergic pathway within the ARC-PVN axis to mediate hypertension. In relation with obesity-related hypertension - which is significantly due to hyperleptinemia-induced hypertension, we found that hypothalamic treatment of glutamate inhibitor kynurenic acid prevented leptin-induced hypertension, just as did the treatment of IKK-beta inhibitor PS-1145. The hypertensive outcome of hypothalamic glutamatergic activation was related to increased sympathetic outflow, as hypothalamic injection of leptin or TNF-alpha similarly upregulated the renal sympathetic pathway. In conclusion, obesity-related hypertension is mechanistically mediated by hypothalamic inflammation-dependent glutamatergic activation and downstream renal sympathetic upregulation.

Supported by: $\mathrm{NIH}$

217-0R

Chronic Activation of Sphingosine 1-Phosphate 1 Receptors Causes Hyperglycemia and Exacerbates Cardiac Fibrosis in Type 1 Diabetic Mice

ZHAO LI, ZHU-OIU JIN, Brookings, SD

Diabetic cardiomyopathy features myocardial hypertrophy and cardiac fibrosis. Sphingosine 1-Phosphate (S1P) is a bioactive small lipid molecule that is involved in multiple cellular functions through binding to five distinct S1P receptors (from S1P1 to S1P5). High plasma level of S1P has been reported in patients with type 1 diabetes. Role of S1P receptors in the onset of diabetic cardiomyopathy remains unknown. Since S1P1 is the dominant receptors in the heart and blood vessels. We tested the hypothesis that chronic modulation of S1P1 may play a critical role in diabetic cardiomyopathy. Type 1 diabetic mouse model was established by low-dose streptozotocin (STZ) induction protocol (50 mg/kg, for 5 days consecutively) in C57BL/6J mice. Mouse tail blood glucose was measured twice: 4 weeks and 11 weeks after STZ injection. SEW2871 $(0.125 \mathrm{mg} / \mathrm{kg})$, a selective S1P1 receptor agonist, was given once a day (i.p.) for 4 weeks (first phase) and 2 weeks (second phase) before evaluation of the cardiac function. STZ increased the fasting blood glucose level at 4 weeks of post-injection $(490.3+/-40.1 \mathrm{mg} /$ $\mathrm{dL}, \mathrm{P}<0.01$ compared with $201.7+/-12.5 \mathrm{mg} / \mathrm{dL}$ in vehicle control group). SEW2871 remained no effect on blood glucose level at 4 weeks (527.7+/$23.8 \mathrm{mg} / \mathrm{dL}, \mathrm{P}=\mathrm{N}$.S. compared with STZ group) but aggravated the glucose level at 11 weeks $(543.3+/-25.4, P<0.05$, compared with $421.5+/-40.0$ in STZ group). SEW2871 dilated the left ventricular lumen area (41.8 +/- 1.8 $\mathrm{mm} 2, \mathrm{P}<0.01$, compared with $17.0+/-1.2$ in control group) and decreased cardiac contractile force at 11 weeks $(0.14+-0.034 \mathrm{~g}, \mathrm{P}<0.01$, compared with $0.54+/-0.055$ in control group). S1P1 receptor expression and cardiac fibrosis were increased in diabetic mice. Enhanced nitrotyrosine in heart tissue was also observed in SEW2871-treated hearts. In conclusion, chronic activation of S1P1 by SEW2871 aggravates cardiac remodeling and cardiac fibrosis and plays a detrimental role in diabetic cardiomyopathy.

218-0R

Hyperglycemia Enhances Myeloid Cell Proliferation and Impairs Atherosclerosis Regression in Diabetes

PRABHAKARA R. NAGAREDDY, ANDREW J. MURPHY, BERND HEWING, LI-SHIN HUANG, ANN MARIE SCHMIDT, EDWARD A. FISHER, ALAN R. TALL, IRA J. GOLDBERG, New York, NY

Diabetes is one of the most potent risk factors for coronary heart diseases. Although atherosclerosis is initiated by deposition of cholesterol-rich lipoproteins in the artery wall, the entry of monocytes into the lesion drive the disease progression and/or impair its resolution. We found that hyperglycemia, independently of cholesterol, drives monocytosis in type I diabetic mouse models. Monocytosis was caused by increased proliferation of progenitor cells in the bone marrow but not in spleen. Hyperglycemia-mediated monocytosis was driven by PU.1 (Purine-rich box-1) and CCAAT/enhancer-binding proteins, $\mathrm{C} / \mathrm{EBP} \alpha$ and $\mathrm{C} / \mathrm{EBP} \varepsilon$, the key transcriptional factors involved in myelopoiesis. Lowering plasma glucose with sodium glucose co-transporter inhibitors de- creased myelopoiesis and suppressed the expression of lineage commitment markers. Mice or bone marrow cells deficient in $\mathrm{S} 100$ calcium binding proteins (S100A8/9) or its receptor, the RAGE (Receptor for Advanced Glycation Endproducts), were protected from diabetes-induced monocytosis in vivo and high glucose conditions in vitro. Enhanced myelopoiesis was not cell-autonomous but mediated by $S 100 A 8 / 9$, secreted predominantly by circulating neutrophils and sensed by RAGE on common myeloid progenitors. Binding of S100A8/A9 to RAGE but not to TLR2/4 (Toll Like Receptor) initiated a signaling cascade via $\mathrm{NF}-\mathrm{KB}$ to induce cytokine release and stimulate proliferation/ differentiation of progenitor cells. Further, in a mouse model of atherosclerotic regression, normalizing plasma glucose reduced lesion area by lowering the number of circulating monocytes as well as their accumulation in the lesion. We propose that hyperglycemia enhances myelopoiesis and that disruption of S100A8/A9RAGE signaling could be a potential therapeutic strategy in the management of diabetic atherosclerosis.

Supported by: NIH (U01-HL087945 and P01-HL54591), CIHR

\section{CLOSED LOOP SYSTEMS}

\section{9-OR}

Factors Affecting Performance of Overnight Closed-Loop Insulin Delivery in Type 1 Diabetes (T1D)

MARIANNA NODALE, DANIELA ELLERI, JANET M. ALLEN, KAVITA KUMARESWARAN, JULIE HARRIS, AHMAD HAIDAR, LALANTHA LEELARATHNA, KAREN CALDWELL, HELEN R. MURPHY, MALGORZATA E. WILINSKA, CARLO L. ACERINI, MARK L. EVANS, DAVID B. DUNGER, ROMAN HOVORKA, Cambridge, United Kingdom, Montreal, OC, Canada

We investigated factors which might influence the performance of overnight closed-loop insulin delivery. 79 subjects with T1D [ 45 female, 11 children, 43 adolescents, 25 adults, BMI $22.4(4.1) \mathrm{kg} / \mathrm{m} 2$, T1D duration $10.6(9.0)$ yrs, A1c 8.1(1.3)\%, total daily insulin 49.0(21.7)U/day; mean(SD)] attended a clinical research facility to undergo closed-loop treatment with basal rates on insulin pump adjusted every $15 \mathrm{~min}$ using a model-predictive-control (MPC) algorithm and real-time sensor glucose levels. Reference plasma glucose (PG) taken every $15 \mathrm{~min}$ to hourly assessed closed-loop performance. Time spent in target glucose range $71-145 \mathrm{mg} / \mathrm{dl}[82 \%$ (63-100), median (IQR)] increased with age and total daily insulin dose, but no relationship was observed with A1c levels or insulin absorption rate [time-to-peak of plasma insulin Tmax; 63.3 (22.5) min]. Time spent below 70mg/dl [0.0 (0.0-0.8) \%] and low blood glucose index [LBGI; 0.5 (0.0-1.7)] increased with Tmax. Mean PG [117 (101-133) mg/dl] and variability of PG [SD of PG 20 (13-22) mg/dl] increased with the controller algorithm effort [ratio of delivered insulin advised by MPC and pre-programmed pump delivery profile $1.1(0.3)]$, while time spent in target range, time spent below target and LBGI all decreased. We conclude that delayed insulin absorption may increase risk of low glucose levels. The MPC controller may be equally effective irrespective of A1C levels, but its performance may be hindered by acutely elevated insulin requirements or by underestimated pump delivery profile.

\begin{tabular}{lcccccc}
\hline Correlation between closed-loop outcomes and explanatory factors \\
\hline & N & $\begin{array}{c}\text { Mean } \\
\text { PG }\end{array}$ & $\begin{array}{c}\text { SD } \\
\text { PG }\end{array}$ & $\begin{array}{c}\text { Time } \\
\text { spent } \\
\text { below } \\
70 \mathrm{mg} / \mathrm{dl}\end{array}$ & $\begin{array}{c}\text { Time in } \\
\text { target range } \\
\text { 71-145 } \\
\text { mg/dl }\end{array}$ & LBGl \\
\hline Age & 79 & -0.03 & -0.01 & -0.06 & $0.23 \dagger$ & 0.04 \\
\hline A1c & 79 & -0.05 & 0.16 & $0.24 \dagger$ & -0.19 & 0.17 \\
\hline Total daily insulin dose & 79 & -0.16 & -0.15 & -0.05 & $0.29 \dagger$ & 0.07 \\
\hline Controller effort & 79 & $0.51^{*}$ & $0.24 \dagger$ & $-0.28 \dagger$ & $-0.40^{*}$ & $-0.42^{*}$ \\
\hline Insulin absorption Tmax & 70 & -0.20 & 0.06 & $0.24 \dagger$ & 0.05 & $0.28 \dagger$
\end{tabular}

${ }^{*} \mathrm{P}<0.05+\mathrm{P}<0.01$

Supported by: JDRF, Diabetes UK, NIH, EC FTP7

220-0R

The Order Effect of the In-Clinic ASPIRE Study: Hypoglycemia Begets Hypoglycemia

SATISH GARG, RONALD L. BRAZG, TIMOTHY S. BAILEY, BRUCE A. BUCKINGHAM, DAVID C. KLONOFF, JOHN SHIN, JOHN B. WELSH, FRANCINE R. KAUFMAN, $A u-$ rora, CO, Renton, WA, Escondido, CA, Stanford, CA, San Mateo, CA, Northridge, CA

In the in-clinic ASPIRE study of induced hypoglycemia with the low glucose suspend (LGS) feature, we examined the effect of the order of experiments on the duration of hypoglycemia. ASPIRE enrolled 50 subjects (48 finished) with type 1 diabetes using the Veo sensor-augmented insulin pump system 
(Medtronic) with the LGS feature on or off. Exercise began after an overnight fast and continued until plasma glucose (YSI) reached $\leq 85 \mathrm{mg} / \mathrm{dL}$. In LGS-ON experiments, the LGS feature stopped the pump for $2 \mathrm{~h}$ when a sensor glucose (SG) value $\leq 70 \mathrm{mg} / \mathrm{dL}$ was detected. Subjects were randomly assigned to Group A (LGS-ON first) or Group B (LGS-OFF first). Experiments were separated by at least $3 \mathrm{~d}$. To achieve pre-defined criteria for success, some experiments had to be repeated. Hypoglycemia was significantly less in successful LGS-ON vs. LGS-OFF experiements (138.3 \pm 76.68 vs $170.7 \pm 75.91 \mathrm{~min}, \mathrm{p}=0.0006)$. The duration of hypoglycemia in successful LGS-ON experiments was 63.7 min less for Group $A$ than for group $B(p<0.01)$. The number of inductions prior to a successful LGS-ON experiment was lower for Group A than for group B $(0.36 \pm 0.64$ vs. $1.57 \pm 0.84, p<0.001)$, as was the cumulative duration of antecedent hypoglycemia (Table). The difference in hypoglycemia duration between Groups A and $B$ was not attributable to differences in $S G$ rates of change, the duration of exercise, or $\mathrm{AUC}<70$ in the $2 \mathrm{~d}$ before the successful induction (all $\mathrm{p}>0.3$ ). The ASPIRE study shows that antecedent hypoglycemia can significantly exacerbate subsequent hypoglycemia. Despite the crossover design leading to an order effect, the LGS feature was shown to reduce hypoglycemia

Hypoglycemia in Successful LGS-ON experiments in the In-Clinic ASPIRE study

\begin{tabular}{lcc}
\hline & $\begin{array}{c}\text { Cumulative hypoglycemia* } \\
\text { duration prior to successful } \\
\text { LGS-ON experiment } \\
\text { (mean } \pm \text { SD, min) }\end{array}$ & $\begin{array}{c}\text { Hypoglycemia* duration } \\
\text { in the successful } \\
\text { LGS-ON experiment } \\
\text { (mean } \pm \text { SD, min) }\end{array}$ \\
\hline Group A N=25 & $16.6 \pm 57.3$ & $107.8 \pm 71.2$ \\
\hline Group B N=23 & $204.6 \pm 123.7$ & $171.5 \pm 67.0$ \\
\hline p value & $<0.001$ & 0.003 \\
\hline
\end{tabular}

${ }^{*}$, defined as $\mathrm{YSI}<70 \mathrm{mg} / \mathrm{dL}$

Supported by: Medtronic, Inc.

221-0R

Automatic Insulin Pump Suspension for Induced Hypoglycemia: The ASPIRE Study

SATISH GARG, RONALD L. BRAZG, TIMOTHY S. BAILEY, BRUCE A. BUCKINGHAM, DAVID C. KLONOFF, JOHN SHIN, JOHN B. WELSH, FRANCINE R. KAUFMAN, Aurora, CO, Renton, WA, Escondido, CA, Stanford, CA, San Mateo, CA, Northridge, $C A$

Automatic insulin pump suspension by the Medtronic Veo insulin pump system may safely and effectively reduce hypoglycemia. The ASPIRE study examined the utility of the low glucose suspend (LGS) feature in hypoglycemia induced by overnight fasting and exercise. Fifty insulin pump users $\left(56 \%\right.$ male, mean $[ \pm S D]$ age $34.3 \pm 12.4 \mathrm{yr}$, BMI $26.9 \pm 4.3 \mathrm{~kg} / \mathrm{m}^{2}$, A1C $7.9 \pm$ $0.6 \%$ ) exercised until their YSI glucose value was $<85 \mathrm{mg} / \mathrm{dL}$ with LGS-ON (set to suspend insulin for 2 hours at sensor glucose values $\leq 70 \mathrm{mg} / \mathrm{dL}$ ) and with LGS-OFF. A successful experiment was defined by YSI glucose values $<70$ and $>50 \mathrm{mg} / \mathrm{dL}$ with an observation period of up to $4 \mathrm{~h}$ that was not interrupted by symptomatic hypoglycemia or protocol deviations. Of the 134 experiments, 98 were successful and 36 failed (Table). Failures were attributable to YSI values falling to $<50 \mathrm{mg} / \mathrm{dL}$, YSI values not reaching $<70$ $\mathrm{mg} / \mathrm{dL}$, and protocol deviations. The mean duration of hypoglycemia in the 98 successful experiments was significantly less with LGS-ON compared to LGS-OFF (138.5 \pm 76.68 vs. $170.7 \pm 75.91 \mathrm{~min}, \mathrm{p}=0.006)$. Mean nadir ( $59.5 \pm$ 5.72 vs $57.6 \pm 5.69 \mathrm{mg} / \mathrm{dL}, \mathrm{p}=0.015)$ and mean end-observation $\mathrm{YSI}$ glucose values $(91.4 \pm 41.84 \mathrm{vs} 66.2 \pm 13.48 \mathrm{mg} / \mathrm{dL}, \mathrm{p}<0.001)$ were both higher with LGS-ON than with LGS-OFF. The ASPIRE study showed the efficacy of the LGS feature in reducing the duration and severity of hypoglycemia without the development of significant rebound hyperglycemia.

\begin{tabular}{|c|c|c|c|c|}
\hline & \multicolumn{4}{|c|}{ ASPIRE Experiments } \\
\hline & $\begin{array}{c}\text { LGS-ON } \\
\text { Experiments (n) }\end{array}$ & $\begin{array}{c}\text { LGS-ON } \\
\text { Subjects (n) }\end{array}$ & $\begin{array}{c}\text { LGS-OFF } \\
\text { Experiments (n) }\end{array}$ & $\begin{array}{c}\text { LGS-OFF } \\
\text { Subjects (n) }\end{array}$ \\
\hline Attempts & 69 & 50 & 65 & 50 \\
\hline $\begin{array}{l}\text { Successful } \\
\text { Attempts }\end{array}$ & $48^{*}$ & $48^{*}$ & 50 & 50 \\
\hline Failed Attempts & 21 & 14 & 15 & 12 \\
\hline $\mathrm{YSI} \geq 70 \mathrm{mg} / \mathrm{dL}$ & 9 & 8 & 5 & 5 \\
\hline $\mathrm{YSI}<50 \mathrm{mg} / \mathrm{dL}$ & 9 & 5 & 8 & 7 \\
\hline Protocol deviation & 3 & 3 & 2 & 2 \\
\hline $\begin{array}{l}\text { * one subject's YSI } \\
\text { YSI fell below } 50 \mathrm{~m} \\
\text { previously complete } \\
\text { further attempts. }\end{array}$ & $\begin{array}{l}\text { did not reach } \\
\text { g/dL on each c } \\
\text { d a successfu }\end{array}$ & $\begin{array}{l}\text { g/dL after } \\
\text { attempts. } \\
\text { S OFF sess }\end{array}$ & $\begin{array}{l}\text { ttempts and on } \\
\text { se } 2 \text { subjects } h \\
\text { and refused to }\end{array}$ & $\begin{array}{l}\text { ne subject's } \\
\text { nad both } \\
\text { undergo }\end{array}$ \\
\hline
\end{tabular}

Supported by: Medtronic, Inc.
222-0R

Automated Management of Blood Glucose in Children With Type 1 Diabetes using a Bi-Hormonal Bionic Pancreas

STEVEN J. RUSSELL, FIRAS H. EL-KHATIB, KENDRA L. MAGYAR, DAVID M. NATHAN, LYNNE L. LEVITSKY, NICOLE A. SHERRY, EDWARD R. DAMIANO, Boston, MA

Automated blood-glucose control with a bionic pancreas could reduce average blood plasma glucose (PG), glucose variability, and hypoglycemia, and improve quality of life for people with type 1 diabetes. Twelve subjects, 12-18 years of age, with type 1 diabetes and no endogenous insulin secretion each participated in a 51-hour closed-loop blood-glucose control experiment, which included six carbohydrate-rich meals and a period of structured exercise. Plasma glucose was managed with a fully automated bionic pancreas using a continuous glucose monitor (CGM) and a compute algorithm controlling subcutaneous delivery of insulin and glucagon with insulin pumps. A partial priming bolus of insulin was given at the beginning of each meal. The priming bolus was preset to $0.08 \mathrm{u} / \mathrm{kg} / \mathrm{meal}$ on the first day, and then automatically adapted on the second day with the goal of providing $45 \%$ of the 4-hour post-prandial insulin for each meal. Plasma glucose control was evaluated with a reference quality measurement on venous blood every 15 minutes. There were no significant differences in either PG or CGM glucose control between the first and second days. The overall mean PG was $166 \mathrm{mg} / \mathrm{dl}$ on the first day and $174 \mathrm{mg} / \mathrm{dl}$ on the second, with $61 \%$ of PG values in the $70-180 \mathrm{mg} / \mathrm{dl}$ range (CGM glucose averaged $162 \mathrm{mg} / \mathrm{dl}$ on the first day and $158 \mathrm{mg} / \mathrm{dl}$ on the second). On average, the partial priming bolus accounted for $33 \pm 5 \%$ of the 4 -hour post-prandial insulin on the first day and $42 \pm 4 \%$ on the second. Hypoglycemia (PG<70 mg/dl) was rare with 10 instances (4 requiring supplemental carbohydrates) during 576 hours of closed-loop control ( $0.7 \%$ of total time). During the nighttime hours, mean PG was $137 \mathrm{mg} / \mathrm{dl}$. An automated, bi-hormonal bionic pancreas achieved safe and effective glycemic control in children with type 1 diabetes with minimal hypoglycemia over two days of continuous use despite high-carbohydrate meals and exercise. A trial testing a wearable version of the system in close to free-living conditions is justified.

223-0R

Dual-Hormone Closed-Loop (CL) System in Adults With Type 1 Diabetes (T1D): Randomized Crossover Trial

AHMAD HAIDAR, MARYSE DALLAIRE, ANNABELLE MATHIEU, AMMAR ALKHATEEB, ADÈLE CORIATI, ANNIE TARDIF, VIRGINIE MESSIER, MAUDE MILLETTE, PEIYAO CHENG, BENOIT BOULET, LAURENT LEGAULT, RÉMI RABASA-LHORET, Montreal, OC, Canada, Farwaniya, Kuwait, Tampa, FL

We are conducting a randomized crossover study to compare dual-hormone CL system with conventional pump therapy (CSII) in regulating glucose levels in 15 adults with T1D. Patients were admitted twice to a clinical facility (CF) and received CL and CSII treatments. Patients received interventions starting from $16 \mathrm{~h} 00$, exercised at $17 \mathrm{~h} 50$ for $30 \mathrm{~min}\left(60 \% \mathrm{VO}_{2 \text { max }}\right)$, ingested a meal at $19 \mathrm{~h} 20$ ( $80 \mathrm{~g} \mathrm{CHO}$ for males and $60 \mathrm{~g} \mathrm{CHO}$ for females), ingested a $15 \mathrm{~g} \mathrm{CHO}$ snack at $22 \mathrm{hOO}$ and stayed in the CF until 7h00 next morning. In $\mathrm{CL}$, based on glucose sensor readings (Sofsensor, Medtronic), subcutaneous insulin and glucagon were delivered following 10-minutes recommendations from a dosing algorithm. The algorithm is based on fuzzy-supervised modelbased predictive controller combined with extended Kalman filter and a set of heuristic rules. Plasma glucose (PG) was measured using YSI2300 STAT Plus Analyser. Results of the first 14 completed subjects (Age $47 \pm 12 y r s$, A1c $7.8 \pm 0.7 \%$, total daily dose $0.6 \pm 0.1 \mathrm{U} / \mathrm{kg} /$ day) demonstrate that $\mathrm{CL}$ increased the percentage of time for which PG is in target range by a median 15\% [-1 to 21] $(P=0.009)$ and decreased the time less than $4 \mathrm{mmol} / \mathrm{L}$ by $2.1 \%[0-10.2](P$ $=0.01)$. Seven patients (50\%) had at least one hypoglycemic event $(\mathrm{PG}<3.0$ $\mathrm{mmol} / \mathrm{L}$ ) requiring oral treatment during CSII intervention compared with one patient $(7 \%)$ in closed-loop $(P=0.04)$, demonstrating a 7 -fold reduction. We conclude that dual-hormone $\mathrm{CL}$ combined with advanced algorithms could reduce the risk of hypoglycemia in adults with T1D.

Comparisons between CL and CSII (median [IOR])

\begin{tabular}{lcccc}
\hline $\begin{array}{l}\text { Time plasma } \\
\text { glucose is }(\%)\end{array}$ & $\mathrm{CL}$ & $\mathrm{CSIl}$ & $\begin{array}{c}\text { Paired } \\
\text { difference }\end{array}$ & P value \\
\hline in target & $69[46-85]$ & $60[25-72]$ & $15[-1$ to 21$]$ & 0.009 \\
\hline$<4.0 \mathrm{mmol} / \mathrm{L}$ & $0.0[0.0-3.0]$ & $6.4[0.0-12.2]$ & $-2.1[-10.2$ to 0.0$]$ & 0.01 \\
\hline$<3.3 \mathrm{mmol} / \mathrm{L}$ & $0.0[0.0-0.0]$ & $2.3[0.0-5.9]$ & $-2.3[-5.9$ to 0.0$]$ & 0.008 \\
\hline above target $\neq$ & $31[11-49]$ & $30[23-63]$ & $-7[-21$ to 3$]$ & 0.14 \\
\hline
\end{tabular}

¥Target range 4-8 $\mathrm{mmol} / \mathrm{L}$ from $21 \mathrm{~h}$ to $7 \mathrm{~h}$ and $4-10 \mathrm{mmol} / \mathrm{L}$ otherwise

Supported by: Diabète Québec, Canadian Diabetes Association, JA Sève Chair, and Medtronic 
224-0R

Comparison of Two Closed Loop Algorithms With Open Loop Control in Type 1 Diabetes

J. HANS DEVRIES, ANGELO AVOGARO, CARSTEN BENESCH, DANIELA BRUTTOMESSO, KAREN CALDWELL, CLAUDIO COBELLI, WERNER DOLL, SIMONE DEL FAVERO, LUTZ HEINEMANN, ROMAN HOVORKA, LALANTHA LEELARATHNA, YOERI M. LUIJF, JULIA MADER, LALO MAGNI, MARIANNA NODALE, JEROME PLACE, ERIC RENARD, CHIARA TOFFANIN, AP@HOME CONSORTIUM, Amsterdam, The Netherlands, Padua, Italy, Neuss, Germany, Cambridge, United Kingdom, Graz, Austria, Pavia, Italy, Montpellier, France

Closed loop systems aim to automate blood glucose control in patients with type 1 diabetes by optimal insulin delivery, driven by a control algorithm based on continuous glucose monitoring (CGM). Blood glucose was controlled for $23 \mathrm{~h}$ in 47 patients in six centers by a Model Predictive Control algorithm developed at the University of Pavia with a Safety Supervision Module from UVA \& UCSB (iAP), or at Cambridge University (CAM), with a patient driven open loop (OL) control session in a randomized three-way cross-over design, during three clinical research center admissions including three meals and an exercise bout. CGM data were provided by the Dexcom Seven Plus (Dexcom Inc, San Diego, CA, USA) and insulin was administered by the Omnipod Insulin Pump (Insulet Corp, Bedford, MA, USA), either automatically with the Artificial Pancreas System (UCSB/Sansum, CA, USA) (three centers) or manually (three centers). For glucose reference measurements the YSI glucose analyzer was used. An intention-to-treat analysis was done using a general linear model for repeated measures. There was no significant difference in time spent in euglycemia (70-144, postmeal 180 $\mathrm{mg} / \mathrm{dL}$ ) between closed loop and open loop (62.6\% for 0L, 59.2\% for iAP and $58.3 \%$ for $C A M$, overall $P=0.377$ ). Mean glucose level was significantly lower in open loop (126 for OL vs. 148 for iAP and $149 \mathrm{mg} / \mathrm{dL}$ for CAM, overall $P=0.001)$. There was significantly less time spent in hypoglycemia $(<70 \mathrm{mg} /$ $\mathrm{dL}$ ) during closed loop $(6.4 \%, 2.1$ and $2.0 \%$, overall $P=0.001)$ with a trend towards less hypoglycemia $\leq 50 \mathrm{mg} / \mathrm{dL}(0.92 \%, 0.43$ and $0.17 \%$, overall $\mathrm{P}=$ 0.072 ). There were no significant differences in any of these outcomes between the two closed loop algorithms. From the largest and first multicenter head-to-head closed loop study performed so far, we conclude that both CAM and iAP algorithms allow safe automated control of blood glucose in a closed loop system, also during meal and exercise challenges, with less hypoglycemia at the expense of a higher mean glucose.

Supported by: European Community Framework Programme 7 (FP7-ICT-2009-4)

\section{PEDIATRIC OBESITY AND TYPE 2 DIABETES- PHENOTYPES AND GENOTYPES PRE- AND POST-DIAGNOSIS}

$\Delta$ 225-0R

HbA1c Diagnostic Categories and $\beta$-Cell Function Relative to Insulin Sensitivity in Overweight/Obese Adolescents

LINDSEY A. SJAARDA, SOJUNG LEE, HALA TFAYLI, FIDA BACHA, ELISA ANDREATTA, SILVA ARSLANIAN, Pittsburgh, PA, Beirut, Lebanon, Houston, TX

The recommended $\mathrm{HbA1c}$ diagnostic categories remain controversial and their utility in doubt in pediatrics. Because impaired fasting glucose (IFG) and impaired glucose tolerance (IGT) in youth are linked to impaired insulin secretion, we hypothesized that alterations in the pathophysiological mechanisms of type 2 diabetes (T2DM) may be evident in the ADA recommended at-risk/prediabetes category. Thus, we compared insulin sensitivity (IS) and $\beta$-cell function in overweight/obese adolescents categorized with prediabetes ( $\mathrm{HbA1C} \geq 5.7-<6.5 \%)$ to those with normal HbA1c $(<5.7 \%)$. Adolescents $(\mathrm{n}=200)$ with body mass index $(\mathrm{BMI}) \geq 85^{\text {th }}$ percentile were evaluated by a 2-h oral glucose tolerance test, body fat by dual energy $x$-ray absorptiometry, IS by a $3-\mathrm{h}\left(80 \mathrm{mu} / \mathrm{m}^{2} / \mathrm{min}\right)$ hyperinsulinemic-euglycemic clamp, and first phase insulin secretion by a $2-\mathrm{h}$ hyperglycemic clamp $(\sim 225 \mathrm{mg} / \mathrm{dL})$. $\beta$-cell function relative to IS was calculated (disposition index, $\mathrm{DI}=\mathrm{IS} \times$ first phase insulin). Differences between normal and prediabetes groups (Table) were adjusted for race since $\mathrm{HbA1c}$ and $\mathrm{DI}$ are known to be higher in blacks (B) than whites (W). The prediabetes group had lower IS, lower $\beta$-cell function relative to IS (DI) and higher \% body fat ( $P=0.003)$. Rates of IGT ( 25 vs. $33 \%$ ) and IFG (23 vs. $36 \%)$ were not different between the two groups $(P>0.10)$. In conclusion, overweight/obese adolescents with $\mathrm{HbA1c}$ in the currently recommended at-risk/prediabetes range manifest metabolic impairments associated with heightened risk for T2DM. The predictive value of $\mathrm{HbA1c}$ for the development of T2DM in youth remains to be determined.

\begin{tabular}{lccc}
\hline & $\begin{array}{c}\mathrm{HbA1c}<5.7 \% \\
\mathrm{n}=161(43 \% \text { male })\end{array}$ & $\begin{array}{c}\mathrm{HbA1c}>5.7-<6.5 \% \\
\mathrm{n}=39(38 \% \text { male })\end{array}$ & $\mathrm{P}$ \\
\hline Age & $14.1 \pm 0.2$ & $14.3 \pm 0.4$ & 0.68 \\
\hline Race $(\% \mathrm{~B} / \mathrm{W})$ & $37 / 63$ & $67 / 33$ & 0.001 \\
\hline BMl $\left(\mathrm{kg} / \mathrm{m}^{2}\right)$ & $33.6 \pm 0.5$ & $37.5 \pm 0.8$ & $<0.001$ \\
\hline Fasting insulin $(\mathrm{mu} / \mathrm{ml})$ & $35.7 \pm 1.4$ & $47.9 \pm 4.1$ & 0.001 \\
\hline IS $(\mathrm{mg} / \mathrm{kg} / \mathrm{min} \mathrm{per} \mathrm{mu} / \mathrm{mL})$ & $2.8 \pm 0.2$ & $2.0 \pm 0.2$ & 0.02 \\
\hline First phase insulin $(\mathrm{mu} / \mathrm{mL})$ & $217 \pm 10$ & $247 \pm 31$ & 0.79 \\
\hline Dl $(\mathrm{mg} / \mathrm{kg} / \mathrm{min})$ & $511 \pm 26$ & $424 \pm 56$ & 0.01 \\
\hline
\end{tabular}

Supported by: NIH (R01HD27503, K24HD01357 and UL1RR024153)

226-OR

Evidence of Early Beta Cell Deficiency in Children who Develop Impaired Fasting Glucose-A 10-Year Longitudinal Study JOANNE HOSKING, BRAD S. METCALF, ALISON N. JEFFERY, ADAM J. STREETER, MOHAMMOD MOSTAZIR, LINDA D. VOSS, TERENCE J. WILKIN, Plymouth, United Kingdom

Blood glucose levels are a combined function of the demand for insulin (insulin sensitivity) and the capacity of the beta cells to supply it. Impaired fasting glucose $(\geq 5.6 \mathrm{mmol} / \mathrm{l})$ is a predictor of future diabetes and increasingly common in children, but the extent to which it results from excess demand or failure of supply is unclear. We compared the trajectories of glucose, BMlsds, insulin resistance (HOMA-IR) and beta cell function (HOMA-B) in children who developed IFG and those whose glucose levels remained within the normal range annually from $5 y$ to $15 y$. We also examined their association with corresponding measures in the parents at baseline. Fiftyfive children showed IFG, mostly after age 11y, the majority of whom (39) were boys. Fasting glucose rose progressively in both groups, but was higher throughout in those who developed IFG compared with those who did not ( $p=$ $0.002)$. IR and BMI correlated ( $r=0.4, p<0.001)$, but there was no difference in BMl at any age between those who showed IFG and those who did not $(p=$ 0.58). IR was biphasic, falling from $5 y$ and rising after $7 y$, but again was the same in both groups at all ages ( $p=0.08)$. HOMA-B, on the other hand, was lower in those who developed IFG even at $5 y$ ( 81.1 v 94.1, $p=0.02$ ). Glucose levels of the parents whose children developed IFG were higher than those whose children did not (mother's glucose: $4.9 \vee 4.6 \mathrm{mmol} / \mathrm{l}, \mathrm{p}<0.001$; father's glucose: $5.0 \mathrm{v} 4.8 \mathrm{mmol} / \mathrm{l}, \mathrm{p}=0.03$ ). There was no difference in the IR of either parent group ( $p \geq 0.81$ ), but the HOMA-B of the mothers whose children had IFG was lower (112.7 v 129.6, $p=0.01$ ), IFG is common in contemporary children, and it appears to be dependent on a defect in beta cell function, which is already present at $5 y$. The parental associations suggest a possible genetic cause, which needs IR to reveal it. As the IR of puberty falls, further weight gain seems likely to be the determinant of progression to diabetes.

227-0R

One Hour Glucose during an Oral Glucose Challenge Prospectively Predicts $\boldsymbol{\beta}$-Cell Deterioration and Prediabetes in Youth

JOON YOUNG KIM, MICHAEL I. GORAN, CLAUDIA TOLEDO-CORRAL, MARC J. WEIGENSBERG, MYUNGHAN CHOI, GABRIEL 0. SHAIBI, Phoenix, AZ, Los Angeles, CA

In adults, a 1-hr glucose concentration of $155 \mathrm{mg} / \mathrm{dl}$ during an Oral Glucose Tolerance Test (OGTT) prospectively predicts the development of type 2 diabetes (T2D). Whether this threshold is useful in younger populations is unknown. The purpose of this study was to examine the utility of a 1-hr glucose threshold $\geq 155 \mathrm{mg} / \mathrm{dl}$ to predict diabetes risk factors in high-risk Latino youth. Obese Latino youth with a family history of T2D (133 M / 100 $\mathrm{F} ; 11.1 \pm 1.7 \mathrm{yrs}$ ) underwent a baseline OGTT and were subsequently divided into two groups based upon 1-hr glucose concentrations (Below155: <155 $\mathrm{mg} / \mathrm{dl}, \mathrm{n}=151$ or Above155: $\geq 155 \mathrm{mg} / \mathrm{dl}, \mathrm{n}=82$ ). Participants were followed for up to 8 years where serial frequently sampled IV glucose tolerance tests were performed to estimate insulin sensitivity $\left(S_{\mathrm{i}}\right)$, acute insulin response $(A I R)$, and $\beta$-cell function using the disposition index ( $D I=S_{i} \times$ AIR). At baseline, no group differences were noted for BMI percentile, total fat mass, or $S_{i}$ while the Above155 group had lower AIR $(1572.9 \pm 1292.7$ vs. $1848.2 \pm$ $1246.4 \mu \mathrm{U} / \mathrm{ml}, \mathrm{p}<0.01)$ and DI $\left(2321.0 \pm 120.0\right.$ vs. $2708.4 \pm 100.0 \times 10^{-4} \mathrm{~min}^{-1}$. $p<0.01)$ compared to the Below155 group. Using linear mixed model analysis, assignment in the Above155 group at baseline was associated with a significantly lower DI over time even after controlling for fasting and 2-hour glucose $(B=-20495.9, p<0.05)$. The pattern of change for the Above155 group was characterized by a steady decline in DI resulting in a $54.8 \%$ decrease by year 8 . In contrast, Below155 was characterized by an initial decrease followed by an increase in $\mathrm{Dl}$ of $28.6 \%$ by year 8 . When data were restricted to 
only participants with normal glucose tolerance at baseline, $70 \%$ of those in the Above155 group progressed to prediabetes compared to only $43 \%$ in the Below155 group $(p<0.01)$. These data suggest a 1-hour glucose threshold of $155 \mathrm{mg} / \mathrm{dl}$ during an OGTT may predict future deterioration of $\beta$-cell function and progression to prediabetes among obese Latino youth.

Supported by: NIH (R01 DK 59211)

228-OR

Is Prevalence of Type 2 Diabetes Increasing in Youth? The SEARCH for Diabetes in Youth Study

DANA DABELEA, ELIZABETH MAYER-DAVIS, JENNIFER W. TALTON, RICHARD F. HAMMAN, RONNY A. BELL, LAWRENCE M. DOLAN, JASMIN DIVERS, GIUSEPPINA IMPERATORE, LENNA L. LIU, BARBARA LINDER, ANWAR MERCHANT, KRIST REYNOLDS, SHARON SAYDAH, FOR THE SEARCH FOR DIABETES IN YOUTH STUDY, Aurora, CO, Chapel Hill, NC, Winston-Salem, NC, Cincinnati, OH, Atlanta GA, Seattle, WA, Bethesda, MD, Columbia, SC, Pasadena, CA

Type 2 diabetes (T2D) among youth is of increasing concern, with some reports suggesting an epidemic. However, there is a paucity of populationbased data to quantify such trends. SEARCH aimed to estimate temporal changes (2001 to 2009) in the prevalence of T2D in youth. Data from four geographic-based SEARCH sites were included (Colorado, South Carolina, Ohio and Washington). Similar methods were used for 2001 and 2009 prevalent cases. Cases had a physician diagnosis of $\mathrm{T} 2 \mathrm{D}$ and were $<20$ years on December 31, 2001 and 2009, respectively. Population denominators were projected from age- race- and sex-specific population counts for youth $<20$ years old using vintage 2009 US census data. Race/ethnicity was categorized as non-Hispanic white (NHW), Hispanic (HISP), African American (AA), Asian/Pacific Islander (API) and American Indian (AI). A total of 382 and 464 youth with T2D prevalent in 2001 and 2009 were included. Completeness of ascertainment was estimated at $91 \%$ in 2001 and $88 \%$ in 2009 using capture-recapture. Since T2D was exceedingly rare below 10 years, prevalence estimates in 2001 and 2009 (per 1000) are presented for youth age $\geq 10$ years, by sex, race/ethnicity, and age-group (Figure). Overall, the prevalence of T2D increased from $0.29(0.27-0.33)$ to $0.36(0.32-0.39, p=0.007)$, a $21 \%$ increase over eight years. The increase was significant in females $(p=0.003)$, HISP ( $p=0.0002)$ and NHW youth ( $p=0.05)$ and not significant among AA, API and Al youth. Our data suggest that the prevalence of diagnosed T2D is increasing in U.S. youth. A longer study period is needed to fully quantify these trends in all racial/ethnic groups.

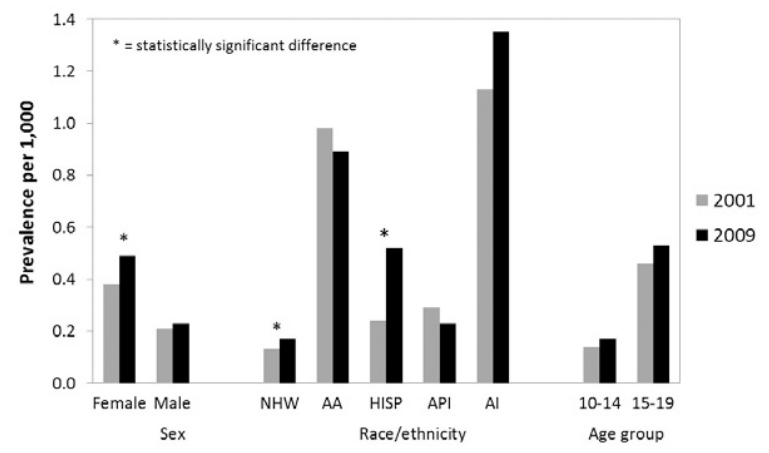

Supported by: The CDC with support from the NIDDK

229-0R

Physical Inactivity and Activity in Youth With Recent Onset of Type 2 Diabetes

ANDREA KRISKA, LINDA DELAHANTY, SHARON EDELSTEIN, NANCY AMODEI, JENNIFER CHADWICK, KENNETH COPELAND, BRYAN GALVIN, LAURE EL GHORMLI, MOREY HAYMOND, MEGAN KELSEY, CHAD LASSITER, ELIZABETH MAYER-DAVIS, KERRY MILASZEWSKI, AMY SYME, Pittsburgh, PA, Boston, MA, Washington, DC, San Antonio, TX, Oklahoma City, OK, Houston, TX, Aurora, CO, Philadelphia, PA, Chapel Hill, NC, New Haven, CT

With the rise of type 2 diabetes (T2D) in youth, it is critical to investigate factors such as physical activity (PA) and sedentary time (inactivity) that may be contributing to this public health problem. We compared PA and inactivity levels in youth with T2D to a similarly aged, obese subset of the NHANES (2005-2006) cohort. The 699 youth (aged 10-17 years) with T2D were part of the Treatment Options for type 2 Diabetes in Adolescents and Youth (TODAY) multi-center study. Accelerometry measurement was similar in TODAY and NHANES. At baseline, accelerometry was completed in 35\% of the TODAY cohort (those who did and did not complete accelerometry did not statistically differ by age, gender, race/ethnicity, or BMI). A comparison of mean (SE) minutes of PA and sedentary time is presented.

A. Age-Adjusted Moderate-Vigorous Physical Activity

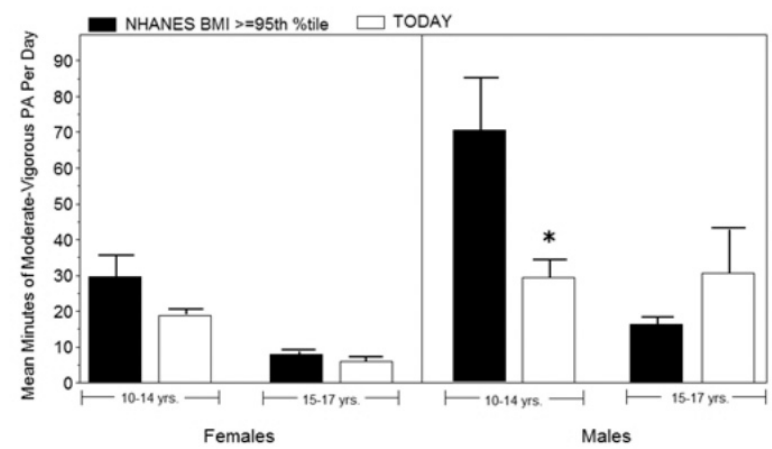

B. Age-Adjusted Sedentary Behavior

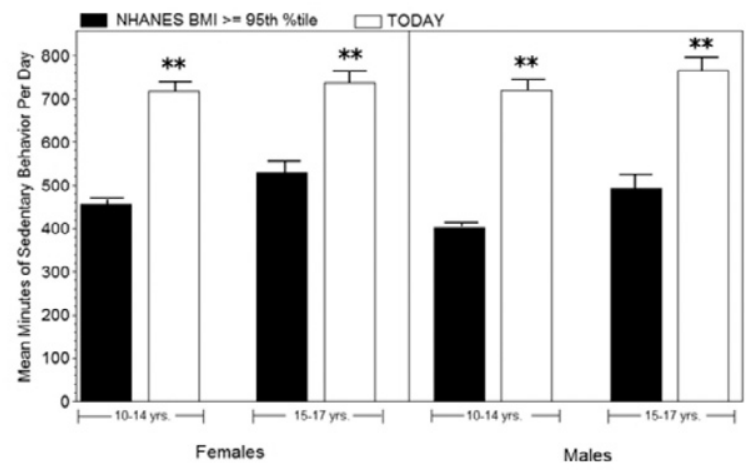

Statistical significance for between group differences is based on means and standard error of the means for NHANES and TODAY, ${ }^{*} P<.05,{ }^{*} P<.01$ Overall, minutes of moderate/vigorous PA were low and similar between the two cohorts. However, relative to the obese NHANES subset, TODAY youth had significantly higher levels of sedentary behavior for all age/gender groups $(p<0.01)$. These data demonstrate that treatment efforts in T2D youth should focus on increasing non-sedentary activities.

Supported by: NIDDK/NIH

230-OR

Body Composition during the TODAY Randomized Clinical Trial KENNETH COPELAND, LAURE EL GHORMLI, FOR THE TODAY STUDY GROUP, Oklahoma City, OK, Washington, DC

TODAY (Treatment Options for type 2 Diabetes in Adolescents and Youth) examined the effects of three treatments on time to glycemic failure in a cohort of 699 youth with recent-onset type 2 diabetes, followed at least 2 years. The treatment groups were (1) metformin alone (M), (2) metformin + rosiglitazone $(M+R)$, and (3) metformin + intensive lifestyle $(M+L)$. Body composition was measured by anthropometry and DEXA. Differences across treatments at 24 months were tested with baseline value in the model as a covariate. Data presented include measures collected prior to glycemic failure - i.e., while the participant was taking the randomized treatment. The table provides results at 24 months for the overall sample. There was a significant treatment effect for BMI, BMI z-score, waist circumference, abdominal height, fat, and percent fat free mass (FFM) (all p-values $<0.05$ ). BMI, BMI z-score, waist circumference, abdominal height, and fat were lowest in the $M+L$ group and highest in the $M+R$ group, whereas FFM was highest in the $M+L$ group but lowest in the $M+R$ group. Similar patterns were seen in males and in females. There were no differences across treatments in the nonHispanic Black subgroup. FFM differed by treatment group among Hispanic participants $(p=0.0034)$, and BMI and waist circumference differed by treatment in non-Hispanic Whites ( $p=0.0044$ and $p=0.0097$, respectively). 
Mean (SD) of Body Composition Measurements at Baseline and 24 Months by Treatment Group

\begin{tabular}{lcccccc}
\hline & \multicolumn{2}{c}{$\begin{array}{c}\text { Metformin } \\
\text { alone }\end{array}$} & \multicolumn{2}{c}{$\begin{array}{c}\text { Metformin + } \\
\text { Rosiglitazone }\end{array}$} & \multicolumn{2}{c}{$\begin{array}{c}\text { Metformin + } \\
\text { Lifestyle }\end{array}$} \\
\hline Measurement & Baseline & 24 Months & Baseline & 24 Months & Baseline & 24 Months \\
\hline BMl & $35.8(8.1)$ & $36.7(9.1)$ & $35.0(7.7)$ & $38.3(8.1)$ & $34.1(7.1)$ & $35.4(8.4)$ \\
\hline BMI z-score & $2.3(0.4)$ & $2.2(0.6)$ & $2.2(0.5)$ & $2.3(0.5)$ & $2.2(0.5)$ & $2.1(0.5)$ \\
\hline Waist circumference $(\mathrm{cm})$ & $110.4(16.6)$ & $110.8(17.5)$ & $109.0(17.1)$ & $114.8(19.1)$ & $106.6(16.2)$ & $108.3(17.5)$ \\
\hline Abdominal height $(\mathrm{cm})$ & $24.9(5.2)$ & $25.0(6.0)$ & $24.3(4.0)$ & $25.9(5.8)$ & $24.8(6.9)$ & $24.5(4.0)$ \\
\hline Total fat mass (kg) & $33.7(9.9)$ & $36.1(12.0)$ & $33.5(10.2)$ & $39.7(10.6)$ & $32.6(9.8)$ & $32.2(9.4)$ \\
\hline Total fat free mass $(\%)$ & $62.7(6.4)$ & $61.6(7.8)$ & $62.4(5.9)$ & $60.0(6.7)$ & $62.2(6.2)$ & $63.8(7.3)$ \\
\hline
\end{tabular}

In conclusion, all treatment groups had increases in almost all measures of body composition within the first two years of the study, with most measures worse in the $\mathrm{M}+\mathrm{R}$ group compared to the other two groups.

Supported by: NIDDK/NIH

\section{$\Delta$}

231-0R

A Common Variant in the TRIB1 Gene is Associated With Triglycerides Levels, Lipoprotein Subclasses and ALT Levels in Obese Children and Adolescents

NICOLA SANTORO, BRIDGET PIERPONT, LEIF GROOP, SONIA CAPRIO, New Haven, CT, Lund, Sweden

Recent genome wide association studies have identified a single nucleotide polymorphism, rs17321515, in a locus at chromosome 8q24 as having the $G$ allele associated with lower levels of plasma triglycerides (TG). This locus contains only one annotated gene, tribbles homolog 1(TRIB1), which has been shown to be involved in lipoprotein metabolism in animal studies. We aimed to determine whether this gene variant might be associated with triglyceride, lipoprotein subclasses and ALT levels in a multiethnic cohort of obese children and adolescents. We studied a multiethnic group of 482 obese children and adolescents (200 Caucasians, 140 African Americans, 142 Hispanics, mean age 13.2+/-3.3 years, mean z-score BMI 2.16+/-0.74). Patients underwent an OGTT, lipoprotein subclasses measurement by proton NMR and a subset of 170 children underwent a fast gradient MRI to measure the Hepatic fat content (HFF\%). Genotyping was performed with the use of matrix-assisted laser desorption-ionization time-of-flight mass spectrometry on the MassARRAY platform (Sequenom). The prevalence of the A allele was 0.55 in Caucasians, 0.45 in African Americans and 0.55 in Hispanics. Subjects homozygote for the A allele $(n=131)$ showed significantly higher TG, $(p=0.004)$, larger VLDL $(p=0.02)$, higher AP0 B100 levels $(p=0.004)$ and smaller LDL $(p=0.02)$ than subjects carrying the other genotypes (AG $\mathrm{n}=267$ and $\mathrm{GG} \mathrm{n}=113$ ) independent of age, gender, $\mathrm{z}$-score BMI and ethnicity. Moreover, subjects carrying the AA genotype showed also higher ALT levels $(p=0.004)$, while no difference in terms of HFF\% was observed across the genotypes $(p=0.20)$. The A allele of the rs17321515 SNP near the TRIB1 gene is associated with a proatherogenic profile as well as with liver damage in obese children and adolescents, thus suggesting that the TRIB1 gene may be a link between hepatic fat metabolism and liver damage occurring in obese youths.

Supported by: NIH, AHA

\section{$\Delta$} 232-0R Interactive Effect Between the PNPLA3 rs738409 and Dietary n6/n3 on Hepatic Fat Accumulation and ALT Levels in Obese Youths GRACE KIM, MARY SAVOYE, SONIA CAPRIO, ELVIRA DURAN, JESSICA KUBAT, KATIE MAROTTO, MELISSA SHAW, NICOLA SANTORO, New Haven, CT

A common missense variant (rs738409) characterized by a C-to-G substitution encoding an isoleucine-to-methionine substitution at the amino acid position 148 , in the patatin like phospholipase 3 (PNPLA3) gene has been recently associated with increased hepatic fat deposition and ALT levels in adults and children. Previous studies describing the relationship between dietary intake and NASH have shown a higher intake of omega-6 to omega-3 fatty acids ratio $(n 6 / n 3)$ among NASH patients. We determined whether the influence of the PNPLA3 rs738409 variant on hepatic fat accumulation is modulated by an excess intake of omega- 6 PUFA relative to omega-3 PUFA. We studied 124 obese children and adolescents (53\% girls; 47\% Caucasians;

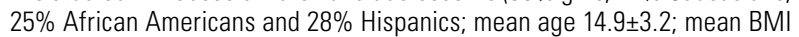
30.7 \pm 7.2). A 3-day food record was obtained by all subjects and dietary composition was assessed with the Nutrition Data System for Research (NDS-R 5.0_35). Subjects underwent an oral glucose tolerance test (OGTT) and a Magnetic Resonance Imaging study to assess the liver fat content (HFF\%) and were genotyped by automatic sequencing for the rs738409 variant. Sixty eight subjects were CC, 38 were $\mathrm{CG}$ and 18 were GG. As previously observed the rs738409 variant was associated with HFF\% ( $p<.001)$ and ALT levels $(p=0.010)$. ALT levels and HFF\% were significantly influenced by the interaction between genotype and $\mathrm{n} 6 / \mathrm{n} 3$ independent of age, gender BMI and ethnicity (0.01 and 0.05, respectively) . HFF\% and ALT levels were, in fact, related to the $n 6 / n 3$ consumption only in subjects homozygote for the $G$ allele of the rs738409 $\left(r^{2}=0.42, p=0.0036\right.$ and $r^{2}=0.38, p=0.0086$, respectively). These findings suggest that obese youths carrying the $G G$ genotype are susceptible to develop hepatic steatosis and liver damage when dietary n6/n3 intake is high. Thus, specific dietary interventions based on genetic predisposition may lead to more effective therapeutic outcomes for NAFLD. Supported by: NIH, AHA

\section{INSULIN ACTION-METABOLISM}

233-0R

Improved Insulin Sensitivity in a Mouse Model of Mitochondrial Dysfunction

DEEPA SATHYASEELAN, DANIEL PULLIAM, NING ZHANG, HANYU LIANG, LAUREN SLOANE, ADAM SALMON, NICOLAS MUSI, HOLLY VAN REMMEN, San Antonio, TX

A decline in the oxidative phosphorylation capacity of mitochondria plays a critical role in the development of insulin resistance. Surfeit locus protein 1 (Surfl) is essential for the assembly of electron transport chain complex IV (Cytochrome c oxidase, COX) and Surff\%- mice have a 50-80\% decrease in COX activity compared to control littermates, yet a significant increase in lifespan. Because mitochondrial dysfunction causes insulin resistance, we asked whether Surf1-- mice with specific down regulation of COX activity have altered insulin sensitivity. Surprisingly, insulin sensitivity was significantly increased in Surf1- mice both under normal chow as well as high fat diet, while glucose tolerance remained unaffected. Surf\% $\%$ mice have a reduced body weight that can be attributed to decreased fat mass due to increased fatty acid oxidation. We also observed a strong up-regulation of PGC-1alpha and PPARalpha as well as increased mitochondrial biogenesis in multiple tissues suggesting the up-regulation of compensatory pathway(s) in response to reduced COX activity. One such pathway is the mitochondrial unfolded protein response (mtUPR), a retrograde signaling response that results in the induction of mitochondrial specific chaperones and proteases involved in refolding misfolded proteins and degrading damaged proteins. Surf ${ }^{-1}$ mice have an increase in the expression of mitochondrial specific chaperone (HSP60) and proteases (CLPP, Lon) that have been linked to the mtUPR. Over-expression of mitochondrial protease Lon in vitro increases fatty acid oxidation as well as glucose uptake. This suggests a novel role of mitochondrial matrix protease Lon, a mtUPR component involved in the maintenance of mitochondrial homeostasis, in insulin sensitivity. In summary, our findings suggest that targeted down regulation of COX activity do not cause insulin resistance, rather improves insulin sensitivity through up-regulation of compensatory pathway(s).

234-0R

Insulin Concentration-Dependent Effect on Mitochondrial Capacity in Obese and Lean Subjects

ESTHER PHIELIX, JULIA SZENDROEDI, GILLES SEQUARIS, TOMAS JELENIK, MARKUS ZEPPETZAUER, MICHAEL RODEN, Düsseldorf, Germany

Mitochondrial function has been suggested to be lower in insulin resistant humans. In vivo assessment of skeletal muscle ATP synthesis showed that impaired insulin stimulation of glucose uptake relates to blunted insulin stimulation of ATP synthetic flux rates. It is yet unclear if insulin stimulates mitochondrial function directly or via increased substrate flux. We examined if ex vivo insulin stimulation directly increases mitochondrial capacity in human muscles. In total, 14 lean and 6 obese glucose tolerant volunteers were included (age: $27.3 \pm 6.2$ and $26.2 \pm 5.4$ years; BMl: $23.5 \pm 3.5$ and $44.6 \pm 6.3$ $\mathrm{kg} / \mathrm{m}^{2}, p<0.05$, respectively). Muscle biopsies were taken from the $m$. vastus lateralis before a hyperinsulinemic-euglycemic clamp was performed and four muscle pieces were incubated with $0,4,40$ or $100 \mathrm{nM}$ insulin for 2.5 h. Mitochondrial capacity was measured in permeabilized fibers using highresolution respirometry in the presence of glycolytic and tricarboxylic acid cycle derived substrates (pyruvate $5 \mathrm{mM}$, glutamate $10 \mathrm{mM}$ and succinate $10 \mathrm{mM}$ ). Obese humans were more insulin resistant than lean participants (M-value: $2.9 \pm 3.2$ v.s. $10 \pm 3.3 \mathrm{mkg}^{-1} \mathrm{~min}^{-1}, p<0.05$ ). Ex vivo insulin stimulation increased mitochondrial capacity $(+11 \%$ and $+21 \%, p<0.05)$ as well as maximal uncoupled respiration $(+11 \%$ and $+18 \%, p<0.05)$ in a concentrationdependent manner between 4 and $40 \mathrm{nM}$ compared with $0 \mathrm{nM}$ of insulin. In contrast, myocellular mitochondrial capacity did not increase upon insulin stimulation in obese insulin resistant glucose tolerant humans. Insulin 
directly stimulates muscular mitochondrial capacity in insulin sensitive humans. Insulin resistance at the mitochondrial level seems to be an early defect in insulin resistant glucose tolerant obese humans.

\section{5-OR}

Importance of Pyruvate Dehydrogenase Kinases in the Regulation of Blood Levels of Glucose and the Metabolism of Ketone Bodies and Fatty Acids

YASMEEN RAHIMI, NAM HO JEOUNG, PEIGFEI WU, MARTHA KUNTZ, PAUL W. LEE, ROBERT A. HARRIS, Indianapolis, IN, Daegu, Republic of Korea, Torrance, CA

The importance of pyruvate dehydrogenase kinases (PDK) 2 and 4 in regulation of the pyruvate dehydrogenase complex (PDC) was assessed in single and double knockout (DKO) mice. PDK2 deficiency caused higher PDC activity and lower blood glucose in the fed state. PDK4 deficiency caused similar effects in the fasting state. DKO intensified these effects in both states. PDK2 deficiency had no effect on glucose tolerance, PDK4 deficiency produced a modest effect, but DKO caused a marked improvement, lowered insulin levels, and increased insulin sensitivity. However, the DKO mice were more sensitive than wild-type mice to long-term fasting, succumbing to hypoglycemia, ketoacidosis, and hypothermia. Stable isotope flux analysis indicated that hypoglycemia was due to a reduced rate of gluconeogenesis and that acetyl-CoA derived from glucose contributed to ketone body synthesis in the DKO mice. DKO mice fed a high fat diet had improved glucose tolerance, decreased adiposity, and were euglycemic $(107 \pm 11 \mathrm{mg} / \mathrm{dl}$ compared to $197 \pm$ $11 \mathrm{mg} / \mathrm{dl}$ in wild-type mice) due to reduction in the rate of gluconeogenesis $(9.7 \pm 0.3 \mathrm{mg} / \mathrm{kg} / \mathrm{min}$ compared to $18.8 \pm 0.8 \mathrm{mg} / \mathrm{kg} / \mathrm{min}$ in wild-type mice; $\mathrm{p}<0.01)$. Like chow-fed DKO mice, high fat fed DKO mice are unusually sensitive to fasting because of ketoacidosis $(\mathrm{pH} 6.87 \pm 0.08)$ and hypothermia $\left(24.4 \pm 0.9^{\circ} \mathrm{C}\right)$. PDK deficiency results in greater PDC activity which limits the availability of pyruvate for oxaloacetate synthesis. Low oxaloacetate results in overproduction of ketone bodies by the liver and inhibition of ketone body and fatty acid oxidation by peripheral tissues, culminating in ketoacidosis and hypothermia. The findings establish that PDK2 is more important in the fed state, PDK4 is more important in the fasted state, survival during longterm fasting depends upon regulation of the PDC by both PDK2 and PDK4, and that the PDKs are important for the regulation of ketone body metabolism and fatty acid oxidation.

Supported by: T32 Diabetes and Obesity Predoctoral Fellowship (T32 DKO 64466)

\section{$\Delta$} 236-OR TRIB3 Mediates Glucose-Induced Insulin Resistance and its Induction by High Glucose Requires the Hexosamine Biosynthetic Pathway

WEI ZHANG, JIARONG LIU, LING TIAN, QINGLAN LIU, YUCHANG FU, W. TIMOTHY GARVEY, Birmingham, AL

Our group has previously demonstrated that Tribbles homolog 3 (TRIB3), a negative regulator of Akt activity, is induced by glucose and mediates glucose-induced insulin resistance in cultured muscle cells. We also reported that TRIB3 expression in fat and muscle is altered during short-term nutrient excess and fasting, supporting its role as a nutrient sensor and regulator. In the current study, we endeavored to determine whether TRIB3 could mediate hyperglycemia-induced insulin resistance in vivo, and whether the induction of TRIB3 by glucose is dependent upon the nutrient-sensing hexosamine biosynthetic pathway (HBP). In STZ-induced diabetic rats, TRIB3 protein expression in skeletal muscle was increased by $92 \%(p<0.05)$ after 10 days of hyperglycemia when compared with controls, and both glycemia and muscle TRIB3 levels were restored towards normal by exogenous insulin therapy. The role of the HBP in the induction of TRIB3 by high glucose was examined in L6 muscle cells. First, the induction of TRIB3 expression by 25 $\mathrm{mM}$ glucose was prevented by azaserine, an inhibitor of GFAT which is the rate-limiting enzyme in the HBP pathway. Second, TRIB3 expression was also substantially stimulated $(62.5 \%)$ by glucosamine $(24 \mathrm{hr}, 0.5 \mathrm{mM})$ which bypasses GFAT, accompanied by a $45 \%$ decrease in the insulin-stimulated glucose transport rate, and both responses were not affected by azaserine. Further, shRNA-mediated knockdown of TRIB3 inhibited the ability of both high glucose and glucosamine to induce insulin resistance when compared with scramble RNA control cells. In conclusion, our results indicate that TRIB3 is induced by high glucose, and this response is mediated by glucose metabolism via the HBP; 2) glucose-induced insulin resistance in muscle cells is dependent upon TRIB3. These data provide the first mechanistic link between the HBP flux and insulin resistance, and point to TRIB3 as a novel target for treatment of glucose-induced insulin resistance.

Supported by: NIH (DK038746, DK083562), DRTC (P60 DK079626) and VA Merit Review Program
Brain Insulin Regulates Systemic Branch-Chain Amino Acid Levels ANDREW C. SHIN, MARTIN FASSHAUER, ELIZABETHZIELINSKI, CLAUDIA LINDTNER, THOMAS SCHERER, JAMES O'HARE, JIANYING ZHOU, WEI-JUN OIAN, CHRISTOPHER B. NEWGARD, CHRISTOPHER J. LYNCH, CHRISTOPH BUETTNER, New York, NY, Richland, WA, Durham, NC, Hershey, PA

Circulating branched-chain amino acid (BCAA) levels are markedly elevated in obese and diabetic individuals, and they are the earliest predictor for the future risk of diabetes. Moreover, Roux-en-Y gastric bypass surgery that commonly improves metabolic control and often cures type 2 diabetes leads to reduced circulating BCAA levels. The cause of increased BCAA levels in obesity and diabetes is likely decreased utilization and/or catabolism and not increased BCAA intake, although the mechanisms are poorly understood. Insulin acutely lowers circulating BCAA levels in normal healthy individuals, but less so in diabetic individuals, implicating insulin resistance as a major cause for the elevated BCAA levels in obesity and diabetes. We first show through euglycemic clamp studies that insulin dose-dependently lowers plasma BCAA levels in rats. Insulin regulates nutrient partitioning through both direct effects on peripheral organs such as the liver, but also indirectly through changes in organ crosstalk. Insulin signaling within the mediobasal hypothalamus (MBH) orchestrates nutrient partitioning for example hepatic glucose production through the autonomic nervous system. Here we demonstrate that brain insulin signaling plays an important role in the regulation of systemic BCAA levels. Infusion of $2 \mathrm{uU}$ of insulin into the $\mathrm{MBH}$ acutely lowered circulating BCAA levels. Metabolomic analysis further demonstrated that circulating degradation products of BCAAs such as short chain acylcarnitines are also decreased. This lowering of BCAA is likely due to the induction of several enzymes participating in BCAA catabolism including branched-chain alpha keto-acid dehydrogenase (BCKDH), the rate-limiting enzyme in BCAA catabolism, as demonstrated through shotgun proteomics. These findings suggest that brain insulin controls circulating BCAA levels at least in part by regulating BCAA catabolism in liver. Elevated plasma BCAA levels in obesity and diabetes may be due to hypothalamic insulin resistance.

238-OR

Nicotinamide N-Methyltransferase (NNMT) is a Novel Regulator of Nuclear Factor Erythroid 2-Related Factor (Nrf2) Activity and Production of Reactive Oxygen Species (ROS) in Adipocytes

OIN YANG, FENGYING GONG, YA-CHIN WANG, DANIEL KRAUS, ODILE D. PERO$\mathrm{NI}$, BRETT P. MONIA, SANJAY BHANOT, BARBARA B. KAHN, Boston, MA, Carlsbad, $C A$

Chronic elevation of ROS in adipocytes plays a causal role in insulin resistance. Nrf2 is a master transcriptional regulator of antioxidant defense genes such as Heme oxygenase-1 (H0-1) and NADPH quinine oxidoreductas-1 (Nq01). Responses of Nrf2 to ROS are critical for maintaining cellular ROS homeostasis. Our data show that despite increased ROS in adipocytes in obesity, the expression of Nrf2 targets $\mathrm{HO}-1$ and Nqo1 are paradoxically reduced, suggesting failure of Nrf2 to respond to ROS. The aim of current study is to determine the mechanism by which Nrf2 fails to respond to ROS in adipocytes in insulin resistant states. Here we show NNMT is a novel inhibitor of Nrf2 activity. NNMT methylates nicotinamide using S-adenosylmethionine as a methyl donor. Adipose NNMT protein is increased in white adipose tissue (WAT) of obese mice such as ob/ob, db/db, and high-fat diet fed mice compared to lean controls. NNMT knockdown in WAT and liver by antisense oligonucleotides (ASO) protects mice from diet-induced obesity and improves insulin sensitivity and glucose tolerance. NNMT knockdown in WAT in vivo increases H0-1 and Nqo1 expression, consistent with Nrf2 activation. The effect appears to be direct since NNMT knockdown in 3T3-L1 adipocytes similarly activates Nrf2. Regulation of Nrf2 by NNMT is further demonstrated with luciferase transcriptional assays. NNMT overexpression suppresses, while NNMT knockdown enhances Nrf2 transcriptional activity. Finally, NNMT knockdown decreases ROS production in 3T3-L1 cells, which is abolished by Nrf2 siRNA treatment. These data indicate NNMT is a novel regulator of Nrf2 activity. Increased adipose NNMT in obesity may be responsible for suppressed Nrf2 activity, leading to chronic ROS elevation and insulin resistance. Thus, targeting NNMT could be a novel strategy for improving insulin sensitivity by regulating Nrf2 activity and ROS production in adipocytes. 
239-OR

Myeloid Expression of ABCA1 Inhibits Macrophage Inflammation and Protects Mice from Insulin Resistance

CHONGREN TANG, YUHUA LIU, TIM S. MCMILLEN, BARBARA A. HOUSTON, ALAN CHAIT, JAY W. HEINECKE, RENEE C. LEBOEUF, Seattle, WA

Cellular cholesterol balance induces changes in the inflammatory status of macrophages, and low grade chronic inflammation is now recognized as a key step in the pathogenesis of atherosclerosis as well as obesity-induced type 2 diabetes mellitus and insulin resistance. ATP-binding cassette transporter A1 (ABCA1), a cell- membrane protein, essential for reverse cholesterol transport and high density lipoprotein (HDL) synthesis, protects against macrophages inflammation and atherosclerosis. In the present study, the effect of ABCA1 deficiency in myeloid cells on diet-induced inflammation and insulin resistance was tested in vivo by transplantation of bone marrow from wild-type (WT) or ABCA1-/- mice into WT DBA/2 background mice. When challenged with high-fat, high-carbohydrate diabetogenic diet with

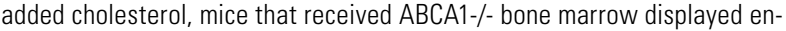
hanced liver and adipose tissue macrophage inflammation as well as insulin resistance and glucose intolerance as compared with animals that received WT bone marrow. In vitro studies indicated that bone marrow derived macrophages (BMDMs) from ABCA1-/- mice had significantly increased expression of markers of the M1 states such as MCP-1, IL-6 and TNF- $\alpha$, while the expression of the markers of $\mathrm{M} 2$ activation, such as mannose receptor and arginase-1 decreased. Moreover, BMDMs or peritoneal macrophages from ABCA1-/- mice showed significantly increased inflammation when induced by mixed fatty acids. Taken together, these studies demonstrated that myeloid ABCA1 protects mouse liver and adipose tissue from macrophage inflammation and overall insulin resistance in diet-induced obesity. Thus, increasing ABCA1 expression in myeloid cells may present a new therapeutic approach for the treatment of type 2 diabetes and insulin resistance.

Supported by: NIH

240-0R

Moderate Vitamin D Repletion Improves Insulin Action and Reduces Adipose Inflammation

SUDHA KOPPAKA, SYLVIA KEHLENBRINK, KEHAO ZHANG, WEIJIE LI, NORICA TOMUTA, ELIZABETH SANCHEZ, MEREDITH HAWKINS, PREETI KISHORE, BronX, NY

We have previously shown that repletion of vitamin $D$ (vitD) in obese, insulin resistant subjects (IR) improved hepatic insulin sensitivity (EGP). Since it is not known which vitD levels are ideal for metabolic benefits, we hypothesized that higher vitD levels (HiD, $50 \mathrm{ng} / \mathrm{ml}$ ) would be metabolically more advantageous than simply normalizing vitD ( $\mathrm{LoD},>30 \mathrm{ng} / \mathrm{ml})$. Euglycemic, hyperinsulinemic pancreatic clamp studies were performed in 5 obese, IR subjects $(4 \mathrm{M} / 1 \mathrm{~F}$, age $=38 \pm 3 \mathrm{yrs}, \mathrm{BMI}=33 \pm 2 \mathrm{~kg} / \mathrm{m} 2$, HOMA-IR=5.5 \pm 1.2$)$ at baseline, and following oral supplementation with vitamin D3 to LoD for first 2 months and then to HiD for the next 2 months. 6-6D-glucose was utilized to examine EGP and peripheral insulin sensitivity (Rd) at insulin infusion rates of 30 and $80 \mathrm{mU} / \mathrm{m} 2 / \mathrm{min}$. The $25(\mathrm{OH}) \mathrm{D}$ levels of $15.6 \pm 1.4 \mathrm{ng} / \mathrm{ml}$ at baseline increased to $37.7 \pm 4.1 \mathrm{ng} / \mathrm{ml}$ over first 2 months and $53.7 \pm 5.3 \mathrm{ng} / \mathrm{ml}$ over the subsequent 2 months (average weekly dose $\sim 30,350 \mathrm{IU}$ and $\sim 40,300 \mathrm{IU}$ respectively). EGP decreased by $29 \%$ with $\mathrm{LOD}$ and by $26 \%$ with $\mathrm{HiD}$ (baseline $=1.48 \pm 0.30, L o D=1.05 \pm 0.22$ and $H i D=1.10 \pm 0.25 \mathrm{mg} / \mathrm{kg} / \mathrm{min}, \mathrm{p}<0.05)$. Rd remained unchanged at both HiD and LoD levels. We hypothesized that vitD deficiency predisposes to adipose tissue inflammation by activating Adipose Tissue Macrophages (ATMs) that are important in IR. The effects of vitD on inflammation were studied in periumbilical subcutaneous adipose tissue biopsies. Following vitD repletion, the expression of TNF $\alpha$ and PAI-1 in fat was significantly reduced $(0.54$, and 0.71 fold change respectively; all $\mathrm{p}<0.05$ ) with a downward trend in iNOS and IL-6 expression. Additionally, pro-inflammatory activation markers expressed in ATMs were significantly reduced (IL6=0.44, iNOS=0.56, PAI-1=0.37; all $p<0.05$ ) and TNF $\alpha$ showed a downward trend. This suggests that correcting vitD deficiency can improve hepatic insulin resistance in association with decreased adipose tissue inflammation, with maximal metabolic effects observed by simply correcting the deficiency to LoD levels.

Supported by: Diabetes Action, NIH
NEW PLAYERS IN ENERGY METABOLISM

241-OR

Lymphocytes: Key Players in the Development of Diet Induced Hepatic Steatosis

ELISAVET KODELA, STAMATIS THEOCHARIS, KIHWA KANG, MARIA MOISIDOU, CHRISTINA KOUSKOUTI, MARK SLEEMAN, KATIA KARALIS, Athens, Greece, Tarrytown, NY, Melbourne, Victoria, Australia, Boston, MA

Obesity is a chronic, active inflammatory disease. The significance of activated macrophages in the pathogenesis of obesity-associated insulin resistance and the metabolic syndrome has been well described. Recent studies in diet-induced obese mice have demonstrated a critical role for lymphocytes in the development of insulin resistance and inflammation in the adipose tissue. The aim of this study was to further elucidate the role of lymphocytes in the pathogenesis of obesity with an emphasis in the development of non-alcoholic fatty liver disease (NAFLD). We compared the responses of the lymphocyte-deficient rag1-/- and wild-type (WT) mice to high-fat diet (HFD) - induced obesity with particular emphasis in liver pathology. Following 8-10 weeks of HFD we found similar body weight changes in both genotypes and comparable serum glucose levels, whereas rag1-/- mice exhibited increased insulin sensitivity, as assessed by the insulin tolerance test. Importantly, rag1-/- mice showed no histological signs of liver steatosis in contrast to the significant infiltration detected in the wild-type mice. Genomic and proteomic analyses of liver and adipose tissues from both groups revealed significant differences in the expression of genes involved in lipid synthesis and oxidation, in agreement with the histological picture. Preliminary findings from HFD-fed rag1-/- reconstituted with lymphocytes from WT mice, show significantly improved liver histology. On-going in vitro studies are also in accordance. These findings provide evidence for a critical role of lymphocytes in hepatic lipid metabolism and insulin sensitivity in hypercaloric states. The exact molecular factors mediating the above effects and their potential use in the development of novel therapeutic approaches for NAFLD are under investigation.

Supported by: EASD

242-0R

Insulin Activates Erk1/2 Signaling in the Dorsal Vagal Complex to Inhibit Glucose Production

BEATRICE M. FILIPPI, CLAIR S. YANG, TONY K. LAM, Toronto, ON, Canada

Hypothalamic insulin signaling inhibits glucose production through the activation of hypothalamic PI3-Kinase in rodents. It is currently unknown whether extra-hypothalamic brain region(s) mediate insulin to regulate glucose homeostasis. We here first report that insulin injection into the hypothalamus or dorsal vagal complex (DVC) of rats independently activated insulin receptors by 2- or 4-fold, respectively. Insulin activated PI3-kinase and AKT in the hypothalamus but not in the DVC. DVC insulin administration instead activated ERK1/2, and the activation was associated with an inhibition of glucose production during the pancreatic (basal insulin)-euglycemic clamps (GP mg/kg/min; DVC Insulin $5.4 \pm 0.5$ vs. saline $8.8 \pm 0.5, p<0.01$ ). Furthermore, inhibition of DVC ERK negated the ability of DVC insulin infusion to lower glucose production (GP mg/kg/min; DVC ERK inhibitor $9.7 \pm 1$ ). Importantly, circulating hyperinsulinemia failed to inhibit glucose production when DVC ERK signaling was disrupted (GP mg/kg/min; DVC ERK inhibitor $5.6 \pm 0.4$ vs. saline $0.6 \pm 0.4, p<0.01$ ), while direct DVC insulin administration failed to activate ERK and lower glucose production (GP $\mathrm{mg} / \mathrm{kg} / \mathrm{min}$; HFD-DVC Insulin $9.4 \pm 0.3$ vs. saline $9.7 \pm 0.6$ ) in high-fat fed rats. In summary, DVC is a novel site of insulin action in which insulin triggers a PI3-kinase independent but an ERK-dependent pathway to inhibit glucose production, and of insulin resistance in high-fat feeding.

243-OR

Reduction of HIF1 $\alpha$ Expression With Antisense Oligonucleotide Lowered Adiposity and Improved Insulin Sensitivity in High-Fat Diet-Fed Mice

XING XIAN YU, LYNNETTA M. WATTS, BRETT P. MONIA, SANJAY BHANOT, Carlsbad, $C A$

Obesity-associated hypoxia in fat tissue is postulated as a possible contributor to obesity-related insulin resistance (IR). HIF1 $\alpha$, a key mediator of hypoxic response, is highly expressed in fat and elevated in obese fat. However, data regarding its role in metabolism remain controversial. To evaluate the role of HIF1 $\alpha$ after pharmacological inhibition in target tissues, we used an antisense approach to reduce its expression in liver and fat tissues. Male C57BL/6 mice fed a high-fat (HF) diet for 4 wk were subsequently treated with a HIF1 $\alpha$-specific antisense oligonucleotide (ASO) or a control (Cnl) ASO 
at $50 \mathrm{mg} / \mathrm{kg} / \mathrm{wk}$, or with saline (Sal), for 9 wk. HIF1 $\alpha$ ASO reduced HIF1 $\alpha$ mRNA levels by $85 \%$ in liver and $62 \%$ in white fat whereas Cnl ASO did not change its expression. Neither ASO changed food intake or HIF2 $\alpha$ expression. However, HIF1 $\alpha$ ASO, but not Cnl ASO, time-dependently lowered BW and adiposity. At wk 9, HIF1 $\alpha$ ASO group was $15.5 \%$ lower in BW, $57.5 \%$ lower in body fat content, and $53 \%$ less in epidydimal fat versus Sal $\mathrm{Cnl}$. Decreased adiposity coupled with unchanged food intake indicates an increased metabolic rate in HIF1 $\alpha$ ASO treated mice. In addition, the treatment caused marked down-regulation of several key lipogenic genes including LPL, FAS, SCD1, SCD2 and DGAT2 in white fat while it had no effect on the expression of AR $\beta 3$, ATGL or HSL, suggesting decreased lipogenesis with unchanged lipolysis. The treatment also caused down-regulation of key hepatic lipogenic genes, including ACC1, ACC2, FAS, SCD1, SCD2, DGAT2 and SREBP1c. Furthermore, HIF1 $\alpha$ ASO lowered plasma glucose (191 \pm 6 vs $242 \pm 9 \mathrm{mg} / \mathrm{dl}$ in Sal) and insulin levels (1.5 \pm 0.2 vs $3.7 \pm 0.8 \mathrm{ng} / \mathrm{ml}$ in Sal), indicating improved insulin sensitivity after the treatment. HIF1 $\alpha$ ASO did not affect liver function. These data demonstrate that HIF1 $\alpha$ plays a critical role in lipid metabolism and energy homeostasis and that antisense reduction of HIF1 $\alpha$ expression could be a novel approach for the treatment of obesity and IR.

244-0R

Hypothalamic Protein Kinase Ikkepsilon (IKKع) has a Key Role in Energy Balance and Hepatic Glucose Metabolism in Db/Db and DIO Mice

LAIS WEISSMANN, PAULA GABRIELE FERNANDES QUARESMA, ANDRESSA C. SANTOS, VINIÍCIUS D'ÁVILA BITENCOURT PASCOAL, CARLOS KIYOSHI KATASHIMA, TAMIRES ZANOTTO, DIOZE GUADAGNINI, ISCIA LOPES CENDES, MARIO J. SAAD, PATRICIA 0. PRADA, Campinas, Brazil

The $I_{K B}$ kinase $\varepsilon$ (IKKE) induces insulin (INS) resistance in liver and fat from DIO mice. IKK\& KO mice are protected from obesity and whole-body INS resistance. However, if IKKE is activated in the hypothalamus (HY) and if it is involved in INS and leptin (LEP) resistance or affects hepatic glucose metabolism in obese mice were not yet investigate. Thus, the aim of the present study was to investigate (1) the expression and activation of IKKE in the HY of $\mathrm{db} / \mathrm{db}$ and DIO mice; the role of hypothalamic IKKE in the regulation of (2) energy balance and, (3) hepatic glucose metabolism in liver. Herein, we observed higher activation of hypothalamic IKKE in $\mathrm{db} / \mathrm{db}$ and DIO mice compared to controls. Intraperitoneal (IP) siRNA with RVG-9dR which binds to microglial cells or intracerebroventricular (ICV) ASO (oligonucleotide antisense) or ICV CAY (CAY10576-pharmacological inhibitor) reduced 80-70\% the activity of IKKE and did not alter $1 \kappa B / I K K \alpha / \beta$ pathway in $H Y$. Inhibition of IKK $\varepsilon$ with ICV ASO or siRNA in DIO and db/db mice reduced fat mass and food intake and enhanced energy expenditure and thermogenesis via higher 02 consumption and UCP1 expression. Pair-feeding obese mice treated with ICV CAY or siRNA improved the anorexigenic effects of LEP and INS. LEP induced LEPR/JAK2/STAT3 phosphorylation was increased in HY. INS induced IRS/Akt/Foxo1 phosphorylation was enhanced in HY. These mice also had lower fasting glycemia and hepatic glucose output, measured by an IP pyruvate tolerance test, which correlated with reduced gluconeogenic expression of Pepck in the liver. In summary, our data provide evidence in vivo that in $\mathrm{db} / \mathrm{db}$ and DIO mice IKKE is upregulated and a short time inhibition improves INS and LEP action/signaling in the HY and reduces hepatic glucose output and gluconeogenesis independently of IKK $\beta$. Thus, our results suggest that hypothalamic IKKE has a key role in energy balance and hepatic glucose metabolism in obese mice and may be a new target to treat obesity and diabetes.

Supported by: Fapesp (2008/55674-8) and CNPq (480131/2009-0)

245-OR

IEX-1 Deficiency Renders Mice Refractory to HFD-Induced Obesity and Insulin Resistance

MOHD SHAHID, AMMAR A. JAVED, WARREN M. ZAPOL, MEI X. WU, Boston, MA

Obesity is characterized by chronic inflammation with recruitment and activation of resident macrophages in tissues. NF-KB is activated upon highfat diet (HFD) feeding leading to chronic inflammation in liver and adipose tissue. Despite strong evidence for the involvement of inflammation in the pathogenesis of obesity, the primary mediator(s) and the site(s) at which the inflammatory response occurs have not yet been established. The immediate early response gene X-1 (IEX-1) is a stress-inducible gene that is a direct downstream target of NF- $\mathrm{\kappa B}$. IEX-1 is also highly expressed in macrophages. We hypothesized that IEX-1 is required for HFD feeding induced-inflammation leading to insulin resistance and obesity. We fed IEX-1-deficient mice (IEX$1 \%$ ) and their wild-type (WT) littermates a HFD (45\% kCal as fat) or normal chow (ND) for 20 weeks. IEX-1\% mice gained markedly less weight on a HFD than did WT mice $(25 \pm 1$ to $37 \pm 2 \mathrm{~g}$ vs $26 \pm 0.5$ to $50 \pm 2 \mathrm{~g}$, respectively, $P<0.01)$. HFD-fed IEX $\%$ mice exhibited less inflammation in adipose tissue and liver (TNF- $\alpha$, IL-6, \& leptin mRNA levels), hepatic steatosis (H\&E staining), and glucose intolerance than did HFD-fed WT mice. IEX-1\% mice had greater food consumption and thermogenesis than did WT mice. Flow cytometry analysis of adipose tissue macrophages (ATM) revealed that HFD feeding increased the percentage of proinflammatory M1 macrophages $(10 \pm 1$ to $37 \pm 1 \%, p<0.001$ ) while decreasing the percentage of anti-inflammatory $M 2$ cells $(39 \pm 3$ to $13 \pm 3 \%, p<0.001)$ in WT mice. In sharp contrast, the ratio of M1 to M2 cells in ATM did not differ in IEX-1\% mice fed a HFD or ND. Annexin staining revealed a similar percentage of $M 1$ apoptotic cells in ATM of WT and IEX-1\% mice, whereas there were significantly fewer apoptotic $\mathrm{M} 2$ cells in IEX-1\% than in WT mice $(11 \pm 2$ vs $23 \pm 3 \%, p<0.01)$. Taken together, these findings suggest that IEX-1 deficiency protects mice from HFD-induced glucose intolerance and obesity by maintaining an anti-inflammatory ATM phenotype in adipose tissue.

Supported by: NIH (AI050822 and Al070785) and AHA (0855762D)

\section{6-OR}

Perilipin 5, a Novel Regulator of Cardiac Lipid Droplet Hydrolysis HONG WANG, DAWEI GONG, URMILLA SREENIVASAN, ANDREW SALADINO, BRIAN POLSTER, KELLY O'CONNELL, ERINNE DABKOWSKI, PETER HECKER, WILLIAM STANLEY, CAROLE SZTALRYD, Baltimore, MD

Maintaining cellular lipid homeostasis is crucial to heart function, and is compromised in diabetic and obesity-induced cardiomyopathies. Lipid droplets (LDs) play a central role in lipid homeostasis by mediating transient storage of fatty acids (FA) in the form of triglyceride (TAG), while preventing high levels of toxic lipid that mediate cellular lipotoxicity. LD and mitochondrial function are intimately related but mechanisms of interaction have not been uncovered, nor underlying genes. Perilipin 5 (Plin5) is the newest member of the perilipin protein family coating LD surfaces and is highly expressed in heart. We reported that Plin5 mediates physical and metabolic interactions between LDs and mitochondria in vitro. Plin5 recruits mitochondria at the LD surface, is a scaffolding protein for key lipolytic enzymes (adipose tissue triglyceride lipase (ATGL), CGI-58 its co-lipase and hormone sensitive lipase (HSL), inhibits LD hydrolysis and facilitate TAG storage These in vitro results suggest that Plin5 regulates cardiac LD hydrolysis and controls local FA flux to protect mitochondria against FA surge in physiological conditions. To investigate plin5 function in vivo, we obtained transgenic mice with heart-specific plin5 over-expression ( $\alpha M H C$-plin5). Results suggest that these mice have a strong cardiac LD phenotype. Hearts from aMHCplin5 mice expressed at least 20-fold higher levels of plin5 and exhibit a 3.5- fold increase in TAG content versus non-transgenic littermate. Cardiac Plin5 over-expression leads to decreased PPAR $\alpha$ targeted gene expression, altered mitochondria morphology and function and altered heart function, phenotype similar to the ATGL ko mice. These studies support the conclusions that Plin5 is a negative regulator of ATGL-mediated LD hydrolysis in vivo and is a promising key gene in the pathways that couple the LD storage compartment to the mitochondria FA utilization compartment and will help unravel the underpinnings of cardiac cellular lipid homeostasis.

Supported by: NIH, AHA, GRECC VA

247-0R

Activated $\alpha \beta$ T Cells Affect Adipose Tissue and Skeletal Muscle Function in Obesity

ILVIRA KHAN, XIAOYUAN PERRARD, JERRY PERRARD, AMIR MANSOORI, C.W. SMITH, HUAIZHU WU, CHRISTIE BALLANTYNE, Houston, TX

Obesity is accompanied by low-grade chronic inflammation, which contributes to pathogenesis of insulin resistance. In addition to classically activated M1 macrophages, $\alpha \beta$ T cells are crucial mediators of adipose tissue inflammation. We have observed that high fat diet (HFD)-fed TCRb-null mice deficient in $\alpha \beta$ T cells have reduced adipose tissue (AT) inflammation compared to obese wild-type (WT) controls. Our current studies show that of the $\alpha \beta$ T cells, CD4+ T helper cells are polarized to T helper I (TH1) cells, as indicated by higher percentage of TH1 cells in AT of obese mice $(6.27 \pm 1.90 \%$ in obese vs $3.02 \pm 1.37 \%$ in lean AT). In fact, obese AT has TH1 polarizing environment with markedly increased IL-2 and IL-12 levels. To determine the functional consequence of increased TH1 cells in AT, we studied the effect of TH1 cell on preadipocyte/adipocyte functions and found that treatment of 3T3-L1 cells with TH1 supernatant inhibited adipogenesis and decreased lipid accumulation, but did not affect lipolysis in mature adipocytes. TH1 supernatant also increased expression of proinflammatory cytokines (TNFo 
and IL-6) and chemokines (MCP-1, RANTES) and reduced level of adiponectin in differentiated 3T3-L1 cells. Consistent with the in vitro data, the size of individual adipocytes in perigonadal AT of HFD-fed TCRb-null mice, which are also TH1 deficient, was significantly larger compared to obese WT controls, suggesting that the absence of TH1 cells could restore AT lipid storage. We found that accumulation of $\alpha \beta$ T cells also occurred in skeletal muscle of obese WT mice $(23.23 \pm 1.39 \%$ of CD $45+$ cells in obese vs $13.79 \pm 3.07 \%$ in lean). Deficiency of $\alpha \beta$ T cells in HFD-fed mice blunted skeletal muscle inflammation with reduced levels of MCP-1, RANTES, IL-6, and IFN $\gamma$. Insulin sensitivity was also improved in skeletal muscle of obese TCRb-null mice. Thus $\alpha \beta$ T cells with TH1 polarization could affect not only AT, but also skeletal muscle function in obesity resulting in insulin resistance.

Hepatic Glucokinase Negatively Regulates BAT Thermogenesis via a Neural Pathway

SOHEI TSUKITA, TETSUYA YAMADA, KENJI UNO, YOSHITOMO OKA, HIDEKI KATAGIRI, Sendai, Japan

We focused on systemic impacts, especially on energy metabolism, of alterations in hepatic glucose metabolism. Since glucokinase (GK) catalyzes the first and rate-limiting step of glycolysis in hepatocytes, we first examined the time course of GK expression levels in the livers of obesity-prone C57BL/6 mice during high-fat (HF) diet feeding. HF feeding markedly up-regulated hepatic GK within 1 week. Then, to examine whether hepatic GK expression actually affects energy metabolism, we overexpressed GK in the livers of chow-fed C57BL/6 mice, using the adenoviral gene transfer system. Hepatic GK expression increased adipocyte sizes in brown adipose tissue (BAT) and decreased expressions of thermogenesis-related genes, such as uncoupling protein-1 (UCP1), in BAT in a manner dependent on hepatic GK expression levels. In addition, hepatic GK expression suppressed c-fos expression in the raphe pallidus of the medulla, attenuated systemic thermogenic response to norepinephrine (NE), and promoted weight gain. Selective hepatic vagotomy abolished these anti-thermogenic effects induced by hepatic GK expression. These findings indicate that hepatic GK expression negatively regulates BAT thermogenesis via a neural pathway, consisting of the vagal afferents from the liver and sympathetic efferents from the medulla. Furthermore, hepatic GK induction of obesity-prone mice during HF feeding was more enhanced than that of obesity-resistant mice, and the enhanced levels of hepatic GK expression were inversely associated with UCP1 expression levels in BAT. Knockdown of hepatic GK induction in obesity-prone mice increased BAT UCP1 expression, enhanced thermogenic response to NE, and suppressed HF diet-induced weight gain. Thus, this novel inter-tissue (liver-to-BAT) system may function as a systemic thrifty mechanism and trigger obesity development on an early stage of over-nutrition.

\section{DEATH OF AN ISLET}

\begin{abstract}
249-0R
Glucokinase Activator Ameliorates $\beta$ Cell ER Stress and Apoptosis by both IRS-2-Dependent and -Independent Pathways

JUN SHIRAKAWA, YASUO TERAUCHI, Yokohama-City, Japan

Endoplasmic reticulum (ER) stress is involved in $\beta$ cell apoptosis and the development of diabetes. Glucokinase (Gck) up-regulates IRS-2 expression in $\beta$ cell, but the role of Gck and IRS-2 in ER stress has been unclear. In this study, we investigated the impact of Gck activator (GKA) on ER stress. Administration of GKA significantly decreased TUNEL-positive $\beta$ cells in Akita (Ins2) mouse, an animal model of ER stress-mediated diabetes. Based on the results of gene expression microarray analysis in islets after treatment with GKA, we validated that GKA significantly increased the expressions of ER stress related genes, such as Sdf2I1, Stc2, Edem2, or Ero1 $\beta$, and significantly decreased the expressions of Chop in islets. In islets from db/db mice, GKAinduced up-regulation of IRS-2, Sdf2I1, or Stc2 was attenuated. Of note, the regulation of expressions of ER stress related genes by GKA were preserved in IRS-2-deficient (IRS-2-/-) islets. MEK inhibitor (U0126), but not Akt inhibitor (Akti-1/2), prevented GKA-induced suppression of CHOP in islets. These results suggested that GKA regulated ER stress-related genes via IRS-2 independent manner. Gck-deficient (Gck+/-) islets and IRS-2-/- islets demonstrated increased expressions of $\mathrm{CHOP}$ and Bax after treatment with thapsigargin, but GKA significantly decreased the elevated expressions of CHOP and Bax. On the contrary, islets from transgenic mice overexpressing IRS-2 $\beta$ cell-specifically ( $\beta \mid R S 2 T g$ ) showed significantly decreased expressions of Bax under ER stress. Furthermore, both Gck+/- Akita mice and IRS-2-/- Akita mice demonstrated severe hyperglycemia at early stage and increased $\beta$
\end{abstract}

cell apoptosis, compared with Akita mice. We also generated $\beta I R S 2 T g$ Akita mice, and these mice depicted decreased $\beta$ cell apoptosis, compared to Akita mice. Taken together, GKA ameliorated ER stress-mediated apoptosis via both IRS-2-dependent pathway and IRS-2-independent regulation of the expressions of genes related to ER stress.

250-0R

Role of Sphingosine Kinase 1 in Pancreatic $\beta$-Cell Survival and Prevention of Diabetes in High Fat Diet-Fed Mice

YANFEI OI, JINBIAO CHEN, PU XIA, Sydney, Australia

Pancreatic $\beta$-cell death resulted from elevated circulating levels of free fatty acids (FFA) is a critical pathogenic event for the development of type 2 diabetes, especially in obese individuals. Attempting to prevent $\beta$-cell death has been considered as a new strategy of prevention and treatment of diabetes. In this study, we aimed to examine the role of sphingosine kinase 1 (SK1) in $\beta$-cell survival under lipotoxic stress in vitro and in mice fed with high fat diet (HFD). Exposure to palmitate resulted in a dose-dependent increase in cell death in both MIN6 and INS-1 $\beta$-cell lines. Lipotoxicity was significantly reduced in the $\beta$-cells overexpressing of SK1 or pre-treated with sphingosine 1-phosphate (S1P), the product of SK1. By blocking the enzyme activity, dominant negative form of SK1 markedly potentiated palmitateinduced $\beta$-cell apoptosis. Moreover, primary islets isolated from SK1-/- mice exhibited more susceptible to FFA-induced apoptosis, compared with the wild-type (WT) controls in vitro. To further investigate the role of SK1 in $\beta$-cell survival under lipotoxic stress, we fed SK1-/- and WT mice with either HFD or normal chow diet. After 24 weeks feeding, HFD induced significant body weight gain and enhanced circulating levels of FFA and lipids in both WT and SK1-/- animals to a similar degree. In strikingly contrast, while HFD feeding induced glucose intolerance and compensatory hyperinsulinemia in WT mice, SK1-/- littermates developed evident diabetes accompanied with a significant reduction in insulin levels. Morphological analysis indicated a dramatic loss of $\beta$-cell mass and increased apoptosis in pancreatic islets of HFD-fed SK1-/- mice. Collectively, these findings for the first time demonstrate a critical role of SK1 in maintaining $\beta$-cell survival under obese or lipotoxic conditions.

Supported by: National Health and Medical Research Council of Australian Government

251-0R

Osteoprotegerin, a Novel Downstream Target of Lactogens, Enhances Human Beta Cell Proliferation Through RANKL Modulation and Improves Human Beta Cell Survival

NAGESHA GUTHALU KONDEGOWDA, CONNIE CHIN, ANAIS MOZAR, RUPANGI

C. VASAVADA, Pittsburgh, PA

Osteoprotegerin (OPG), a member of the tumor necrosis factor receptor superfamily, is expressed in beta cells, and its expression is increased in three models of rodent beta cell expansion. The current study shows that OPG expression is increased 22-fold in transgenic mouse islets expressing placental lactogen in the beta cell. OPG mRNA is also increased 6-fold in INS1 cells treateåd with prolactin, identifying $\mathrm{OPG}$ as a novel downstream target of lactogens in beta cells. Based on this increase in OPG expression, we hypothesized that OPG may directly enhance beta cell proliferation. To examine this, human islet cell cultures ( $n=3-5)$ were treated with 25-200ng of OPG in the presence of bromodeoxyuridine (BrdU) and human beta cell proliferation was assessed by insulin-BrdU co-staining. There was a significant 2.5-3.0 fold increase in human beta cell proliferation with the higher OPG doses. OPG mediates its effects in bone is through inhibition of the receptor activator of nuclear factor kappa-B ligand (RANKL) pathway. To examine the importance of this pathway on human beta cell proliferation, we treated human islet cell cultures with Denosumab (1-200ng/ml), a human monoclonal RANKL antibody, which acts as a partial functional equivalent of OPG. Denosumab significantly increased human beta cell proliferation by 2 -fold, demonstrating the importance of the RANKL pathway in human beta cell proliferation. We next examined if OPG had a direct effect on human beta cell survival against glucolipotoxicity (GLT) and cytokines. OPG significantly ameliorated by $\sim 45 \%$ and $\sim 50 \%$, the 3.5 -fold increase in GLT and cytokineinduced human beta cell death, respectively, as measured by insulin and TUNEL co-staining $(n=3)$, demonstrating a direct pro-survival effect of OPG on human beta cells. Our studies reveal OPG to be a novel downstream target of lactogens, which can independently and directly enhance human beta cell proliferation and survival.

Supported by: DK078060, DK072264 


\section{DEATH OF AN ISLET}

252-0R CXCL10 Antagonism Protects from High Fat Diet-Induced Hyperglycemia and Improves Beta-Cell Function and Mass

KATHRIN MAEDLER, ERNA DOMSGEN, JENNIFER BERGEMANN, PIA JANSSEN, NADINE STAPPENBECK, PINA CARDARELLI, PAYAL SHAH, Bremen, Germany, Sunnyvale, $C A$

Sub-clinical inflammation participates in the pathophysiology of type 2 diabetes (T2DM) and leads to $\beta$-cell failure and impaired function. The proinflammatory chemokine CXCL10 is expressed in islets in T1DM and T2DM and leads to $\beta$-cell apoptosis and impaired function. In the present study we investigated whether CXCL10 antagonism improves glycemia, $\beta$-cell function and survival in vivo in mice and in vitro in human islets. A murine CXCL10 antibody prevented diabetes progression in C57BL/6J mice fed a high fat/ high sucrose diet (HFD) for 16 weeks. Anti-CXCL10 improved glucose tolerance and glucose stimulated insulin secretion. HFD feeding reduced stimulatory index by 2.8-fold, but anti-CXCL10 treatment completely restored GSIS While anti-CXCL10 treated mice showed a 1.8-fold increased $\beta$-cell mass compared to normal diet (ND) controls, $\beta$-cells were unable to compensate for the higher insulin demand in response to the HFD in vehicle treated mice. Analysis of $\beta$-cell apoptosis showed an induction of TUNEL-positive $\beta$-cells in the HFD mice compared to ND mice $(0.2 \%$ vs. $0.05 \% ; p<0.05)$ in HFD treated mice, which was inhibited in anti-CXCL10 treated mice. CXCL10 mRNA was 5 -fold up-regulated in epididymal fat and normalized by anti-CXCL10. In contrast, in the hypothalamus, neither CXCL10 nor IL-1 $\beta$ were changed in HFD or anti-CXCL10 treated mice. The protective effects of anti-CXCL10 were also confirmed in human islets. The cytokine mixture IL-1 $\beta / I F N-\gamma$ highly induced IL-1 $\beta$ production and secretion of IL-1 $\beta$, which was inhibited by antiCXCL10, possibly through a negative feedback mechanism. In parallel, cytokine-induced apoptosis was also inhibited. Our results show that CXCL10 antagonism improved $\beta$-cell survival and function and support a potential role for CXCL10 in the treatment of diabetes.

$$
\text { Supported by: JDRF }
$$

253-0R

Deletion of ER Cochaperone P58IPK Causes ER Stress and Oxidative Stress in Pancreatic Beta Cells that is Rescued by Either Antioxidant Treatment or Chop Deletion

JAESEOK HAN, BENBO SONG, SUNG HOON BACK, JUSTIN R. HASSLER, VAMSI KODALI, SHIYU WANG, RANDAL J. KAUFMAN, Ann Arbor, MI, La Jolla, CA, Ulsan, Republic of Korea, San Diego, CA

Improper protein folding results in accumulation of misfolded or unfolded protein in the ER, which is detrimental to cell survival. Given that molecular chaperones are required for proper protein folding, we investigated the requirement for the ER cochaperone P58 ${ }^{\mathrm{IPK}}$ in $\mathrm{ER}$ homeostasis and insulin production in pancreatic $\beta$ cells. P58/PK- mice displayed a diabetic phenotype including elevated blood glucose levels with reduced insulin and proinsulin levels in the serum. In parallel, islets from P58PK-F mice showed impaired Glucose-stimulated insulin secretion (GSIS), likely due to a decreased number of insulin containing granules as measured by electron microscopy (EM) and reduced expression levels of $\beta$ cell-specific genes compared to wildtype islets. In addition, there was increased cell death in P58/PKK- islets and in established $P 58^{\mid P K-F} \beta$ cell lines under ER stress, that was associated with induction of the unfolded protein response (UPR) including the pro-apoptotic gene Chop compared to wild-type islets and $\beta$ cell lines. We also found that there were elevated levels of oxidative stress in $P 58^{\mid P K-} \propto$ islets. Significantly, feeding mice a chow diet supplemented with the antioxidant butylated hydroxyanisole (BHA) restored blood glucose levels and serum insulin levels in P58 $8^{\mid P K} \%$ mice within one week. GSIS of BHA-treated P58/PK- islets was significantly improved with increased pancreatic insulin content and insulin containing secretory granules. Furthermore, treatment with phenylbutyric acid (PBA), a chemical chaperone that relieves ER stress, as well as deletion of Chop, rescued diabetic phenotype with improved $\beta$ cell function and survival of $P 58^{\mid P K-}$ mice. These results suggest that the ER cochaperone P58 $8^{\mathrm{PK}}$ is essential to maintain the secretory capacity of the $\beta$ cell. Significantly, impaired protein folding in the ER of the $\beta$ cell causes oxidative stress that results in $\beta$ cell failure and apoptosis.

254-0R

p53 Plays an Essential Role in Glucolipotoxicity-Induced $\beta$-Cell Death

JUAN C. ALVAREZ-PEREZ, LAURA C. ALONSO, GABRIELLA P. CASINELLI, TAYLOR C. ROSA, ADOLFO GARCIA-OCANA, Pittsburgh, PA

High concentrations of free fatty acids and glucose (gluco-lipotoxicity, GLT) are detrimental for pancreatic $\beta$-cells, resulting in reduced insulin content, defective insulin secretion, and $\beta$-cell death. However, the mechanisms involved in GLT-induced $\beta$-cell death are not completely understood. p53 plays a critical role in responding to cellular stresses by regulating a myriad of cellular effects including growth arrest, DNA repair, mitochondrial function, energy metabolism and apoptosis. However, whether p53 is upregulated in $\beta$-cells exposed to GLT conditions and whether p53 has any role in GLT-mediated $\beta$-cell death is unknown. INS-1 cells grown in $11 \mathrm{mM}$ glucose and exposed to $0.1-0.5 \mathrm{mM}$ palmitate (GLT conditions) for 24h displayed increased apoptosis in a dose dependent manner, as assessed by TUNEL staining and western blot analysis of caspase-3 activation. Interestingly, GLT also increased dose-dependently the expression levels of p53 and the p53-responsive genes p21 and the pro-apoptotic protein Bax. Moreover, GLT induced the phosphorylation of p53 on Ser15 and Ser20, known to promote both the accumulation and transactivation of $p 53$. To analyze whether $p 53$ transactivation could participate in GLT-induced $\beta$-cell death, we tested the ability of the p53 transactivation inhibitor Pifithrin- $\alpha$ (PIF) to ameliorate INS1 cell apoptosis induced by GLT. PIF $(25 \mu \mathrm{M})$ significantly $(\mathrm{p}<0.05)$ diminished INS-1 cell apoptosis induced by GLT. Mouse islets exposed to GLT conditions also displayed a dramatic upregulation of p53 expression. Importantly, p53-null mouse $\beta$-cells in culture were resistant to GLT-induced apoptosis compared to $\beta$-cells from normal littermates. Preliminary studies suggest that GLT also upregulates p53 levels in human islets and that PIF treatment ameliorates human $\beta$-cell apoptosis in this setting. Collectively, these studies indicate that p53 plays an important role in GLT-induced $\beta$-cell death and may contribute to the $\beta$-cell failure observed in obesity-associated type 2 diabetes.

Supported by: NIH/NIDDK (DK067351) to A.G.O.

255-0R mt-ND2a Protects Human $\beta$ Cells from Immune-Mediated Destruction

YAIMA L. LIGHTFOOT, JING CHEN, CLAYTON E. MATHEWS, Gainesville, FL

Mitochondria play essential roles in both the function and death of pancreatic $\beta$ cells. These organelles produce ATP required for optimal insulin secretion and participate in $\beta$ cell death induced by mechanisms linked to both Type 1 and Type 2 Diabetes. Accordingly, mutations in the mitochondrial DNA (mtDNA) have been linked to diabetes. A single nucleotide polymorphism within the mitochondrially encoded gene for NADH dehydrogenase subunit 2 (mt-ND2) has been linked to Type 1 Diabetes resistance in both humans and mouse. In the mouse, this allele provides enhanced $\beta$ cell protection from apoptosis. The goal of this report was to study the impact of the human alleles on $\beta$ cell death. For this study we developed cybrid human $\beta$ cell lines with equal nuclear genomes yet differing by the mtDNA haplotype, encoding either the diabetes-resistance allele, $m t-N D 2^{\mathrm{a}}$, or the $m t-N D 2^{\mathrm{c}}$ allotype associated with diabetes susceptibility. Cybrid cell lines were developed by first depleting the human $\beta$ cell line, $\beta$ lox5, of mtDNA with low dose Ethidium Bromide, and then fusing the resulting cell line with human donor platelets with $m t-N D 2^{\mathrm{a}}$ or $m t-N D 2^{\mathrm{c}}$. This process produced the $\beta$ lox5-ND2 ${ }^{\mathrm{a}}$ and $\beta l o x 5-N D 2^{\circ}$ cell lines. $\beta$ lox5-ND2 and $\beta$ lox5-ND2 ${ }^{\circ}$ cells were incubated with the cytokine combination of TNF $\alpha$ and IFN $\gamma$, or the combination of IFN $\gamma$ and $\alpha$-Fas for $48 \mathrm{~h}$ before assaying cell death, mitochondrial ROS production, and changes in mitochondrial membrane potential (MMP). $\beta$ lox5-ND2a cells showed increased resistance to cytokine and Fas killing compared to $\beta l o x 5$ $N D 2^{c}$. Resistance to apoptosis correlated with lower levels of ROS generation and increased resistance to MMP changes. Our results indicate that, similar to findings using mouse systems, mt-ND2 a protects human $\beta$ cells from insults associated with Type 1 Diabetes progression by suppressing $\mathrm{ROS}$ produced by mitochondria in response to pro-death signals.

Supported by: R01 DK074656

256-0R

Prevention of Human Islet Apoptosis Activation by Co-Culture With Bone Marrow (BM) Cells

JUNPING WANG, JOHN ZQ LUO, LUGUANG LUO, Providence, RI

Apoptosis is one of major factors for human islet transplantation failure. The activation of inflammatory factors such as interleukin (IL-1 $\beta$ ) is a significant factor in the apoptosis process. The present study specifically focuses on evaluating the anti-apoptotic effect of BM on human islet cells and the mechanisms. Two groups were used: 1) human islets cultured under normal conditions and 2) human islets co-cultured with bone marrow cells. BM cells co-cultured with human islets reduced apoptosis (percentage of apoptotic cells: $76 \% \pm 6 \%$ normal culture VS $13 \% \pm 2.1 \%$ co-culture for 7 hours; $97 \% \pm$ $3 \%$ VS $21 \% \pm 1.5 \%$ for 21 days culture respectively, $p<0.001)$. The measurement of IL-1 $\beta$ within the culture medium in both culture systems showed that 
BM co-culture significantly decreased IL- $\beta$ levels $(0.022 \pm 0.001$ for normal culture, $0.026 \pm 0.002$ for co-culture at day $8 ; 2.266 \pm 0.054,0.048 \pm 0.011$ $\mathrm{ng} / \mathrm{ml}$ at day 63, $\mathrm{p}<0.001)$. Reduction in inflammatory factor levels in coculture may be related to a decrease in external cellular ATP levels, which has been reported as a major cause in inducing IL-1 $\beta$ production and release. Reduced ATP accumulation (111.49 \pm 5.99 normal culture VS $100.68 \pm 5.99$ co-culture on day 8; $25382.43 \pm 2617.60$ normal culture VS $1673.65 \pm 111.42$ co-culture on day $63, p<0.001$ ) was detected and correlated with a reduction in ATP receptor (P2X7) expression. This study demonstrated that human islet $\beta$-cells undergo apoptosis spontaneously upon isolation. Co-culturing islets with BM cells postpones islet deterioration and apoptosis by reducing extracellular ATP levels, inflammatory factor production, and $\beta$-cell apoptosis.
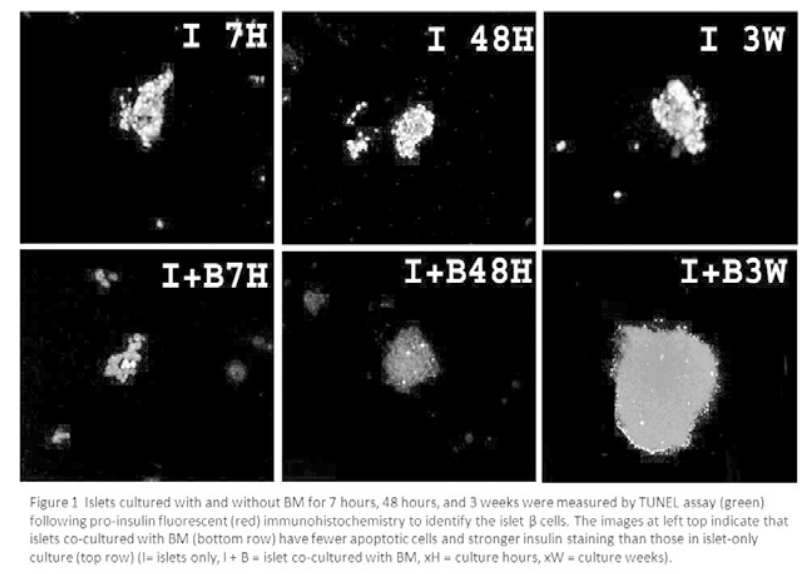

Supported by: JDRF

\section{DIABETIC RETINOPATHY-PATHOGENESIS AND NEW APPROACHES TO TREATMENT}

257-0R

Slow Progression of Diabetic Retinopathy (DR) in Patients With 50 or More Years of Type 1 Diabetes

ABUMERE AKINWALE, JENNIFER K. SUN, HILLARY A. KEENAN, LLOYD P. AIELLO, GEORGE L. KING, Boston, MA

Over $40 \%$ of patients with type 1 diabetes (DM) for $\geq 50$ years (Medalists) have no-mild nonproliferative DR (NPDR) even after five or more decades of hyperglycemia. An important question with mechanistic implications is whether this long term protection from advanced DR is due to slowed DR progression or regression from more severe DR levels. We evaluated 158 Medalists (316 eyes) with longitudinal follow-up at Joslin Diabetes Center (mean \pm SD follow-up: $16 \pm 13$ yrs; $27 \pm 33$ total visits) by retrospective chart review. Evaluation of all available standard care ETDRS protocol fundus photographs was performed for eyes with moderate or severe NPDR that did not progress to PDR $(\mathrm{N}=43)$ to document $\mathrm{DR}$ regression or progression. The Medalists had a mean age of $10 \pm 6 y$ rs at diagnosis, $58 \pm 6 y$ rs DM duration and current hemoglobin A1c (A1c) of $7.2 \pm 0.9 \%$. In eyes with PDR, worse current renal function $(p=0.003)$, older current age $(p=0.0002)$, shorter DM duration $(p<0.0001)$ and higher current A1c $(p=0.03)$ were significantly related to faster progression to PDR, but of these, only worse current renal function $(p=0.003)$ was related to PDR as an outcome in the whole group. Eyes without baseline DR that did not progress to PDR $(\mathrm{N}=152)$ had a slower rate of DR worsening than eyes that progressed to PDR ( $N=61, p<0.001)$, and once a patient reached 20yrs of DM, only 2 eyes worsened from baseline thereafter. In eyes with moderate or severe NPDR that did not progress to PDR, regression of some DR lesions (hemorrhages, microaneurysms, hard exudates and cotton wool spots) was evident over time, but there was not substantial ( $\geq 2$ steps on the clinical ETDRS scale) regression of DR severity in any eye. These results suggest that Medalists protected from PDR have slow DR onset and/or progression rather than increased DR regression and are unlikely to experience DR worsening after 20 years of DM. Thus, protective factors may exist in some Medalists that ameliorate hyperglycemia-induced retinal pathology and prevent progression to neovascular DR.

Supported by: JDRF, R24 DK083957-01, P30DK036836-2, Brehm, Beatson, Eli Lilly

For author disclosure information, see page 797.
$\Delta$

258-0R

Therapeutic Effects of Fenofibrate Eye Drops in Diabetic Retinopathy YING CHEN, Oklahoma City, OK

Recent FIELD studies showed that oral administration of fenofibrate, a safe and low-cost drug that lowers VLDL, has displayed impressive efficacy in slowing the progression of diabetic retinopathy (DR) in type 2 diabetic patients. The purpose of this study was to explore whether fenofibrate topical administration has therapeutic effect on DR in type 1 diabetes. Fenofibrate eye drops delivery capacity to retina was detected by HPLC/MS/MS. Adult $\mathrm{BN}$ rats were given a single intraperitoneal injection of streptozotocin (STZ) to induce diabetes. Newborn Brown Norway rats at postnatal day 7 were exposed to hyperoxia $\left(75 \% 0_{2}\right)$ for 5 days and then returned to normoxia to induce oxygen-induced retinopathy (OIR). Vascular permeability was quantified by measuring leakage of Evans blue dye-albumin complex from the blood vessels into the retina. Full-field electroretinogram (ERG) was recorded using the Espion $E^{2} E R G$ system. Fenofibrate eye drops can efficiently delivery to the retina. Fenofibrate eye drops reduced retinal vascular leakage in both the OIR and STZ-diabetes models. Fenofibrate eye drops have significantly potent effects on retinal neovascularization in OIR rats. Fenofibrate eye drops down-regulated the expression of VEGF and ICAM-1 in the retina. ERG and histological analysis after the eye drops administration showed that Fenofibrate eye drops did not cause detectable functional and morphological abnormalities in ocular tissues. Fenofibrate eye drops demonstrate a novel administration method of fenofibrate and have therapeutic potential on DR in type 1 diabetes.

259-0R

Fenofibrate: Prevention and Treatment of LDL-Enhanced Diabetic Retinopathy in STZ-Induced Diabetic Mice

JING ZHANG, SHIHE YANG, DONGXU FU, MINGYUAN WU, MEI DU, KENNETH WILSON, TIMOTHY LYONS, Oklahoma City, OK

Fenofibrate, a PPAR- $\alpha$ agonist, is used to treat hypertriglyceridemia. Beneficial effects of fenofibrate on diabetic retinopathy (DR) have been described in two recent large prospective studies, but mechanisms are unknown. To evaluate fenofibrate action on modified LDL-enhanced DR in a STZ-diabetic rodent model, diabetic and control mice (C57BL/6J, 8 weeks old) were given a single, unilateral, intra-vitreal injection of fenofibrate $(125 \mu \mathrm{M}, 1 \mu \mathrm{l} / \mathrm{eye})$, then maintained for 2 months on a diet supplemented with fenofibrate $(0,100,300 \mathrm{mg} /$ $\mathrm{kg} \cdot \mathrm{d})$. Then, to enhance DR, some animals received an intra-vitreal injection of "highly oxidized glycated" (HOG-) LDL ( $5 \mu \mathrm{g} / \mu \mathrm{l}, 1 \mu \mathrm{l} / \mathrm{eye})$, with native LDL (N-LDL) or PBS injections as controls. Seven days later, ERG, H\&E staining, flat mounts, and retinal vascular permeability assays were performed, and markers of inflammation, apoptosis, and ER stress were determined (western blot). In non-diabetic mice, neither LDL injection nor fenofibrate had any effect on normal retinal structure and function. In contrast, in diabetic mice, seven days after intra-vitreal injection, HOG-LDL (but not N-LDL) disrupted retinal histology, impaired retinal function (ERG), and increased blood-retinal barrier breakdown. High- but not low-dose dietary fenofibrate protected both structure and function; significantly attenuating vascular leakage and inflammation, and blocking apoptosis and ER stress. Interestingly, the early, single, intravitreal injection of fenofibrate also robustly protected retinal structure and function 9 weeks later in the diabetic mice treated with intra-vitreal HOG-LDL. In conclusion, both dietary and intra-vitreal fenofibrate countered the stress imposed by intra-vitreal injection of $L D L$ in diabetic animals. The data suggest the protective effect of fenofibrate operates directly within the retina, independent of systemic effects.

260-0R

Acetylation of Retinal Histones in Diabetes Increases Inflammatory Proteins: Effects of Minocycline, HAT and HDAC

TIMOTHY S. KERN, CHANDRA S. KADIYALA, YUNPENG DU, MASARU MIYAGI, Cleveland, $\mathrm{OH}$

Evidence accumulates that inflammatory changes contribute to the development of diabetic retinopathy. We investigated the effect of diabetes on acetylation of retinal histones, and the relation of this acetylation to the increased expression of pro-inflammatory proteins in diabetes/elevated glucose. Studies were conducted in vivo (2 mos diabetes) and in vitro (retinal Muller cell line, rMC-1). Sites and extent of acetylation on lysines were determined by mass spectrometric methods and western blots. Minocycline was administered in vivo and in vitro, and HAT inhibitors and HDAC activators and inhibitors were administered to the rMC-1 cells. To inhibit expression of the HAT p300, siRNA against p300 was administered to rMC-1 cells. Diabetes or $25 \mathrm{mM}$ glucose increased acetylation of retinal histones on specific lysines, and this increase was significantly inhibited by minocycline. Mino- 
cycline also inhibited ICAM expression in vivo. In vitro, minocycline as well as inhibitors of HAT (including antisense against p300) or activators of HDAC inhibited both histone acetylation and expression of the pro-inflammatory proteins, ICAM and iNOS. Hyperglycemia-induced acetylation of histones or other proteins contribute to the diabetes-induced upregulation of at least some pro-inflammatory proteins in retina, and thereby likely contribute to the development of diabetic retinopathy.

261-OR High Glucose-Induced Mitochondrial Dysfunction and its Role in the Metabolic Memory Phenomenon

THOMAS TIEN, SAYON ROY, Boston, MA

Currently it is unknown whether changes in mitochondrial morphology and subsequent functional alterations participate in the development of the metabolic memory phenomenon. Our recent studies indicate high glucose (HG)-induced mitochondrial fragmentation promotes retinal vascular changes seen in diabetic retinopathy. In this study, we have examined whether HG-induced mitochondrial dysfunction, in particular, mitochondrial morphology changes, results in irreversible mitochondrial function that can promote the metabolic memory phenomenon. To determine if $\mathrm{HG}$-induced mitochondrial changes are retained, rat retinal endothelial cells (RRECs grown in $\mathrm{HG}(30 \mathrm{mM})$ medium for 4 days were shifted to normal $(\mathrm{N}, 5 \mathrm{mM})$ medium for 3 days; in parallel, RRECs were grown as control in $\mathrm{N}$ medium and HG medium for 7 days. Cells were stained with MitoTracker Red, a mitochondrial specific dye, and examined live under confocal microscope. Images were analyzed for mitochondrial morphology using Form Factor (FF) and Aspect Ratio (AR) values. Oxygen consumption rate (OCR) of the cells was also measured by XF24 bioenergetic assay. Cells exposed to HG showed significantly increased mitochondrial fragmentation compared to cells grown in $\mathrm{N}$ medium ( $\mathrm{FF}=2.3 \pm 0.3$ vs $1.7 \pm 0.2, \mathrm{p}<0.001 ; \mathrm{AR}=2.3 \pm 0.2$ vs $2.0 \pm 0.1, \mathrm{p}<$ $0.001)$. Cells grown in $\mathrm{HG}$ medium for 4 days followed by $\mathrm{N}$ medium for 3 days $(F F=1.8 \pm 0.3 ; A R=2.1 \pm 0.2)$ retained the $H G$-induced increased mitochondrial fragmentation ( $p<0.005, p<0.05$, respectively). No significant differences in $\mathrm{FF}$ and $\mathrm{AR}$ values were observed between cells grown in $\mathrm{HG}$ medium and those grown in $\mathrm{HG}$ medium and shifted to $\mathrm{N}$ medium. Furthermore, XF24 bioenergetic assay showed that cells grown in $\mathrm{HG}$ and shifted to $\mathrm{N}$ medium maintained $\mathrm{HG}$-induced decrease in OCR. Findings from this study indicate that $\mathrm{HG}$-induced mitochondrial fragmentation and altered OCR are irreversible changes that may contribute to the development and progression of the metabolic memory phenomenon

\section{$\Delta$}

262-0R

Activating Transcription Factor 4 Regulates Endothelial Inflammatory Signaling in Diabetic Retinopathy

JOSHUA J. WANG, GUANGJUN JING, MICHAEL DANESHFAR, SARAH X. ZHANG, Oklahoma City, OK

We previously reported that endoplasmic reticulum (ER) stress is a key player in retinal inflammation and vascular leakage in diabetes. However, the cellular mechanisms and signaling pathways of ER stress in diabetic retinopathy remain unclear. Activating transcription factor-4 (ATF4) is a transcriptional factor induced by various cellular stresses. The objective of this study is to delineate the role of ATF4 in endothelial inflammatory response and vascular dysfunction. Immortalized rat retinal endothelial (TRi-BRB) and primary mouse brain endothelial cells were exposed to high glucose $(25$ $\mathrm{mM})$, TNF- $\alpha(10 \mathrm{ng} / \mathrm{ml})$, or LPS $(250 \mathrm{ng} / \mathrm{ml})$ to induce ER stress and inflammation. ATF4 activity was manipulated by adenoviruses expressing wildtype (WT) or a dominant negative mutant (DN) of ATF4. Mouse models of diabetes were induced in ATF4 knockout mice. Inflammatory gene expression and signaling pathways, including JNK, STAT3, and NF- $\mathrm{KB}$, were analyzed. Our results showed that ER stress was induced, followed by ATF4 activation, in retinal and brain endothelial cells exposed to high glucose, TNF- $\alpha$ or LPS. Inhibiting ATF4 activity by Ad-ATF4DN, or deletion of ATF4 gene significantly attenuates high glucose- or LPS-induced ICAM-1, TNF- $\alpha$ and VEGF expression, and MCP-1 secretion. Conversely, over-expressing ATF4 was sufficient to upregulate inflammatory genes in retinal endothelial cells in vitro and in vivo. This was in part through activation of the JNK and STAT3 pathways. Downregulation of ATF4 remarkably reduced retinal adhesion molecule (ICAM-1) and inflammatory cytokine (MCP-1, IL-1 $\beta$ and VEGF) expression, and mitigated retinal vascular permeability induced by diabetes or introvitreal injection of LPS. Taken together, our data indicate that ATF4 mediates ER stress-associated inflammation in vascular endothelial cells. Inhibition of ATF4 activation may provide a new therapeutic target in diabetic retinopathy and other diabetic complications.

Supported by: NIH EY019949, AHAF, OCAST
263-0R

The Potential Regulation of C/EBP- $\beta$ by AMPK and their Involvement in ER Stress and Inflammation

JOSE M. CACICEDO, BENOIT VIOLLET, NEIL B. RUDERMAN, YASUO IDO, Boston, MA, Paris, France

The C/EBP family of transcription factors plays a critical role in ER stressinduced pancreatic beta cell apoptosis and the activation of inflammatory genes including many that are increased with diabetes and atherosclerosis. Of its six members, C/EBP- $\beta$ is thought to be the most critical since it accumulates in the $\beta$-cells of diabetic animals in which it activates ER stress. Also, its genetic ablation prevents both damage to the $\beta$-cells and the development of diabetes. In the present study, we investigated the role of $\mathrm{C} / \mathrm{EBP}-\beta$ in ER stress and inflammation in retinal pericytes (PCs). PCs are sensitive to both ER stress and inflammation and are among the first cells to die during the course of diabetic retinopathy. In addition, we examined the regulation of C/EBP- $\beta$ by AMP-activated protein kinase (AMPK). Incubation of cultured bovine retinal pericytes with the saturated fatty acid palmitate, but not the monounsaturated fatty acid oleate, induced the upregulation of C/EBP- $\beta$ while simultaneously activating ER stress and apoptosis. Activation of AMPK with AICAR prevented all of these changes. Subsequent studies performed in wild-type mouse embryonic fibroblasts (MEFS) treated with thapsigargin (to release ER Ca2+ stores and induce ER stress) also demonstrated an increase in C/EBP- $\beta$ as did the inflammatory cytokine TNF-alpha. Activation of AMPK not only decreased the ER stress response and inflammation in these cells, it also prevented the increase in C/EBP- $\beta$ levels. Use of double AMPK alpha-1 and -2 knockout MEFs revealed that the decrease in $\mathrm{C} /$ EBP- $\beta$ levels was dependent on the presence of AMPK and that AMPK leads to a shuttling of C/EBP- $\beta$ into the cytosol when stimulated with TNF-alpha. It is unclear, at this time, whether AMPK leads to the active export of $\mathrm{C}$ / EBP- $\beta$ from the nucleus to the cytosol or tags it for degradation. The specific interactions between AMPK and C/EBP- $\beta$, as well as the importance of $\mathrm{C} /$ EBP- $\beta$ in ER stress and inflammation remain to be determined.

264-0R

Decreased Bone Marrow Neuronal Support in Type 1 Diabetes Shifts Normal Hematopoiesis Towards the Generation of (C-C Motif) Receptor 2 (CCR2) Monocytes

ASHAY D. BHATWADEKAR, PING HU, SUGATA HAZRA, SERGIO CABALLERO, SUSANNE MOHR, STEVEN F. ABCOUWER, DANIEL R. SABAN, TAILOI CHAN-LING, JULIA V. BUSIK, MARIA B. GRANT, Gainesville, FL, Camperdown, Australia, East Lansing, MI, Ann Arbor, MI, Boston, MA

Dynamic control of hematopoietic stem cell (HSC) release is regulated by the neurotransmitters in bone marrow (BM). Previously, we showed that in both Type 1 diabetes (T1D) and Type 2 diabetes (T2D), reduced levels of norepinephrine lead to abnormal release of HSCs from BM into the circulation. In the present study, we examined the role of key neurotransmitters/ neuropeptides (NT/NP) in T1D in the support, differentiation and the fate of HSCs. Immunohistochemical analysis of NT/NP in T1D long bones was conducted. Quantification of the side population (SP) of the bone marrow cells was performed using colony forming assays and flow cytometry. Chimeric mice (transplanted with gfp-expressing HSC) were made T1D and their retinas were evaluated for contribution and characterization of BM derived cell using flat-mounts and by flow cytometry of enzymatically-dissociated retinal cells. In T1D mice, immunodetection of calcitonin gene related peptide was reduced by $65 \%(p<0.05)$, neuropeptide $Y$ by $67 \%(p<0.05)$, acetylcholine by $51 \%(p<0.05)$, and somatostatin by $50 \%(p<0.05)$. BM cells isolated $\mathrm{T} 1 \mathrm{D}$ mice showed depletion of SP-HSCs but increased numbers of monocyte/ macrophage colony forming units. BM supernatant of T1D mice showed increased levels (2.5-fold, $\mathrm{p}<0.002)$ of macrophage-colony stimulating factor (M-CSF/CSF-1, a stimulator of monocyte generation) and increased levels IL-1 $\beta$ (2-fold, $p<0.05$ ) and IL-27 ( 3 -fold, $p<0.01)$. Retinas of T1D mice showed a 10 -fold increase in CD11b+/CD45hi/ (C-C motif) receptor 2 (CCR2+) populations while lba+ microglia exhibited a ramified morphology suggesting an inflammatory activation. Our data suggests that loss of BM neurotransmitter regulation may shift hematopoiesis towards the generation of excessive numbers of CCR2 monocytes. This inflammatory retinal phenotype combined with the loss of key reparative cells contributes towards retinal pathology in diabetes.

Supported by: Thomas H. Maren Junior Investigator Award (A.B.), EY007739, EY012601 (M.B.G.) 


\section{CLINICAL NUTRITION}

\section{CLINICAL NUTRITION}

265-OR

A High-Fiber Diet Improves M1/M2 Phenotype as well as the Vascular Function in the Patients With Type 2 Diabetes Mellitus

KEIKO KONDO, YOSHIHIKO NISHIO, ATSUSHI ISHIKADO, MOTOYUKI KONDO, KEIKO NAKAO, KATSUTARO MORINO, TAKESHI YOSHIZAKI, OSAMU SEKINE, SATOSHI UGI, ATSUNORI KASHIWAGI, HIROSHI MAEGAWA, Otsu, Japan, Kagoshima, Japan

The monocyte/macrophage system has mainly two distinct phenotypes of differentiation: proinflammatory (M1) and anti-inflammatory (M2). We reported that a high-fiber diet improved the vascular dysfunction in the patients with type 2 diabetes mellitus (T2DM). In this study, we investigated whether a high-fiber diet affects M1/M2 phenotype of peripheral blood monocytes (PBM) as well as the vascular function in the patients with T2DM. We evaluated the mRNA expression of CD11c/CD163 ratio as a marker of M1/M2 phenotype of PBM and the vascular function in 10 patients with T2DM $(59.2$ years, $\mathrm{M} / \mathrm{F}=3 / 7$, diet $/ \mathrm{OHA}=8 / 2$ ) and age-matched 8 subjects with normal glucose tolerance (NGT) (58.0 years, $M / F=4 / 4)$. In addition, the diabetic patients were followed for 16 weeks assigned to two periods of 8 weeks of either a high-fiber diet or control diet in a randomized crossover design. Before and after each period, vascular function and CD11c/CD163 ratio in PBM were evaluated. The vascular function assessed with either peak forearm blood flow (FBF) ( $417 \pm 209$ vs. $800 \pm 458 \%, p=0.026)$ or flow debt repayment (FDR) (48 \pm 18 vs. $104 \pm 68 \%, p=0.015)$ was significantly low in the diabetic patients as compared with NGT. The ratio of CD11c/CD163 expression levels was higher in the diabetes patients as compared with NGT. The intervention with a high-fiber diet for 8 weeks improved $39.2 \%$ of peak FBF $(p=0.075)$, and $63.5 \%$ of FDR $(p=0.006)$, but not with a control diet. Total energy intake was unchanged throughout the study periods. The intake of dietary fiber in the high-fiber diet period was higher than that in the baseline or control period ( $23.9 \pm 4.3$ vs. $12.8 \pm 3.0$ vs. $12.4 \pm 2.8 \mathrm{~g} /$ day, $p<0.0001)$. Body weight $(69.7 \pm 12.9$ vs. $68.5 \pm 13.2 \mathrm{~kg}, \mathrm{p}=0.007)$ and $\mathrm{HbA} 1 \mathrm{c}(7.0 \pm 0.6$ vs. $6.8 \pm 0.6 \%$, $p=0.036$ ) levels were decreased, and CD11c/CD163 ratio showed a 20\% reduction ( $p=0.022)$ after the high-fiber diet period. A high-fiber diet improved the vascular function, and might alter the gene expression of PBM in the patients with T2DM.

266-OR

Dietary Fat Acutely Increases Glucose Levels and Insulin Requirements in Type 1 Diabetes

HOWARD A. WOLPERT, ASTRID ATAKOV-CASTILLO, STEPHANIE A. SMITH, GARRY M. STEIL, Boston, MA

Current guidelines for intensive treatment of type 1 diabetes (T1D) base the meal-time insulin bolus calculations exclusively on carbohydrate counting. There is strong evidence that free fatty acids impair insulin sensitivity, and we hypothesized that patients with T1D would require more insulin coverage for higher fat meals than lower fat meals with identical carbohydrate content. We used a cross-over design comparing two 18-hour periods (6 PM to 12 noon) of closed loop (CL) glucose control following high fat (HFD) vs. low fat dinners (LFD; 6 PM). Each dinner had identical carbohydrate and protein content, but different fat content ( 60 vs. 10 grams, respectively). Breakfast meals (8 AM) were identical. The CL system utilized the external Physiologic Insulin Delivery (PID) algorithm. Seven T1D subjects (age $55 \pm 12$ years, A1c $7.2 \pm 0.8 \%$ ) successfully completed the protocol. The HFD required more insulin $(15.2 \pm 7.5$ vs. $10.3 \pm 4.7 \mathrm{U} ; \mathrm{p}=0.01)$, and despite the additional insulin caused more hyperglycemia (Figure, AUC above $180 \mathrm{mg} / \mathrm{dL}=55.8 \pm 8.2$ vs. $33.3 \pm 4.8 \mathrm{~g} / \mathrm{dL} \cdot \mathrm{min} ; \mathrm{p}=0.0003$ ). Calculated carbohydrate-to-insulin ratio increased from $9 \pm 2$ to $13 \pm 3$ grams/U (HFD vs. LFD, respectively; $p=0.01$ ). There were marked inter-individual differences in fat sensitivity, with one subject requiring a more than two-fold $(108 \%)$ increase in insulin dose to cover the HFD. These findings: 1) Highlight the limitations of the current carbohydrate counting-based approach to bolus insulin dose calculation, and 2) Suggest that dietary fat intake is an important nutritional consideration for glycemic control in individuals with type 1 diabetes.
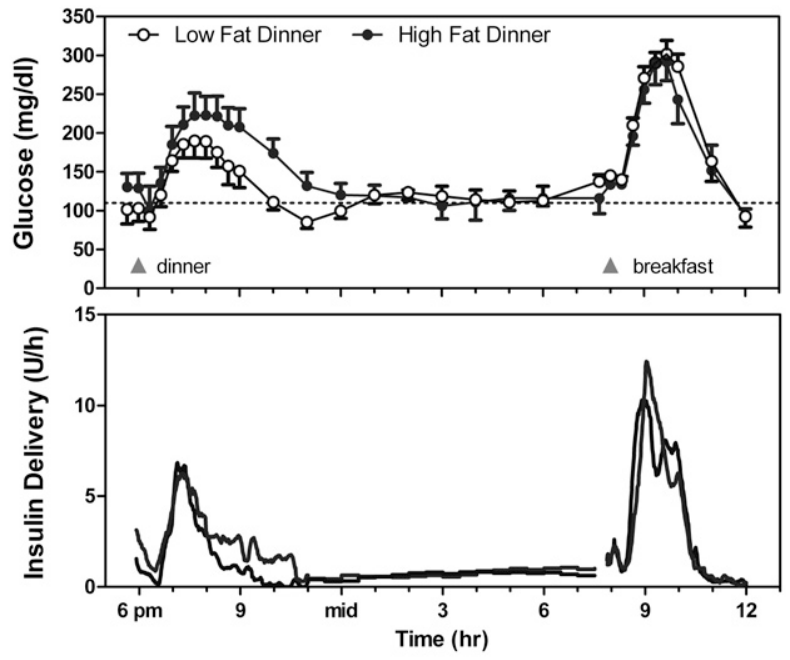

267-0R

Protein-Rich Meal Replacement Significantly Reduces Insulin Demand, HbA1c and Weight Long-Term in Type 2 Diabetes Mellitus Patients With >100 U Insulin/Day

KERSTIN KEMPF, NANETTE C. SCHLOOT, BABETTE GÄRTNER, RUDOLF KEIL, PETER SCHADEWALDT, STEPHAN MARTIN, Düsseldorf, Germany, Köln, Germany, Bad Homburg, Germany, Grevenbroich, Germany

Despite application of high insulin doses good glycaemic control is often lacking in type 2 diabetes mellitus (T2DM) patients and new therapeutic options are needed. We analyzed if a protein-rich meal replacement (PRMR) could be successful in reducing insulin demand, $\mathrm{HbA1c}$ and body weight. During the 1st week obese T2DM patients ( $n=22)$ with insulin doses $>100 \mathrm{U} /$ day replaced 3 main meals by $50 \mathrm{~g}$ PRMR (Almased-Vitalkost) each $(=1100$ $\mathrm{kcal} / \mathrm{day})$. In 2nd-4th week 2 meals were replaced and a protein-rich lunch was allowed. In 5th-12th week only dinner was replaced. Clinical parameters were determined at baseline, after 4, 8 and 12 weeks as well as after 1.5 years of follow up. Wilcoxon signed rank test was used for the intention-totreat analysis and Mann-Whitney test for subgroup analyses. The 12 week program was completed by 15 participants (68\%). Already after 1 week mean insulin dose could be reduced from $147 \pm 75 \mathrm{U}$ to $91 \pm 55 \mathrm{U} /$ day $(-56$ $\mathrm{U} ; \mathrm{p}=0.0001)$ and further decreased to $65 \pm 32 \mathrm{U}(-82 \mathrm{U} ; \mathrm{p}<0.0001)$ after 12 weeks of study. During the study HbA1c decreased from $8.8 \pm 1.4 \%$ to $8.1 \pm$ $1.6 \%(-0.8 \pm 1.4 \% ; p=0.048)$ and weight from $118.0 \pm 19.7 \mathrm{~kg}$ to $107.4 \pm 19.2$ $\mathrm{kg}(-7.9 \pm 3.5 \mathrm{~kg} ; p<0.0001)$. Significant improvements were also seen for body mass index $(-2.6 \pm 1.3 \mathrm{~kg} / \mathrm{m} 2 ; \mathrm{p}<0.0001)$, waist $(-7.1 \pm 6.6 \mathrm{~cm} ; \mathrm{p}=0.0003)$ and hip circumference $(-3.3 \pm 6.2 \mathrm{~cm} ; \mathrm{p}=0.035)$, fasting blood glucose $(27.6 \pm$ 39.6; $p=0.027)$, triglycerides $(-70.3 \pm 85.1 \mathrm{mg} / \mathrm{dl} ; \mathrm{p}=0.0001)$ and $\mathrm{HDL}$ cholesterol $(2.2 \pm 3.8 \mathrm{mg} / \mathrm{dl} ; \mathrm{p}=0.049)$. After 1.5 years insulin demand and weight remained significantly lower than baseline. Participants who continued PRMR further reduced their HbA1c, weight and insulin dose. Two patients could stop insulin therapy. In sum, PRMR was effective in reducing insulin demand of T2DM patients with intensified insulin therapy accompanied by reduction of $\mathrm{HbA1c}$, weight and other cardiometabolic risk factors. With continuous use of PRMR glycaemic control might be improved long-term.

Supported by: Almased Wellness GmbH

268-0R

Eating Vegetables Before Carbohydrates for Test Meals Improves Postprandial Glucose Excursions Assessed by Continuous Glucose Monitoring System Both in Patients With Type 2 Diabetes and in Subjects With Normal Glucose Tolerance

SAEKO IMAI, MICHIAKI FUKUI, NEIKO OZASA, ONKOO RHEE, SHIZUO KAJIYAMA, Osaka, Japan, Kyoto, Japan, Nagoya, Japan

We evaluated whether eating test meals of vegetables before carbohydrates could reduce the postprandial glucose excursions assessed by continuous glucose monitoring system (CGMS) in Japanese patients with type 2 diabetes mellitus (DM) and subjects with normal glucose tolerance (NGT). Seventeen patients with DM $(65.7 \pm 9.6$ yrs, HbA1c $7.2 \pm 1.0 \%)$ and 16 subjects with NGT $(28.6 \pm 12.0 \mathrm{yrs})$ were assigned to use CGMS (Medtronic Minimed Gold) for 4 days (72 hours) of either eating test meals of vegetables before carbohydrates or carbohydrates before vegetables in a randomized 


\section{CLINICAL NUTRITION}

crossover design. The participants consumed each test meal at a fixed time on the $2^{\text {nd }}$ and $3^{\text {rd }}$ day. The subjects ate the first dish of vegetables for $5 \mathrm{~min}$, then the main dishes, and consumed rice or bread with a 10-min interval between the vegetables and carbohydrates in each test meal, or eating vice versa. The glucose fluctuations were assessed by the following parameters obtained from CGMS and compared between two different days: the mean blood glucose (MBG), standard deviation (SD), mean amplitude of glycemic excursions (MAGE), and the largest amplitude of glycemic excursions (LAGE). The levels of SD, MAGE, and LAGE were significantly reduced when the subjects ate the test meals of eating vegetables before carbohydrates compared to the reverse regimen. These results suggest that the eating order of vegetables before carbohydrates reduces the postprandial glucose excursions both in patients with DM and in subjects with NGT.

\begin{tabular}{|c|c|c|c|c|c|c|}
\hline \multicolumn{7}{|c|}{$\begin{array}{l}\text { Characteristics of glycemic excursions in patietns with DM and subjects with } \\
\text { NGT }\end{array}$} \\
\hline & $\mathrm{DM}(\mathrm{n}=17)$ & $(n=17)$ & & NGT $(n=16)$ & $(n=16)$ & \\
\hline & $\begin{array}{c}\text { Vegetables } \\
\text { before } \\
\text { carbohy- } \\
\text { drates }\end{array}$ & $\begin{array}{c}\text { Carbohy- } \\
\text { drates } \\
\text { before } \\
\text { vegetables }\end{array}$ & $p$ & $\begin{array}{l}\text { Vegetables } \\
\text { before } \\
\text { carbohydrate }\end{array}$ & $\begin{array}{c}\text { Carbohy- } \\
\text { drates } \\
\text { before } \\
\text { vegetables }\end{array}$ & $p$ \\
\hline$\overline{M B G}(\mathrm{mg} / \mathrm{dl})$ & $141 \pm 29$ & $144 \pm 29$ & n.s. & $91 \pm 7$ & $96 \pm 9$ & $p<0.05$ \\
\hline $\mathrm{SD}(\mathrm{mg} / \mathrm{dl})$ & $31 \pm 13$ & $42 \pm 21$ & $p<0.01$ & $12 \pm 4$ & $15 \pm 6$ & $p<0.01$ \\
\hline MAGE (mg/dl) & $78 \pm 35$ & $114 \pm 58$ & $p<0.01$ & $28 \pm 11$ & $42 \pm 19$ & $p<0.01$ \\
\hline LAGE (mg/dl) & $125 \pm 42$ & $164 \pm 67$ & $p<0.01$ & $57 \pm 17$ & $78 \pm 30$ & $p<0.01$ \\
\hline
\end{tabular}

Supported by: Scientific Research Grants from Japan and Osaka Prefecture University

269-0R

Effects of Brown Rice Diet on Metabolic Parameters and Inflammatory Markers in Chinese American With Prediabetes

BIN WANG, RAJ MEDAPALLI, JIN XU, WEIJING CAI, XUE CHEN, JOHN C. HE, JAIME URIBARRI, New York, NY

Epidemiological studies have shown an association between consumption of white rice and prevalence of insulin resistance. We wanted to test the effect of substituting brown rice for white rice on insulin resistance and expression of inflammatory markers in pre-diabetic Chinese patients with a usual high intake of white rice. 100 Chinese American with diagnosis of pre-diabetes in the area of Flushing, New York City, were randomized to either continue their usual white rice intake or change to same amount of brown rice for 3 months. Fasting blood was obtained at baseline and end of study in both groups for measurement of circulating and cellular (peripheral mononuclear cells) metabolic and inflammatory markers. Only 57 patients finished the study. The brown rice group experienced significant weight loss $(64.9 \pm 8 \mathrm{~kg}$ to $63.4 \pm 8 \mathrm{~kg}$; mean \pm SD) and fall of both systolic and diastolic blood pressure $(123 \pm 10 \mathrm{mmHg}$ to $114 \pm 13 \mathrm{mmHg}$ and $75 \pm 6 \mathrm{mmHg}$ to $72 \pm$ $6 \mathrm{mmHg}$, respectively). Insulin $(6.3 \pm 5 \mathrm{U} / \mathrm{ml}$ to $5.5 \pm 5 \mathrm{U} / \mathrm{ml})$, HOMA $(1.5 \pm 1.2$ to $1.3 \pm 1.2$ ) serum Advanced Glycation Endproducts (CML $6.6 \pm 2 \mathrm{U} / \mathrm{ml}$ to 5.4 $\pm 1 \mathrm{U} / \mathrm{ml}$ and $\mathrm{MG} 0.89 \pm 0.16 \mathrm{nmol} / \mathrm{ml}$ to $0.73 \pm 0.20 \mathrm{nmol} / \mathrm{ml})$, 8-isoprostane $(102 \pm 32 \mathrm{pg} / \mathrm{ml}$ to $87 \pm 24 \mathrm{pg} / \mathrm{ml})$ and TNF protein $(71 \pm 36 \mathrm{pg} / \mathrm{mg}$ to $55 \pm 24$ $\mathrm{pg} / \mathrm{mg}$ ) decreased, while SIRT1 mRNA increased in the brown rice group as compared to the white rice group. In conclusion, substituting brown rice for white rice in a population with high daily consumption of rice for a period of 3 months has a significant beneficial effect in improving their metabolic risk factors. Elucidation of the mechanisms of these effects will require further studies.

\section{WITHDRAWN}

271-OR

An Isocaloric High Fat Diet Induces an Inflammatory Cytokine Response in Healthy Humans Without Increasing Body Weight ANNE-CATHRIN SELTMANN, MICHAEL KRUSE, SILKE HORNEMANN, DANIELA HOFFMANN, MARTIN OSTERHOFF, ANDREAS BUSJAHN, ANDREAS F. PFEIFFER, Nuthetal, Germany, Berlin, Germany

Consuming hypercaloric high fat diets leads to obesity and cytokine release from adipocytes, which is a risk factor for diabetes and cardiovascular diseases. It is unclear whether the inflammatory response relates to weight gain or to the dietary fat itself. This study analysed the effect of a nutritional switch from a low fat to a high fat diet under isocaloric conditions on body weight (BW), fat mass (FM), CRP and gene expression of cytokines in subcutaneous adipose tissue (SAT) in healthy humans. 46 healthy individuals, age 18-70 years, were investigated for 12 weeks. An isocaloric diet rich in carbohydrates (55\% carbohydrates, $15 \%$ protein, $30 \%$ fat, LF) was applied for 6 weeks followed by an isocaloric diet rich in saturated fat ( $40 \%$ carbohydrates, $15 \%$ protein, $45 \%$ fat, HF) for another 6 weeks. Serum concentration of CRP, interleukin-6 (IL-6), visfatin (VS) and chemerin (CM) were measured after the LF period, after one week of HF (HF1wk) and at the end of the HF period (HFGwk). Gene expression of IL-6, CM and VS was assessed in biopsies from SAT. Body composition was determined using a DEXA scan. BW and DEXA FM were constant over the time of intervention: BW: LF $69.44 \pm 1.94 \mathrm{~kg}$, HF1wk $69.45 \pm 1.91 \mathrm{~kg}$, HF6wk $69.92 \pm 1.96 \mathrm{~kg}$, FM: LF $23.56 \pm 1.04 \%$, HF6wk $23.77 \pm 1.01 \%$. CRP was increased after 6 wk on HF $(1.90 \pm 0.43 \mathrm{mg} / \mathrm{dl})$ compared to LF and HF1wk $(0.97 \pm 0.19$ and $1.04 \pm 0.21 \mathrm{mg} / \mathrm{dl}$, both $\mathrm{p}<0.05)$. Serum VS was increased in HF6wk $(0.71 \pm 0.13 \mathrm{ng} / \mathrm{ml})$ compared to $L F(0.48 \pm 0.08 \mathrm{ng} / \mathrm{ml}, \mathrm{p}<0.05)$, but not in HF1wk. Serum CM was increased in HF1wk $(143.22 \pm 4.40 \mathrm{ng} / \mathrm{ml})$ and HF6wk $(139.65 \pm 3.63 \mathrm{ng} / \mathrm{ml})$ compared to LF $(133.62 \pm 4.52 \mathrm{ng} / \mathrm{ml})$, both $\mathrm{p}<0.05$. There was no difference in serum IL-6. In SAT, gene expression of VS was increased 2.06-fold in HF1wk and 2.08-fold in HFGwk (both $p<0.05$ ) and gene expression of CM was 1.25 fold increased in HF1wk and 1.28fold increased in HF6wk compared to LF (both $p<0.01$ ). Despite constant BW an isocaloric HF diet leads to an inflammatory cytokine response in SAT in normal weight healthy humans.

Supported by: Federal Ministry of Education and Research, Germany

272-0R

Grape Polyphenols Prevent High-Fructose Diet-Induced Insulin Resistance in Obese 1st-Degree Relatives of T2 Diabetic Patients by Modulating Mitochondrial Function and Oxidative Stress

ANTOINE AVIGNON, MARIE HOKAYEM, EMILIE BLOND, JEAN-FRÉDÉRIC BRUN, CHRISTINE FEDOU, JENNIFER RIUSSET, HUBERT VIDAL, JACQUES MERCIER, CHRISTINE FEILLET-COUDRAY, CHARLES COUDRAY, JEAN-PAUL CRISTOL, ANNE-MARIE DUPUY, MARTINE LAVILLE, Montpellier, France, Lyon, France

Recent evidence supports that grape polyphenols (GPP) improve insulin resistance (IR) in high-fructose-fed rats. There is no equivalent data in humans. We treated 35 healthy, obese 1 st degree relatives of T2 diabetics with placebo (P) or 2g/day GPP in a randomized double-blind study for $9 \mathrm{wk}$. During the last wk, both groups received a $3 \mathrm{~g} / \mathrm{kg}$ lean body mass fructose load (FL)/day. Very few clinical and metabolic changes were noted after the first 8 wk of GPP. After the FL, the glucose infusion rate (GIR) measured using the hyperinsulinemic euglycemic clamp $(7.38 \pm 0.57$ vs $6.57 \pm 0.55, p=0.026)$ and fasting hepatic insulin sensitivity index $(11.94 \pm 1.52$ vs $9.57 \pm 0.88$, $p=0.025)$ decreased in the P group and muscle PAkt/Akt tended to decrease (13.13 \pm 1.49 vs $6.16 \pm 0.7, p=0.068$ ). Such alterations were not noted in the GPP group. The FL significantly increased protein muscle carbonylation, muscle TBARS and urinary F2isoprostanes in the P group, leaving the GPP group spared. Considering the global population, urinary F2isoprostanes were 
inversely correlated with GIR before the $F L(r=-0.46, p<0.005)$ and urinary F2-isoprostanes fluctuations during $\mathrm{FL}$ were negatively correlated with the variation of $\mathrm{GIR}(r=-0.38, p=0.02)$. Muscle mitochondrial fonction tended to decrease in the P group after FL but significantly increased in the GPP group. In the $\mathrm{P}$ group, mitochondrial function after FL was associated with urinary F2isoprostanes $(r=0.79, p<0.05)$ and negatively with $G I R(r=-0.97, p<0.001)$. Moreover, we noted a downward trend in UCP3 protein in the P group after $\mathrm{FL}(59 \%$ decrease, $\mathrm{p}=0.08)$ and an increase in mitochondrial biogenesis in the GPP group as evidenced by a significant rise in PGC-1a mRNA and a higher citrate synthase activity compared to the P. In conclusion, we demonstrate that GPP protect against fructose induced IR by modulating mitochondrial function and oxidative stress.

Supported by: ANR - Agence Nationale de la Recherche

\section{MATERNAL DIABETES- EXPOSURE, EPIGENETICS, AND EMBRYOPATHY}

273-OR

Oxidative Stress Inhibits Differentiation-Associated DNA Methylation Patterns that are Necessary for Embryo Gene Expression DAN WEI, MARY R. LOEKEN, Boston, MA

Neural tube defects (NTD) are among the most common malformations caused by diabetic pregnancy in humans and animal models. We have previously shown that maternal hyperglycemia-induced oxidative stress inhibits expression of Pax3, a gene that is expressed in neuroepithelium and required for neural tube closure, and is sufficient to explain these NTD. The precise mechanisms by which oxidative stress inhibits Pax 3 expression are not known. We hypothesize that induction of Pax3 during differentiation requires epigenetic modification of transcription regulatory elements, and that oxidative stress inhibits these changes. We tested the involvement of Pax3 $\mathrm{CpG}$ island methylation using murine embryonic stem cells (mESC). mESC can differentiate into Pax3-expressing neuronal precursors, and antimycin $A$ $(\mathrm{AA})$, a chemical inducer of oxidative stress, inhibits Pax3 expression mESC. We report here that a DNA methyltransferase inhibitor, AzaC, induced Pax3 expression in undifferentiated (UD) mESC. Upon differentiation, methylation of a promoter-proximal Pax3 CpG island decreased, and AA blocked this decrease. Of the 3 DNA methyltransferases (DNMTs), DNMT1, DNMt3a, and DNMT3b, only expression of DNMT3b changed (decreased) with differentiation. However, AA did not affect DNMT3b expression. Using inducible shRNA vectors to knock down expression of the DNMTs, we found that only DNMT3b regulates Pax3 expression and $\mathrm{CpG}$ island methylation. Moreover, expression of DNMT3b was required for AA to inhibit Pax 3 expression. These results indicate that Pax3 expression is silenced in UD early embryo cells by $\mathrm{CpG}$ island methylation caused by DMT3b. Upon differentiation, induction of Pax3 expression requires loss of $\mathrm{CpG}$ methylation caused by decreased DNMT3b expression. Oxidative stress appears to block differentiation-induced Pax3 expression by increasing activity of DNMT3b, thereby maintaining an UD pattern of $\mathrm{Pax} 3 \mathrm{CpG}$ island methylation.

Supported by: R01 DK58300 to M.R.L.

274-0R

Diabetes and Obesity Modify the DNA Methylation Pattern of Human Trophoblast Genes

DAVID SERRE, MARICELA HAGHIAC, MATTHEW CANNON, HADLEY MILLER, SYLVIE HAUGUEL-DE MOUZON, Cleveland, $\mathrm{OH}$

Background: Epigenetic marks are subjected to variation in response to changes in metabolic homeostasis. This study tested the hypothesis that obesity and gestational diabetes (GDM) alter the genome-wide DNA methylation patterns of the human placenta. Methods: Placentas were obtained at term elective $\mathrm{C}$-section of 15 pregnancies with GDM (BMI 38.0 0.6$)$ and healthy lean (BMI 22.0+2.0) or obese (BMI 38.4+0.6) women. The genome wide patterns of DNA methylation was assessed in isolated trophoblasts and whole placenta. Two complementary approaches were used: 1) precipitation of densely methylated DNA using recombinant Methyl-CpG Binding Domain protein 2 followed by massively parallel sequencing and 2) reduced representation bisulfite sequencing (RRBS). Results : Over 500,000 genomic $100 \mathrm{bp}$ regions were differentially methylated ( $\mathrm{FDR}<5 \%$ ) between placenta and trophoblast cells. 1,329 windows were methylated only in placenta while 6,987 were methylated only in trophoblasts. This translated into 67 gene promoters being solely methylated in placenta and 304 in trophoblasts. Trophoblast-specific DNA methylation marks included genes encoding known cytokines, growth factors and transcriptional regulators (LEP, RELT, TGFB1, APBB2, GDF6, NR2F2, FOXC1, WNT3A, WNT7). RRBS analysis of DNA methylation patterns of trophoblasts from lean, obese and GDM women generated 25 million $36 \mathrm{bp}$ reads. Within the 1,000 most differentially methylated regions, 670 were significantly overmethylated in obese and GDM vs lean $(p<0.05)$. The overmethylated genes were located close $<2 k b$ away) to genes regulating nutrient transporters (G00031323, $\left.\mathrm{p}=2.96 \times 10^{-8}\right)$ and Wnt receptor signaling pathway (G00016055, $\left.p=7 \times 10^{-3}\right)$. Conclusion: The human trophoblast harbors a cell type specific methylome distinct from whole placental tissue. Obesity and GDM modify trophoblast epigenetic marks and delineate the canonical Wnt signaling pathway, as a novel regulatory candidate.

Supported by: RO1 HD-22965

275-OR

Paternal Prenatal Undernutrition Alters Fetal Liver Development in F2 Offspring

YUSUKE ADACHI, ELVIRA ISGANAITIS, ARISTIDES LYTRAS, VICENCIA SALES, MICHAEL CHEN, MARY-ELIZABETH PATTI, Boston, MA

Prenatal exposure to nutritional and metabolic stress increases risk of diabetes, obesity and cardiovascular disease in offspring in both humans and animal models. We have previously shown in mice that prenatal undernutrition (UN) during pregnancy results in glucose intolerance and obesity in first-generation (F1-UN) offspring. Moreover, second-generation (F2) offspring of F1-UN males and normal females have glucose intolerance and increased adiposity, despite normal nutrition during the second-generation pregnancy. To evaluate mechanisms contributing to early-life metabolic phenotypes in F2 offspring of F1-UN males, we analyzed liver from E16.5 F2 fetuses generated from breeding: (1) control females with F1-UN males (F2-CU) and (2) control females with control males (F2-CC). There were no significant differences in liver weight; however, examination of H\&E-stained sections demonstrated that erythrocyte islands, prominent components of fetal liver at this stage, were substantially reduced in F2-CU mice as compared with F2-CC controls. Immounohistochemistry demonstrated reductions in CD34+ cells in F2-CU, confirming abnormal development of hematopoietic cells in F2-CU liver. In addition, the number of cells positive for the cell proliferation marker Ki67 and the mitosis marker phospho-histone $\mathrm{H} 3$ were decreased in F2-CU mice, indicating potential downregulation of the cell cycle in F2CU mice. To assess this possibility, we examined gene expression patterns (qRT-PCR) in fetal liver, observing marked downregulation of genes related to regulation of the cell cycle, such as AurkA and AurkB (24-51\% decrease, $p<0.01)$. Interestingly, similar gene expression patterns were also observed in fetal liver of F1 mice. Taken together, these results suggest that paternal nutritional exposures may regulate cell cycle patterns in offspring, potentially via durable epigenetic marks.

276-OR

Maternal Insulin Resistance Impacts Endocrine and Metabolic Programming in the Offspring

SEVIM KAHRAMAN, ERCUMENT DIRICE, DARIO F. DE JESUS, JIANG HU, ROHIT N. KULKARNI, Boston, MA

Studies in humans and rodents suggest maternal diabetes during fetal life leads to a higher risk of impaired glucose tolerance and obesity during adulthood. However, the impact of insulin resistance (IR) in the mother on glucose homeostasis in the offspring is not fully explored. We aimed to determine the consequences of $I R$ in the mother on fetal growth and fetal endocrine pancreas in the female liver-specific insulin receptor knockout (LIRKO) mouse model of maternal IR. Female LIRKO mice were hyperinsulinemic (LIRKO $8.1 \pm 1.1$ vs controls $1.4 \pm 0.1 \mathrm{ng} / \mathrm{mL}, \mathrm{n}=3, \mathrm{p}<0.01$ ) and developed spontaneous gestational diabetes (LIRKO $196.3 \pm 55.3$ vs controls $109.4 \pm 9$ $\mathrm{mg} / \mathrm{dL}, \mathrm{n}=3, \mathrm{p}<0.05$ ). We examined control offspring of LIRKO mothers (CL), LIRKO offspring of LIRKO mothers (LL), and control offspring of control mothers (CC) at embryonic (E) days 13.5, 15.5, 17.5, and postpartum (P) days 0,4 , 10, 21 (n=3-10). Although, all embryos of LIRKO mothers exhibited low body weights at $E 17.5$ (CL, $0.7 \pm 0.03 ; \mathrm{LL}, 0.65 \pm 0.02$ vs $C C, 0.8 \pm 0.05 \mathrm{~g} ; \mathrm{p}<0.05$ each), they rapidly gained weight after birth compared to $\mathrm{CC}$ at $\mathrm{P4}$ (CL, 2.68 \pm 0.08 ; $\mathrm{LL}, 2.28 \pm 0.15$ vs $C C, 1.94 \pm 0.14 \mathrm{~g} ; \mathrm{p}<0.01, p<0.05$ respectively). At $\mathrm{P} 4$, the $\mathrm{CL}$ but not $\mathrm{LL}$ embryos were hyperinsulinemic $(\mathrm{CL}, 2.5 \pm 0.5$; $\mathrm{LL}, 1.5 \pm 0.5$ vs

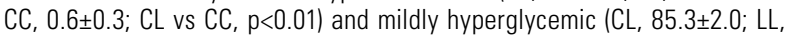
$81.0 \pm 6.5$ vs CC, $67.4 \pm 5.5$; $C L$ vs $C C, p<0.05$ ). Analyses of the endocrine pancreas at $\mathrm{P} 4$ revealed significantly reduced islets per $\mathrm{mm} 2$ of pancreas in $\mathrm{CL}$ but not in $\mathrm{LL}$ compared to $\mathrm{CC}$ (CL, $7.7 \pm 0.5 \mathrm{LL}, 9.6 \pm 1.6$ vs CC, $9.8 \pm 0.6$; $\mathrm{CL}$ vs CC, $\mathrm{p}<0.01)$. While there was no change in beta cell size, the beta cell content was increased in all offspring of LIRKO mothers compared to CC, and tended to be higher in $\mathrm{LL}$ (CL, 80.4\%; $\mathrm{LL}, 82.7 \%$ vs CC, $75.3 \%$; $\mathrm{n}=4$ ). Together these data indicate that maternal IR is important in determining fetal beta cell 
mass. The relatively greater islets and beta cells in the $\mathrm{LL}$ compared to $\mathrm{CL}$ embryos suggest cell autonomous epigenetic mechanisms in the regulation of islet growth and development.

\begin{abstract}
$\Delta$
277-0R

Hyperglycemia Induces Embryopathy In Vivo, Even in the Absence of Systemic Maternal Diabetes

ANDREW W. NORRIS, MICHELLE L. BAACK, CHUNLIN WANG, lowa City, IA

The Petersen-Freinkel hypothesis proposes that excesses of glucose and perhaps other fuels produce the embryopathy of maternal diabetes. In vitro, hyperglycemia is sufficient to induce embryopathy and teratogenesis in cultured embryos, but corresponding in vivo tests have been elusive. Thus we exposed developing embryos in vivo to hyperglycemia, but not systemic maternal diabetes, by infusing $4 \mathrm{mg} / \mathrm{min}$ glucose into the left uterine artery on gestation days 7-9 in healthy pregnant rats. Maternal, and thus right uterine horn, euglycemia was maintained despite pathologic hyperglycemia in the left uterine horn. Embryopathy prevalence and severity per an established 0-10 embryopathy score were determined on gestational day 13. Embryopathy was more prevalent among hyperglycemia exposed (i.e. left sided) than control (right sided) fetuses, at $43 \pm 16 \%$ versus $6.9 \pm 3.6 \%$ ( $p<0.0001, n=7$ mothers, 88 fetuses). Likewise, the mean embryopathy score was higher in the left $(4.0 \pm 1.6)$ versus right fetuses $(0.63 \pm 0.36, p<0.00005)$. For comparison, additional mothers received the teratogen retinoic acid $(25$ $\mathrm{mg} / \mathrm{kg}$ ) systemically on gestational day 7.5. These mothers exhibited identical embryopathy prevalence (19\%) and severity (1.3-1.7, $p=0.7, n=6$ mothers, 93 fetuses) between uterine horns. Among a third set of mothers, dual exposure to retinoic acid and hyperglycemia produced embryopathy rates $(48 \pm 16 \%)$ and scores $(4.5 \pm 1.8)(n=6$ mothers, 40 fetuses) not significantly different than hyperglycemia alone. Embryopathy produced by either retinoic acid or hyperglycemia was manifest by a range of malformations and varied degrees of embryo resorption. We conclude that hyperglycemia alone, even in the absence of systemic maternal diabetes, is sufficient to produce in vivo embryopathy during organogenesis, as predicted by the Petersen-Freinkel hypothesis.
\end{abstract}

Supported by: NIH R01 DK081548

278-OR

PGC1 $\alpha$ Overexpression in Transgenic Mice Reduces Maternal Diabetes-Induced Neural Tube Defects

CHENG XU, XUEZHENG LI, FANG WANG, E. ALBERT REECE, PEIXIN YANG, Baltimore, $M D$

Maternal diabetes increases the risk of neural tube defects (NTD). Hyperglycemia-induced metabolic stress and apoptosis cause NTD; however, the molecular intermediates that lead to these adverse events have not been well characterized. The peroxisome proliferator-activated receptor (PPAR)- $\gamma$ transcriptional coactivator PGC- $1 \alpha$ is an important regulator of mitochondrial biogenesis and function. PGC-1 $\alpha$ is abundantly present in the central nervous system. Furthermore, overexpression of PGC- $1 \alpha$ suppresses apoptosis, whereas reduced levels of PGC-1 $\alpha$ sensitize cells to apoptosis. Because mitochondrial dysfunction and apoptosis are two interdependent and causative events in diabetic embryopathy, we aimed to determine whether PGC-1 $\alpha$ plays a role in this disease process. Levels of all three PGC-1 family members, PGC-1 $\alpha$, PGC-1 $\beta$ and PRC, were determined. PGC-1 $\alpha$ and PRC were significantly reduced by maternal diabetes at embryonic day 8.5 (E8.5), while PGC-1 $\beta$ levels remained unchanged. To determine the function role of PGC-1 $\alpha$ reduction, rat nestin promoter driven PGC-1 $\alpha$ transgenic $(\mathrm{Tg})$ mice were generated. PGC-1 $\alpha$ overexpression occurred at E8 onwards, and specifically in the neural tube. Wildtype (WT) diabetic female mice were mated with male PGC1 $\alpha-\operatorname{Tg}$ mice to generate WT and PGC-1 $\alpha$ overexpressed embryos. Under diabetic conditions, the NTD rate of PGC1 $\alpha$ overexpressed embryos was only $2.3 \%$, which was significantly lower than that of WT embryos (25\%). Under non-diabetic conditions, the NTD rates in both WT and $\mathrm{PGC1} \alpha$ overexpressed embryos were $0.0 \%$. To delineate the mechanism underlying maternal diabetes-reduced PGC-1 $\alpha$ and PRC expression, we used targeted deletion of Protein Kinase $C$ alpha gene (PKC $\alpha$ ), whose activation mediates the teratogenicity of diabetes. Targeted deletion of PKC $\alpha$ abrogated diabetes-reduced PGC-1 $\alpha$ and PRC expression. We conclude that PGC-1 $\alpha$ reduction, which is likely downstream of $\mathrm{PKC} \alpha$, is a causative event leading to NTD in diabetic embryopathy.

Supported by: NIH R01 DK083243 to P.Y. and R01 DK083770 to E.A.R.
SOURCE OF BETA CELLS IMPROVING ISLET FUNCTION

279-OR

Functional In Vivo $\beta$-Cell Differentiation of Expanded Human Pancreatic Duct Cells D

PHILIPPE A. LYSY, FRANCISCO CABALLERO, TAKATSUGU YAMADA, AMEDEO VETERE, RYOSUKE MISAWA, PIOTR WITKOWSKI, JI LEI, JAMES MARKMANN, SUSAN BONNER-WEIR, Boston, MA, Chicago, IL

A major goal for $\beta$-cell replacement therapy is to find an adequate supply of functional $\beta$-cells. Pancreatic duct cells have $\beta$-cell differentiation potential but their role as candidates for transplantation has been uncertain Our aim was to extensively expand human pancreatic duct cells and then evaluate their potential for in vitro and in vivo $\beta$-cell differentiation. Human pancreatic CA19-9+ duct cells from 5 donors achieved up to 18 population doublings after monolayer culture in growth medium EGM-2-MV. In this medium, the cells became spindle-shaped and most duct cell markers were down-regulated. These human pancreatic duct-derived cells (HDDCs) had features of partial epithelial-mesenchymal transition but differed from bona fide mesenchymal stromal cells or fibroblasts. In vitro differentiation protocols reprogrammed these HDDCs towards a $\beta$-cell-like phenotype, with up to $3.1 \%$ of the cells stained for insulin but without glucose-responsive insulin secretion. After this differentiation protocol, 106 HDDCs were transplanted under the kidney capsule of STZ-induced diabetic $(570 \pm 30 \mathrm{mg} / \mathrm{dl})$ NOD-SCID mice. By 33 days the fed blood glucose levels of 4 of 6 mice were below $200 \mathrm{mg} / \mathrm{dL}$ while similar transplantation of undifferentiated HDDCs (n=8) did not lower blood glucoses. During IPGTTs, the 4 "cured" mice had basal human insulin values of $2.2 \pm 0.3 \mu \mathrm{U} / \mathrm{mL}$ that increased to $4.6 \pm 0.5$ and $11.9 \pm$ $2.7 \mu \mathrm{U} / \mathrm{mL}$, respectively, at 15 and $30 \mathrm{~min}$ after glucose injection. Grafts from these mice consisted mainly of insulin positive cells $(78.1 \pm 7.3 \%) \mathrm{co}$ expressing c-peptide, synaptophysin, and chromogranin A with no glucagon, somatostatin, or pancreatic polypeptide expression observed. In conclusion, HDDCs are potential candidates for $\beta$-cell replacement therapy since they have great capacity for expansion, acquire glucose-responsiveness in vivo and lower blood sugars of diabetic animals to near-physiological levels.

280-0R

Stimulating Beta Cell Replication and Improves Islet Graft Function by a GPR40 and GPR120 Dual Agonist

JIE GAO, GUOBIN WENG, TIMOTHY D. O'BRIEN, ANSARULLAH, YAN LU, MARTHA KOENIG, BRITTANY DERUYTER, ZHIGUANG GUO, Beijing, China, Ningbo, China, Minneapolis, MN, Sioux Falls, SD

GPR40 is abundantly expressed in $\beta$ cells. GPR40 and GPR120 are expressed on the intestinal $L$ cells. In this study, we investigated whether GW9508 (GW), a GPR40 and GPR120 dual agonist, can directly stimulate $\beta$-cell replication in vitro and in vivo and improve islet graft function in diabetic mice. To determine $\beta$-cell proliferation in vitro, C57BL/6 mouse islets were cultured for 4 days, with and without GW. BrdU was added on day 3 for overnight. Insulin and BrdU immunofluorescence staining was performed on intact islets and $\mathrm{BrdU}+\beta$ cells were accounted using confocal microscope. To determine islet graft function, $100 \mathrm{C} 57 \mathrm{BL} / 6$ mouse islets were transplanted under the left kidney of each streptozotocin induced-diabetic C57BL/6 mouse. These recipient mice were given BrdU and with or without $\mathrm{GW}$ at $10 \mathrm{mg} / \mathrm{kg} /$ day, starting from the day of transplantation. Islet graft function was monitored by measuring blood glucose level. At 4 weeks, Islet grafts were collected to determine $\beta$-cell replication. Plasma GLP-1 was measured. Insulin ${ }^{+}$and $\mathrm{BrdU}^{+} \beta$ cells in each cultured islet were $13.6 \pm 1.2$ cells with 0.1 $\mu \mathrm{M} \mathrm{GW}$; $18.3 \pm 1.5$ cells with $1 \mu \mathrm{M} \mathrm{GW}$; and $27.4 \pm 2.4$ cells with $10 \mu \mathrm{M} \mathrm{GW}$ $(\mathrm{P}<0.01$, vs. $10.5 \pm 0.6$ cells in each untreated islet). After islet transplantation, vehicle treated mice achieved normoglycemia in $14.4 \pm 2.9$ days $(\mathrm{N}=10)$, while $\mathrm{GW}$ treated mice only required $6.4 \pm 2.9$ days $(\mathrm{N}=10, \mathrm{P}<0.01)$. The percentage of insulin ${ }^{+}$and $\mathrm{BrdU}^{+} \beta$ cells in islet grafts were significantly higher in GW treated mice than in vehicle treated mice. The mean percentage of insulin ${ }^{+}$and $\mathrm{BrdU}^{+} \beta$ cells in islet grafts was $23.6 \pm 6.4 \%$ in $\mathrm{GW}$ treated mice and $6.7 \pm 3.4 \%$ in vehicle treated mice $(P<0.01)$. At 30 minutes treatment, plasma GLP-1 was $9.3 \pm 2.6 \mathrm{pM} / \mathrm{L}$ in vehicle treated mice and $14.7 \pm 4.7 \mathrm{pM} / \mathrm{L}$ in $\mathrm{GW}$ treated mice $(\mathrm{P}<0.05)$. Our data demonstrated that $\mathrm{GW}$ can stimulate $\beta$-cell replication and improve islet graft function. Targeting GPR40 and/or GPR120 is a novel therapeutic approach to stimulate $\beta$-cell replication and to improve islet graft function. 
281-OR

Human Hair Follicle Cells Induce Localized Immune Privilege Against Allogeneic Islet Responses In Vitro

XIAOJIE WANG, JIANQIANG HAO, I-FANG LEE, ZILIANG AO, BRUCE VERCHERE, GARTH WARNOCK, JERRY SHAPIRO, KEVIN MCELWEE, Vancouver, BC, Canada

Type 1 diabetes is one of the most common chronic diseases. Islet transplantation offers a potential cure, but its widespread use is challenged by the increased tumor risk and opportunistic infections from long-term usage of immunosuppressive drugs. Therefore, reduced-risk, localized immunomodulatory therapy is urgently needed. Hair follicle derived mesenchymal dermal sheath cells (DSCs) exhibit natural immune privilege due to the secretion of immuno-regulatory factors and a lack of MHC class I and II expression. The objective of this study is to test whether non-histocompatible islets, in close association with DSCs, will escape immuno-surveillance. We first showed that cultured DSCs retained immune privilege properties. Compared to fibroblasts, an upregulation of immune privilege associated genes lactivin A, $\alpha M S H$, and TGF- $\beta 2$ showed a 3.0-, 12.6-, and 3.2-fold increase, respectively) and downregulation of MHC class I molecules (HLA-A, B, and $C$ showed a 4.6-, 2-, 4.8-fold decrease, respectively by quantitative PCR) were observed on DSCs. Furthermore, in allogeneic responses co-cultured with human peripheral blood mononuclear cells (PBMCs, as responders) and purified human islets (as stimulators), the secretion of IFNg from PBMCs was significantly reduced in the presence of DSCs $(23.0 \mathrm{vs} .5 .7 \mathrm{pg} /$ $\mathrm{ml}, \mathrm{p}<0.01$ ) or DSC-conditioned medium (18.5 vs. $2.15 \mathrm{pg} / \mathrm{ml}, \mathrm{p}<0.04)$. The function of T-effector- (CD8+IFNg+, $64.2 \%$ vs. $42.8 \%, p<0.01)$ and T-helper 1 -cells (Th1, CD4+IFNg+, $29.8 \%$ vs. $20.4 \%, \mathrm{p}<0.03$ ) was inhibited by $36 \%$ and $32 \%$, respectively, indicating a hyporesponsiveness to alloantigen stimulation. This study demonstrates that human islet/hair follicle dermal sheath cell constructs exhibit localized immunosuppressive properties, suggesting that transplanted islets may be benefited from the immune privilege status. Using naturally immune privileged cells may reduce the need for chronic systemic immunosuppression in clinical islet transplantation.

282-OR

Effect of Organ Transplantation on Cellular Senescence in Type 1 Diabetic Patients

ROBERTO LUPI, SILVIA DEL GUERRA, MORENA GABRIELE, PIERO MARCHETTI, STEFANO DEL PRATO, Pisa, Italy

Measurement of the length of telomeres could provide a marker for changes in cell senescence after transplantation procedures. We have determined telomere length (TL) and telomerase activity (TA) in diabetic patients before and after pancreas transplantation. We have recruited 19 non-diabetics (ND), 19 type 1 diabetes (T1DM) and 27 T1DM from the waiting list for transplantation (L-TX) and 15 transplanted T1DM (TX). TL, telomerase subunits HTERT and HTERC, and CCR2 gene expression was determined by Real-Time qPCR. In the study population as a whole, TL was inversely associated with age $(r=0.240, p=0.035), B M l(r=0.258, p=0.041)$, total cholesterol $(r=0.290$, $p=0.021)$, LDL $(r=0.281, p=0.025)$, and $C$-peptide $(r=0.315, p=0.007)$ (all $p<0.05$ or less), while the correlation was positive with $\mathrm{HDL}(\mathrm{r}=0.610, p<0.001)$, and $\mathrm{DD}(r=0.421, p=0.004)$ (both $p<0.05$ or less). By multiple regression analysis, $\mathrm{DD}$ and $\mathrm{HDL}$ remained independently associated with telomere length. TL was higher in $\mathrm{L}-\mathrm{TX}(0.217 \pm 0.028)$ than in ND $(0.153 \pm 0.015 ; \mathrm{p}<0.05)$. Moreover, HTERT expression was higher $(0.0354 \pm 0.0009$ and $0.164 \pm 0.029$, respectiveIy) in T1DM and L-TX than in ND $(0.010 \pm 0.002$; all $p<0.05$ or less). Upon normalization of $T L$ by $T A(T / T)$, a significant telomere shortening became apparent in T1DM and L-TX $(8.07 \pm 1.48$ and $12.57 \pm 0.49$, respectively) as compared

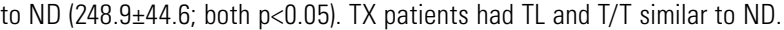
Finally, CCR2 gene expression was greater in L-TX $(+549 \pm 100 \%$; $p<0.001)$ as compared to ND, while there was no difference in TX. CCR2 expression correlated with $T / T$ values $(r=0.458, p=0,047)$ and HTERT component $(r=0.896$, $\mathrm{p}<0.001)$. Our data demonstrate an apparent effect of T1DM on markers of cellular senescence with TL being affected by duration of diabetes and $\mathrm{HDL}$ levels. TX may reduce inflammatory status and the telomere shortening process. Assessment of TL may improve risk stratification in T1DM and provide a marker of outcomes of pancreas transplantation.

Supported by: Cari Lucca Foundation

283-OR

Umbilical Cord-Derived Mesenchymal Stem Cell for Treatment of Newly-Onset Type 1 Diabetes Mellitus in NOD Mice

YANGANG WANG, WENLONG YU, WEI XU, Qingdao, China

The aim of this study was to examine the ability of umbilical cord-derived mesenchymal stem cells(UC-MSCs) to prevent and treat type 1 diabetes in the NOD mice model. Prevention of diabetes was evaluated after transplantation with culture expanded MSCs $\left(1 \times 10^{6}\right.$ per mouse $)$ in 9-weekold female NOD mice by lateral tail vein injection. Three months later, the incidence of type 1 diabetes was observed. And the frequency of reactive $\mathrm{CD} 4^{+}, \mathrm{CD} 8^{+} \mathrm{T}$ cells and $\mathrm{CD} 4^{+} \mathrm{CD} 25^{+}$regulatory $\mathrm{T}$ cells in the blood of male NOD recipients were also quantified by flow cytometry. In addition, Cytokine production like IL-2, IL-10 and TNF- $\alpha$ were assessed by ELISA.We also observe lymphocyte infiltration and $\alpha / \beta$ cell levels of islets. At the end of 12 weeks after transplantation, we found that the incedence of type 1 diabetes was $75 \%$ for the control group and $30 \%$ for the treatment group. The CD4+ cell levels and $\mathrm{CD}^{+} / \mathrm{CD}^{+}$ratio were more in the stem cell therapy group than in the disease group $(P<0.05)$, whereas less than in the normal group $(P<0.05)$. Accordingly the $\mathrm{CD}^{+} \mathrm{CD} 25^{+}$cell levels were higher in the stem cell therapy group than in the disease group $(P<0.01)$,whereas lower than in the normal group $(\mathrm{P}<0.01)$. IL-10 was up-regulated $(\mathrm{P}<0.01)$, and TNF- $\alpha$ was downregulated $(P<0.01)$ in the murine transplantation model. Importantly immunochemistry confirmed that a more complete structure of islet cell where lymphocyte infiltration was significantly reduced and $\alpha / \beta$ cells were consistently increased in the treatment group.This study suggests that MSCs reverse hyperglycemia and reduce the use of insulin which may thus offer a novel cell-based approach for the prevention of autoimmune diabetes and for islet cell transplantation.

284-0R

Upregulation of MafA Promotes Maturation of Mouse Embryonic Progenitor-Derived Insulin-Producing Cells and Improves their In Vivo Function

SHIYING SHAO, WATARU NISHIMURA, YONG MONG BEE, ARUN SHARMA, GUODONG LI, Singapore, Singapore, Boston, MA

Shortage of pancreas donors has impeded transplantation therapy for curing type 1 diabetes. We have generated a dozen of glucose-responsive insulin-producing cell lines (e.g. MEPI and EROSHK) by differentiating mouse embryonic stem or progenitor cells. Transplantation of these cells into diabetic animals could correct hyperglycemia, but they displayed features of immaturity such as induction of moderate hypoglycaemia and continuous proliferation in vivo. We found the expression of MafA, a master transcription factor implicated in controlling islet $\beta$-cell development and glucose responsiveness, was significantly lower in these cells than that in mouse islets. Thus we transfected mouse embryonic progenitor-derived insulinproducing (MEPI)-1 cells with vectors expressing MafA to upregulate its levels close to that in islets. MafA restoration significantly improved glucose responsiveness of MEPI-1 cells, e.g. metabolism, membrane potential depolarization, cytosolic $\mathrm{Ca}^{2+}$ rise and insulin secretion. MafA upregulation significantly slowed MEPI-1 cell growth by prolonging the doubling time by $20 \%$ and increasing the cells at G1 phase. Accordingly, the expression of p27, an inhibitor of CDKs controlling cell cycle, was enhanced by 1.5 fold. The in vivo function of MafA-restored MEPI-1 cells was assessed by peritoneal implantation in immune-competent diabetic mice following alginate encapsulation. The growth of encapsulated MafA-restored MEPI-1 cells reached a peak at 90 days after implantation, markedly slower than the encapsulated control cells at 30 days. Importantly, the period of normoglycemia after implantation of MafA-restored MEPI-1 cells was significantly longer than that by control cells $(105 \pm 5$ vs. $69 \pm 3$ days; $n=8 ; p<0.01)$ before relapse of hyperglycemia. These data indicate that MafA can promote embryonic stem cell-derived insulin-producing cells toward maturation and enhance their in vivo function.

Supported by: National Medical Research Council of Singapore

285-0R

Long-Term Function of Marginal Mass of Encapsulated Islet AIlografts in the Omentum of Immune-Competent Diabetic Rats RAJESH A. PARETA, JOHN P. MCQUILLING, JUAN ARENAS-HERRERA, SITTADJODY SIVANANDANE, NATHANIEL T. MARSHALL, ALAN C. FARNEY, EMMANUEL C. OPARA, Winston-Salem, NC

Immunoisolation is a strategy to overcome barriers to routine islet transplantation. However, the ideal site for engraftment of encapsulated islets has not been established. Microencapsulated islets have been transplanted into the general peritoneal cavity, but with variable success and an inability to recover islet grafts for analysis. Our study aim was to determine the viability of encapsulated islet allografts in an alternative site, the omentum pouch, made in immune-competent diabetic rats. Islets isolated from Wistar-Furth rats were encapsulated in microcapsules (300 - $400 \mu \mathrm{m}$ in diameter) made with $1.5 \mathrm{wt} \%$ ultrapurified high M alginate (LVM). Following perm-selective coating with 0.1 wt $\%$ Poly-L-Ornithine, they were finally coated with high 
G alginate (1.25 wt $\%$ LVG). The inner LVM core of the microcapsules was chelated with $55 \mathrm{mM}$ of sodium citrate for $2 \mathrm{~min}$. A marginal mass of the encapsulated islets $(2000$ islets $/ \mathrm{kg})$ was transplanted in an omentum pouch made in each of 5 STZ-diabetic Lewis rats whose blood glucose, plasma Cpeptide, and body weights were monitored for 90 days along with those of a control group $(n=5)$ which received empty capsules (no islets). The control group received daily insulin injections to keep blood glucose $<500 \mathrm{mg} / \mathrm{dL}$ during follow-up. Although normoglycemia was not achieved with the marginal mass, the islet recipients had a $12 \%$ reduction in their mean blood sugar levels compared to controls $(p<0.001)$, and increased their body weight from the diabetic baseline in contrast to the control group. Also, C-Peptide (Mercodia ELISA kit) increased from a non-detectable level to a range of 200-600 $\mathrm{pmol} / \mathrm{L}$ in the islet recipients, but not in the control group during the 3-month period. These data show for the first time that a marginal mass of encapsulated islet transplants have long-term function in an omentum pouch making it a possible alternative site for encapsulated islet transplantation in large animals and humans with abundant omental tissue.

Supported by: R01DK080897

286-0R Islet Allograft Rejection Recognizes a P2X7R-Mediated Mechanism

ANDREA VERGANI, CARMEN FOTINO, SARA TEZZA, RUTH MOLANO, MICHELE PODETTA, ANTONIO SOLETI, FABIO GRASSI, CAMILLO RICORDI, MOHAMED SAYEGH, ANTONELLO PILEGGI, PAOLO FIORINA, Boston, MA, Milan, Italy, Miami, FL, Torino, Italy, Bellinzona, Switzerland

ATP, released during cell damage/activation, is sensed by $P 2 X 7$ receptor (P2X7R) on lymphocytes and promotes T-cell activation. Novel P2X7R inhibitors are available (e.g. periodate-oxidized ATP [0ATP]), rendering P2X7R target a potential therapy. We demonstrated that P2X7R is induced in murine islet allograft-infiltrating lymphocytes. P2X7R targeting on murine CD4+ T-cells by oATP inhibited Th1 and Th17 differentiation and anti-CD3- antiCD28-Ig-induced IFN $\gamma$ production (number of spots, ELISPOT: oATP $=164 \pm 14$ vs. Control $=401 \pm 12, n=5 ; p=0.0002) ;$ OATP was not effective on P2X7R- $/$ cells, confirming drug specificity. WB analysis revealed inhibition of STAT3 phosphorylation; the use of Colivelin restored STAT3 phosphorylation, thus mitigating oATP effects. Short-term oATP treatment $(250 \mu \mathrm{g}$ day 0 to day 15 i.p.) in allogeneic islet transplantation (BALB/c into hyperglycemic $\mathrm{C} 57 \mathrm{BL} / 6$ mice), reduced the frequency of peripheral and intra-graft Th1/Th17 cells, reduced T-cell STAT3 phosphorylation, inhibited alloantigen-specific T-cell activation, thus prolonging graft survival (Mean Survival Time [MST]: OATP treated $=22$ days vs. Untreated $=14$ days, $n=10 ; p=0.0001$ ) with indefinite graft survival achieved in 3 mice out of 10 . Treated mice were immunocompetent (as assessed by Ovalbumin rechallenge). Graft survival prolongation was observed in P2X7R-/- recipient, although compensatory P2X1R/P2X4R expression limited the benefit of P2X7R genetic targeting. Tor-inhibitors increased P2X7R expression on murine and human T-cells and the use of Rapamycin synergized with oATP preventing murine CD4+ T-cells activation (IFN $\gamma$, number of spots: Rapamycin(-)/oATP(-) $=348 \pm 29$, Rapamycin(+)/oATP()$=111 \pm 16$; Rapamycin $(+) / 0 A T P(+)=49 \pm 2, n=3 ; p<0.01$ vs. all) and promoting in definite graft survival in 5 out of 7 recipients ( $<<0.01$ vs. Rapamycin). These data demonstrate the central role of purinergic system in priming Th1/Th17 response during islet rejection possibly through STAT3 phosphorylation.

\section{NOVEL AGENTS FOR DIABETES MANAGEMENT}

\author{
$\Delta$ \\ 287-0R \\ Targeting Insulin Resistance via the Immune Modulation of Cord \\ Blood-Derived Multipotent Stem Cells by the Stem Cell Educator \\ Therapy \\ YONG ZHAO, ZHAOSHUN JIANG, MINGLIANG YE, ZHAOHUI YIN, HENG LI, YA- \\ LIN DIAO, MARK HOLTERMAN, THEODORE MAZZONE, Chicago, IL, Jinan, China, \\ Peoria, IL \\ The prevalence of type 2 diabetes (T2D) is increasing worldwide, high- \\ lighting the need for a better understanding of the pathogenesis of the \\ disease and the development of innovative therapeutic approaches for the \\ prevention and cure of the condition. Mounting evidence points to the in- \\ volvement of immune dysfunction in insulin resistance in T2D, suggesting \\ that immune modulation may be a useful tool in treating the disease. We de- \\ veloped an innovative procedure for Stem Cell Educator therapy in which a \\ patient's blood is circulated through a closed-loop system that separate lym- \\ phocytes from the whole blood and briefly co-cultures them with adherent \\ human cord blood-derived multipotent stem cells (CB-SCs), and returns the
}

educated lymphocytes (but not the CB-SCs) to the patient's circulation. In an open-label, phase1/phase 2 study, patients $(n=25)$ with T2D received one treatment with the Stem Cell Educator. Median age was 50 years (range, 29 to 66), and median diabetic history was 9 years (range, 1 to 25). Notably, we found that T2D patients achieve improved metabolic control and reduced inflammation. Median glycated hemoglobin $\left(\mathrm{HbA}_{1} \mathrm{C}\right)$ was significantly reduced from $8.47 \% \pm 0.99$ at baseline to $7.87 \% \pm 1.07$ at 4 weeks post treatment $(p=$ $0.022)$, and to $7.1 \% \pm 0.6$ at 12 weeks post treatment $(p=1.6 \mathrm{E}-05)$. More than $80 \%$ of subjects achieved the $<7 \%$ standard recommended by the ADA. Homeostasis model assessment of insulin resistance (HOMA-IR) and HOMApancreatic islet beta-cell function (HOMA-B) demonstrate that insulin sensitivity have been improved post treatment. Mechanistic studies revealed this therapy can correct the immune dysfunction, as demonstrated by balancing the Th1/Th2/Th3 cytokine productions. Thus, Stem Cell Educator therapy is safe, and in individuals with moderate or severe $\mathrm{T} 2 \mathrm{D}$, a single treatment produces lasting improvement in metabolic control, without the safety and ethical concerns related to conventional stem cell-based therapies.

Supported by: JDRF, UIC CCTS Grant, Chinese Government Funding

FGF21-Adnectin-Pharmacokinetic Enhancer (FGF21-AdPKE): A Novel Protein Candidate With Uniquely Extended Pharmacokinetic Profile for the Treatment of Diabetes and Dyslipidemia

RANJAN MUKHERJEE, PAUL MORIN, JOSEPH TAYLOR, DENNIS FARRELLY, DEBORAH HAGAN, BIN HE, DANIEL COHEN, JOHN KRUPINSKI, MARK KIRBY, MICHAEL GOSSELIN, HONG SHEN, CHRISTINE HUANG, BRADLEY ZINKER, Pennington, $N J$

FGF21 treatment has been shown to improve the diabetic, insulin resistant and dyslipidemic state in several animal models of diabetes and obesity without inducing hypoglycemia, mitogenesis or edema. Its short half life (30 minutes in mice) would require multiple, daily injections to be used as a therapeutic agent. We hypothesized that extending the half life of FGF21 would lead to improved efficacy and require less frequent dosing. Adnectins are a new family of proteins based on the 10th type III domain of human fibronectin that can be designed to bind to targets of interest with high affinity and specificity. We have created a novel protein by fusing human FGF21 to an adnectin pharmacokinetic enhancer (FGF21-adPKE) which binds to human and monkey but not mouse serum albumin. This novel FGF21-adPKE retains the property of inducing ERK phosphorylation in $\beta$-klotho-expressing cells and glucose uptake in adipocytes. In ob/ob mice injected q.d., s.c., for 7 days with FGF21-adPKE $(1 \mathrm{mg} / \mathrm{kg})$ or native FGF21 $(0.3 \mathrm{mg} / \mathrm{kg})$, non-fasting glucose was lowered by $29 \%$ and $34 \%$ respectively. When the FGF21-adPKE was coinjected with human albumin, glucose lowering was $41 \%$ and the increased efficacy was associated with prolonged exposure. After 14 days of q.d. codosing of FGF21-adPKE with human albumin, vehicle subtracted HbA1c was lowered by $0.94 \%$. In cynomolgus monkeys where FGF21-adPKE bound to endogenous monkey serum albumin, the half life was 97 hours, significantly greater than native human FGF21 (4 hours). FGF21-adPKE is produced in E.coli, has low viscosity and displays preclinical pharmacokinetic properties that would support convenient dosing regimens (including once weekly) in humans. In conclusion, FGF21-adPKE represents a promising candidate for the treatment of diabetes and attendant dyslipidemic disorders.

289-0R

SRT3025, a Novel SIRT1 Activator, Reverses Metabolic Dysfunction Induced by a High Fat Diet through Transcriptional and Post Transcriptional Modulation of Multiple Metabolic Pathways

VIPIN SURI, YONG OI, MEGHAN L. DAVIS, OING NIE, MARC O. JOHNSON, ANGELA M. COTE, ELDEN LAINEZ, GEORGE P. VLASUK, JAMES L. ELLIS, Cambridge, $M A$

Activation of the protein deacetylase SIRT1 through transgenic overexpression in mice confers resistance to body weight gain, insulin resistance, dyslipidemia and hepatic steatosis induced by a high fat diet. We report here the discovery of SRT3025, a novel small molecule activator of SIRT1, which reverses several aspects of metabolic dysfunction induced by a high fat diet. SRT3025 enhanced the deacetylase activity of SIRT1 by 4-fold, with half maximal activation at $200 \mathrm{nM}$. Diet Induced Obese (DIO) mice treated with $100 \mathrm{mg} / \mathrm{kg}$ SRT3025 for 7 weeks weighed $13 \%$ less than vehicle treated mice and showed significant reductions in fed glucose $(-17 \%)$ and insulin $(-77 \%)$, fasted glucose $(-23 \%)$ and insulin $(-62 \%)$, and serum $(-41 \%)$ and hepatic lipids $(-37 \%)$. Indirect calorimetry indicated that SRT3025 reduced body weight by increasing energy expenditure through stimulation of both carbohydrate and lipid oxidation. Euglycemic-Hyperinsulinemic clamp studies showed that SRT3025 increased hepatic insulin sensitivity as well as insulin-stimulated 
glucose uptake in skeletal muscle and adipose tissue. Transcriptional profiling of tissues from SRT3025 treated mice revealed striking suppression of hepatic gluconeogenic and lipogenic pathways as well as alterations in mitochondrial pathways in muscle and kidney. Metabolomic profiling confirmed the changes in activity of gluconeogenic and lipogenic pathways, and also revealed efficient fatty acid oxidation in SRT3025 treated DIO mice. Our data provides mechanistic insight into how pharmacological activation of SIRT1 impacts metabolic regulation in the setting of DIO. Small molecule activators of SIRT1 may have unique therapeutic potential in metabolic dysfunction through their ability to positively impact body weight, insulin sensitivity and lipid metabolism.

\section{0-0R}

Anti-Diabetic Potential of Novel, Small Molecule GPR119 Agonists DHANAPALAN NAGARATHNAM, KANTHIKIRAN VARANASI, KASIVISWANATH ROUTHU, MEYYAPPAN MUTHUPPALANIAPPAN, GAYATRISWAROOP MERIKAPUDI, SRIDHAR VEERARAGHAVAN, PRASANNA R, SRIKANT VISWANADHA, SWAROOP VAKKALANKA, Hamden, CT, Hyderabad, India, La Chaux de Fonds, Switzerland

Insulin release via GLP1 and cAMP induction by GPR119 agonists represents a novel strategy for achieving optimal glycemic control. Herein, we describe the biological and pharmacokinetic properties of RP9056, a novel and small molecule GPR119 agonist with scope to be further developed as a clinical candidate for Type-2 diabetes. Induction of cAMP in hGPR119 transfected HEK-293 cells was determined by a Homogenous Time Resolved Fluorescence assay (Cisbio, MA). Potency of the compound was further corroborated in a cAMP assay using the high GPR119 expressing HIT-T15 insulinoma cells. Ability of the compound to induce insulin secretion in HITT15 cells was determined. Pre-clinical efficacy of the molecule $(10 \mathrm{mg} / \mathrm{kg})$ was corroborated in an oral glucose tolerance test (OGTT) in C57BL/6J mice. Metabolic stability of the compound was evaluated in microsomes obtained from mouse, rat, dog, monkey, and human. Pharmacokinetic behaviour of compounds in plasma after single dose oral administration was determined in female Balb/c mice and Wistar rat. RP9056 induced cAMP concentrations in hGPR119 transfected HEK-293 as well as HIT-T15 cells with $\mathrm{EC}_{50}$ of 12.2 and $23.5 \mathrm{nM}$ respectively. Half-maximal induction of insulin release from HIT-T15 cells was achieved at a concentration of $34.5 \mathrm{nM}$. Treatment of mice with $10 \mathrm{mg} / \mathrm{kg}$ RP9056 resulted in an overall improvement of glucose tolerance by $50 \%$ when compared to the control group. Pharmacokinetic studies indicated good oral bioavailability of RP9056 with a favourable half-life in mouse and rat. Further, RP9056 was metabolically stable across the species studied besides showing no significant CYP inhibition in human liver microsomes. Results demonstrate the potential of RP9056 as an effective treatment for symptoms associated with diabetes. Additional in vivo studies in models relevant to diabetes and insulin resistance are currently in progress.

291-OR

The GPR119 Agonist JNJ-38431055/APD597 Improves Blood Glucose Control in Rats and Cynomologous Monkeys

FUYONG DU, CHRIS CARROLL, ZHI-LIANG CHU, JUERG LEHMANN, ROBERT M. JONES, JUNE XU, JAMES LEONARD, TROY SARICH, PATRICIA ANDRADE-GORDON, KEITH DEMAREST, YIN LIANG, Spring House, PA, San Diego, CA

GPR119 acts directly on pancreatic $\beta$-cells and enteroendocrine $L$ - and $\mathrm{K}$-cells to increase insulin and incretin release, respectively. GPR119 agonists are therefore potentially novel therapeutics for the treatment of type 2 diabetes. JNJ-38431055 (also termed APD597) is a potent GPR119 agonist with superior pharmaceutical properties. The present study characterized the efficacy of JNJ-38431055 in normal and diabetic animal models. JNJ38431055 induced cAMP production in CHO-hGPR119 and CH0-rGPR119 cells with an $\mathrm{EC}_{50}$ of $24 \mathrm{nM}$ and $380 \mathrm{nM}$, respectively. In isolated human pancreatic islets, this compound stimulated insulin release in a glucose-dependent manner; control and JNJ-38431055-treated islets secreted 8.6 and $16.9 \mathrm{uU} / \mathrm{hr} / 5$ islets, respectively, when incubated in culture media supplemented with $8 \mathrm{mM}$ glucose. JNJ-38431055 also enhanced GLP-1 release from GLUTag cells and significantly increased plasma GLP-1 levels in C57/B6 mice. In ob/ob mice and ZDF rats, JNJ-38431055 significantly improved oral glucose tolerance. Four-week treatment of ZDF rats with JNJ-38431055 reduced fed blood glucose levels and HbA1c at all doses tested, but decreased body weight gain only at higher doses. In cynomolgus monkeys, JNJ38431055 markedly reduced blood glucose AUC by $17 \%(P<0.05)$, and increased plasma GLP-1 AUC by $212 \%(P<0.01)$. The Insulin ( $\left.\mathrm{UU} / \mathrm{mL}^{*} 100\right) / \mathrm{Blood}$ Glucose (mg/dL) Index at 30 min after glucose challenge was increased from $28.0 \pm 8.8$ in the vehicle group to $44.3 \pm 11.3$ in the compound-treated group. In summary, JNJ-38431055 is a dual secretagogue for both insulin and GLP-1 release in rodents and in non-human primates. GPR119 agonists therefore show promise as novel pharmaceutical anti-hyperglycemic agents to treat patients with type 2 diabetes.

292-0R

Recent Data from DIA-AID 1, a Global Phase III Clinical Study in Newly Diagnosed Type 1 Diabetes Patients

SHLOMO DAGAN, ANETTE G. ZIEGLER, PAOLO POZZILLI, THOMAS LINN, GUNTRAM SCHERNTHANER, FRANÇOIS BONNICI, DANA ELIAS, RACHEL EREN, IRUN R. COHEN, ITAMAR RAZ, 901 STUDY GROUP, Yavne, Israel, München, Germany, Rome, Italy, Giessen, Germany, Vienna, Austria, Cape Town, South Africa, Rehovot, Israel, Jerusalem, Israel

DIA-AID 1 is a randomized, double-blind, placebo-controlled phase III study to evaluate safety and efficacy of DiaPep277 ${ }^{\circledR}$ in newly diagnosed type 1 diabetes (T1D) patients. Major inclusion criteria: age 16-45, not more than 3 months after diagnosis of T1D, fasting C-peptide $>0.2 \mathrm{nmol} / \mathrm{L}$, and positive islet autoantibodies. Subjects received $1 \mathrm{mg}$ DiaPep $277^{\circledR}$ or placebo at 0, 1, 3 , $6,9,12,15,18,21$ months. Efficacy primary endpoint was defined as change from baseline to end of study in stimulated C-peptide area under curve (AUC) secretion measured by the glucagon stimulated test. Key secondary endpoints include change from baseline to end of study in Mixed-Meal-stimulated Cpeptide AUC secretion; proportion of subjects who maintain HbA1c treat-totarget of $\leq 7 \%$ at end of study and change from baseline to endpoint in basal fasting C-peptide. Four hundred and fifty seven patients were randomized. The target population for efficacy included subjects who entered the study according to the major inclusion / exclusion criteria and received at least 1 dose of study medication (MITT population, $n=422$ ). Results from patients treated with DiaPep $2777^{\circledR}$ show significant preservation of C-peptide levels compared to placebo $(23.4 \%$ relative change, $p=0.037)$. This preservation is even more significant in patients who completed two years of therapy in full compliance with the study protocol $(29 \%$ relative change, $p=0.011)$. Furthermore, the percentage of patients who maintained HbA1c levels $\leq 7 \%$ at study end was significantly higher in the DiaPep $277^{\circledR}$ treated group as compared to placebo $(56 \%$ vs $44 \%, p=0.035)$. These initial results indicate that the study has met its primary endpoint. Additional data are currently being evaluated. DiaPep $277^{\circledR}$ was well tolerated. No significant differences in drug-related adverse events were reported between the treatment and placebo groups. A second confirmatory phase III clinical study, DIA-AID 2 is being conducted worldwide in newly diagnosed adult T1D patients.

293-OR

GKM-001, a Liver-Directed/Pancreas-Sparing Glucokinase Modulator (GKM), Lowers Fasting and Post-Prandial Glucose Without Hypoglycemia in Type 2 Diabetic (T2D) Patients

VIKRAM RAMANATHAN, NIMISH VACHHARAJANI, RAKESH PATEL, RASHMI BARBHAIYA, Bangalore, India, Ahmedabad, India

Glucokinase (GK) is involved in glucose homeostasis through control of insulin secretion in pancreas and glucose disposal in liver. Although GKAs have shown antihyperglycemic effects, hypoglycemia (HG) due to their dual action of insulin release and hepatic glucose disposal remains a concern. GKM-001 is designed to selectively target liver GK and spare pancreatic GK, and thereby eliminate the risk of HG. GKM-001 has shown robust glucose lowering both acutely and chronically in animal models of diabetes. Safety, pharmacodynamics, and pharmacokinetics (PK) of GKM-001 were investigated in single (SAD) and multiple ascending dose (MAD) studies in healthy and T2D subjects. In SAD study, GKM-001 50-2000 mg OD doses in healthy $(n=30)$ and $50-600 \mathrm{mg}$ OD doses in T2D ( $n=16)$ were investigated. GKM-001 was safe (no HG), well tolerated, and exhibited dose-proportional PK with $\sim 20 \mathrm{~h}$ half-life. About $30 \%$ dose was excreted unchanged in urine. In MAD study, GKM-001 25-1000 mg was administered $\mathrm{QD}$ for 14 days to T2D ( $\mathrm{n}=60)$. Mean HbA1c, FPG and BMI at screening were $9.0 \%, 176 \mathrm{mg} / \mathrm{dL}$ and $26 \mathrm{~kg} / \mathrm{m}^{2}$, respectively. Plasma glucose, insulin, and C-peptide were measured after OGTT and mixed meal tolerance tests (MMTT) at baseline, Day 1 and Day 14. Fasting plasma glucose (PG) was determined on multiple days and PK on Days 1 - 14. No HG or clinically relevant changes in laboratory tests (including lipids) and vital signs were observed. GKM-001 provided dose dependent reduction in fasting $\mathrm{PG}$, glucose excursions to MMTT and $24 \mathrm{~h}$ glucose. The reduction in mean PG ranged from 23 to $46 \mathrm{mg} / \mathrm{dL}$. Even at the highest 1000 $\mathrm{mg}$ dose, there was no meaningful increase in insulin level. Reduction in glucose across doses was not attributable to increased insulin. Overall, GKM001 represents a novel mechanism to enhance glucose disposal specifically via liver with potential beneficial pancreas $\beta$-cell sparing action. A Phase Ilb study of GKM-001 will soon be initiated. 


\section{DIABETES COMPLICATIONS AND MORTALITY-RISK FACTORS, TRENDS, AND SEX/RACE DISPARITIES}

294-0R

Fruit Intake and Incident Retinopathy in Japanese Patients With Type 2 Diabetes-Nutritional Analysis in the Japan Diabetes Complications Study (JDCS)

SHIRO TANAKA, YUKIO YOSHIMURA, RYO KAWASAKI, CHIEMI KAMADA, SACHIKO TANAKA, CHIKA HORIKAWA, YASUO OHASHI, ATSUSHI ARAKI, HIDEKI ITO, YASUO AKANUMA, NOBUHIRO YAMADA, HIDETOSHI YAMASHITA, HIROHITO SONE, THE JAPAN DIABETES COMPLICATIONS STUDY GROUP, Kyoto, Japan, Tokushima, Japan, Yamagata, Japan, Ibaraki, Japan, Tokyo, Japan

Antioxidants and dietary fiber are postulated to have preventive effects on diabetic retinopathy (DR), but evidence for encouraging fruit intake to prevent DR is lacking. We aimed to investigate this association in a cohort with type 2 diabetes. After excluding non-responders to a dietary survey or patients who had DR or a major ocular disease at baseline, 978 patients were included for analysis. Baseline dietary intake was assessed by the Food Frequency Questionnaire based on food groups and 24-hour dietary records. Primary outcome was time to incident DR following international DR severity scales. Hazard ratios (HRs) for dietary intake were estimated by quartile Cox regression adjusted for $\mathrm{HbA}_{1}, \mathrm{BMI}$, energy intake and other confounders. Mean fruit intake in quartiles ranged from 22.3 to $253.0 \mathrm{~g}$ per day, with similar increasing trends for vitamin $\mathrm{C}$, vitamin $\mathrm{E}$, carotene, retino equivalent, dietary fiber, potassium, and sodium. $\mathrm{HbA}_{1 \mathrm{C}}, \mathrm{BMI}, \mathrm{TG}$ and SBP were well controlled. During the median follow-up of 8.0 years, incident DR occurred most frequently in the $4^{\text {th }}$ quartile (Figure 1). The HRs for $2^{\text {nd }}, 3^{\text {rd }}$, and $4^{\text {th }}$ quartiles compared to the $1^{\text {st }}$ quartile were $0.66195 \%$ confidence interval, 0.48 to 0.93$), 0.60(0.42$ to 0.84$)$, and $0.50(0.34$ to 0.74$)$, respectively (trend $p<0.01$ ). Significant decreasing trends were noted for vitamin $C$ and carotene. There was no significant effect modification in subgroup analysis. In conclusion, increase in fruit intake in a range commonly consumed is associated with reduced incident DR within a low-fat energy-restricted diet.

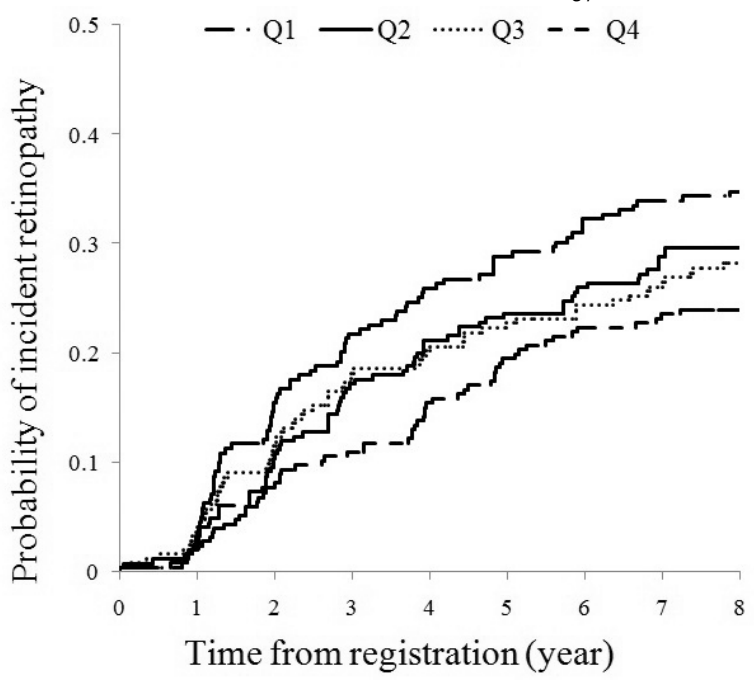

Supported by: The Ministry of Health, Labor and Welfare, Japan

295-0R

Risk of Mortality in LADA May Be Higher than in Type 2 Diabetes LISA OLSSON, ANDERS AHLBOM, TOMAS ANDERSSON, VALDEMAR GRILL, KRISTIAN MIDTHJELL, SOFIA CARLSSON, Stockholm, Sweden, Trondheim, Norway

Knowledge on mortality in latent autoimmune diabetes in adults (LADA) is limited. We analyzed the risk of all-cause and cardiovascular mortality in LADA and compared the risk to that of subjects without diabetes and with type 2 diabetes. The study cohort $(n=46,304)$ emerges from the Norwegian HUNT Study and includes 2,699 cases of diabetes. Subjects diagnosed at $\geq 35$ years were classified with LADA if they were anti-GAD positive $(n=150)$ and type 2 diabetes if anti-GAD negative $(n=2,073)$. Information on mortality from all-cause, cardiovascular disease and ischemic heart disease during follow-up 1995-2008 was obtained by linkage to the cause of death registry. Hazard ratios (HR) with $95 \% \mathrm{Cl}$ were calculated using Cox proportional hazards models. All-cause mortality was increased in LADA compared to subjects without diabetes (HR 1.63, 95\% Cl 1.30-2.04) as was cardiovascular mortality $(1.92,1.44-2.56)$ (Table 1). The excess risk was seen in both men and women, was not explained by BMI or lifestyle factors and was primarily seen in cases with $\mathrm{HbA} 1 \mathrm{C} \geq 6.5 \%$. Compared to type 2 diabetes, LADA was associated with higher all-cause mortality $(1.21,0.95-1.54)$. Adjustment for $\mathrm{HbA1C}$ attenuated this excess mortality $(1.15,0.90-1.47)$ (relative excess risk $29 \%$ ). Adjustment for titer of anti-GAD did not significantly affect risk estimates. All-cause and cardiovascular mortality is increased in LADA compared not only to subjects without diabetes but also to subjects with type 2 diabetes. Increased risk is associated with worse glucose control but not with degree of autoimmune activity. To diagnose LADA will be clinically important for assessing correct prognosis and therapy.

Table 1. Risk of mortality in LADA and type 2 diabetes compared to subjects without diabetes

\begin{tabular}{ccccccccc}
\hline & \multicolumn{2}{c}{ Men $^{1}$} & \multicolumn{3}{c}{ Women $^{1}$} & \multicolumn{3}{c}{ All subjects $^{2}$} \\
\hline No. & HR & $95 \%$ & No. & HR & $95 \%$ & No. & HR & $95 \%$ \\
deaths & & Cl & deaths & & Cl & deaths & Cl \\
\hline
\end{tabular}

\begin{tabular}{|c|c|c|c|c|c|c|c|}
\hline \multicolumn{8}{|c|}{ All-cause mortality } \\
\hline \multicolumn{8}{|c|}{$\begin{array}{lll}\text { All-cause mortainity } & & \\
\text { No diabetes } & 4676 & 1\end{array}$} \\
\hline$\overline{L A D A}$ & 42 & $1.501 .10-2.03$ & 34 & $1.85 \quad 1.32-2.59$ & 76 & 1.63 & $1.30-2.04$ \\
\hline Type 2 diabetes & 371 & $1.27 \quad 1.14-1.41$ & 401 & $1.48 \quad 1.34-1.64$ & 772 & 1.38 & $1.28-1.48$ \\
\hline
\end{tabular}

\section{Cardiovascular mortality}

\begin{tabular}{lrlrrllrll}
\hline No diabetes & 2318 & 1 & 1949 & 1 & 4267 & 1 & \\
\hline LADA & 27 & 1.83 & $1.25-2.69$ & 21 & 2.24 & $1.45-3.44$ & 48 & 1.92 & $1.44-2.56$ \\
\hline Type 2 diabetes & 225 & 1.48 & $1.29-1.70$ & 253 & 1.80 & $1.56-2.03$ & 478 & 1.63 & $1.49-1.80$
\end{tabular}

Mortality from ischemic heart disease

\begin{tabular}{lrlrrrrrrr}
\hline No diabetes & 1163 & 1 & & 695 & 1 & & 1858 & 1 & \\
\hline LADA & 12 & 2.72 & $1.54-4.81$ & 10 & 4.26 & $2.28-7.96$ & 22 & 3.29 & $2.16-5.02$ \\
\hline Type 2 diabetes & 134 & 1.79 & $1.49-2.14$ & 116 & 2.61 & $2.14-3.18$ & 250 & 2.10 & $1.84-2.40$
\end{tabular}

${ }^{1}$ Adjusted for age. ${ }^{2}$ Adjusted for age and sex.

296-OR

Micro-Vascular Disease Among Chinese With Newly Diagnosed Diabetes: A 23-Year Follow-Up of the Da Oing Diabetes Study OIUHONG GONG, PING ZHANG, JINPING WANG, YALI AN, EDWARD GREGG, GOJKA ROGLIC, WENYING YANG, BO ZHANG, YANYAN CHEN, YINGHUA HU, PETER BENNETT, GUANGWEI LI, Beijing, China, Atlanta, GA, Daqing, China, Geneva, Switzerland, Phoenix, AZ

Among the estimated 100 million persons with diabetes in China, little is known about the occurrence of micro-vascular complications and their natural history. We sought to document the incidence of micro-vascular disease (retinopathy, nephropathy and neuropathy) in the 630 persons with newly diagnosed diabetes (NDMs), and in a sample of 519 with normal glucose tolerance (NGT), who had been identified initially in 1986 by screening $50 \%$ of the Da Qing population aged 25 years and over. In 2009 among those with NDM, 593 (94\%), and 491 (95\%) with NGT, were traced. Clinically significant micro-vascular disease was ascertained by direct or proxy interviews and review of medical records up to the time of death, loss to follow-up or December 31st, 2009, and among the survivors 252/260 (97\%) of the NDMs and $374 / 392$ (95\%) of the NGTs were examined. Among the NDMs over the 23year follow-up period, severe retinopathy, defined as proliferative retinopathy, having received retinal photocoagulation, or blindness, was identified in $24.9 \%(95 \% \mathrm{Cl} 21.4-28.4)$, and severe nephropathy, defined as receiving renal replacement therapy or with urinary albumin/creatinine ratio(ACR) $\geq 300$ $\mathrm{mg} / \mathrm{g}$ at the follow-up examination, had developed in $24.8 \%$ (95\% $\mathrm{Cl} 19.3$ $30.3)$. Both the severe retinopathy and nephropathy developed mainly after 10 years or more duration of diabetes. Among the NDM survivors, $22.7 \%$ had ACR $\geq 300 \mathrm{mg} / \mathrm{g}$, and $27.3 \%$ (95\% Cl 21.4-33.2) had evidence of neuropathy (10g monofilament test) at the follow-up examination. In the NGT group, only $3.9 \%(95 \% \mathrm{Cl} 2.2-5.6)$ had severe retinopathy, 3.1\% (95\% Cl 1.2-4.0) severe nephropathy, and $11.2 \%$ (95\% Cl 7.7-14.7) had neuropathy at the 2009 followup examination. Severe diabetic retinopathy and nephropathy are common long-term complications of type 2 diabetes in China, which, given the huge numbers of persons with recent onset diabetes, will lead to major medical and economic challenges to the country in the future.

Supported by: CDC, WHO, China-Japan Friendship Hospital 
297-OR

Declining Rates of Hospitalization for Acute Myocardial Infarction and Stroke Among Adults Aged $\geq \mathbf{4 5}$ Years With Diagnosed Diabetes, United States, 1988-2009

NILKA R. BURROWS, YANFENG LI, EDWARD W. GREGG, LINDA S. GEISS, Atlanta, GA

Diabetes is a major cause of heart disease and stroke. We examined trends in rates of hospitalization for diabetes-related acute myocardial infarction (AMI) and stroke among U.S. adults. Using data from the National Hospital Discharge Survey, we estimated the number of discharges having AMI (ICD-9 410) or stroke (ICD-9 430-434, 436-438) as first-listed diagnosis among those having diabetes (ICD-9 250) as a secondary diagnosis. Hospitalization rates for adults aged $\geq 45$ years by sex and by race (whites, blacks) were calculated using estimates of the population with diagnosed diabetes and age-adjusted to the 2000 U.S. standard population. Joinpoint regression was used to analyze trends and calculate an average annual percentage change (AAPC). The age-adjusted rates for AMI and for stroke changed little from 1988 to 1996 and then declined significantly from 1996 to 2009 (Figure). From 1988 to 2009, the age-adjusted hospitalization rate per 1000 diabetic population declined from 24.7 to 7.7 (AAPC: $-5.3 \%$ per year) for AMI, and from 18.9 to 9.2 (AAPC: $-3.9 \%$ per year) for stroke. Similarly, the age-specific rates and the age-adjusted rates by sex and by race for AMI and for stroke showed significant declines throughout the period. Hospitalization rates for $\mathrm{AMI}$ and stroke in the diabetic population have declined. This decline may be due to a number of factors, including a reduction in prevalence of risk factors, new and or more aggressive treatment of cardiovascular risk factors, or other factors.

Age-adjusted Hospitalization Rate for AMI and Stroke Among Adults Aged $\geq 45$ Years With Diagnosed Diabetes, United States, 1988-2009

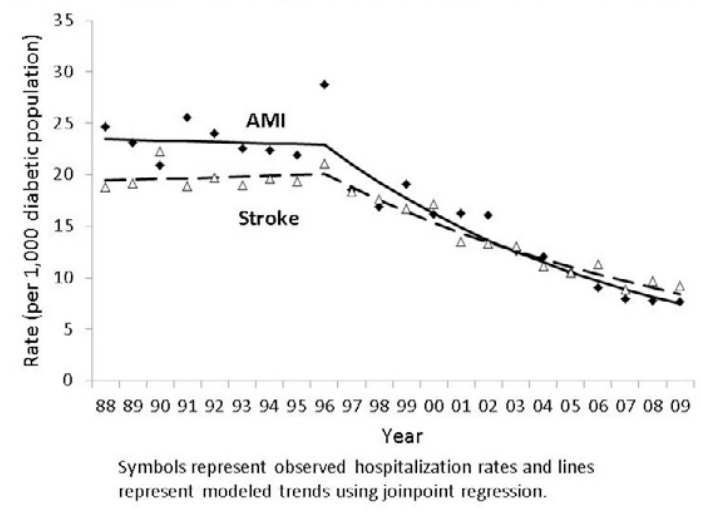

298-OR

Association between Glycated Haemoglobin and the Risk of Congestive Heart Failure in Diabetes Mellitus-Systematic Review and Meta-Analysis

SEBHAT EROOU, CHEE-TIN CHRISTINE LEE, MATHEW SUFFOLETTO, JUSTIN B. ECHOUFFO-TCHEUGUI, RUDOLF A. DE BOER, JOOST P. VAN MELLE, AMANDA I. ADLER, Pittsburgh, PA, Toronto, ON, Canada, Atlanta, GA, Groningen, The Netherlands, Cambridge, United Kingdom

Background: Although emerging evidence suggests that the degree of glycemia is associated with the risk of congestive heart failure (CHF), the magnitude of the risk has not been adequately quantified, and clinical trials to date have not provided definitive evidence regarding the effects of glucose lowering on CHF risk. We synthesized the available prospective epidemiological data on the association between glycemia measured by hemoglobin $\mathrm{A} 1 \mathrm{c}(\mathrm{HbA1c})$ and the risk of $\mathrm{CHF}$ in individuals with diabetes. Methods: We searched electronic databases and reference lists of relevant articles for prospective epidemiological studies that measured $\mathrm{HbA1c}$ level at baseline and assessed incident CHF as an outcome. We abstracted data from relevant studies using standardized forms and obtained additional data from investigators when required. Study-specific relative risk estimates were pooled using random-effects model meta-analysis. Results: Of the $1044 \mathrm{ci}-$ tations identified, we included 10 studies comprising 178,929 participants with diabetes and 14,176 incident CHF cases. The overall adjusted risk ratio (RR) for CHF was $1.15(95 \% \mathrm{Cl}, 1.10-1.21)$ for each percentage point increase in HbA1c. There was substantial heterogeneity across the ten studies (I2:
$83 \%$; $95 \% \mathrm{Cl}, 69-91 \% ; \mathrm{p}<0.001)$. After exclusion of one study involving participants with type 1 diabetes and another study reporting an inverse association between $\mathrm{HbA1C}$ and $\mathrm{CHF}$, the heterogeneity was no longer significant (I2: $49 \%, 0-79 \% ; p=0.08$ ). Among these remaining eight studies involving 149,484 predominantly type 2 diabetic participants and 12,891 incident CHF cases, the pooled adjusted RR for CHF was 1.15 (1.11-1.19). The corresponding RR for the single study comprising participants with type 1 diabetes was 1.30 (1.21-1.40). Conclusions: In observational studies of individuals with diabetes, a higher $\mathrm{HbA1c}$ level was associated with a significantly increased risk of CHF

299-OR

\section{WITHDRAWN}

Effects of Sex and Race on Risk of Congestive Heart Failure, Coronary Heart Disease, and Mortality in Older Adults With Diabetes VARSHA G. VIMALANANDA, MARY LOU BIGGS, JAMES L. ROSENZWEIG, MERCEDES R. CARNETHON, JAMES B. MEIGS, EVAN L. THACKER, DAVID S. SISCOVICK, KENNETH J. MUKAMAL, Boston, MA, Seattle, WA, Chicago, IL

The relative and absolute risks of coronary heart disease (CHD) incidence and mortality associated with diabetes are greater in women as compared with men. We evaluated whether these sex differences extend to older adults, to risk of congestive heart failure (CHF), and are present in black and white individuals. We followed participants in the Cardiovascular Health Study, a cohort study of community-dwelling adults aged $\geq 65$ from 4 US communities. We used Cox proportional-hazards models to assess the association of diabetes with incident CHF, CHD, and total mortality among 2923 women (16\% black) and 1894 men (15\% black). We tested whether any interaction by sex was present within black and white race groups, adjusting for age and clinic site. Over a median follow-up of 12.5 years, diabetes was similarly associated with CHF among white women (adjusted hazard ratio [aHR] 2.10:95\% confidence interval [CI] 1.68-2.63) and white men (aHR 2.07:95\% Cl 1.67-2.56, $p$ for interaction $=0.91$ ). Findings were similar for $\mathrm{CHD}$ and mortality. Among blacks, diabetes was associated with CHF among women (aHR $2.42 ; 95 \% \mathrm{Cl} 1.70-3.43$ ) but not among men (aHR 1.39; 95\% Cl 0.83-2.34, $p$ interaction $=0.08$ ). This also held for CHD ( $p$ interaction 0.17 ). In competing risk analyses of a composite endpoint (CHF + CHD), the sex interaction was unchanged. We conclude that older black women have markedly higher relative risks of $\mathrm{CHF}$ and $\mathrm{CHD}$ associated with diabetes than do older black men, but that this sex disparity is absent in older white adults. 
Association of diabetes with CHF, CHD and mortality by sex and race

\begin{tabular}{lcccc}
\hline & \multicolumn{2}{c}{ White women } & \multicolumn{2}{c}{ White men } \\
\hline & $\begin{array}{c}\text { No diabetes } \\
(\mathrm{n}=2196)\end{array}$ & $\begin{array}{c}\text { Diabetes } \\
(\mathrm{n}=251)\end{array}$ & $\begin{array}{c}\text { No diabetes } \\
(\mathrm{n}=1368)\end{array}$ & $\begin{array}{c}\text { Diabetes } \\
(\mathrm{n}=247)\end{array}$ \\
\hline $\mathrm{CHF}^{*}$ & 18.6 & 36.0 & 26.0 & 49.7 \\
\hline $\mathrm{CHD}^{*}$ & 15.3 & 30.1 & 28.1 & 47.9 \\
\hline Total mortality & 42.8 & 69.6 & 58.2 & 88.9 \\
\hline \multicolumn{4}{c}{ Black women } & \multicolumn{2}{c}{ Black men } \\
\hline $\mathrm{CHF}^{*}$ & $\begin{array}{c}\text { No diabetes } \\
(\mathrm{n}=365)\end{array}$ & $\begin{array}{c}\text { Diabetes } \\
(\mathrm{n}=111)\end{array}$ & $\begin{array}{c}\text { No diabetes } \\
(\mathrm{n}=207)\end{array}$ & $\begin{array}{c}\text { Diabetes } \\
(\mathrm{n}=72)\end{array}$ \\
\hline $\mathrm{CHD}^{*}$ & 20.8 & 46.3 & 24.2 & 31.9 \\
\hline Total mortality $^{*}$ & 14.7 & 31.9 & 25.8 & 38.5 \\
\hline${ }^{*}$ & 42.7 & 64.6 & 63.5 & 85.8 \\
\hline
\end{tabular}

*rate per 1000 person-years

Supported by: RO1HLO94555

301-0R

All-Cause and Cause-Specific Mortality by Race in Low Income Blacks and Whites With Type 2 Diabetes

BAOIYYAH N. CONWAY, MICHAEL E. MAY, LISA B. SIGNORELLO, WILLIAM J.

BLOT, Nashville, TN

Our objective was to estimate overall and cause-specific mortality by race in a low SES population of Blacks and Whites with type 2 diabetes. We conducted a mortality follow up of a cohort of low income Blacks and White participants aged 40-79 with type 2 diabetes (defined as diagnosed with diabetes at/after age 30; $n=12,498)$ and without diabetes $(n=49,914)$ at cohort entry. Multivariable Cox models were used to compute $\mathrm{HRs}$ and $95 \% \mathrm{Cl}$ for cause-specific mortality based on both underlying and contributing causes of death from death certificates. During follow-up (mean 5.9 years), $13.5 \%$ of those with and $7.3 \%$ of those without diabetes died. Blacks and Whites with diabetes were at a similarly increased all-cause mortality risk compared to those without diabetes ( $\mathrm{HR}=1.8195 \% \mathrm{Cl}=1.64-2.00$ in Blacks; $\mathrm{HR}=1.82$ 95\% $\mathrm{Cl}=1.53-2.15$ in Whites). Of the common diabetes-related causes of death, coronary artery disease (CAD) was the leading cause of death in both Blacks and Whites, followed by renal failure and then heart failure in Blacks and sepsis and respiratory failure in Whites. Compared to Whites, Blacks with diabetes were at lower all-cause ( $\mathrm{HR}=0.85,95 \% \mathrm{Cl}=0.72-0.99)$ and $\mathrm{CAD}$ mortality ( $\mathrm{HR}=0.64,95 \% \mathrm{Cl}=0.47-0.86)$, but had higher or similar HRs (Cls) for mortality from heart failure (1.67 [1.01-2.76]), stroke (1.66 [0.87-3.19]), renal failure (1.60 [0.95-2.71]), respiratory failure (1.09 [0.68-1.74]), and sepsis (1.03 [0.64-1.67]). Risk factors were similar by race, with the exception of a significantly greater impact of baseline CAD on all-cause (interaction $\mathrm{p}$-val$u e=0.009$ ) and CAD mortality (interaction $p$-value $=0.03$ ) in Blacks compared to Whites. In this population with a similarly low SES and access to health care, Blacks and Whites with type 2 diabetes are at a similarly increased all-cause mortality risk, while Blacks with type 2 diabetes are a moderately but significantly lower mortality risk compared to Whites due mainly to the lower CAD mortality, the leading cause of death in both populations.

Supported by: R01 CA 092447 from the National Cancer Institute

\section{PREDICTORS AND CONSEOUENCES OF DIABETIC KIDNEY DISEASE}

Markers of Inflammation are Associated With Development of $\mathbf{A b}$ normal Albuminuria in Type 1 Diabetics

MARIA F. LOPES-VIRELLA, NATHANIEL L. BAKER, KELLY J. HUNT, PATRICIA A. CLEARY, GABE VIRELLA, THE DCCT/EDIC GROUP OF RESEARCHERS, Charleston, SC, Washington, DC

We measured inflammatory markers in 641 type 1 diabetics at baseline of DCCT and studied their association with abnormal albuminuria during $14-20$ years of follow-up in DCCT/EDIC. Of these, $426(67 \%)$ patients had albumin excretion rates $(A E R)<40 \mathrm{mg} / 24 \mathrm{~h}$ at baseline and during follow-up (persistent normal albuminuria); 155 (24\%) had AER $<40 \mathrm{mg} / 24 \mathrm{~h}$ at baseline and progressed to $\geq 40 \mathrm{mg} / 24 \mathrm{~h}$ but remained less than $299 \mathrm{mg} / 24 \mathrm{~h}$ during follow-up (microalbuminuria); and 60 (9\%) progressed to AER >299 mg/24 $\mathrm{h}$ (macroalbuminuria). To test the association of log transformed biomarkers, sample weighted multinomial logistic regression models were use where normal, micro and macro albuminuria were the outcomes of interest. Models were adjusted for DCCT treatment group, baseline retinopathy cohort, age, gender, and baseline measures of AER, LDL, HDL, and HbA1c \%. A 1 unit increase in the log transformed measure of CRP was associated with an 32 $\%$ increase in the odds to develop macroalbuminuria and a 1 unit increase in the measure of E-selectin lead to a nearly $59 \%$ increase in the odds to develop macroalbuminuria (Table). Additionally, increased levels of ICAM I and TNFR 2 at baseline were indicative of increased odds to develop abnormal albuminuria during DCCT/EDIC. Higher levels of CRP, E-selectin, ICAM I, and TNFR 2 were associated with increased odds to develop abnormal albuminuria. Our study indicates that high levels of inflammatory markers including CRP, E-selectin, ICAM I, and TNFR II are important predictors of abnormal albuminuria in patients with type 1 diabetes.

\begin{tabular}{lccc}
\hline \multicolumn{4}{c}{$\begin{array}{l}\text { Table. Odds of abnormal albuminuria as compared to persistent normal } \\
\text { albuminuria for a } 1 \text { unit increase in biomarkers listed }\end{array}$} \\
\hline \multicolumn{1}{c}{ Biomarkers } & $\mathrm{n}$ & Micro Albuminuria & Macro Albuminuria \\
\hline CRP & 608 & $1.01(0.89-1.14)$ & $1.32(1.04-1.67)^{*}$ \\
E-selectin & 631 & $1.11(0.85-1.44)$ & $1.59(1.02-2.48)^{*}$ \\
ICAM 1 & 635 & $1.56(1.01-2.41)^{*}$ & $0.90(0.45-1.83)$ \\
Interlukin 6 & 583 & $0.83(0.63-1.08)$ & $0.98(0.64-1.53)$ \\
PAl 1 Active & 622 & $0.93(0.75-1.16)$ & $0.89(0.57-1.39)$ \\
PAI 1 Total & 639 & $1.10(0.82-1.48)$ & $0.98(0.52-1.85)$ \\
TNFR 1 & 636 & $1.13(0.81-1.58)$ & $0.99(0.54-1.81)$ \\
TNFR 2 & 639 & $1.76(1.03-3.01)^{*}$ & $1.85(0.79-4.33)$ \\
\hline
\end{tabular}

Models include DCCT Treatment group, baseline retinopathy cohort, age, gender, HDL-C, LDL-C, AER and $\mathrm{HbA} 1 \mathrm{c} \%$ as adjustment variables. Any Measure $\geq 40$ is listed as abnormal albuminuria and any measure $\geq 300$ as macro albuminuria. Biomarkers are natural logarithm transformed and individually entered into separate models. SAS Proc Surveylogistic was used to calculate the weighted regression parameters and associated $95 \%$ Confidence intervals. ${ }^{*} \mathrm{p}<0.05$

Supported by: DHHS/NIH/NIDDK R01 DK081352-02

303-0R

SOD2 Polymorphisms and Risk of Kidney Complications in Subjects With Type 1 Diabetes

KAMEL MOHAMMEDI, SULIYA MAIMAITIMING, NATHALIE EMERY, NAIMA BELLILI-MUÑOZ, RONAN ROUSSEL, FRÉDÉRIC FUMERON, SAMY HADJADJ, MICHEL MARRE, GILBERTO VELHO, Paris, France, Poitiers, France

Manganese superoxide dismutase (SOD2) catalyzes the dismutation of superoxide, regulates reactive oxygen species metabolism in the mitochondria and is highly expressed in the kidney. We investigated associations of allelic variations of SOD2 with diabetic nephropathy in type 1 diabetic subjects. Eight SNPs in the SOD2 region were analyzed in 1285 Caucasian type 1 diabetic patients from the SURGENE prospective study ( $n=340$; 10 -year followup), Genesis France-Belgium ( $n=501)$ and GENEDIAB $(n=444)$ cross-sectional studies. Proliferative retinopathy was present in $10 \%, 44 \%$ and $82 \%$ of the participants of the three cohorts, respectively. Cox proportional hazards and logistic regression analyses were used to estimate hazard ratios (HR) or odds ratios (OR) for incidence and prevalence of nephropathy. All analyses were adjusted or stratified by retinopathy stages. $p \leq 0.01$ was significant. In the SURGENE study, the risk allele of a functional variant (rs4880, V16A) was associated with the prevalence of established/advanced nephropathy at baseline (OR 9.81, 95\% $\mathrm{Cl} 1.51-76.21, \mathrm{p}=0.01)$, with the incidence of microalbuminuria (HR 1.54,95\% Cl $1.11-2.11, p=0.01$ ), with the incidence of renal events (defined as progression to a more severe stage of nephropathy: HR $1.42,95 \% \mathrm{Cl} 1.12-1.77, \mathrm{p}=0.004)$ and with glomerular filtration rate decline during follow-up (TT: $-3.96 \pm 0.89$, CT: $-1.57 \pm 1.05$, CC: $-0.64 \pm 1.12 \mathrm{ml} /$ $\mathrm{min} /$ year, $\mathrm{p}=0.003$ ). Similar results were observed for rs2758329 and rs8031. After stratification for retinopathy stages, these variants were similarly associated with established/advanced nephropathy in participants of Genesis and GENEDIAB studies without proliferative retinopathy. In summary, SOD2 allelic variations were associated with the prevalence and incidence of albuminuria and with a faster decline in GFR in type 1 diabetic subjects. These results are consistent with a major role for SOD2 in the protection against oxidative stress and kidney disease in type 1 diabetic patients.

Supported by: Association L'Aide Aux Jeunes Diabétiques

304-0R

Advanced Glycation End Products May Predict Progression of Diabetic Nephropathy

PAUL J. BEISSWENGER, SCOTT HOWELL, MICHAEL MAUER, GREG RUSSELL, MIKE MILLER, STEPHEN RICH, Lebanon, NH, Hanover, NH, Minneapolis, MN, WinstonSalem, NC, Charlottesville, VA

Increased advanced glycation end products (AGEs) and oxidation products (OPs) are proposed to lead to diabetic nephropathy (DN). We investigated the relationship between AGEs, OPs and progression of DN in 103 subjects 
with type 1 diabetes participating in the Natural History of Diabetic Nephropathy Study. The subjects were $16.8 \pm 6.2$ years old, had diabetes duration of $8.1 \pm 4.3$ years, and most were normoalbuminuric. Change in glomerular basement membrane (GBM) width from baseline to 5 years, measured in electron micrographs of renal biopsies, was our primary endpoint, since it is the best early structural predictor of DN clinical progression. Mesangial fractional volume was also measured. Fast progressors (FP) were defined as the upper quartile $(\mathrm{n}=24)$ of GBM thickening and others as slow progressors (SP). AGEs [3-deoxyglucosone and methylglyoxal hydroimidazolones (DG3H1, MGH1)] and carboxymethyl and ethyl lysine (CML, CEL), and oxidation products [methionine sulfoxide and 2-Aminoadipic Acid] were measured by liquid chromatography, triple quadruple mass spectroscopy on 10 $\mathrm{K}$ plasma filtrates at year 5 . We found that MGHI, CEL, and CML levels were significantly higher in GBM-defined FP relative to SP. No AGE or OP predicted mesangial expansion. HbA1c at year 5 accounted for $4.7 \%$ of the variation in GBM width ( $\mathrm{r} 2$ ). The proportion of variation in GBM width was increased to $11.6 \%$ when $\mathrm{MGHI}$, CEL, and CML were added to the model (7.9\% increase), and MGHI was a significant independent predictor of GBM increase. These results suggest that these three major AGEs, in addition to $\mathrm{HbA1c}$, may be early indicators of progression of important DN lesions.

\begin{tabular}{lccc}
\hline Biomarker(All nM ) & FPMean \pm SD & SPMean \pm SD & P- Value (Wilcoxon) \\
\hline CML & $0.088 \pm 0.022$ & $0.075 \pm 0.023$ & 0.003 \\
\hline MGHI & $0.200 \pm 0.099$ & $0.165 \pm 0.127$ & 0.040 \\
\hline CEL & $0.058 \pm 0.015$ & $0.049 \pm 0.015$ & 0.026 \\
\hline
\end{tabular}

Supported by: JDRF

305-OR

Correlation of Renal Physiology and Pathology in Deceased Donor Kidneys Following 50-Years of Type 1 Diabetes (T1DM): 50-Year Medalist Study

HILLARY A. KEENAN, ISAAC E. STILLMAN, STEPHANIE HASTINGS, SARA TUREK, ROBERT STANTON, JENNIFER K. SUN, GEORGE L. KING, Boston, MA

The 50-Year Medalist Study has characterized renal function and pathology of 22 Medalist donors to document the extent of glomerulopathy after $50+y$ of T1DM. This study examines individuals $(n=685$, male $=48.7 \%$, duration $=58.6 \pm 6.6$ y age $=69.7 \pm 8.5$ y) with $50+y$ of T1DM by clinical exam and extensive medical history and has found low rates of microvascular complications including microalbuminuria (11.8\%; ACR $>30 \mathrm{mcg} / \mathrm{mg}$ ) and chronic kidney disease (4.28\%; CKD >4: CKD-EPI). Whole kidneys were procured post-mortem from 22 Medalists and classified by an experienced nephropathologist into diabetic nephropathy (DN) class using the method of Tervaert et al (JASN 2010) by degree of mesangial expansion $(0+\mathrm{l}, \mathrm{ll}+\mathrm{a}+\mathrm{b})$ and presence of Kimmelstiel-Wilson lesions (III) $(n=6$, class $0+1$; 8 , class Ila; 3 , class IIb; 5 , class III). Surprisingly, $28 \%$ of these longstanding T1DM patients had no to only mild, nonspecific changes by light and electron microscopy (Class $0+1$ ) even though $33 \%$ of these had co-existing proliferative diabetic retinopathy (PDR). There were no differences in sex, A1c, age, duration, age at diagnosis, BMI, lipids or c-peptide across DN classes ( $>>0.05)$. Mean eGFRs declined across class, $0+$ I: $58.5 \pm 27.2$; Ila: $53.8 \pm 13.0$, Ilb: $47.7 \pm 6.5$, III: $35.0 \pm 18.2 \mathrm{~mL} / \mathrm{min} / 1.73 \mathrm{~m}^{2}$. However, ACR did not consistently increase across the classes: $0+1$ : $18.0 \pm 10.9$, Ila: $17.8 \pm 16.2$, Ilb: $142.4 \pm 179.3$, III: 1016.4 $\pm 1743.6 \mathrm{mcg} / \mathrm{mg}$. Two of the 22 cases were positive for $\lg A ; 10$ had score 1 tubulo-interstitial scarring, 11 had score 2 and 1 had score 3. Medalists' kidneys classified in class $0+1$ demonstrate that it is possible to have normal glomerular morphology even after 50+ y of T1DM. The presence of PDR in the Class $0+1$ group indicates protective factors for diabetic kidney disease may differ from those of the retina. Finally, greater use of the kidney biopsy in diabetic patients with normal ACR and low GFR might provide unexpected diagnoses and new pathophysiologic insights.

306-OR

Gastrointestinal-Mediated Glucose Disposal, Incretin Effect and Glucagon Responses in Patients With End-Stage Renal Disease THOMAS IDORN, FILIP K. KNOP, JENS J. HOLST, MADS HORNUM, BO FELDTRASMUSSEN, Copenhagen, Denmark, Hellerup, Denmark

Non-diabetic patients with end-stage renal disease (ESRD) depending on dialysis treatment exhibit disturbed glucose metabolism. The underlying pathophysiological mechanisms remain elusive. We aimed to evaluate gastrointestinal-mediated glucose disposal (GIGD), incretin effect and glucagon- and glucagon-like peptide-1 (GLP-1) secretory responses in patients with ESRD. Three groups matched by age, gender and BMI (ESRD patients with normal glucose tolerance (NGT, N=10) or impaired glucose tolerance
(IGT, N=10) and 11 healthy control subjects (CTRL)) were investigated using $75 \mathrm{~g}$ OGTT and isoglycemic intravenous glucose infusion (IIGI) on separate days. Plasma glucose, insulin, glucagon and total GLP-1 were measured repeatedly and GIGD, based on glucose amounts utilized $\left(100 \% \times\right.$ (glucose OGTT $_{\text {- }}$ glucose $\left._{\| G G}\right) /$ glucose $\left._{O G T T}\right)$ and incretin effect, based on insulin responses (area under curve (AUC)) $\left(100 \% \times\left(\mathrm{AUC}_{0 \mathrm{OGTT}}-\mathrm{AUC}_{\| \mathrm{GG}}\right) / \mathrm{AUC}_{\mathrm{OGTT}}\right)$ were calculated. GIGD was significantly $(P<0.02)$ diminished in both ESRD groups $(38 \pm 7 \%$ (NGT) and $31 \pm 3 \%$ (IGT), $P=0.4$ ) compared to the CTRL group $(59 \pm 3 \%)$. Incretin effects amounted to $64 \pm 16 \%$ (CTRL), $54 \pm 15 \%$ (NGT) and $38 \pm 26 \%$ (IGT), with significant difference between CTRLS and IGTS $(P=0.01)$. Fasting GLP-1 and glucagon levels were significantly higher in ESRD patients compared to CTRLS $(P=0.007$ and $P<0.0001$, respectively). Furthermore, maximal glucagon suppression (\% of basal) was significantly $(P<0.0001)$ impaired in ESRD patients (NGT: $75 \pm 4 \%$ (OGTT) and $76 \pm 3 \%$ (IIGI), $P=0.8 ;$ IGT: $77 \pm 5 \%$ (OGTT) and $61 \pm 5 \%$ (IIGI), $P=0.04$ ) compared to CTRLs $(19 \pm 4 \%$ (OGTT) and $16 \pm 1 \%$ (IIGI), $P=0.4)$. ESRD patients are characterized by 1) reduced GIGD, 2) decreased incretin effect (IGT patients), 3) elevated fasting GLP-1 and 4) severe fasting hyperglucagonemia that seems irrepressible in response to oral and iv glucose stimuli. These perturbations may constitute important mechanisms underlying the disturbed glucose metabolism in ESRD.

\section{Glycemia and Death in Dialysis Patients}

307-0R AMANDA ADLER, ANNA CASULA, MARTIN WILKIE, DAMIAN FOGARTY, CHARLIE TOMSON, Cambridge, United Kingdom, Bristol, United Kingdom, Sheffield, United Kingdom, Belfast, United Kingdom

The National Kidney Foundation advocates that $\mathrm{HbA} 1 \mathrm{c}$ "be $<7.0 \%$ (53 $\mathrm{mmol} / \mathrm{mol}$ ) irrespective of the presence or absence of CKD ". Regarding dialysis, the Renal Association (UK) advises an $\mathrm{HbA1c}$ between 6.5\% and $7.5 \%$. Yet, glucose-lowering trials on which the guidance is based excluded patients with end stage renal disease (ESRD), who tend to have complications including hypo-unawareness. Using national UK Renal Registry data, we tested whether glycemia $(\mathrm{HbA1c})$ is associated with death in adults with diabetes on hemo- or peritoneal dialysis for ESRD of any cause. We included patients starting dialysis 1 Jan 1997 to 31 Dec 2006, and followed for at least 6 months. From a cohort of 7814, we excluded those who within the first 6 months died $(n=797)$, were transplanted $(n=228)$, were lost/recovered $(n=57)$ and who had no measure of HbA1c $(n=3386)$, ethnicity $(n=172)$ or hemoglobin $(\mathrm{Hb})(\mathrm{n}=17)$. We categorized HbA1c measured $+/-6$ months of starting dialysis as $<6.5 \%, 6.5 \%$ to $7.4 \%$ (reference), $7.5-8.4 \%$, and $\geq 8.5 \%$. We adjusted in proportional hazards models for age, sex, ethnicity, deprivation, year, dialysis mode and $\mathrm{Hb}$. We could not adjust for weight, cardiovascular disease, or diabetes type, duration or treatment. Of 3157 patients with a mean age of $60 \mathrm{yrs}$ followed a median of $2.7 \mathrm{yrs}, 1688$ died. For patients aged $\geq 60 \mathrm{yrs}$, we found no association between $\mathrm{HbA1c}$ and death; for those $<60 \mathrm{yrs}$, relative to patients with $\mathrm{HbA} 1 \mathrm{c}$ values $6.5-7.4 \%$, the hazard ratio was 1.2 (95\% $\mathrm{Cl} 0.9$ to 1.5$)$ for $\mathrm{HbA1c} 7.5-8.4 \%$, and 1.5 (1.2 to 1.9) for $\mathrm{HbA1c}$ $>8.5 \%$. In the absence of trials and confounding notwithstanding, these observational data support tighter glycemic control in younger patients prior to and following dialysis.

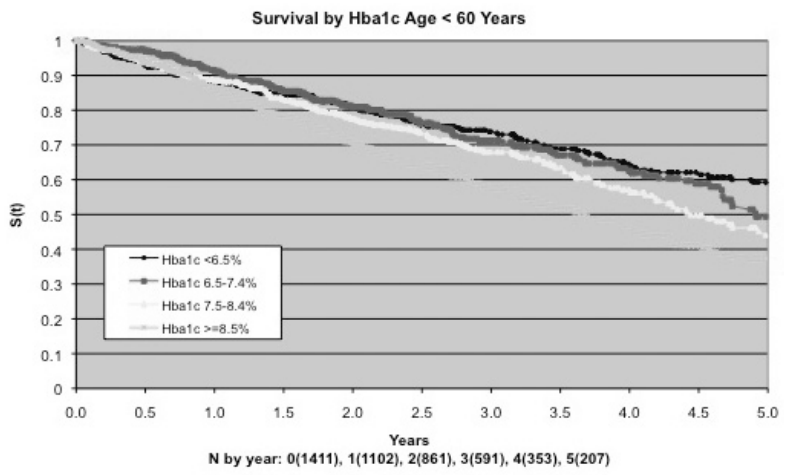




\section{IMPROVING HEALTH CARE DELIVERY AND HEALTH OUTCOMES FOR DIABETES}

308-0R

New Risk Score for Predicting 5-Year Incidence of Type 2 Diabetes in Japan: The Toranomon Hospital Health Management Center Study YORIKO HEIANZA, YASUJI ARASE, HIROSHI TSUJI, SAKIKO YOSHIZAWA, KAZUMI SAITO, SHIUN DONG HSIEH, SATORU KODAMA, NOBUHIRO YAMADA, SHIGEKO HARA, HIROHITO SONE, Mito, Japan, Tokyo, Japan

Whether adding information on HbA1c into a prediction model that includes plasma glucose and other potential predictors would lead more accurate prediction of subsequent diabetes has not been clarified. We assessed the clinical significance of introducing $\mathrm{HbA1c}$ into a prediction rule for diabetes and developed a scoring system to predict the incidence of diabetes in Japanese individuals. Studied were 10250 non-diabetic individuals aged 2480 years who underwent a baseline health examination and a reexamination 5 years later. Diabetes was indicated by fasting plasma glucose (FPG) $\geq 7.0$ $\mathrm{mmol} / \mathrm{L}, \mathrm{HbA} 1 \mathrm{C} \geq 6.5 \%$, or self-reported clinician-diagnosed diabetes. We assessed the predictive ability of conventional predictors of diabetes and whether adding $\mathrm{FPG}, \mathrm{HbA1c}$, or both $\mathrm{FPG}$ and $\mathrm{HbA1c}$ would improve risk prediction. The conventional predictive model included age, male sex, parental history of diabetes, current smoking, body mass index, hypertension, resting heart rate and triglycerides (area under the receiver-operating characteristic curve (AROC) 0.734 ). The addition of FPG significantly improved discrimination (AROC 0.875) and then adding $\mathrm{HbA1C}$ further improved discrimination (AROC 0.917). After multivariate logistic analysis, we developed a risk scoring system based on points (P) that consisted of parental history of diabetes (2P), smoking (1P), obesity (1P), FPG 5.3-5.5 mmol/L (2P) or 5.6-6.9 mmol/L (7P) and $\mathrm{HbA1C} 5.3-5.4 \%$ (4P) or 5.5-6.4\% (8P) (AROC 0.888). At an optimal cut-off of 11 points, sensitivity and specificity were high at $82.5 \%$ and $80.2 \%$. We validated the diabetes risk score in a separate group of 1856 non-diabetic individuals (AROC 0.909, sensitivity $95.3 \%$ and specificity $77.2 \%$ ). By adding information on $\mathrm{HbA1c}$ into the prediction model, we developed a simple, sensitive and specific algorithm to predict the 5-year risk of diabetes. Our algorithm would be useful in identifying individuals likely to benefit from early interventions.

309-0R

Hospitalization Patterns in Type 1 Diabetes (T1DM) for First 20 Years after Diagnosis

VASUDEV MAGAJI, THOMAS J. SONGER, TREVOR J. ORCHARD, Pittsburgh, PA

Health care utilization experiences are important for clinicians, policy planners and researchers to characterize T1DM prognosis and resource need. We thus examined the natural history of hospitalization, post diagnosis in a subgroup ( $n=73$; males=38; females=35) of the Pittsburgh Epidemiology of Diabetes Complications (EDC) study, a prospective follow up of childhood onset T1DM. Cases were diagnosed with T1DM, or seen within 1 year of diagnosis, at Children's Hospital of Pittsburgh from January 1978 to May 1980 and were $<17$ years and on insulin at diagnosis. At EDC enrollment, these subjects had T1DM for $<10$ years without complications. Hospitalization data, from diagnosis were obtained by self report from subjects by biennial surveys. There were 258 admissions (Females 68\% (pregnancy related $20 \%$ ) vs Males $32 \%$ ) after 20 years of T1DM diagnosis. Overall, the cumulative incidence at 20 years duration was $77 \%(73.6 \%$ in males vs. $80 \%$ in females). Figure 1 details the cumulative incidence of hospitalization by duration and reason for admission. Hospitalization for acute complications increased linearly and stabilized at 15 years of T1DM diagnosis. Acute admissions were higher in females ( $60 \%$ vs $39 \%, p=0.03$ ). Admissions for infection progressively increased whereas for chronic conditions were low and began at 10 years duration. Hospitalization for acute complications and infection account for $64 \%$ of all admissions by 20 year duration. This analysis highlights the need for improvement in clinical care required to prevent hospitalizations and provides insight for resource allocation to prevent and control T1DM complications.

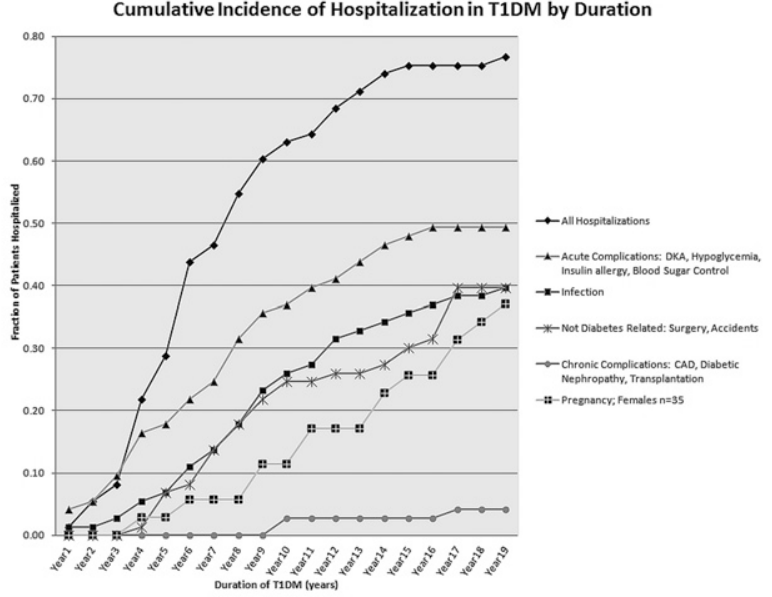

310-0R

Trend in Racial Disparity of Diabetes Monitoring Among the U.S. Non-Institutionalized Population in the Last Decade (2000-2009) YINGNAN ZHAO, OIAN SHI, LIZHENG SHI, Hartford, CT, New Orleans, LA

The aim of this study was to examine the racial disparity of diabetes monitoring over years 2000 to 2009 among individuals with diabetes in the United States (US). We analyzed the national representative samples of the US non-institutionalized population from the Medical Expenditure Panel Survey (MEPS) 2000-2009. "Good monitoring" was defined as >=2 A1c tests along with $>=1$ eye or foot exam annually, according to the ADA guidelines. A1c test, foot and eye exam, and overall "good monitoring" were assessed among the whole US population with diabetes, and compared between nonHispanic whites and minority populations. From 2002 to 2009, the overall rate of "good monitoring" decreased from $71 \%$ to $66 \%$. Specifically, the percentage of individuals with $>=2 \mathrm{~A} 1 \mathrm{c}$ tests annually declined in general over the last 10 years, at the level of $73 \%$ in both 2008 and 2009 compared to $78 \%$ in 2000 . Foot exam varied between $65-72 \%$, with a continuous decrease from 2002 to 2008. Eye exam maintained at level of $61-63 \%$. Among non-Hispanic whites, the percentages of good monitoring and specific items were consistently higher than that of minority populations in years 2000 to 2009. The racial gap did not show improvement till 2006 or 2007, and yet deteriorated especially in 2008 and 2009. Over the past decade, the racial gap of $5 \%$ in good monitoring was in 2002 and $15 \%$ in 2009; $5 \%$ in A1c test in 2000 and $12 \%$ in 2009; $4 \%$ in foot exam in 2000 and $7 \%$ in 2009; $6 \%$ in eye exam in 2002 and $11 \%$ in 2009. Our analyses have identified the racial disparity in the last decade in the US, which has not been improved, but deteriorated in recent years.

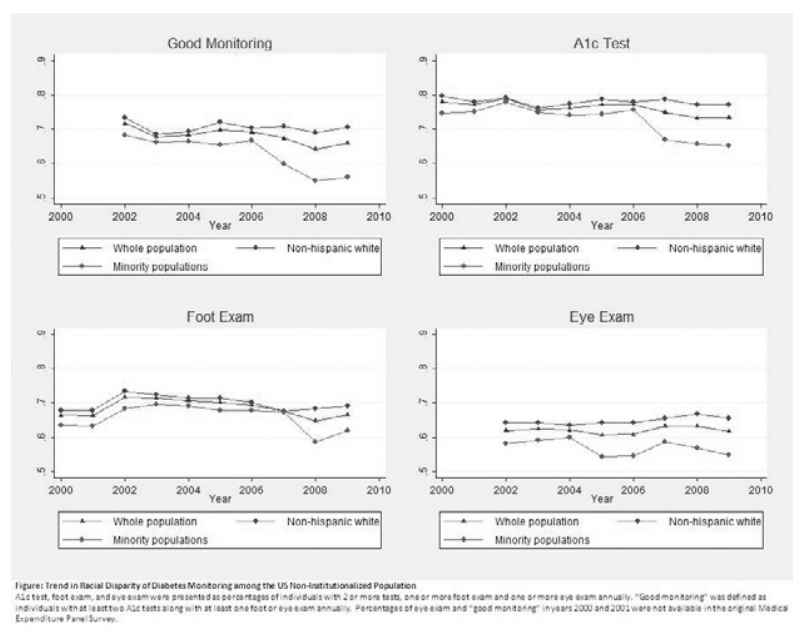


311-OR

Primary Care Physician Impact on Treatment of Patients With Diabetes FRITHA MORRISON, MARIA SHUBINA, ALEXANDER TURCHIN, Boston, MA

It is known that patients whose primary care is provided by multiple physicians have worse outcomes, but the reasons are not well understood. This study compares critical process measures between PCPs and covering providers during encounters with patients with uncontrolled diabetes. We conducted a retrospective cohort study to determine if PCPs are more likely than covering providers to intensify medications, provide lifestyle counseling, and have shorter intervals to next encounter for patients with elevated A1c, LDL, or blood pressure. The study included 27,225 patients with 601,330 face-to-face and remote encounters in primary care settings. Patients had at least two years of follow-up with primary care practices affiliated with two teaching hospitals between $1 / 1 / 2000$ and 1/1/2010. In multivariable analysis, the odds of medication intensification were $52 \%(p<0.0001)$ and $27 \%$ $(p=0.0004)$ higher for PCPs than covering physicians and mid-level providers, respectively (Table 4). Odds of lifestyle counseling were $89 \%(p<0.0001)$ higher for PCPs than covering physicians and $23 \%(p=0.0021)$ higher than mid-level providers. Compared to PCPs, the hazard ratios for time to next encounter after a visit without acute complaints were 1.09 and 1.18 for covering physicians and mid-level providers, respectively ( $p<.0001$ for both). PCPs perform better than other providers on several process measures tightly linked to diabetes outcomes. These findings suggest a mechanism for the observed effect of continuity of care on outcomes in diabetes and point to critical gaps that should be addressed in the settings where exclusive care by PCPs is not feasible.

\begin{tabular}{|c|c|c|c|c|}
\hline \multicolumn{5}{|c|}{ Effects of Provider Type on Key Process Measures in Treatment of Diabetes } \\
\hline Encounter Type & Provider Category & $\begin{array}{c}\text { Medication } \\
\text { Intensification } \\
\text { (odds ratios) }\end{array}$ & $\begin{array}{c}\text { Lifestyle } \\
\text { Counseling } \\
\text { (odds ratios }\end{array}$ & $\begin{array}{l}\text { Time to Next } \\
\text { Encounter } \\
\text { (hazard ratios) }\end{array}$ \\
\hline \multirow[t]{3}{*}{ No acute complaints } & PCP & 1.00 & 1.00 & 1.00 \\
\hline & Non-PCP MD & 0.66 & 0.53 & 1.09 \\
\hline & Mid-level provider & 0.79 & 0.82 & 1.18 \\
\hline \multirow[t]{3}{*}{ With acute complaints } & PCP & 1.00 & 1.00 & 1.00 \\
\hline & Non-PCP MD & 0.72 & 0.92 & 0.99 \\
\hline & Mid-level provider & 0.79 & 0.75 & 0.97 \\
\hline
\end{tabular}

Supported by: 1R18HS017030, 1RC1LM010460 and DAREF

\section{2-0R}

Comparison of Tele-Health With Usual Case Management in Patients With Diabetes

NACIDE ERCAN-FANG, KIRANJOT GUJRAL, STEPHEN K. FIRESTONE, EMILY HAGEL, BRENT TAYLOR, AREEF ISHANI, Minneapolis, MN, St. Cloud, MN

In patients with diabetes, compared to usual care, nurse case management (CM) demonstrated significant improvement in Hba1c, blood pressure, lipids, patient satisfaction and perceived quality of care. In other chronic diseases, CM using Tele-health system (CMTH) further improved patient accessibility and timeliness of intervention, with lesser hospitalizations and death. There have been no direct comparison studies between CMTH and CM programs for management of diabetes. The aim of our study is to compare the outcomes of the two management programs on Hbalc. A two center randomized controlled trial of CMTH $(n=94)$ versus $\mathrm{CM}(\mathrm{n}=93)$ in patients with Hba1c $>9.0 \%$, who were followed for a 9 month period. The primary outcome was mean decrease in Hbalc at 9 months. Secondary outcomes included percentage of patients achieving $\mathrm{Hba1c}<8.0 \%$, percentage of patients achieving goal $\mathrm{BP}<130 / 80 \mathrm{mmHg}$, total number of successful contacts and mean duration of each contact. Differences between groups were analyzed using t tests and Pearson [chi square] tests. The mean decrease in Hba1c was similar between the groups $(1.25 \%$ vs. $1.43 \%$ in the CMTH vs. CM groups, respectively; $p=0.47$ ). The number of patients who attained goal Hba1c $<8 \%$ was 33 vs. 30 , in the CMTH vs. CM groups, respectively; $p=0.68$. The number of patients achieving goal BP $<130 / 80 \mathrm{mmHg}$ was similar ( 34 vs. 36 in the CMTH vs. CM groups, respectively; $p=0.72$ ). The total number of successful contacts per person was higher in the CMTH group (17 vs. 10 in the CMTH vs. CM groups, respectively; $p=<0.0001)$. The mean duration of contact was shorter in the CMTH (8.6 min vs. $10.2 \mathrm{~min}$ in the CMTH vs. CM groups, respectively; $p=0.01$ ). Our results suggest that $C M T H$ is not superior to CM program in management of hyperglycemia. While CMTH increased the number of successful contacts and reduced the mean contact duration, the total contact time was increased. Addition of Tele-health system for hyperglycemia management does not offer improvements over standard CM.

Supported by: Veterans Integrated Service Network 23 Minimizing Risks of Hypoglycemia in High Risk Patients: A Proposal for a Glycemic Overtreatment Indicator

CHIN-LIN TSENG, ORYSYA SOROKA, MIRIAM MANEY, DAVID C. ARON, LEONARD M. POGACH, East Orange, NJ, Newark, NJ, Cleveland, $\mathrm{OH}$

Diabetes measurement has focused upon undertreatment, however, hypoglycemia is a common and potentially serious yet avoidable risk of therapy. Insulin (I) and sulfonyurea (SU) have been associated with increased hospitalizations in the elderly. Known risk factors include decreased renal function, cognitive impairment (CI) and dementia, and tight glycemic control. Although the National Committee for Quality Assurance has excluded some high risk individuals from meeting an A1c threshold $<7 \%$, there is no surveillance of A1c $<7 \%$ in vulnerable persons. Our objective was to evaluate the prevalence of possible overtreatment among veterans receiving care in the Veterans Administration in 2009. Our study population was veterans receiving I or SU in 2009 with one of the following: age $>70$ years, serum creatinine $>1.7 \mathrm{mg} / \mathrm{dl}$, or ICD-9CM diagnosis of $\mathrm{Cl} / \mathrm{dementia}$ in 2009. Potential overtreatment was assessed using A1c thresholds of $<7 \%,<6.5 \%$, and $<6 \%$ based on the last A1c value in FY 2009. Of the identified 286,904 high risk individuals ( $26 \%$ of all veterans with diabetes), $98.7 \%$ were male, $82.7 \%$ were older than 70 years old, $26.2 \%$ had a serum cretainine $>1.7 \mathrm{mg} / \mathrm{dl}$, and $14.8 \%$ had $\mathrm{Cl}$ or dementia. Slightly less than half $(47.4 \%)$ were on insulin and the rest were on SU but not insulin. Overall, $48.4 \%, 27.2 \%$, and $10.5 \%$ of patients had the last A1c value in $\mathrm{FY} 2009$ lower than 7\%, 6.5\%, and 6\%, respectively. The rates were higher among patients treated with SU but not on insulin: $57.9 \%, 33.0 \%$, and $12.3 \%$, while lower among patients treated with l: $37.9 \%$, $20.7 \%$, and $8.6 \%$. The difference in rates between I and SU groups were significant $(p<0.001)$ across three A1c thresholds. Additionally, we evaluated a small subgroup of patients $(n=62,055)$ who were older than 70 years old and had either $\mathrm{Cl} /$ dementia or a serum creatinine $>1.7 \mathrm{mg} / \mathrm{dl}$. The rates for the three different thresholds were $51.4 \%(<7 \%), 30.25(<6.5 \%)$, and $12.3 \%$ $(<6 \%)$. In sum, the rates of potential overtreatment were markedly elevated among high risk patients.

Supported by: VAHSRD IIR08-355-2

314-0R

A Randomized Study to Evaluate Peer Support in Type 2 Diabetic Patients Receiving Structured Care

JULIANA CHAN, YI SUI, HARRIET CHUNG, REBECCA WONG, SAMSONLY W. CHAN, MAGGIE S. LAU, GRACE L. LIN, JENNY ZHANG, NICOLA BROWN, BRIAN OLDENBURG, ED FISHER, GARY KO, Hong Kong, China, Melbourne, Australia, Chapel Hill, NC

In a multicentre randomized study, we examined the effects of peer support on physical-psychological health in Type 2 diabetic (T2D) patients receiving structured care through a web-based program (JADE: Joint Asia Diabetes Evaluation Program). The latter incorporates a validated risk engine and protocols for comprehensive assessment to generate reports to doctors and patients with risk prediction, treatment targets and decision support. The peer support group (PEARL: Peer Support Empowerment And Remote Communication Linked by Information Technology) received 6-10 phone calls from trained mentors in a 10:1 ratio for practical tips and social support. Outcome measures included risk factor control and psychometric scales. In 2009-2010, 628 Chinese T2D patients [mean \pm SD age: $54.5 \pm 9.9$ years, $59 \%$ men, disease duration: $9.2 \pm 7.8$ years, A1c:8.2 $\pm 1.7 \%$; systolic BP:136 $\pm 19 \mathrm{mmHg}$, LDL-C: $2.90 \pm 0.81 \mathrm{mmol} / \mathrm{l}$, insulin-treated $(40.2 \%)$, cardiovascular-renal complications (17.4\%)] were randomized to PEARL ( $P ; n=312$ ) or control group $(\mathrm{C} ; \mathrm{n}=316)$. All patients received 4 -monthly JADE reports by mail showing trends of A1c, BP, LDL-C and body weight (BW). At one year, both groups had significant reduction in risk factors compared to baseline: A1c $\geq 0.5 \%$ : (P:35\%, C:34\%); systolic BP $\geq 10 \mathrm{mmHg}$ (P:36\% C:34\%): LDL-C $\geq 30 \%$ (P:17\% C:20\%); BW $\geq 3 \%$ (P:22\% C:18\%). Depression (PH09), distress (DSS17), anxiety-stress (DASS21) scales improved similarly in both groups. In this integrated program, regular communication of personalized risk with decision support improved risk factor control and psychological well-being. 
Structured care (JADE) and peer support (PEARL) in type 2 diabetes $\left({ }^{*} p<0.05, \#<0.01\right)$

\begin{tabular}{lcccc}
\hline & \multicolumn{2}{c}{ PEARL+JADE } & \multicolumn{2}{c}{ JADE only } \\
\hline & Baseline & 1 year & Baseline & 1 year \\
\hline A1c $<7 \%$ & $24.7 \%$ & $30.4 \%^{*}$ & $25.0 \%$ & $32.8 \%^{*}$ \\
\hline BP $<130 / 80 \mathrm{mmHg}$ & $31.7 \%$ & $41.9 \%^{*}$ & $32.9 \%$ & $43.4 \%^{*}$ \\
\hline LDL-C $<2.6 \mathrm{mmol} / \mathrm{L}$ & $41.3 \%$ & $56.1 \%^{*}$ & $40.2 \%$ & $56.9 \%^{*}$ \\
\hline DASS21<median & $50.2 \%$ & $40.5 \%^{\#}$ & $53.0 \%$ & $42.9 \%^{\#}$ \\
\hline DSS17<median & $53.4 \%$ & $45.1 \%^{\#}$ & $51.3 \%$ & $41.9 \%^{\#}$ \\
\hline PH09<median & $56.6 \%$ & $45.7 \%^{\#}$ & $58.1 \%$ & $48.6 \%^{\#}$ \\
\hline
\end{tabular}

Supported by: Peers for Progress

315-0R

Reducing Glycaemic Burden Through Improved Access to Community Based Specialist Services: A Review of a Tier 2 Clinical Service

ABDUL Z. MOHAMED, REBECCA F. PARKER, SYLVIA MILES, SALMAN HASSOUN, DEVAKA J. FERNANDO, Sutton in Ashfield, United Kingdom

The value of managing people with Type 2 diabetes through an early goalorientated strategy with intention to treat to target through frequent visits with a health care provider based in the community, who has specialist competencies are known to lead to reductions in $\mathrm{HbA1c}$ levels. Implementing this strategy remains a challenge due to economic restrictions making specialist care scarce in community settings. We report a 12 month experience of moving from hospital to a community based specialist diabetes service where glycaemic burden was used to asses long term glycaemic control in 100 patients with type 2 diabetes (mean age60.4 SD 13.8 years, 51 males) with type 2 Diabetes all having $\mathrm{HbA1C}>7.5$ referred to the service. Glycaemic control was prospectively monitored, three monthly, using HbA1c. Glycaemic burden (Current HBA1c-7.5) was calculated for each patient and plotted against time giving a graphical representation of risk potential end organ damage. HBA1c targets were assessed at baseline 6 months and 12 months. HBA1c fell from 9.5 SD $1.4 \%$ at baseline to 8.9 SD $1.5 \%$ at 6 months $(p=0.004,95 \% \mathrm{Cl} 0.18-0.94)$ and $8.6 \mathrm{SD} 1.4 \%$ at 12 months $(p=0.001,95 \% \mathrm{Cl}$ 0.5-1.2). Glycaemic Burden fell from 1.95 SD 1.4 at baseline to 1.4 SD 1.5 at 6 months ( $p=0.0495 \% \mathrm{Cl} 0.18-0.94)$ and 1.07 SD 1.4 at 12 months ( $p=0.001$, $95 \% \mathrm{Cl} 0.5-1.2)$. Targets of $<7.5 \mathrm{HbA1C}$ were achieved in 6 months in $16(16 \%)$ and $22(22 \%)$ in one year. The intermediate care clinic achieved reductions in glycaemic burden equivalent to a $1 \%$ reduction in $\mathrm{HBa} 1 \mathrm{c}$ giving an expected reductions of in mortality (21\%) and a legacy effect in a cost effective manner through reorientation of existing health services, if results of the United Kingdom Prospective Diabetes Study are extrapolated to this population.

\section{IMMUNOMETABOLISM}

\begin{abstract}
$\Delta$ Lipid-Sensing NKT Cells in Adipose Tissue Promote M2 Macrophage Polarization and Glucose Homeostasis via IL-4/STAT6 in Obesity YEWEI JI, LING QI, Ithaca, NY

Inflammation in adipose tissue plays an important role in the pathogenesis of obesity-associated complications. However, the underlying mechanism by which inflammation develops during obesity remains largely unknown. Here we show that adipose tissues of both mice and humans contain a unique and previously unidentified population of lipid-sensing type-1 natural killer T (NKT) cells, whose abundance decreases with increased adiposity. Remarkably, in response to 4 day high-fat-diet (HFD) feeding, NKT cells are activated and promote an IL-4-mediated TH2 response in adipose tissue. Using several gain- and loss-of-function mouse models, our data demonstrate that NKT cells in adipose tissue play a key role in modulating the TH1/TH2 response and macrophage polarization in adipose tissue as well as systemic glucose homeostasis, in part via the IL-4/STAT6 signaling axis at both the onset and late stages of obesity. Thus, our data identify NKT cells in adipose tissue as a key regulatory hub linking excess lipids to inflammation and glucose homeostasis in obesity, suggesting a novel therapeutic target for the treatment of obesity-associated inflammation and type-2 diabetes.

Supported by: NIH
\end{abstract}

\section{7-0R}

T Cell Activation and Proliferation in Adipose Tissue of Obese Mice ERLIE JIANG, XIAOYUAN DAI PERRARD, JERRY L. PERRARD, C.W. SMITH, CHRISTIE M. BALLANTYNE, HUAIZHU WU, Houston, TX, Tianjin, China

Increased accumulation of T cells, particularly $\mathrm{CD} 8^{+} \mathrm{T}$ cells, in adipose tissue (AT) is a hallmark of obesity-linked AT inflammation. T cells are traditionally thought to become activated and to proliferate when they encounter antigens presented by antigen-presenting cells (APCs) in lymphoid organs. By using Edu labeling techniques in mice and cell culture, we studied the potential for activation and proliferation of $\mathrm{CD}^{+}{ }^{+} \mathrm{T}$ cells in mouse AT. C57BL/6 mice fed high-fat diet for 3 months were used as an obesity model, with mice on normal diet as lean controls. $C D 8^{+} T$ cells and related cytokines were examined in AT by flow cytometry or quantitative RT-PCR. Compared to lean mice, obese mice had increased $C D 8^{+} T$ cell numbers in AT, with even higher relative ratios of $\mathrm{CD} 44^{+} / \mathrm{CD} 62 \mathrm{~L}^{-}$effector memory $\mathrm{T}$ cells and $\mathrm{CD} 44^{+} / \mathrm{CD} 69^{+}$ effector T cells and increased mRNA levels of granzyme B and IFN- $\gamma$, implying activation of $\mathrm{CD} 8^{+} \mathrm{T}$ cells in AT of obese mice. At 3 hours after intraperitoneal injection of Edu, no Edu+ ${ }^{+}$cells appeared in blood but we found $\mathrm{CD}^{+} / \mathrm{Edu}^{+} \mathrm{T}$ cells in AT, indicating that AT CD8 ${ }^{+} \mathrm{T}$ cells proliferated with Edu incorporation. Compared to lean mice, obese mice had increased percentage of proliferating $\mathrm{CD}^{+} \mathrm{T}$ cells $\left(\mathrm{Edu} \mathrm{u}^{+}\right)$in $\mathrm{AT}(2.1 \pm 0.1 \%$ in obese vs. $1.4 \pm 0.1 \%$ in leans, $\mathrm{P}<0.01)$. IL-2, IL-12 and IL-18 were all increased in AT of obese mice. To examine the potential of these cytokines to activate T cells in AT, we treated $\mathrm{CD}^{+} \mathrm{T}$ cells isolated from AT with IL-2, IL-12 and IL-18 in vitro (in the absence of antigens and APCs) and found that these cytokines induced proliferation and increased expression of IFN- $\gamma$ and CD69 in AT CD $8^{+}$T cells. Compared to those from lean mice, $C D 8^{+} T$ cells from AT of obese mice showed increased response to cytokine stimulation with more cells expressing IFN- $\gamma(11.3 \pm 1.1 \%$ in obese vs. $3.5 \pm 0.2 \%$ in leans, $P<0.01$ ). In conclusion, we demonstrated that the combined action of inflammatory cytokines led to local activation and proliferation of $\mathrm{CD}^{+} \mathrm{T}$ cells in AT, which may contribute significantly to AT inflammation observed in obesity.

Supported by: R01HL098839 (H.W.) and R01DK078847 (C.M.B.)

318-0R

B Lymphocytes are Master Regulators of a Pro-Inflammatory T Cell Balance in Obesity and Glucose Intolerance

BARBARA NIKOLAJCZYK, ANNA C. BELKINA, JENNIFER SNYDER-CAPPIONE, MADHUMITA JAGANNATHAN-BOGDAN, JORDAN CARR, JASON DEFURIA, DOUGLAS MARKHAM, JESSICA ALLEN, JACOUELINE BOUCHARD, YANINA NERSESOVA, AMANDA WATKINS, GERALD DENIS, MARIE MCDONNELL, CAROLINE APOVIAN, Boston, MA

Lymphocytes play key roles in the chronic inflammation critical for T2D pathogenesis. We have shown T2D patients have an elevated ratio of pro- to anti-inflammatory T cells, and that B cells from T2D patients fail to produce anti-inflammatory IL-10 but produce elevated levels of IL-8. New data show that $B$ cells from the diet-induced obesity (DIO) mouse model of T2D secrete a pro-inflammatory balance of cytokines, including relatively high MIP-2 (an IL-8 ortholog) and low IL-10, which mirrors our findings in T2D patients. Although recent work indications that T2D has an auto-immune component, DIO B cells do not to secrete auto-antibodies, as shown by anti-nuclear antigen staining. Complementary metabolic studies show that B cell-null mice resist the development of glucose intolerance in response to DIO, despite weight, fasting serum glucose, RER and whole body percentage of adipose tissue that are indistinguishable from WT mice. Increased glucose tolerance is likely explained by elevated insulin secretion in B cell-null mice responding to a glucose bolus. Taken together, these data support the conclusion that a pro-inflammatory B cell cytokine balance, rather than antibodies, promotes T2D. To further define roles for B cells in T2D, we immunophenotyped DIO B cell-null splenocytes. Although B cell-null and WT mice on low fat diet have similar percentages of regulatory T cells (Tregs), Tregs expand in DIO B cellnull, while Tregs fail to expand in DIO WT mice. These data are consistent with new human studies showing B cells from T2D (but not healthy) subjects support pro-inflammatory T cell function, and indicate clinical relevance of published studies showing decreased Th1 function in DIO B cell-null vs. WT mice. We conclude $B$ cells are critical regulators of inflammation in T2D due to 1. Secretion of a pro-inflammatory cytokine balance; and 2. An ability to promote pro-inflammatory T cell function. Thus B cell depletion may represent a valuable tool in the T2D clinic.

Supported by: NIH/NIDDK, BADERC 
319-OR

Toll-Like Receptor 4 Deficiency Impairs Insulin Sensitivity During the Progression of Autoimmune-Mediated Diabetes in Non-Obese Diabetic Mice

ANNA L. REINBECK, GILLES SÉOUARIS, HANS-JOACHIM PARTKE, PETER NOWOTNY, JÖRG KOTZKA, BIRGIT KNEBEL, VOLKER BURKART, MICHAEL RODEN,

Düsseldorf, Germany

Recent studies on the pathogenesis of type 1 diabetes implicate that mechanisms controlling energy metabolism are involved in disease development. Certain aspects of carbohydrate and lipid metabolism are under control of the toll-like receptor 4 (TLR4), initially identified as receptor for lipopolysaccharide on innate immune cells. We hypothesized that TLR4 determines a diabetes-modulating metabolic phenotype in non-obese diabetic (NOD) mice. We monitored the age of diabetes onset and whole-body metabolism using metabolic chambers and indirect calorimetry in female TLR4-expressing (TLR4+) and TLR4-deficient (TLR4) NOD mice. Glucose tolerance was assessed in $6 \mathrm{~h}$ fasted mice by intraperitoneal glucose tolerance tests (ipGTT). Mitochondrial function was determined by high-resolution respirometry. TLR4-NOD mice developed diabetes earlier in life $(152 \pm 25 \mathrm{~d})$ than TLR4 + NOD mice $(208 \pm 40 d, p<0.001)$. Prediabetic TLR4 animals had a lower respiratory quotient (TLR4- $0.89 \pm 0.3$; TLR4 ${ }^{+} 0.98 \pm 0.05, p<0.001$ ) than TLR4+mice in periods of activity. Moreover, TLR4mice had a lower glucose tolerance (area under the glucose curve: TLR4 $587 \pm 152$, TLR4+

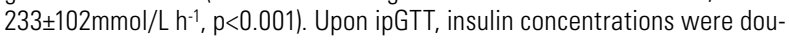
bled in TLR4 compared to TLR4+mice. Fasting serum free fatty acids were 40\% higher in TLR4- than in TLR4+mice. In TLR4-NOD mice, hepatic but not myocellular mitochondria showed increased $\mathrm{O}_{2}$ flux through complex I and II (TLR4- $54 \pm 14$, TLR4 $^{+} 29 \pm 7\left(\mathrm{pmol}^{\mathrm{mg}} \mathrm{s}^{-1}\right) / \mathrm{mtDNA}$ copy number, $\left.\mathrm{p}<0.05\right)$ and higher maximal respiratory capacity (TLR4 $87 \pm 18$, TLR $^{+} 56 \pm 23\left(\mathrm{pmol}^{-1} \mathrm{mg}^{-1}\right.$ $\left.\mathrm{s}^{-1}\right) / \mathrm{mtDNA}$ copy number, $\left.\mathrm{p}<0.01\right)$ compared to TLR4+NOD mice. Taken together, TLR4 deficiency decreases insulin sensitivity resulting in increased substrate availability and subsequently enhanced $\mathrm{O}_{2}$-flux in liver mitochondria. This indicates that TLR4 controls the progression of immune-mediated diabetes in NOD mice by impairing insulin sensitivity.

320-0R The Important Role of MyD88 in Dendritic Cells in Obesity and Metabolic Syndrome

YUFEI XIANG, XIAOYAN XIAO, CHEN CHAO, JIAN PENG, YIPENG WANG, ZHIGUANG ZHOU, ROBERT SHERWIN, RUSLAN MEDZHITOV, F. SUSAN WONG, LI WEN, New Haven, CT, Changsha, China, Cardiff, United Kingdom

To study innate immunity and dendritic cells in obesity and metabolic syndrome, we generated transgenic mice expressing MyD88 only in CD11c+cells, designated as CD11CMyD88+/+MyD88-/- mice. We used a standard high fat diet induced obesity (HFDIO) model. Young male CD11cMyD88+/+MyD88-/mice ( $n=18)$, control MyD88-/- mice ( $n=18)$ and wild type B6 mice ( $n=24)$ were fed with HF diet for 16 weeks. A further 3 groups of age and gender matched mice were fed with conventional diet ( $C D, n=13-17 /$ group). We found that the body weight (BW) of MyD88-/- mice was significantly lower than B6 mice after HFD for 6 weeks $(P<0.05)$, whereas there was no obvious difference in BW between B6 and MyD88-/- mice on CD. CD11cMyD88+/+MyD88-/mice and $\mathrm{B} 6$ mice gained a similar amount of weight on HFD and they were significantly heavier than MyD88-/- mice $(P<0.05)$ after 6 wks on HFD. We further investigated metabolic function after HFDIO. Glucose (IPGTT) and insulin tolerance tests (ITT) showed that MyD88-/- mice were sensitive to insulin regulation, whereas WT B6 and CD11cMyD88+/+MyD88-/- mice showed more insulin resistance and there was no difference between $B 6$ and CD11cMyD88+/+MyD88-/- mice. Similarly, serum levels of triglyceride, total cholesterol, HDL-ch, LDL-ch, adiponectin, leptin, frbrinogen and insulin, were significantly different in MyD88-/- mice compared to B6 or CD11CMyD88+/+MyD88-/- mice but there was no difference between B6 and CD11cMyD88+/+MyD88-/- mice. Furthermore, we found that primary epididymal adipose stromal cells from B6 and CD11cMyD88+/+MyD88-/- differentiated into more mature adipoctyes than cells from MyD88-/- mice. B cell response from B6 and CD11cMyD88 +/+MyD88-/- mice were also stronger compared to MyD88-/- mice but the number of Treg cells were lower in B6 or CD11cMyD88+/+MyD88-/- mice than that from MyD88-/-B6 mice. Our study suggests that expression of MyD88 only in DCs affects the phenotype of HFDIO and metabolic syndrome. Further functional studies will determine the mechanism by which this occurs.

Supported by: NIH and JDRF
Selective PI3Kס Inhibitors as Anti-NASH Agents

SWAROOP VAKKALANKA, MEYYAPPAN MUTHUPPALANIAPPAN, BABU G, KANTHIKIRAN VARANASI, KASIVISWANATH ROUTHU, SEETA NYAYAPATHY, SRIDHAR VEERARAGHAVAN, SRIKANT VISWANADHA, DHANAPALAN NAGARATHNAM, La Chaux de Fonds, Switzerland, Hyderabad, India, Hamden, CT

Non-alcoholic steatohepatitis (NASH) represents a stage of advanced hepatic injury associated with metabolic disorders such as obesity, Type-2 diabetes, and dyslipidemia. The objective of this study was to explore the therapeutic potential of RP5090, a novel, selective, small molecule PI3K $\delta$ inhibitor in the pathogenesis of NASH. Activity of RP5090 on individual PI3K isoforms was determined by a Homogenous Time Resolved Fluorescence assay (Millipore, Billerica, MA) with modifications. Potency of the compound on the delta isoform was further corroborated in FceR1 induced CD63 expression studies using human whole blood and anti-lgM induced human B-cell proliferation assays. Ability of the compound to suppress LPS induced pro-inflammatory cytokines such as TNF $\alpha$ and IL-1 $\beta$ in THP-1 cells was determined. Pre-clinical efficacy of the molecule $(20 \mathrm{mg} / \mathrm{kg} / \mathrm{bid})$ was studied in a methionine-choline deficient diet induced NASH model in C57BL/6J mice. Pharmacokinetic behaviour of compounds in plasma after single dose oral administration was determined in Balb/c mice and Wistar rat. RP5090 demonstrated significant potency against PI3K $\delta(18.3 \mathrm{nM})$ with several fold selectivity over the $\alpha$ $(>485), \beta(>60)$, and $\gamma(>50)$ isoforms. Additionally, the compound inhibited B-cell proliferation (63.5 $\mathrm{nM}$ ) and FceR1 induced CD63 expression in human whole blood basophils $(94.7 \mathrm{nM})$ indicating specificity towards the delta isoform. Half-maximal inhibition of LPS-induced TNF $\alpha$ and IL- $1 \beta$ by RP5090 was achieved at 132.2 and $103.1 \mathrm{nM}$ respectively. RP5090 displayed excellent efficacy in inhibiting MCD-induced NASH in mice manifested by a significant reduction in liver weight ratio (88\%) and liver hydroxyproline content (100\%). Pharmacokinetic studies indicated good oral bioavailability of RP5090 with a favourable half-life in mouse and rat. Results demonstrate the therapeutic potential of selective PI3K $\delta$ blockade by small molecule inhibitors in NASH. Additional in vivo studies in other NASH models are in progress prior to initiation of clinical trials.

322-0R

Activation-Induced Cytidine Deaminase Deficiency Exacerbates Diet-Induced Obesity in Mice

HEYUAN DING, CHANGYUN HU, JIAN PENG, XIAOJUN ZHANG, F. SUSAN WONG, LI WEN, New Haven, CT, Cardiff, United Kingdom

Activation-induced cytidine deaminase (AID) is a B lymphocyte specific DNA deaminase and plays an important role in antibody class switch and the formation of the secondary antibody repertoire of B cells. AID, therefore, is critical for immune responses and immune diseases. However, little is known the role of AID and obesity and its associated metabolic dysfunction. We hypothesize that $B$ cell repertoire is associated with inflammation and may be important in obesity and its associated metabolic dysfunction. To test our hypothesis, we evaluated metabolic and inflammatory phenotypes of high-fat diet induced obesity (DIO) in AID-/- and control wild type (WT) mice. Interestingly, we found that AID-/- mice are more susceptible to DIO. AID deficiency promotes insulin resistance, glucose intolerance and obesity-induced hepatic steatosis. The AUC for ITT was $1650.8 \pm 101.2 \mathrm{mg} / \mathrm{dL}$ . $\mathrm{h}$ in DIO AID-/- mice compared with $1198.5 \pm 112 \mathrm{mg} / \mathrm{dL} \cdot \mathrm{h}$ in DIO WT mice $(P<0.05)$. The AUC for IPGTT was $9971.7 \pm 922.8 \mathrm{mg} / \mathrm{dL} \cdot \mathrm{h}$ in AID-/- mice compared with $6142.4 \pm 850.1 \mathrm{mg} / \mathrm{dL} \cdot \mathrm{h}$ in WT mice $(P<0.05)$. AID deficiency increases the level of IgM antibody and immune cell activation. In high-fat diet (HFD)-treated AID-/-mice, the serum IgM concentrations were significantly higher than those in WT mice, and as expected, serum IgA, IgG1, IgG2a, $\lg \mathrm{G} 2 \mathrm{~b}$ and IgG3 levels were almost absent in AID-/-mice. Interestingly, we found the similar Ig profiles in the intestinal contents. Furthermore, AID-/mice had a more inflammatory phenotype than WT mice, which included higher levels of macrophage $(\mathrm{CD} 11 \mathrm{~b}+\mathrm{CD} 11 \mathrm{c}+\mathrm{F} 4 / 80+)$ infiltration in white adipose tissue (WAT); higher number of CD103+CD11c+ DCs in Payer's patches (PPs); higher levels of IFN- $\gamma+$ T cells (both CD4+ and CD8+) in both lymphoid tissue and WAT and higher levels of circulating inflammatory cytokines. In summary, our study demonstrates that the repertoire of class switched immunoglobulins play an important role in obesity-induced inflammation and metabolic dysfunction.

Supported by: NIH and JDRF 
323-OR

Vaccination Against FABP4 for Preventing High-Fat-Induced Insulin Resistance and Atherosclerosis in Mice

XINYE JIN, DAJIN ZOU, Shanghai, China

Adipocyte fatty-acid-binding protein(FABP4) is expressed in adipocytes and macrophages. Studies have shown that inhibiting its function can improve metabolic syndrome. Here we constructed vaccines against FABP4. Two carriers were selected, which were Virus-like particles formation by modified Hepatitis $B$ virus core $(\mathrm{HBC})$ and keyhole limpet hemocyanin $(\mathrm{KLH})$, and 3 types of vaccines were constructed: FABP4,HBc-FABP4 and FABP4-KLH. C57 mice were immunized respectively, and produced antibody in all groups,among which antibody titer in FABP4-immunized group was the highest. FABP4immunized mice with high-fat diet (treating group) were compared with adjuvant-injected mice with high-fat diet(control group) and mice with normal diet(normal group) in the 20th week after first immunization. Weight,foodintake,triglyceride,total cholesterol, fasting insulin,HOMA-IR,120' bloodglucose and insulin levels in treating group were lower than those in control group $(\mathrm{P}<0.05)$, while had no difference with those in normal group $(P>0.05)$.There was no difference in fasting blood-glucose levels between treating and control group $(P>0.05)$,which were higher than that in normal group $(P<0.05)$ but still within the normal limit. There was no difference in free fatty-acid levels among the groups. After executing mice,we made pathological sections with their livers, pancreases and thoracic aortas, which showed that in treating group,the areas of fat deposition in liver and arterial plaque in aortas were smaller than those in control group $(\mathrm{P}<0.05)$. But such difference was not found in pancreas. In conclusion,we successfully constructed vaccines against FABP4 which could induce specific humoral immunity and protect mice against the development of obesity, hyperlipoidemia, hyperinsulinemia,fatty liver and atherosclerosis induced by high-fat diet.0ur study has offered theoretical and experimental basis for a brand-new immunologic intervention targetting to FABP4.

Supported by: CMA

\section{ADIPOCYTE BIOLOGY}

324-OR

Adipose Tissue-Specific Deletion of BMP-Receptor Type 1A Results in a Reduced Macrophage Infiltration Profile and Improved Systemic Insulin Sensitivity

TIM J. SCHULZ, LINDSAY E. MCDOUGALL, TIAN LIAN HUANG, YUJI MISHINA YU-HUA TSENG, Boston, MA, Ann Arbor, MI

Adipose tissue inflammation is a hallmark process during obesity that is thought to contribute to insulin resistance and the development of diabetes. Recent findings have implicated signaling through type $1 \mathrm{~A}$ BMP-receptor (BMPR1A) in adipose tissue development and indicated its potential impact on human obesity. To investigate the function of BMPR1A in adipose tissue physiology and whole body energy metabolism, we generated a mouse model with conditional deletion of BMPR1A using the Cre/loxP system: Two mouse strains with adipocyte-specific expression of Cre-recombinase, under the control of either the adiponectin- or the fatty acid binding protein (FABP)4-promoter, were intercrossed with a mouse strain carrying a floxed allele of the Bmpr1A gene. The resultant knock-out mice displayed significantly improved insulin sensitivity and reduced expression of inflammatory cytokines, such as interleukins and interferon- $\gamma$. A concomitant reduction of macrophage marker expression, such as F4/80 and CD68, and macrophage infiltration in adipose tissue was observed during high fat diet feeding. Moreover, lower levels of adipose expression of leptin and reduced circulating levels of leptin and insulin were observed in knock-out mice. Aside from its central role in appetite regulation, leptin is known to act as a pro-inflammatory factor. We therefore propose that reduced leptin production in BMPR1A-deficient adipocytes leads to reduced macrophage infiltration which counteracts the onset of insulin resistance in diet-induced obesity. This effect in turn is associated with enhanced systemic insulin sensitivity during high fat feeding in mice with impaired BMP signaling. Taken together, these findings reveal a novel role for BMP signaling in the regulation of systemic glucose homeostasis by regulating the endocrine function of adipocytes and the inflammatory response during metabolic challenges such as obesity.
325-OR

RBP4 Inhibits Insulin Signaling in Adipocytes by Inducing Pro-Inflammatory Cytokines in Macrophages through a JNK- and TLR4Dependent and Retinol-Independent Mechanism

JULIE NORSEEN, TETSUYA HOSOOKA, ANN HAMMARSTEDT, MARK M. YORE, SHASHI KANT, PRATIK ARYAL, HIROSHI MARUYAMA, BETTINA J. KRAUS, ANNY USHEVA, ROGER J. DAVIS, ULF SMITH, BARBARA B. KAHN, Boston, MA, Gothenburg, Sweden, Worcester, MA

Retinol-binding protein 4 (RBP4) is secreted from adipocytes and liver. Serum RBP4 levels correlate highly with insulin resistance and cardiovascular risk factors. Elevated serum RBP4 levels cause insulin resistance. Our aim was to determine the molecular mechanisms for this. We show that RBP4 induces pro-inflammatory cytokines (TNF $\alpha$, IL6, MCP1) in mouse and human macrophages and this causes insulin resistance in co-cultured adipocytes. Incubation of adipocytes alone with RBP4 does not alter insulin-stimulated Akt phosphorylation but RBP4 incubation of adipocytes co-cultured with macrophages inhibits insulin signaling in adipocytes. Blocking antibodies or soluble receptors against TNF $\alpha$, IL6 and MCP1 block RBP4-induced insulin resistance in adipocytes co-cultured with macrophages. This effect occurs partially by activation of the JNK and Toll Like Receptor 4 (TLR4) pathways since RBP4-induced cytokine production is markedly attenuated by JNK inhibitors and in both JNK1-/-/JNK2-/- macrophages and TLR4-/macrophages. In preliminary studies, chronic RBP4 treatment in normal mice increases cytokine expression and macrophage infiltration in perigonadal fat and causes systemic insulin resistance. Since retinol metabolites are signaling molecules, we investigated if these effects are retinol-dependent Unexpectedly, retinol-free RBP4 (apo) induces pro-inflammatory cytokines as potently as retinol-bound RBP4 (holo) in human and mouse macrophages. These effects are not mediated by the RBP4 receptor, STRA6, since STRA6 is not expressed in mouse or human macrophages. Our results show that RBP4 may cause insulin resistance by contributing to the development of an inflammatory state in adipose tissue through activation of pro-inflammatory cytokines in macrophages. This process reveals a novel JNK- and TLR4-dependent and retinol-independent mechanism of action for RBP4.

Supported by: NIDDK F32 DK091041 (to J.N.) and NIDDK R37 DK43051 (to B.B.K)

3-Phosphoinositide-Dependent Protein Kinase 1 (Pdk1) Regulates Macrophage Migration, Polarization, and Insulin Sensitivity YOSHINAGA KAWANO, JUN NAKAE, HIROSHI ITOH, Tokyo, Japan

The 3-phosphoinositide-dependent protein kinase 1 (Pdk1) plays important roles in the regulation of glucose and energy homeostasis. However, little is known about Pdk1 in adipose tissue macrophage (ATM) and resident Kupffer cell in liver. We have reported that Foxo1, which is downstream of and inhibited by Pdk1, in ATM induces $C$ - $C$ motif chemokine receptor 2 (Ccr2) expression and insulin resistance. To investigate the role of the Pdk1/Foxo1 pathway in macrophages, we generated macrophage/granulocyte-specific Pdk1 knockout (LysMPdk1\%) and/or transactivation-defective Foxo1 ( $\Delta 256 \mathrm{Fox} 01)$ transgenic $\left(\triangle 256\right.$ LysMPdk1 $\left.{ }^{-}\right)$mice. LysMPdk1\% exhibit glucose intolerance and insulin resistance under normal chow diet. Insulin-stimulated IRS1 or IRS2 and Akt phosphorylation were significantly decreased in epididymal fat and liver of $\mathrm{LysMPdk}^{-}$compared to control mice. F4/80+CD11 $\mathrm{c}^{+} \mathrm{CD} 206^{-}$cells in epididymal fat of $L y s M P d k 1 \%$ were significantly increased compared to control mice. However, F4/80+Kupffer cells in liver were significantly decreased. Furthermore, expression levels of II4 and II10 were significantly decreased and bone marrow derived $\mathrm{Cd}_{1} 1 \mathrm{~b}^{+}$cells were significantly increased in liver of LysMPdk1\%. LysMPdk1\% exhibit increased Ccr2 expression in stromal vascular fraction (SVF) and peritoneal macrophage. Transwell migration assays revealed that Pdk1-deficient bone marrow derived macrophages (BMDM) exhibited significantly increased capacity of migration compared to BMDM from control. Moreover, deletion of Pdk1 or activation of Foxo1 in BMDM abolished insulin- or IL-4-stimulated induction of genes for alternative activation. Overexpression of transactivation-defective Foxo1 rescues these phenotypes. These data suggested that Pdk1-Foxo1 pathway regulates migration and polarization of macrophage and plays an important role in the regulation of insulin sensitivity in vivo. 
Targeting Neutrophils in Adipose Tissue Inflammation and Type 2 Diabetes

NOZOMU KAMEI, YASUHIKO YAMAMOTO, TAKESHI SHIMADA, HYUEK JONG LEE, JONGSOON LEE, STEVEN E. SHOELSON, Boston, MA, Tokyo, Japan, Kanazawa, Japan, Wakayama, Japan

Elevated WBC counts predict incident T2D and CVD and poor outcomes. More recent studies focused on leukocytes in adipose tissue (AT). Macrophages (ATMs) predominate, but T and B cells also increase with obesity in rodents. These findings suggest that obesity-induced inflammation is more similar to 'classical' inflammation - the response to infections and sterile necrosis - than previously recognized. In acute inflammation, neutrophils (Neu) are recruited in hours, and macrophages are called in later. Despite a relative paucity in AT, we hypothesized that Neu play important roles in insulin resistance and T2D and that macrophage accumulation in AT depends on the earlier recruitment of $\mathrm{Neu}$. Using flow cytometry we found that Neu increase $4-5$ fold during the induction of obesity at earlier times than ATMs. To test if Neu are important for insulin resistance and T2D, we used 6 distinct manipulations: Ab neutralization, 2 knockout models, and 3 different drugs. None affected body weight or feeding under study conditions but all improved glycemia and lowered insulin levels. Ly6G Ab is often used to selectively deplete Neu; numbers in the circulation and spleen were reduced $50-75 \%$. There was no affect in chow fed mice but in HFD mice both glucose (71 vs 82 $\mathrm{mg} / \mathrm{dl}$, Ly6G vs Ctrl Ab) and insulin (0.37 vs. $0.72 \mathrm{ng} / \mathrm{ml})$ levels were reduced $(P<0.05)$. The leukotriene LTB4 is a potent Neu chemoattractant. LTA4H catalyzes its formations, and BLT1 is its receptor. Ltb4-/- and -/+ HFD mice and Blt1-/- and -/+ HFD mice had dramatically lower glucose and insulin levels than littermate wt mice. Drugs also target LTB4: zileuton and bestatin inhibit biosynthetic enzymes 5-LO (5-lipoxigenase) and LTA4H, whereas CP105696 inhibits the receptor BLT1. Each drug reduced AT Neu number and blood glucose and insulin levels $(P<0.05)$. These findings suggest that AT neutrophils have previously unrecognized local roles in AT inflammation and T2D and provide potential new therapeutic avenues for treatment.

328-0R

Epigenetic Regulation of Macrophage Polarization by DNA Methylation in Obesity

BINGZHONG XUE, XIANFENG WANG, HANG SHI, Winston-Salem, NC

Obesity is associated with increased classically activated M1 adipose tissue macrophages (ATMs) and reduced alternatively activated M2 ATMs, which contributes to insulin resistance. However, the underlying mechanism is unclear. Epigenetic mechanisms play important roles in complex diseases including obesity and insulin resistance. However, little is known whether and how epigenetic mechanisms regulate macrophage polarization and inflammation in obesity. Here we find that inhibiting DNA methylation pharmacologically by 5 -aza-2'-deoxycytidine (5-azadC) or genetically using macrophages from myeloid-specific DNA methyltransferase 1 (DNMT1) knockout mice (MD1KO) results in M2 macrophage polarization, evidenced by up-regulation of M2 marker, such as arginase I (ARG1), mannose receptor, Dectin-1, programmed cell death 1 ligand 2, interleukin 1 receptor antagonist, interleukin 10, and the nuclear receptor peroxisome proliferator-activated receptor g1 (PPARg1), key regulator of M2 macrophage activation; whereas over-expressing DNMT1 profoundly suppresses interleukin 4-induced ARG1 and PPARg1 expression. PPARg1 promoter is enriched with $\mathrm{CpG}$ sites. Inhibiting DNA methylation by 5 -azadC significantly decreases, whereas saturated fatty acids (SFAs) and pro-inflammatory cytokines, levels of which are usually elevated in obesity, significantly increase PPARg1 promoter DNA methylation. Finally, MD1KO mice have significantly improved insulin sensitivity without altering body weight. In summary, DNA methylation plays an important role in regulating macrophage polarization. Inhibiting DNA methylation at PPARg1 promoter promotes M2 macrophage polarization; whereas in obesity, elevated SFAs and pro-inflammatory cytokines enhance PPARg1 promoter DNA methylation, which contributes to deregulated ATM polarization, inflammation and insulin resistance in obesity.

Supported by: AHA

329-0R

Peroxisomal Lipid Biosynthesis Regulates Adiposity and Metabolism through PPARy Signaling

IRFAN J. LODHI, LI YIN, ANNE P. JENSEN-URSTAD, TREY COLEMAN, CLAY F. SEMENKOVICH, St. Louis, MO

Options for treating obesity and diabetes are limited. Peroxisome proliferator-activated receptors (PPARs) are ligand-activated transcription factors involved in metabolism and inflammation. This receptor family was originally

For author disclosure information, see page 797.

identified and named based on activation by chemicals promoting peroxisomal proliferation. PPAR $\gamma$ is critical for normal adipose tissue function and is activated by glitazones, diabetes drugs with side effects that include increased adiposity and heart failure. Endogenous pathways of PPAR $\gamma$ activation are unclear. We demonstrate that a lipogenic pathway encompassing the multifunctional enzyme fatty acid synthase (FAS) and an enzyme encoded by a cDNA not previously cloned in mammals, PexRAP (for Peroxisomal Reductase Activating PPAR $\gamma$ ), modulates endogenous PPAR $\gamma$ activation. Mice lacking FAS in adult adipose tissue resisted diet-induced adipocyte hypertrophy and obesity through mechanisms that did not involve altered food intake. There was no effect on hypothalamic FAS in these animals. FAS knockdown in embryonic fibroblasts decreased PPAR $\gamma$ transcriptional activity and adipogenesis. FAS-dependent alkyl ether phosphatidylcholine species were associated with PPAR $\gamma$ protein in mass spectrometry experiments, and treatment of 3T3-L1 cells with one such ether lipid increased PPAR $\gamma$-dependent transcriptional activity. PexRAP, a protein required for alkyl ether lipid synthesis, was physically associated with peroxisomes and its abundance was induced during adipogenesis. PexRAP knockdown in cells decreased PPAR $\gamma$ transcriptional activity and adipogenesis. In mice fed a high fat diet, antisense oligonucleotide-mediated knockdown of PexRAP decreased expression of PPAR $\gamma$-dependent genes, reduced adiposity, increased leanness, and improved insulin sensitivity as compared to control mice. These findings suggest that inhibiting PexRAP or related lipogenic enzymes could treat obesity and diabetes.

330-0R

Adipose Tissue Specific Deletion of the Mitochondrial Transcription Factor A (TFAM) Causes Lipodystrophy and Hepatosteatosis CECILE VERNOCHET, C.R. KAHN, Boston, MA

Mitochondrial dysfunction in adipose tissue has been reported in obesity, type 2 diabetes and metabolic syndrome, but whether this dysfunction participates in the development or is a result of these disorders remains an open question. Mitochondrial transcription factor A (Tfam) plays a key role in mitochondrial function by controlling both mtDNA stability and transcription. To investigate the physiological consequences of mitochondrial impairment in adipose tissue, we generated a mouse deficient for Tfam specifically in adipocytes (Ad-TFKO) using Adiponectin-Cre and Tfam floxed allele. Ad-TFKO mice are leaner than control mice on normal chow diet (CD) by $16 \%$ and $28 \%$ less after 6 weeks upon HFD. In the CD, Ad-TFKO mice brown adipose tissue was almost inexistent and completely overtaken by white adipose tissue, whereas subcutaneous white fat was reduced by $61 \%$ and perigonadal fat by $52 \%$. Ad-TFKO mice also had $14 \%$ decreased in basal energy expenditure with no significant change in spontaneous activity. Interestingly, fed glucose levels in Ad-TFKO mice were elevated by 35\% compared to controls but fasting glucoses were similar. Ad-TFKO also exhibited a similar glucose clearance during an insulin tolerance test but remained hyperglycemic compared to control throughout. Ad-TFKO has a 2 fold increase in liver mass and histology revealed marked triglycerides accumulation. Thus TFAM KO in fat impaired white and brown adipose tissue expansion and provoked lipid localization to the liver leading to hepatosteatosis and systemic insulin resistance. These results thus suggest that mitochondria dysfunction in adipose tissue could indeed participate and accelerate development of metabolic syndrome.

331-0R

Effect of a Point Mutation in the c-fos Gene Promoter in a Patient With Congenital Generalized Lipodystrophy

BIRGIT KNEBEL, JÖRG KOTZKA, STEFAN LEHR, SYLVIA JACOB, ULRIKE NITZGEN, DIRK MÜLLER-WIELAND, Düsseldorf, Germany, Hamburg, Germany

Congenital generalized lipodystrophy (CGL) are rare genetic syndromes characterized by the absence of adipose tissue. CGL patients' show pathological systemic lipid levels, lipid accumulation in non-adipose tissues mainly the liver and most complications of the metabolic syndrome. Therefore genetic defects associated to CGL resemble candidate genes also for the metabolic syndrome. We examined hormone activated signal transduction pathways in isolated fibroblasts of a CGL patient and identified a postreceptor defect altering expression of the immediate early gene c-fos. This signaling defect can be reconstituted by transfecting a wtc-fos promoter thus focusing on a genetic defect and excluding further upstream signaling alterations. Sequence analyses revealed a single homozygous point mutation in the patient's c-fos promoter. Functional analyses of this promoter revealed a by $90 \%$ reduced basal transcription activity Furthermore, stimulatory effects of growth factors and insulin were abolished. The point mutation was located upstream of the well characterized promoter elements in a region with no homology to any known cis-elements. In gel retardation experiments 
the mutation facilitates the formation of a novel and specific protein/DNA complex. Using a mass spectrometry we have identified proteins of this novel complex which belong to the glucose regulated protein family (Grp 78, Grp 90), heterogeneous nuclear ribonucleoproteins hNRNP families and 14-3-3 protein. The results indicate that a genetic defect influencing the regulation of a ubiquitous transcription factor can be valuable to dissect novel mechanisms and candidate genes involved in metabolic syndrome.

\section{DIABETES AND MACROVASCULAR DISEASE- FROM BIOMARKERS TO THERAPEUTICS}

332-0R

Insulin Sensitizers May Reduce the Risk of Peripheral Arterial Disease in Type 2 Diabetes: Results from the Bypass Angioplasty Revascularization Investigation 2 Diabetes (BARI 2D) Trial

ANDREW D. ALTHOUSE, MARIA M. BROOKS, J. DAWN ABBOTT, ALAN FORKER, LUISA V. BUITRON, JEAN-CLAUDE TARDIF, KIM SUTTON TYRRELL, THE BARI 2D STUDY GROUP, Pittsburgh, PA, Providence, RI, Kansas City, MO, Mexico City, Mexico, Montreal, QC, Canada

Patients with type 2 diabetes mellitus (T2DM) have a high risk of developing peripheral arterial disease (PAD). It is thought that insulin sensitizing medications may confer an advantage over insulin providing medications for prevention of $\mathrm{PAD}$, but this has never been proven in a randomized controlled trial. The BARI 2D trial randomly assigned T2DM patients to insulin sensitizing (IS) or insulin providing (IP) glycemic control strategy. We hypothesized that patients assigned to IS therapy would experience fewer incident cases of PAD than patients assigned to IP therapy. PAD status was assessed annually using the ankle-brachial index (ABI). Patients with abnormal $A B I$ at study entry were excluded from this analysis. Incident PAD was defined as a new low $\mathrm{ABI}(<0.9)$ during follow-up. Secondary endpoints include lower extremity revascularization and amputation. We analyzed the incidence of PAD in 1,389 BARI 2D patients that had normal $A B I(0.9<A B \mid<1.3)$ at study entry and at least one follow-up $A B I$ measurement. 293 of these patients $(21.1 \%)$ were diagnosed with incident PAD during an average of 4.6 years of follow-up. The incidence of PAD was significantly lower among patients assigned to IS therapy than those assigned to IP therapy (17.6\% vs. $24.6 \%$, $\mathrm{p}=0.001)$. The difference was statistically significant with stratification for baseline insulin use (IS vs. IP difference for patients on insulin at baseline: $21.7 \%$ vs. $33.5 \%, p=0.013$; IS vs. IP difference for those not on insulin at baseline: $16.2 \%$ vs. $21.6 \%, p=0.026)$. Patients assigned to IS therapy also required fewer amputations $(0.1 \%$ vs. $1.9 \%, \mathrm{p}=0.001)$ and fewer lower extremity revascularizations $(1.2 \%$ vs. $2.4 \%, p=0.069)$ than patients assigned to IP therapy. In summary, a glycemic control strategy of IS therapy resulted in a significantly lower incidence of PAD and related clinical outcomes than a strategy of IP therapy in this cohort of patients with T2DM.

Supported by: NHLBI with support from NIDDK, GSK, and other pharmaceutical companies

333-OR

The GLP-1 Metabolite GLP 9-37 Increases Atherosclerotic Lesions In Mice and is Associated With Coronary Atherosclerosis in Humans KATJA PIOTROWSKI, MELANIE BECKER, JULIA ZUGWURST, INGEBORG BILLERFRIEDMANN, GERALD SPÖTTL, JULIA MÖLLMANN, MARTIN GREIF, ALEXANDER W. LEBER, ALEXANDER BECKER, ANNE TAILLEUX, RÜDIGER P. LAUBENDER, CORINNA LEBHERZ, BART STAELS, BURKHARD GÖKE, NIKOLAUS MARX, KLAUS G. PARHOFER, ULI C. BRÖDL, MICHAEL LEHRKE, Munich, Germany, Aachen, Germany, Lille, France

GLP-1 (7-36 amide or 7-37) is a gastrointestinal hormone which improves glucose metabolism by augmenting glucose dependent insulin secretion from the pancreatic beta cell. In addition, a variety of extra-pancreatic effects have been described for GLP-1 which include vasoprotective and anti-inflammatory actions. GLP-1 is rapidly inactivated in a DPP-4 dependent manner. The thereby created GLP-1 metabolite (GLP 9-36 amide or 9-37) is unable to activate the GLP-1 receptor but still has overlapping cardiovascular effects, suggesting the presence of an additional receptor system. The cardiovascular relevance of the GLP-1 metabolite in human biology is unknown. Total circulating GLP-1, mainly consisting of the GLP-1 metabolite (9-36 amide or 9-37), was assessed in a cohort of 303 patients receiving CT angiography due to typical or atypical chest pain. GLP-1 levels were significantly associated with total atherosclerotic plaque burden in simple (OR: 1.22; $95 \% \mathrm{Cl}$ : 1.03-1.48; $p=0.03$ ): and fully adjusted models (OR of 1.30 (95\% Cl: $1.06-1.65 ; \mathrm{p}=0.02$ ). To determine whether GLP-1 or its metabolite play a role in atherogenesis we overexpressed both, GLP-1 (7-37) and GLP (9-37) in atherosclerosis-prone LDL receptor deficient mice. GLP (9-37) but not GLP-1
(7-37) increased atherosclerotic lesion formation in mice. This was at least partially attributable to elevation of non-HDL serum cholesterol concentrations in GLP (9-37) treated animals. The GLP-1 metabolite (9-37) causes atherosclerosis in mice and is associated with atherosclerosis in humans. These initial observations should prompt further investigations to clarify the relevance of the GLP-1 metabolite in cardiovascular disease.

Supported by: EFSD and the DFG

334-0R

Relationship Between Pericardial, Mediastinal Fat and Whole Body Band Myocardial Glucose Uptake

MARJORIE MOLINA-WILKINS, GEOFFREY D. CLARKE, SANDRA MARTINEZ, JOHN R. KINCADE, FENG DONG, JESUS GARDUNO-GARCIA, SAMIA B. GEEVARGHESE, VERNA MENDEZ, ADRIANA MONROY, RUCHA J. MEHTA, CAROLINA SOLIS-HERRERA, MUSTAFA KANAT, AURORA MEROVCI, STEVEN R. BAILEY, ROBERT J. CHILTON, PATRICIA IOZZO, MUHAMMAD ABDUL-GHANI, RALPH A. DEFRONZO, San Antonio, TX, Pomona, CA, Pisa, Italy

Previous studies have demonstrated a strong relationship between mesenteric fat and whole body insulin resistance. No previous study has examined the relationship of thoracic and pericardial fat to insulin resistance. In the present study, we measured whole body insulin sensitivity, myocardial glucose uptake, pericardial fat and mediastinal fat in 25 healthy NGT individuals (age $=45 \pm 10$ year, $\mathrm{BMI}=26.6 \pm 3.8 \mathrm{~kg} / \mathrm{m}^{2}, \mathrm{HbA} 1 \mathrm{c}=5.4 \pm 0.3 \%$ ) and 25 type 2 diabetic individuals $\left(52 \pm 9\right.$ years, BMl $31.5 \pm 4.3 \mathrm{~kg} / \mathrm{m}^{2}, \mathrm{HbA} 1 \mathrm{c}=$ $7.1 \pm 1.6 \%)$ and examined the relationship between pericardial fat content and whole body insulin sensitivity and myocardial glucose uptake. As expected, glucose infusion rate during the euglycemic insulin clamp was significantly decreased in T2DM compared to NGT subjects ( $3.9 \pm 1.6$ vs. $7.6 \pm 2.8$ $\mathrm{mg} / \mathrm{kg} / \mathrm{min}, \rho<0.005)$. Myocardial glucose uptake measured with FDG and PET scan also was significantly decreased in T2DM compared to healthy individuals $(0.27 \pm 0.13$ vs $0.40 \pm 0.16 \mathrm{mmol} / \mathrm{min} / \mathrm{mL}$ tissue, $\rho=0.01)$. Conversely, pericardial and mediastinal content measured with MRI were significantly greater in T2DM compared to healthy individuals $(14.4 \pm 3.5$ and $32.4 \pm 11.9$ vs $8.8 \pm 2.8$ and $17.6 \pm 7.6 \mathrm{~cm}^{2}$, respectively, both $\rho<0.005$ ). Whole body insulin sensitivity was strongly and inversely correlated with both pericardial $(r=-$ $0.69)$ and mediastinal fat $(r=-0.72),(\rho<0.001$ for both). However, surprisingly, myocardial glucose uptake did not have significant correlation either with pericardial or mediastinal fat content. These results demonstrated that pericardial and mediastinal fat exert differential effect on myocardial and whole body glucose uptake.

Supported by: Takeda Pharmaceuticals

335-0R

Increased Arterial Stiffness is Independently Associated With Cerebral Infarctions and White Matter Lesions in Patients With Type 2 Diabetes Despite Good Blood Pressure and Lipid Control

ESBEN LAUGESEN, PERNILLE HØYEM, BRIAN S. GRØN, ANDERS MIKKELSEN, WON Y. KIM, SAMUEL THRYS $\emptyset E$, MOGENS ERLANDSEN, JENS S. CHRISTIANSEN, SØREN T. KNUDSEN, KLAVS W. HANSEN, TROELS K. HANSEN, PER L. POULSEN, Aarhus, Denmark

Patients with type 2 diabetes (T2DM) have increased risk of cardiovascular disease (CVD) including stroke. The risk of CVD is traditionally assessed using office blood pressure (BP) and lipid profile. Increased arterial stiffness predicts cardiovascular events in the general population. We investigated if arterial stiffness was associated with cerebrovascular disease in patients with T2DM and sex- and age-matched controls. 90 patients with newly diagnosed T2DM and 90 sex- and age- matched controls were examined. Arterial stiffness was assessed by aortic pulse wave velocity (PWV), and cerebrovascular disease by cerebral infarctions and severity of white matter lesions (WMLs) on MRI scans of cerebrum. A blinded reviewer rated WMLs a.m. Breteler (no/slight changes $=0$, moderate $=1$, severe $=2$ ). Antihypertensive treatment and lipid lowering treatment was more frequent in diabetic patients, who consequently had lower office BP $(126+/-12$ vs $131+/-14 \mathrm{mmHg}$ systolic, $p=0.01$ ) and lower lipid levels. Despite this, diabetic patients had significantly higher PWV compared to controls, $(9.2+/-2.0$ vs $8.0+/-1.6 \mathrm{~m} / \mathrm{s}$, $p<0.0001)$. PWV was higher in patients with cerebral infarctions ( 9.9 vs 8.5 $\mathrm{m} / \mathrm{s}, \mathrm{p}=0.002$ ) and PWV increased across Breteler categories (8.2+/- $1.7 \mathrm{vs}$ $9.3+/-2.0$ vs $9.4+/-2.1 \mathrm{~m} / \mathrm{s}, \mathrm{p}<0.001$ for trend). PWV remained independently associated with severity of WMLs $(p<0.01)$ and cerebral infarctions, $(p<0.02)$ after adjustment for the following covariates: age, sex, diabetes, mean arterial pressure, smoking, statins and BMI in multivariate regression. Thus, in conclusion, despite good BP and lipid control, PWV was substantially higher in T2DM patients. PWV is independently associated with WMLs and cerebral infarctions. PWV may represent a clinically relevant parameter in the evaluation of CVD risk in T2DM. 
336-OR

Long Term Complications and Mortality in Youth Onset Diabetes: Type 2 Diabetes is More Lethal than Type 1

MARIA CONSTANTINO, LYNDA MOLYNEAUX, FRANZISKA H. GISLER, ABDULGHANI AL SAEED, CONNIE LUO, TED WU, DENNIS K. YUE, JENCIA WONG, Sydney, Australia

Despite an increasing prevalence of youth onset type 2 diabetes (T2DM), few data exist on the long term complications or mortality in this group. We examined both outcomes in 138 patients with T2DM of onset between 15 and 30 years and duration of diabetes $>15$ years $\left(\mathrm{T}^{30}\right)$ and in 208 patients with T1DM selected by identical criteria (T130). Subjects had all attended for a complications assessment. Mortality data were extracted from the National Death Index. Retinopathy was not different between groups but $T 2^{30}$ had higher neuropathy scores and albuminuria ( $p<0.0001$ for both). Despite a greater use of statins and antihypertensives (both $p<0.0001$ ), $T 2^{30}$ show an excess of IHD $(p<0.0001)$, stroke $(p<0.008)$ with reduced survival (Fig 1, $\mathrm{p}=0.002$ ) and a shorter duration before death. Risk factors HbA1c, systolic $\mathrm{BP}, \mathrm{Tg}, \mathrm{HDL}$ and BMI were all worse in $\mathrm{T} 2^{30}$ (Table). By using the same age range for diabetes onset, this study has been able to compare the detrimental long term effects of youth onset T2DM and T1DM, minimizing the otherwise unavoidable confounding effects of age and diabetes duration. These data are evidence for the greater metabolic derangement and long term detrimental effect of youth onset T2DM, highlighting this group for aggressive intervention.

Table 1. Clinical characteristics of T230 and T130 groups

\begin{tabular}{lccl}
\hline $\mathrm{n}=346$ & $\begin{array}{c}\text { Type 2 Diabetes } \\
\mathrm{n}=138 \% \text { males }=54.3\end{array}$ & $\begin{array}{c}\text { Type 1 Diabetes } \\
\mathrm{n}=208 \% \text { males }=61.1\end{array}$ & Statistics \\
\hline Duration of Diabetes (yrs) & $24.1[19-30]$ & $22.9[19-30]$ & $\mathrm{Z}=1.4 ; \mathrm{p}=0.2$ \\
\hline Age of Onset (yrs) & $25.6 \pm 3.8$ & $21.3 \pm 4.2$ & $\mathrm{t}=9.9 ; \mathrm{p}<0.0001$ \\
\hline Age (yrs) & $51.7 \pm 9.0$ & $46.1 \pm 8.6$ & $\mathrm{t}=5.9 ; \mathrm{p}<0.0001$ \\
\hline HbA1c \% & $8.9 \pm 2.0$ & $8.3 \pm 1.6$ & $\mathrm{t}=2.8 ; \mathrm{p}=0.006$ \\
\hline BMl kg/m² & $31.7 \pm 8.0$ & $25.8 \pm 4.0$ & $\mathrm{t}=7.8 ; \mathrm{m}=<0.0001$ \\
\hline Systolic BP $(\mathrm{mm} / \mathrm{Hg})$ & $134 \pm 20$ & 128.19 & $\mathrm{t}=2.4 ; \mathrm{p}=0.02$ \\
\hline $\begin{array}{l}\text { Triglycerides mmol } \\
\text { L HDL mmol/L }\end{array}$ & $1.8[1.2-3.0] 1.2 \pm 0.3$ & $1.1[0.8-1.5] 1.5 \pm 0.5$ & $\mathrm{Z}=6.7 ; \mathrm{p}<0.0001$ \\
& & & $\mathrm{t}=-6.0 ; p<0.0001$ \\
\hline
\end{tabular}

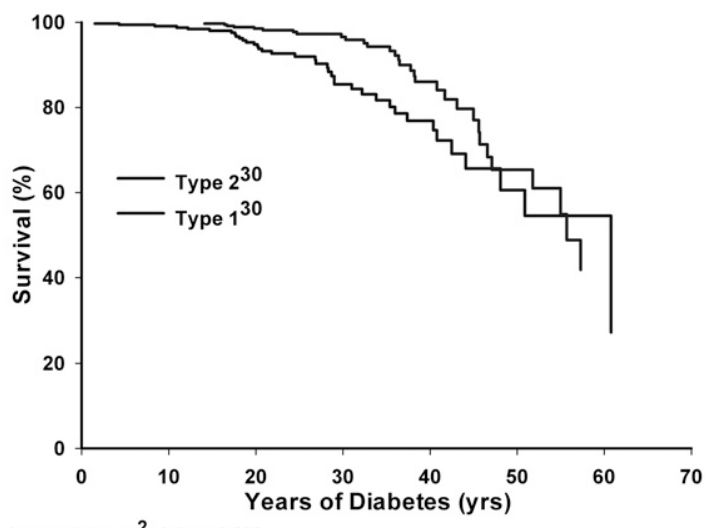

Logrank test $\mathrm{x}^{2}=9.3 ; \mathrm{p}=0.002$

Figure 1. Kaplan Meier Survival Curve to show reduced survival in early onset type 2 diabetes.

\section{$\Delta$} al Dysunction artormin-Induced Changes in Markers of Endotheliin the Diabetes Prevention Program

ELLA TEMPROSA, KIEREN J. MATHER, TREVOR J. ORCHARD, GEORGE A. BRAY, ABBAS E. KITABCHI, SANTICA M. MARCOVINA, LEIGH PERREAULT, ROBERT E. RATNER, RONALD B. GOLDBERG, DPP RESEARCH GROUP, Rockville, MD, Indianapolis, IN, Pittsburgh, PA, Baton Rouge, LA, Memphis, TN, Seattle, WA, Aurora, CO, Hyattsville, MD, Miami, FL

Endothelial dysfunction is present early in the course of type 2 diabetes, where it may be a forerunner of diabetic vascular complications. The relationship of endothelial dysfunction with diabetes development is not well understood. It is also unknown whether interventions that slow diabetes development also improve endothelial dysfunction. We have previously reported that baseline levels of $\mathrm{sE}$-selectin and sICAM, circulating markers of endothelial dysfunction, are strongly associated with incident diabetes in the placebo group. Here we assessed whether 1-year changes in $\mathrm{SE}$ selectin and sICAM induced by lifestyle and metformin interventions, are associated with diabetes incidence over 3.2 years of follow-up, compared to the placebo group. Baseline sE-selectin $(46 \mathrm{ng} / \mathrm{mL})$ and sICAM $(251 \mathrm{ng} / \mathrm{mL})$ concentrations were not different across the 3 groups. At 1 year, sE-selectin fell by $8 \%$ in the lifestyle group (demographic adjusted $p<0.05$ vs placebo and metformin groups), and sICAM fell by $6 \%$ in the lifestyle group ( $p<0.05$ vs placebo and metformin groups) and by $4 \%$ in the metformin group ( $p<0.05$ vs placebo). In separate Cox models for each treatment group with adjustment for baseline levels and demographic factors, change in sICAM was not associated with diabetes development, but a decrease of $11 \mathrm{ng} / \mathrm{ml}(\sim 1 \mathrm{sd})$ in sE-selectin was associated with a reduction in diabetes risk of $14 \%$ to $28 \%$ in the 3 groups. The association between sE-selectin and diabetes development was however no longer significant after adjustment for 1 year changes in insulin sensitivity (1/FI) or BMI in all 3 treatment groups, with greater attenuation of the association with BMI. Thus sE-selectin is not only a significant predictor of incident diabetes at baseline, but its reduction by both lifestyle and metformin is associated with the intervention-driven decreases in diabetes incidence and is explained principally by weight change.

Supported by: NIDDK (U01-DK048489)

\section{BEHAVIORAL DIABETES INTERVENTIONS- RESULTS FROM THE LATEST CONTROLLED TRIALS}

338-OR

Communicating Cardiovascular Risk to Latinos With Type 2 Diabetes Using a Spanish Language-Based Multimedia Program PARIS ROACH, CHANDAN SAHA, DAVID G. MARRERO, Indianapolis, IN

Cardiovascular disease (CVD) is the leading cause of mortality in Latinos with type 2 diabetes (T2DM), yet CVD risk factors (RF) are under-treated in this population. This may result from low awareness of CVD and low literacy among patients and from linguistic barriers, limited encounter time, and "clinical inertia" among providers. To address these barriers, we developed a Spanish-language, tablet computer-based multimedia CVD risk communication intervention consisting of a series of video presentations that patients viewed just prior to outpatient encounters. Each presentation uniquely incorporated individual CVDRF data and 10-year CVD risk based on UKPDS Risk Engine estimates. The program also generated color graphic 1-page printouts showing updated CVDRF status for use during the encounter. The hypothesis that this intervention would stimulate therapeutic intervention (TI), i.e. the discussion of CVD risk during the visit (determined via exit interviews) or a change in medication directed toward CVDRFs (determined via chart audit) was tested in a randomized controlled comparison of the intervention vs usual care in four urban primary care clinics providing care to the local Spanish-speaking Latino population. Participants $(n=144)$ had a mean age of 47 years, $69 \%$ had less than a high school education, and $87 \%$ had annual income less than $\$ 40 \mathrm{~K}$. Patients in the intervention group $(\mathrm{n}=86)$ were exposed to the intervention one to three times during the 12-month follow-up period, and compared to control patients $(n=58)$, had an overall TI rate that was $87 \%$ higher, based on a Poisson regression model: 19.6 vs. $10.5 \mathrm{Tl}$ per patient per 12 months, $\mathrm{p}=0.023 .94-100 \%$ of intervention group patients felt that it facilitated interactions with their providers. This multimedia CVD risk communication intervention resulted in a significant increase in TI aimed at reducing CVDRFs in a Spanish-speaking population with T2DM and was used successfully within the primary care environment.

Supported by: Robert Wood Johnson Foundation

339-0R

Impact of Communicating Genetic and Phenotypic Risk of Type 2 Diabetes (T2D) on Objectively-Measured Physical Activity (PA): The Diabetes Risk Communication Trial

JOB G. GODINO, ESTHER VAN SLUIJS, THERESA M. MARTEAU, STEPHEN SUTTON, STEPHEN J. SHARP, SIMON J. GRIFFIN, Cambridge, United Kingdom

Information about risk of T2D is widely available, yet effects are uncertain and regulation of providers has been proposed. We aimed to assess effects of providing such information on risk reducing health behaviors, anxiety, and cognitive and emotional antecedents of behavior change. We recruited 569 adults aged 39 to 61 years from a population-based observational study in Cambridgeshire, UK. Participants underwent clinical, anthropometric, and 
psychosocial measurements, were genotyped for 23 SNPs associated with T2D, and wore a combined heart rate and movement sensor (Actiheart) continuously for 6 days and nights to assess PA. They were randomized to receive either standard lifestyle advice alone (control group, $\mathrm{n=190)}$ ), or in combination with a genetic $(n=189)$ or phenotypic $(n=190)$ risk estimate for T2D. PA (energy expenditure in $\mathrm{kJ} / \mathrm{kg} /$ day), fruit and vegetable (FV) consumption (g/ day), self-reported weight $(\mathrm{kg})$, intention to increase PA and FV consumption (5-point scale), anxiety (State-Trait Anxiety Inventory), and diabetes related worry (24-point scale) were remeasured 8 weeks after randomization. We assessed intervention effects by regression analysis, adjusted for baseline values. $557(98 \%)$ participants completed the trial. Comparing the combined intervention groups with the control group, no effect was observed on PA $(\beta=1.15,95 \% \mathrm{Cl}:-1.34$ to $3.65, p=0.37)$, FV consumption ( $\beta=-4.08,95 \% \mathrm{Cl}$ 37.3 to 29.1$)$, self-reported weight ( $\beta=-0.06,95 \% \mathrm{Cl}:-0.63$ to 0.51$)$, intentions (PA: $\beta=0.01,95 \% \mathrm{Cl}$ : -0.09 to 0.11 ; FV consumption: $\beta=0.02,95 \% \mathrm{Cl}:-0.07$ to 0.11$)$, anxiety ( $\beta=-0.29,95 \% \mathrm{Cl}:-1.77$ to 1.19$)$, and diabetes related worry $(\beta=0.16,95 \% \mathrm{Cl}:-0.12$ to 0.45$)$. There was no difference in outcomes between intervention groups. Information about genetic or phenotypic risk of T2D is not associated with changes in behavior or anxiety in the short term, and it is unlikely to enhance T2D prevention strategies.

340-0R

The Effect of Community-Based Peer Support in Achieving and Maintaining Weight Loss Following Lifestyle Intervention

GRETCHEN A. PIATT, MIM C. SEIDEL, ROBERT POWELL, JANICE C. ZGIBOR, Ann Arbor, MI, Pittsburgh, PA

The obesity epidemic continues to rise worldwide, putting the health of communities in jeopardy. Effective, sustainable models should be identified and implemented at the community level to address this burden. We aimed to determine the effectiveness of a community-based peer support (CBPS) model in achieving and maintaining weight loss following a lifestyle intervention in 8 rural communities in southwestern, Pennsylvania. Communities and their eligible participants ( $\mathrm{n}=493$; mean age: $51 \mathrm{yrs}, 87.6 \%$ female, $94.1 \%$ Caucasian, $86.8 \% \mathrm{BMI} \geq 30 \mathrm{~kg} / \mathrm{m}^{2}$ ) were assigned to 4 lifestyle groups: Face to Face $(n=119), D V D(n=113)$, internet $(n=101)$, and self-selection $(n=101)$. Self selection participants were empowered to select the modality $(60 \%$ chose face to face, $40 \%$ chose internet, $0 \%$ chose DVD). CBPS was defined as support provided within the context of the community, not within a healthcare organization. Each of the 8 communities had one peer leader $(\mathrm{PL})$ to support participants. Three of the $8 \mathrm{PL}$ were community-based. Data were collected at baseline, 3, 6, and 12 months. At 3 months, CBPS participants lost an average of $1.6 \mathrm{lbs}$ more than non-CBPS participants $(p=0.12)$. As support continued over time, CBPS participants lost an average of $2.2 \mathrm{lbs}$ more at 6 -months ( $p=0.09$ ) and an average of $2.8 \mathrm{lbs}$ more at 12 -months ( $p=0.02)$. After controlling for baseline weight, age, CBPS, SF12 scores, exercise and lifestyle group, CBPS participants were 3.6 times $(\mathrm{p}=0.03)$ more likely to achieve significantly more weight loss at 3 -months, 4.6 times $(p=0.04)$ at 6 -months, and 4.5 times $(p=0.04)$ at 12 -months compared to non-CBPS participants. Despite the lifestyle group, participants who received CBPS achieved and maintained significantly greater weight loss compared to non-CBPS participants. As programs that include PLs are implemented worldwide, increased attention should be placed on the importance of the PL within the context of the community, not just the health system.

Supported by: The Air Force Surgeon General

341-0R

Weight Loss for Metabolic Syndrome in Primary Care: Results from the SHINE Telephone Translation of the Diabetes Prevention Program (DPP)

RUTH S. WEINSTOCK, PAULA M. TRIEF, DONALD CIBULA, LINDA DELAHANTY, PHILIP MORIN, Syracuse, NY, Boston, MA

SHINE (Support, Health Information, Nutrition and Exercise) is a randomized weight loss trial ( $n=257)$ of 2 telephone interventions, based on the DPP, delivered through 5 primary care sites. Telephone delivery was used to increase reach. Participants were randomized to receive the DPP lifestyle change program, participating individually by phone (solo; $n=129 ; 114$ received intervention) or in a group by conference call $(n=128 ; 123$ received intervention). The 16 session DPP curriculum was delivered over 1 year by trained primary care staff, with continued contact in year 2. Six and 12 month data are presented. Participants' data were: mean age $51.8 \mathrm{yrs}, 74.3 \%$ female, $85.6 \%$ white, $12.8 \%$ African American, 1.2\% Hispanic, 44.1\% employed, $22.4 \%$ retired, $53.2 \%$ married, $46.3 \%$ high school graduates, $36.9 \%$ annual income $<\$ 30,000$. At baseline, participants had metabolic syndrome (without diabetes) with mean weight $107.5 \mathrm{~kg}$, BMI $39.3 \mathrm{~kg} / \mathrm{m}^{2}$, waist circumference $118.4 \mathrm{~cm}$, fasting glucose $99.6 \mathrm{mg} / \mathrm{dl}$, blood pressure 128.8/75.6 $\mathrm{mm} \mathrm{Hg}$, triglycerides $145.3 \mathrm{mg} / \mathrm{dl}$, HDL-chol $42.4 \mathrm{mg} / \mathrm{dl}$, LDL-chol $108.1 \mathrm{mg} / \mathrm{dl}$. Intention-to-treat mixed linear model regression using repeated measures was performed. At 6 and 12 months solo treatment participants adjusted mean weight (waist circumference) decreased by $4.1 \mathrm{~kg}(3.4 \mathrm{~cm})$ and $4.6 \mathrm{~kg}$ $(5.1 \mathrm{~cm})$, and in group treatment decreased by $4.8 \mathrm{~kg}(2.7 \mathrm{~cm})$ and $6.0 \mathrm{~kg}(6.0$ $\mathrm{cm})$; all adj $\mathrm{p} \leq .01$. At 1 year, mean $\%$ weight loss from baseline was $4.6 \%$ in both treatments; $40.3 \% / 46.6 \%$ lost at least $5 \%$ weight; $26.9 \% / 37.7 \%$ lost at least $7 \%$ weight and $17.9 \% / 26 \%$ lost at least $10 \%$ weight in the solo/group treatments respectively. There were no significant differences in weight loss between treatment groups. Glucose, lipid and blood pressure levels did not significantly improve. In conclusion, primary care staff can be trained to deliver a DPP translation by telephone that is effective for achieving significant weight loss in both individual and group formats.

Supported by: NIDDK (R18 DK078553)

342-0R

Reducing Diabetes Distress: First Results From The REDEEM Trial LAWRENCE FISHER, DANIELLE HESSLER, JOSEPH MULLAN, RUSSELL GLASGOW, PATRICIA AREAN, UMESH MASHARANI, San Francisco, CA, Bethesda, MD

Diabetes distress (DD) refers to the emotional burdens that are part of the spectrum of patient diabetes experience. DD is common, is distinct from depression and has been associated with a variety of glycemic and behavioral markers. Reducing Distress and Enhancing Effective Management (REDEEM) was a 3 -arm clinical trial to reduce DD among patients with type 2 diabetes. Following baseline assessment, patients were randomized to one of three interventions: a Computer Automated Self-Management program (CASM) that helps establish behavioral goals, address barriers and monitor progress: CASM plus problem-solving therapy (CAPS), a CBT program to address DD directly; and Leap Ahead (LA), an attention control health risk screening. Patients then received 4 live support calls at 2, 4, 6 and 8 weeks, followed by a 4-month assessment, a booster at 5 months, 4 more live support calls at weeks $24,28,36,44$, and final assessment at 12 months. Inclusion criteria were T2DM $>12$ months, moderate DD (>1.5 DDS score), age $>21$, read and speak English, screen out for clinical depression (PH08 $<15)$. Using MLM, ITT analyses analyzed changes in DD at 0-4, and 4-12 months on the Regimen Distress (RD) and Emotional Burden (EB) subscales of the Diabetes Distress Scale. With 0-12 month attrition at 8.4\% and controlling for demographics, significant decreases in both $\mathrm{RD}$ and $\mathrm{EB}$ occurred for the entire sample at 0-4 and 4-12 months ( $p<.002)$, but no significant between-group differences in reductions in $\mathrm{RD}$ or EB occurred for either time period. However, a significant Group By Baseline RD interaction at $0-4$ months $(p=.009)$ indicated that those with high DD at baseline achieved greater reductions in RD in CAPS than in CASM $(p=.01)$ or $L A(p=.007)$. These reductions were uniformly maintained between 4-12 months. There was no significant Group By Baseline EB interaction. Findings indicate that management support alone does not decrease $\mathrm{DD}$, but that a combination of management support and focused DD problem solving significantly reduces $\mathrm{DD}$, with maintenance over time.

Supported by: NIH (DK061937)

343-0R

Diabetes Nurse Case Management and Motivational Interviewing for Change (DYNAMIC Study): Results of a 2-Year RCT

RAQUEL M. ANEL-TIANGCO, ALAN ADELMAN, CHERYL DELLASEGA, ROBERT A. GABBAY, Hershey, PA

Nurse case management (NCM) and motivational interviewing (MI) are two approaches that have been used in the management of chronic medical conditions like diabetes. NCM is a collaborative process that provides and coordinates health care to decrease fragmentation. $\mathrm{Ml}$ is a teachable method to counsel patients on behavior change by enhancing the intrinsic motivation to change. The data thus far on their effects on clinical outcomes have been inconclusive. The aim of this study was to determine if the addition of nurse case management and motivational interviewing to usual primary care would result in improved outcomes over two years in high risk patients with type 2 diabetes. A 2-year randomized controlled trial was conducted in diabetes patients from 12 primary care clinics. Patients were randomized to usual care control $(n=313)$ or the intervention $(n=232)$ who received additional NCM care, including self-management education, MI-guided behavior change counseling and prompting PCPs to implement diabetes guidelines. All nurse case managers received intensive training in $\mathrm{MI}$ with an ongoing assessment of fidelity. At the end of 2 years, systolic BP was better in the intervention group than the control group $(131 \pm 15.9$ vs. $135 \pm 18.2, p<0.05)$. $\mathrm{HbA1c}, \mathrm{LDL}$, and diastolic BP improved in both groups: HbA1c (control group 
$9.1 \%$ to $8.0 \%$, intervention group $8.8 \%$ to $7.8 \%$ ), LDL (control group 127 to $100 \mathrm{mg} / \mathrm{dL}$, intervention group 128 to $102 \mathrm{mg} / \mathrm{dL}$ ), diastolic BP (control group 78 to $74 \mathrm{~mm} \mathrm{Hg}$, intervention group 80 to $74 \mathrm{~mm} \mathrm{Hg}$ ). Screening for complications improved significantly in the intervention group compared to the control group: foot exam, ophthalmologic exam, and nephropathy screening. $\mathrm{NCM}$ and $\mathrm{Ml}$ improved systolic BP and complications screening. The large decrease in $\mathrm{HbA1C}$ and $\mathrm{LDL}$ in the control group may have obscured any further intervention effect. Although nurses prompted providers for medication titration, strategies to reduce provider clinical inertia might also be needed. Supported by: NIDDK (R18-DK067495)

344-0R

Internet Programs for Youth With Type 1 Diabetes (T1D) Improve Outcomes

MARGARET GREY, ROBIN WHITTEMORE, SANGCHOON JEON, SARAH JASER, KATHRYN MURPHY, MELISSA FAULKNER, ALAN DELAMATER, New Haven, CT, Philadelphia, PA, Tucson, AZ, Miami, FL

The purpose of this trial was to compare the effectiveness of TEENCOPE ${ }^{\mathrm{TM}}$ (internet coping skills training) with MANAGING DIABETES (internet education program) for youth with T1D on A1C, self-management, self-efficacy, stress, coping, quality of life (0OL), \& family conflict over 12 month followup. Mediators of improved outcomes were also explored. Teens with T1D $(n=320,11-14$ years) from 4 sites were randomly assigned to one of the programs \& completed baseline, 3,6 , and 12 month data via the internet. A1C was obtained from clinic records. Teens were $12.3 \pm 1.1$ yrs old, with T1D duration $6.1 \pm 3.5$ yrs, A1C $8.3 \pm 1.5 \%, 60 \%$ on pump therapy, $55 \%$ female \& $62 \%$ non-Hispanic White. Groups were comparable at baseline. Participation (78\% completed 4 of 5 sessions) \& satisfaction were high. Data were analyzed with mixed models. Teens in both groups reported a significant increase in diabetes problem-solving $(p<.01)$, goal setting $(p<.01)$, selfefficacy $(p<.001)$, and $00 \mathrm{~L}(p<.001)$ along with a decrease in collaboration with parents $(p<.001)$ and stress $(p<.01)$ after controlling for race, income, gender, age, duration, therapy type, and site. Teens participating in MANAGING DIABETES reported a significant improvement in social acceptance over time $(p<.001)$ and less family conflict $(p<.05)$ than those in TEENCOPE. Teens participating in TEENCOPE reported better communication $(p<.05)$ and coping ( $\mathrm{p}<.01)$ over time. A1C did not deteriorate in either group. Improvements in self-management, self-efficacy, perceived stress, and coping mediated the effect of TEENCOPE on QOL. Improvements in self-efficacy, social competence, and family conflict mediated the effect of MANAGING DIABETES on QOL. Participation in both internet programs was excellent \& led to improved psychosocial outcomes after 12 months in ethnically diverse youth with T1D. Thus, young teens may benefit from both diabetes-specific coping and educational internet programs. Results of the mediation analysis begin to identify the mechanisms by which programs improve $00 \mathrm{~L}$.

Supported by: NINR \#R01NR004009

$\triangle$ 345-OR

Can You Hear (and See) Me Now? Skype-Based Intervention for Teens With Poorly Controlled Diabetes

MICHAEL A. HARRIS, KURT A. FREEMAN, DANNY C. DUKE, BETH HIRSCHFIELD, BRUCE BOSTON, Portland, OR

This study examined the relative effectiveness of a behavioral-based family therapy intervention for diabetes (BFST-D) delivered via tele-health (SKYPE) or face-to-face (CLINIC) for youth with poorly controlled diabetes $<$ $9.0 \%$ ) and their families. Seventy three youth (M age 15.1 years, SD=1.8) and their parents participated. Of youth in the study, $85 \%$ of were Caucasian, $44 \%$ were female, and $36 \%$ resided in single-parent homes. They had mean duration of $\mathrm{DM}$ of 6.8 years (SD=3.7) and a mean baseline $\mathrm{HbA1c}$ of $11.2 \%$ $(\mathrm{SD}=1.7 \%)$. At baseline and post-treatment, youth and parents completed the Diabetes Self Management Profile (DSMP), an assessment of diabetes treatment adherence, and $\mathrm{HbA1c}$ values were collected. Results showed no difference in the number of BFST-D treatment sessions completed across SKYPE (9.9) vs CLINIC (6.9). Three separate ANCOVAs were performed with treatment condition serving as the independent variable and baseline $\mathrm{HbA1c}$ value and youth and parent-reported DSMP total scores serving as the covariates. From baseline to post-treatment, $\mathrm{HbA1c}$ declined by an average of $0.76 \%$ for the CLINIC group and an average of $0.40 \%$ for the SKYPE group. ANCOVA yielded no significant between-group differences. From baseline to end of treatment, youth-reported DSMP scores increased by an average of 5.8 points for the CLINIC group and by an average of 2.9 points for the SKYPE group. ANCOVA identified no significant between-group difference. From baseline to end of treatment, parent-reported DSMP scores increased by an average of 28 points for the CLINIC group and by an average of 21 points for the SKYPE group. ANCOVA identified no significant between-group differences for parent or youth-reported DSMP scores. Findings suggest no statistically significant differences between SKYPE and CLINIC based delivery of BFST-D for youths with poorly controlled T1DM, supporting either delivery method as effective.

\section{INSULIN ANALOGS}

346-OR

The Novel Long-Acting Insulin LY2605541 is Superior to Insulin Glargine in Lowering Intra-Day Glucose Variability and Hypoglycemia Event Rate from Continuous Glucose Monitoring (CGM) in Patients With Type 2 Diabetes

EDWARD J. BASTYR, RICHARD M. BERGENSTAL, JULIO ROSENSTOCK, MELVIN J. PRINCE, YONGMING QU, SCOTT J. JACOBER, Indianapolis, IN, Minneapolis, MN, Dallas, TX

The basal insulin analog LY2605541 (LY) is PEGylated insulin lispro designed to have a large hydrodynamic size which delays insulin absorption and reduces clearance, resulting in prolonged duration of action. Hypoglycemia and glucose variability were assessed with CGM of interstitial glucose $(I G)$ in a subset of patients from a Phase 2, randomized, open-label, parallel study of $L Y(n=51)$ or insulin glargine (GL, $n=25)$. CGM was conducted on 3 consecutive days (72-84 hrs) during the week before Week 0, 6, and 12 study visits. IG intra-patient intra- and inter-day standard deviations (SD) during nocturnal (2400-0600 hrs) and diurnal (0600-2400 hrs) periods were calculated to assess glucose variability. A hypoglycemia episode was defined as IG $\leq 70 \mathrm{mg} / \mathrm{dL}$ and continued until IG $>70 \mathrm{mg} / \mathrm{dL}$ for $15 \mathrm{~min}$ (or 3 time points). All statistical comparisons were tested at 2-sided alpha $=0.1$. At 12 weeks, LY-treated patients spent less time with IG below $70 \mathrm{mg} / \mathrm{dL}$ than GL-treated patients during the nocturnal period ( $11 \pm 5 \vee 38 \pm 13 \mathrm{~min}, \mathrm{p}=.024$ ) and during the 24 -hr period $(25 \pm 6$ v $83 \pm 16$ min, $p<.001)$. Significantly fewer LY- than GL-treated patients experienced any hypoglycemia $(50.0 \%$ v $78.3 \%, p=.036)$, including nocturnal hypoglycemia (20.5\% v $47.8 \%, p=.027)$, based on CGM. At 12 weeks, both treatments resulted in similar mean glucose values, as indicated by the area under the IG curve, during the 24-hr period (LY: 11601 $\mathrm{mmol} / \mathrm{L} \cdot \mathrm{min} ; \mathrm{GL}: 11286 \mathrm{mmol} / \mathrm{L} \cdot \mathrm{min})$. LY-treated patients had significantly lower intra-day glucose SD at 12 weeks compared to GL-treated patients for both nocturnal $(1.00 \pm 0.07 \vee 1.35 \pm 0.16 \mathrm{mmol} / \mathrm{L}, \mathrm{p}=.061)$ and diurnal $(2.03 \pm$ $0.10 \vee 2.50 \pm 0.18 \mathrm{mmol} / \mathrm{L}, \mathrm{p}=.039$ ) periods. In conclusion, by CGM, LY treatment compared to GL resulted in: less time spent in hypoglycemia, fewer patients experiencing hypoglycemia, and lower intra-day glucose variability.

347-0R

Reduced Nocturnal Hypoglycemia and Weight Loss With Novel Long-Acting Basal Insulin LY2605541 Compared With Insulin Glargine in Patients With Type 2 Diabetes

RICHARD M. BERGENSTAL, JULIO ROSENSTOCK, RICHARD F. ARAKAKI, MELVIN J. PRINCE, YONGMING QU, VIKRAM P. SINHA, DANIEL C. HOWEY, SCOTT J. JACOBER, Minneapolis, MN, Dallas, TX, Honolulu, HI, Indianapolis, IN

The basal insulin analog LY2605541 (LY) is PEGylated insulin lispro designed to have a large hydrodynamic size which delays insulin absorption and reduces clearance, resulting in prolonged duration of action. This 12 wk, Phase 2, randomized, open-label, parallel study evaluated whether selfmonitored FBG was lower with once-daily LY than insulin glargine (GL). Patients (pts) converted to AM insulin administration during lead-in and were randomized 2:1 to $A M$ administration of $L Y(n=195)$ or $G L(n=93)$ from basal insulin (GL: $\mathrm{n}=248, \mathrm{NPH}: \mathrm{n}=39$ ). At baseline, for $\mathrm{LY} \vee \mathrm{GL}$, mean $\pm \mathrm{SE} \mathrm{FBG}$ was $147 \pm 3 \vee 140 \pm 4 \mathrm{mg} / \mathrm{dL}, A 1 \mathrm{C}$ was $7.7 \pm 0.1 \vee 7.8 \pm 0.1 \%$, and weight was 90.7 $\pm 1.4 \vee 89.7 \pm 2.1 \mathrm{~kg}$. At wk 12, LY v GL resulted in similar mean FBG $(118 \pm 2$ v $117 \pm 3 \mathrm{mg} / \mathrm{dL}, \mathrm{p}=.433)$, and mean $\mathrm{A} 1 \mathrm{C}(7.0 \pm 0.06 \vee 7.2 \pm 0.09 \%, p=.279)$. Intra-day BG variability, as measured by 8-point self-monitored BG standard deviation, was reduced with $\mathrm{LY}(34 \pm 1$ v $39 \pm 2 \mathrm{mg} / \mathrm{dL}, \mathrm{p}=.031)$. LY pts had significant mean weight loss $(-0.58 \pm 0.16 \mathrm{~kg}, \mathrm{p}=.007)$, while $\mathrm{GL}$ pts gained weight $(0.31 \pm 0.18 \mathrm{~kg}, p=.66$; LS mean difference: $-0.84 \mathrm{~kg}, p=.001)$. Mean hypoglycemia rate (\#/30d) with $\mathrm{LY}$ was not different compared to $\mathrm{GL}$ (total: $1.34 \vee 1.52 ; p=.80$; nocturnal: $0.25 \vee 0.39, p=.18$ ). After adjusting for baseline nocturnal hypoglycemia, $L Y$ pts had a $48 \%$ rate reduction in nocturnal hypoglycemia events compared to $\mathrm{GL}(\mathrm{p}=.021)$. Adverse events were similar across treatments. Mean increases in ALT and AST were higher with LY v GL $(p<.01)$, but remained in normal range. Triglyceride (TG) change from baseline was not significant for either group, but endpoint TGs were higher with $L Y$ v $\mathrm{GL}(p<.01)$. There was no difference in LDL-C or HDL-C between treatments. In conclusion, LY provided similar glycemic control, with reduced intra-day 
variability and lower nocturnal hypoglycemic events compared to $\mathrm{GL}$ in pts with T2D. While improving glycemic control, LY resulted in weight loss.

348-0R

Altering the Time of Day of Once-Daily Dosing of Insulin Degludec Achieves Similar Glycemic Control and Safety Compared to Dosing the Same Time of Day in People With Type 1 Diabetes

DAVID L. RUSSELL-JONES, PRISCILLA HOLLANDER, BRESTA MIRANDA-PALMA, JOHN G. COOPER, EDWARD FRANEK, STEPHEN BAIN, CHRISTIAN B. DJURHUUS, SØREN C. TAMER, CHANTAL MATHIEU, Guildford, Surrey, United Kingdom, Dallas, TX, Miami, FL, Stavanger, Norway, Warsaw, Poland, Swansea, United Kingdom, Bagsvaerd, Denmark, Leuven, Belgium

To date, consistent timing of basal insulin injection is needed to ensure stable day to day glycemic control. Insulin degludec (IDeg) provides an ultralong, flat action profile, enabling flexible dosing to accommodate an individual patient's lifestyle. In this 26-week, open-label, treat-to-target trial in people with type 1 diabetes (T1DM), once-daily (OD) IDeg (N=165), administered with the evening meal, was compared to a forced-flexible dosing regimen of IDeg (Flex) ( $\mathrm{N}=164)$ where subjects alternated insulin administration timing between morning and evening to create intervals of 8 to $40 \mathrm{hrs}$ between insulin doses. Mean baseline values for $\mathrm{HbA1c}$ (both groups 7.7\%), FPG (172.7 vs $179.4 \mathrm{mg} / \mathrm{dL}$ ), disease duration (17.3 vs $20.0 \mathrm{yrs}$ ), age (42.6 vs $44.5 \mathrm{yrs})$, and BMI (27.0 vs $\left.26.4 \mathrm{~kg} / \mathrm{m}^{2}\right)$ were comparable between IDeg Flex and IDeg OD groups, respectively. At 26 weeks, $84 \%$ of individuals in both groups completed the trial. Both IDeg Flex and IDeg OD reduced HbA1c by $0.4 \%$ (estimated treatment difference [ETD] Flex-0D: 0.01\% [95\% Cl: -0.13 ; $0.14])$. FPG reductions were greater with IDeg OD $(-23.0$ and $-45.7 \mathrm{mg} / \mathrm{dL}$, respectively (ETD: 17.1 [2.6; 31.6]; $p=0.0207$ ). Overall hypoglycemia rates ( $\mathrm{PG}<56 \mathrm{mg} / \mathrm{dL}$ or severe hypoglycemia) were similar (82.4 vs 88.3 events/ patient-yr; estimated rate ratio (ERR) Flex/OD: 0.92 [0.76; 1.12]). Nocturnal hypoglycemia rates (6.2 vs 9.6) were significantly lower with IDeg Flex (ERR: $0.63[0.46 ; 0.86] ; p=0.0031$ ). Severe hypoglycemia rates were similar for both groups, as were adverse event rates. Final IDeg doses were 0.42 (Flex) and $0.38(\mathrm{OD}) \mathrm{U} / \mathrm{kg} /$ day. IDeg can be administered in a once-daily regimen but at a different time from day to day with no difference in glycemic control or safety compared to standard OD dosing. Greater dosing flexibility may represent a major improvement in patient convenience by allowing injection times to be changed daily according to needs of the individual.

349-0R Insulin Degludec $200 \mathrm{U} / \mathrm{ml}$ is Ultra-Long-Acting and has a Flat and Stable Glucose-Lowering Effect

TIM HEISE, LESZEK NOSEK, ULRIKE HÖVELMANN, SUSANNE G. BØTTCHER, HANNE HASTRUP, HANNE L. HAAHR, Neuss, Germany, Søborg, Denmark

Insulin degludec (IDeg) is a new-generation, ultra-long-acting basal insulin, which is developed as both $100 \mathrm{U} / \mathrm{ml}$ and $200 \mathrm{U} / \mathrm{ml}$ in order to accommodate the wide range of insulin dose requirements characteristic of diabetes mellitus, especially type 2 diabetes. With IDeg $200 \mathrm{U} / \mathrm{ml}$, up to $160 \mathrm{U}$ of IDeg can be administered in a single injection using a newly developed prefilled pen. We investigated the pharmacodynamic and pharmacokinetic properties of $0.6 \mathrm{U} / \mathrm{kg} \mathrm{IDeg} 200 \mathrm{U} / \mathrm{ml}$ in subjects with type 2 diabetes $(\mathrm{n}=16$, mean: BMI, $30 \mathrm{~kg} / \mathrm{m}^{2} ; \mathrm{A} 1 \mathrm{C}, 7.3 \%$ ) who received IDeg $200 \mathrm{U} / \mathrm{ml}$ once daily over 6 days. On Day 6 while at steady state, a 26-h euglycemic glucose clamp was conducted (Biostator; clamp blood glucose level: $90 \mathrm{mg} / \mathrm{dl}$ ). As previously shown for IDeg 100 U/ml (Nosek et al. Diabetologia 2011:54, Suppl.1:S429), the mean glucose infusion rate (GIR) profile for IDeg $200 \mathrm{U} / \mathrm{ml}$ was flat and stable over the dosing interval $\tau$ (Fig. 1). The glucose-lowering effect of IDeg was evenly distributed over the dosing interval, with $\mathrm{AUC}_{\mathrm{GIR}}$ for each of the two 12 -h intervals being approximately $50 \%$ of the total AUC $\left(A \cup C_{G I R, T S}\right)$. The effect of IDeg extended beyond $26 \mathrm{~h}$ in all subjects, as blood glucose stayed close to the target level throughout the clamp. The terminal half-life at steady state was $26.2 \mathrm{~h}$. IDeg $200 \mathrm{U} / \mathrm{ml}$ was well tolerated and safe, with no injection site reactions. In conclusion, IDeg $200 \mathrm{U} / \mathrm{ml}$ has a flat and stable glucose-lowering effect with a duration of action beyond $26 \mathrm{~h}$ in people with type 2 diabetes.

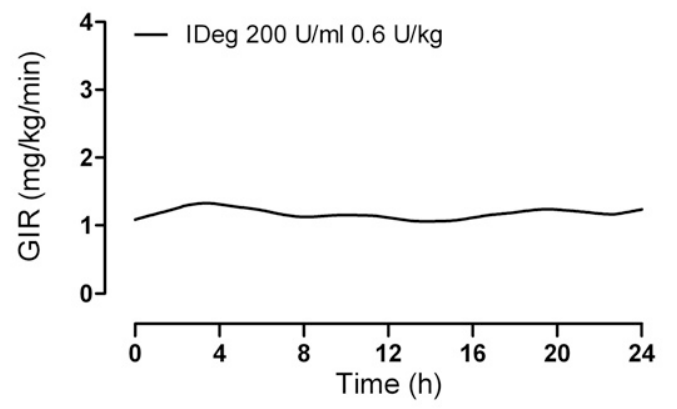

Fig. 1: Mean 24-hour GIR profile of IDeg $200 \mathrm{U} / \mathrm{ml}$ at steady state

Supported by: Novo Nordisk A/S

350-0R

Evaluations of Modified Ultra-Rapid Acting Linjeta ${ }^{\mathrm{TM}}$ Formulations BIOD-105 and BIOD-107 in Patients With Type 1 Diabetes

JESSICA R. CASTLE, LINDA MORROW, AMY PITTS, JOSEPH EL YOUSSEF, LORI BLANCHFIELD, PHILIP PICHOTTA, MARCUS HOMPESCH, W. KENNETH WARD, ALAN KRASNER, Portland, OR, Chula Vista, CA, Danbury, CT

Human insulin formulations containing citrate and EDTA (Linjeta) are more rapidly absorbed but cause increased injection site discomfort compared to insulin lispro (IL). BIOD-105 and BIOD-107 are formulations similar to Linjeta but with the addition of calcium to lessen subcutaneous EDTA-calcium binding post-injection, which we hypothesized as the cause of discomfort. These formulations were compared to IL in two single-center, randomized, doubleblind, three-period crossover euglycemic glucose clamp trials. Study 1 was completed in 18 and Study 2 in 8 subjects with type 1 diabetes. In Study 1, 0.15 $\mathrm{u} / \mathrm{kg}$ of each insulin was administered by SC injection and in Study 2, the same dose was given via insulin pump. Insulin profiles in Study 1 showed more rapid initial absorption of BIOD-105 and -107 relative to IL ( $\mathrm{t}_{1 / 2}$-early $=15.3$, 16.6, and 23.7 min, respectively, $\mathrm{p}<0.001)$. BIOD-105 and -107 exhibited lower peak metabolic effects relative to IL $\left(G I R_{\max }=5.8,6.4\right.$, and $\left.7.2 \mathrm{mg} / \mathrm{kg} / \mathrm{min}, p<0.05\right)$ and slower declines in metabolic action ( $\mathrm{t}_{1 / 2}$ GIR-late $=279,281$, and $211 \mathrm{~min}$, $\mathrm{p}<0.001)$. When given via pump, BIOD-105 also showed faster absorption and lower peak effect relative to IL. The BIOD-107 peak was similar in magnitude but longer in duration vs. IL. In Study 1, there were no significant differences in injection site tolerability as assessed by visual analog scores between BIOD105, BIOD-107 and IL (LS means $4.8 \pm 1.5,2.5 \pm 1.5$, and $2.2 \pm 1.5 \mathrm{~mm}$ ). Study 2 showed no significant differences in bolus or basal infusion discomfort. In summary, BIOD-105 and BIOD-107 resulted in local injection discomfort comparable to that of IL. While these formulations exhibited more rapid initial absorption, lower peaks and longer durations of effect compared to IL were seen. We conclude that the choice or concentration of the cation associated with the chelating agent EDTA can affect the PK/PD and tolerability of human insulin. Alternative formulation variants are currently being explored.

351-0R

Liver Preferential Effects of an Insulin Analog Demonstrated by Insulin Receptor Phosphorylation and Glucose Dynamics in Insulin Target Tissues In Vivo

CHRISTIAN L. BRAND, STEPHAN D. BOUMAN, JONAS KILDEGAARD, THOMAS KJELDSEN, PETER MADSEN, HELLE NAVER, CLAUS B. JEPPESEN, JOHANNES J. FELS, JEPPE STURIS, ERICA NISHIMURA, Maaloev, Denmark

Compared to the distribution of endogenously secreted insulin throughout the body, subcutaneous (sc) insulin is distributed in a non-physiological manner to the insulin target tissues with the peripheral tissues being relatively overexposed to insulin compared to the liver, resulting in increased risk of hypoglycemia. The present study investigates a novel insulin analog (insulin 327) and its potential liver preferential effects compared to human insulin $(\mathrm{HI})$ in rodents. Insulin receptor phosphorylation (pIR) was measured in liver, muscle and fat tissues at 0, 5, 15, 60,120 and 180 min after intravenous (iv) injection of either $\mathrm{HI}$ or insulin 327 in conscious male NMRI mice. For similar glucose lowering, insulin 327 exerted a liver preferential pIR pattern as the response in liver accounted for $71 \%$ of the combined pIR response (AUC) in all tissues compared to $43 \%$ for $\mathrm{HI}$. The dynamic rates of glucose uptake (GU) and hepatic glucose production (HGP) were determined by ${ }^{3} \mathrm{H}$-glucose techniques during 5 hrs of three different constant iv infusion rates of either $\mathrm{HI}$ or insulin 327 and variable glucose infusion rates (GIR) to maintain euglycemic clamp in conscious male Sprague Dawley rats. The potency of insulin 327 relative to $\mathrm{HI}$ was calculated as the ratio between their $\mathrm{ED}_{50}$ values calculated by fitting 
the insulin infusion rates and the corresponding response values to Sigmoidal curves. The potency of insulin 327 on GIR during the last hr of the clamp was $59 \%$ relative to $\mathrm{HI}$. The potency of insulin 327 relative to $\mathrm{HI}$ on $\triangle \mathrm{AUCS}$ for HGP and GU was $77 \%$ and $37 \%$, respectively, indicating a more potent suppression of HGP than stimulation of GU. These data demonstrate that insulin 327 exerts liver preferential pIR and glucose dynamic patterns compared to $\mathrm{HI}$ in rodents, and may therefore represent a novel basal insulin regimen with with a potential for improving the hypoglycemia safety margin.

352-0R

Effect of Peripheral Delivery of a Liver Preferential Insulin Analog on Glucose and Fat Metabolism

DALE S. EDGERTON, MELANIE SCOTT, JOSHUA ROOP, DOSS NEAL, PHILLIP WILLIAMS, THOMAS KJELDSEN, PETER MADSEN, HELLE NAVER, CLAUS B. JEPPESEN, ERICA NISHIMURA, CHRISTIAN L. BRAND, ALAN D. CHERRINGTON, Nashville, TN, Copenhagen, Denmark

Endogenous insulin secretion exposes the liver to concentrations of insulin which are 2.5 -fold greater than at non-hepatic tissues. Subcutaneous insulin delivery eliminates this gradient and is associated with metabolic abnormalities. Thus, restoring the physiologic gradient may provide a therapeutic benefit. The hepato-selectivity of a novel insulin analog (insulin 327) was tested. Dogs with arterial, portal vein and hepatic vein catheters were studied after an $18 \mathrm{~h}$ fast. $3 \mathrm{H}$-glucose was infused from $-140 \mathrm{~min}$. After a basal period (-40 to $0 \mathrm{~min}$ ) somatostatin and basal portal glucagon were infused (0 to $300 \mathrm{~min})$. At the same time insulin 327 or human insulin (HI) was infused into a peripheral vein $(7.2$ or $1.8 \mathrm{pmol} / \mathrm{kg} / \mathrm{min}$, respectively; $n=5 /$ group) and euglycemia was maintained by glucose infusion. Arterial insulin 327 and $\mathrm{HI}$ levels increased to $10870 \pm 2543$ and $95 \pm 8 \mathrm{pmol} / \mathrm{l}$, respectively. Although the glucose infusion rates were similar in insulin 327 and $\mathrm{HI}(3.1 \pm 0.3$ vs $3.1 \pm 1.6$ $\mathrm{mg} / \mathrm{kg} / \mathrm{min}$, respectively, last $3 \mathrm{~h}$ ), insulin 327 had a greater effect on the liver and a lesser effect on non-hepatic tissues compared to HI. Relative to the basal period, insulin 327 suppressed net hepatic glucose balance and glucose $\mathrm{Ra}(\mathrm{mg} / \mathrm{kg} / \mathrm{min}$; last $3 \mathrm{~h}$ ) by $1.9 \pm 0.1$ and $1.7 \pm 0.1$, respectively, while $\mathrm{HI}$ reduced these by only $0.5 \pm 0.6$ and $1.1 \pm 0.3$. On the other hand, insulin 327 only increased non-hepatic glucose uptake and glucose $\mathrm{Rd}(\mathrm{mg} / \mathrm{kg} / \mathrm{min}$; last $3 \mathrm{~h}$ ) by $1.0 \pm 0.4$ and $1.5 \pm 0.3$, respectively, while $\mathrm{HI}$ increased these by $2.4 \pm 1.2$ and 2.5 \pm 1.1 . Suppression of lipolysis was delayed with insulin 327 compared to $H$ : during the 1 st hour plasma NEFA levels $(\mu \mathrm{mol} / \mathrm{l})$ decreased by $91 \pm 37 \mathrm{vs}$ $419 \pm 42$, respectively, and blood glycerol levels ( $\mu \mathrm{mol} / \mathrm{l})$ fell by $0 \pm 7$ vs $39 \pm 5$, respectively. During the last $3 \mathrm{~h}$, however, there were no differences in the suppression of lipolysis by insulin 327 compared to $\mathrm{HI}$. These results demonstrate the efficacy of a peripherally delivered insulin analog designed to preferentially target liver glucose metabolism.

353-OR

Human Hyaluronidase + Rapid Analog Insulin (RAI) Improves Postprandial Glycemic Control in Type 1 Diabetes (T1DM) Compared to Insulin Lispro Alone

IRL B. HIRSCH, JAY S. SKYLER, SATISH GARG, THOMAS BLEVINS, DANIEL E. VAUGHN, DOUGLAS B. MUCHMORE, Seattle, WA, Miami, FL, Aurora, CO, Austin, TX, San Diego, CA

Recombinant human hyaluronidase (rHuPH20) accelerates absorption \& action of prandial insulins. We compared control in T1DM using prandial lispro or RAl+rHuPH2O (Analog-PH20). After a 4-6 week run-in using prandial glulisine + bid glargine, 117 subjects (age $43 \pm 14$ years, BMI $27.3 \pm 4.7$, A1C 7.0 \pm ) were randomized (double blind crossover) to lispro+rHuPH2O or aspart+rHuPH2O vs lispro alone for two $12 \mathrm{wk}$ intensive management periods; prandial doses were immediately before meals. Primary endpoint of A1C noninferiority (.4\% margin) was achieved with no treatment difference $(95 \% \mathrm{Cl}-.05,+.15)$. Mean postmeal (90 min) excursions were reduced by $82 \%$ ( $p=.0044)$ over the 12 week period with more subjects consistently (at least $2 / 3$ of meals) achieving goals:

\begin{tabular}{lccc}
\hline & \multicolumn{3}{c}{ (\% of Subjects at Goal) } \\
\hline 90 min Postprandial Goal $(\mathrm{mg} / \mathrm{dL})$ & Analog-PH20 & Lispro & p-value \\
\hline$<180$ Breakfast & 70.5 & 54.0 & .003 \\
\hline$<180$ All Meals & 70.8 & 59.3 & .016 \\
\hline$<140$ Breakfast & 21.4 & 10.6 & .007 \\
\hline$<140$ All Meals & 15.0 & 8.8 & .089 \\
\hline
\end{tabular}

Continuous monitoring over 3 days at the end of treatment showed improved excursion profiles (Fig.). Overall hypoglycemic rates $(\leq 70 \mathrm{mg} / \mathrm{dL}$ or symptoms) were reduced $5 \%(p=.035)$ and events $<56 \mathrm{mg} / \mathrm{dL}$ reduced $7 \%$ $(p=045)$. Total daily insulin dose $(54 \pm 27$ for Analog-PH20 vs $56 \pm 27 \mathrm{U}$ for lispro, $\mathrm{p}=.057)$ and weight gain difference $(-.57 \mathrm{lb}, \mathrm{p}=.27)$ showed favorable trends.
Adverse events were comparable between treatments and Analog-PH2O was well tolerated.
Breakfast

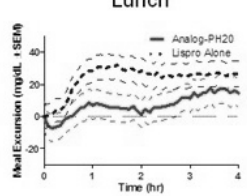

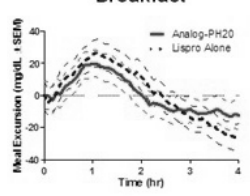

Dinner

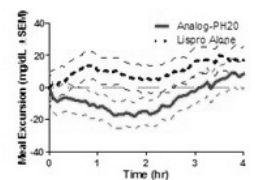

\section{GENOTYPE-PHENOTYPE CORRELATIONS}

354-0R

Meta-Analysis of Type 2 Diabetes Genome-Wide Association Studies in African Americans: The MEDIA Consortium

MAGGIE C. NG, DANIEL SHRINER, PING AN, LOREN L. ARMSTRONG, LAWRENCE F. BIELAK, SUZETTE J. BIELINSKI, INGRID BORECKI, BRIAN H. CHEN, YIII-DER I. CHEN, WEI-MIN CHEN, STEVEN R. CUMMINGS, DANIEL S. EVANS, XIUQING GUO, GEOFFREY HAYES, RICHARD A. JENSEN, EDMOND K. KABAGAMBE, CARL D. LANGEFELD, JIANKANG LIU, SIMIN LIU, JIRONG LONG, MICHAEL A. NALLS, NICHOLETTE D. PALMER, SANJAY R. PATEL, LESLIE J. RAFFEL, MICHELE SALE, YAN V. SUN, LAURA J. RASMUSSEN-TORVIK, LISA R. YANEK, LINGYAO YANG, THE MEDIA CONSORTIUM, Winston-Salem, NC, Bethesda, MD, St. Louis, MO, Chicago, IL, Ann Arbor, MI, Rochester, MN, Los Angeles, CA, Charlottesville, VA, San Francisco, CA, Seattle, WA, Birmingham, AL, Jackson, MS, Nashville, TN, Cleveland, $\mathrm{OH}$, Atlanta, GA, Baltimore, $M D$

The prevalence of type 2 diabetes (T2D) in African American ( $A f A$ ) adults is almost twice that of European Americans (18.7\% vs. 10.2\%). Despite this disparity, efforts to identify T2D genes in AfA have been restricted partly due to available sample sizes. We formed the "Meta-analysis of Type 2 Diabetes in African Americans (MEDIA) Consortium" to perform meta-analysis of $17 \mathrm{ge}$ nome-wide association studies (GWAS) for T2D in a large well phenotyped and comprehensively genotyped AfA sample. Discovery analysis included 8,272 T2D cases diagnosed by the ADA 2004 criteria and 16,079 controls. In each study, up to $1 \mathrm{M}$ SNPs were genotyped. To increase genomic coverage and account for admixture in AfA, we performed imputation with combined HapMap Phase II+III CEU and YRI reference data and carried out principal components analysis. Using up to 2.9M genotyped and imputed SNPs, we tested for association with T2D under the additive genetic model, adjusting for age, sex, study site, population structure, and with or without BMI. Each study was corrected for genomic control $(\lambda=1.01-1.10)$ before association results were combined using an inverse variance-weighted fixed effect model. Combined results were corrected for residual population stratification by a second genomic control $(\lambda=1.06)$. We identified 30 loci associated with T2D at $p \leq 1 \times 10^{-5}$, of which 26 were novel. Genome-wide significant SNPs $\left(p<5 \times 10^{-8}\right)$ were observed at the known T2D loci TCF7L2 (rs7903146) and KCNO1 (rs2283228), with odds ratios (ORs) of $1.36\left(p=2.2 \times 10^{-26}\right)$ and $1.24(p=$ $4.3 \times 10^{-8}$ ), respectively. SNPs at the known loci INS-IGF2 (rs3842770) and HMGA2 (rs343092) also showed strong associations, with ORs of 1.18 ( $p=$ $\left.7.8 \times 10^{-8}\right)$ and $1.18\left(p=7.3 \times 10^{-7}\right)$, respectively. Replication studies are underway. These findings provide insight into the genetic architecture of T2D in AfA, demonstrating the potential for new discovery from genetic association studies in populations with predominantly African ancestry.

\section{5-OR}

Meta-Analysis of 211,221 Individuals Identifies Novel Waist-Hip Ratio Loci Including Those Involved In Adipogenesis and Type 2 Diabetes

DMITRY SHUNGIN, TERESA FERREIRA, DAMIEN C. CROTEAU-CHONKA, THOMAS W. WINKLER, REEDIK MAGI, JOSEPH WU, RONA J. STRAWBRIDGE, ADAM E. LOCKE, KRISTA FISCHER, TSEGASELASSIE WORKALEMAHU, PAULA J. GRIFFIN, CHARLES C. WHITE, ANNE U. JACKSON, FELIX DAY, M. CAROLA ZILLIKENS, CAROLINE S. FOX, ERIK INGELSSON, MARK I. MCCARTHY, KARI E. NORTH, ELIZABETH K. SPELIOTES, PAUL W. FRANKS, ADRIENNE L. CUPPLES, LU QI, IRIS M. HEID, RUTH J. LOOS, INES BARROSO, KAREN L. MOHLKE, CECILIA M. LINDGREN, GIANT CONSORTIUM THE GENETIC INVESTIGATION OF ANTHROPOMETRIC TRAITS, Malmo, Sweden, Umea, Sweden, Oxford, United Kingdom, Chapel Hill, NC, Regensburg, Germany, Boston, MA, Stockholm, Sweden, Ann Arbor, MI, Tartu, Estonia, Cambridge, United Kingdom, Rotterdam, The Netherlands, Framingham, MA, Cambridge, MA, Hinxton, United Kingdom

Waist-hip ratio (WHR), a measure of adipose tissue distribution, is associated with risk of type 2 diabetes (T2D) and other metabolic and cardiovascular 
disease traits independent of total adiposity. We genotyped 67,163 individuals from 28 studies using the Metabochip, a targeted genotyping array comprising SNPs pre-selected for cardiometabolic traits. Each SNP was tested for additive association with WHR, adjusted for body mass index. All 14 previously established WHR loci replicated with directionally consistent effects (13 at $\left.P<5.0 \times 10^{-8}\right)$. SNPs selected to follow up suggestive WHR signals from the discovery meta-analysis by Heid et al., 2010, showed more directional consistency than expected by chance $\left(1,409\right.$ of 2,377 independent SNPs $\left(r^{2}<0.1\right)$ compared to 1,189 expected, $\left.P=2.01 \times 10^{-19}\right)$. We than performed fixed-effects metaanalysis of the Metabochip studies together with genome-wide association studies (GWAS) from the published discovery meta-analysis $(n=72,919)$, and a further 24 new GWAS $(n=71,139)$. We identified 28 novel WHR loci $\left(P<5.0 \times 10^{-8}\right)$, including SNPs near PPARG $\left(P=8.2 \times 10^{-9}\right)$ and CEBPA $\left(P=2.0 \times 10^{-9}\right)$. The CEBPA signal is located near two subunits of the transcription factor C/EBP, which is essential for adipocyte differentiation. C/EBP also binds promoters of adiponectin and leptin, suggesting a range of effects on downstream pathways. Variants in PPARG have previously been associated with T2D. We identified two new WHR index SNPs, one $5^{\prime}$ and the other $3^{\prime}$ of $P P A R G$, which are in low linkage disequilibrium with each other $\left(r^{2}=0.18\right)$ and with the Pro12Ala variant, an established T2D locus ( $r^{2}=0.11$ and 0.07 respectively); this is of interest, as $P P A R G$ binds the thiazolidinedione insulin sensitizing class of drugs commonly used to treat T2D and that induce weight gain. This meta-analysis from the $\mathrm{Gl}$ ANT consortium of 211,221 GWAS and Metabochip samples further elucidates the genetic architecture of adipose distribution and T2D.

356-0R

Novel Loci Associated With Birth Weight Provide Biological Links Between Intrauterine Growth, Postnatal Growth and Metabolic Traits

DENNIS O. MOOK-KANAMORI, HANIEH YAGHOOTKAR, MOMOKO HORIKOSHI ULLA SOVIO, NICOLAS TIMPSON, M. KAAKINEN, DIANE BERRY, JONATHAN BRADFIELD, DIANE COUSMINER, M. KERKHOF, M. KIRIN, E. KREINER-MØLLER, V. LAGOU, E. THIERING, NICOLE WARRINGTON, J. ZHAO, H. TAAL, MARK MCCARTHY, M.R. JARVELIN, RACHEL FREATHY, INGA PROKOPENKO, VINCENT JADDOE, GEORGE DAVEY SMITH, THE EGG CONSORTIUM INVESTIGATORS, Doha, Oatar, Rotterdam, The Netherlands, Exeter, United Kingdom, Oxford, United Kingdom, London, United Kingdom, Bristol, United Kingdom, Oulu, Finland, Philadelphia, PA, Helsinki, Finland, Groningen, The Netherlands, Edinburgh, United Kingdom, Copenhagen, Denmark, Neuherberg, Germany, Perth, Australia

The genetic loci influencing birth weight (BW) and their relationship to postnatal growth and adult disease are poorly characterized. Two loci have been reported, CCNL1 and ADCY5, which contain variants associated with $B W$. To identify additional loci associated with BW we performed a genome wide association study (GWAS) in 26,836 European descent individuals from 18 studies and followed up 20 loci in up to 42,472 individuals. We identified five novel and confirmed two loci containing variants associated with BW at $p<5 \times 10-8$. These loci fell into 4 broad categories. First, two loci represented the same signals as known type 2 diabetes (T2D) signals - in or near ADCY5 $(p=5.5 \times 10-20)$ and CDKAL1 ( $p=1.5 \times 10-18)$. In both loci, the BW-lowering allele is also associated with greater T2D risk. This is consistent with the fetal insulin hypothesis, which proposes that common genetic variation influencing insulin secretion is a key link between lower BW and later life T2D risk. Second, two associations shared loci with known adult height signals, in or near HMGA2 ( $p=1.4 \times 10-19)$ and LCORL $(p=5.9 \times 10-11)$ genes. For both loci, the height-increasing allele also was the BW-increasing allele. Third, one association represented the same signal as that known between variation at ADRB1 and blood pressure $(p=3.6 \times 10-8)$. The effect of this signal on fetal BW appears to be mediated through maternal genotype. Finally, the previously reported association near the CCNL1 gene ( $p=3.6 \times 10-38)$, represents the strongest association with BW. The effect on BW seems to be through neonatal adiposity and this locus has no known overlap with adult metabolic traits. Separate to these groups, the association at $5 q 11.2,(p=4.6 \times 10-8)$ has no recognized associations with other traits. Our analysis of the genetic component contributing to BW highlights genetic loci contributing to growth processes and to metabolic trait regulation.

357-0R

Fine-Mapping of Type 2 Diabetes Susceptibility Loci With “Metabochip"

ANDREW P. MORRIS, TERESA FERREIRA, TANYA M. TESLOVICH, MICHAEL BOEHNKE, MARK I. MCCARTHY, DIAGRAM CONSORTIUM, Oxford, United Kingdom, Ann Arbor, MI

Despite the success of genome-wide association studies (GWAS) in identifying susceptibility loci for type 2 diabetes (T2D), most of these signals map to regions containing many transcripts and potential functional variants. In this study, we combined "Metabochip" and GWAS data from 34,840 cases and 114,981 controls (European descent, except 1,178 cases and 2,472 controls of Pakistani descent) to fine-map causal variants across 35 T2D susceptibility loci. We detected two loci with independent association signals $\left(r^{2}<0.05\right)$ at genome-wide significance $\left(p<5 \times 10^{-8}\right)$ that lie outside the recombination interval containing the lead SNP. Both signals at KCNQ1 (rs163184, $p=1.2 \times 10^{-11}$; rs231361, $p=1.2 \times 10^{-9} ; r^{2}=0.01$ ) have been previously reported, but at CDKN2A/B ( $r s 10811661, p=3.7 \times 10^{-27} ; r s 944801, p=2.4 \times 10^{-9} ; r^{2}=0.01$ ), the secondary signal is novel. We also observed independent association signals $\left(p<10^{-5}\right)$ at two further loci: $D G K B$ (rs17168486, $p=5.9 \times 10^{-11} ;$ rs6960043, $p=3.4 \times 10^{-7} ; r^{2}=0.01$ ) and MC4R (rs12970134, $\left.p=1.2 \times 10^{-8} ; r s 11873305, p=3.8 \times 10^{-7} ; r^{2}=0.02\right)$. Approximate conditional analyses also provided evidence of additional association signals in the same recombination interval as the lead SNP at KCNO1 (rs74046911, $p=3.7 \times 10^{-9}, r^{2}=0.06$ ) and CDKN2A/B (rs10757283, $p=4.7 \times 10^{-30}$ $r^{2}=0.36$ ). Across the 35 susceptibility loci, we found no evidence that lead Metabochip SNPs were characterised by substantially lower allele frequency and higher odds ratio than previously reported GWAS SNPs. At 24 loci, the GWAS SNP was the same, or highly correlated $\left(r^{2}>0.8\right)$, with the lead Metabochip SNP (of which 17 have $r^{2}>0.95$ ). Of the remaining 11 loci, the lead Metabochip and GWAS SNPs were uncorrelated at just two, $D G K B\left(r^{2}=0.00\right)$ and KCNO1 $\left(r^{2}=0.03\right)$, because they map to the independent association signals described above. Our fine-mapping analyses suggest that multiple independent association signals are widespread at T2D susceptibility loci and indicate that the underlying causal variants are common.

358-0R

Fine-Mapping of Type 2 Diabetes Susceptibility Loci via Trans-Ethnic Meta-Analysis

MOMOKO HORIKOSHI, STEVEN WILTSHIRE, TERESA FERREIRA, NORIHIRO KATO, JEROEN HUYGHE, ANUBHA MAHAJAN, FUMIHIKO TAKEUCHI, YIK YING TEO, ELEFTHERIA ZEGGINI, ANDREW P. MORRIS, MARK I. MCCARTHY, T2D-GENES CONSORTIUM, Oxford, United Kingdom, Tokyo, Japan, Ann Arbor, MI, Singapore, Singapore, Cambridge, United Kingdom

Over 60 type 2 diabetes (T2D) susceptibility loci have been identified: however, the causal variant(s) been characterized at very few of these. One approach to refine the localisation of causal variants shared across major ethnic groups is to combine genome-wide association data across populations to leverage differences in linkage disequilibrium. We examined 20,058 T2D cases and 38,789 controls from five ethnic groups (30\% European, 34\% South Asian, 29\% East Asian, 2\% Hispanic, 5\% African American), focusing on five T2D loci: CDKAL1, CDKN2A/B, FTO, IGF2BP2 and KCNQ1. We combined GWAS data (imputed to a "cosmopolitan" reference panel incorporating variants from the 1000 Genomes August 2010 release) using both fixed-effects and Bayesian (MANTRA) methods. Fine-mapping resolution was most obviously improved at CDKALI, highlighting two SNPs that jointly account for $98.8 \%$ of the posterior probability of association: rs 9368222 (fixed-effects $\left.p=2.4 \times 10^{-22}\right)$ and $\mathrm{rs} 2206734\left(p=9.8 \times 10^{-16}\right)$. At KCNO1, there are two distinct signals. One of these (lead SNP rs234864) showed marked heterogeneity in effect size with a stronger effect in E Asian (OR=1.27 [1.20-1.35]) than European, $\mathrm{S}$ Asian and Hispanic descent populations (combined, $\mathrm{OR}=1.11$ [1.06-1.15]) (fixed-effects $p_{\text {het }}=0.002$, MANTRA $\log _{10}$ Bayes' factor 2.67). The second locus (lead SNP rs2237892) featured homogeneous effect sizes (OR = 1.21 [1.16-1.26]), but differences in risk-allele frequency led to marked reduction in the strength of the association signal in European and S Asian samples (minor allele frequency $2-7 \%, p=2.5 \times 10^{-6}$ ) compared to $\mathrm{E}$ Asians (minor allele frequency $\left.29-44 \%, p=2.9 \times 10^{-16}\right)$. At the other three loci, there was much more modest improvement in localisation. Our results highlight the potential advantages of trans-ethnic meta-analysis for fine-mapping causal variants, and show promise for localizing potential targets for further functional investigation.

359-0R

Genotype-Phenotype Correlation in $\mathbf{4 4 0}$ Children With Congenital Hyperinsulinism (HI)

KARA SNIDER, ARUPA GANGULY, SUSAN BECKER, LINDA BOYAJIAN, SHOWLING SHYNG, COURTNEY MACMULLEN, NKECHA HUGHES, KARTHIK GANAPATHY, TRICIA BHATTI, AMANDA LEE, CHARLES STANLEY, Philadelphia, PA, Port land, $O R$

Congenital hyperinsulinism (HI) is associated with 8 genetic disorders of insulin secretion. These include recessive, loss of function mutations of the genes encoding the KATP channel (ABCC8 and KCNJ11), resulting in either focal or diffuse diazoxide-unresponsive HI. Dominant, loss of function KATP channel mutations may cause either diazoxide -responsive or -unresponsive 
HI. We evaluated the correlations between genotype and phenotype as a guide for clinical management of in a large series of $440 \mathrm{HI}$ cases. Mutation analysis was completed in all cases for the genes commonly associated with HI (ABCC8, KCNJ11, GLUD1 and GCK). Additional genes were screened in children negative for mutations in the above genes (HNF4a, UCP2 and SCHAD). Mutations could be identified in 272 diazoxide-unresponsive probands (91\%); 265 had KATP channel mutations (145 focal HI, 109 diffuse $\mathrm{HI}, 11$ did not have surgery). GCK mutations were identified in 6 diazoxideunresponsive children, 2 of whom had surgery for diffuse disease. In 119 diazoxide-responsive probands, mutations were found in 45\%: 24 GLUD1 mutations, 23 dominant KATP mutations, 2 SCHAD mutations, 2 UCP2 mutations, and 2 HNF4a mutations. Interpretation of the significance of KATP mutations was complicated by the fact that $70 \%$ of the defects identified were novel variants. Inheritance of 2 recessive KATP mutations predicted diffuse- $\mathrm{HI}$ with $100 \%$ sensitivity and specificity. Inheritance of a single, paternal recessive KATP mutation predicted focal-HI with $100 \%$ sensitivity and $92 \%$ specificity. These correlations between genotype to phenotype in children with $\mathrm{HI}$ indicate that mutation analysis can be especially useful in children unresponsive to diazoxide in distinguishing focal versus diffuse $\mathrm{HI}$ and selecting patients who are potentially curable by surgery.

Supported by: R37-DK-056268 and R01-DK-066485

360-0R

The HLA-DR2, DOB1*0602 Haplotype is Associated With Lack of Disease Progression and Lower Autoantibody Prevalence in the TrialNet Natural History Study

ALBERTO PUGLIESE, DAVID BOULWARE, SUNANDA BABU, ANDREA STECK, CRAIG BEAM, DOROTHY BECKER, GEORGE S. EISENBARTH, CARLA GREENBAUM, AND THE TYPE 1 DIABETES TRIALNET STUDY GROUP, Miami, FL, Tampa, FL, Denver, CO, Pittsburgh, PA, Seattle, WA

The TrialNet NHS has screened $>99,000$ relatives of T1D patients for autoantibodies (AAb, to GAD65, ICA-512, insulin, and islet cell antibodies, ICA); 2,147 relatives (1,179 females, 954 males, 14 unknown gender, mean age $18.1 \pm$ SD 13.7 years) were HLA-typed because they had $1 \mathrm{AAb}$ confirmed or $>2 A A b$, at screening or on follow-up. ZnT8 AAb were measured in 1,031 HLA-typed relatives. We analyzed the association of the T1D-protective HLA-DR2, DQB1*0602 haplotype with disease progression and AAb status in AAb-positive relatives. There was a negative association with T1D: 16\% $(343 / 2,147)$ of relatives developed T1D, but only $0.5 \%(2 / 343)$ carried this haplotype compared to $6.9 \%(128 / 1859)$ of nondiabetic relatives $(p<0.0001)$; $35(27 \%)$ relatives with DR2, DQB1*0602 had DR4 (17 had DRB1*0401) and only 1 (with DRB1*0402) developed T1D. Table 1 shows the frequency of individual $A A b$ and $A A b$ number in 1,031 relatives also tested for ZnT8 AAb in relation to $\mathrm{DR} 2, \mathrm{DQB} 1{ }^{*} 0602$.

\begin{tabular}{lccl}
\hline Table 1 & $\begin{array}{c}\text { HLA DR2, } \\
\text { DQB1*0602 negative }\end{array}$ & $\begin{array}{c}\text { HLA DR2, } \\
\text { DQB1*0602 positive }\end{array}$ & p-value \\
\hline ZnT8 & $318 / 967(32.9 \%)$ & $6 / 64(9.4 \%)$ & $<0.0001$ \\
\hline GAD65 & $739 / 967(76.4 \%)$ & $47 / 64(73.4 \%)$ & 0.59 \\
\hline ICA512 & $328 / 967(33.9 \%)$ & $11 / 64(17.2 \%)$ & 0.006 \\
\hline Insulin & $399 / 967(41.3 \%)$ & $15 / 64(23.4 \%)$ & 0.005 \\
\hline ICA & $359 / 959(37.4 \%)$ & $16 / 64(25.0 \%)$ & 0.04 \\
\hline $1 \mathrm{AAb}$ & $406 / 961(42.2 \%)$ & $46 / 64(71.9 \%)$ & 0.000002 \\
\hline $2 \mathrm{AAb}$ & $210 / 961(21.8 \%)$ & $12 / 64(18.8 \%)$ & 0.12 \\
\hline $3 \mathrm{AAb}$ & $148 / 961(15.4 \%)$ & $1 / 64(1.6 \%)$ & 0.0003 \\
\hline $4 \mathrm{AAb}$ & $113 / 961(11.8 \%)$ & $3 / 64(4.7 \%)$ & 0.03 \\
\hline $5 \mathrm{AAb}$ & $84 / 961(8.7 \%)$ & $2 / 64(3.1 \%)$ & 0.05 \\
\hline
\end{tabular}

The frequency of GAD65 AAb was similar, while other AAb were less frequent, with ZnT8 AAb being the least frequent, in relatives with DR2, $\mathrm{DQB1}{ }^{*} 0602$. They more often had $1 \mathrm{AAb}$ and rarely $\geq 3 \mathrm{AAb}(\mathrm{p}=0.0001)$. Among relatives with DR2, DQB1*0602 who also had DR4, 11/14 (78\%) had 1 autoantibody, 1 had 2, none had 3, 2 had 4 and none had 5. Thus, in NHS autoantibody-positive relatives, DR2, DQB1*0602 is associated with strong protection from T1D, which appears dominant over DR4 susceptibility, and the presence of fewer AAb, even in the presence of DR4; ZnT8 AAb are the least frequent $A A b$ in relatives with this protective haplotype.

Supported by: NIH and JDRF
$\Delta$ 361-0R

T-Cell Receptor Alpha and Beta Chain Skewing in the Islets of PreDiabetic MAD Rats: Germline-Encoded TCR Repertoire as a Determinant of Type 1 Diabetes Susceptibility

RYAN A. EBERWINE, LAURA CORT, FRANK BEAROFF, ZHIJUN LIU, MICHAEL HABIB, JOHN MORDES, ELIZABETH BLANKENHORN, Philadelphia, PA, Worcester, $M A$

Autoimmune type 1 diabetes (T1D) is common among inbred rat strains with a high-risk MHC-II. Prior genetic studies have mapped a robust diabetes susceptibility locus (/ddm14) using five T1D susceptible (including the MAD strain) and three resistant rat strains (e.g. WF). We then identified a T-cell receptor (TCR) beta chain variable region gene, $T c r b-V 13$, as the probable Iddm14 gene. We have shown that deletion of $V \beta 13+T$ cells prevents T1D (Diabetes, In press), suggesting that TCR-V $\beta 13+T$-cells recognize a critical islet autoantigen. If true, we should detect clonal expansion of $\mathrm{V} \beta 13+\mathrm{T}$ cells in prediabetic islets. We sequenced $V \beta 13+$ CDR3 regions from T cells isolated from spleen and islets, and T cells cultured ex vivo from islets of pre-diabetic MAD rats. We observed significant skewing of the TCR $\beta$ repertoire, with pauciclonal expansion of V $\beta 13-C D R 3$ sequences from islet T-cells compared to a high diversity of V $\beta 13$-CDR3 regions in spleen. These data indicate that an oligoclonal V $\beta 13$ response to pancreatic beta cells exists early in progression to autoimmune diabetes. In NOD/LtJ mice, TCR-V $\alpha 5.4$ is frequently used in the T-cell response to islet antigen and recognizes insulin B:9-23 in combination with multiple TCR $\beta$ chains. We analyzed rat V $\alpha 5.4+$ CDR3 sequences from pre-diabetic islets, T cell exfiltrates from cultured pre-diabetic islets, and V $\beta 13+$ and V $\beta 13$ - sorted exfiltrates. We observed significant TCR skewing among islet homing V $\alpha 5.4+$ T-cells, suggesting an autoimmune response to insulin occurs early in rat T1D pathogenesis. Additionally we show the presence of TCR-V $\alpha 5$ repertoire skewing among islet homing TCR- V $\beta 13+$ cells. We conclude that the TCR genotype is a critical genetic determinant of T1D susceptibility.

\section{METABOLISM IN VIVO (HUMANS)}

\section{2-0R}

The Size of the Incretin Effect is Dependent on the Small Intestinal Glucose Load

CHINMAY S. MARATHE, CHRISTOPHER K. RAYNER, KAREN L. JONES, MICHAEL HOROWITZ, Adelaide, Australia

The incretin effect, mediated by GLP-1 and GIP, accounts for $~ 50 \%$ of postprandial insulin secretion in health, and is deficient in type 2 diabetes. Previous studies have not assessed the potential impact of gastric emptying (GE), for which there is low intra-individual but substantial inter-individual variation (range 1-4 kcal/min). When GE is relatively more rapid, the postprandial rise in glucose is greater. We hypothesized that the incretin effect would be greater at higher rates of intraduodenal (ID) glucose delivery. 10 healthy men (age $47 \pm 3$ years, BMI $29.3 \pm 1.0$ ) were each studied on four days. They received ID glucose infusions at 2 or $4 \mathrm{kcal} / \mathrm{min}$ for $120 \mathrm{~min}$ in random order, and corresponding isoglycemic intravenous (IV) glucose infusions. Blood glucose and serum insulin were measured to 240 min and incremental area under the curves (iAUC) calculated. Data are mean \pm SEM. The insulin iAUC (mU/L.min) was substantially greater in response to $4 \mathrm{kcal} / \mathrm{min}$ than $2 \mathrm{kcal} /$ min ID glucose $(5390 \pm 1338 \mathrm{v} 1836 \pm 528, \mathrm{P}<0.005)$, so that the blood glucose iAUC ( $\mathrm{mmol} / \mathrm{L} . \mathrm{min})$ was not significantly different between the two $(238 \pm$ $34 \mathrm{v} 200 \pm 20$ ). As expected, the insulin iAUC was greater after ID than IV glucose for the $2 \mathrm{kcal} / \mathrm{min}(1836 \pm 528$ v $779 \pm 213, \mathrm{P}<0.01)$ and $4 \mathrm{kcal} / \mathrm{min}$ $(5390 \pm 1338 \vee 1289 \pm 554, P<0.005)$ studies, but the size of this incretin effect (\%insulin response attributable to the gut) was substantially greater for $4 \mathrm{kcal} / \mathrm{min}$ than $2 \mathrm{kcal} / \mathrm{min}(80 \pm 4 \%$ v $51 \pm 5 \%, \mathrm{P}<0.005)$. Our results suggest that in healthy subjects, the rate of glucose entry into the small intestine, within the physiological range of $\mathrm{GE}$, has a major impact on the size of the incretin effect. 


\section{Serum Insulin levels in response to ID and IV infusions}

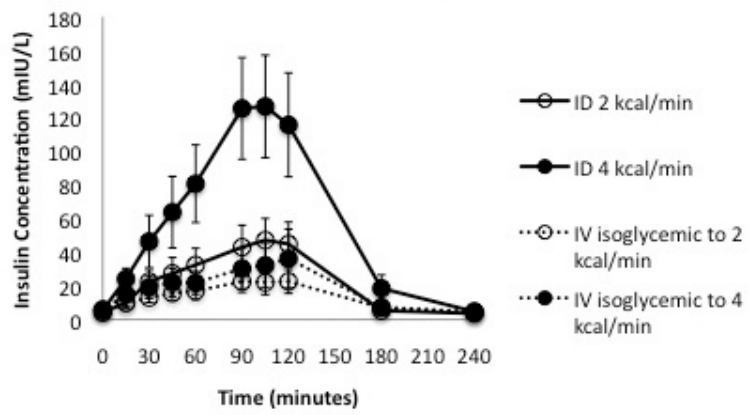

Supported by: NHMRC, Australia

\section{$\Delta$}

363-0R

Defective Insulin Secretion in Humans With Type 2 Diabetes Mellitus (T2DM) May Reflect an Impaired Response to Cholinergic Rather than GIP Input

SARA CHOWDHURY, SONGYAN WANG, ERIN LACINY, BRUCE W. PATTERSON DOMINIC N. REEDS, BURTON M. WICE, St. Louis, MO

Impaired insulin secretion is a hallmark feature of T2DM. Glucagon-like peptide-1 (GLP1) and glucose-dependent insulinotropic polypeptide (GIP) are intestinal peptides secreted with meal ingestion and amplify insulin secretion rates (ISRs). In contrast to GLP-1, it is thought that beta cells in T2DM are resistant to GIP precluding its use as a therapeutic target. Xenin-25 (Xen) is a 25 -amino acid neurotensin related peptide. We previously showed that Xen amplifies GIP-mediated insulin release in mice via a cholinergic relay in the periphery. We now explore this relay in humans with normal (NGT) or impaired (IGT) glucose tolerance and T2DM.Each subject underwent 4 separate graded glucose infusions (GGIs) with continuous infusion of albumin, GIP, Xen, or GIP plus Xen. Based on published work, plasma pancreatic polypeptide (PP) levels reflect Xen-mediated cholinergic input to islets. Fasting PP levels were similar in all groups. During the first 40 min of the GGIs, GIP rapidly and transiently increased ISR incremental area under the curve (iAUCs) in humans with NGT (4.6X) and IGT (6.1X). Contrary to dogma, GIP also increased the iAUC for ISR 8.7X in humans with T2DM. Increased ISR occurred without significant changes in plasma glucose and PP levels. In contrast, Xen had no effect on ISR or plasma glucose in any group but increased PP levels $>10 \mathrm{X}$ in humans with NGT, IGT, and T2DM (iAUCs = 4.6, 5.8 , and $6.3 \mathrm{ng} / \mathrm{mL} / 40 \mathrm{~min}$, respectively). During GGIs with GIP plus Xen, ISRs were further increased in NGT (1.3X) and IGT (1.5X), but not T2DM compared to GIP alone. However, with GIP plus Xen, PP levels similarly increased in subjects with NGT, IGT, and T2DM (iAUCs = 11.8, 13.2, and $12.8 \mathrm{ng} / \mathrm{mL} / 40$ $\mathrm{min}$, respectively). Thus, increased cholinergic input alone is not sufficient to augment ISRs in humans but can amplify GIP-mediated ISRs in humans with NGT and IGT, but not T2DM. Thus, beta cells in T2DM may exhibit an impaired response to cholinergic rather than GIP input.

Supported by: NIH/NIDDK (RC1DK086163 and R01DK088126), The Blum Kovler Foundation

\section{Reduced Incretin Effect in Truncal Vagotomized Subjects}

364-0R

ASTRID PLAMBOECK, SIMON VEEDFALD, CAROLYN DEACON, ANDRÈ WETTERGREN, LARS SVENDSEN, SØREN MEISNER, CLAUS HOVENDAHL, JENS HOLST, FILIP KNOP, TINA VILSBøLL, Hellerup, Denmark, Copenhagen, Denmark, Odense, Denmark

Rapid degradation of glucagon-like peptide-1 (GLP-1) by dipeptidyl peptidase 4 (DPP-4) suggests that GLP-1 may act locally (through vagal afferents) before being degraded. We aimed to clarify the role of vagal innervation on the incretin effect. Ten truncally vagotomized subjects (due to duodenal ulcer) with pyloroplasty ( $68 \pm 2$ years; fasting plasma glucose (FPG): $6.0 \pm 0.2$ $\mathrm{mM}), 10$ subjects treated for oesophageal cancer with cardia resection including truncal vagotomy/pyloroplasty $(65 \pm 2$ years; FPG: $5.8 \pm 0.3 \mathrm{mM})$ and 10 control subjects $(67 \pm 1$ years; FPG: $5.3 \pm 0.1 \mathrm{mM})$ underwent $4 \mathrm{~h} 50$ g-OGTT \pm concomitant DPP-4 inhibition (DPP-4i) and isoglycemic glucose infusion (IIGI). Isoglycemia during the oral and iv glucose administration was obtained. Peak plasma levels of GLP-1 were about 5 -fold higher $(109 \pm 19$ and $140 \pm 40$ vs. $24 \pm 3$ pM, $P<0.02$ ) and gastric emptying faster (time to peak (ac- etaminophen): $38 \pm 7$ and $33 \pm 7$ vs. $77 \pm 8 \mathrm{~min}, \mathrm{P}<0.002$ ) in vagotomized subjects after OGTT compared to controls. Control subjects suppressed plasma glucagon concentrations similarly irrespectively of the route of glucose administration (total area under the curve (tAUC): $1184 \pm 147$ (IIGI) vs. $1292 \pm 180$ 4h×pM (OGTT), P=NS) whereas vagotomised subjects suppressed glucagon during $\| G \mid$ and exhibited hyperglucagonemic responses following OGTT (duodenal ulcer: $1374 \pm 174$ vs. $1891 \pm 2764 \mathrm{~h} \times \mathrm{pM}, \mathrm{P}<0.05$; cardia resection: $1125 \pm 139$ vs. $1608 \pm 2374 \mathrm{~h} \times \mathrm{pM}, \mathrm{P}<0.05$ ). The incretin effect (calculated using the incremental AUC for C-peptide) was reduced in the vagotomized groups (48 $\pm 7 \%$ (duodenal ulcer), $45 \pm 5 \%$ (cardia resection, $P=N S$ ) compared to the controls $(64 \pm 6 \%, \mathrm{P}<0.05)$. The incretin effect was diminished in vagotomized subjects despite 5 -fold higher OGTT-induced GLP-1 levels. Futhermore, the vagotomized subjects had an inappropriate glucagon response after an oral glucose load. These findings may indicate that an intact vagal innervation is important for the effect of GLP-1 and thereby maintenance of normal glucose homeostasis.

Supported by: EFSD/Novo Nordisk Grant

$365-0 R$

Mechanisms of Impaired Incretin Effects in Type 2 Diabetes

ANDREA MARI, JONATAN I. BAGGER, ELE FERRANNINI, JENS J. HOLST, FLLIP K KNOP, TINA VILSBøLL, Padova, Italy, Hellerup, Denmark, Pisa, Italy, Copenhagen, Denmark

To characterize the incretin effects (IE) on insulin secretion (ISR), we used 25,75 , and $125 \mathrm{~g} \mathrm{OGTTs}$ and isoglycemic intravenous glucose infusions (IIGI) in 8 type 2 diabetic (T2D) and 8 matched nondiabetic (C) subjects ( 3 males; age $57 \pm 1$ years; BMI $\left.29 \pm 0.3 \mathrm{~kg} / \mathrm{m}^{2}\right)$, and a $\beta$-cell mathematical model embedding a dose-response (slope=glucose sensitivity, GS), an early response (rate sensitivity, RS), and potentiation (POT, time-related response increase relative to basal). During IIGI in C, both GS $\left(42 \pm 4,46 \pm 6,43 \pm 7 \mathrm{pmol} \mathrm{min}^{-1} \mathrm{~m}^{-2} \mathrm{~L}\right.$ $\mathrm{mmol}^{-1} ; 25,75,125 \mathrm{~g}$ dose, respectively) and $\mathrm{RS}(441 \pm 118,446 \pm 109,481 \pm 115$ pmol m$\left.{ }^{-2} \mathrm{Lmol}^{-1}\right)$ were dose-independent, while POT increased $(0.99 \pm 0.07$ to $1.35 \pm 0.12,25$ vs $125 \mathrm{~g}, P<0.01)$. In T2D, GS and RS were also dose-independent (GS: $16 \pm 2,17 \pm 2,17 \pm 2 ; R S: 28 \pm 16,12 \pm 10,7 \pm 7$ ), but severely impaired vs $C$ (both $P<0.0001$ ). In C, IE (as the 0GTT/IIGI total ISR ratio) increased with the glucose dose $(1.3 \pm 0.1,1.7 \pm 0.2,2.2 \pm 0.2, P<0.02$ vs 1 for all doses); this effect was mediated by a dose-dependent GS increase $(1.9 \pm 0.4,2.4 \pm 0.4$, $3.1 \pm 0.4$ fold of $\| G I)$, and a dose-independent RS enhancement (1091 \pm 293 , $510 \pm 157,1112 \pm 415 \mathrm{pmol} \mathrm{m}^{-2} \mathrm{~L} \mathrm{mmol}^{-1}$ absolute increase vs IIGI). POT also increased at the highest doses $(0.97 \pm 0.06,1.45 \pm 0.20,1.24 \pm 0.16$ fold of IIGI). In T2D, IE was significantly increased only at $125 \mathrm{~g}(1.0 \pm 0.1,1.1 \pm 0.1,1.3 \pm 0.1)$ and impaired vs $C$ across doses $(P<0.0001)$. GS showed the same pattern $(1.2 \pm 0.2,1.3 \pm 0.2,2.0 \pm 0.2$ fold of $\|G\|$, while RS increased already at $25 \mathrm{~g}$ (369 $\pm 136,338 \pm 84,260 \pm 100 \mathrm{pmol} \mathrm{m}^{-2} \mathrm{~L} \mathrm{mmol}^{-1}$ increase vs IIGI). However, OGTT did not stimulate POT above IIGI $(0.94 \pm 0.04,0.89 \pm 0.06,1.06 \pm 0.09$ fold of IIGl; $P<0.01$ vs C). On the OGTT, total GIP and GLP-1 levels increased dose-dependently, and similarly in C and T2D. In conclusion, 1) In nondiabetic subjects, dose-dependent IE increase results mainly from GS and POT; 2) In T2D, IE is blunted vs $C$ at all glucose doses and significant at $125 \mathrm{~g}$ only, but enhanced early ISR is detectable at all doses; 3 ) the IE differences cannot be explained by GLP-1/GIP responses.

Earlier Glucose and Insulin Peaks and Postprandial Hyperglycemia after Gastric Bypass (GBP) in Patients With and Without Diabetes: Results from the Longitudinal Assessment of Bariatric Surgery (LABS) Study

JONATHAN Q. PURNELL, MYRLENE STATEN, BRUCE WOLFE, ANITA P. COURCOULAS, DAVID R. FLUM, DAVID E. CUMMINGS, KAREN FOSTER-SCHUBERT, BRET SHEPPARD, Portland, OR, Bethesda, MD, Pittsburgh, PA, Seattle, WA

Patients with type 2 diabetes (T2DM) have impaired islet secretory capacity compared to those without. Hypothesizing that insulin secretion during a mixed meal would improve in patients with T2DM undergoing GBP (indicating improved insulin secretory capacity) but decrease in those without T2DM (as insulin sensitivity improved with weight loss), we studied 41 subjects with and 20 subjects without T2DM (similar in age, sex, BMI) before and 6 mo after GBP. Each subject drank $360 \mathrm{Kcal}$ (BoostPlus) with timed sampling over $240 \mathrm{~min}$. Fasting glucose and insulin levels, and AUC glucose, significantly decreased by $6 \mathrm{mo}$ in both groups (figure). However, at 6 mo the average postprandial (PP) peak glucose levels remained unchanged in T2DM, increased in controls $(+19 \mathrm{mg} / \mathrm{dL}, \mathrm{P}=0.001)$, and occurred a median of 30-min sooner in both groups $(P<0.001)$. Insulin responses mirrored the glucose responses in both timing and peak, resulting in a more rapid late PP decline in glucose levels at 6 mo. The average AUC insulin decreased in patients with 
T2DM $(-21 \%, P<0.001)$ and did not change in controls $(-9.5 \%, P=0.38)$. GBP improves fasting and PP AUC glucose levels, but also leads to rapid glucose absorption, transient PP hyperglycemia, a concomitant rapid release of insulin, and subsequent prompt reduction in glucose levels in obese patients with and without T2DM. These data raise the possibility that PP hyperinsulinemic hypoglycemia after GBP may be more a function of altered glucose absorption than exaggerated insulin secretion.
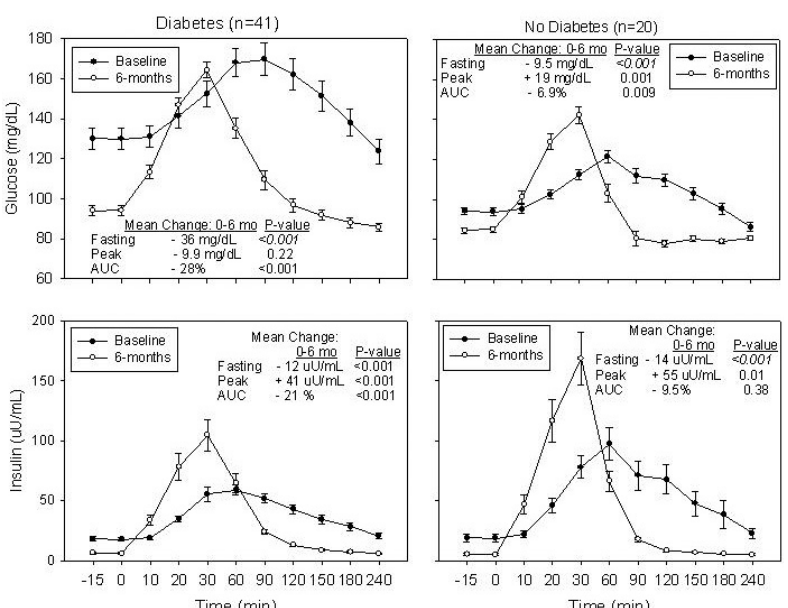

Supported by: NIH/NIDDK

367-0R

Changes in Beta Cell Function and Insulinotropic Effect of the Incretin Hormones 1 Week after Roux-en-Y Gastric Bypass Surgery in Subjects With Normal Glucose Tolerance

CARSTEN DIRKSEN, KIRSTINE N. BOJSEN-MØLLER, NILS B. JØRGENSEN, SIV H. JACOBSEN, DORTE WORM, DORTE L. HANSEN, JENS J. HOLST, STEN MADSBAD, Hvidovre, Denmark, Copenhagen, Denmark

In this study, "intrinsic" beta cell function as well as the insulinotropic effects of glucagon-like peptide-1 (GLP-1) and glucose-dependent insulinotropic polypeptide (GIP) were examined before and early after Roux-en-Y gastric bypass (RYGB). Seven obese subjects (age $40.0 \pm 3.8$ yrs (mean \pm SEM), BMI $40.9 \pm 1.8 \mathrm{~kg} / \mathrm{m}^{2}$ ) with normal glucose tolerance (HbA1c $5.5 \pm 0.2$ $\%$ ) underwent 3 hyperglycemic clamps (1 hr, plasma glucose $9 \mathrm{mmol} / \mathrm{L})$ with bolus injection of arginine after $45 \mathrm{~min}$ and infusion of GLP-1 $(1.0 \mathrm{pmol} / \mathrm{kg} /$ $\mathrm{min})$, GIP (1.5 pmol $/ \mathrm{kg} / \mathrm{min})$ or saline before as well as within the first week after RYGB. Acute insulin response to glucose from 0-10 min (AIRg), second phase insulin response from 10-44 min and acute insulin response to arginine (AIRarg) were assessed. Disposition indices were calculated based on HOMA-IR (hepatic) and M/I (peripheral) (30-44 min). Before RYGB, HOMA-IR was $2.8 \pm 0.4$ and tended to decrease during the first postoperative week $(-23 \pm$ $13 \%, p=0.07)$, whereas $M /$ did not change. AlRg and second phase insulin response, but not AIRarg, were significantly higher during infusion of GLP-1 and GIP compared to saline at the preoperative visits. After RYGB, AIRg and second phase insulin response were significantly reduced during GLP-1 infusion $(-24 \pm 8, p=0.02$ and $-38 \pm 18 \%, p=0.04$, respectively), but not during saline and GIP infusions. AlRarg was reduced during all 3 infusions, although most pronounced during GLP-1 infusion (GLP-1 $-38 \pm 11, p<0.01$; GIP $-19 \pm$ $6, p=0.01$; saline $-24 \pm 14 \%, p=0.10$ ). Hepatic and peripheral disposition indices calculated from both AIRg and AIRarg did not change significantly postoperatively during any of the infusions. In conclusion, "intrinsic" beta cell function does not change significantly during the first week after RYGB. Unadjusted insulin secretion during GLP-1 stimulation declined significantly, possibly indicating reduced insulinotropic effect of GLP-1 postoperatively.

368-0R

Effect of Bariatric Surgery on Components of $\beta$ Cell Function in Obese Patients Without Diabetes

KAREN HERSHKOP, ANDREI KEIDAR, CHAYA SCHWEIGER, LIOR HECHT, LIMOR MARKO, RAM WEISS, Jerusalem, Israel

Hormonal induced $\beta$ cell boosting has been suggested to explain glucose metabolism improvement following bariatric procedures. Obese patients with fasting glucose between $90-110 \mathrm{mg} / \mathrm{dl}$ suffer from insulin resistance and $\beta$ cell stress and may similarly benefit from improved $\beta$ cell function.
We aimed to measure different $\beta$ cell function components before and 3 months after bariatric surgery in order to identify the effects of "metabolic surgery" on specific components of $\beta$ cell function in patients at significant risk for diabetes development. Fourteen obese patients underwent a modified hyperglycemic clamp including 180 min hyperglycemic clamp (glucose $200 \mathrm{mg} / \mathrm{dl}$ ), an additional oral glucose load- 30 gram glucose at $120 \mathrm{~min}$ for estimation of the incretin effect, and IV Arginine at $180 \mathrm{~min}$ for assessment of $\beta$ cell capacity. This procedure was preformed before and 3 months after bariatric surgery sleeve gatsrectomy $(n=10)$ or Roux-en- $Y$ gastric bypass ( $n=$ 4). All patients had significant weight loss 3 months after surgery (mean $21.6 \pm 5.8 \mathrm{~kg}$ ). No difference was detected in glucose levels during the clamp. Fasting glucose significantly dropped from $96 \pm 15$ to $81 \pm 8 \mathrm{mg} / \mathrm{dl}$ between baseline and follow up respectively, $p=0.003$ ). First phase insulin secretion dropped significantly after surgery $(p=0.02)$, yet incremental first phase insulin was not changed. Second phase insulin response reduced significantly after surgery as expected due to improved insulin sensitivity $(p<0.001)$. The incretin effect was significantly improved following surgery $(100.9 \pm 136$ vs. $291.1 \pm 207.5 \mathrm{pmol} / \mathrm{li}^{*} \mathrm{~min}$ for baseline vs. follow up, $\left.\mathrm{p}<0.001\right)$. No difference was observed in beta cell capacity compared to baseline. No difference was detected between the effects of both procedures. In obese non-diabetic patients, bariatric surgery has a positive effect on $\beta$ cell function only in regards to beta cell sensitivity to the incretin stimuli. No improvement was detected in first phase insulin secretion or in maximal $\beta$ cell capacity.

$$
\text { Supported by: EFSD }
$$

369-0R

Effects of Daily Intake of Lactobacillus Reuteri on Incretins, Insulin Secretion and Insulin Sensitivity in Human Subjects

MARIE-CHRISTINE SIMON, BETTINA NOWOTNY, PETER NOWOTNY, FARIBA ZIVEHE, JONG-HEE HWANG, HUBERT KOLB, PETER STEHLE, COLIN MACKENZIE, BIRGIT HENRICH, NANETTE SCHLOOT, MICHAEL RODEN, Düsseldorf, Germany, Bonn, Germany, Bad Homburg, Germany

Ingestion of probiotics can modify gut microbiota composition and also insulin resistance and diabetes development in rodents. In humans, faecal transfer from lean into obese subjects was reported to improve insulin sensitivity. We tested the hypothesis that enrichment of microbiota with daily intake of Lactobacillus (L.) reuteri over 4 weeks increases insulin sensitivity and secretion in humans by improving gut hormone secretion. A prospective, double-blind, randomized, parallel-group trial in 18 glucose tolerant humans ( 9 obese; age $51 \pm 2$ years, BMI $36.0 \pm 4.8 \mathrm{~kg} / \mathrm{m}^{2} ; 9$ lean; $49 \pm 4$ years, $\left.23.6 \pm 1.9 \mathrm{~kg} / \mathrm{m}^{2}\right)$ was performed. Participants ingested twice/day $10^{10} \mathrm{~L}$. reuteri (Nutraceutix, WA, USA) or placebo over 4 weeks. Oral glucose tolerance and matching "isoglycemic" i.v. glucose infusion tests were used to assess incretin and insulin secretion, euglycemic-hyperinsulinemic clamps combined with isotopic dilution $\left(6,6\left[{ }^{2} \mathrm{H}_{2}\right]\right.$ glucose) to measure peripheral (M) and hepatic insulin sensitivity. Ectopic (muscular, hepatic) lipids were assessed by ${ }^{1} \mathrm{H}$ magnetic resonance spectroscopy. Stool samples from persons ingesting $\mathrm{L}$. reuteri were positive tested by real time PCR, placebo treated persons stayed negative. Ingestion of $L$. reuteri did not affect body mass, ectopic lipid content, incretin or insulin secretion. M was 37\% lower $(p<0.01)$ in obese than in lean volunteers and remained unchanged $(6.7 \pm 2.6$ vs. $9.5 \pm 2.2 \mathrm{mg} / \mathrm{kg}^{*} \mathrm{~min}$ ). Endogenous glucose production (EGP) was comparable between groups at baseline and during insulin suppression. L. reuteri treatment neither affected EGP at baseline $\left(1.9 \pm 0.4 \mathrm{vs} .1 .6 \pm 0.3 \mathrm{mg} / \mathrm{kg}^{*} \mathrm{~min}\right)$ nor EGP suppressed $(-63 \pm 16 \%$ vs. $-62 \pm 22 \%)$. In conclusion, daily ingestion of $L$. reuteri over 4 weeks enriched gut microbiota by $L$. reuteri but had no effects on insulin secretion and insulin sensitivity in non-diabetic humans. These results will be of critical importance in the emerging research of the acute use of probiotics in insulin resistance.

Supported by: HHU Düsseldorf; the DZD e.V. and an unrestricted grant by Danone

\section{ISLET GROWTH AND DEVELOPMENT}

T3 Acts as a Physiological Regulator of Maturation by Directly Binding to Mafa

CRISTINA AGUAYO-MAZZUCATO, ANN MARIE ZAVACKI, ILHAM EL-KHATTABI, THOMAS S. SCANLAN, P. REED LARSEN, GORDON C. WEIR, ARUN SHARMA, SUSAN BONNER-WEIR, Boston, MA, Portland, OR

Neonatal beta cells lack glucose-responsive insulin secretion and so are considered immature. We previously showed that thyroid hormone $\left(T_{3}\right)$ effectively upregulates the transcription factor MafA, which is key for the 
functional maturation of beta cells. Here we tested the hypothesis that $T_{3}$ regulates MafA transcription through direct interaction of its receptor with the MafA promoter region. INS1 cells cultured in the presence of $\mathrm{T}_{3}$ for $14 \mathrm{~h}$ showed a significant increase in MafA mRNA, which was sensitive to actinomycin D-dependent inhibition of RNA polymerase. These results suggest that MafA may be a direct target of thyroid hormone. Agonists for the different isoform thyroid receptors, either CO 23 (THRA agonist) or GC1 (THRB agonist), were added to P7 rat islets cultured for 4 days in RPMI (11.1 mM glucose) containing 10\% Charcoal Stripped-FBS. GC1 effectively increased MafA mRNA levels while $\mathrm{CO} 33$ failed to, suggesting that at this postnatal period $\mathrm{T}_{3}$ activates THRB to enhance MafA transcription. Potential thyroid response elements (TRE) in a $20 \mathrm{~kb}$ fragment of the MafA mouse gene were identified. Two regions, one upstream and one downstream of the MafA coding region were identified as having greater than $75 \%$ conservation across mouse, rat and human MafA gene. Eight potential binding sites, including the two conserved sites, were further characterized for their ability to bind THRB by chromatin immunoprecipitation followed by site-specific PCR amplification. Cross-linked chromatin from untreated or $T_{3}(10 n M)$ treated MIN6 cells was immunoprecipitated using anti-THRB antibodies. PCR analysis confirmed the binding of THRB to at least one putative TRE in the MafA promoter Overall, these results indicate that MafA transcription can be regulated by $\mathrm{T}_{3}$ via THRB directly bound to a MafA TRE. Thus, thyroid hormone can function as a direct physiological regulator of MafA expression and subsequently drive the functional maturation of beta cells.

371-0R KRAS Inhibition of Beta-Cell Growth Occurs Through a MENINRASSF1 Regulatory Network

CHESTER E. CHAMBERLAIN, MICHAEL S. GERMAN, San Francisco, CA

KRAS is a regulated GDP/GTP molecular switch that controls several signaling networks. Although activating missense mutations in the KRAS gene are a major cause of accelerated growth in many epithelial cancers, KRAS signaling can elicit diverse, cell-type specific responses, and its function in the normal growth and physiology of the pancreatic beta-cell remains unknown. We discovered that mice heterozygous for a null allele of the KRAS gene have an expanded beta-cell population at birth and improved glucose tolerance as adults. We showed that the additional beta-cells come from two sources: increased numbers of Neurogenin3-expressing endocrine progenitors during embryogenesis and increased beta-cell proliferation during the perinatal period. Increased KRAS signaling directly suppressed both sources of new beta-cells (neogenesis from non-beta-cell progenitors, and proliferation of pre-existing beta-cells) and activated the opposing MAPK and RASSF1 downstream effector pathways in beta-cells. The block of beta-cell growth caused by KRAS activity depends on the expression of the endocrine tumor suppressor MENIN. In support of this conclusion, endocrine cells from the parathyroid and pituitary glands, two tissues known to develop tumors in patients carrying heterozygous mutations in MENIN (Multiple Endocrine Neoplasia Type 1, MEN1), similarly showed increased proliferation in KRAS heterozygous null mutants. Our data suggest a model in which KRAS activates both the pro-proliferative MAPK pathway, and the anti-proliferative RASSF1 pathway. In the tissues susceptible to the MENIN gene mutation, MENIN prevents the MAPK effector pathway from driving beta-cell proliferation while leaving the inhibitory RASSF1 effector pathway intact. In this model, loss of MENIN causes proliferation due to removal of the block in the proliferative drive mediated by MAPK signaling, while loss of KRAS signaling increases proliferation by decreasing the unopposed RASSF1 activity in susceptible cells.

Supported by: Larry L. Hillblom Foundation, Nora Eccles Treadwell Foundation, JDRF, NIH/NIDDK

372-OR MafA Can Reverse Beta-Cell Dysfunction Resulting from Pdx1 Haplo-Insufficiency

ARCHANA KAPOOR, WANZHU JIN, MICHAEL KARADIMOS, CONNOR FITZPATRICK, WATARU NISHIMURA, SUSAN BONNER-WEIR, ARUN SHARMA, Boston, $M A$

Although MafA was identified as a glucose-responsive insulin gene transcription factor, during pancreatic development its expression follows that of insulin in newly formed beta cells. This observation suggests that MafA regulates function, but not the specification, of insulin-producing cells. Consistent with this, MafA-null mice have normal looking islets with insulinexpressing cells at birth and only develop hyperglycemia with age. Additionally, several animal models with beta cell dysfunction had reduced MafA expression suggesting that MafA is essential for normal beta cell function.
While we recently showed that MafA over expression in isolated neonatal islets enhanced their glucose responsiveness and functional maturation, the question of whether MafA is sufficient to enhance beta cell function in vivo remains unresolved. Therefore we tested whether enhancing MafA expression in vivo in a model of beta cell dysfunction (Pdx1 haplo-insufficient mice) would improve beta cell function. Pdx1tTA/+ and tet0MafA mice were crossed and doxycycline (DOX) was given in drinking water throughout gestation. Then DOX was stopped at birth to trigger induction of MafA transgene in $\mathrm{Pdx} 1 \mathrm{tTA} /+$; tet0MafA (bigenic) offspring. Bigenic pups were born at the expected Mendelian ratio with normal pancreatic morphology. At P0 both bigenic and Pdx1 haplo-insufficient mice had reduced Pdx1 and MafA expression compared to controls, and they did not express transgene. By one-month transgene expression was only seen in beta cells of bigenic mice. These mice had significantly improved glucose tolerance, and increased MafA, GLUT2, insulin and even Pdx1 levels compared to Pdx1tTA/+ littermates. These results indicate that enhancing MafA expression in vivo is sufficient to overcome beta-cell dysfunction and the associated changes in gene expression in Pdx1 haplo-insufficient mice. Thus, MafA should be considered as a master regulator of beta-cell function.

Supported by: NIH/NIDDK, JDRF, Herbert Graetz Fund, Shirley and William Fleischer family

$\Delta$ 373-0R

Fate Mapping of the Murine Pancreas Precursors Reveals a Multipotent Ventral Foregut Progenitor

JESSE ANGELO, MAR-ISEL GUERRERO-ZAYAS, JESSE MAGER, KIMBERLY D. TREMBLAY, Amherst, MA

The definitive endoderm is the germ layer that gives rise to the endodermal organs including the thyroid, lung, liver and pancreas. The endodermal origin of the dorsal and ventral pancreas buds remains enigmatic, yet understanding how these tissue buds are induced and formed is an essential step in understanding normal pancreas development. We have used physical fate mapping combined with whole embryo culture to uncover the endodermal origin of both pancreas buds uncovering remarkable differences between the origins of each. The dorsal bud progenitors are located in a single medial endodermal position from the 2 somite (S) stage through 11S. The endoderm forming the 9.5dpc ventral pancreas bud is found in 2 distinct domains: the predominant contribution from the domain on the left and right lateral endoderm caudal to the ventral lip by $6 S$, while a second domain located in the ventral midline of the endoderm lip (VMEL) contributes to a small population in the midline of the 9.5dpc ventral pancreas bud. Finally we find that the VMEL population contributes in a defined temporal manner to the thyroid bud, rostral cap and ventral midline of the liver bud as well as the midline of the ventral pancreas bud. These results are the first to discern the endodermal origins of the dorsal and ventral pancreas buds as well as those of the thyroid in the pre-specified endodermal sheet. Furthermore, these data suggest that the early ventral foregut tissue buds are at least in part, descended from the same progenitor population.

374-0R

The Diabetes-Associated Gene Glis3 Regulates Beta Cell Differentiation in Utero via Neurog3 Transactivation but Modulates Beta Cell Function in Adults via Non-Neurog3-Mediated Control of Insulin Production

YISHENG YANG, BENNY CHANG, LAWRENCE CHAN, Houston, TX

Glis3, a member of the Krüppel-like family of transcription factors, is highly expressed in pancreatic beta cells. GLIS3 mutations were found to cause sporadic neonatal diabetes. Recent genome-wide association studies (GWAS) in adult populations identified GLIS3 also as a candidate gene for type 1 diabetes, and as a gene that is associated with type 2 diabetes. Moreover, the GLIS3 locus is linked to altered fasting glucose level in healthy children and adolescents. To gain insight into the functional roles of Glis3, we generated Glis $3^{--}$mice, which died neonatally with severe hyperglycemia and ketoacidosis. Using in vivo and in vitro analyses, we show that Glis3 is involved in the differentiation of endocrine progenitor cells through direct and indirect transcriptional control of Neurog3 expression, which is essential to islet formation. To determine if sustained Glis 3 expression is required for the normal functioning of beta cells in adult animals, we generated condi-

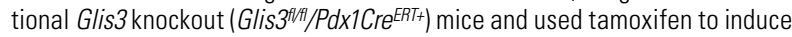
Glis3 deletion in adult mice. Tamoxifen-mediated beta cell-specific inactivation of Glis 3 induces diabetes acutely by downregulating insulin expression, leading to hyperglycemia and subsequently enhanced beta cell apoptosis, an effect that appears to be largely independent of Glis3's Neurog3 transactivating function. We conclude that [1] Glis3 controls fetal islet differentiation 


\section{ISLET GROWTH AND DEVELOPMENT}

via Neurog3 which perturbation causes neonatal diabetes, and [2] Glis3 expression is required for normal pancreatic beta cell function in adults, which impairment leads to defective insulin production and diabetes.

Supported by: NIH (DK-68037)

375-0R

Postnatal Duct-Specific Pdx1 Deletion Results in Formation of Pdx1 Negative Insulin Positive Immature Beta Cells

LILI GUO, AKARI INADA, CRISTINA AGUAYO-MAZZUCATO, JENNIFER HOLLISTER-LOCK, YOSHIO FUJITANI, CHRISTOPHER V. WRIGHT, ARUN SHARMA, SU-

SAN BONNER-WEIR, Boston, MA, Fukuoka, Japan, Tokyo, Japan, Nashville, TN

Pancreatic duodenal homeobox $-1(\mathrm{Pdx} 1)$ is a transcription factor required for pancreatic development and maintenance of beta-cell function. Our goal was to test whether $P d x 1$ expression in pancreatic ducts is necessary for the islet neogenesis seen during the first postnatal month. With crossing CAllCre and $\mathrm{Pdx} 1^{\mathrm{fl} / \mathrm{fl}}$ mice, $\mathrm{Pdx} 1$ was efficiently excised postnatally from pancreatic duct cells starting around birth. While in the first 2 days after birth bigenic mice had normal fed blood glucose values, they were moderately hyperglycemic (4 wk: $271 \pm 11$ vs control $176 \pm 6 \mathrm{mg} / \mathrm{dl}$ ) until 8-10 weeks. At 10 weeks, CAllCre;Pdx $1^{\mathrm{fl} / \mathrm{fl}}$ mice had near normal morning fed blood glucose $(181 \pm 10 \mathrm{mg} / \mathrm{dl}$ vs $152 \pm 4 \mathrm{mg} / \mathrm{dll})$. However, upon acute glucose challenge these mice had severely impaired glucose tolerance and reduced plasma insulin levels; insulin tolerance did not differ from control littermates. At 4 and 10 weeks, neither islet nor beta cell mass differed from littermate controls. Within the same duct-specific Pdx1 deficient pancreas, there was a mixed population of islets with $\mathrm{Pdx} 1$ and MafA protein expression being normal in some and severely diminished in others. Many insulin ${ }^{\text {positive }}$ cells coexpressed PP/PYY/NPY, which is characteristic of embryonic and neonatal pancreas and not seen in adult islets even from chronic hyperglycemic animals. There were even MafBpositive Glucagon ${ }^{\text {negative }}$ cells, also reflecting an early stage of beta cell development. Insulin secretion of isolated islets from 10 wk old duct-specific Pdx1 deficient mice was severely impaired in response to glucose. This lack of glucose responsiveness is consistent with the observed impaired glucose tolerance seen in these mice and is characteristic of immature beta cells. In conclusion, $\mathrm{Pdx} 1$ is not required for the formation of new beta cells by neogenesis from ducts in the postnatal period, but the resultant $\mathrm{Pdx} 1^{\text {negative }}$ insulin ${ }^{\text {positive }}$ cells have the functional and morphological characteristics of immature beta cells.

Supported by: JDRF

376-0R

Islet Endothelial Cells Secrete Factors that Enhance Beta Cell Glucose-Stimulated Insulin Secretion

AMY W. LIU, REBECCA L. HULL, Seattle, WA

Islet endothelial cells secrete growth factors that are important for islet endocrine cell development and can stimulate adult beta-cell proliferation. We hypothesize that islet endothelial cells also release paracrine factors that are important for beta-cell secretory function. To test this, conditioned media were generated from immortalized murine islet endothelial cells (MS1) following 48 hours of culture in $5.5 \mathrm{mM}$ glucose. Glucose levels in conditioned media were determined and matched to that of non-conditioned media (not previously exposed to cells) prior to the performance of subsequent studies, so as to avoid the potential confounding effect of different glucose concentrations. Islets from C57BL/6J mice were then cultured for 48 hours in conditioned or non-conditioned media. Glucose-stimulated insulin release, insulin content and mRNA levels of insulin and Pdx1 were measured ( $\mathrm{n}=2-4$ per condition). Culture of islets in media previously exposed to MS-1 cells resulted in no change in basal insulin release $(24.8 \pm 4.0$ vs. $25.6 \pm 3.0$ $\mathrm{pM} / 5$ islets), but a significant increase in glucose-stimulated insulin release compared to non-conditioned media $(88.2 \pm 11.7$ vs. $135.7 \pm 11.5 \mathrm{pM} / 5$ islets, $p<0.05)$. Insulin content tended to increase $(7.9 \pm 2.5$ vs. $16.0 \pm 2.8 \mathrm{nM} / 5$ is lets), as did insulin and Pdx1 mRNA levels (1.2 \pm 0.04 and $1.3 \pm 0.1$ fold increase above non-conditioned media, $p=0.06$ and $p=0.10$ respectively). Our data suggest that islet endothelial cells secrete factors that enhance glucosestimulated insulin release and may also upregulate key beta-cell genes such as insulin and Pdx1. The mechanism(s) by which this occurs, and whether this beneficial effect is diminished under conditions of endothelial dysfunction as seen in diabetes, needs to be determined.

Supported by: NIH (R01DK088082)
377-0R

Pancreatic Parasympathectomy Impacts Hypothalamic and $\beta$-Cell Adaptation to a High Fat Diet MINA PESHAVARIA, AIDA HABIBOVIC, KYLA LAROCK, BASANTHI SATISH, THOMAS JETTON, Colchester, VT

The vagus nerves mediate homeostatic processes such as glucose tolerance, compensatory $\beta$-cell proliferation, and the regulation of visceral inflammation. However, their potential roles in adaptive $\beta$-cell and hypothalamic responses to diet-induced obesity (DIO) have not been adequately explored. In B6 mice, weight gain, mild hyperglycemia, and hyperinsulinemia are apparent by one week on a high-fat diet (HFD). Recent mouse DIO studies demonstrate that hypothalamic inflammation (HI) precedes weight gain suggesting the primacy of the hypothalamus in early adaptive processes in response to nutrient stress. To examine whether $\beta$-cell adaptation and $\mathrm{HI}$ in response to a HFD are dependent on intact vagus nerves to the celiac plexus and pancreas, we subjected mice to a bilateral celiac vagus transection (CVX) or sham surgery, followed by either a HFD or control chow one week later through 5 weeks. At week 1 in CVX-chow mice, hypothalamic tnf-a expression was increased, whereas islet inflammatory markers did not change. By 2 weeks, tnf-a expression was markedly decreased in the CVXchow group that normalized to that of the sham-chow group by 5 weeks. Although CVX surgery did not significantly alter weight gain or worsen glucose or insulin tolerance, both fed and fasting glucose concentrations were elevated in CVX-HFD mice compared to the other groups. Interestingly, the CVX-HFD group exhibited a 2-fold increase over sham-HFD mice in tnf-a expression at 2 weeks, though no further increase was observed at 5 weeks. Thus CVX appears to have led to a premature enhancement of HI. Although early $\beta$-cell functional compensation due to a high-energy diet is moderately impacted by a selective parasympathectomy, CVX significantly affects $\mathrm{HI}$ differentially depending on diet. These data collectively point to a complex integration of the hypothalamus and vagus nerves of the autonomic nervous system with several tissues to maintain energy homeostasis in the context of nutrient overabundance and obesity development. 